\title{
雨 \\ 흘 \\ Die Nutzung \\ von Evaluationen \\ in den Schweizer \\ Parlamenten
}

Daniela Eberli 
Daniela Eberli Die Nutzung von Evaluationen in den Schweizer Parlamenten 

Daniela Eberli

\section{Die Nutzung
von Evaluationen
in den Schweizer \\ Die Nutzung
von Evaluationen
in den Schweizer \\ Die Nutzung
von Evaluationen
in den Schweizer Parlamenten}

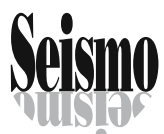


Die vorliegende Arbeit wurde von der Philosophischen Fakultät der Universität Zürich im Herbstsemester 2017 auf Antrag der Promotionskommission, Prof. Dr. Thomas Widmer (hauptverantwortliche Betreuungsperson),

Prof. Dr. Fritz Sager und Prof. Dr. Daniel Kübler, als Dissertation angenommen.

Publiziert mit Unterstützung des Schweizerischen Nationalfonds zur Förderung der wissenschaftlichen Forschung.

Texte (C) Die Autorin 2019

Publiziert von

Seismo Verlag AG

Zähringerstrasse 26, CH-8001 Zürich

E-Mail: buch@seismoverlag.ch

http ://www.seismoverlag.ch

ISBN 978-3-03777-205-8 (Print)

ISBN 978-3-03777-726-8 (PDF)

DOI 10.33058/seismo.30726

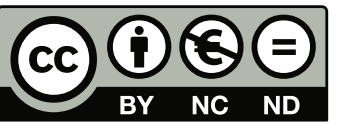

Dieses Werk ist lizenziert unter einer Creative

Commons Namensnennung - Nicht kommerziell

- Keine Bearbeitungen (CC BY-NC-ND 4.0)

BY NC ND

International Lizenz

Layout und Gestaltung: Fabian Elsener, Zürich

Umschlag: Wessinger und Peng GmbH 


\section{Inhalt}

$\begin{array}{ll}\text { Tabellen } & 7\end{array}$

Abbildungen 9

Abkürzungen $\quad 11$

$\begin{array}{ll}\text { Vorwort } & 13\end{array}$

1 Einleitung $\quad 15$

2 Ausgangspunkt: Nutzung von systematisch generiertem Wissen 23 in der Politik

2.1 Verständnis und Konzepte von systematisch generiertem Wissen und dessen Nutzung

2.2 Erklärung der Nutzung von systematisch generiertem Wissen 29

2.3 Besonderheiten des parlamentarischen Kontexts 34

2.4 Besonderheiten des schweizerischen Kontexts 41

2.5 Zwischenfazit: Folgerungen aus der bisherigen Forschung 49

3 Theoretischer Rahmen: Evaluationsnutzung im Parlament 51 und ihre Erklärungsfaktoren

3.1 Evaluationsnutzung: Analytisch und politisch 51

3.2 Individuelle Erklärungsfaktoren der Evaluationsnutzung 54

3.3 Kontextuelle Erklärungsfaktoren der Evaluationsnutzung 61

$4 \quad$ Analyse der individuellen Erklärungsfaktoren der Evaluationsnutzung $\quad 73$

4.1 Grundlage: Befragung der schweizerischen Parlamentsmitglieder $\quad 73$

4.2 Beschreibung der Evaluationsnutzung und der individuellen 77 Erklärungsfaktoren

4.3 Quantitative Analyse der individuellen Erklärungsfaktoren 83

4.4 Diskussion der Ergebnisse der quantitativen Analyse 106

5 Analyse der kontextuellen Erklärungsfaktoren der Evaluationsnutzung $\quad 114$

5.1 Vorgehen: Fallstudien von Gesetzgebungsprozessen 114

5.2 Verbrauchsabhängige Heiz- und Warmwasserkosten- 127 abrechnung (VHKA)

5.3 Steuersystem für energieeffiziente Fahrzeuge 148

$\begin{array}{ll}5.4 \text { Schulsozialarbeit } & 175\end{array}$

5.5 Basis- respektive Grundstufe 198

5.6 Vergleich und Diskussion der Beobachtungen in den Fallstudien 228 
6 Synthese und Diskussion der Ergebnisse 253

6.1 Analytische und politische Evaluationsnutzung, 254 meist durch Kommissionsmitglieder

6.2 Erfahrung, Professionalisierung und Konfliktivität bedeutsam 256 für Nutzung

6.3 Diskussion des Forschungsansatzes und Tragweite der Ergebnisse 259

7 Ausblick: Was ist die Rolle der Evaluation in den Parlamenten? 263

8 Literatur 266 


\section{Tabellen}

Tabelle 1: Erklärungsfaktoren der Evaluationsnutzung aus der Literatur

Tabelle 2: Beteiligung an der Befragung nach Parlament

Tabelle 3: Übersicht und Fragetext der Nutzungsarten als abhängige Variable

Tabelle 4: Zusammenhang der Nutzungsarten

Tabelle 5: Messung der individuellen Erklärungsfaktoren

Tabelle 6: Zusammenhänge Nutzungsarten und individuelle Erklärungsfaktoren

Tabelle 7: Resultate der Regressionsmodelle zur instrumentellen Nutzung

Tabelle 8: Resultate der Regressionsmodelle zur konzeptionellen Nutzung

Tabelle 9: Resultate der Regressionsmodelle zur legitimierenden Nutzung

Tabelle 10: Resultate der Regressionsmodelle zur persuasiven Nutzung

Tabelle 11: Eigenschaften der Kantone Bern und Zürich sowie deren Parlamente

Tabelle 12: Phasen eines Gesetzgebungsprozesses und dazugehörige

Dokumentengrundlage

Tabelle 13: Evaluationsergebnisse zur Wirkung der VHKA

Tabelle 14: VHKA im Kantonsrat Zürich - Übersicht der Nutzung

Tabelle 15: VHKA im Berner Grossen Rat - Übersicht der Nutzung

Tabelle 16: Evaluationsergebnisse zur Wirkung des Steuersystems für energieeffiziente Fahrzeuge

Tabelle 17: Bonussystem im Kantonsrat Zürich - politische Nutzung durch Parlament und Exekutive

Tabelle 18: Bonus-Malus-System im Berner Grossen Rat - politische Nutzung durch Parlament und Exekutive

Tabelle 19: Bonussystem im Kantonsrat Zürich - Übersicht der Nutzung

Tabelle 20: Bonus-Malus-System im Berner Grossen Rat - Übersicht der Nutzung 174

Tabelle 21: Evaluationsergebnisse zur Wirkung der Schulsozialarbeit 180

Tabelle 22: Schulsozialarbeit im Berner Grossen Rat - politische Nutzung durch Parlament und Exekutive

Tabelle 23: Schulsozialarbeit im Kantonsrat Zürich - Übersicht der Nutzung

Tabelle 24: Schulsozialarbeit im Berner Grossen Rat - Übersicht der Nutzung 
Tabelle 25: Evaluationsergebnisse zur Wirkung der Basis- respektive Grundstufe 202

Tabelle 26: Grundstufe im Kantonsrat Zürich - politische Nutzung durch Parlament und Exekutive

Tabelle 27: Basisstufe im Berner Grossen Rat - politische Nutzung durch Parlament und Exekutive

Tabelle 28: Grundstufe im Kantonsrat Zürich - Übersicht der Nutzung

Tabelle 29: Basisstufe im Berner Grossen Rat - Übersicht der Nutzung

Tabelle 30: Analytische Nutzung - Einordnung der Beobachtungen aus den Fallstudien

Tabelle 31: Politische Nutzung - Einordnung der Beobachtungen aus den Fallstudien

Tabelle 32: Übersicht der Beobachtungen der Nutzung aus den Fallstudien

Tabelle 33: Verfügbarkeit der Evaluationen und identifizierbare, genutzte Quellen 246

Tabelle 34: Häufigkeit der Nutzung und Übereinstimmung der Evaluationsergebnisse 


\section{Abbildungen}

Abbildung 1: Theoretischer Rahmen - Evaluationsnutzung und ihre Erklärungsfaktoren

Abbildung 2: Arten und Unterscheidung der analytischen und politischen Nutzung

Abbildung 3: Häufigkeit der Nutzung für Budgetentscheide, Aufsicht und Gesetzgebung

Abbildung 4: Häufigkeit der Nutzungsarten für die Gesetzgebung

Abbildung 5: Durchschnittliche Nutzung in den einzelnen Schweizer Parlamenten

Abbildung 6: Wahrscheinlichkeiten der Nutzung nach Erfahrung

Abbildung 7: Unterschiede der persuasiven Nutzung nach Parteizugehörigkeit

Abbildung 8: Unterschiede der legitimierenden Nutzung nach Parteizugehörigkeit (Differenz zu SP)

Abbildung 9: Wahrscheinlichkeiten der Nutzung nach Professionalisierung

Abbildung 10: Wahrscheinlichkeiten der persuasiven Nutzung nach Erfahrung und Professionalisierung

Abbildung 11: Unterschiede der konzeptionellen Nutzung nach Bildungsgrad

Abbildung 12: Unterschiede der legitimierenden Nutzung nach Geschlecht

Abbildung 13: Unterschiede der legitimierenden Nutzung nach Kandidatur und Alter

Abbildung 14: Übersicht der ausgewählten Gesetzgebungsprozesse nach Auswahlkriterien

Abbildung 15: Wirkungsmodell der VHKA

Abbildung 16: Zeitliche Verfügbarkeit der Evaluationen zur VHKA im Kantonsrat Zürich

Abbildung 17: Zeitliche Verfügbarkeit der Evaluationen zur VHKA im Berner Grossen Rat

Abbildung 18: Wirkungsmodell des Steuersystems für energieeffiziente Fahrzeuge 150

Abbildung 19: Zeitliche Verfügbarkeit der Evaluationen zum Bonussystem im Kantonsrat Zürich

Abbildung 20: Zeitliche Verfügbarkeit der Evaluationen zum Bonus-Malus-System im Berner Grossen Rat

Abbildung 21: Wirkungsmodell der Schulsozialarbeit

Abbildung 22: Zeitliche Verfügbarkeit der Evaluationen zur Schulsozialarbeit 
Abbildung 23: Zeitliche Verfügbarkeit der Evaluationen zur Schulsozialarbeit 193 im Berner Grossen Rat

Abbildung 24: Wirkungsmodell der Basis- respektive Grundstufe

Abbildung 25: Zeitliche Verfügbarkeit der Evaluationen zur Grundstufe im Kantonsrat Zürich

Abbildung 26: Zeitliche Verfügbarkeit der Evaluationen zur Basisstufe im Berner Grossen Rat

Abbildung 27: Häufigkeit der Nutzung nach Wissenschaftsnähe 236

Abbildung 28: Häufigkeit der Nutzung nach Grad der Konfliktivität 


\section{Abkürzungen}

\begin{tabular}{|c|c|}
\hline $\mathrm{ACF}$ & Advocacy Coalition Framework \\
\hline AGEVAL & interdepartementale Arbeitsgruppe Gesetzesevaluation \\
\hline AIC & Aikaikes Informationskriterium \\
\hline AJB & Amt für Jugend und Berufsberatung \\
\hline AL & Alternative Liste \\
\hline asa & Vereinigung der Strassenverkehrsämter \\
\hline auto-schweiz & Vereinigung Schweizer Automobil-Importeure \\
\hline AWEL & Amt für Abfall, Wasser, Energie und Luft \\
\hline BDP & Bürgerlich-Demokratische Partei \\
\hline BFE & Bundesamt für Energie \\
\hline $\mathrm{BFH}$ & Berner Fachhochschule \\
\hline BIC & Bayessches Informationskriterium \\
\hline BSFG & Gesetz über die Besteuerung der Strassenfahrzeuge \\
\hline BVE & Bau-, Energie- und Verkehrsdirektion \\
\hline CERN & European Organization for Nuclear Research \\
\hline CVP & Christlichdemokratische Volkspartei \\
\hline EBP & Evidenzbasierte Politikgestaltung \\
\hline EDK & Schweizerische Konferenz der kantonalen Erziehungsdirektoren \\
\hline EDK-Ost & $\begin{array}{l}\text { Erziehungsdirektoren-Konferenz der Ostschweizer Kantone und } \\
\text { des Fürstentums Liechtenstein }\end{array}$ \\
\hline EDK-Ost 4bis8 & $\begin{array}{l}\text { Erziehung und Bildung in Kindergarten und Unterstufe } \\
\text { im Rahmen der EDK-Ost }\end{array}$ \\
\hline EDU & Eidgenössisch-Demokratische Union \\
\hline EnDK & Konferenz Kantonaler Energiedirektoren \\
\hline ERZ & Erziehungsdirektion des Kantons Bern \\
\hline ETHZ & Eidgenössisch Technische Hochschule Zürich \\
\hline EVP & Evangelische Volkspartei \\
\hline FDP & FDP.Die Liberalen \\
\hline $\mathrm{FiG}$ & $\begin{array}{l}\text { Gesetz über die Finanzierung der Jugendhilfe und } \\
\text { Sonderschulung }\end{array}$ \\
\hline GEAK & Gebäudeenergieausweis der Kantone \\
\hline GOLM & generalisiertes ordinales logistisches Regressionsmodell \\
\hline
\end{tabular}




$\begin{array}{ll}\text { HarmoS } & \begin{array}{l}\text { Interkantonale Vereinbarung über die Harmonisierung } \\ \text { der obligatorischen Schule }\end{array} \\ \text { HEV } & \text { Hauseigentümerverband des Kantons Bern } \\ \text { KBIK } & \text { Kommission für Bildung und Kultur } \\ \text { KEnG } & \text { Kantonales Energiegesetz } \\ \text { KEVU } & \text { Kommission für Energie, Verkehr und Umwelt } \\ \text { KGV } & \text { Kantonaler Gewerbeverband Zürich } \\ \text { KMU } & \text { Kleine und mittlere Unternehmen } \\ \text { MuKEn } & \text { Mustervorschriften der Kantone im Energiebereich } \\ \text { NPM } & \text { New Public Management } \\ \text { NW EDK } & \text { Nordwestschweizerische Erziehungsdirektorenkonferenz } \\ \text { OLM } & \text { ordinales logistisches Regressionsmodell } \\ \text { PM } & \text { Parlamentsmitglied } \\ \text { POM } & \text { Polizei- und Militärdirektion } \\ \text { PPOM } & \text { Partial Proportional Odds Model } \\ \text { PVK } & \text { Parlamentarische Verwaltungskontrolle } \\ \text { REVOS 2012 } & \text { Revision Volksschulgesetz 2012 } \\ \text { RFA } & \text { Regulierungsfolgenabschätzung } \\ \text { SP } & \text { Sozialdemokratische Partei } \\ \text { SSA } & \text { Schulsozialarbeit } \\ \text { SVP } & \text { Schweizerische Volkspartei } \\ \text { SVSA } & \text { Strassenverkehrs- und Schifffahrtsamt des Kantons Bern } \\ \text { TCS } & \text { Touring-Club Schweiz } \\ \text { UVEK } & \text { Eidgenössisches Departement für Umwelt, Verkehr, Energie } \\ \text { Verw. } & \text { und Kommunikation } \\ \text { VHKA } & \text { Verwaltung } \\ \text { VIF } & \text { Verbrauchsabhängige Heiz- und Warmwasserkostenabrechnung } \\ \text { VSG-BE } & \text { Varianzinflationsfaktor } \\ \text { VSG-ZH } & \text { Volksschulgesetz des Kantons Bern } \\ \text { WAK } & \text { Volksschulgesetz des Kantons Zürich } \\ & \text { Kommission für Wirtschaft und Abgaben } \\ & \end{array}$




\section{Vorwort}

Das Thema der Evaluation begleitet mich bereits seit einiger Zeit. Gegen Ende des Bachelorstudiums wurde mir klar, dass mich solch eine angewandte Tätigkeit ausserordentlich interessiert. Mir schien nichts naheliegender, als mit den analytischen Werkzeugen, die sich mir allmählich erschlossen, einen praktischen Beitrag an die Politik zu leisten. Die Idee, Politik von ihrem Konzept über ihre Realisierung bis hin zu ihren Wirkungen zu untersuchen und damit wirksame, zielgerichtete Massnahmen zu identifizieren, finde ich auch heute noch spannend und ist gleichzeitig grundlegend für die vorliegende Untersuchung.

Nicht nur in Bezug auf das Thema, auch in vielerlei Hinsicht sonst hatte ich Glück mit meinem Dissertationsvorhaben. Zuerst möchte ich daher meinem Hauptbetreuer Prof. Dr. Thomas Widmer danken. Ich schätze es sehr, dass ich mich jederzeit mit ihm austauschen konnte. Er hat sich viel Zeit genommen, meine Arbeit zu begleiten und mich mit seinen kritischen Kommentaren herausgefordert und motiviert. Wertvolle Rückmeldungen habe ich auch von Prof. Dr. Fritz Sager erhalten. Ich bin sehr dankbar für die Möglichkeiten zur Präsentation meiner Arbeit an Konferenzen und in Publikationen, die sich mir auch aufgrund seiner Bemühungen ergaben. Seine Unterstützung ist für mich von Bedeutung, da er es war, der mich zur Evaluation brachte: In seiner Vorlesung hatte er angeregt, ein Praktikum bei einem Evaluationsbüro zu absolvieren. Wie Thomas Widmer und Fritz Sager hat mich auch Prof. Dr. Daniel Kübler seit dem ersten Jahr als Mitglied der Prüfungskommission unterstützt. Zumal er Arbeiten betreut hat, die für mich von besonderer Relevanz sind, freue ich mich sehr über sein Mitwirken.

Mit Glück verbunden war auch, dass ich meine Arbeit im Rahmen des vom Schweizerischen Nationalfonds finanzierten Projekts "Policy Evaluation in the Swiss Political System - Roots and Fruits" schreiben konnte (Projektnummer 141893). Die alljährlichen Workshops waren für mich positiver, notwendiger Druck und anregender Austausch zugleich. Die gehaltvollen Kommentare der beteiligten Doktorandinnen und Doktoranden sowie der Projektleiterinnen und Projektleiter waren sehr hilfreich. Besonderer Dank gebührt dem Projektteam am Institut für Politikwissenschaft der Universität Zürich (IPZ), dem neben Thomas Widmer auch Kathrin Frey und Pirmin Bundi angehörten. Kathrins Hinweise und ihre Arbeiten waren für mich äusserst wertvoll. Mit Pirmin konnte ich erfreulicherweise viele Ereignisse zusammen erleben. Seine Kommentare zur vorliegenden Arbeit und anderen Papieren haben mein Dissertationsvorhaben weitergebracht. Neben dem Dissertationsprojekt unterstützte der Schweizerische Nationalfonds auch meinen Auslandsaufenthalt an der Université Laval in der Stadt Québec. Prof. Dr. Steve Jacob hat mich dort sehr freundlich empfangen. In den gemeinsamen Mittagessen gab er mir Einblick in den kanadischen (Forschungs-)Alltag und teilte mit mir seine Sichtweisen zur Evaluation, was ich sehr geschätzt habe.

Glücklich bin ich auch über die Unterstützung meines beruflichen Umfelds. Aktuelle sowie ehemalige IPZlerinnen und IPZler haben an meiner Arbeit und den oft kleinen Fortschritten Anteil genommen. Mit Pirmin, Christine und zuletzt Thomi habe ich 
sehr gerne das Büro geteilt. Dank gebührt abseits des IPZ Martina Brägger und Charles Landert. In ihrem Büro konnte ich erstmals einen Einblick in die Evaluation erhalten und danach bei weiteren Projekten in sehr spannenden Themenfeldern mitwirken. Ihren Zuspruch, ihre Unterstützung und die notwendige Flexibilität schätze ich sehr.

Neben der finanziellen, fachlichen und beruflichen Unterstützung durfte ich auch auf mein privates Umfeld zählen. Substanziell zur vorliegenden Arbeit beigetragen hat mein Bruder Andreas. Er hat einen Grossteil der Arbeit gelesen und dabei auf inhaltliche Unstimmigkeiten sowie auf kleinere oder grössere sprachliche Vergehen hingewiesen. Auch Lineo und die Konstanzer Kathrin haben mir sehr hilfreiche Inputs gegeben. Meinen Eltern danke ich für die Unterstützung während der Doktorarbeit und in anderen Lebenslagen. Ein herzlicher Dank geht schliesslich an Michael und allen meinen Freundinnen und Freunden. Sie haben mich abgelenkt, aufgemuntert und mich mit ihrer unbeirrten Zuversicht angespornt.

Verschiedene Personen tragen also zur vorliegenden Arbeit bei, zu guter Letzt sei aber den Protagonistinnen und Protagonisten der folgenden Seiten gedankt. In der Befragung haben die Parlamentsmitglieder ihre Sicht auf die Evaluation mit unserem Team geteilt und uns eine umfassende Datengrundlage ermöglicht. Für die Interviews haben mich die Kantonsrätinnen und Kantonsräte, Grossrätinnen und Grossräte in ihre Büros oder gar Wohnzimmer eingeladen. Ihre Ausführungen sind entscheidend für das Verständnis der studierten Gesetzgebungsprozesse und bereichernd für die Lektüre. 


\section{Einleitung}

Eignet sich eine politische Massnahme zur Lösung eines Problems? Erreicht sie die gesteckten Ziele? Welche Wirkungen entfaltet sie? Wie ist ihr Wert und Nutzen im Vergleich mit den aufgewendeten Ressourcen zu beurteilen? Solche, für parlamentarische Tätigkeiten relevante Fragen sind Gegenstand von Evaluationen. Basierend auf wissenschaftlichen Methoden beurteilen Evaluationen die Konzipierung, Umsetzung oder Wirkungen einer Politik. Dies macht sie zu einem vielfältig nutzbaren Instrument für Parlamentsmitglieder (vgl. Bättig und Schwab 2015; Speer et al. 2015). Dank Evaluationen können Parlamentsmitglieder gesetzliche Lücken erkennen und diese schliessen. Indem Evaluationen ebenso zur Identifikation von Mängeln oder Verbesserungspotenzial bestehender Massnahmen beitragen, können Parlamentsmitglieder sie nicht nur bei Neuerlassen, sondern auch bei Gesetzesrevisionen berücksichtigen. Mit diesen Informationen über die Umsetzung einer Politik eignen sich Evaluationen zugleich für die parlamentarische Aufsicht über die Tätigkeiten der Regierung und Verwaltung. Letztlich erlauben Evaluationen eine wirkungsorientierte Budgetierung der öffentlichen Finanzen. Folglich unterstützen sie Parlamentsmitglieder bei der Erfüllung ihrer zentralen Aufgaben der Gesetzgebung und Aufsicht sowie im Rahmen ihrer Budgetkompetenzen (Janett 2004). Eine solche Unterstützung scheint umso bedeutsamer angesichts der komplexen, internationalisierten, von zahlreichen Beteiligten beeinflussten Problemlagen, mit denen Parlamentsmitglieder heute konfrontiert sind (vgl. Janett 2009).

Evaluationen können nicht nur als vielfältig einsetzbares, politiknahes Instrument zur Erfüllung der zentralen parlamentarischen Aufgaben beitragen, sondern Parlamentsmitglieder müssen sich durchaus der Forderung stellen, Evaluationen zu nutzen. Parlamentsmitglieder handeln zwar als von den Stimmberechtigten gewählte Volksvertreterinnen und Volksvertreter. Diese Input-Legitimation, auch «Herrschaft durch das Volk» genannt, rechtfertigt jedoch Entscheide heutiger Parlamente als moderne Gesetzgeber nicht ausschliesslich (Scharpf 1975; 2000, 16-23; 2006; vgl. Sager et al. 2017). Entscheide der Parlamente, und generell staatliches Handeln, werden vielmehr auch an den daraus resultierenden Ergebnissen gemessen. Um diese Output-Legitimation oder «Herrschaft für das Volk» zu erreichen, müssen Fragen der Wirkung in die parlamentarischen Tätigkeiten einbezogen werden (vgl. dazu auch Widmer 2009, 358f.). Diese Leistungsund Wirkungsorientierung ist zentraler Bestandteil von Modernisierungsbestrebungen wie New Public Management oder der evidenzbasierten Politikgestaltung, die vor der Jahrtausendwende aufkamen und mit ihrem umfassenden Charakter auch die Parlamente betreffen (vgl. Van Dooren 2008; Vedung 2010). "What counts is what works" als Leitsatz der evidenzbasierten Politikgestaltung fasst diese Forderung nach der Wirkung als zentraler Orientierungspunkt für politische Entscheide prägnant zusammen (vgl. Davies et al. 2000b, 1; Pawson 2006, 20; Sanderson 2002, 3).

Trotz dieser offenkundigen Relevanz der Evaluation wird in der Literatur hinterfragt, welche Rolle sie im parlamentarischen Alltag einnimmt. Wenn auch Evaluationen nützlich erscheinen, dürften verschiedene Gepflogenheiten und Logiken des parlamen- 
tarischen Handelns die Bedeutsamkeit von Evaluationen einschränken (vgl. Hird 2009; Pollitt 2006; Weiss 1987, 1989; Whiteman 1995; Zwaan et al. 2016). Balthasar (2005, 66; vgl. auch 2009) bemerkt dazu etwa:

Ob die implementierten Massnahmen die Ziele erreichen, ist für Politikerinnen und Politiker nicht das Mass aller Dinge. Politikerinnen und Politiker müssen nach vorne schauen. Sie müssen Ideen entwickeln, um zu zeigen, dass sie die Probleme ernst nehmen, welche die Presse und die Wählerinnen und Wähler heute beschäftigen.

Eine andere, wichtige Einschränkung der Rolle der Evaluation ist in der Aufgabe der Parlamente selbst begründet. Parlamentsmitglieder sind demokratisch gewählte Volksvertreterinnen und Volksvertreter. Informationen aus Evaluationen bilden daher nur einen Gesichtspunkt, den Parlamentsmitglieder in ihre Entscheide einbeziehen können. Ideologien, Interessen oder andere Informationen etwa der Wählerinnen und Wähler sind ebenfalls legitime Grundlagen für Entscheide (vgl. Guston et al. 1997; Schwab 2005; Webber 1987; Weiss 1987, 1989, 1999). Es kann deshalb auch argumentiert werden, dass es einer Gesamtsicht aller Beteiligten und Betroffenen bedarf statt einer alleinigen Sicht des Fachwissens aus Evaluationen, um letztlich eine erfolgreiche Politik im Sinne des Gemeinwohls zu gestalten (Albaek 1995, 94; Weiss 1990, 173). Diese Erläuterungen zeigen folglich ein Spannungsverhältnis zwischen Wissenschaft und Politik auf, dem Parlamente als demokratisch gewählte Volksvertretungen besonders ausgesetzt sind. Systematisch generiertes Wissen stellt mittels der angestrebten Objektivität und Rationalität gewissermassen das Gegenstück zur ideologischen, interessenbasierten Politik dar (vgl. Cairney 2016; Head 2010). Gleichzeitig ist gerade mit der Forderung, Entscheide vorrangig auf Evaluationen oder anderem systematisch generierten Wissen und weniger auf Ideologien oder Interessen zu stützen, die Befürchtung verbunden, die demokratisch legitimierten parlamentarischen Entscheide zu technokratisieren (vgl. Whiteman 1995).

Übereinstimmend mit dieser kritisch diskutierten praktischen Bedeutung wird in der Literatur eine einseitige Verwendung von Evaluationen in den Parlamenten erwartet. Anstatt Entscheide rational auf Evaluationen gestützt zu treffen, sollten Parlamentsmitglieder demgemäss Evaluationen für ihre eigenen Zwecke instrumentalisieren und sie als politisches, argumentatives Mittel einsetzen, um Überzeugungsarbeit zu leisten, der eigenen Position Legitimität zu verleihen oder Entscheidungsprozesse zu bremsen (vgl. Balthasar 2005; Freiburghaus und Zimmermann 1985; Jenkins-Smith und Sabatier 1993, 53f.; Weiss 1989; Whiteman 1985b). Diesem Argument folgend gehört es im politischen Umfeld der Parlamente für alle Seiten dazu, die eigenen Ansichten mit Evaluationen zu untermauern und dabei zu übertreiben, zu verfälschen oder über Grenzen der Evaluationen hinwegzusehen (Boswell 2009, 98 f.; Frey 2012, 81, 89; Weiss 1990, 180).

$\mathrm{Ob}$ und wie Parlamentsmitglieder Evaluationen oder anderes systematisch generiertes Wissen tatsächlich verwenden, wurde bisher kaum empirisch untersucht. Die Forschungsbeiträge deuten generell darauf hin, dass Parlamentsmitglieder durchaus positiv gegenüber Evaluationen eingestellt sind (vgl. Boyer und Langbein 1991; Hird 2005b, 
2009). Eine systematische Verwendung lässt sich aber nicht beobachten, vielmehr variiert sie je nach Gegenstand des parlamentarischen Entscheidungsprozesses und nach Phase dieses Prozesses selbst (vgl. Florio et al. 1979; Frey 2012; Mooney 1991a, 1993; Whiteman 1985a, 1985b). Parlamentarische Kommissionen, die Geschäfte vorberaten und sich dazu vertieft mit ihnen auseinandersetzen, spielen eine wesentliche Rolle für die Evaluationsnutzung (Weiss 1987, 1989). Vereinzelt beschäftigen sich gerade Kommissionsmitglieder differenziert mit Evaluationen oder anderem systematisch generierten Wissen (vgl. Frey 2012; Mooney 1991b, 2012; Whiteman 1995). Solches Wissen findet zudem erwartungsgemäss Verwendung als politisches Mittel im Rahmen von Debatten, Hearings oder der Diskussion parlamentarischer Fragen (Esterling 2007, 2011; Parlamentarische Verwaltungskontrolle 2005; Shulock 1998, 1999; Whiteman 1995; vgl. Bundi 2016, 2018a; Speer et al. 2015; Zwaan et al. 2016). Gemäss den existierenden Forschungsbeiträgen trifft folglich weder das Ideal evidenzbasierter Entscheide noch die Erwartung einer einseitigen politischen Instrumentalisierung zu (vgl. Shulock 1998, 1999; Whiteman 1985a, 1995).

Die Forschungsbeiträge, auf denen diese bisherigen Erkenntnisse beruhen, befassen sich nur mit wenigen Parlamenten und fokussieren nicht spezifisch auf Evaluationen. Der Grossteil der bisherigen Forschung konzentriert sich auf die Verwendung von Policy-Analysen im US-Kongress oder in Parlamenten der US-Bundesstaaten (z. B. Shulock 1998; 1999; Whiteman 1985a, 1985b, 1995). Daneben widmen sich wenige Arbeiten der Nutzung von Instrumenten des New Public Management in europäischen oder kanadischen Parlamenten (z. B. Johnson und Talbot 2007; McDavid und Huse 2012). Auch in der Schweiz befassen sich Forschende mit der Bedeutung und Verwendung von Instrumenten des New Public Management im Parlament (z. B. Demaj et al. 2012; Summermatter und Demaj 2012; Widmer und Rüegg 2005). Die schweizerischen Parlamente waren zudem Teilgegenstand von empirischen Untersuchungen zur Verwendung von Evidenz oder Expertise im gesamten Gesetzgebungsprozess (z. B. Blatter et al. 2015; Frey 2012; Himmelsbach 2014). Ferner ist die Rolle der Evaluation in der Bundesversammlung in Erfahrungsberichten aus der Praxis dokumentiert (Bättig und Schwab 2015; Janett 2001, 2003a, 2004, 2009).

Vergleichsweise kaum erforscht sind die Parlamente vor allem angesichts der enormen Aufmerksamkeit, die der übergeordneten Frage der Verwendung und Bedeutung von systematisch generiertem Wissen in der Politik zukommt. Die Untersuchung dieser Frage im Rahmen der so genannten Nutzungsforschung (utilization research) nahm ihren Anfang mit den grossen Reformprogrammen in den USA Mitte der 1960er-Jahre (vgl. Backer 1991; Hense und Widmer 2013). Verschiedene, sich teilweise überlappende Forschungstraditionen wie die Forschung über Evaluation oder die Policy-Forschung widmen sich dieser Frage und entwickelten sich mit dem Aufkommen neuer Konzepte weiter (Radaelli 1995). Im Zentrum der Nutzungsforschung steht aber die Verwendung von systematisch generiertem Wissen durch Entscheidungsträgerinnen und träger, die Programme oder Massnahmen in der Regierung, der Verwaltung oder anderen (staatlichen) Organisationen umsetzen (Boyer und Langbein 1991, 512; Pollitt 2006, 41; Speer et al. 2015, 39). Zudem fokussiert die Nutzungsforschung auf die Verwendung von systema- 
tisch generiertem Wissen als Informations- oder Entscheidungsgrundlage, während die Verwendung zu politischen Zwecken selten diskutiert wird (vgl. Johnson et al. 2009, 388; Nutley et al. 2007).

Die Verwendung von Evaluationen und damit auch deren Untersuchung interessiert besonders, da Evaluationen äusserst politiknahes, handlungsorientiertes Wissen bereitstellen sollen. Evaluation lässt sich allgemein gemäss Scriven $(1991,1)$ wie folgt definieren: "Evaluation is the process of determining the merit or worth or value of things, and evaluations are the products of that process." Dieses Buch befasst sich mit Evaluationen im Sinne von Politikevaluationen, die staatliches Handeln in Form etwa von Programmen oder Massnahmen als Gegenstand (things) haben. Der Prozess, auf dem Evaluationen basieren, ist gekennzeichnet durch eine systematische und transparente Vorgehensweise. Evaluationen beruhen demnach auf wissenschaftlichen Ansätzen, ihre Ergebnisse sind nachprüfbar (Klöti 1997, 40). Der Wert staatlichen Handelns (merit or worth or value) wird in Evaluationen anhand verschiedener Kriterien beurteilt. ${ }^{1}$ Gängige Kriterien stellen etwa die Zweckmässigkeit, Zielerreichung, Wirksamkeit oder Wirtschaftlichkeit dar (Balthasar 2005; Widmer und De Rocchi 2012). Dieses Element der Bewertung, das Evaluationen definitionsgemäss beinhalten, zeichnet sie aus (Alkin und Taut 2003, 3). Da sich Evaluationen mit einem spezifischen Gegenstand befassen und zudem häufig auf Auftragsbasis erstellt werden, liefern sie gezielte, handlungsorientierte Informationen. Evaluationen stehen daher nicht nur in engem Bezug zur Politik, auch zielt ihre Erstellung bereits auf ihre Nutzung ab (vgl. James und Jorgensen 2009; Leviton und Hughes 1981; Weiss 1970; Widmer und De Rocchi 2012).

Im Zentrum von Evaluationen steht stets die Wirkungsweise staatlichen Handelns (Klöti 1997, 46). Wesentlich für Evaluationen sind daher Wirkungsmodelle, die beschreiben, wie eine Politik erwartungsgemäss funktioniert. Sie umfassen die Ressourcen und Prozesse, die damit realisierte Politik sowie die beabsichtigten und unbeabsichtigten Wirkungen bei der Zielgruppe dieser Politik wie auch darüber hinaus (Knoepfel und Bussmann 1997; Sager und Rüefli 2005; WK Kellogg Foundation 2004). Die Analyse der Wirkungsweise staatlicher Massnahmen kann vorausschauend bei der Planung einer Politik, aber auch begleitend oder rückblickend bei ihrer Umsetzung erfolgen. Ebenso existieren verschiedene Ansätze der Evaluation (Beywl und Widmer 2009, 16 f.; Widmer und De Rocchi 2012, 21-31). Im vorliegenden Buch wird folglich von einem breiten Verständnis der Evaluation ausgegangen.

Die Schweiz eignet sich bestens, um die Rolle der Evaluation im Parlament zu untersuchen. Evaluationen haben sich in der Schweiz fest etabliert und finden auf Bundes- sowie Kantonsebene in diversen Bereichen der öffentlichen Politik statt (vgl. Balthasar et al. 2017; Balthasar und Strotz 2017; Frey et al. 2016). International gesehen gilt die Schweiz aktuell als Vorreiterin der Evaluation (Jacob et al. 2015). Dies gilt auch für die schweizerische Bundesversammlung, die mit der Parlamentarischen Verwaltungskontrolle (PVK) über einen eigenen Evaluationsdienst verfügt und gemäss Artikel $170 \mathrm{der}$

1 Für eine Diskussion der Begriffe merit und worth, die hier im deutschen Begriff «Wert» zusammengefasst werden, siehe Lincoln und Guba (1980). 
Bundesverfassung für die Überprüfung der Wirksamkeit der Massnahmen des Bundes sorgt (vgl. Janett 2001, 2004, 2009; Mader 2009; Widmer 2007). Hingegen ist kaum bekannt, welche Rolle die Evaluation in den kantonalen Parlamenten einnimmt (vgl. Horber-Papazian 2006).

Angesichts dieser Überlegungen bezweckt das vorliegende Buch, die Nutzung von Evaluationen in den schweizerischen Parlamenten umfassend zu betrachten. ${ }^{2}$ Dabei orientiert sich die Untersuchung an den folgenden zwei zentralen Fragen:

\section{Wie nutzen die schweizerischen Parlamentsmitglieder Evaluationen?}

Was sind Erklärungsfaktoren für die Nutzung von Evaluationen?

Die erste Frage nach dem Wie zielt auf die unterschiedlichen Formen der Evaluationsnutzung ab. Damit verbunden sind die verschiedenen Zwecke, zu denen Parlamentsmitglieder Evaluationen verwenden können: Informieren sich Parlamentsmitglieder anhand von Evaluationen? Nutzen Parlamentsmitglieder Evaluationen als Grundlagen für ihre Entscheide? Stützen sie sich auf Evaluationen in ihrer Argumentation? Kritisieren sie Evaluationen? Dabei soll davon ausgegangen werden, dass Evaluationsnutzung ein komplexes Phänomen ist, das diverse Formen annehmen kann, die schwierig unterscheidbar sind oder sich überlappen können (vgl. Kirkhart 2000; Mark und Henry 2004; Weiss 1979).

Die zweite Frage ist auf die Erklärung dieser Evaluationsnutzung gerichtet. Dafür werden Eigenschaften des individuellen Parlamentsmitglieds, der Organisation innerhalb eines Parlaments, der Situation eines Gesetzgebungsprozesses sowie der zugrundeliegenden Evaluationen berücksichtigt: Gibt es Unterschiede bei der Verwendung von Evaluationen, die auf die Parteizugehörigkeit zurückgeführt werden können? Haben die individuelle Professionalisierung und die Amtserfahrung der Parlamentsmitglieder einen Einfluss auf die Evaluationsnutzung im schweizerischen Milizsystem? Erfolgt die Verwendung von Evaluationen bei umstrittenen Fragen nur als politisches Mittel oder auch als Entscheidungsgrundlage? Welche Rolle spielt die Aktualität einer Evaluation oder die Ergebnislage der verfügbaren Evaluationen? Bei dieser zweiten Frage interessieren Erklärungsfaktoren, die im parlamentarischen Kontext besonders relevant sein sollten. Die Auswahl dieser Erklärungsfaktoren berücksichtigt zudem die bisherige Forschung zu anderen politischen Entscheidungsträgerinnen und -trägern (vgl. Cousins und Leithwood 1986; Nutley et al. 2007).

Zur empirischen Untersuchung der beiden Fragen werden quantitative und qualitative Methoden kombiniert. Eine umfassende Sicht auf die Verwendung von Evaluationen in den schweizerischen Parlamenten ermöglicht einerseits die quantitative Auswertung einer Befragung der Mitglieder der Kantonsparlamente und der Bundesversammlung. Angesichts kaum existierender empirischer Kenntnisse ist von besonderem Interesse, wie die schweizerischen Parlamentsmitglieder überhaupt zur Evaluation stehen. Eine realis-

2 An dieser Stelle sei darauf hingewiesen, dass dieses Buch naturgemäss Aussagen aus früheren, verwandten Publikationen aufgreift (besonders: Eberli 2018; Eberli und Bundi 2017; Eberli et al. 2014). 
tische Abbildung der Evaluationsnutzung in den Parlamenten erlauben anderseits auf qualitativen Methoden basierende Fallstudien von Gesetzgebungsprozessen. Die Untersuchung konkreter Gesetzgebungsprozesse ist sinnvoll, da die Verwendung von Evaluationen situationsabhängig ist (vgl. Esterling 2004; Frey 2012; Shulha und Cousins 1997; Whiteman 1995). Die Fallstudien fokussieren auf die bisher kaum erforschten Kantonsparlamente. Dies ist vielversprechend, da sich die föderale Struktur der Schweiz für Vergleiche zwischen den Kantonen eignet und die Kantonsparlamente zudem über weitgehende Gestaltungskompetenzen in Bereichen verfügen, in denen auch Evaluationen verbreitet sind, wie beispielsweise die Bildungspolitik (vgl. Balthasar und Rieder 2009; Widmer und De Rocchi 2012). Gegenstand der Untersuchung bilden die Parlamente der beiden grössten Kantone Bern und Zürich. Wie diese beiden Kantone ihre Politik gestalten, kann nicht nur als Vorbild für kleinere Kantone der Schweiz dienen, sondern ist auch von internationaler Relevanz. Mit Hilfe der quantitativen und der qualitativen Auswertung wird also Evaluationsnutzung in diesem Buch gleichzeitig umfassend und vertieft betrachtet.

Der Fokus der Untersuchung liegt auf der parlamentarischen Aufgabe der Gesetzgebung. Diese Aufgabe gehört zum Kern der Tätigkeiten der Parlamente als legislative, das heisst gesetzgebende Gewalt. Dies gilt auch für die Parlamente in der schweizerischen Demokratie, in der die Stimmberechtigten nur über einen vergleichsweise kleinen Bruchteil der Gesetzesentwürfe im Rahmen von Referenden entscheiden (Sciarini 2014). Zudem ist die Verwendung von Evaluationen im Rahmen der Gesetzgebung gewissermassen mit anderen zentralen Aufgaben der Parlamente verbunden. Befassen sich Parlamentsmitglieder etwa anlässlich einer Gesetzesrevision mit der Frage, wie eine Massnahme umgesetzt wird, erhalten sie zugleich einen Einblick in die Tätigkeiten der Regierung und Verwaltung, die Gegenstand ihrer Aufsichtsfunktion sind. Bei der gesetzgeberischen Beratung relevant ist aber nicht nur die inhaltliche Ausgestaltung einer Massnahme, sondern auch deren Finanzierung, welche die Parlamentsmitglieder im Rahmen ihrer Budgetkompetenz beschliessen.

Mit der Untersuchung der Evaluationsnutzung in den Parlamenten am Beispiel der Schweiz leistet dieses Buch einen Beitrag zu mehreren Forschungstraditionen. Es widmet sich den bisher vernachlässigten Parlamentsmitgliedern als Nutzerinnen und Nutzer von systematisch generiertem Wissen und zeigt auf, welche Faktoren im Parlament im Vergleich zu anderen politischen Kontexten bedeutsam sind. Damit bildet dieses Buch eine wertvolle Ergänzung des breiten Fundus an Beiträgen zur Nutzungsforschung. Es trägt zugleich zur Weiterentwicklung der Nutzungsforschung bei, da es differenziert Bezüge zwischen der Nutzung in ihren Formen und den Erklärungsfaktoren herstellt, was in der bisherigen Literatur kaum geschieht (Nutley et al. 2007). Die Forschung über Evaluation in der Schweiz erweitert dieses Buch um eine parlamentsspezifische Perspektive. Zwar ist die schweizerische Forschung zur Verwendung von Evaluationen relativ ausgebaut, sie fokussiert aber grösstenteils auf die Exekutive (Widmer et al. 2016). Forschende befassen sich zudem ausgiebig mit dem Zusammenhang zwischen den Besonderheiten des schweizerischen politischen Systems und der Verbreitung von Evaluationen (vgl. Bussmann 2008; Sager et al. 2017b; Sager und Rissi 2011; Widmer 2007; Widmer und Neu- 
enschwander 2004). Unklar ist aber, wie sich diese Besonderheiten wie die Konsensorientierung, die direkte Demokratie oder das Milizsystem auf die Rolle der Evaluation in den Parlamenten auswirken. Generell erhielten die schweizerischen Parlamente lange Zeit relativ wenig Beachtung in der politikwissenschaftlichen Forschung, gerade die Kantonsparlamente erlangten erst kürzlich besondere Aufmerksamkeit (z. B. Bundi et al. 2017; Feh Widmer 2015; Schmidt 2008; Strebel 2014). Folglich trägt dieses Buch auch zur schweizerischen Parlamentsforschung bei.

Die praktische Relevanz der Frage nach der Nutzung und damit auch dem Nutzen von Evaluationen ist hoch. Angesichts der Ressourcen, die in politische Massnahmen und deren Evaluationen investiert werden, drängt sich konkret die Frage auf, was mit Evaluationen geschieht (vgl. Balthasar 2015). Darüber hinaus greift die Untersuchung der Nutzung von Evaluationen im Parlament, wie bereits erläutert, die übergeordnete Frage nach der Verwendung und der Bedeutung von systematisch generiertem Wissen in der Politik auf. Fragen zum Verhältnis von Wissenschaft und Politik werden bereits lange und eingehend diskutiert (vgl. Albaek 1995). In der heutigen, modernen Informations- und Wissensgesellschaft scheint ihre Relevanz offensichtlich: Welchen Stellenwert hat systematisch generiertes Wissen für demokratisch gewählte Milizpolitikerinnen und Milizpolitiker in einem Zeitalter der Vermessung (staatlicher) Leistungen? Dieses Buch beleuchtet diese Frage und kann Möglichkeiten zu einer Steigerung des Einbezugs von systematisch generiertem Wissen aufzeigen, aber auch Grenzen desselbigen skizzieren. Dies wiederum kann letztlich zur Diskussion anregen, welche Rolle systematisch generiertem Wissen in der Politik zukommen soll.

Das vorliegende Buch befasst sich in insgesamt sieben Kapiteln mit der Evaluationsnutzung durch die schweizerischen Parlamentsmitglieder. Nachdem dieses erste Kapitel in die zugrundeliegenden Fragen einführte, diese abgrenzte und deren Relevanz aufzeigte, ordnet das folgende, zweite Kapitel die Fragen in die bestehende Literatur und Forschung ein. Ausgehend von der übergeordneten Frage, wie politische Entscheidungsträgerinnen und -träger systematisch generiertes Wissen verwenden und wie sich dies erklären lässt, präsentiert es die relevanten Konzepte sowie Erklärungsansätze und fasst die wichtigsten Forschungserkenntnisse zusammen. Dabei setzt es sich mit dem für das Buch massgebenden parlamentarischen und schweizerischen Kontext auseinander. Darauf basierend legt das dritte Kapitel den theoretischen Rahmen der vorliegenden Untersuchung dar. Es erläutert das zugrundeliegende Verständnis der Evaluationsnutzung und ihren Formen und leitet Hypothesen zum Verhältnis zwischen diesen Formen der Evaluationsnutzung und den Erklärungsfaktoren her. Den analytischen Teil eröffnet das vierte Kapitel: Darin werden Zusammenhänge zwischen individuellen Merkmalen der Parlamentsmitglieder und der Evaluationsnutzung mittels der quantitativen Analyse von Daten einer schweizweiten Befragung untersucht. Auf situationsabhängige, kontextuelle Merkmale konzentrieren sich die qualitativen Fallstudien von acht Gesetzgebungsprozessen in den Kantonen Bern und Zürich im fünften Kapitel. Beide Kapitel des analytischen Teils erläutern jeweils das methodische Vorgehen, präsentieren dann die Ergebnisse, die abschliessend zusammengefasst und in Bezug auf die postulierten Hypothesen kritisch diskutiert werden. Zwei weitere, kurze Kapitel schliessen das Buch ab. Während das sechste Kapitel 
die zentralen Befunde beider Untersuchungen zusammenfasst und deren Grenzen diskutiert, leitet das siebte Kapitel Folgerungen für die Frage der Bedeutung von systematisch generiertem Wissen in Parlamenten ab. 


\section{Ausgangspunkt: Nutzung von systematisch generiertem Wissen in der Politik}

Wie nutzen politische Entscheidungsträgerinnen und -träger systematisch generiertes Wissen? Wie lässt sich diese Nutzung erklären? Diese beiden Fragen stecken den grösseren Rahmen der vorliegenden Untersuchung zur Nutzung von Evaluationen in Parlamenten $\mathrm{ab}$ und leiten den folgenden Literatur- und Forschungsüberblick. Zunächst werden dazu Grundlagen zur Nutzung von systematisch generiertem Wissen und deren Erklärung vorgestellt, danach wird der spezifische Kontext der Parlamente und der Schweiz aufgezeigt. Ein abschliessendes Zwischenfazit hält die wichtigsten Punkte in Bezug auf die Nutzung von Evaluationen in den Schweizer Parlamenten fest.

Dieses gesamte Kapitel stützt sich auf verschiedene Forschungsstränge, besonders aber auf die Nutzungsforschung. Die Nutzungsforschung hat ihren Ursprung in den USA. Angeregt wurde sie durch die weitreichenden Reformprogramme in der Bildungs, Gesundheits- und Sozialpolitik Mitte der 1960er-Jahre und die damit verbundenen Fragen zur Wirksamkeit und Steuerung (vgl. Backer 1991; Hense und Widmer 2013). ${ }^{3}$ Zur Nutzungsforschung trägt einerseits die Policy-Forschung zu knowledge utilization sowie anderseits die Forschung über Evaluation ${ }^{4} \mathrm{zu}$ evaluation utilization bei (Hofstetter und Alkin 2003, 204). Die Forschungsbeiträge können jedoch nicht immer klar einer dieser beiden Forschungstraditionen zugeordnet werden.

\subsection{Verständnis und Konzepte von systematisch generiertem Wissen und dessen Nutzung}

Wie nutzen politische Entscheidungsträgerinnen und -träger systematisch generiertes Wissen? Zur Beleuchtung dieser Frage bedarf es zunächst einer Klärung des Verständnisses von systematisch generiertem Wissen und Evaluation. Danach wird das vielfältige Konzept der Nutzung von systematisch generiertem Wissen erläutert. Ein Überblick zum Forschungsstand des Ausmasses der Nutzung schliesst die Ausführungen zu dieser Frage ab.

\subsubsection{Evaluationen und anderes systematisch generiertes Wissen}

Evaluationen können als eine von verschiedenen Formen von systematisch generiertem Wissen betrachtet werden. Grundlegend gemeinsam haben diese Formen, dass sie auf einer systematischen und transparenten Vorgehensweise beruhen. Andere Formen systematisch generierten Wissens sind etwa Forschung, Evidenz oder Expertise respektive

3 Die Nutzungsforschung ist abzugrenzen von der Verwendungsforschung der deutschsprachigen Soziologie der 1980er-Jahre (Beck und Bonß 1985; Hense et al. 2013a, 254).

4 In Anlehnung an Hense et al. (2013b, 9-10) wird der Begriff «Forschung über Evaluation» gewählt, da «Evaluationsforschung» teilweise auch als Synonym für «Evaluation» verwendet wird. 
Fachwissen (verwendete Begriffe in z. B. Boswell 2009; Ledermann 2014, 456; Lundin und Öberg 2014, 27; Nutley et al. 2007; Weible 2008, 615). Das Verständnis dieser Formen ist relativ offen. Forschung bezeichnen etwa Nutley et al. $(2007,21)$ als "any investigation towards increasing the sum of knowledge based on planned and systematic enquiry". Als weitere Formen des systematisch generierten Wissens zählen die Policy-Analyse, Policy-Information oder Performanzinformation (dazu siehe z. B. Pollitt 2006, 39; Sager et al. 2017a, 67-68; Webber 1991; Widmer und De Rocchi 2012). Diese Formen beziehen sich wie die Evaluation stärker auf Politikinhalte und prozesse sowie deren Steuerung. Die Forschenden verwenden jedoch unterschiedliche Definitionen und Bezeichnungen für die Formen systematisch generierten Wissens. ${ }^{5}$ Zudem weisen sie darauf hin, dass die Abgrenzung von systematisch und unsystematisch generiertem Wissen von der Betrachtungsweise der Entscheidungsträgerinnen und -träger abhängt (Boswell 2009, 25; Nutley et al. 2007, 25; Weible 2008, 616).

Mehrere Eigenschaften charakterisieren Evaluationen gleichzeitig als eigenständige Form systematisch generierten Wissens. Evaluationen zeichnen sich vor allem durch den Aspekt der Bewertung aus, der bereits in ihrem Begriff enthalten ist (vgl. Alkin und Taut 2003). Indem sich Evaluationen mit einem Evaluationsgegenstand befassen, beispielsweise ein konkretes Programm, können sie zudem als kontext-spezifisches Wissen betrachtet werden. Folglich ist ihr Anspruch an Generalisierbarkeit verhältnismässig gering (Alkin und Taut 2003; Hofstetter und Alkin 2003, 219). Damit übereinstimmend bemerken Widmer und De Rocchi $(2012,16)$, dass Evaluationen besonders häufig im Auftragsverhältnis entstehen. Schliesslich ist bei Evaluationen, teilweise auch bei der Policy-Analyse, der Nutzungsaspekt zentral. Diese zweckorientierte, normative Prägung beschreiben James und Jorgensen $(2009,142)$ treffend wie folgt: "The findings and recommendations are intended to be useful and used" (Howlett 2009, 154; Nutley und Webb 2000; Sager et al. 2017a, 67-68; Widmer und De Rocchi 2012).

Folglich bilden Evaluationen eine kontextspezifische, handlungsorientierte Form von systematisch generiertem Wissen, politische Entscheidungsträgerinnen und träger haben aber gemäss der bisherigen Literatur eine breitere Auffassung davon, was als systematisch generiertes Wissen zählt (vgl. Cairney 2016, 22; Fornerod 2001, 72; Lomas und Brown 2009, 914; Webber 1987, 612; Whiteman 1995, 158). "For the most part, 'policy information,' 'research,' 'analysis,' and 'knowledge' are synonymous from the perspective of policy makers (Webber 1991, 11). Entsprechend dürfte auch ihr Verständnis von Evaluationen relativ offen sein. Verbunden damit stellt sich die Frage, inwiefern politische Entscheidungsträgerinnen und träger systematisch generiertes Wissen als objektiv ansehen oder dies verlangen (vgl. Bimber 1996, 5; Cairney 2016; Mooney 1992, 349-350; Weiss 1989, 416).

5 Im Folgenden wird Evaluation verwendet, wenn sich die angeführte Literatur explizit auf Evaluationen bezieht. Dies gilt auch für andere Wissensformen, falls sich eine Passage spezifisch auf diese bezieht. Wenn sich die angeführte Literatur mit unterschiedlichen Formen systematisch generierten Wissens befasst (z. B. Evidenz, Policy-Analyse), wird systematisch generiertes Wissen verwendet. 


\subsubsection{Verständnis und Formen der Nutzung}

Das Konzept der Nutzung ist gerade in der Forschung über Evaluation mit verschiedenen Begrifflichkeiten verbunden. Englischsprachige Forschende verwenden für die Nutzung oder Verwendung die Begriffe use oder utilization in der Regel als Synonyme (vgl. Alkin und Taut 2003; Henry und Mark 2003; Johnson et al. 2009). Vereinzelt wird aber utilization als technischere, engere und use als offenere Bezeichnung aufgefasst (Alkin und King 2016, 577; Vedung 1997, 289; Weiss 1980, 381; 1981, 18). Der Begriff der Nützlichkeit (utility) bezeichnet weiter, inwiefern eine Evaluation für eine entsprechende Frage relevant ist (Leviton und Hughes 1981). Evaluationsstandards zur Nützlichkeit sollen folglich dafür sorgen, dass die Prozesse und Produkte der Evaluation den Bedürfnissen der Beteiligten und Betroffenen entsprechen (Widmer et al. 2000; Yarbrough 2011). Der Begriff des Nutzens (usefulness) stellt schliesslich die subjektive Beurteilung der Qualität und der Nützlichkeit einer Evaluation dar (Stockbauer 2000, 16-17) womit die Verwertung der Evaluationsergebnisse durch die evaluierte Institution mit konkreten Konsequenzen als Folge der Evaluationsergebnisse gemeint ist. Ob Verwertung bzw. Nutzung passiert, ist objektiv messbar (z. B. durch Beobachtung.

Aus der Vielzahl an Versuchen, die Nutzung von systematisch generiertem Wissen zu typologisieren, hat sich basierend auf den Arbeiten der Nutzungsforschung der 1970er- und 1980er-Jahre eine dreiteilige Unterscheidung etabliert (vgl. Alkin und King 2016, 572 f.; Henry und Mark 2003, 194; Herbert 2014, 389). Sie unterscheidet zwischen den folgenden Formen der Nutzung (Cousins und Shulha 2006; Johnson 1998):

- Instrumentelle Nutzung (instrumental use)

Direkte Verwendung von systematisch generiertem Wissen, um Handlungen zu ergreifen oder Entscheide zu treffen. Im Vordergrund bei dieser Form steht die Problemlösung dank den Erkenntnissen aus systematisch generiertem Wissen.

- Konzeptionelle Nutzung (conceptual use)

Indirekte Verwendung von systematisch generiertem Wissen, die neue Denkweisen, Verständnisse oder Einstellungen eröffnet oder bestehende verändert. Sie wird auch enlightenment genannt (Weiss 1977).

- Symbolische Nutzung (symbolic use)

Verwendung von systematisch generiertem Wissen zur Unterstützung einer bereits vorgefassten Position, um diese Position zu legitimieren, zu begründen oder andere davon zu überzeugen. Die Bezeichnungen variieren, Forschende sprechen auch von persuasive, legitimative oder political use. (Henry 2000, 91; Kirkhart 2000, 10).

Die dreiteilige Typologie der instrumentellen, konzeptionellen und symbolischen Nutzung dient zwar als Referenzpunkt, verschiedene Forschende erweiterten oder differenzierten sie aber und zeigen so die vielfältigen Formen der Nutzung auf. Die wohl bedeutendste Erweiterung aus der Forschung über Evaluation bildet die prozessorientierte Nutzung (process use) (Patton 1997, 90). Diese Nutzungsform bezieht sich explizit auf die Änderungen, die aufgrund des Prozesses und nicht aufgrund der Resultate einer 
Evaluation geschehen. Eine andere Differenzierung und Erweiterung zugleich stellen die fünf Typen der instrumentellen, konzeptionellen, interaktiven, legitimierenden und taktischen Nutzung nach Vedung (1997, 267-276) dar. Interaktive Nutzung geschieht im Austausch von Evaluierenden und Personen aus der Praxis, aber auch zwischen den Praktikerinnen und Praktikern. Legitimierende und taktische Nutzung können als Formen der symbolischen Nutzung interpretiert werden: Während Evaluationen bei der legitimierenden Nutzung zur Stärkung des Standpunkts dienen, sollen bei der taktischen Nutzung mittels der Veranlassung beziehungsweise Durchführung von Evaluationen Entscheide verschoben werden. ${ }^{6}$

Gerade neuere Beiträge basieren dagegen auf reduzierten Typologien und lenken damit die Aufmerksamkeit auf die gemeinsamen Handlungslogiken der Nutzungsformen. Ausgehend von der Feststellung, dass eine instrumentelle, problemlösende Denkweise in der bisherigen Nutzungsforschung dominiert und diese auch in der Betrachtung der konzeptionellen und symbolischen Nutzung inhärent ist, unterscheidet Boswell (2009, 35-36) grundsätzlich zwischen instrumentalistischer und symbolischer Funktion von Fachwissen. Als instrumentalistische Funktion bezeichnet sie die Nutzung von Fachwissen, um Leistungen einer Politik anzupassen und zu verbessern (Boswell 2009, 55). Die symbolische Funktion teilt sie in die legitimierende und die substanziierende Funktion. Während Nutzende Fachwissen in der legitimierenden Funktion verwenden, um ihre Entscheidungskompetenz hervorzuheben und die rationale Grundlage dieser Entscheide zu zeigen, soll Fachwissen in der substanziierenden Funktion Positionen unterstützen und dadurch in der Entscheidfindung etwas bewirken (Boswell 2009, 72, 81). Damit fokussiert Boswell auf die symbolische Nutzung und begründet diese auf eine neue Weise. Auf eine Zweiteilung beschränkt sich auch Frey $(2012,64)$, die anstatt von Nutzungsformen von zwei Verwendungsrationalitäten spricht: Unterliegt die Nutzung einer analytischen Verwendungsrationalität, geschieht sie mit einer verbesserungsorientierten, ergebnisoffenen Absicht, wobei die Nutzenden die Evidenz unterschiedlich systematisch aufbereiten können. Bei einer politischen Verwendungsrationalität fehlt hingegen die Verbesserungsorientierung, die Aufbereitung und Nutzung erfolgt stattdessen opportunistisch und dient der Überzeugung oder taktischen Zwecken. Obwohl ihre Unterscheidung auf der Absicht der Nutzenden beruht, betont Frey $(2012,63)$ jedoch, dass Nutzende verschiedene Absichten verfolgen können und diese nur schwer abzugrenzen sind.

Andere Beiträge befassen sich mit der Frage, was als Nutzung zählt, und verdeutlichen damit, dass Nutzungsformen nacheinander erfolgen oder sich gegenseitig bedingen können. Um die Nutzung zu erfassen, betrachten verschiedene Forschende sie als Prozess in mehreren Stufen (Dunn 1983, 127-129; Nutley et al. 2007, 46f.). Ein Beispiel eines solchen Stufenmodells präsentieren Knott und Wildavsky (1980, 546), die zwischen sieben Stufen unterscheiden. ${ }^{7}$ Den Erhalt des systematisch generierten Wissens definieren sie als erste Stufe. Als letzte Stufe sehen sie an, wenn die aufgrund des systematisch generierten

6 Weitere Typologien: z. B. Whiteman (1985b; 1995, 170) oder Weiss (1979).

7 Für ein anderes Beispiel eines Stufenmodells siehe Rich (1997, 20-21). Für eine umfassende Definition von Nutzung inklusive ihrer Formen siehe Alkin und King $(2017,5)$. 
Wissens verabschiedete und umgesetzte Politik spürbare Wirkungen entfaltet. Damit ist ihr Modell klar auf eine instrumentelle Denkweise der Nutzung ausgerichtet (vgl. Nutley et al. 2007, 51). Dies zeigt, dass Stufenmodelle zwar den Prozesscharakter der Nutzung aufzeigen, sich bei ihnen aber stets die Frage nach dem Ziel oder Endpunkt des Prozesses stellt.

Zuweilen kritisch diskutiert wird die Nutzung in der Forschung über Evaluation seit den 2000er-Jahren anhand der Konzepte der missbräuchlichen Nutzung (misuse) und des Einflusses (influence). Die missbräuchliche Nutzung kann gemäss Christie und Alkin (1999, 2-3) als die absichtliche Zweckentfremdung des Prozesses oder der Ergebnisse einer Evaluation verstanden werden (vgl. Cousins und Shulha 2006, 269). Entsprechend muss aber keine Nutzung von Evaluationen nicht missbräuchlich sein (Cousins 2004). Das zweite, weitaus intensiver diskutierte Konzept des Einflusses soll die vielfältigen Effekte von Evaluationen gesamthaft umfassen (vgl. Cousins und Shulha 2006, 269). Erstmals eingeführt hat das Konzept des Einflusses Kirkhart (2000). Ihre Idee weiterentwickelt haben etwa Henry und Mark (2003) in ihrem prozessbasierten Modell des Einflusses (vgl. Henry 2000; Mark und Henry 2004). Das Plädoyer, anstelle der Nutzung das Konzept des Einflusses zu verwenden, gründet auf der Kritik am historisch gewachsenen Konzept der Nutzung. Demgemäss ist die Nutzung einseitig auf die Resultate, die instrumentelle Funktion und kurzfristige Effekte fokussiert (Kirkhart 2000). Zudem können sich die Nutzungsformen - etwa resultat- oder prozessbasierte konzeptionelle Verwendung - überlappen (Alkin und Taut 2003; Mark und Henry 2004). Folglich setzen sich die Konzepte des Einflusses, und auch der missbräuchlichen Nutzung, kritisch auseinander mit dem historischen und positiv konnotierten Konzept der Nutzung als «heiligem Gral» (Henry 2000, 95) oder «Dogma» (Vedung 1997, 265) der Evaluation. Die beiden Konzepte sind aber vor allem theoretisch wertvoll, da sie bisher kaum Anwendung in der empirischen Forschung finden (Cousins und Shulha 2006, 286; Herbert 2014, 389, 407).

\subsubsection{Nutzung zur wirkungsorientierten Steuerung der Politik}

Die Nutzung von systematisch generiertem Wissen ist auch ein zentrales Element zweier prominenter Reformbewegungen, die eine Verbesserung der Handlungsfähigkeit des Staats durch eine resultat- und wirkungsorientierte Steuerung postulieren: New Public Management und evidenzbasierte Politikgestaltung. New Public Management (NPM, Neue Verwaltungsführung) verbreitete sich in den 1980er- und 1990er-Jahren von den angelsächsischen Staaten aus (Rieder und Widmer 2007; Sanderson 2001; Van Dooren 2008; Widmer und De Rocchi 2012). Generell beruht NPM auf der Übertragung von Methoden und Prinzipien des Managements in den öffentlichen Sektor, die konkrete Anwendung von NPM variiert aber deutlich in den verschiedenen Ländern und ihren Gliedstaaten (Pollitt und Bouckaert 2004, 96-98; Wollmann 2003, 3). Wesentliches Element von NPM ist die output-basierte Steuerung und Kontrolle durch die Messung der Performanz staatlicher Leistung (Rieder und Widmer 2007, 15; Van Dooren 2008, 1; Vedung 2010, 272; Widmer und De Rocchi 2012). Evaluation kommt in NPM eine zentrale Rolle zur Messung der Resultate und Wirkungen sowie der Rechenschaftslegung zu, weswegen Vedung (2010) NPM als dritte Welle der Verbreitung der Evaluation bezeich- 
net (vgl. Sanderson 2001, 301; Wollmann 2003, 4-8). Das Konzept der evidenzbasierten Politikgestaltung (EBP, evidence-based policymaking) als zweite Bewegung beruht auf dem Grundsatz, aussagekräftiger Evidenz bei der Entscheidfindung Priorität einzuräumen und die Wirkungen der Politik durch den systematischen Einbezug der Evidenz zu verbessern (vgl. Frey 2012; Nutley et al. 2010; Pawson 2006; Widmer 2009). Die EBP hat ihren Ursprung in der evidence-based medicine und zeichnet sich durch ihren Fokus auf rigorose Evidenz aus. Zentral sind daher randomized controlled trials (RCT) und systematic reviews, die bestehende Forschung zu einer Frage zusammenfassen und kritisch bewerten (Pawson 2006; Vedung 2010). Ausgebreitet hat sich die EBP Ende der 1990er-Jahre von Grossbritannien aus, wo sich New Labour unter der Philosophie what counts is what works für die EBP aussprach (vgl. Nutley und Webb 2000; Parsons 2002; Solesbury 2001). Die EBP bildet gemäss Vedung (2010) die vierte Welle der Verbreitung von Evaluation, indem sie stark auf die Wirkungen der Politik fokussiert (vgl. Nutley und Webb 2000; Sanderson 2002).

Die beiden Bewegungen erweitern die Betrachtung des Nutzungskonzepts, indem sie die Aufmerksamkeit auf die Leistungs- und Wirkungsorientierung und damit die Output-Legitimation staatlichen Handelns lenken, sie werden aber auch kritisch diskutiert (vgl. Widmer 2009; Widmer und Rüegg 2005). Beide Bewegungen entwickelten sich in einer Zeit, in der Fortschritte in der Informationstechnologie sowie in den Analysemethoden eine sophistiziertere Betrachtung der Leistungen von Politik ermöglichten und der Anspruch nach einem Nachweis der wirkungsvollen Leistungen des öffentlichen Handelns zunahm (Davies et al. 2000a, 1-2; Head 2010; Nutley et al. 2010, 133; Pawson 2006; Solesbury 2001, 4; Van Dooren 2008). Gleichzeitig geben technokratische Elemente der Konzepte Anlass zu Kritik. Einerseits wird hinterfragt, inwiefern Leistungen und Wirkungen komplexer Massnahmen auf einseitige, einfache Indikatoren des NPM reduziert werden können (Sanderson 2001). Anderseits wird die EBP in ihrer Idealform als Modell gesehen, in dem Politik - von Parsons $(2002,54)$ zugespitzt ausgedrückt ideologie- und interessenfrei würde. Da die Annahme eines rationalen Umgangs als realitätsfern gesehen werden kann und andere Gesichtspunkte die Politikgestaltung ebenso prägen, wird bisweilen von policy-based evidence gesprochen (Cairney 2016; Marmot 2004). Deswegen merken etwa Nutley et al. (2010) oder Head (2015) an, dass Bezeichnungen wie evidence-informed, evidence-inspired oder evidence-aware besser zutreffen. Zudem machen sie darauf aufmerksam, dass solch rigorose Evidenz nur eine relevante Art des Wissens für Politikgestaltung ist (Nutley et al. 2003).

\subsubsection{Forschungsstand: Ausmass der Nutzung hängt von Konzeptualisierung ab}

Ausgangspunkt der empirischen Forschung der Nutzung von systematisch generiertem Wissen bilden sowohl die Nutzenden als auch das systematisch generierte Wissen selbst. Einerseits befassen sich die Arbeiten damit, wie ausgewählte Entscheidungsträgerinnen und -träger oder Organisationen systematisch generiertes Wissen generell verwenden oder in einem spezifischen Fall verwendet haben. Anderseits setzen die Arbeiten bei konkreten Evaluationen oder anderen Studien an und betrachten deren Nutzung (Cousins 
und Leithwood 1986; Johnson et al. 2009; Nutley et al. 2007, 67). Die Untersuchung der Nutzung erfolgt teilweise anhand eines qualitativen Fallstudiendesigns, am häufigsten stützen sich die Forschenden auf Befragungen (Cousins und Leithwood 1986, 341; Newman und Head 2015, 386; Nutley et al. 2007, 66). Folglich ist die Forschung stark auf die Angaben der Befragten angewiesen. Erinnerungslücken, sozial erwünschtes Antworten oder übertriebene Antworten können diese Selbsteinschätzungen aber beeinflussen (Leviton 2003, 526).

Bisher im Zentrum der Forschung standen die instrumentelle und konzeptionelle Nutzungsform. Die ersten Arbeiten fokussieren klar auf die instrumentelle Verwendung (Albaek 1995; Weiss 1981, 25). Diese Untersuchungen zeigen, dass systematisch generiertes Wissen nur selten so direkt Anwendung findet (vgl. Hofstetter und Alkin 2003, 212-213): "Much of this use is not deliberate, direct, and targeted, but a result of longterm percolation of social science concepts, theories, and findings into the climate of informed opinion" (Weiss 1977, 534; vgl. Weiss 1980, 1999). Folglich weitete sich bald der Fokus der Untersuchungen auf die konzeptionelle Nutzung aus. Der symbolischen Nutzung widmen sich hingegen wenige Beiträge und sie wurde anfänglich - und gemäss Mark $(2011,109)$ teilweise immer noch - negativ gewertet (vgl. Johnson et al. 2009, 382; Leviton und Hughes 1981, 530). Zudem ist die Forschung über Evaluation auf die Nutzung der Ergebnisse und nicht des Prozesses der Evaluation fokussiert (Johnson et al. 2009, 388).

Das beobachtete Ausmass der Nutzung fällt je nach Konzeption und Objekt der Forschung unterschiedlich aus (vgl. Frey 2012, 40-41). Wie zuvor erwähnt, fokussiert die anfängliche Forschung auf die direkte, unmittelbare Anwendung von systematisch generiertem Wissen und stellt kaum Nutzung fest. Je weiter das Verständnis der Nutzung, aber auch des Wissens gefasst wird, desto eher ist eine Verwendung feststellbar. Förderlich für die Beobachtung der Nutzung ist zudem das Betrachten eines längeren Zeithorizonts (vgl. Hofstetter und Alkin 2003). Weiter variiert das beobachtete Ausmass je nach Untersuchungsgegenstand: Direkte, instrumentelle Nutzung ist eher in lokalen Settings oder spezifischen Programmen beobachtbar (Hofstetter und Alkin 2003, 213; James und Jorgensen 2009, 155). Ebenso unterscheidet sich das Ausmass der Evaluationsnutzung in verschiedenen Politikbereichen (vgl. Askim 2008; Weiss 1999). Generell zeigen die Untersuchungen auf, dass politische Entscheidungsträgerinnen und -träger systematisch generiertes Wissen durchaus schätzen und es ein Teil davon verwendet (vgl. Newman und Head 2015).

\subsection{Erklärung der Nutzung von systematisch generiertem Wissen}

Wie lässt sich die Nutzung von systematisch generiertem Wissen erklären? Diese Frage wird im Folgenden aus der Perspektive der Nutzungsforschung sowie theoretischen Ansätzen des Politikprozesses aus der Policy-Forschung diskutiert. Ausgangspunkt der Erklärungsansätze der Nutzungsforschung ist dabei die Frage, wieso bereitgestelltes, systematisch generiertes Wissen so geringe Anwendung in der Politik findet (vgl. Cous- 
ins und Shulha 2006, 269; James und Jorgensen 2009, 144). Theoretische Ansätze des Politikprozesses hingegen konzeptualisieren das Verhalten der Entscheidungsträgerinnen und träger bei der Politikgestaltung und können so aufzeigen, wann, wozu oder wie systematisch generiertes Wissen in Politikprozessen Verwendung finden kann. Ihr Beitrag zur Erklärung der Nutzung liegt folglich gerade darin, dass sie im Gegensatz zur Nutzungsforschung nicht bei der Nutzung ansetzen und sie zumeist nicht von einer direkten Anwendung ausgehen (Cairney 2016, 30; 2018).

\subsubsection{Nutzungsforschung: Empirische Identifikation diverser Erklärungsfaktoren}

Die Arbeiten der Nutzungsforschung befassten sich bisher weniger mit der Entwicklung umfassender theoretischer Erklärungsansätze der Nutzung, vielmehr widmeten sie sich der empirischen Erforschung von förderlichen oder hinderlichen Faktoren der Nutzung (James und Jorgensen 2009, 143; Oh 1997, 5; vgl. Shulha und Cousins 1997, 196; Whiteman 1995, 179). Diese diversen Erklärungsfaktoren sind für die verschiedenen Forschungstraditionen in überblicksartigen Darstellungen zusammengefasst. ${ }^{8}$

Aus diesen überblicksartigen Darstellungen geht hervor, dass die bisherigen Arbeiten trotz unterschiedlicher Forschungstraditionen und Herangehensweisen übereinstimmend eine Reihe von Erklärungsfaktoren der Nutzung systematisch generierten Wissens identifizieren (Newman und Head 2015, 386; Nutley et al. 2007, 67). Gestützt auf die Einteilung von Cousins und Leithwood (1986) zur Nutzung von Evaluationen präsentiert Tabelle 1 daher beispielhaft verschiedene Erklärungsfaktoren, die sie zwei Dimensionen zuordnen: Dem Evaluations- und dem Nutzungskontext. Dies entspricht folglich der Angebotsseite sowie der Nachfrageseite von Evaluationen beziehungsweise systematisch generiertem Wissen.

$\mathrm{Zu}$ verschiedenen der Erklärungsfaktoren gemäss Cousins und Leithwood (1986, 347348) können auch relevante Erklärungsansätze zugeordnet werden. Ein Erklärungsansatz, der mehrere Erklärungsfaktoren des Evaluationskontexts umfasst, stellen die Truth Tests and Utility Tests (Weiss und Bucuvalas 1980) dar. Demgemäss hängt die Nutzung der Entscheidungsträgerinnen und -träger mit ihrer Bewertung der Forschung zusammen, die aus dem Zusammenspiel verschiedener Erklärungsfaktoren entsteht. Neben der Relevanz der Forschung für den Verantwortungsbereich der Entscheidungsträgerinnen und träger spielt dabei die Vertrauenswürdigkeit der Forschung (Truth Test) eine Rolle, die beeinflusst wird von der Qualität der Forschung und der Übereinstimmung der Resultate mit den Erwartungen der Entscheidungsträgerinnen und -träger. Wenn die Resultate nicht den Erwartungen entsprechen, bedarf es einer hohen Qualität, damit Forschung Beachtung findet. Für die Beurteilung der Forschung relevant ist weiter deren Nützlichkeit (Utility Test), die auf der Handlungsorientierung der Ergebnisse sowie ihrer Übereinstimmung mit dem gegenwärtigen Zustand gründet. Es müssen sich umso klarere

8 Forschung über Evaluation: z. B. Cousins und Leithwood (1986), Johnson et al. (2009), Shulha und Cousins (1997); EBP und evidence-based practice Nutley et al. (2007, 61-90); NPM: Askim (2008). 
Handlungen ableiten lassen, je stärker die Ergebnisse mit dem gegenwärtigen Zustand übereinstimmen (Weiss und Bucuvalas 1980).

\section{Tabelle 1: Erklärungsfaktoren der Evaluationsnutzung aus der Literatur}

\begin{tabular}{|c|c|c|c|}
\hline & Evaluationskontext & & Nutzungskontext \\
\hline & $\begin{array}{l}\text { Evaluationsqualität bezogen auf den Prozess, } \\
\text { d. h. verwendete Methoden oder Evaluationsmodelle, } \\
\text { Stringenz des Vorgehens }\end{array}$ & , & $\begin{array}{l}\text { Informationsbedürfnisse der Zielgruppe(n) der } \\
\text { Evaluationen, d.h. Art der Information, Anzahl } \\
\text { Zielgruppen, Bedarf nach Evaluation }\end{array}$ \\
\hline , & $\begin{array}{l}\text { Glaubwürdigkeit des Evaluationsprozesses oder der } \\
\text {-resultate, Objektivität, Bewertungskriterien }\end{array}$ & , & $\begin{array}{l}\text { Charakteristika des Entscheids, d. h. Wichtigkeit } \\
\text { und Art des Entscheids, Neuheit des Programms }\end{array}$ \\
\hline , & $\begin{array}{l}\text { Relevanz der Evaluation für die Bedürfnisse der } \\
\text { Entscheidungsträgerinnen und -träger }\end{array}$ & , & $\begin{array}{l}\text { Politisches Klima, d. h. Position der Auftrag- } \\
\text { gebenden, Budget- und Machtkämpfe }\end{array}$ \\
\hline , & $\begin{array}{l}\text { Kommunikationsqualität der Resultate, } \\
\text { d. h. Klarheit, Stil, Verbreitung der Resultate }\end{array}$ & , & $\begin{array}{l}\text { Konkurrierende Information von anderen Quellen } \\
\text { als Evaluationen }\end{array}$ \\
\hline & $\begin{array}{l}\text { Art der Resultate, d. h. positiv, negativ, übereinstim- } \\
\text { mend mit Erwartungen der Entscheidungsträgerin- } \\
\text { nen und träger }\end{array}$ & , & $\begin{array}{l}\text { Persönliche Charakteristika der Entscheidungs- } \\
\text { träger und -trägerinnen, d.h. sozialstrukturelle } \\
\text { Merkmale, Erfahrung, Informationsverarbeitung }\end{array}$ \\
\hline & $\begin{array}{l}\text { Rechtzeitigkeit der Verbreitung der Evaluations- } \\
\text { resultate }\end{array}$ & , & $\begin{array}{l}\text { Einstellung gegenüber Evaluation der Entschei- } \\
\text { dungsträgerinnen und -träger, Zustimmung zu und } \\
\text { Einbezug in Evaluation }\end{array}$ \\
\hline
\end{tabular}

Entnommen und sinngemäss übersetzt aus Cousins und Leithwood (1986, 347-348).

Erklärungsfaktoren des Nutzungskontexts stehen hingegen im Zentrum des Analyserahmens der Four Is von politischen Entscheidungsprozessen. Gemäss Weiss (1995, 573 f.; vgl. auch 1999) resultieren Entscheide aus den folgenden vier Elementen, die in einer Wechselbeziehung stehen:

- Interessen (interests): Primär Eigeninteressen, etwa Wiederwahl oder sonstiger Erhalt der eigenen Position.

- Ideologie (ideology): Ethische und moralische Prinzipien, Werte und politische Positionen, die sowohl langfristig als auch eher ad hoc sein können.

- Informationen (information): Das gesamte Wissen und die Vorstellungen, die Entscheidungsträgerinnen und -träger zur Information über die gegenwärtige Situation benötigen. Dieses Wissen kann systematisch oder nicht systematisch generiert sein.

- Institutionen (institutional rules and culture): Struktur, Kultur, Prozesse und Entscheidregeln der entscheidungsbefugten Organisation oder Institution. Interessen, Ideologie und Informationen sind darin eingebettet. 
Diese vier Elemente zeigen auf, dass systematisch generiertes Wissen nur eine Komponente in der Politikgestaltung bildet. Entsprechend kann systematisch generiertes Wissen kaum unmittelbar zu grossen Veränderungen führen und direkt angewendet werden.

Die Aufmerksamkeit auf den Kontakt zwischen dem Evaluations- und dem Nutzungskontext lenken dagegen die kritisch diskutierten Ansätze der frühen Nutzungsforschung unter dem Begriff der Two Communities Theory. ${ }^{9}$ Forschung und Politik stellen gemäss diesen Ansätzen zwei separate, selten interagierende Systeme dar. In diesen Systemen divergieren die Werte sowie Sprachen und das Handeln folgt unterschiedlichen Logiken (Caplan 1979; Caplan et al. 1975, 50). Während sich die Forschenden demgemäss über Jahre mit abstrakten Konzepten und theoretischen Erklärungen befassen, benötigen die handlungsorientierten Politikerinnen und Politiker zeitnahe Lösungen für praktische Probleme (Newman und Head 2015). Folglich impliziert dieser Erklärungsansatz, dass sich der Kontakt von Forschung und Politik förderlich auf die Nutzung auswirkt. Gleichzeitig wird er aufgrund des vereinfachten Bilds von nur zwei Gemeinschaften, die homogen sind, grundlegend kritisiert (vgl. Lindquist 1990, 30 f.; Newman und Head 2015, 386 f.).

Die vorangehende Diskussion zentraler Erklärungsansätze und die empirisch festgestellten, diversen Erklärungsfaktoren der Nutzung zeigen die Grenzen einer umfassenden Erklärung der Nutzung auf. Viele Beiträge haben zudem unterschiedliche Verständnisse von Nutzung und gehen generell von Nutzung aus. Es bleibt unklar, welche Erklärungsfaktoren für welche Formen der Nutzung relevant sind und wie die verschiedenen Erklärungsfaktoren insgesamt zueinander stehen (vgl. Frey 2012, 84; Nutley et al. 2007, 67). Gleichzeitig scheint die Identifikation von einigen wenigen Schlüsselfaktoren bedingt aussichtsreich, da sich viele der Erklärungsfaktoren auf den Kontext beziehen (Nutley et al. 2007, 89; Shulha und Cousins 1997, 197). Auch steht eine Auflistung und möglicherweise Quantifizierung von Erklärungsfaktoren im Widerspruch mit dem komplexen, interaktiven Vorgang der Nutzung (Nutley et al. 2007, 84; Weiss 1998, 23). Ein wesentlicher Teil der Nutzungsforschung untersucht schliesslich die Erklärungsfaktoren aus einer individuellen Perspektive der Nutzenden (Nutley et al. 2007, 84-89). Die Erklärungsansätze der Four Is oder der Two Communities weisen aber darauf hin, dass die Betrachtung der Nutzung als individueller Prozess zu kurz greift.

\subsubsection{Theoretische Ansätze des Politikprozesses: Politik und daran Beteiligte im Zentrum}

Indem sich theoretische Ansätze des Politikprozesses mit den verschiedenen, an der Politikgestaltung beteiligten Akteurinnen und Akteuren, Institutionen und Strukturen befassen, beinhalten sie gleichzeitig Annahmen zur Funktion von systematisch generiertem Wissen in der Politik (Cairney 2016). Ausgangspunkt in die Einführung solcher Ansätze bildet oft das Modell des Policy-Cycle. Dieses stellt den Politikprozess als linearen Zyklus mit aufeinanderfolgenden Phasen dar, namentlich Agenda Setting, Politikformulierung,

9 Cousins und Leithwood (1986) verorten den Einbezug der Entscheidungsträgerinnen und -träger in die Evaluation als Teil des Nutzungskontexts als "User commitment and/or receptiveness to evaluation", in der Tabelle 1 unter «Einstellung gegenüber Evaluation» zusammengefasst. 
«Entscheidung, Umsetzung, Evaluation und abschliessend die Terminierung oder Weiterführung der Politik» (vgl. Sager et al. 2017a). Diese Modellierung eines geordneten Prozesses der Politik in sequenzielle, trennbare Phasen wird jedoch weitgehend nicht als realistisch angesehen (Cairney 2016; Sager et al. 2017a). Entsprechend wurden andere Ansätze zur Erklärung von politischen Prozessen entwickelt. In Verbindung mit der Nutzung von systematisch generiertem Wissen werden namentlich das Advocacy Coalition Framework (ACF) (Sabatier 1988; Sabatier und Jenkins-Smith 1993b) oder seltener etwa der «Multiple-Streams-Ansatz» (Kingdon 1995) verwendet (Cairney 2016, 13-43; Radaelli 1995; Weible 2008).$^{10}$ Generell ist aber die Literatur zu theoretischen Ansätzen des Politikprozesses kaum mit derjenigen zur Nutzungsforschung verbunden (James und Jorgensen 2009, 147; Radaelli 1995, 159). Wie empirische Anwendungen zeigen, kann mit Hilfe solcher theoretischen Ansätze aber gerade die in der Nutzungsforschung aufgeworfene Frage erläutert werden, wieso systematisch generiertes Wissen kaum oder gar keine Rolle spielt (Cairney und Yamazaki 2018; Rietig 2018; Ritter et al. 2018). Da viele theoretischen Ansätze des Politikprozesses die Verwendung von systematisch generiertem Wissen eher implizit modellieren, wird im Folgenden nur auf den ACF eingegangen, der sich vergleichsweise stark auf die Nutzung von systematisch generiertem Wissen stützt und auch Hypothesen zur Nutzung postuliert (vgl. James und Jorgensen 2009).

Im Zentrum der Konzeptualisierung des Politikprozesses gemäss dem ACF stehen die Advocacy Coalitions, in denen Akteurinnen und Akteure ihre beliefs grundsätzlich teilen und sich für deren Umsetzung durch die Politik einsetzen. Die Advocacy Coalitions bestehen dabei in verschiedenen Policy-Subsystemen oder Politikbereichen. Die beliefs der Akteurinnen und Akteure sind in drei Schichten strukturiert: Die innerste Schicht bilden die (deep) core beliefs, die fundamentale und allgemeine Werte darstellen und nur sehr schwer veränderbar sind. Policy core beliefs stellen die mittlere Schicht dar. Damit meint Sabatier $(1993,31)$ die grundsätzlichen Positionen in Politikbereichen, die aber auch sehr resistent gegenüber Veränderungen sind. Zur äussersten Schicht gehören die secondary aspects, die zur Umsetzung der policy core beliefs dienen und am ehesten veränderbar sind.

Die Nutzung von systematisch generiertem Wissen ist im ACF primär beschrieben in der konzeptionellen Form bei policy-orientiertem Lernen (policy-oriented learning), das von kontextuellen Faktoren abhängt und zu Politikwandel führen kann (vgl. James und Jorgensen 2009, 143; Sabatier 1988, 129; Weible 2008, 616; Weible et al. 2009, 122). ${ }^{11}$ Policy-orientiertes Lernen und Politikwandel sind gemäss dem ACF relativ langfristige Prozesse (Jenkins-Smith und Sabatier 1993, 42-44; Weible et al. 2009, 124). Durch konzeptionelle Nutzung von Policy-Analyse oder technischer Information ändern sich secondary aspects und policy core beliefs über Jahre bis Jahrzehnte graduell. Policy-orientier-

10 Einen Überblick über theoretische Ansätze des Politikprozesses bieten generell Sager et al. (2017a) oder mit Bezug auf die Verwendung von systematisch generiertem Wissen Cairney (2016), Radaelli (1995) oder Weible (2008).

11 Der zweite Auslöser für Politikwandel sind externe Ereignisse (Sabatier 1993). In einer Revision des ACF nennen Sabatier und Weible (2007, 204f.) Policy-Subsystem-interne Ereignisse sowie Vereinbarungen zwischen zwei oder mehr Koalitionen als weitere Wege zu Politikwandel. 
tes Lernen findet wahrscheinlicher in einer Advocacy Coalition statt, es kann aber auch zwischen Advocacy Coalitions geschehen und so zu Politikwandel führen (Jenkins-Smith und Sabatier 1993, 48). Gemäss Jenkins-Smith und Sabatier (1993, 50-54) wird dieses policy-orientierte Lernen durch drei Faktoren begünstigt: (1) ein moderater Konfliktgrad einer Frage, (2) gute analytische Erfassbarkeit einer Frage sowie (3) die Existenz eines professionalisierten Forums, wobei sie Parlamente als Beispiel eines nicht professionalisierten, offenen Forums nennen. Folglich fokussiert der ACF auf die Erklärung der Nutzung durch kontextuelle Faktoren (vgl. Heintz und Jenkins-Smith 1988, 267).

Indem der ACF von langfristigen, indirekten Prozessen ausgeht, zeichnet er ein realitätsnahes Bild der Nutzung in Politikprozessen, verschiedene Aspekte können aber auch kritisch diskutiert werden. Generell ist die Bildung und das Verhalten der Koalitionen im ACF weniger klar konzeptualisiert (vgl. Schlager 1995). Zudem weisen Anwendungen des ACF auf europäische Länder auf seine starke Ausrichtung auf die USA hin (Kübler 2001). Als Reaktion auf diese Kritik erweiterten Sabatier und Weible (2007, 199) den ACF um die coalition opportunity structures. Damit bezeichnen sie namentlich die Offenheit und Konsensorientierung eines politischen Systems, welche das Verhalten und die Ressourcen der Koalitionen beeinflussen. So kann etwa der Föderalismus ein System offener machen, indem sich den Koalitionen mehr Eintrittspunkte eröffnen (vgl. Analyse von Kübler 1999; Kübler 2001). In Bezug auf die Nutzung ist der ACF vor allem auf die konzeptionelle Form ausgerichtet, obwohl er auch annimmt, dass die Koalitionen systematisch generiertes Wissen symbolisch verwenden (James und Jorgensen 2009, 145). Ebenso konzentriert sich der ACF auf die Rolle von systematisch generiertem Wissen bei policy-orientiertem Lernen und es bleibt unklar, ob und wie systematisch generiertes Wissen bei anderen Formen von Politikwandel vorkommt (James und Jorgensen 2009, 147). Schliesslich bestehen Unklarheiten über die Rolle von Expertinnen und Experten als Beteiligte am Politikprozess. Erweiterungen und empirische Anwendungen weisen darauf hin, dass Expertinnen und Experten je nach Konstellation der Koalitionen unterschiedlich zentral für eine Koalition sowie das gesamte Subsystem sein können (Ingold und Gschwend 2014; Weible et al. 2010). Zudem können Expertinnen und Experten unterschiedlichen Koalitionen angehören und voneinander abweichende Positionen vertreten (Montpetit 2011).

\subsection{Besonderheiten des parlamentarischen Kontexts}

Die bisherigen, generelleren Ausführungen haben sich mit der Nutzung von systematisch generiertem Wissen durch politische Entscheidungsträgerinnen und -träger befasst; was aber sind Besonderheiten, wenn auf Parlamentsmitglieder als Nutzerinnen und Nutzer fokussiert wird? Der folgende Abschnitt präzisiert daher das Verständnis der Nutzung für diesen spezifischen Kontext, indem er sich mit dem Umgang mit Informationen in Parlamenten und mit der symbolischen Nutzung befasst, die in Parlamenten besonders zentral sein sollte. Die darauffolgenden Abschnitte präsentieren den Forschungsstand zu Ausmass und Erklärungsfaktoren der Nutzung von systematisch generiertem Wissen in Parlamenten. 


\subsubsection{Nutzung: Kommissionen, mündliche Kommunikation und politische Instrumentalisierung zentral}

Verbunden mit der Frage der Nutzung sind auch die Frage des Zugangs und der Verarbeitung von systematisch generiertem Wissen und generell Informationen im Parlament. Aufgrund der Arbeitsteilung in Parlamenten sind Kommissionen, in denen sich die Mitglieder spezialisieren, zentral für diese Aufgaben (Bimber 1991; Sabatier und Whiteman 1985; Zwier 1979). Zweck dieser Spezialisierung in den Kommissionen ist gemäss der informational theory (Gilligan und Krehbiel 1987; Krehbiel 1991) hauptsächlich die Informationsgewinnung. ${ }^{12}$ Indem das Parlament den Kommissionen Privilegien einräumt, setzt dies einen Anreiz zur aufwändigen Spezialisierung. Dies dient dem Parlament, da die von der Kommission erworbenen Informationen die Unsicherheit über die Politik reduzieren und die restlichen Parlamentsmitglieder ihre Entscheide auf die Hinweise der Kommissionsmitglieder abstützen können (Gilligan und Krehbiel 1987, 287-289; Sabatier und Whiteman 1985, 395-396). Damit geht die informational theory von einem rationalistischen Verständnis von Informationen und deren Nutzung aus (Shulock 1998). Insgesamt führt die Arbeitsteilung in Parlamenten dazu, dass sich zumeist nur spezialisierte Parlamentsmitglieder vertieft mit einer Frage auseinandersetzen (vgl. Mooney 2012).

Kommissionen sind folglich zentral für die Informationsgewinnung und -verarbeitung, sie unterscheiden sich aber. Grundsätzlich lassen sich Arbeits- und Redeparlamente voneinander abgrenzen. Während Kommissionen in Arbeitsparlamenten wie etwa in der Schweizer Bundesversammlung oder im US-Kongress eine zentrale Stellung haben, kommt dem Plenum in Redeparlamenten wie etwa in Grossbritannien eine grössere Bedeutung zu (Steffani 1979). Starke Kommissionen ermöglichen einem Parlament generell, Einfluss auf die Politikgestaltung einzunehmen (Mattson und Strøm 1995, 250). Die Stärke der Kommissionen variiert aber nicht nur zwischen Arbeits- und Redeparlamenten, sondern hängt von diversen Faktoren ab wie etwa das Verhältnis der Sitzanteile der Parteien oder die Rechte der Kommissionen (vgl. Mattson und Strøm 1995; Shaw 1979, 391). Die Rechte der Kommissionen können auch innerhalb eines Parlaments verschieden sein. Unterschiede innerhalb eines Parlaments ergeben sich zudem aufgrund des Tätigkeitsgebiets und damit der Einflussmöglichkeiten der Kommissionen (Fenno 1973; vgl. Smith und Deering 1990, 86). Schliesslich können Parlamente über ständige oder nichtständige Kommissionen verfügen. Während ständige Kommissionen feste Mitgliedschaften und Zuständigkeitsgebiete haben, werden nichtständige Kommissionen für spezifische Geschäfte oder Untersuchungen einberufen und nach Erledigung ihrer Aufgabe wieder aufgelöst (Mattson und Strøm 1995, 259).

Relevant für den Zugang und die Verarbeitung von Informationen und systematisch generiertem Wissen sind weiter die Mitarbeitenden oder parlamentseigenen Dienste (vgl. Bimber 1991). Sie bilden gemäss Arbeiten zu den US-Parlamenten und zum Euro-

12 Neben der Informationsgewinnung erfüllen Kommissionen zudem auch andere Funktionen (Martin 2014). Andere Erklärungsansätze für die Bildung von Kommissionen siehe Damgaard (1995); Lüthi (1997); Shaw (1979, 428-430); Weingast und Marshall (1988). 
päischen Parlament eine der wichtigsten Quellen der Parlamentsmitglieder für systematisch generiertes Wissen (Guston et al. 1997; Hird 2005b; Jackson-Elmoore 2014; Pegan 2017). Die Mitarbeitenden suchen und bereiten solche Informationen auf, Guston et al. $(1997,466)$ und Florio et al. $(1979,82)$ diskutieren aber kritisch, inwiefern sie ein differenziertes Verständnis von systematisch generiertem Wissen haben. Zudem können die Mitarbeitenden nicht nur bei der Informationsgewinnung, sondern auch bei der Politikgestaltung im Parlament eine zentrale Rolle einnehmen (vgl. Högenauer und Neuhold 2015; Whiteman 1995; Winzen 2011). Folglich hat ihr Umgang mit systematisch generiertem Wissen doppelte Konsequenzen für die Rolle dieses Wissens im Parlament (vgl. Patashnik und Peck 2016, 289). Da die Mitarbeitenden systematisch generiertes Wissen suchen und aufbereiten, sollten Parlamentsmitglieder damit vergleichsweise selten in direktem Kontakt sein (Whiteman 1995).

Darüber hinaus weisen mehrere Forschende darauf hin, dass die mündliche Kommunikation bei der Vermittlung von Informationen besonders bedeutsam ist in Parlamenten (Hird 2009; Raudla 2012, 1009; Weiss 1987, 98; 1989, 414). Die Parlamentsmitglieder erhalten zwar viele schriftliche Dokumente, sie erfahren aber gemäss Whiteman $(1995,158)$ von systematisch generiertem Wissen vielmehr in Briefings, Präsentationen im Rahmen von Hearings oder anderen Veranstaltungen sowie im direkten Gespräch (vgl. Raudla 2012).

Der wenig erforschten symbolischen Nutzung kommt besondere Bedeutung im Parlament zu: "Certainly in Congress - or any legislative setting - strategic use of policy analysis cannot be ignored" (Whiteman 1985b, 222). In parlamentarischen Entscheidungsprozessen sollten ideologische Positionen und Interessen vorrangig sein. Daher kann generell angenommen werden, dass Parlamentsmitglieder systematisch generiertes Wissen kaum zur Meinungsbildung verwenden. Erwartungsgemäss sollte systematisch generiertes Wissen im Parlament vielmehr vorwiegend oder sogar nur politische oder taktische Zwecke erfüllen: Etwa verwenden Parlamentsmitglieder Evaluationen, um ihre bereits vorgefassten Positionen zu bekräftigen oder gegnerische Positionen zu entkräften, aber auch um Entscheide zu verzögern (vgl. Mark 2011; Weiss 1987, 1989). Zudem gehen verschiedene Forscherinnen davon aus, dass Parlamentsmitglieder systematisch generiertes Wissen immer selektiv so verwenden können, dass es die eigene Position unterstützt oder sie zumindest Kritik daran üben können (vgl. Boswell 2009, 98 f.; Weiss 1990, 180).

Die Funktion der symbolischen Nutzung wird kontrovers diskutiert. Wie bereits erwähnt, wird sie in der Forschung über Evaluation teilweise als unangemessen betrachtet (vgl. Johnson et al. 2009, 382; Leviton und Hughes 1981, 530; Mark 2011, 109). Mehrere Forschende widersprechen aber einer negativen Konnotation der symbolischen Nutzung. In ihrer Sichtweise beschränkt sich die Funktion von systematisch generiertem Wissen nicht auf die Problemlösung, sondern sie kann etwa auch als Mittel zum Erhalt der Legitimität dienen (Boswell 2009, 30). Shulock (1999, 229), welche sich spezifisch mit dem parlamentarischen Kontext befasst, bezeichnet die Funktion von Policy-Analyse als Instrument zur Beschaffung von Ideen, zur Gewinnung von öffentlichem Interesse, zur Begründung von Handlungen und genereller als Symbol einer rationalen Entscheid- 
findung. Dies gründet auf der Logik, dass Policy-Analyse nicht für sich steht, sondern Argumente für eine Politik liefert, welche diese bedarf: "[O]bjective analysis, unassisted by advocacy and persuasion, is seldom sufficient to achieve major policy innovations" (Majone 1989, 36; vgl. Albaek 1995; Cairney 2018; Henry 2000; Shulock 1998, 1999). ${ }^{13}$ Dies impliziert auch, dass symbolische Nutzung durchaus folgenreich für die Politik sein kann, weswegen Whiteman (1985a; 1985b, 205) für das Ersetzen des Begriffs symbolische durch den Begriff strategische Nutzung plädiert (vgl. Mark und Henry 2004).

\subsubsection{Forschungsstand: Systematisch generiertes Wissen geschätzt, Nutzung variiert nach Phase und Rolle des Parlamentsmitglieds}

Dem parlamentarischen Kontext widmen sich in der bisherigen Forschung nur wenige Beiträge, als Nutzende im Zentrum stehen vielmehr andere Entscheidungsträgerinnen und -träger, namentlich Beschäftigte des öffentlichen Diensts (vgl. Pollitt 2006, 41; Speer et al. 2015, 39). Die Beiträge mit Fokus auf den Parlamenten befassen sich vorwiegend mit der Verwendung von Evaluationen oder Policy-Analyse im US-Kongress oder in einem oder mehreren Parlamenten der US-Bundesstaaten (z. B. Apollonio und Bero 2017; Bogenschneider et al. 2013; Guston et al. 1997; Hird 2005a, 2005b, 2009; Weiss 1987, 1989; Whiteman 1985a, 1985b, 1995, 1997). In Europa und Kanada befasst sich ein verwandter, neuerer Forschungszweig mit der parlamentarischen Verwendung von Evaluationen, aber auch mit der Nutzung von Evidenz sowie Performanzinformation (z. B. Hardy 2016; Lawrence et al. 2017; McDavid und Huse 2012; Speer et al. 2015; van Dooren 2004). Gerade Beiträge mit einem Fokus auf Letzterem fokussieren aber auf die Tätigkeiten zu Budgetfragen sowie der Rechenschaftslegung (vgl. Johnson und Talbot 2007; Raudla 2012; van Dooren 2004; Zwaan et al. 2016). Zudem beschränken sich die Beiträge auf wenige Parlamente. ${ }^{14}$

Die Nutzungsforschung zum Parlament stützt sich auf ähnliche Methoden wie die generelle Nutzungsforschung, allerdings erfolgt die Datenerhebung nicht immer in direktem Kontakt mit den Parlamentsmitgliedern. Die Nutzung wird hauptsächlich mit Befragungen, leitfadengestützte Interviews sowie Analysen von parlamentarischen Dokumenten oder Evaluationen erfasst. Teilweise stützen sich die Arbeiten auch auf eine Kombination dieser Methoden, etwa in Fallstudien (z. B. Whiteman 1985a, 1985b; Whiteman 1995). Parlamentsmitglieder selbst bezieht aber nur ein Teil der Arbeiten in die Erhebungen mit ein. Andere Arbeiten beruhen auf den Angaben der parlamentarischen Mitarbeitenden Weiss (1987, 1989); Whiteman (1985a, 1985b). Diesen Verzicht auf einen direkten Kontakt mit den Parlamentsmitgliedern begründet etwa Weiss $(1989,412)$ mit dem einfacheren Zugang, aber auch mit der zentralen Stellung, die Mitarbeitende in der Suche und Aufbereitung von systematisch generiertem Wissen haben (vgl. 2.3.1).

13 Eine solche Sichtweise steht im Zentrum der so genannten argumentativen Wende in der Policy-Analyse (Fischer und Forester 1993).

14 Gemäss der Literaturrecherche: Europäisches Parlament, Parlamente von Deutschland, Estland, Kanada und Grossbritannien, subnationale Parlamente von British Columbia, Flandern und Schottland. Abseits von Europa zudem zum philippinischen Parlament (Tabuga 2017). Die Forschung zu den Schweizer Parlamenten wird in einem separaten Kapitel präsentiert (vgl. 2.4.3). 
Die Forschung zeigt insgesamt, dass Parlamentsmitglieder systematisch generiertes Wissen überwiegend schätzen und dieses grundsätzlich auch verwenden. Gemäss der Literaturrecherche lässt sich in keiner Arbeit eine negative Einstellung der Parlamentsmitglieder gegenüber systematisch generiertem Wissen feststellen. Die Arbeiten zeigen vielmehr mehrheitlich, dass Parlamentsmitglieder systematisch generiertes Wissen nützlich finden und es auch als relevant ansehen (Boyer und Langbein 1991, 528; Guston et al. 1997, 454; Hird 2009, 527; Webber 1987, 617). Allerdings schliessen mehrere Forschende, dass Parlamentsmitglieder systematisch generiertes Wissen als eines von verschiedenen Instrumenten zur Erfüllung ihrer Tätigkeiten betrachten (Hird 2009, 527; Johnson und Talbot 2007, 126). Die Einschätzung der Nutzung variiert wie in der generellen Nutzungsforschung nach Konzeption, aber auch nach Betrachtungsgegenstand. So unterscheidet sich die Nutzung in den von Whiteman (1985b, 1995) untersuchten Gesetzgebungsprozessen deutlich. Systematisch generiertes Wissen findet gemäss der Forschung also Verwendung im Parlament - wenn auch teilweise in geringem Ausmass. Mehrere Beiträge weisen zudem darauf hin, dass grundsätzlich verschiedene Formen der Nutzung im Parlament vorkommen (vgl. Boyer und Langbein 1991, 529; Tabuga 2017, 9; Whiteman 1985a, 299). Allerdings scheinen instrumentelle Nutzung und Lernen aus Evaluationen generell seltener zu sein und die deutlich sichtbare symbolische Nutzung zu dominieren (vgl. Borrás und Højlund 2015, 109; Shulock 1999, 227; Webber 1987, 615; Weiss 1987, 105; 1989, 425). Teilweise stellen Forschende aber auch fest, dass die konzeptionelle Nutzung überwiegt und Parlamentsmitglieder sich von systematisch generiertem Wissen durchaus neue Perspektiven erhoffen (vgl. Bogenschneider et al. 2013, 274; Hird 2005b, 144; Morshed et al. 2017, 8). Schliesslich deuten Fallstudien darauf hin, dass die Nutzung in verschiedenen Gesetzgebungsprozessen klar variiert.

Die Forschungsbeiträge illustrieren, dass Nutzung in verschiedenen schriftlichen Quellen beobachtbar ist. Dokumentenanalysen etwa zeigen, dass Parlamentsmitglieder Fragen zu systematisch generiertem Wissen stellen. Wie oft dies geschieht, ist unklar: Während van Dooren $(2004,518)$ in 52 Prozent der analysierten Fragen einen Hinweis auf Performanzinformation im flämischen Regionalparlament findet, stellen Speer et al. $(2015,47)$ für dasselbe Parlament in 3.5 Prozent ihrer analysierten Fragen einen Bezug zu Evaluationen fest. Verweise auf systematisch generiertes Wissen enthalten zudem auch Kommissionsberichte und Protokolle der Beratungen oder Anhörungen, wobei in Letzteren gerade externe Teilnehmende, namentlich Fachpersonen oder Mitglieder der Regierung und Verwaltung auf solches Wissen aufmerksam machen (vgl. Apollonio und Bero 2009, 6; Esterling 2011; Marnoch 2008, 8; Michaels und Gruszczynski 2016, 141). Die Arbeiten von Mooney (1991a, 449; 1992) zeigen, dass Parlamentsmitglieder teilweise auch schriftliche Informationen konsultieren, obschon andere, mündliche Information überwiegt.

Einhellig geht aus der bisherigen Forschung hervor, dass die Nutzung im Parlament deutlich nach Phase sowie Akteur variiert. Während Parlamentsmitglieder für die abschliessende Entscheidung kaum systematisch generierte Informationen bedürfen und diese vorwiegend zu strategischen Zwecken nutzen, sind sie in früheren Phasen durchaus offener für solches Wissen und instrumentelle oder konzeptionelle Nutzung scheint 
eher möglich (Florio et al. 1979, 82; Whiteman 1985a, 299). Auch nutzen Parlamentsmitglieder systematisch generiertes Wissen nicht routinemässig und systematisch (vgl. Webber 1987). Die Nutzung konzentriert sich zudem auf die Kommissionsmitglieder oder Parlamentsmitglieder, die ein Geschäft initiieren (Mooney 1993, 191; 2012, 31 f.; Songer 1988, 380). Ein Grund dafür ist gemäss Mooney $(1993,195)$ der engere Bezug zu Geschäften: "Legislators get the most benefit from succeeding in their committee work, on bills they sponsor, and on important bills, and it is here that legislative organization and incentives inspire them to search hardest for information." Dieser Anreiz verstärke sich noch, da Parlamentsmitglieder wenn möglich an jenen Kommissionen teilnehmen, bei denen sie ein starkes Interesse haben (Mooney 1993, 187; 2012, 11-12). Ebenso haben Kommissionen mit den Hearings einen privilegierten Zugang zu systematisch generierten Wissen.

In Übereinstimmung mit den Ergebnissen zur Nutzung von systematisch generiertem Wissen ist auch dessen Bedeutung weniger fassbar und variiert nach Geschäft. Einen substantiellen Einfluss scheint solches Wissen generell nicht zu haben und sich - wenn überhaupt - auf einzelne Geschäfte oder Phasen zu beschränken (Florio et al. 1979, 84; Malen et al. 1988; Weiss 1989, 429; Whiteman 1995, 181). Demgemäss ist systematisch generiertes Wissen auf subtilere Art im Parlament bedeutsam (Hird 2005a, 2005b). Dies äussert sich etwa in einem Einfluss von systematisch generiertem Wissen auf Debatten (Shulock 1999; Whiteman 1985a). Ebenso kann systematisch generiertes Wissen einen (symbolischen) Beitrag an die umfassendere Kontrollfunktion der Parlamente leisten (vgl. McDavid und Huse 2012, 21; Speer et al. 2015, 240).

\subsubsection{Forschungsstand: Erklärungsfaktoren des Kontexts zentraler als Angebot an systematisch generiertem Wissen}

Analog zur generellen Nutzungsforschung identifizieren auch die Forschungsbeiträge mit Fokus auf das Parlament eine Vielzahl an Erklärungsfaktoren der Nutzung. Gemäss der zuvor vorgestellten Einteilung von Cousins und Leithwood (1986) betreffen diese Erklärungsfaktoren einerseits Eigenschaften der Angebotsseite des systematisch generierten Wissens. Wiederholt weisen die Forschungsbeiträge auf die folgenden begünstigenden Erklärungsfaktoren hin: Relevanz für das zugrunde liegende Problem, eine hohe Aktualität, eine adäquate Aufbereitung sowie Präsentation der Ergebnisse in kurzem Bericht mit konziser, gut verständlicher Sprache (vgl. Apollonio und Bero 2017; Boyer und Langbein 1991; Brownson et al. 2016; Florio et al. 1979; Guston et al. 1997; Raudla 2012). Insgesamt scheint aber die Bedeutung dieser Erklärungsfaktoren für die parlamentarische Nutzung fraglich, da die Parlamentsmitglieder selten in direktem Kontakt mit einem Bericht sind (vgl. 3.2.1; Raudla 2012; Whiteman 1995). Zudem machen mehrere Forschende auf relevante Eigenschaften der Angebotsseite aufmerksam, die sich nicht auf einzelne Berichte beziehen. Demgemäss nutzen Parlamentsmitglieder systematisch generiertes Wissen häufiger bei Fragen, zu denen viel Forschung existiert (vgl. Apollonio und Bero 2009; Hird 2005b; Whiteman 1995). Gemäss Esterling (2004) hängt weiter die Nutzung 
in den Parlamenten davon ab, ob innerhalb der Forschung ein Konsens über ein Problem sowie dessen Lösungsansätze besteht.

Anderseits identifiziert die Nutzungsforschung zu Parlamenten diverse Erklärungsfaktoren auf der Nachfrageseite des Nutzungskontexts. Eine Gruppe dieser Erklärungsfaktoren bezieht sich auf die individuelle Ebene der Parlamentsmitglieder. Wenn auch mit teilweise divergierenden Resultaten, offenbaren die Beiträge Unterschiede in der Nutzung nach Ideologie, Amtserfahrung, Professionalisierung sowie Geschlecht (vgl. Bogenschneider et al. 2013; Hird 2005b; Mooney 1993; Raudla 2012; Speer et al. 2015). Weiter zeigen sich Unterschiede zwischen den Parlamentsmitgliedern in ihrer Einstellung gegenüber systematisch generiertem Wissen und ihrem Interesse daran (vgl. Guston et al. 1997, 462; Webber 1987). Bogenschneider und Corbett (2010, 137-141) erklären dies mit der Motivation der Parlamentsmitglieder. Mehr für Evidenz interessieren sollten sich so Parlamentsmitglieder, die sich stärker an Politikinhalten und weniger an der Wiederwahl orientieren. Andere Forschende vermuten, dass gebildetere Parlamentsmitglieder eine positivere Einstellung gegenüber systematisch generiertem Wissen und ein höheres Interesse daran haben (vgl. Esterling 2007, 102-103; Hardy 2016, 121).

Andere Erklärungsfaktoren der Nachfrageseite betreffen die Ebene des Parlaments. Die Professionalisierung, die (Macht-)Position des Parlaments sowie die Fluktuation seiner Mitglieder können die Nutzung gemäss der Forschung beeinflussen (vgl. Guston et al. 1997; Hird 2005b; Hird 2009; Raudla 2012). Weiter lassen Studien zum US-Kongress auf Unterschiede zwischen Parlamentskammern sowie zwischen Kommissionen schliessen (vgl. Esterling 2011, 190; Michaels und Gruszczynski 2016, 140; Weiss 1989, 422). Zu den Erklärungsfaktoren auf Ebene der Parlamente können daneben Eigenschaften gezählt werden, die generell die Funktionsweise der Parlamente beschreiben. Parlamentsmitglieder sind einem relativ starken Zeitdruck ausgesetzt. Sie müssen relativ rasch auf aktuelle Fragen reagieren können und beschäftigen sich mit verschiedenen Geschäften gleichzeitig (vgl. Hird 2009, 531; Weiss 1987, 97). Dies widerspricht dem langsamen, sorgfältigen Prozess der systematischen Generierung von Wissen. Verschiedene Forschende stellen schliesslich fest, dass direkter Kontakt mit den Parlamentsmitgliedern die Nutzung begünstigen kann (vgl. Bogenschneider und Corbett 2010, 132-134; Florio et al. 1979, 67; Hardy 2016, 120; Hird 2009, 533).

Zusätzliche Erklärungsfaktoren der Nachfrageseite beziehen sich auf die Art der diskutierten Frage. Viel Aufmerksamkeit schenkt die Nutzungsforschung zu Parlamenten dem Konfliktgrad einer Frage. Die Forschungsbeiträge zeigen, dass ein gewisser Konfliktgrad und damit verknüpft eine höhere Salienz und eine grössere öffentliche Aufmerksamkeit die Nutzung begünstigen kann (vgl. Mooney 1993; Shulock 1999; Zwaan et al. 2016). Allerdings weisen die Ergebnisse auch darauf hin, dass Parlamentsmitglieder ab einer gewissen Konfliktivität systematisch generiertes Wissen vorrangig symbolisch nutzen (vgl. Tabuga 2017; Whiteman 1985a; Whiteman 1985b). Verbunden mit dem Konfliktgrad stellt Shulock $(1999,238)$ für den US-Kongress fest, dass mehrfache Zuständigkeiten von Kommissionen zu einer höheren symbolischen Nutzung führen. Ein anderer diskutierter Erklärungsfaktor betrifft die Komplexität einer politischen Frage und die mit ihr verbundene Unsicherheit, die beide die Verwendung begünstigen sollten (Mooney 
1993, 191; Weiss 1989, 422). Schliesslich variiert die Nutzung von systematisch generiertem Wissen in Parlamenten auch gemäss Politikfeldern, die wenigen Beiträge dazu offenbaren aber kein eindeutiges Muster (vg. Speer et al. 2015; van Dooren 2004). Die Forschung zu EBP weist zudem darauf hin, dass sich die Eigenschaften der Parlamentsmitglieder und ihre Einstellung zu Forschung nach Interessengebiet unterscheiden können (Apollonio und Bero 2017, 4-5; vgl. Brownson et al. 2016, 1037).

\subsection{Besonderheiten des schweizerischen Kontexts}

Neben Besonderheiten des parlamentarischen Kontexts gilt es auch Besonderheiten des schweizerischen politischen Systems sowie der schweizerischen Parlamente zu identifizieren, die relevant sind für die Nutzung von systematisch generiertem Wissen durch die Parlamentsmitglieder. Dazu zählt die Entwicklung der Evaluation in der Schweiz sowie deren Wechselwirkung mit den Eigenschaften des politischen Systems sowie der Parlamente. Der letzte Abschnitt befasst sich mit dem Stand der Nutzungsforschung in der Schweiz.

\subsubsection{Stand der Evaluation in der Schweiz und ihren Parlamenten}

Die Evaluation entwickelte sich in der Schweiz massgeblich in den 1990er-Jahren und hat sich heute klar etabliert (vgl. Bussmann 2008; Widmer 2017). Diese Dynamisierung (Widmer 2007, 89) der Evaluation wesentlich gefördert haben die interdepartementale Arbeitsgruppe Gesetzesevaluation (AGEVAL), die das Eidgenössische Justiz- und Polizeidepartement (EJPD) von 1987 bis 1991 einsetzte, sowie das Nationale Forschungsprogramm zur Wirksamkeit staatlicher Massnahmen (NFP 27), das von 1990 bis 1995 auf Vorschlag des Bundesrats lief. Weitere Meilensteile in der Entwicklung der Evaluation sind die Gründung der Schweizerischen Evaluationsgesellschaft (SEVAL) 1996 und die Verabschiedung ihrer ersten Evaluationsstandards 2001 sowie die Aufnahme des Artikels 170 in die Bundesverfassung (vgl. Balthasar 2009b, 228; Bussmann 2008; Mader 2005, 31; Spinatsch 2002, 386 f.; Widmer und Neuenschwander 2004, 389). ${ }^{15}$ In Kraft seit 2000, lautet der Artikel wie folgt: «Die Bundesversammlung sorgt dafür, dass die Massnahmen des Bundes auf ihre Wirksamkeit überprüft werden.» Heute gehört die Schweiz international gesehen zu den führenden Staaten in der Evaluation. Eine Besonderheit im internationalen Vergleich bildet gerade die Verankerung der Evaluation in der Verfassung (Jacob et al. 2015, 13). ${ }^{16}$

Neben der institutionellen Verankerung etablierte sich auch die Evaluationstätigkeit in der Schweiz auf hohem Niveau, sie unterscheidet sich aber nach Politikbereich (Balthasar 2007, 2009a; 2015, 121 f.). Die meiste Evaluationstätigkeit findet in den Bundesäm-

151999 führte der Bundesrat weiter die Regulierungsfolgenabschätzung (RFA) sowie 2006 die vertiefte RFA ein (Parlamentarische Verwaltungskontrolle 2005, 13; Rissi und Sager 2013; Sager und Rissi 2011).

16 In früheren Studien nahm die Schweiz noch eine Position in der zweiten Hälfte ein (Furubo et al. 2002). Diese Einschätzung wurde aber kritisch diskutiert (vgl. Janett 2003b; Varone und Jacob 2004). 
tern statt (vgl. Widmer 2007). Die Intensität, mit der die Bundesämter Evaluationen durchführen, und auch ihr genereller Umgang mit Evaluationen variiert aber deutlich (vgl. Widmer und Neuenschwander 2004; Widmer et al. 2001, 83) Balthasar Strotz 2017. Als regelmässig bezeichnet Balthasar (2009a, 497) die Evaluationsaktivität in etwa einem Drittel der Bundesämter, wobei Evaluationen besonders verbreitet sind in Fragen zur Entwicklungszusammenarbeit, zu den Sozialversicherungen sowie zur Arbeitsmarkt-, Gesundheits- oder Energiepolitik (für eine Aktualisierung siehe Balthasar 2015, 121 f.) Balthasar Strotz 2017.

In der Bundesversammlung ist die Evaluation gesetzlich sowie organisatorisch verankert. Die Institutionalisierung der Evaluation in der Bundesversammlung begann zunächst im Rahmen der Oberaufsicht. Als Reaktion auf mehrere Krisen der Verwaltungsführung schuf die Bundesversammlung mit der PVK ihren eigenen Evaluationsdienst, der 1991 die operative Tätigkeit aufnahm. Als Fachstelle der Geschäftsprüfungskommissionen soll die PVK zu einer professionelleren, systematischeren und wirkungsvolleren Oberaufsicht beitragen (Janett 2004, 139; 2009, 125 f.). Seit Anfang der 2000er-Jahre ist die Evaluation zudem stärker in den gesetzlichen Grundlagen des Parlaments verankert (Schwab 2005). Artikel 170 der Bundesverfassung, der erst in den parlamentarischen Beratungen eingefügt wurde, richtet sich etwa konkret an die Bundesversammlung (vgl. Schwab 2005, 40; Widmer 2007, 77). Dies bedeutet jedoch laut Mader $(2009,55)$ nicht, dass die Bundesversammlung selbst Evaluationen durchführen, sondern vielmehr die Überprüfung der Wirksamkeit sicherstellen muss. Das Parlamentsgesetz ${ }^{17}$, das ab 2003 in Kraft trat, sieht weiter die Überprüfung der Wirksamkeit als Aufgabe der Kommissionen vor und verankert die prospektive Evaluation (vgl. Bussmann 2008; Mader 2009, 56 f.). ${ }^{18}$ Insgesamt ist diese Stellung der Evaluation im schweizerischen Parlament international gesehen aussergewöhnlich stark: Unter 19 untersuchten Ländern der OECD erhielt nur die Schweiz den Maximalwert für die Institutionalisierung im Parlament (Jacob et al. 2015, 13, 19).

Mit der Institutionalisierung der Evaluation in der Bundesversammlung verbunden ist auch die Frage nach deren praktischer Bedeutung. Bättig und Schwab $(2015,20)$ stellen fest, dass sich die Evaluation zwar klar als Instrument der Oberaufsicht etabliert hat, ihr aber (noch) nicht eine umfassende Bedeutung zukommt (vgl. Janett 2004, $147 \mathrm{f}$.; Schwab 2005). Für die praktische Relevanz der Evaluation in der Bundesversammlung spricht hingegen, dass die Parlamentsmitglieder selbst Evaluationen anregen (vgl. Mader 2009, 61). Ein Mittel dafür stellt der Erlass von Evaluationsklauseln dar, die jedoch meistens bereits im Gesetzesentwurf der Exekutive integriert sind (Bussmann 2010, 282; Wirths 2017, 496). Ein anderes und oft verwendetes Mittel zur Anregung von Evaluationen sind parlamentarische Vorstösse, besonders Postulate (Bundi et al. 2016; Eberli und

17 Bundesgesetz über die Bundesversammlung vom 13. Dezember 2002 (Parlamentsgesetz, ParlG), SR 171.10 .

18 Das neue Parlamentsgesetz ermöglicht auch den Legislativkommissionen, die PVK mit Evaluationen zu beauftragen (Janett 2009, 126). 
Bundi 2017; Janett 2004, 145). ${ }^{19}$ Wie häufig Parlamentsmitglieder dieses Mittel verwenden, unterscheidet sich je nach Parlamentsmitglied und Politikfeld. Parlamentsmitglieder regen etwa besonders Evaluationen mittels Vorstössen an, wenn sie auf die Funktion der Aufsicht fokussieren (Bundi 2016). Auch ist die Nachfrage besonders ausgeprägt in den Politikfeldern der Infrastruktur, Bildung und der öffentlichen Finanzen, wobei die politikfeldspezifische Nachfrage nach Partei variiert (Bundi 2018b; Eberli und Bundi 2017; Eberli et al. 2014).

Die wenigen Arbeiten zum Stand der Evaluation in den Kantonen deuten darauf hin, dass die Evaluation auf kantonaler Ebene ebenfalls etabliert ist, wenn auch in geringerem Masse. Mehrere Erhebungen zeigen, dass die Evaluation in den Kantonen gesetzlich verankert ist (Horber-Papazian 2006, 137 f.; Wirths und Horber-Papazian 2016, 489) Wirths et al. 2017. Die Evaluationstätigkeit fällt aber deutlich geringer als beim Bund aus. Balthasar und Rieder $(2009,409,423)$ stellen fest, dass lediglich in den Kantonen Zürich, Bern, Genf und - wenn auch in deutlich geringerem Ausmass - St. Gallen von einer systematischen Evaluationstätigkeit gesprochen werden kann (vgl. Balthasar 2010). Die Evaluationen auf Kantonsebene konzentrieren sich hauptsächlich auf den Bildungsbereich (vgl. Frey et al. 2016, 513). Auch variiert gemäss der Untersuchung von Dolder et al. 2017 zu Basel-Stadt, Bern und Luzern die Evaluationskultur stärker nach Politikbereich denn nach Kanton. Im zeitlichen Vergleich stellen sie zudem durchaus Entwicklungen in der Evaluationskultur fest, aber nicht nur Weiter- sondern auch Rückentwicklungen. Bis auf die Studien von Dolder et al. 2017 sind detaillierte Informationen über die Evaluationspraxis in einzelnen Kantonen oder in den Städten und Gemeinden aber nur vereinzelt verfügbar, namentlich zu dem Kanton und der Stadt Bern sowie zum Kanton Genf (Delley 2005; Holzer et al. 2003; Nuspliger 2005; Ritz und Sager 2010).

Die Stellung der Evaluation in den Kantonsparlamenten ist kaum erforscht. Ähnlich wie ihre Pendants in der Bundesversammlung regen die Mitglieder der Kantonsparlamente relativ häufig Evaluationen an (Eberli und Bundi 2017). Auch veranlassen sie in seltenen Fällen die Integration von Evaluationsklauseln in ein Gesetz (Wirths und Horber-Papazian 2016, 496). Zudem gab es im Zuge der wirkungsorientierten Verwaltungsführung als schweizerische Auslegung des NPM verschiedene Reformen der Parlamente, die ihnen eine Leistungs- und Wirkungsorientierung ermöglichen sollten (vgl. Schmidt 2008; Widmer und Rüegg 2005). Da die Wirkungsorientierung zentral in der schweizerischen Auslegung des NPM ist, verstärkten die NPM-Reformen auch die Stellung der experimentellen Gesetzgebung und der Evaluation (Arnold 2008, 136-136; Schedler und Schmucki 2009, 9-10; Widmer 2002). Allerdings ist unklar, was diese Reformen für den parlamentarischen Alltag bedeuten. Verschiedene Forschende weisen darauf hin, dass die Parlamentsmitglieder in den Kantonen nach den NPM-Reformen mehr Leistungs- und Wirkungsinformation erhalten, aber die geänderte Arbeitsweise eine grössere

19 Die folgenden Abschnitte verweisen teilweise auf Arbeiten, die im selben Forschungsprojekt wie die vorliegende Untersuchung entstanden sind. Namentlich: Bundi (2016, 2018a, 2018b); Bundi et al. (2017, 2018); Bundi et al. (2016); Eberli (2018); Eberli und Bundi (2017); Eberli et al. (2014); Eberli et al. (2018). Die Verweise beziehen sich aber ausschliesslich auf Fragen, die in der folgenden Untersuchung nicht oder nicht ausführlich abgedeckt werden. 
zeitliche Belastung für sie bedeutet (Haering 2012, 12; Rieder und Widmer 2007; Summermatter und Demaj 2012, 171).

\subsubsection{Evaluation in der Wechselwirkung mit den politischen Institutionen der Schweiz}

Angesichts des Sonderfalls Schweiz befassen sich schweizerische Beiträge intensiv mit der Frage, inwiefern sich die Besonderheiten des politischen Systems auf die Entwicklung der Evaluation und deren Nutzung auswirken (vgl. Rissi und Sager 2013). ${ }^{20}$ Generell lassen die Beiträge darauf schliessen, dass das politische System der Schweiz ein Hemmnis für die Evaluation bildet, die Wirkung der einzelnen, zentralen Elemente wie des Föderalismus und der direktdemokratischen Mitwirkungsrechte wird aber kontrovers diskutiert (vgl. Bussmann 2008; Widmer und Neuenschwander 2004). Besondere Beachtung in der Literatur zur Evaluation im politischen System der Schweiz erhält die Konsensdemokratie. Institutionalisierte Konfliktlösungsmechanismen wie namentlich das Vernehmlassungsverfahren dienen dazu, eine mehrheitsfähige Lösung zu finden und letztlich ein Referendum oder zumindest dessen Annahme zu verhindern. Dies begrenzt auch den möglichen Spielraum einer (mehrheitsfähigen) Lösung und beschränkt damit den Beitrag von Evaluationen und ihren Ergebnissen (vgl. Bussmann 2008, 501; Klöti und Schneider 1989, 235; Widmer und Neuenschwander 2004, 391). Das Verfahren der Vernehmlassung vermindert gerade den Bedarf und das Interesse für prospektive Evaluationen wie die RFA (Rissi und Sager 2013; Sager und Rissi 2011). Gleichzeitig kann die Konsensdemokratie Chancen für die Evaluation bieten. Die längere Dauer der Entscheidungsprozesse sowie der vielfältige Einbezug von Akteurinnen und Akteuren schaffen auch mehr Eintrittspunkte und Nutzungsgelegenheiten für die Evaluation (Frey 2010, 668; 2012, 306; Mader 2009, 62; Sager et al. 2017b). Ebenso bedarf es möglicherweise Evaluationen, um eine mehrheitsfähige Lösung zu finden (Widmer 2007, 89).

Neben diesen generellen Rahmenbedingungen des schweizerischen politischen Systems stellt sich auch die Frage, wie sich die Besonderheiten der Schweizer Parlamente auf die Rolle und Nutzung von Evaluationen auswirken. Mehrere Eigenschaften sprechen für formell starke Schweizer Parlamente. Die Parlamente haben verfassungsrechtlich eine starke Stellung und die Gewaltenteilung ist ausgeprägt (Lüthi 2014, 170; Vatter 2014, 264). Für die Evaluation und deren Nutzung besonders wichtig sind die Kommissionen, die in den Schweizer Arbeitsparlamenten eine zentrale Rolle einnehmen (Lüthi 2005, 4; vgl. 2.3.1; Mattson und Strøm 1995, 299). Die beiden Räte der Bundesversammlung verfügen seit der Reform von 1992 über ständige Legislativkommissionen. Dazu kommen die ebenfalls ständigen Geschäftsprüfungs- und Finanzkommissionen, die sich der Oberaufsicht widmen (Lüthi 2014, 174). Die Rechte und Stellung der Kommissionen in den Kantonsparlamenten sind grundsätzlich ähnlich wie auf Bundesebene, die Bezeichnungen, das System und die Stärke der Kommissionen unterscheiden sich aber (vgl. Heierli 2000; Trippolini 2007). Trippolini $(2007,26)$ identifiziert drei Typen von Kommissionensystemen in den Kantonsparlamenten: (1) Systeme mit ständigen Legis-

20 Für eine detaillierte Diskussion siehe Sager et al. (2017b). 
lativkommissionen, (2) Mischsysteme mit ständigen und nichtständigen Legislativkommissionen und (3) Systeme mit nichtständigen Legislativkommissionen. Die Aufsichtskommissionen sowie die Querschnittskommissionen, die sich etwa der Redaktion von Erlassen widmen, sind in der Regel ständig. Gemäss der letzten Aktualisierung von Ladner und Leuenberger $(2012,5)$ hat etwa die Hälfte der Kantone ein System mit ständigen Legislativkommissionen, während je ein Viertel der Kantone über ein Mischsystem beziehungsweise über ein System mit nichtständigen Legislativkommissionen verfügt. ${ }^{21}$ Es ist aber - gerade im Zuge von NPM - ein Trend zu ständigen Kommissionsystemen beobachtbar (Ladner und Leuenberger 2012, 27; Lienhard et al. 2011, 107; Lüthi 2005, 4). ${ }^{22}$

Die Schweizer Parlamente verfügen zugleich über mehrere Eigenschaften, die diese formelle Stärke relativieren und auch die Rolle der Evaluation einschränken dürften. Die Parlamente greifen nach dem langen Vorbereitungs- und Konsultationsprozess zu einem relativ späten Zeitpunkt in das Gesetzgebungsverfahren ein (Lüthi 2014, 188). Weiter haben die Stimmberechtigten bei gewissen Fragen die letzte Entscheidungskompetenz. Gerade in den Kantonen verfügen die Stimmberechtigten über umfangreiche direktdemokratische Rechte und wählen auch die Regierung (Bochsler et al. 2004, 29; Vatter 2014).

Die geringe Professionalisierung in den schweizerischen Milizparlamenten vermindert weiter ihre formelle Stärke und möglicherweise ihre Evaluationskapazität. Gemäss Z'graggen $(2009,156)$ ist die Schweiz insgesamt das am zweitwenigsten professionalisierte Parlament der OECD-Staaten (vgl. Z'graggen und Linder 2004, 18). Die Institution der Bundesversammlung ist kaum professionalisiert, da sie verhältnismässig wenig Mitarbeitende zählt (vgl. Z'graggen 2009, 161). Bezogen auf die einzelnen Mitglieder und ihren Zeitaufwand kann hingegen mindestens von einem Halbberufsparlament (Riklin und Ochsner 1984, 110) gesprochen werden. Bereits in den 1970er-Jahren beobachtet Kerr (1981, 239 f.), dass die Parlamentsmitglieder im Schnitt 55 Prozent ihrer Zeit für das Mandat verwenden. Gemäss seiner Definition waren damals insgesamt drei Viertel der Bundesparlamentsmitglieder keine Milizparlamentsmitglieder, da sie mehr als ein Drittel ihrer Zeit in das Parlamentsmandat investierten. Neuere Erhebungen weisen auf noch klarere Gegensätze zum Milizsystem hin. In der 49. Legislatur von 2011 bis 2015 investierten die befragten Mitglieder des Nationalrats im Median 70 Prozent ihrer Zeit in das Mandat, während die befragten Mitglieder des Ständerats 77 Prozent ihrer Zeit für das Mandat aufwendeten (Bütikofer 2013, 69; Sciarini et al. 2017, 34). Damit überein stimmt die Beobachtung, dass sich Parlamentsmitglieder - wenn auch zögerlich - immer häufiger als Berufspolitikerin oder Berufspolitiker bezeichnen (Bütikofer 2013, 74; Pilotti 2017, 304; Pilotti et al. 2010, 237; Riklin und Ochsner 1984, 100).

In den Kantonsparlamenten ist die Professionalisierung wenig erforscht, sie können aber insgesamt klar als Milizparlamente bezeichnet werden. Die Institutionen der

21 Gemäss der aktualisierten, nicht publizierten Darstellung von Gander $(2017,14)$ haben 16 Kantonsparlamente ständige, 6 Kantonsparlamente nichtständige Legislativkommissionen und 4 Kantonsparlamente ein gemischtes System.

Zur Stärke der Kommissionen noch vor den besagten Parlamentsreformen siehe Heierli (2000). 
Kantonsparlamente verfügen kaum über ausgebaute Parlamentsdienste, die nicht immer dem Parlament unterstellt sind und sich vorrangig organisatorischen Tätigkeiten widmen (Trippolini 2007, 29f.). Weiter sind die Fraktionsbeiträge relativ gering (Bochsler et al. 2004, 36 f.). Gemäss der Befragung, die auch für die folgende Untersuchung verwendet wird, beträgt der durchschnittliche Zeitaufwand für ein kantonales Parlamentsmandat 20 Prozent. Zwischen den Kantonen sind aber deutliche Unterschiede sichtbar: Parlamentsmitglieder in der lateinischen Schweiz sowie in grossen Parlamenten investieren verhältnismässig mehr Zeit für ihr Mandat (Bundi et al. 2017, 9 f.; 2018; Eberli et al. in press). Ein historischer Vergleich dieser Ergebnisse ist angesichts fehlender Daten kaum möglich, nur Blum (1978) liefert Anhaltspunkte für die Professionalisierung in einzelnen Kantonsparlamenten.

\subsubsection{Forschungsstand: Nutzung beobachtbar, gewichtige Stellung der Verwaltung in der Konsensdemokratie}

Die Frage der Nutzung von Evaluationen oder anderem systematisch generierten Wissen ist auch in der Schweiz, besonders auf Bundesebene, relativ breit erforscht. Meist fokussieren die Beiträge auf die Verwaltung und die Regierung als Nutzerinnen von systematisch generiertem Wissen bei der Formulierung oder Umsetzung von Politik. ${ }^{23}$ Die Ansätze und Methoden der Beiträge zur Schweiz sind ähnlich wie in der internationalen Forschung (2.1.4; vgl. Frey und Ledermann 2017).

Während die Forschenden in den ersten Untersuchungen kaum Nutzung in der Bundesverwaltung beobachten, zeichnen die späteren Forschungsbeiträge ein positiveres Bild (vgl. Balthasar 2007; Frey 2012; Klöti und Schneider 1989; Zimmermann 1990). Fallstudien und Befragungen zeigen, dass die Bundesverwaltung Evaluationen instrumentell nutzt, wobei sie dies häufiger bei der Umsetzung als bei der Formulierung der Politik tut (Balthasar 2007; 2009a, 493; Fornerod 2001, 31; Frey 2012, 278; Frey und Ledermann 2017, 234; Ledermann 2014, 475). Anderseits dienen Evaluationen der Bundesverwaltung auch zur Mobilisierung von Unterstützung. Dies geschieht nicht nur im Abstimmungskampf (vgl. Schlaufer und Stucki 2017, 74; Stucki 2016, 499), sondern bereits in frühen Phasen des Gesetzgebungsprozesses. Mehrere Forschende weisen daraufhin, dass bereits die Beschaffung von systematisch generiertem Wissen interessengetrieben erfolgt, um die Position zu stärken (Frey 2012, 271; Himmelsbach 2014, 263; Klöti und Schneider 1989, 233). Zudem werden nicht nur Ergebnisse von Evaluationen, sondern auch deren Durchführung als politisches Mittel verwendet (Rissi und Sager 2013, 361). In den Kantonen ist die Frage der Nutzung dagegen wenig erforscht. Eine Untersuchung von sechzig Gesetzgebungsprozessen weist daraufhin, dass kantonale Verwaltungen Evidenz selten systematisch aufbereiten (Balthasar und Müller 2014, 88; vgl. Blatter et al. 2015).

23 Einen ausführlichen Überblick über die Forschung zur Nutzung durch Regierung und Verwaltung geben Frey und Ledermann (2017), siehe auch Widmer et al. (2016). 
Die Forschungsbeiträge zeigen insgesamt auf, dass die Bundesverwaltung eine wegweisende Rolle in der Beschaffung und Verwendung von systematisch generiertem Wissen einnimmt: "The ability to access and control scientific expertise is strongly concentrated in the hands of the federal administration" (Himmelsbach 2014, 266; vgl. Balthasar 2007, 2009; Frey 2012). Neben ihr bringen auch Fachpersonen aus der Forschung, Fachstellen oder ausserparlamentarischen Kommissionen systematisch generiertes Wissen in den Politikprozess. Interessenorganisationen, Kantone und Gemeinden hingegen tragen wenig zu einer evidenzbasierten Politikgestaltung bei (Frey 2012, 302-304; Himmelsbach 2014, 255).

Weiter lässt sich insgesamt schliessen, dass systematisch generiertem Wissen in der schweizerischen Politik durchaus eine Bedeutung zukommt. Direkte Umsetzungen einer Evaluation oder Prozesse im Sinne einer evidenzbasierten Politik stellen aber eine Minderheit dar (Frey 2012, 273; Nideröst 2002). Zudem befasst sich ein kleiner Kreis mit Fachwissen (Himmelsbach 2014, 259). Dennoch kann es Diskussionen auslösen und in diese einfliessen, was wiederum zu einem informierten Diskurs beitragen kann (vgl. Frey und Ledermann 2017; Schlaufer 2018; Stucki 2018).

Auch die Beiträge zur Schweiz weisen auf eine Vielzahl an Faktoren hin, die zur Erklärung der Nutzung beitragen. Neben anderen Faktoren, die auch die internationale Nutzungsforschung identifiziert (2.2.1), betonen Forschende im schweizerischen konsensorientierten Kontext besonders die Wichtigkeit des Konfliktgrads (vgl. Blatter et al. 2015; Frey 2012). Ist eine Frage konfliktiv, erschwert dies die Berücksichtigung von systematisch generiertem Wissen in der Regel bereits in frühen Phasen und führt zu einer selektiven, symbolischen Verwendung (vgl. Freiburghaus und Zimmermann 1985; Frey 2010, 684 f.; Rissi und Sager 2013, 360). Einzelne Studien weisen aber auch daraufhin, dass ein hoher Konfliktgrad die Nutzung bereits zu Beginn des Gesetzgebungsprozesses blockieren kann (Ledermann 2014). Andere einzelne Studien zeigen demgegenüber auf, dass Evaluationen bei einem hohen Konflikt und Veränderungsdruck ein Umdenken bringen können (Ledermann 2012, 173; Nideröst 2002, 50).

Im Gegensatz zur Exekutive waren die Parlamente bisher selten Gegenstand der Nutzungsforschung über die Schweiz. Explizit der Bundesversammlung widmen sich vor allem Erfahrungsberichte zur Einführung und Entwicklung der PVK (Bättig und Schwab 2015; Janett 2001, 2003a, 2004, 2009; Schwab 2005). Daneben geben Fallstudien zur Verwendung von systematisch generiertem Wissen in Gesetzgebungsprozessen Einblick in die parlamentarische Nutzung (Frey 2010, 2012; Frey und Widmer 2011, 2013; Himmelsbach 2014). Verhältnismässig besser erforscht sind die kantonalen Parlamente, wobei diese Analysen auf die Verwendung von NPM-Instrumenten fokussieren (Brun 2003; Brun und Siegel 2006; Demaj et al. 2012; Horber-Papazian 2011; Summermatter und Demaj 2012). ${ }^{24}$ Methodisch stützen sich die Forschenden auf Dokumentenanalysen, Interviews oder Befragungen, in einem Fall auch auf ein Experiment (Demaj et al. 2012).

24 Brun (2003) sowie Brun und Siegel (2006) befassen sich mit den Parlamenten auf Bundes- und Kantonsebene. 
Übereinstimmend mit den Ergebnissen für andere Parlamente lassen diese Forschungsarbeiten darauf schliessen, dass die Mitglieder der Bundesversammlung Evaluationen grösstenteils für nützlich halten (Balthasar 2009a; Bättig und Schwab 2015; Eberli und Bundi 2017; Eberli et al. 2014; Janett 2001, 2009). Einzelne Beiträge weisen aber darauf hin, dass sie Evaluationen als theoretisch einschätzen und anderen Informationsinstrumenten wie namentlich der Vernehmlassung ein höheres Gewicht geben (Parlamentarische Verwaltungskontrolle 2005; Schwab 2005). Weiter betont Fornerod (2001, 71), dass die Parlamentsmitglieder Evaluationen vor allem als Instrument der Oberaufsicht sehen. An systematisch generiertes Wissen gelangen gemäss Eberli und Bundi $(2017,262)$ fast sämtliche der befragten Parlamentsmitglieder der Bundesversammlung. Am häufigsten erfahren sie über die parlamentarischen Kommissionen oder die Parlamentsdienste von Evaluationen. Gemäss den Fallstudien von (Frey 2012, 288) und namentlich Himmelsbach $(2014,255,258)$ verfügt die Verwaltung dennoch über eine gewisse Definitionsmacht der Expertise im Parlament, da das Parlament und Parteien nur punktuell selbst Expertise organisieren.

Die Beiträge zeigen weiter auf, dass Evaluationen in der Bundesversammlung in verschiedenen Formen Verwendung finden und ihnen damit eine gewisse Bedeutung zukommt. Parlamentsmitglieder lesen aber meist Zusammenfassungen, selten ganze Evaluationsberichte (Eberli und Bundi 2017, 263; Parlamentarische Verwaltungskontrolle 2005). Zudem stellt Fornerod $(2001,73)$ fest, dass Evaluationen die Meinung der Parlamentsmitglieder nicht verändern, während sich Parlamentsmitglieder in einzelnen Fallstudien von Frey $(2012,306)$ durchaus an Evaluationen erinnerten und sich davon auch überzeugen liessen. Im Gegensatz zu diesen unterschiedlichen Ergebnissen zur instrumentellen oder konzeptionellen Nutzung beobachten die Forschenden übereinstimmend symbolische Nutzung in Form eines Aufgreifens von Evaluationen in den Beratungen (vgl. Frey 2012; Janett 2001). Auf die symbolische Nutzung taktischer Art macht weiter Bundi (2018a) in seiner Analyse der Anregung von Evaluationen mittels parlamentarischer Vorstösse aufmerksam. Insgesamt deuten die Beiträge auch in der Schweiz kaum auf einen substantiellen Einfluss von systematisch generiertem Wissen, wobei dieses Urteil für die Tätigkeiten der PVK positiver ausfällt (Bättig und Schwab 2015, 13 f.; Janett 2004, 141). Indem es aber teilweise Eingang in die Debatte findet und mitunter sensibilisierend wirkt, dient es auch im Parlament als eine Erweiterung der Entscheidungsgrundlagen (Frey 2012, 318; vgl. Janett 2001; Parlamentarische Verwaltungskontrolle 2005).

Gemäss der bisherigen Forschung schätzen auch die kantonalen Parlamentsmitglieder Evaluationen und verwenden diese. Übereinstimmend mit den Befunden zur Bundesversammlung, haltet mit über zwei Dritteln die Mehrheit der befragten kantonalen Parlamentsmitglieder Evaluationen generell für nützlich und liest Zusammenfassungen. Allerdings variieren diese Angaben deutlich zwischen Partei und Kantonen: Befragte Parlamentsmitglieder aus der lateinischen Schweiz halten Evaluationen für deutlich nützlicher als Befragte Parlamentsmitglieder aus der Deutschschweiz. Besonders positiv gegenüber Evaluationen eingestellt sind zudem die befragten SP-Parlamentsmitglieder, während sich SVP-Parlamentsmitglieder zurückhaltender äussern. Auch lässt sich 
feststellen, dass die befragten Mitglieder der Bundesversammlung Evaluationen häufiger lesen und als nützlicher befinden als Mitglieder der kantonalen Legislativen (Eberli und Bundi 2017, 263-265). Diese Ergebnisse decken sich auch mit der Untersuchung zu NPM-Instrumenten in den Räten der Kantone Aargau und Zürich (Demaj et al. 2012, 38). Einerseits verwenden die Mitglieder dieser Parlamente solche Instrumente, um sich einen Überblick zu verschaffen oder um Vorstösse zu formulieren (Demaj et al. 2012, 38 f.; Summermatter und Demaj 2012, 177). Anderseits ist für diese Parlamentsmitglieder auch relevant, dass die Exekutive mit diesen Instrumenten Rechenschaft über ihre Tätigkeiten ablegen muss (Summermatter und Demaj 2012, 176 f.). Eine symbolische Nutzung in Form von Verweisen stellen Horber-Papazian $(2011,140)$ und Blatter et al. (2015) schliesslich nur selten fest.

Die (geringe) Forschung zu schweizerischen Parlamenten weist auf viele verschiedene Erklärungsfaktoren hin und stützt damit die internationale Forschung. Als Hindernisse für die Evaluationsnutzung im schweizerischen Milizsystem sehen die Forschenden die Position des Parlaments gegenüber der Verwaltung, Zeitdruck sowie die Fluktuation der Mitglieder (vgl. Demaj et al. 2012, 46; Frey und Widmer 2011, 509; Horber-Papazian 2011, 139). Der direkte Kontakt mit beispielsweise Fachstellen stellt daher ein Mittel dar, damit systematisch generiertes Wissen Eingang in das Parlament findet (Frey 2012, 316).

\subsection{Zwischenfazit: Folgerungen aus der bisherigen Forschung}

Welche Schlüsse lassen sich aus der präsentierten Literatur und Forschung in Bezug auf die Nutzung von Evaluationen in den Schweizer Parlamenten ziehen? Da Evaluationen als eine von verschiedenen Formen von systematisch generiertem Wissen betrachtet werden, kann sich die Erforschung dieser Frage auf die Erkenntnisse der breiteren Nutzungsforschung stützen. Der Überblick zu dieser Nutzungsforschung verdeutlicht, dass das Konzept der Nutzung historisch gewachsen, vielschichtig und schwer fassbar ist. Es gibt diverse Herangehensweisen, um unterschiedliche Formen der Nutzung zu typologisieren (vgl. 2.1.2, z. B. Boswell 2009, 55; Frey 2012; Vedung 1997). Diese verschiedenen Herangehensweisen veranschaulichen die Schwierigkeit, die Nutzung greifbar zu machen und deren Formen klar zu trennen. Trotz der unterschiedlichen Typologien ist zudem die bisherige Forschung auf die die instrumentelle sowie die konzeptionelle Nutzung fokussiert (vgl. Albaek 1995; Johnson et al. 2009). Auch wird gerade in der Forschung über Evaluation die positive Konnotation der Nutzung kontrovers diskutiert (Henry 2000, 95).

Die bisherige Literatur und Forschung lässt erwarten, dass Nutzung von Evaluationen oder anderem systematisch generierten Wissen auch im parlamentarischen Kontext beobachtbar sein sollte, jedoch besonders häufig in symbolischer Form. Wie die Four Is aufzeigen, beeinflussen ideologische und interessenbasierte Gesichtspunkte Entscheidungen der Parlamentsmitglieder als Volksvertreterinnen und Volksvertreter ebenso (Weiss 1995, 1999). Folglich sollten Parlamentsmitglieder systematisch generiertes Wissen vorrangig als politisches Mittel verwenden, um ihre Positionen zu stützen und durchzusetzen (vgl. Shulock 1999; Weiss 1987, 1989; Whiteman 1985b). 
Der bisherige Wissensstand zum Zugang und zur Vermittlung von Informationen in Parlamenten legt nahe, dass wenige, spezialisierte Mitglieder in den Kommissionen sowie die indirekte, mündliche Vermittlung zentral für die Nutzung von Evaluationen im Parlament sein sollten (vgl. Mooney 1993, 2012; Webber 1986; Weiss 1987, 1989). Kommissionen befassen sich vertieft mit einer Frage und nehmen gerade in den Schweizer Arbeitsparlamenten eine wichtige Stellung zur Erfüllung der parlamentarischen Tätigkeiten ein (Lüthi 2005, 2014). Parlamentsmitglieder erfahren zwar in den Kommissionen von Evaluationen, aber Fachpersonen und in den schweizerischen Milizparlamenten auch die Verwaltung sollten eine wesentliche Rolle bei der Vermittlung und Aufbereitung von Fachwissen spielen (vgl. Frey 2012; Himmelsbach 2014; Whiteman 1995). Ausserdem dürfte die mündliche Kommunikation in Parlamenten bedeutsam sein (Hird 2009; Raudla 2012, 1009; Weiss 1987, 98; 1989, 414).

Der Überblick zur Forschung und Literatur zählt weiter eine Vielfalt von Erklärungsfaktoren der Nutzung von systematisch generiertem Wissen auf. Diese Erklärungsfaktoren betreffen sowohl das Angebot wie auch die Nachfrage von systematisch generiertem Wissen oder Evaluationen. Dieser Nutzungskontext auf der Nachfrageseite scheint zentral, da er Charakteristika der Nutzenden und ihrer Organisation sowie Eigenschaften der Entscheidungsprozesse umfasst. Was Schlüsselfaktoren sind und welchen Effekt die Erklärungsfaktoren auf die verschiedenen Nutzungsformen haben, bleibt aber unklar, gerade da die bisherigen Beiträge Nutzung unterschiedlich messen und zumeist von Nutzung generell ausgehen (Nutley et al. 2007). Zudem ist die bisherige Nutzungsforschung vor allem auf die konzeptionelle und instrumentelle Nutzung und nicht auf die im Parlament erwartungsgemäss zentrale symbolische Nutzung fokussiert (Johnson et al. 2009, 388).

Zur Erklärung der parlamentarischen Nutzung dürften Eigenschaften des Nutzungskontexts bedeutender sein als Eigenschaften auf der Angebotsseite, zumal sich Parlamentsmitglieder selten und direkt vertieft mit Evaluationsberichten befassen (Frey 2012; Whiteman 1995). In den schweizerischen Parlamenten sind zudem spezifische Einflüsse bei der Erklärung der Nutzung zu beachten. Etwa könnte das Milizsystem die Evaluationskapazität der Parlamentsmitglieder beeinflussen, ebenso prägen die konsensdemokratischen Mechanismen zur Konfliktlösung auch die parlamentarischen Gesetzgebungsprozesse.

Mit diesen Ausführungen zu den zentralen Punkten aus der bisherigen Literatur und Forschung schliesst dieses Kapitel. Sie bilden zugleich den Rahmen für die theoretischen Erläuterungen im folgenden Kapitel, in dem zunächst die Evaluationsnutzung konzipiert und danach Erklärungsfaktoren hergeleitet werden, die im parlamentarischen und schweizerischen Kontext bedeutsam sein sollten. 


\section{Theoretischer Rahmen: Evaluationsnutzung im Parlament und ihre Erklärungsfaktoren}

Im Folgenden wird der theoretische Rahmen erläutert, den Abbildung 1 zusammenfassend darstellt. Im Zentrum dieses Buchs steht die Evaluationsnutzung in den schweizerischen Parlamenten als zu erklärende Variable. Grundsätzlich wird zwischen der analytischen und politischen Form der Nutzung unterschieden, die verschiedene Arten annehmen können. Diese Konzeptualisierung beschreibt und diskutiert das folgende Unterkapitel. Die Auswahl der Faktoren zur Erklärung der Nutzung sowie die dazugehörigen Hypothesen werden danach erläutert. Analog zur bisherigen Literatur wird von einer Vielzahl an Erklärungsfaktoren ausgegangen. Im theoretischen Rahmen sind daher einerseits Merkmale des Parlamentsmitglieds als individuelle Erklärungsfaktoren integriert. Anderseits umfasst der theoretische Rahmen kontextuelle Erklärungsfaktoren: Merkmale des Parlaments als institutionelle Kontextfaktoren, Merkmale der Gesetzgebungsprozesse als politische Kontextfaktoren sowie evaluationsspezifische Kontextfaktoren. Anhand dieser vielfältigen Erklärungsfaktoren wird die Evaluationsnutzung umfassend betrachtet. Gleichzeitig fokussiert die Auswahl auf wesentliche Merkmale, die für die Evaluationsnutzung in den schweizerischen Parlamenten bedeutsam sein sollten.

Abbildung 1: Theoretischer Rahmen - Evaluationsnutzung und ihre Erklärungsfaktoren

Erfahrung
Parteizugehörigkeit
Professionalisierung
Bildungsgrad
Kommissionensystem
Wissenschaftsnähe
Konfliktivität
Rechtzeitigkeit und Aktualität
Übereinstimmung der
Evaluationsergebnisse

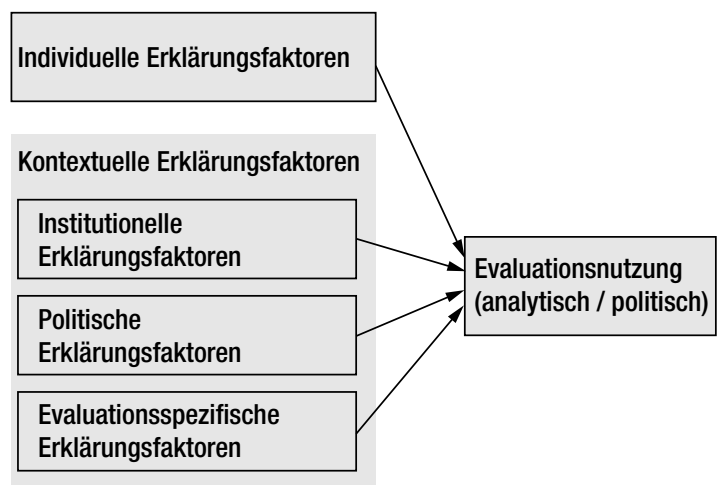

\subsection{Evaluationsnutzung: Analytisch und politisch}

Im Folgenden werden zwei Formen der Evaluationsnutzung unterschieden: Analytische und politische Nutzung (vgl. Abbildung 2). Bei der analytischen Nutzung dienen Evaluationen Entscheidungsträgerinnen und trägern dazu, sich zu informieren und ihre politische Position zu formen. Analytische Nutzung bedingt eine Offenheit gegenüber den Resultaten der Evaluationen unabhängig von der eigenen Position. Damit verbunden 
erfordert sie ebenso die Bereitschaft, die eigene Position zu überdenken. Boswell (2009, 248) spricht in diesem Zusammenhang von einer problemlösenden Absicht, Frey (2012, 61-64) von einer verbesserungsorientierten Absicht: Eine Politik soll mit einem analytischen Zugang durch die Nutzung von Evaluationen verbessert werden. Im Gegensatz dazu dienen Evaluationen Entscheidungsträgerinnen und trägern bei der politischen Nutzung als strategisches Mittel, um ihre Position zu unterstützen. Dazu zählt die Verwendung von Evaluationen zur Begründung oder Legitimation eines Standpunkts, aber auch zur Überzeugung anderer. Weiter können Entscheidungsträgerinnen und träger Evaluationen politisch verwenden, indem sie diese fordern, um einen Entscheid zu verzögern. Folglich bedarf die politische Verwendung keiner Offenheit gegenüber den Resultaten der Evaluationen und keiner Bereitschaft, die eigene Position zu überdenken. Die problemlösende Absicht fehlt ebenso und Evaluationen dienen bei der politischen Nutzung nicht als (inhaltliche) Grundlage zur Verbesserung.

\section{Abbildung 2: Arten und Unterscheidung der analytischen und politischen Nutzung}

\begin{tabular}{|l|l|l|}
\hline $\begin{array}{l}\text { Analytisch } \\
\text { Position formen }\end{array}$ & $\begin{array}{l}\text { Politisch } \\
\text { Position stützen } \\
\text { (symbolisch) }\end{array}$ \\
$\begin{array}{l}\text { Konzeptionell } \\
\text { sich informieren, Verständnis } \\
\text { erhalten } \\
\text { Instrumentell } \\
\text { Entscheid treffen }\end{array}$ & $\begin{array}{l}\text { bereit, eigene Position zu } \\
\text { überdenken } \\
\text { Persuasiv } \\
\text { andere überzeugen } \\
\text { Legitimierend } \\
\text { Entscheid begründen } \\
\text { offen gegenüber Evaluations- } \\
\text { ergebnissen }\end{array}$ & $\begin{array}{l}\text { Taktisch } \\
\text { Entscheid herauszögern }\end{array}$ \\
\hline
\end{tabular}

Diese Untersuchung fokussiert auf die Parlamentsmitglieder als Nutzerinnen und Nutzer von Evaluationen, was sich in zweierlei Hinsicht auf die Konzeption der Nutzung auswirkt. Erstens bezieht sich die Nutzung auf die Ergebnisse und weniger den Prozess einer Evaluation. Parlamentsmitglieder sind selten in Evaluationen involviert und verwenden Evaluationen entsprechend kaum in Folge ihrer Beteiligung an Evaluationsaktivitäten. Zweitens kann die Nutzung unterschiedlich geschehen. Parlamentsmitglieder können Evaluationen verwenden, indem sie Evaluationsberichte oder deren Kurzfassungen lesen. Informationen über Evaluationen vermitteln können ihnen auch Berichte von etwa Medien, Interessengruppen oder Nichtregierungsorganisationen (NGOs). Parlamentsmitglieder können ebenso Evaluationen nutzen, indem sie über Fachpersonen, Beteiligte aus der Exekutive oder andere Dritte von Evaluationen erfahren. Im Vergleich zu anderen Kontexten dürften mündliche Vermittlungsarten besonders bedeutsam im Parlament sein (vgl. 2.3.1; Mooney 1991b, 434; Weiss 1987, 98; 1989, 414; Whiteman 1995, 158). Insgesamt können also die Parlamentsmitglieder unterschiedlich stark beteiligt sein an der Suche, Aufbereitung und Anwendung von Informationen bei der Nutzung von Evaluati- 
onen. Auch müssen sie sich nicht systematisch mit Evaluationen auseinandersetzen, um diese zu verwenden. Zur Nutzung sollte aber ein Abwägen gehören, ob eine Evaluation oder ihre Ergebnisse relevant für analytische und beziehungsweise oder politische Zwecke sind. Neben den Parlamentsmitgliedern können schliesslich auch Beteiligte aus Verwaltung, Regierung sowie andere Dritte Evaluationen in parlamentarischen Gesetzgebungsprozessen nutzen.

Die im Folgenden verwendete zweiteilige Unterscheidung zwischen analytischer und politischer Nutzung bezieht sich auf den grundlegenden Gegensatz zwischen einer analytisch-verbesserungsorientierten und einer politisch-strategischen Logik der Nutzung. Wie Abbildung 2 zeigt, können sich die beiden Nutzungsformen in unterschiedlichen Arten äussern. Die analytische Nutzung subsumiert die in der bisherigen Literatur diskutierten Arten der instrumentellen und konzeptionellen Verwendung, während die politische Nutzung sämtliche Arten der symbolischen oder je nach Begriffsanwendung auch persuasiven, legitimierenden oder taktischen Verwendung zusammenfasst. Damit knüpft die vorliegende Konzeption an die bisherige Nutzungsforschung an, wobei sie sich besonders auf die ebenfalls zweiteiligen Unterscheidungen von Boswell $(2008,5)$ und Frey $(2012,63)$ bezieht (vgl. 2.1.2). Die Terminologie orientiert sich an Frey (2012, 63), wobei sie genauer von analytischer und politischer Verwendungsrationalität spricht. Trotz dieses grundlegenden Gegensatzes gestaltet sich die Abgrenzung und Beziehung zwischen analytischer und politischer Nutzung als komplex. Einerseits sind die Nutzungsformen empirisch schwierig beobacht- und unterscheidbar (Boswell 2009, 82; Frey 2012, 63; Weiss 1990, 183). Demzufolge stellt auch die Erforschung der Beziehung verschiedener Nutzungsformen eine Herausforderung dar (Amara et al. 2004, 100; Cousins und Shulha 2006, 279). Anderseits können die analytische und politische Nutzung auch theoretisch nicht vollständig losgelöst voneinander betrachtet werden. Die analytische und politische Verwendung schliessen sich nicht aus und können gleichzeitig oder sequentiell auftreten (Amara et al. 2004; Frey 2012; Whiteman 1985a, 1985b, 1995). Auch bedingen sie sich gewissermassen, wie Boswell $(2009,249)$ herausstreicht, da die politisch-symbolische Nutzung ihre Wirkung entfaltet, indem sie sich auf das instrumentell-problemlösende Bild von systematisch generiertem Wissen und dessen Nutzung stützt. Es ist daher fraglich, ob Parlamentsmitglieder Evaluationen überhaupt analytisch verwenden können, ohne dies mit der Absicht zu verbinden, diese Evaluationen oder deren analytische Verwendung eventuell auch politisch zu nutzen. Es scheint zwar möglich, dass ein Parlamentsmitglied ergebnisoffen und mit relativ wenig gefestigten Positionen Evaluationen analytisch nutzt, um sich zu informieren, allerdings kann erwartet werden, dass eine Absicht zur (möglichen) politischen Nutzung immer auch vorhanden ist (Frey 2012, 316). Daher kann generell erwartet werden, dass die analytische Nutzung die politische Nutzung fördert. Wie bereits im vorherigen Kapitel erläutert, kann gemäss der Literatur generell erwartet werden, dass politische Verwendung im Parlament verbreiteter ist als analytische Verwendung (vgl. 2.3.1).

$\mathrm{Zu}$ diesen grundsätzlichen Schwierigkeiten bei der Abgrenzung der analytischen und politischen Nutzung kommt hinzu, dass erwartungsgemäss nicht alle Arten dieser beiden Formen gleich stark zusammenhängen. Die Erwartung, dass die analytische Nut- 
zung die politische Nutzung fördert, betrifft besonders die politische Verwendung in Form von Verweisen oder Zitaten von Evaluationen zur Begründung oder Überzeugung: Es erscheint intuitiv, dass ein Parlamentsmitglied eine Evaluation auch in einer Rede zitiert, wenn es sich anhand der Evaluation informierte (vgl. Whiteman 1985b, 206). Andere politische Nutzungsarten können ohne analytische Nutzung vorkommen. Dies betrifft die taktische Forderung einer Evaluation oder die Kritik an Evaluationen, die keine Kenntnis von Evaluationen erfordern. Verweise auf Evaluationen scheinen zwar ebenso ohne eine analytische Auseinandersetzung mit der Evaluation möglich, da sich Parlamentsmitglieder bei Reden oder Vorstössen auch auf Informationen von Interessengruppen oder anderen Dritten stützen können (Hall und Deardorff 2006). Ob dies aber in den schweizerischen Parlamenten - gerade auf kantonaler Ebene - häufig vorkommt, ist unklar. ${ }^{25}$

Gemäss den obigen Erläuterungen fördert die analytische zwar die politische Nutzung, aber aufgrund der unterschiedlichen Logik dieser beiden Formen ist ihr Auftreten anders begründet. Bei der analytischen Nutzung mit einer verbesserungsorientierten Logik stellen sich grundsätzlich die folgenden Fragen: Wann sind Parlamentsmitglieder offen gegenüber Evaluationen und bereit zur Reflektion ihrer Position? Wann setzen sie sich mit Evaluationen effektiv auseinander und schreiben ihnen eine vergleichsweise hohe Relevanz zu? Mit der politischen Nutzung und ihrer strategischen Logik hingegen sind generell die folgenden Fragen verbunden: Wann können Parlamentsmitglieder einen Vorteil aus Evaluationen ziehen und so ein für sie wünschenswertes Ergebnis erreichen? Wann können Evaluationen ihren Standpunkten mehr Legitimität verleihen? Die Begründung des Zusammenhangs zwischen den Erklärungsfaktoren und Evaluationsnutzung stützt sich auf diese Fragen. Die Begründungen zur politischen Nutzung fokussieren jedoch nur auf die strategische Logik und nicht auf Veränderungen der politischen Nutzung aufgrund der analytischen Nutzung. Aufgrund des Zusammenhangs der beiden Nutzungsformen widmet sich für jeden Erklärungsfaktor je eine Hypothese der analytischen respektive der politischen Nutzung.

\subsection{Individuelle Erklärungsfaktoren der Evaluationsnutzung}

Verschiedene Forschende betrachten individuelle Merkmale der Entscheidungsträgerinnen und träger als bedeutende - teilweise als bedeutendste - Erklärungsfaktoren für die Verwendung systematisch generierten Wissens (Askim 2009, 37; Cousins und Shulha 2006, 273-274; Weiss 1990, 177). Entsprechend bilden individuelle Merkmale Gegenstand vieler Studien der Nutzung. Diese Studien weisen aber nur auf wenige eindeutige Befunde (Cousins und Leithwood 1986, 356f.; Johnson et al. 2009, 382; Nutley et al. 2007, 72f.). Angesichts dieser unklaren Ergebnisse sowie der geringen Nutzungsforschung zu Parlamentsmitgliedern liegt eine Untersuchung der individuellen Erklärungsfaktoren also nahe.

25 Für den Nationalrat stellt Bailer $(2011,312)$ etwa fest, dass die Verbindung zu Interessengruppen keinen Beitrag zur Erklärung der Häufigkeit von parlamentarischen Fragen leistet. 
Die Untersuchung fokussiert auf individuelle Erklärungsfaktoren, die vergleichsweise konstant über verschiedene Gesetzgebungsprozesse bleiben: Gegenstand der Untersuchung sind die Erfahrung, die Parteizugehörigkeit, die Professionalisierung und der Bildungsgrad eines Parlamentsmitglieds. Diese vier Erklärungsfaktoren haben eine zentrale Bedeutung für die Erfüllung parlamentarischer Tätigkeiten, wobei die Professionalisierung und der Bildungsgrad besonders aufgrund des schweizerischen Milizsystems relevant sind. Die Einstellung gegenüber Evaluationen wird im Folgenden hingegen nicht als Erklärungsfaktor berücksichtigt. Grund dafür ist die erwartete Nähe der Einstellung gegenüber Evaluationen zur Nutzung von Evaluationen und der Evaluationspraxis. Es kann angenommen werden, dass die Einstellung gegenüber Evaluationen von überwiegend denselben Faktoren beeinflusst wird wie die Nutzung. Dies illustrieren Beiträge, welche die Nutzung mit Angaben zur Einstellung gegenüber Evaluationen messen (Bogenschneider et al. 2013; Caplan et al. 1975; Cousins und Leithwood 1986, 346; Webber 1986). Zudem dürfte die Einstellung gegenüber Evaluationen im Gegensatz zu den anderen untersuchten Merkmalen auch davon abhängen, wie verbreitet Evaluationen sind. Schliesslich ist sie im Gegensatz zu den anderen Merkmalen nicht grundlegend von Bedeutung zur Erfüllung verschiedener parlamentarischer Tätigkeiten.

\subsubsection{Erfahrung eines Parlamentsmitglieds}

Der Erfahrungsschatz der Parlamentsmitglieder ist zentral für das Funktionieren der Parlamente: Mit zunehmender Erfahrung eignen sich die Parlamentsmitglieder die notwendigen Kompetenzen sowie informellen Regeln im Parlament an und können so an Einfluss gewinnen (Bailer et al. 2013; Bell und Price 1975; Best und Vogel 2014; Feh Widmer 2015). Diese Adaptation der Neumitglieder an die erfahrenen Parlamentsmitglieder wird als parlamentarische Sozialisierung gesehen. Aufgrund dieser Sozialisierung sieht Webber $(1986,269)$ die Erfahrung als wegweisenden Erklärungsfaktor der Nutzung an: Sind Evaluationen nicht nützlich im Parlament, sollten laut seiner Argumentation erfahrene Parlamentsmitglieder dies durch ihre Nicht-Nutzung anzeigen.

Ein Zusammenhang zwischen analytischer Evaluationsnutzung und Erfahrung ist plausibel, da sich im Laufe der Amtszeit ändern sollte, zu welchen Informationen Parlamentsmitglieder Zugang haben und wie sie mit diesen Informationen umgehen. Einerseits ist es daher möglich, dass die analytische Nutzung mit zunehmender Erfahrung steigt. Da Erfahrene vertrauter mit Evaluationen werden und rascher die für sie relevanten Informationen finden sowie verarbeiten, sollten sie sich einfacher mit Evaluationen auseinandersetzen können. Auch komplexe und viele Informationen sollten erfahrene Parlamentsmitglieder schneller einordnen können als Neugewählte (Mooney 1993, 187 f.; 2012, 14; Raudla 2012, 1004; vgl. Askim 2009; Sabatier und Jenkins-Smith 1993). Anderseits gibt es Argumente, die für einen negativen Zusammenhang zwischen analytischer Evaluationsnutzung und Erfahrung sprechen. Mit zunehmender Erfahrung sollte die Relevanz von Evaluationen abnehmen, da sich erfahrene Parlamentsmitglieder in ihrer Amtszeit bereits mit verschiedenen Fragen auseinandergesetzt haben und über gefestigte Positionen verfügen (Weiss 1989, 413). Entsprechend stützen sie sich stärker 
auf ihre Erfahrung als auf Evaluationen, um bei neuen Fragen ihre Positionen zu formen. Da Erfahrene öfter führende, einflussreiche Funktionen im Parlament besetzen und häufiger Kontakte zu Interessengruppen haben, erschliessen sich ihnen auch andere, informelle Informationsquellen (Gava et al. 2017; Miquel und Snyder 2006, 350, 371; Webber 1986, 269). Umgekehrt bilden folglich Evaluationen gerade für Neulinge eine relevante Informationsquelle, zu der sie Zugang haben. Aufgrund ihrer mangelnden Erfahrung sollten Neulinge weniger vorgefestigte Positionen und ein grösseres Informationsbedürfnis haben, weswegen sie Evaluationen umso häufiger analytisch verwenden sollten (Raudla 2012, 1004; Weiss 1989, 418).

Zwischen politischer Nutzung und Erfahrung ist ein Zusammenhang aufgrund der unterschiedlichen Stellung der Parlamentsmitglieder begründet. Evaluationen können eher für unerfahrene Parlamentsmitglieder als Instrument dienen, um die Rechtmässigkeit ihres Standpunkts zu unterstreichen, während erfahrene Parlamentsmitglieder Legitimität durch ihre in der Amtszeit erworbene Expertise erlangen (vgl. Weiss 1989). Zudem sollten sich erfahrene Parlamentsmitglieder gerade aufgrund ihres grösseren Einflusses zusätzlicher Mittel bedienen können, um Entscheide zu beeinflussen oder zu verzögern. Auch wenn erfahrene Parlamentsmitglieder aus Evaluationen eher einen politischen Vorteil ziehen können, weil sie vertrauter damit sind, lässt sich somit aufgrund der strategischen Logik deutlicher für einen negativen Zusammenhang zwischen politischer Nutzung und Erfahrung argumentieren.

Bisherige Studien haben sich ausschliesslich dem Zusammenhang zwischen der Erfahrung von Parlamentsmitgliedern und analytischer Nutzung oder Nutzung generell gewidmet. Im schweizerischen Kontext zeigen Studien zum Umgang mit Informationsund Planungsinstrumenten in Kantonsparlamenten, dass erfahrene Parlamentsmitglieder diese besser verstehen, als nützlicher befinden und zur Information verwenden (Demaj et al. 2012, 45, 50). Für die US-Bundesstaaten stellt Mooney $(2012,47)$ dagegen fest, dass erfahrene Parlamentsmitglieder politische Geschäfte nicht differenzierter betrachten. Keinen Zusammenhang stellt auch Webber $(1986,277)$ im selben Kontext fest. Für einen negativen Zusammenhang sprechen die Befunde von Raudla $(2012,1009)$ für Estland. Gemäss ihren Interviews haben die Parlamentsmitglieder als Neulinge Performanzinformationen gründlicher gelesen. Laut den Befunden zu anderen politischen Entscheidungsträgerinnen und trägern suchen schliesslich Erfahrene weniger nach systematisch generiertem Wissen und sind weniger offen demgegenüber eingestellt (vgl. Askim 2008, 132; Askim 2009, 36; Lester 1993, 277; Oliver et al. 2014, 6).

Insgesamt ist bei der politischen Nutzung ein negativer Zusammenhang aufgrund der unterschiedlichen Position der Parlamentsmitglieder klar begründet, während bei der analytischen Nutzung verschiedene Zusammenhänge plausibel sind. Auch wegen der Möglichkeit zur politischen Nutzung sollte aber der Anreiz für unerfahrene Parlamentsmitglieder hoch sein, sich trotz geringer Vertrautheit mit Evaluationen auseinanderzusetzen. Dies ergibt die folgenden Hypothesen: 


\section{$H_{1 a}: \quad J e$ erfahrener ein Parlamentsmitglied, desto seltener nutzt es Evaluationen analytisch. \\ $H_{1 p}$ : Je erfahrener ein Parlamentsmitglied, desto seltener nutzt es Evaluationen politisch.}

\subsubsection{Parteizugehörigkeit eines Parlamentsmitglieds}

Eine zentrale Rolle bei der Erfüllung parlamentarischer Tätigkeiten kommt auch den Parteien zu, die entweder alleine oder mit anderen Parteien zusammen eine Fraktion im Parlament bilden. Die Definition, Funktion und Ziele von Parteien sind vielfältig (Beyme 1984, 25; Saalfeld und Strøm 2014, 272; White 2006, 6). Parteien dienen der Vertretung unterschiedlicher Werte der Wählerinnen und Wähler sowie der Artikulation verschiedener gesellschaftlicher Interessen. Sie übernehmen aber auch eine wichtige Funktion bei der Rekrutierung neuer Parlamentsmitglieder, deren Einführung und Sozialisierung. Ihren Mitgliedern ermöglichen Parteien, die Wahlchancen zu erhöhen, an Einfluss zu gewinnen und sich zu spezialisieren (Saalfeld und Strøm 2014, 376, 381, 383). Angesichts dieser zentralen Aufgaben liegt auch nahe, dass die Parteizugehörigkeit bedeutsam für die Evaluationsnutzung ist.

Besonders aufgrund der Ideologie lassen sich unterschiedliche Begründungen herleiten, wieso sich Parlamentsmitglieder je nach Parteizugehörigkeit in ihrer Offenheit gegenüber Evaluationen und deren Ergebnissen unterscheiden sollten. Da sich Evaluationen mit staatlichen Massnahmen befassen, scheint einerseits wichtig, wie die Parteien gegenüber staatlichen Eingriffen eingestellt sind. Parlamentsmitglieder linker Parteien sollten demgemäss offener gegenüber Evaluationen sein und diese eher berücksichtigen, da sie an staatlichen Massnahmen interessiert sind und deren möglichen Ausbau befürworten (Askim 2008, 133; Hird 2005a, 91; Webber 1986, 268). Umgekehrt könnte aber von Parlamentsmitgliedern bürgerlicher Parteien erwartet werden, dass sie einen besonders hohen Anreiz haben, sich mit Evaluationen auseinanderzusetzen, um staatliche Massnahmen zu kontrollieren und gegebenenfalls zu reduzieren (Askim 2008, 134). Anderseits kann argumentiert werden, dass die Offenheit gegenüber Evaluationsergebnissen weniger von der Richtung als der Stärke der Ideologie abhängt. Je ideologischer eine Partei, desto weniger Spielraum bleibt, um Positionen zu formen und Evaluationen zu berücksichtigen. Daher sollten besonders Parlamentsmitglieder moderater Parteien offen für Evaluationen sein und diese häufiger analytisch nutzen als Parlamentsmitglieder am linken oder rechten Ende des Spektrums (Frey 2012, 91; vgl. auch Askim 2008, 134). Neben diesen ideologisch basierten Erklärungen dürften schliesslich Evaluationen für gewisse Parteien relevanter sein, da sie historisch damit verbunden sind. So hat etwa New Labour in Grossbritannien EBP stark geprägt (vgl. 2.1.3). Eine solche Verbindung, etwa in Bezug auf Reformen des NPM, besteht in der Schweiz hingegen nicht (vgl. Rieder und Widmer 2007).

In Verbindung mit der Richtung und der Stärke der Ideologie können ebenso Erklärungen zur politischen Nutzung entwickelt werden. Da sie skeptisch gegenüber staatli- 
chen Eingriffen sind, sollten Bürgerliche Evaluationen zur Kontrolle und Abschaffung öffentlicher Massnahmen fordern. Parlamentsmitglieder linker Parteien wiederum sollten Evaluationen fordern, um Kosten für staatliche Eingriffe zu rechtfertigen (Balthasar und Rieder 2009, 416). Bei der Argumentation sollten aber vor allem moderate Parteien Vorteile haben: Die weniger ideologischen Positionen der Parlamentsmitglieder dieser Parteien erlauben es, Evaluationen häufiger und glaubwürdiger anzuführen. Zudem sollten Parlamentsmitglieder aus der Mitte eher Evaluationen bedürfen, um zu rechtfertigen, wieso sie sich auf die eine und nicht die andere Seite stellen.

Mit der Frage des Zusammenhangs von Parteizugehörigkeit und Evaluationsnutzung befassten sich die Studien bisher nur am Rande. Für die Bundesversammlung stellt Frey $(2012,139,279)$ in ihren Fallstudien fest, dass Parlamentsmitglieder der Mitteparteien offener gegenüber Evidenz sind und Interessengruppen bei diesen Mitgliedern auch gezielt mit Evidenz zu überzeugen versuchten. Demaj et al. $(2012,48)$ bemerken weiter, dass Parlamentsmitglieder der SVP Informations- und Planungsinstrumente häufiger ablehnen. Studien zu den Parlamenten der US-Bundesstaaten zeigen, dass Mitglieder der Demokraten häufiger Evaluationen verwenden als Mitglieder der Republikaner (Bogenschneider et al. 2013, 271; Hird 2005a, 98; 2005b, 147,150). Speer et al. (2015, 52-54) finden in ihrer Analyse des flämischen und deutschen Parlaments zwar parteispezifische Unterschiede in der Häufigkeit, in der Parlamentsmitglieder Fragen zu Evaluationen stellen, allerdings können sie dies nicht erklären. Befunde zur Nutzung anderer politischer Entscheidungsträgerinnen und träger sind nicht eindeutig (Askim 2008, 134; Balthasar und Müller 2014, 88; Oliver et al. 2014, 6). Keine Studie hat sich spezifisch zur politischen Nutzung und der Parteizugehörigkeit befasst. Für das Schweizer Parlament stellt Pedrini $(2014,279)$ allgemein fest, dass Parlamentsmitglieder von rechten Parteien, namentlich der SVP, ihre Aussagen im Gegensatz zu Parlamentsmitgliedern moderater Parteien ${ }^{26}$ seltener begründen. Dies könnte bedeuten, dass sie Evaluationen seltener politisch verwenden.

Basierend auf der Ideologie der Parteien können diverse Hypothesen zur analytischen und politischen Nutzung abgeleitet werden. Insgesamt sind die Erklärungen aufgrund der Stärke der Ideologie kohärenter mit den Logiken der beiden Nutzungsformen: Nicht nur scheint bei Parlamentsmitgliedern moderater Parteien aufgrund ihrer flexibleren Positionen eher eine Offenheit gegenüber den Evaluationsergebnissen plausibel, auch bedürfen sie Evaluationen, um ihre flexiblen Positionen zu rechtfertigen. Für die Parteizugehörigkeit wird also erwartet:

\section{$H_{2 a}: \quad$ Ein Parlamentsmitglied einer moderaten Partei nutzt Evaluationen häufiger analytisch als ein Parlamentsmitglied einer anderen Partei. \\ $H_{2 p}$ : Ein Parlamentsmitglied einer moderaten Partei nutzt Evaluationen häufiger politisch als ein Parlamentsmitglied einer anderen Partei.}




\subsubsection{Professionalisierung eines Parlamentsmitglieds}

In den schweizerischen Milizparlamenten kommt der individuellen Professionalisierung der Parlamentsmitglieder eine besondere Bedeutung zu: Da die Parlamentsmitglieder kaum auf Unterstützung durch Mitarbeitende des Parlaments zählen können, ist es besonders relevant, wie viel Ressourcen sie für die Erfüllung ihrer parlamentarischen Aufgaben investieren können. ${ }^{27}$ Bisherige Studien zur Bundesversammlung und den Kantonsparlamenten weisen darauf hin, dass grosse Unterschiede im zeitlichen Aufwand der Parlamentsmitglieder bestehen (vgl. Bundi et al. 2017; Bütikofer 2013; Sciarini et al. 2017). Es stellt sich daher die Frage, inwiefern sich diese Unterschiede auf die Nutzung von Evaluationen auswirken.

Ein Zusammenhang zwischen der analytischen Nutzung und der Professionalisierung liegt nahe, da der zeitliche Aufwand die Kapazität zur Informationsbeschaffung und -verarbeitung mitbestimmt. Je mehr Zeit ein Parlamentsmitglied dem Mandat widmen kann, desto mehr Zeit bleibt für vertiefte Recherchen oder für die Konsultation zusätzlicher Informationsquellen. Ein weiterer Grund für einen positiven Zusammenhang kann im Selbstverständnis der Parlamentsmitglieder liegen. Professionalisierte Parlamentsmitglieder dürften eher den Anspruch haben, informiert zu sein. Weniger professionalisierte Parlamentsmitglieder üben dagegen vermutlich ihr Mandat zu einem gewissen Grad auch bewusst nebenberuflich aus. Diesem Selbstverständnis entsprechend dürften sie Evaluationen eine weniger hohe Relevanz zuschreiben, wenn sie ihre Positionen formen.

Im Gegensatz zur analytischen Nutzung ist ein Zusammenhang von politischer Nutzung und Professionalisierung nicht naheliegend. Zwar bedarf es auch für die politische Nutzung von Evaluationen, etwa bei der Vorbereitung von Reden, zeitliche Kapazitäten. Zudem kann argumentiert werden, dass professionalisierte Parlamentsmitglieder Evaluationen eher als politisches Mittel verwenden, um ihre Professionalität zu unterstreichen. Die Erfahrungen aus der Schweiz stellen diese Argumentation aber in Frage: Selbst in der Bundesversammlung mit zeitintensiven Mandaten wird vermieden, von Berufsparlament zu sprechen (Pfister und Ruffieux 1975; Riklin und Möckli 1991, 157; Wurz 2014). ${ }^{28}$ Dies weist gleichzeitig darauf hin, dass sich auch für wenig professionalisierte Parlamentsmitglieder kaum ein politischer Vorteil aus der Verwendung von Evaluationen ergibt.

Nur vereinzelt untersuchten Studien bisher den Zusammenhang zwischen Professionalisierung und analytischer Nutzung. In seiner Befragung stellt Hird (2005b, 149) für die untersuchten Parlamente der US-Bundesstaaten einen positiven Zusammenhang fest: Parlamentsmitglieder, die verhältnismässig mehr Zeit für ihr Mandat aufwenden, investieren auch mehr Zeit in das Erlangen von Expertise und nutzen öfters Policy-Analyse, um die Funktionsweise einer Politik zu verstehen. Dieser Zusammenhang könnte jedoch auch durch die geringere, institutionelle Professionalisierung des Parlaments erklärt werden (Hird 2005b, 149). Einen solchen Erklärungsversuch äussert ebenso (Mooney 1993,

27 Der Zeitaufwand ist nur ein möglicher Indikator für die Professionalisierung eines Parlaments (2.4.2; vgl. Z'graggen 2009, 26).

28 Auf kantonaler Ebene ist der Zeitaufwand geringer, so dass Profis eine Ausnahme bilden (Bundi et al. 2017). 
192), der bei den Mitgliedern von drei Parlamenten der US-Bundesstaaten unterschiedlich hohe Nutzung findet. Spezifisch zum Zusammenhang zwischen politischer Verwendung und Professionalisierung liegen keine Studien vor. Schliesslich sind keine Studien zur Evaluationsnutzung in der Schweiz bekannt, die den Aspekt der Professionalisierung vertieft untersuchen. ${ }^{29}$

Insgesamt stützen die theoretischen Überlegungen klar einen positiven Zusammenhang von analytischer Nutzung und Professionalisierung. Keine stichhaltigen Hinweise gibt es für einen Zusammenhang mit der politischen Nutzung. Dies ergibt folgende Hypothesen:

\section{$H_{3 a}: \quad J e$ professionalisierter ein Parlamentsmitglied, desto häufiger nutzt es Evaluationen analytisch. \\ $H_{3 p}: \quad$ Die Professionalisierung und die politische Nutzung hängen nicht zusammen.}

\subsubsection{Bildungsgrad eines Parlamentsmitglieds}

Generell erscheint es offensichtlich, dass der Bildungsgrad mit der Einstellung gegenüber systematisch generiertem Wissen und dem Umgang damit zusammenhängt (Nutley et al. 2007, 72). Während Parlamentsmitglieder in der Regel über einen hohen Ausbildungsabschluss verfügen und daher kaum Unterschiede im Nutzungsverhalten erwartbar sind, scheint der Bildungsgrad gerade in den schweizerischen Milizparlamenten relevant (vgl. Mooney 2012, 16).$^{30}$ Zwar haben auch die Mitglieder der Bundesversammlung deutlich häufiger einen universitären Abschluss als die Stimmberechtigten. Die Quote der Parlamentsmitglieder mit universitärem Hintergrund unterscheidet sich aber zwischen den Parteien und ist bei gewissen Parteien, namentlich der SVP, sogar rückläufig (Pilotti 2015, 251-252). Bezüglich des Bildungsniveaus der Mitglieder der Kantonsparlamente ist wenig bekannt. Die Befragung von Feh Widmer $(2015,226)$ weist aber darauf hin, dass das gesamte Spektrum der Bildungsabschlüsse in den Kantonsparlamenten vertreten ist.

Die unterschiedliche Kapazität zur Verarbeitung von systematisch generiertem Wissen und die Einstellung gegenüber solchem Wissen weisen auf einen Zusammenhang zwischen analytischer Nutzung und dem Bildungsgrad. Je gebildeter ein Parlamentsmitglied, desto eher ist es gewohnt und sprichwörtlich dazu geschult, analytische Informationen in grossen Mengen aufzunehmen und zu verwerten (Esterling 2007, 94; Mooney 2012, 16; vgl. auch Askim 2009, 30). Gleichzeitig sollten Parlamentsmitglieder mit einem höheren Bildungsgrad eine positivere Einstellung gegenüber solchen Informationen haben und diesen mehr vertrauen (vgl. Askim 2007, 467; Askim 2009, 32; Nideröst 2002, 46).

29 Nideröst $(2002$, 51) erwähnt in seiner Fallstudie einen positiven Effekt des Milizsystems auf die Evaluationsnutzung. Er stützt seine Aussage aber auf ein einzelnes Parlamentsmitglied.

30 Die Ausbildung hängt auch mit der Professionalisierung zusammen. So sind ein hoher Ausbildungsgrad sowie eine standardisierte Ausbildung auch ein Zeichen davon (vgl. Z'graggen 2009: 104-105). 
Für einen negativen Zusammenhang zwischen Bildungsgrad und analytischer Nutzung spricht wenig. Ein mögliches Argument ist, dass höher gebildete Parlamentsmitglieder Evaluationen weniger relvant befinden und seltener analytisch verwenden, da sie sich bereits informiert fühlen (vgl. Askim 2009, 39).

Weniger deutlich erscheint ein Zusammenhang zwischen politischer Nutzung und dem Bildungsgrad. Da höher gebildete Parlamentsmitglieder selbst empfänglicher für evaluations-basierte Argumente sein sollten, kann argumentiert werden, dass sie Evaluationen eher als strategisches Mittel zur Begründung oder Überzeugung einsetzen (Esterling 2007, 95, 106). Ebenso kann erwartet werden, dass höher gebildete Parlamentsmitglieder eher Evaluationen kritisieren, da sie mit den Forschungsmethoden eher vertraut sein sollten.

Die bisherige Evidenz zum Effekt des Bildungsgrads der Parlamentsmitglieder konzentriert sich vorwiegend auf die analytische Nutzung, ist aber nicht schlüssig. Gebildete Parlamentsmitglieder der US-Bundesstaaten geben häufiger an, sich bei Entscheiden nicht genügend informiert zu fühlen und Policy-Analyse vermehrt zu verwenden, wäre sie verfügbar (Hird 2005b, 150). Demgegenüber findet Mooney $(2012,31)$ ebenso für die US-Bundesstaaten keinen Zusammenhang zwischen dem Bildungsgrad und der Differenziertheit, mit der sich ein Parlamentsmitglied mit einer politischen Massnahme auseinandersetzt. Studien zu anderen politischen Entscheidungsträgerinnen und trägern sprechen gegenteilig mehrheitlich für einen negativen Zusammenhang zwischen Bildung und analytischer Nutzung (Askim 2009; Lester 1993; Oliver et al. 2014). Der Zusammenhang zwischen politischer Nutzung und dem Bildungsgrad ist kaum spezifisch erforscht. Esterling $(2007,106)$ stellt fest, dass Parlamentsmitglieder, die über eine hohe analytische Kapazität verfügen, in Debatten öfter auf analytische Aspekte einer politischen Massnahme Bezug nehmen.

Die theoretischen Überlegungen weisen mehrheitlich auf einen positiven Zusammenhang zwischen analytischer sowie politischer Nutzung. Aus diesen Gründen werden folgende Hypothesen untersucht:

\section{$H_{4 a}: \quad$ Je gebildeter ein Parlamentsmitglied, desto häufiger nutzt es Evaluationen analytisch. \\ $H_{4 p}: \quad J e$ gebildeter ein Parlamentsmitglied, desto bäufiger nutzt es Evaluationen politisch.}

\subsection{Kontextuelle Erklärungsfaktoren der Evaluationsnutzung}

Wie der Überblick zur Literatur und Forschung im vorangehenden Kapitel verdeutlicht, ist der Kontext für die Erklärung der Nutzung zentral (vgl. Cousins und Shulha 2006, 272; Nutley et al. 2007, 89). Da der Kontext diverse Erklärungsfaktoren umfasst, wird er in diesem Buch dreigeteilt: Erstens wird der institutionelle Kontext untersucht, der sich auf Eigenschaften der Institution und Organisation des Parlaments bezieht. Zweitens wird der politische Kontext analysiert, das heisst Eigenschaften des Gesetzgebungspro- 
zesses. Drittens wird der evaluationsspezifische Kontext betrachtet. Dieser beschreibt im Gegensatz zu den anderen Kontextfaktoren die Angebotsseite der Evaluationen.

\subsubsection{Institutioneller Kontext}

Aufgrund der Unterschiede zwischen den Kantonen und ihren Parlamenten scheint ein Effekt des institutionellen Kontexts in der föderalistischen Schweiz naheliegend. Die Schweizer Arbeitsparlamente unterscheiden sich in ihrer Organisation. Das Kommissionensystem ist dabei eine wesentliche Eigenschaft, zumal Kommissionen zentral sind für die Arbeitsfähigkeit der Parlamente und gemäss bisheriger Forschung auch für die Nutzung von systematisch generiertem Wissen (vgl. Mooney 1992, 2012; Shulock 1998, 1999; Weiss 1987, 1989, 1999; Whiteman 1985a, 1985b, 1995). Ein weiterer möglicher Erklärungsfaktor in den schweizerischen Parlamenten stellt die Professionalisierung der Parlamente dar. Von einer Untersuchung dieses Aspekts auf institutioneller Ebene wird aber abgesehen, da er bereits als individueller Erklärungsfaktor betrachtet wird und zudem in den Kantonsparlamenten aufgrund ihrer generell wenigen Ressourcen kaum bedeutsam sein dürfte (vgl. 3.2.3; Trippolini 2007, 29 f.).

\section{Kommissionensystem eines Parlaments}

Das Kommissionensystem ist eine der folgenreichsten Unterscheidungen in der Organisation eines Parlaments (Mattson und Strøm 1995, 258; Shaw 1979, 380). Ob eine Kommission ständig ist oder nicht, beeinflusst den Anreiz der Mitglieder, sich zu spezialisieren. Zwar dienen auch nichtständige Kommissionen der Spezialisierung, indem sie ihnen zugeteilte Geschäfte bearbeiten. Da nichtständige Kommissionen wieder aufgelöst werden, haben die Parlamentsmitglieder aber keine Sicherheit, beim nächsten, thematisch ähnlichen Geschäft wieder in der Kommission zu sitzen (Lüthi 1997, 148; Mattson und Strøm 1995, 270; Yläoutinen und Hallerberg 2009, 150). Dies sollte ihren Anreiz zur Spezialisierung verringern. Die Spezialisierung dient gemäss der informational theory (Gilligan und Krehbiel 1987; Krehbiel 1991) der Informationsgewinnung, was einen Zusammenhang von Kommissionensystem und Evaluationsnutzung plausibel macht (vgl. 2.3.1).

Ein Zusammenhang zwischen analytischer Nutzung und dem Kommissionensystem kann direkt durch den Anreiz zur Spezialisierung ihrer Mitglieder begründet werden. Mitglieder ständiger Kommissionen sollten Evaluationen öfter analytisch nutzen als Mitglieder nichtständiger Kommissionen, weil sich die Auseinandersetzung mit Evaluationen mehr lohnt, da sie den daraus gewonnenen Sachverstand in mehreren Geschäften anwenden können. Der Aufbau eines permanenten Sachverstands macht sich umso mehr bezahlt, zumal ständige Kommissionen die Gesetzgebung aktiver mitgestalten können. Ebenso sollten sie dank ihrem vertieften Verständnis auch Evaluationen zu komplexen Fragen besser verarbeiten können (Lienhard et al. 2011, 106; Lüthi 1997, 168; Trees et al. 2013, 49; Trippolini 2007, 28). Die ständige Zusammensetzung erlaubt zudem mehr Möglichkeit für Konsens, da die Mitglieder eher bereit sind, bei einem Geschäft ihre Position anzupassen, wenn sie dafür bei einem anderen Geschäft belohnt werden (Lüthi 1997, 148). Dies sollte den Parlamentsmitgliedern erlauben, offener gegenüber Evidenz zu sein. 
Gleichzeitig wird in der Literatur von einer gewissen Verantwortung gesprochen, als Mitglied einer ständigen Kommission informiert zu sein (Lüthi 1997, 177; Mooney 2012, 10). Dieser Anreiz zur Spezialisierung und sein Effekt können auch kritisch betrachtet werden. So kann argumentiert werden, dass die Parlamentsmitglieder meist ohnehin bei Kommissionen in ihren Themenbereichen teilnehmen (vgl. Lüthi 1997, 167). Die Kommissionsgrösse, der Umfang des Zuständigkeitsbereichs oder Mehrfachmitgliedschaften in Kommissionen beeinflussen ausserdem auch, ob sich ein Parlamentsmitglied spezialisiert (Lüthi 1997, 32 f.; Mattson und Strøm 1995, 268-271; Shulock 1998, 317). Schliesslich könnten nichtständige Kommissionen sogar häufiger Evaluationen analytisch nutzen, da die Mitglieder dieser Kommissionen auch Zeit und Interesse haben, um das behandelte Geschäft vertieft zu betrachten. Weiter könnte gerade die Entwicklung eines fundierten Sachverstands in ständigen Kommissionen zu gefestigten Positionen führen, was die Offenheit gegenüber Evaluationen vermindern würde (Weiss 1989, 413).

Im Gegensatz zur analytischen Nutzung scheint ein Zusammenhang zwischen dem Kommissionensystem und politischer Nutzung wenig offensichtlich. Zwar können Mitglieder ständiger Kommissionen Evaluationen eher politisch einsetzen, da sie diese aus früheren Geschäften kennen und dann zur Unterstützung ihrer Position wieder verwenden können. Allerdings stellt sich die Frage, inwiefern ständige Kommissionsmitglieder Evaluationen überhaupt als politisches Mittel bedürfen. Ihre Mitgliedschaft in der ständigen Kommission und der damit einhergehende Status eines Experten oder einer Expertin verleiht ihren Standpunkten bereits Legitimität. Diesem Argument folgend sollten die Mitglieder von nichtständigen Kommissionen Evaluationen eher politisch verwenden.

Die Evidenz kann wenig zu diesen theoretischen Überlegungen beitragen. Nach bestem Wissen befasste sich bisher keine Studie mit dem Verhältnis der Nutzung systematisch generierten Wissens und des Kommissionensystems. Studien zum US-Kongress oder Parlamenten der US-Bundesstaaten zeigen aber deutlich, dass analytische Nutzung in den Phasen der Kommissionberatungen wahrscheinlicher ist und sich Kommissionsmitglieder differenziert mit Geschäften auseinandersetzen (Florio et al. 1979, 83; Mooney 1993, 191; 2012, 31 f.; Whiteman 1985a, 308). Kommissionen verwenden Evaluationen auch politisch, wie weitere Untersuchungen zum US-Kongress belegen (vgl. Esterling 2011; Shulock 1998, 1999). Diese Ergebnisse dürften aber nicht vollständig auf die Schweiz übertragbar sein, da Hearings von Kommissionen im US-Kongress häufig öffentlich sind (vgl. Esterling 2011, 175).

Insgesamt deuten vielfältige theoretische Gründe klar auf einen positiven Zusammenhang zwischen analytischer Nutzung und ständigen Kommissionensystemen hin: Deren Mitglieder sollten einen erhöhten Anreiz zur Spezialisierung haben, was sich letztlich in Unterschieden zwischen den Parlamenten ausdrücken sollte. Für politische Nutzung existieren nur wenige Vermutungen. Dies führt zu den folgenden Hypothesen:

\section{$H_{5 a}: \quad$ Parlamentsmitglieder in Parlamenten mit ständigen Kommissionen nutzen Evaluationen häufiger analytisch als Parlamentsmitglieder in Parlamenten mit nichtständigen Kommissionen. \\ $H_{5 p}: \quad$ Das Kommissionensystem und die politische Nutzung hängen nicht zusammen.}




\subsubsection{Politischer Kontext}

Die bisherige Forschung, besonders die Policy-Forschung, schreibt dem politischen Kontext eine zentrale Rolle zur Erklärung der Nutzung systematisch generierten Wissens zu (vgl. Frey 2012; Weiss 1999; Whiteman 1985a). Als Erklärungsfaktoren des politischen Kontexts vertieft diskutiert werden in diesem Buch zwei Eigenschaften des Gesetzgebungsprozesses: die Wissenschaftsnähe und die Konfliktivität. ${ }^{31}$ Diese zwei Eigenschaften sind auch Gegenstand des ACF (Jenkins-Smith und Sabatier 1993; vgl. auch Schrefler 2010; Weible 2008). Ein Fokus auf diese Erklärungsfaktoren ist aber vor allem sinnvoll, da diese zu einem gewissen Grad voneinander abhängig sind (Schrefler 2010, 325; Weible et al. 2009, 130). Während sich die Wissenschaftsnähe stärker auf die inhaltliche Dimension einer Politik bezieht (policy), greift die Konfliktivität die prozessbezogene Dimension auf (politics).

\section{Wissenschaftsnähe einer Massnahme}

Mit Wissenschaftsnähe ist im Folgenden gemeint, wie erfassbar Gegenstand, Ziel sowie Wirkungen einer politischen Massnahme sind (vgl. Frey 2012). Je direkter, objektiver Ziele einer politischen Massnahme erfasst und je besser ein kausaler Wirkungszusammenhang festgestellt werden können, desto höher ist die Wissenschaftsnähe. Diese Erfassund Erforschbarkeit der Politik sollte auch die Möglichkeiten der Nutzung beeinflussen. Verbunden mit der Erfassbarkeit sind auch Fragen der Methode und Messung von Zielen und Wirkungen sowie der Forschungsdisziplinen. So wird angenommen, dass Naturwissenschaften eher quantitative, harte Erkenntnisse liefern, während Erkenntnisse aus Sozialwissenschaften kontroverser sind, da sie sich mit komplexen Zusammenhängen befassen (Boswell 2009, 243 f.; Jenkins-Smith und Sabatier 1993, 52; Klöti und Schneider 1989, 40; Nutley et al. 2007, 21). Mit der Weiterentwicklung der Forschung kann sich die Wissenschaftsnähe aber über Zeit verändern (Frey 2012, 316; Gormley 1986, 599). Insgesamt ist die Wissenschaftsnähe mit verschiedenen Konzepten aus der bisherigen Forschung verwandt, wie der analytischen Erfassbarkeit im ACF (Jenkins-Smith und Sabatier 1993, 50-52), der Komplexität oder der Unsicherheit (Boswell 2009; Radaelli 1995; vgl. auch Dunlop und Radaelli 2018; Gormley 1986). Auch zur Literatur $\mathrm{zu}$ wicked problems bestehen Verbindungen. Diese Probleme gelten als "complex, openended, intractable" (Head 2008b, 101) und sind daher schwer erfassbar.

Aufgrund der quantitativen Erfassung sowie der naturwissenschaftlichen Ausrichtung kann ein positiver Zusammenhang zwischen analytischer Evaluationsnutzung und der Wissenschaftsnähe angenommen werden (vgl. Frey 2012; Jenkins-Smith und Sabatier 1993). Bei Fragen zu naturwissenschaftlichen Themen kann erwartet werden, dass Parlamentsmitglieder weniger vertraut mit ihnen sind und weniger Informationen dazu haben, etwa aus ihrer Erfahrung, von der Wählerschaft oder anderen Quellen (Mooney 1993, 188; vgl. auch Lester 1993, 279) . Bei solchen Fragen sind ihre Meinungen weniger vorgefasst und entsprechend sind die Parlamentsmitglieder evidenzoffener. Sie

31 Diese Terminologie wird von Frey (2012) übernommen, Bezeichnungen und Konzeptualisierungen dieser Erklärungsfaktoren variieren aber in der Literatur. 
können daher Evaluationen verwenden, um sich zu informieren und komplexe Themen $\mathrm{zu}$ begreifen. Weiter wird generell argumentiert, dass quantitative Daten glaubwürdiger seien und den Naturwissenschaften mehr Vertrauen geschenkt werde (Fornerod 2001, 27; Jenkins-Smith und Sabatier 1993, 52; Klöti und Schneider 1989, 39; Nutley et al. 2007, 68). Gleichzeitig scheint es möglich, dass Parlamentsmitglieder gar keiner analytischen Verwendung von Evaluationen bedürfen, da gut erfassbare Gegenstände und Wirkungszusammenhänge auch einfacher zu erklären und zu verstehen sein dürften.

In Bezug auf die politische Nutzung sind im Gegensatz dazu verschiedene Zusammenhänge mit der Wissenschaftsnähe plausibel. Einerseits sollte die forschungs- oder evaluationsbasierte Argumentation bei wissenschaftsnahen Fragen auch mehr Autorität geniessen und ein höheres Gewicht haben (vgl. Boswell 2009). Weiter sollten Parlamentsmitglieder Evaluationen zur Erklärung ihres Standpunkts bedürfen, wenn sie diese bereits benötigen, um wissenschaftsnahe Themen zu verstehen. Anderseits kann ein negativer Zusammenhang erwartet werden. Indem Probleme schwierig definierbar und Zielgrössen oder Wirkungszusammenhänge schwer erfassbar sind, öffnen sich auch Möglichkeiten zur politischen Nutzung. Die verwendeten Methoden und Zielgrössen in Evaluationen sind so stärker der Kritik ausgesetzt. Zudem können Parlamentsmitglieder bei schwer erfassbaren Zielgrössen und Wirkungszusammenhängen ganz verschiedene Standpunkte mit Evaluationen untermauern (vgl. Boswell 2009, 93; Jenkins-Smith 1988, 198, 204). Fraglich ist jedoch, ob die evaluationsbasierten Argumente auch schlagkräftig sind und Parlamentsmitglieder daraus einen politischen Vorteil ziehen können.

Im Gegensatz zur generellen Nutzungsforschung beschäftigen sich die Beiträge zu den Parlamenten nur am Rande mit der Wissenschaftsnähe oder verwandten Konzepten. In einer Befragung von Parlamentsmitgliedern in drei US-Bundesstaaten findet Mooney $(1993,191)$ heraus, dass diese bei technisch komplexen Geschäften eher schriftliche Informationen nutzen, um einen Entscheid zu treffen, ein Geschäft zu gestalten oder andere Mitglieder zu überzeugen. Damit übereinstimmend beobachtet Frey (2012, $294 \mathrm{f}$., 308) vor allem in Gesetzesrevisionen mit hoher Wissenschaftsnähe eine analytische Verwendungsrationalität bei schweizerischen Parlamentsmitgliedern. In diesen Fällen verwendeten die Parlamentsmitglieder Evaluationen auch politisch, aber in unterschiedlichem Ausmass (Frey 2012, 279-281). Ohne auf die Parlamentsmitglieder zu fokussieren, stellen Weible et al. $(2009,130)$ im Überblick zur bisherigen Forschung zum ACF fest, dass policy-orientiertes Lernen bei analytisch erfassbaren Fragen eher wahrscheinlich ist, wobei eine hohe Konfliktivität dies begrenzt.

Insgesamt sprechen die theoretischen Überlegungen für einen positiven Zusammenhang zwischen analytischer Verwendung und Wissenschaftsnähe. Bei der politischen Verwendung sind verschiedene Zusammenhänge denkbar. Da Parlamentsmitglieder bei einer geringen Wissenschaftsnähe Evaluationen vielfältig politisch verwenden können, sollen folgende Hypothesen untersucht werden: 


\section{$H_{6 a}: \quad J e$ grösser die Wissenschaftsnähe einer Massnahme, desto häufger nutzt ein Parlamentsmitglied Evaluationen analytisch. \\ $H_{\sigma p}: \quad J e$ grösser die Wissenschaftsnähe einer Massnahme, desto seltener nutzt ein Parlamentsmitglied Evaluationen politisch.}

\section{Konfliktivität einer Massnahme}

Die Konfliktivität stellt gemäss der bisherigen Nutzungsforschung ein zentraler Erklärungsfaktor dar, da sie Entscheide massgeblich prägt und damit auch die Rolle beeinflusst, die Evaluationen zukommen kann. Mit der Konfliktivität ist im Folgenden generell gemeint, inwiefern sich Parlamentsmitglieder in ihren Ansichten bezüglich einer Frage unterscheiden. ${ }^{32}$ Der Grad der Konfliktivität hängt von verschiedenen Faktoren ab, etwa von der Anzahl und der Machtposition der an einem Konflikt Beteiligten (vgl. Esterling 2011). Entscheide, die saliente Fragen und damit viele Beteiligte betreffen, sollten mit einer relativ hohen Konfliktivität einhergehen (Schrefler 2010, 316 f.). Eine hohe Konfliktivität ist folglich auch zu erwarten, wenn Entscheide grundsätzliche Werte und Interessen betreffen (Jenkins-Smith und Sabatier 1993, 49). In solchen Situationen gibt es beschränkte Möglichkeiten für eine mehrheitsfähige Lösung (vgl. Boswell 2009, 74; Frey 2012; Weiss 1999). Wie die Wissenschaftsnähe kann sich aber die Konfliktivität im Verlauf der Zeit verändern und in Entscheidungsprozessen zu ähnlichen Fragen variieren (vgl. Schrefler 2010).

Es existieren verschiedene Argumente, wie die analytische Nutzung und die Konfliktivität zusammenhängen. Zum einen kann argumentiert werden, dass die analytische Verwendung mit der Konfliktivität sinkt. Ist eine Frage wenig umstritten, sind die Positionen flexibler und die Parlamentsmitglieder offener gegenüber Evaluationen. Bei konfliktiven Fragen hingegen sind die Positionen festgefahren, sodass die Parlamentsmitglieder weder offen gegenüber Evaluationen sind noch diese zur Information benötigen (Frey 2012, 73; Whiteman 1985a, 305). ${ }^{33}$ Zudem scheint eine analytische Nutzung der Parlamentsmitglieder bei hoher Konfliktivität aufgrund des schweizerischen politischen Systems unwahrscheinlich. In solchen Fällen wird bereits in der vorparlamentarischen Phase eine konsensfähige Lösung gesucht, um ein Referendum oder dessen Annahme zu vermeiden. Dies sollte die Rolle der Evaluation im vorparlamentarischen Prozess einschränken, was sich auch auf den parlamentarischen Prozess auswirken dürfte (vgl. 2.4.2; Frey 2012, 73; Ledermann 2014, 477; Sager und Rissi 2011, 158; Widmer und Neuenschwander 2004, 391). Gleichzeitig unterstützen einige Forschende einen gegenteiligen Zusammenhang. So erwartet Mooney $(1993,188)$, dass sich Parlamentsmitglieder bei konfliktiven Geschäften eher informieren, da diese eine hohe Relevanz haben und von der Wählerschaft sowie Interessengruppen aufmerksam verfolgt werden. Ebenso können

32 Diese Frage kann die Einführung oder Ausgestaltung einer politischen Massnahme betreffen, beispielsweise aber auch die Verteilung des Budgets oder Wahlen.

33 Vgl. Balthasar und Müller (2014, 75); Jenkins-Smith und Sabatier (1993, 49); Klöti und Schneider (1989, 33); Nutley et al. (2007, 76); Weiss (1999, 482 f.). 
Evaluationen in politischen Krisen mit entsprechendem Handlungsdruck verwendet werden, um einen Konsens zu finden (vgl. Ledermann 2012, 163; Nutley et al. 2007, 76; Valovirta 2002, 75-76). Schliesslich kann argumentiert werden, dass die besten Bedingungen für eine analytische Verwendung bei einer moderaten Konfliktivität bestehen: In dieser Situation sollten die Parlamentsmitglieder nicht ganz so stark an ihrer Position festhalten und offen gegenüber Evaluationen sein. Zugleich sollte die Relevanz von Fragen mit einer moderaten Konfliktivität einen Anreiz geben, sich damit auseinanderzusetzen (Jenkins-Smith und Sabatier 1993, 49 f.).

Auch für die politische Nutzung sind verschiedene Argumente plausibel. Häufig wird postuliert, dass die politische Verwendung mit zunehmender Konfliktivität steigt. Ist eine Frage umstritten, können Parlamentsmitglieder Evaluationen als vielfältiges politisches Mittel verwenden, um die eigene Position zu begründen, andere zu überzeugen oder gegebenenfalls einen Entscheid hinauszuzögern (vgl. Boyer und Langbein 1991, 510 f.; Frey 2012, 73).Wie Jenkins-Smith und Sabatier $(1993,49)$ es ausdrücken: "All sides have great incentives to make their 'best case' and to give as little ground as possible." Zudem könnte die höhere öffentliche Aufmerksamkeit bei konfliktiven Fragen die politische Nutzung zur Begründung der Position fördern (Shulock 1998, 313; vgl. Esterling 2011, 180). Analog zur analytischen Nutzung kann aber auch für die politische Nutzung argumentiert werden, dass eine moderate Konfliktivität diese besonders begünstigt, da Parlamentsmitglieder in dieser Situation überhaupt offen für evaluationsbasierte Argumente sein sollten. Bei einer hohen Konfliktivität hingegen sollten Parlamentsmitglieder häufiger grundsätzliche Werte statt Evaluationen thematisieren (Esterling 2011, $171 \mathrm{f}$;; vgl. auch Boswell 2009, 97). Bei einer geringen Konfliktivität ist vermutlich der Diskurs wenig intensiv und politischer Vorteil ist kaum aus der Verwendung von Evaluationen zu ziehen.

Übereinstimmend mit ihrer zentralen Rolle untersuchten zahlreiche Forschende den Zusammenhang von Konfliktivität und Nutzung empirisch, wobei die Empirie nicht eindeutig ist. Einerseits unterstützen die Fallstudien von Whiteman (1985a, 308) zum US-Kongress die Argumentation, dass eine geringe Konfliktivität die analytische Verwendung begünstigt. Ledermann $(2014,474)$ bestätigt in ihren Fallstudien zum Beizug von Evidenz und Expertise bei der Erarbeitung der Vernehmlassungsvorlagen weiter die Annahme, dass sich die Konfliktivität bereits (früh) im vorparlamentarischen Prozess auswirkt. Anderseits weisen mehrere Beiträge zu Parlamentsmitgliedern und anderen politischen Entscheidungsträgerinnen und trägern darauf hin, dass eine hohe Konfliktivität die analytische Verwendung begünstigt (Balthasar und Müller 2014, 188; Mooney 1993, 196). Auch stellt Frey (2012, 308 f.) fest, dass sich Parlamentsmitglieder bei einzelnen Gesetzesrevisionen mit hoher Konfliktivität von der Evidenz überzeugen liessen. Schliesslich stützen Studien, die sich dem ACF bedienen, die dritte Argumentation, dass eine moderate Konfliktivität am ehesten zu policy-orientiertem Lernen der politischen Entscheidungsträgerinnen und träger führt (Sabatier und Jenkins-Smith 1993a, 218; Weible et al. 2009, 130). Auch bei der politischen Nutzung stützen die Befunde unterschiedliche Zusammenhänge. Einen deutlich positiven Zusammenhang zwischen politischer Nutzung und der Konfliktivität bemerkt Whiteman (1985a, 305; 1985b, 219) für die USA 
und Frey $(2012$, 308) für die Schweiz (vgl. Rissi und Sager 2013, 359). Keinen Einfluss der Konfliktivität auf die Nutzung in allen Formen stellen schliesslich Boyer und Langbein $(1991,527)$ fest, die sich auf Angaben der Mitarbeitenden des US-Kongress stützen. Theoretisch sind insgesamt verschiedene Zusammenhänge zwischen Konflitkivität und analytischer sowie politischer Nutzung plausibel. Bei Fragen, zu denen sich das Parlament äussert, kann generell von einer gewissen Konfliktivität ausgegangen werden (Frey 2012, 73). Gleichzeitig dürfte der Verhandlungsspielraum begrenzt und die Positionen der Parlamentsmitglieder relativ stark festgelegt sein in Situationen mit einer hohen Konfliktivität, was die analytische Nutzung hindern sollte. Im Gegensatz dazu sollte eine hohe Konfliktivität die politische Verwendung fördern, da Parlamentsmitglieder vor allem dann Evaluationen in ihrer Vielfältigkeit als politisches Mittel einsetzen können.

\section{$H_{7 a}: \quad$ Ein Parlamentsmitglied nutzt Evaluationen am häufgsten analytisch bei einer moderaten Konfliktivität einer Massnahme. \\ $H_{7 p}$ : Je höher die Konfliktivität einer Massnahme, desto häufiger nutzt ein Parlamentsmitglied Evaluationen politisch.}

\subsubsection{Evaluationsspezifischer Kontext}

Zu den kontextuellen Erklärungsfaktoren der Nutzung zählen in dieser Untersuchung auch Eigenschaften der Evaluationen. Angesichts des möglicherweise indirekten Kontakts mit Evaluationen sollten diese Eigenschaften auch relevant sein, wenn Parlamentsmitglieder über Dritte von Evaluationen erfahren (vgl. 2.3.1; Whiteman 1995, 162). Als Erklärungsfaktor untersucht wird daher die Rechtzeitigkeit und Aktualität der Evaluationen, welche sich Parlamentsmitgliedern rasch erschliessen sollte. Die Übereinstimmung der Evaluationsergebnisse bildet der zweite Erklärungsfaktor. Im Gegensatz zur Rechtzeitigkeit und Aktualität bezieht sich dieser Erklärungsfaktor nicht auf eine Evaluation, sondern auf alle verfügbaren Evaluationen zu einem Thema.

\section{Rechtzeitigkeit und Aktualität einer Evaluation}

Rechtzeitigkeit, d. h. das Vorliegen der Evaluation zum Zeitpunkt der Nutzung, kann als Grundvoraussetzung für Evaluationsnutzung angesehen werden, während sich Aktualität darauf bezieht, wie kürzlich eine Evaluation zu einem gewissen Zeitpunkt erschienen ist (vgl. Frey 2012, 75). Im Folgenden werden die Rechtzeitigkeit und Aktualität gemeinsam betrachtet, da beides zeitbezogene Eigenschaften sind, die sich in der englischen Forschung unter dem Begriff der timeliness zusammenfassen lassen. In der Forschung wird mehrfach betont, dass der zeitliche Faktor für politische Entscheidungsträgerinnen und träger zentral sei. Die Logik der Politik sei opportunistisch, reaktiv und auf die Aktualität und Zukunft ausgerichtet (Askim 2008, 129; Balthasar 2009a, 496). Folglich sollten zeitbezogene Eigenschaften von Evaluationen eine besondere Relevanz für die Evaluationsnutzung im Parlament haben. 
Es kann angenommen werden, dass Evaluationen bereits früh im Gesetzgebungsprozess vorliegen müssen, um rechtzeitig für die analytische Nutzung zu sein (Frey 2012, 75; Klöti und Schneider 1989, 35; Nideröst 2002, 44). Parlamentsmitglieder dürften im Verlauf des Gesetzgebungsprozesses zwar mehrfach mit einer politischen Massnahme in Berührung kommen. Sie sollten aber besonders in frühen Phasen des Gesetzgebungsprozesses offener gegenüber Evidenz sein, sich informieren und ihre Positionen formen. Nach den Kommissionsberatungen, in denen sich Parlamentsmitglieder etwa über Hearings (weiter) informieren können, sollten die Positionen spätestens bezogen sein (vgl. Whiteman 1985a, 299, 308). Da Parlamentsmitglieder Informationen von der Exekutive, Mitarbeitenden oder auch von Dritten erhalten, erscheint es am idealsten, wenn die Evaluationen vorliegen, bevor ein Geschäft im Parlament beraten wird. In Bezug auf die Aktualität kann weiter argumentiert werden, dass Parlamentsmitglieder neuerschienene Evaluationen eher analytisch verwenden. Aktuelle Evaluationen sind stärker von Relevanz, da sie eher auf die momentane Situation übertragbar sind. Allerdings erschwert eine hohe Aktualität die Aufbereitung der entsprechenden Evaluation für das Parlament. Ähnliche Überlegungen zur Rechtzeitigkeit und Aktualität gelten für die politische Nutzung. Generell scheint es auch für die politische Nutzung vorteilhaft, wenn die Evaluationen spätestens bei den Kommissionsberatungen verfügbar sind. Parlamentsmitglieder verwenden Evaluationen erwartungsgemäss zwar gerade in späteren Phasen des Gesetzgebungsprozesses, um die gefassten Positionen zu begründen oder Entscheide zu verzögern (Whiteman 1985a, 302). Es kann jedoch angenommen werden, dass Parlamentsmitglieder vor allem in den Kommissionen von Evaluationen erfahren. Ist eine Evaluation nicht nur rechtzeitig, sondern auch aktuell, kann dies ebenso als förderlich für die politische Nutzung gesehen werden. ${ }^{34}$ Die Ergebnisse erhalten durch ihre Aktualität Überzeugungskraft, die benötigt wird, um aus Evaluationen einen politischen Vorteil zu ziehen. Auch ist die Argumentation mit einer aktuellen Evaluation weniger angreifbar - zumindest aufgrund ihres Erscheinungsdatums.

Die bisherige Forschung unterstützt die Erwartungen zur Verteilung der Evaluationsnutzung im Gesetzgebungsprozess deutlich: Beobachtungen der analytischen Nutzung konzentrieren sich eher auf die frühen Phasen des Gesetzgebungsprozesses, während Beobachtungen der politischen Nutzung in späteren Phasen des Gesetzgebungsprozesses dominieren (Florio et al. 1979, 83; Whiteman 1985a, 308). Auch sind es die frühen Phasen, in denen sich Parlamentsmitglieder gemäss Mooney (1991a, 451 f.) aus vielfältigen, darunter auch externen Quellen informieren.

Bisherige Studien zeigen, dass der Rechtzeitigkeit und Aktualität klar grosse Bedeutung für die Evaluationsnutzung im Parlament zukommt, sie differenzieren aber nicht zwischen den einzelnen Nutzungsformen. Ebenso bleibt in den Studien weitgehend offen, was Rechtzeitigkeit und Aktualität bedeutet. Die Ergebnisse zum US-Kongress

34 Nutzen Parlamentsmitglieder Evaluationen wie erwartet überwiegend in frühen Phasen des Gesetzgebungsprozesses analytisch und später politisch, verstreicht mehr Zeit zwischen Nutzung und Erscheinen der Evaluation bei der politischen Nutzung. Dieser zeitliche Unterschied sollte jedoch in der Regel vernachlässigbar sein. 
sind gespalten: Während das Timing in der Analyse von Florio et al. $(1979,69)$ eines der am häufigsten genannten Hindernisse der Nutzung darstellt, sind Evaluationen gemäss Boyer und Langbein $(1991,519)$ grösstenteils rechtzeitig. Weiter legt die bisherige Evidenz nahe, dass es einer gewissen Aktualität bedarf: "To use old data is reckless, particularly in light of the fact that if your computer systems are up and working, you can have up-to-the-month [information]", zitieren Guston et al. $(1997,461)$ ein Parlamentsmitglied des US-Bundesstaats Minnesota. Gleichzeitig stellen mehrere Forschende fest, dass Evaluationen erst nach einer gewissen Vorlaufzeit in die politische Debatte aufgenommen werden (Ginsburg und Rhett 2003, 492; Leviton und Hughes 1981, 535).

Insgesamt basiert diese Untersuchung auf der Annahme, dass Evaluationen rechtzeitig für die analytische und politische Nutzung sind, wenn sie spätestens in den frühen Phasen der Kommissionsberatungen verfügbar sind. Bezüglich der Aktualität deuten die theoretischen Überlegungen auf einen positiven Zusammenhang mit beiden Nutzungsformen hin. Da die Aktualität im politischen Umfeld generell zentral ist und die höhere Überzeugungskraft sowohl die politische als auch die analytische Nutzung begünstigen sollte, werden folgende Hypothesen untersucht:

\section{$H_{8 a}: \quad$ Je aktueller Evaluationen sind, desto häufiger nutzen Parlamentsmitglieder diese analytisch. \\ $H_{s p}$ : Je aktueller Evaluationen sind, desto häufiger nutzen Parlamentsmitglieder diese politisch.}

\section{Übereinstimmung der Evaluationsergebnisse}

Die Übereinstimmung der Evaluationsergebnisse drückt aus, ob die verfügbaren Evaluationen zu eindeutigen oder widersprüchlichen Erkenntnissen zu den Wirkungen einer politischen Massnahme kommen. Da sich diese Eigenschaft folglich auf die Gesamtheit der verfügbaren Evaluationen bezieht, hängt sie auch davon ab, wie lange eine Massnahme bereits diskutiert wird und wie erforscht sie ist (vgl. Esterling 2004, 214). Die Übereinstimmung der Ergebnisse sollte für die Evaluationsnutzung bedeutsam sein, da sie bestimmt, was für Aussagen zu den Wirkungen einer Massnahme gemacht werden können. Wenn die Ergebnisse übereinstimmen, sollten Parlamentsmitglieder gemäss Esterling $(2004,110,237)$ mehr Sicherheit über die Effekte einer politischen Massnahme erhalten. Dadurch können sie das Risiko von unbeabsichtigten Wirkungen oder Politikversagen und damit allfällige negative Folgen auf ihre Wiederwahl reduzieren. Generell sollte eine Sammlung von Evaluationen den Bedürfnissen der Parlamentsmitglieder eher entsprechen. Mehrere Evaluationen - anstatt einer - können eine umfassendere Perspektive bieten (vgl. auch Cousins und Shulha 2006, 272 f.; Ginsburg und Rhett 2003, 490; Leviton 2003, 527). Auch weisen mehrere Studien darauf hin, dass Parlamentsmitglieder systematisch generiertes Wissen häufiger verwenden, wenn vergleichsweise viel Forschung zu einem Thema existiert (vgl. Apollonio und Bero 2009; Hird 2005b; Whiteman 1995). Mehrere Gründe sprechen dafür, dass Parlamentsmitglieder Evaluationen öfter analytisch nutzen, wenn die Ergebnisse übereinstimmen. Aus übereinstimmenden Ergebnissen kön- 
nen klare Schlüsse gezogen werden (vgl. Nutley et al. 2007, 72). Bei stark widersprüchlichen Ergebnissen ist hingegen damit zu rechnen, dass nicht nur ein wissenschaftlicher Konsens zu den Effekten einer Massnahme fehlt, sondern umstritten ist, was überhaupt aussagekräftige Evidenz darstellt (Nutley et al. 2007, 68 f.). Übereinstimmende Ergebnisse und ein wissenschaftlicher Konsens sollten zudem dazu führen, dass Evaluationen im Verhältnis zu anderen Informationen relevanter werden. Parlamentsmitglieder sollten sich weniger vor übereinstimmenden Ergebnissen verschliessen können. So kann ein wissenschaftlicher Konsens zu einer Massnahme ändern, welche Priorität ihr Parlamentsmitglieder zuschreiben und wie sie diese auffassen (vgl. Albaek 1995, 92 f.).

Für die politische Nutzung erscheinen hingegen verschiedene Zusammenhänge möglich. Stimmen die Ergebnisse der Evaluationen überein, sollte dies zu einer einseitigen politischen Nutzung führen. Übereinstimmende Ergebnisse dienen vor allem der Seite, die durch die Ergebnisse unterstützt wird. Entsprechend sollte diese Seite auch Evaluationen politisch in ihrer Argumentation verwenden. Ihre gegnerische Seite hat hingegen wenig Anreiz, Evaluationen als politisches Mittel einzusetzen, da diese ihnen übereinstimmend widerspricht (vgl. auch Esterling 2004, 244; Frey 2012, 76 f.). Ebenfalls scheint das Kritisieren von Evaluationen bei übereinstimmenden Ergebnissen erschwert (vgl. auch Weiss und Bucuvalas 1980). Sind die Ergebnisse hingegen widersprüchlich, sollte die politische Nutzung von allen Seiten steigen. Jede Seite kann ihre Position mit Evaluationen untermauern, auch können sich alle aufgrund der unschlüssigen Ergebnisse kritisieren (Esterling 2004, $92 \mathrm{f}$.). Da die Ergebnisse unschlüssig sind und verschiedene Positionen unterstützen, sollten evaluationsbasierte Argumente jedoch auch an Schlagkraft verlieren (vgl. auch Boswell 2009).

Nur wenige Studien untersuchen, was die Übereinstimmung der Ergebnisse für die Nutzung im Parlament bedeutet. Frey $(2012,272,275,309)$ stellt in ihren Fallstudien zu Gesetzesrevisionen in der Schweiz fest, dass sich übereinstimmende Ergebnisse förderlich auf die EBP auswirken. Parlamentsmitglieder liessen sich in einzelnen Fällen von Evidenz überzeugen, auch da diese stichhaltig war. Allerdings sind übereinstimmende und umfassende Ergebnisse nicht in allen Fällen entsprechend eingeflossen. Die bisherigen Befunde zur politischen Nutzung sind nicht eindeutig. Frey $(2012,282)$ stellt fest, dass die Parlamentsmitglieder Evaluationen unabhängig ihrer Position und der Übereinstimmung der Ergebnisse als politisches Mittel verwendeten. Esterling (2004, $235 \mathrm{f}$.) hingegen beobachtet in seinen Fallstudien, dass übereinstimmende Ergebnisse zu einer einseitigen Nutzung führten: Nur diejenige Seite, die klar durch die Ergebnisse gestützt wurde, verwendete sie in ihrer Argumentation. Seine Fallstudien zeigen aber auch, dass die gegnerische Seite trotz Übereinstimmung die Ergebnisse kritisieren kann, gerade wenn es kaum Studien dazu gibt. Neben diesen zwei konkreten Untersuchungen werden in der Literatur übereinstimmende Ergebnisse generell als fördernd für die Nutzung systematisch generierten Wissens angesehen (Head 2010, 81; Nutley et al. 2007, 69).

Die theoretischen Überlegungen stützen insgesamt einen positiven Zusammenhang zwischen der Übereinstimmung der Ergebnisse und analytischer Nutzung. Bei der politischen Nutzung scheint es generell plausibel, dass Parlamentsmitglieder eher einen politischen Vorteil aus Evaluationen ziehen können, wenn deren Ergebnisse die eigene 
Position unterstützen. Da folglich übereinstimmende Ergebnisse zu einseitiger Nutzung führen, während alle Parlamentsmitglieder widersprüchliche Ergebnisse politisch verwenden können, soll ein negativer Zusammenhang angenommen werden. Dies ergibt folgende Hypothesen:

$H_{9 a}: \quad$ Je übereinstimmender die Ergebnisse, desto häufger nutzt ein Parlamentsmitglied Evaluationen analytisch.

$H_{9 p}: \quad$ Je übereinstimmender die Ergebnisse, desto seltener nutzt ein Parlamentsmitglied Evaluationen politisch. 


\section{Analyse der individuellen Erklärungsfaktoren der Evaluationsnutzung}

In diesem Kapitel steht die Analyse der individuellen Erklärungsfaktoren der Evaluationsnutzung im Zentrum. Gemäss den vorangehenden, theoretischen Erläuterungen sollte die Evaluationsnutzung mit der Erfahrung, der Parteizugehörigkeit, der Professionalisierung und dem Bildungsgrad eines Parlamentsmitglieds zusammenhängen. Im Folgenden werden diese Zusammenhänge anhand einer quantitativen Analyse untersucht. Ein solches Vorgehen eignet sich, da die individuellen Erklärungsfaktoren über verschiedene Gesetzgebungsprozesse relativ konstant bleiben. Grundlage für die quantitative Analyse bilden Daten aus einer Online-Befragung von Mitgliedern der Kantonsparlamente und der Bundesversammlung. Diese Datengrundlage liefert einen umfassenden Blick auf die Rolle der Evaluation in den schweizerischen Parlamenten.

Der folgende Abschnitt präsentiert die Datengrundlage der Analyse. Danach wird die Messung der Nutzung von Evaluationen sowie der individuellen Erklärungsfaktoren beschrieben und erste Zusammenhänge werden betrachtet. Das Herzstück der Untersuchung der individuellen Erklärungsfaktoren bildet die multivariate Analyse. Darin werden die Effekte verschiedenen individuellen Erklärungsfaktoren auf die Evaluationsnutzung gleichzeitig untersucht und anhand von Wahrscheinlichkeiten illustriert. Eine Zusammenfassung der Ergebnisse und eine Diskussion der Hypothesen schliesst das Kapitel ab.

\subsection{Grundlage: Befragung der schweizerischen Parlamentsmitglieder}

Die quantitative Analyse stützt sich massgeblich auf die Befragung «Parlamente und Evaluationen», die Mitte 2014 in sämtlichen Kantonsparlamenten und in der Bundesversammlung stattfand. Die nächsten Abschnitte präsentieren zuerst kurz den Ablauf und Inhalt der Befragung. Danach folgt eine Übersicht über die Stichprobe der quantitativen Analyse und eine Diskussion der Aussagekraft dieser Daten. Zusätzliche, vertiefte Informationen über die vorliegende Datengrundlage liefert der Methodenbericht zur Befragung (Bundi et al. 2014).

\subsubsection{Ablauf und Inhalte der Befragung Parlamente und Evaluationen}

Die Online-Befragung richtete sich an sämtliche Parlamentsmitglieder des Bundes sowie der Kantone. Verfügbar war sie in Deutsch, Französisch und Italienisch. Für jedes Parlament wurde eine eigene Version der Befragung erstellt. Dies ermöglichte, Sprachversionen und spezifische Begriffe in den Parlamenten zu berücksichtigen sowie einen geeigneten Zeitpunkt für die Befragung zu wählen (Bundi et al. 2014, 3).

Um den Parlamentsmitgliedern einen Orientierungsrahmen zu bieten, wurde vor Beginn der Befragung «Evaluation» wie folgt definiert: 


\begin{abstract}
Als Evaluationen werden in diesem Zusammenhang Studien, Berichte oder andere Dokumente verstanden, welche eine staatliche Massnahme mit einem systematischen und transparenten Vorgehen bezüglich ihrer Wirksamkeit, Wirtschaftlichkeit oder Zweckmässigkeit beurteilen. (Bundi et al. 2014, 2)
\end{abstract}

Die Befragung begann mit Fragen zum Umgang der Parlamentsmitglieder mit Evaluationen. Danach folgten Fragen zur generellen parlamentarischen Tätigkeit, die beispielsweise den Arbeitsaufwand, die Erfahrung in politischen Ämtern oder die Mitgliedschaft in Kommissionen betreffen. Der letzte Teil beinhaltete Fragen zu demographischen und sozialstrukturellen Merkmalen. Der Original-Fragebogen in allen Sprachen befindet sich im Methodenbericht (Bundi et al. 2014).

\title{
4.1.2 Beteiligung, Stichprobe und Aussagekraft der Daten
}

Tabelle 2 listet Informationen zu Grundgesamtheit, Stichprobe und Rücklauf für die Befragung und die quantitative Analyse auf. Sämtliche damals aktiven 2841 Parlamentsmitglieder des Bundes und der Kantone wurden gebeten, sich an der Befragung zu beteiligen. Insgesamt folgten 1570 Parlamentsmitglieder dieser Bitte, was einer Rücklaufquote von 55.3 Prozent entspricht, wobei die Beteiligung zwischen den Parlamenten stark variiert (Eberli et al. 2014, 2). Die Stichprobe für die quantitative Analyse der vorliegenden Untersuchung beschränkt sich aber auf 1191 der 1570 befragten Parlamentsmitglieder. Im Folgenden werden nur die fünf grössten Parteien CVP, FDP, die Grünen, SP und SVP betrachtet. Der Fokus auf die fünf grössten Parteien ist theoretisch sinnvoll, da die kleineren Parteien nur bedingt gruppiert werden können und nicht in allen Parlamenten vertreten sind. ${ }^{35}$ Die Beschränkung auf die fünf grössten Parteien führt aber dazu, dass Parlamentsmitglieder des Kantonsrats Appenzell-Innerrhoden nicht in die vorliegende Analyse einbezogen werden, da das Geschäftsreglement dieses Parlaments keine Fraktionen vorsieht, sodass die Parteizugehörigkeit teilweise unbekannt ist. ${ }^{36}$ Eine weitere Beschränkung der Stichprobe resultiert aus fehlenden Angaben weniger Parlamentsmitglieder zu den Erklärungsfaktoren der Erfahrung, der Professionalisierung oder dem Bildungsgrad. Daher macht die Stichprobe letztlich 50.4 Prozent aller angeschriebenen Parlamentsmitglieder der fünf grössten Parteien aus.

Insgesamt kann die vorliegende Rücklaufquote von über 50 Prozent bei einer Befragung von Parlamentsmitgliedern je nach Vergleichsbasis als zufriedenstellend bis hoch betrachtet werden. Im Vergleich mit den relativ häufigen Befragungen der Bundesversammlung ist eine Rücklaufquote von über 50 Prozent eher tief. Ältere Befragungen erreichten sehr hohe Quoten von 84 Prozent (Riklin und Möckli 1991, 147) oder eine fast ausnahmslose Teilnahme mit 96 Prozent (Kerr 1981, 23). Auch neuere Befragungen

35 Bei kleinen Parteien besteht zudem statistisch gesehen das Problem, dass die Varianz der Antworten aufgrund der geringen Fallzahl insgesamt oder pro Parlament zu klein wäre. Damit wäre das statistische Modell vordeterminiert (vgl. Long und Freese 2014, 320).

Geschäftsreglement des Grossen Rates vom 21. November 1994, GS AI 171.210. 
Tabelle 2: Beteiligung an der Befragung nach Parlament

\begin{tabular}{|c|c|c|c|c|c|c|c|}
\hline & \multirow{2}{*}{$\begin{array}{r}\text { Anzahl } \\
\text { Sitze }\end{array}$} & \multicolumn{3}{|c|}{ Befragung } & \multicolumn{3}{|c|}{ Quantitative Analyse } \\
\hline & & $\begin{array}{l}\text { Grund- } \\
\text { gesamtheit }\end{array}$ & Stichprobe & Rücklauf & $\begin{array}{l}\text { Grund- } \\
\text { gesamtheit }\end{array}$ & Stichprobe & Rücklauf \\
\hline Aargau & 140 & 140 & 96 & $68.6 \%$ & 118 & 76 & $64.4 \%$ \\
\hline Appenzell-Innerrhoden & 49 & 49 & 38 & $77.6 \%$ & 0 & 0 & - \\
\hline Appenzell-Ausserrhoden & 65 & 64 & 42 & $65.6 \%$ & 41 & 22 & $53.7 \%$ \\
\hline Bern & 160 & 160 & 84 & $52.5 \%$ & 110 & 58 & $52.7 \%$ \\
\hline Basel-Landschaft & 90 & 90 & 48 & $53.3 \%$ & 78 & 36 & $46.2 \%$ \\
\hline Basel-Stadt & 100 & 100 & 52 & $52.0 \%$ & 77 & 39 & $50.6 \%$ \\
\hline Freiburg & 110 & 110 & 55 & $50.0 \%$ & 103 & 50 & $48.5 \%$ \\
\hline Genf & 100 & 100 & 56 & $56.0 \%$ & 71 & 43 & $60.6 \%$ \\
\hline Glarus & 60 & 60 & 35 & $58.3 \%$ & 50 & 25 & $50.0 \%$ \\
\hline Graubünden & 120 & 115 & 55 & $47.8 \%$ & 85 & 35 & $41.2 \%$ \\
\hline Jura & 60 & 57 & 30 & $52.6 \%$ & 46 & 21 & $45.7 \%$ \\
\hline Luzern & 120 & 120 & 67 & $55.8 \%$ & 114 & 60 & $52.6 \%$ \\
\hline Neuenburg & 115 & 114 & 66 & $57.9 \%$ & 100 & 53 & $53.0 \%$ \\
\hline Nidwalden & 60 & 60 & 36 & $60.0 \%$ & 60 & 32 & $53.3 \%$ \\
\hline Obwalden & 55 & 54 & 28 & $51.9 \%$ & 46 & 22 & $47.8 \%$ \\
\hline St. Gallen & 120 & 120 & 87 & $72.5 \%$ & 111 & 78 & $70.3 \%$ \\
\hline Schaffhausen & 60 & 60 & 37 & $61.7 \%$ & 48 & 24 & $50.0 \%$ \\
\hline Solothurn & 100 & 100 & 62 & $62.0 \%$ & 93 & 55 & $59.1 \%$ \\
\hline Schwyz & 100 & 100 & 43 & $43.0 \%$ & 98 & 40 & $40.8 \%$ \\
\hline Thurgau & 130 & 130 & 77 & $59.2 \%$ & 110 & 58 & $52.7 \%$ \\
\hline Tessin & 90 & 90 & 47 & $52.2 \%$ & 67 & 35 & $52.2 \%$ \\
\hline Uri & 64 & 64 & 38 & $59.4 \%$ & 62 & 33 & $53.2 \%$ \\
\hline Waadt & 150 & 150 & 83 & $55.3 \%$ & 136 & 65 & $47.8 \%$ \\
\hline Wallis & 130 & 130 & 51 & $39.2 \%$ & 116 & 35 & $30.2 \%$ \\
\hline Zug & 80 & 80 & 39 & $48.8 \%$ & 71 & 34 & $47.9 \%$ \\
\hline Zürich & 180 & 179 & 106 & $59.2 \%$ & 138 & 75 & $54.3 \%$ \\
\hline Total Kantonsparlamente & 2608 & 2596 & 1458 & $56.2 \%$ & 2224 & 1104 & $49.6 \%$ \\
\hline Bundesversammlung & 246 & 245 & 112 & $45.7 \%$ & 213 & 87 & $40.8 \%$ \\
\hline Total & 2854 & 2841 & 1570 & $55.3 \%$ & 2362 & 1191 & $50.4 \%$ \\
\hline
\end{tabular}

Quelle: Tabelle teilweise entnommen aus Eberli et al. (2014). Quantitative Analyse: Befragte der Parteien CVP, FDP, Grüne, SP und SVP sowie mit Angaben zu Erfahrung, Professionalisierung und Bildungsgrad. Aufgrund der Beschränkung auf die fünf Parteien keine Berücksichtigung von Appenzell-Innerrhoden. 
weisen immer noch hohe Quoten von rund 65 Prozent aus (Bütikofer 2014, 45; Krüger et al. 2001, 17) ${ }^{37}$ Die Befragungen fokussierten aber auf die Bundesebene, setzten daher teilweise verschiedene Mittel zur Erinnerung ein oder wurden zum Teil im Auftrag des Parlaments durchgeführt, was die Rücklaufquote begünstigt haben dürfte. Die Bereitschaft der Bundesversammlung zur Teilnahme an Befragungen scheint aber zurückzugehen, zumal die Studie von Sciarini et al. $(2017,51)$ im Auftrag der Parlamentsdienste einen vergleichsweise tiefen Rücklauf von 52 Prozent erreichte. Die Rücklaufquoten von Befragungen der Bundesversammlung liegen zudem teilweise auch unter 50 Prozent (13.4 Prozent bei Brun [2003], 35.8 Prozent bei Milic et al. [2016, 2]).

Verglichen mit anderen Befragungen auf kantonaler Ebene ist die vorliegende Rücklaufquote hoch. Kürzlich waren die Kantonsparlamente Gegenstand von mehreren Befragungen, die sich aber meist auf ausgewählte Parlamente oder Parlamentsmitglieder beschränkten. Diese Befragungen erreichten Rücklaufquoten von 51.4 Prozent (Feh Widmer 2015, 230), 39.5 Prozent (Strebel 2014, 129) oder 21.3 Prozent (Brun 2003, 69, 82). Milic et al. (2016, 2), deren Befragung zusätzlich zur Bundesversammlung sämtliche Kantonsparlamente umfasste, berichten über einen Rücklauf von 40.5 Prozent. Folglich sind die Rücklaufquoten auf Kantonsebene generell geringer als auf Bundesebene.

Im internationalen Vergleich kann die vorliegende Rücklaufquote ebenfalls als hoch bezeichnet werden, wobei die Rücklaufquoten klar variieren. Bei Befragungen der nationalen und teilweise subnationalen Parlamente aus 15 europäischen Ländern erzielen Deschouwer et al. $(2014,10)$ beispielsweise Rücklaufquoten zwischen 12.0 und 43.3 Prozent. Für Legislativen der US-Bundesstaaten stellen Maestas et al. $(2003,92)$ in ihrer Übersichtsarbeit dagegen deutlich höhere durchschnittliche Rücklaufquoten von 44 bis 47 Prozent fest. Ähnlich zu den Beobachtungen zur Bundesversammlung vermutet schliesslich Bailer $(2014,185)$ in ihrem internationalen Vergleich generell rückläufige Antwortquoten, da Online-Befragungen aufgrund ihrer Einfachheit zunehmen.

Die vorliegende Stichprobe ist sehr repräsentativ bezüglich der Sprachregion der Parlamentsmitglieder, in einem geringeren Mass trifft dies auf das Geschlecht und die Parteizugehörigkeit zu. ${ }^{38}$ Der Vergleich zwischen angeschriebenen und in der Stichprobe eingeschlossenen Parlamentsmitgliedern zeigt, dass Angehörige der Grünen und besonders der SP deutlich häufiger an der Befragung teilnahmen, während sich Angehörige der SVP deutlich seltener beteiligten. Die CVP und die FDP sind zwar auch untervertreten, aber nur in sehr geringem Masse. Zudem beantworteten Frauen die Befragung häufiger als Männer. Der Duncan Index der Dissimilarität bestätigt diese Ungleichheiten der Verteilung in Stichprobe und Grundgesamtheit (vgl. Bailer 2014, 185; Duncan und Duncan 1955). Dieser Index reicht von absoluter Ähnlichkeit (0) bis absolute Unähnlichkeit (100). Mit einem Duncan Index der Dissimilarität von 6.98 sind die fünf Parteien am wenigsten ähnlich verteilt. Dieser Wert ist aber im Vergleich mit anderen Befragungen relativ gering (vgl. Deschouwer et al. 2014, 10). In einem solchen Vergleich hingegen als

37 Weitere Befragungen: Riklin et al. (1977) oder Wiesli und Linder (2000).

38 Die Repräsentativität der gesamten Stichprobe der Befragung diskutieren Bundi et al. (2014, 6-8). 
hoch einzustufen ist der Duncan Index der Dissimilarität von 5.27 für das Geschlecht. Für die Sprachregion sind die Verteilungen fast identisch (Duncan Index von 0.44).

Zusätzliche Einschätzungen zur Aussagekraft der Befragung liefern Bundi et al. (2016) in ihrer Analyse der Evaluationsnachfrage durch die Bundesversammlung. Sie untersuchen, inwiefern die Angaben, welche die Parlamentsmitglieder zur Nachfrage nach Evaluationen in der Befragung gemacht haben, mit den Angaben übereinstimmen, die Bundi et al. $(2016,5)$ aus einer Dokumentenanalyse der parlamentarischen Vorstösse gewonnen haben. Sie stellen fest, dass Parlamentsmitglieder, die sich an der Befragung beteiligten, nicht häufiger mittels eines parlamentarischen Vorstosses nach Evaluationen gefragt haben als jene Parlamentsmitglieder, die nicht an der Befragung teilnahmen. Folglich besteht keine systematische Selbstselektion in die Befragung und die Stichprobe ist in Bezug auf die Nachfrage von Evaluationen repräsentativ (Bundi et al. 2016, 7). Gleichzeitig zeigt der Vergleich, dass das Antwortverhalten nicht mit der Dokumentenanalyse übereinstimmt. Zwar kommen Befragung und Dokumentenanalyse insgesamt auf eine ähnliche Quote an parlamentarischen Vorstössen, in denen eine Evaluation gefordert wurde. Allerdings überschätzten 41.3 Prozent der Parlamentsmitglieder die Anzahl ihrer parlamentarischen Vorstösse im Vergleich mit der Dokumentenanalyse, während sie 13.4 Prozent der Parlamentsmitglieder unterschätzten (Bundi et al. 2016, 7). Diese falsche Einschätzung ist mit der Einstellung gegenüber Evaluationen verbunden. Bundi et al. (2016, 13) erklären sich den Zusammenhang zwischen Einstellung und Antwortverhalten sowohl mit sozialer Erwünschtheit als auch mit Erinnerungslücken. Einerseits dürften Parlamentsmitglieder, die gegenüber Evaluationen positiv eingestellt sind, sozial erwünschtes Antworten stärker spüren und daher ihre Nachfrage nach Evaluationen überschätzen. Anderseits dürfte eine negative Einstellung gegenüber Evaluationen eher zu Erinnerungslücken und damit zur Unterschätzung der Nachfrage nach Evaluationen beitragen. Dies bedeutet, dass der Rolle der Einstellung gegenüber Evaluationen sowie der sozialen Erwünschtheit auch in der folgenden Analyse besondere Aufmerksamkeit zukommen sollte. Dagegen kann analog zu den Ergebnissen zur Nachfrage nach Evaluation für die Nutzung erwartet werden, dass sich Parlamentsmitglieder, die Evaluationen häufiger verwenden, nicht öfter an der Befragung beteiligten.

\subsection{Beschreibung der Evaluationsnutzung und der individuellen Erklärungsfaktoren}

Zur Vorbereitung der quantitativen Analyse wird im Folgenden erläutert, wie die Evaluationsnutzung und die individuellen Erklärungsfaktoren gemessen werden, wie sie ausgeprägt sind und zusammenhängen. ${ }^{39}$ Der erste Abschnitt befasst sich mit der zu erklärenden Variable der Evaluationsnutzung. Zunächst wird erklärt, wie sich diese Variable zusammensetzt, danach werden Häufigkeiten allgemein sowie in den einzelnen Parlamenten präsentiert. Der zweite Abschnitt widmet sich den individuellen Erklärungsfaktoren und deren Zusammenhängen mit der Evaluationsnutzung.

39 Da die Antworten zu sämtlichen Fragen nicht metrisch skaliert sind, basieren die im Folgenden präsentierten Tests und Zusammenhänge zweier Variablen auf nichtparametrischen Kennzahlen. 


\subsubsection{Evaluationsnutzung: Messung und Häufigkeiten}

Die zu erklärende Variable dieser Untersuchung - die Evaluationsnutzung - setzt sich aus zwei Bestandteilen zusammen. Ein Bestandteil stellt die allgemeine Nutzung von Evaluationen für die Aufgabe der Gesetzgebung dar. Abbildung 3 illustriert die Angaben zur Nutzungshäufigkeit für die Gesetzgebung sowie für Budgetentscheide und die Aufsicht. Neben der Wahlfunktion, für die Evaluationen weniger relevant sein dürften, stellen dies die wichtigsten drei Aufgaben der Parlamente dar (vgl. Lüthi 2014; Riklin und Ochsner 1984). Gemäss der Befragung ist die Häufigkeit der Nutzung für alle drei Aufgaben etwa gleich gross. Prozentual geben die Befragten etwas häufiger an, Evaluationen für die Aufsicht oder Budgetentscheide als für die Gesetzgebung zu verwenden. Auch korrelieren die Antworten zur Nutzung für Budgetentscheide und die Aufsicht am stärksten. Allerdings sind aber die Korrelationen der Antworten zu den drei Aufgaben stets signifikant und ähnlich hoch. ${ }^{40}$ Insgesamt beantworteten die Parlamentsmitglieder die Frage zur Aufsicht am seltensten. Möglicherweise nehmen Befragte also den Bezug zwischen Evaluationen und ihren Aufsichtsaufgaben weniger deutlich wahr als zwischen Evaluationen und ihrer Gesetzgebungstätigkeit oder Budgetkompetenz.

\section{Abbildung 3: Häufigkeit der Nutzung für Budgetentscheide, Aufsicht und Gesetzgebung}

\begin{tabular}{|c|c|c|c|c|c|c|}
\hline Budgetentscheide ( $N=1132)$ & 11.8 & & 35.2 & & 40.5 & 12.5 \\
\hline Aufsicht $(\mathrm{N}=1113)$ & 11.4 & & 36.6 & & 37.4 & 14.6 \\
\hline Gesetzgebung $(\mathrm{N}=1141)$ & 10.4 & & 40.1 & & 37.7 & 11.7 \\
\hline & & 20 & 40 & 60 & 80 & 100 \\
\hline
\end{tabular}

Die Formen der Evaluationsnutzung für die Aufgabe der Gesetzgebung stellen den zweiten Bestandteil der zu erklärenden Variable dar. Parlamentsmitglieder, die gemäss ihren Angaben Evaluationen eher selten bis sehr oft für die Gesetzgebung verwenden, wurden gefragt, wie sie diese verwenden. Eine Übersicht über die Formulierung der Fragen gibt Tabelle $3 .^{41} \mathrm{Da}$ die zwei Formen der analytischen und politischen Nutzung sich unterschiedlich ausdrücken können, wurden sie anhand je zwei Nutzungsarten gemessen. Die Arten der instrumentellen sowie der konzeptionellen Nutzung sollen die analytische Nut-

40 Rangkorrelation (Kendalls $\tau_{\mathrm{b}}$ ), 0.33-0.43, Signifikanzniveau $\mathrm{p}<0.001$. Interpretation der Effektstärke nach Cohen (1988, 77 f.): Ein Wert von 0.1 entspricht einer schwachen, ein Wert von 0.3 einer moderaten und Werte grösser als 0.5 einer starken Korrelation.

41 Die Formulierungen basieren auf bisherigen Befragungen zur Verwendung von systematisch generiertem Wissen durch Entscheidungsträgerinnen und träger (vgl. Askim 2007, 2009; Bogenschneider und Corbett 2010; Caplan et al. 1975; Demaj et al. 2012; Hird 2005b; Lester 1993; McDavid und Huse 2012; Webber 1986; Weiss und Bucuvalas 1980). 
zung erfassen, während die legitimierende sowie die persuasive Art die politische Nutzung abbilden sollen. Zwar erörtert etwa Vedung $(1997,267,276)$ auch eine taktische Art der politischen Nutzung von Evaluationen mit der Absicht, Entscheide zu verzögern (vgl. auch Frey 2012; Webber 1986; Widmer 2008, 274). Viele Forschende diskutieren aber vor allem die Verwendung von Evaluationen als Überzeugungs- oder Begründungsmittel (vgl. Johnson 1998; Shulock 1999; Weiss 1998; Whiteman 1995). Daher wurden diese beiden Arten der politischen Verwendung in der Befragung vorgezogen.

\section{Tabelle 3: Übersicht und Fragetext der Nutzungsarten als abhängige Variable}

\begin{tabular}{lll}
\hline Nutzungsform & Nutzungsart & Frage: Bei der Gesetzgebung nutze ich Evaluationen, ... \\
\hline Analytische Nutzung & instrumentell & $\ldots$ um Entscheide zu konkreten Sachfragen zu treffen. \\
& konzeptionell & $\ldots$ um ein politisches Thema generell besser zu verstehen. \\
Politische Nutzung & $\begin{array}{l}\text { legitimierend } \\
\text { persuasiv }\end{array}$ & $\ldots$ um einen bereits getroffenen Entscheid zu begründen. \\
& ... um andere von meiner Haltung zu überzeugen. \\
\hline
\end{tabular}

Gültige Antwortmöglichkeiten: nie [1], eher selten [2], eher häufig [3], sehr oft [4]. Daneben möglich waren auch die folgenden Antworten: weiss nicht, keine Antwort.

Abbildung 4 illustriert die Verteilung der zu erklärenden Variable, die diese beiden Bestandteile kombiniert. Die Häufigkeit der Evaluationsnutzung setzt sich folglich aus Antworten zur Frage der Nutzungsarten sowie Antworten zur allgemeinen Nutzung für die Aufgabe der Gesetzgebung zusammen. Für Befragte, die Evaluationen allgemein nie für die Aufgabe der Gesetzgebung nutzen, wurde die Nutzung in den einzelnen Arten ebenfalls als «nie» codiert. Dies scheint berechtigt, zumal nur sechs Befragte gemäss ihren Angaben Evaluationen eher selten oder häufiger für die Aufgabe der Gesetzgebung verwenden, aber bei allen vier Arten der Nutzung mit «nie» antworteten. ${ }^{42}$ Da diese Codierung aber auf einer relativ starken Annahme beruht, werden mögliche Auswirkungen auf die Ergebnisse in der folgenden Analyse besonders berücksichtigt. Analog zu den Fragen zur Nutzung für die Gesetzgebung und zu den Nutzungsarten nimmt die erklärende Variable also vier Ausprägungen an: nie (1), eher selten (2), eher häufig (3) und sehr oft (4). Weil die Ausprägungen klar geordnet werden können, aber die Distanzen zwischen den Ausprägungen unklar sind, kann die abhängige Variable als ordinal skaliert bezeichnet werden.

Insgesamt zeigt die Verteilung, dass die Befragten häufiger angeben, Evaluationen für analytische als für politische Zwecke zu verwenden. Die Verteilung ist bei den analytischen Nutzungsarten schiefer als bei den politischen Nutzungsarten. Evaluationen eher häufig bis sehr oft zu verwenden geben 63.6 Prozent der Befragten für die instrumentelle und 57.5 Prozent der Befragten für die konzeptionelle Art der analytischen Nutzung an, während dies 48.3 Prozent der Befragten für die legitimierende und 54.5 Prozent der

42 Die Antworten dieser sechs Befragten blieben unverändert im Datensatz, da die abgefragten Nutzungsarten nicht erschöpfend sind, wie etwa die Bemerkungen zur taktischen Nutzung nahelegen. 
Befragten für die persuasive Art der politischen Nutzung tun. Gemäss dem bisherigen Forschungsstand sowie der zuvor geäusserten Erwartung sollte politische Verwendung im Parlament dagegen häufiger als analytische Verwendung vorkommen (vgl. Kap. 3.1; Frey 2012; Weiss 1989; Whiteman 1985b). Eine mögliche Erklärung für diesen Widerspruch könnte sozial erwünschtes Antworten sein. Bundi et al. (2016) vermuten für die vorliegende Befragung, dass soziale Erwünschtheit bei einer positiven Einstellung gegenüber Evaluationen zu einer Überschätzung der Evaluationsnachfrage geführt haben könnte. Folglich könnten (einige) der Parlamentsmitglieder auch sozial erwünscht zur Evaluationsnutzung geantwortet haben, so dass sie die analytische Nutzung überschätzt und oder - die politische Nutzung unterschätzt haben.

\section{Abbildung 4: Häufigkeit der Nutzungsarten für die Gesetzgebung}

\section{Analytische Nutzung}

Instrumentell: Entscheide zu konkreten

Sachfagen treffen $(\mathrm{N}=1160)$

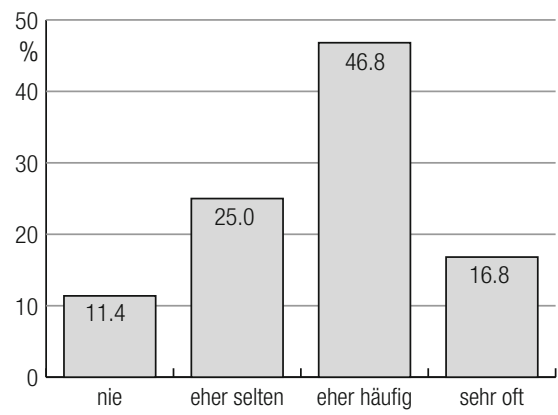

\section{Politische Nutzung}

Legitimierend: einen bereits getroffenen

Entscheid begründen $(\mathrm{N}=1151)$

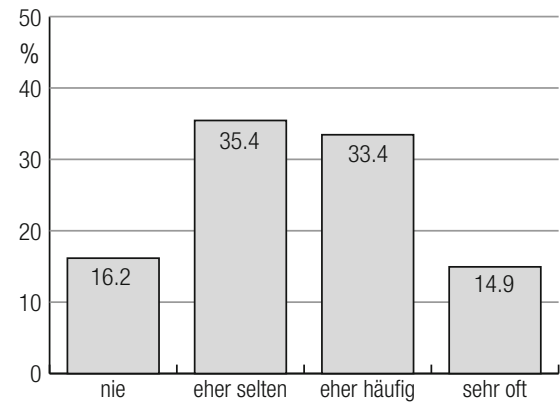

Konzeptionell: ein politisches Thema besser verstehen $(\mathrm{N}=1154)$

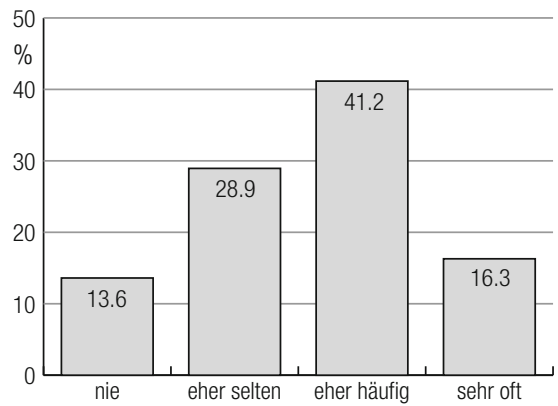

Persuasiv: andere von der eigenen Haltung überzeugen $(\mathrm{N}=1141)$

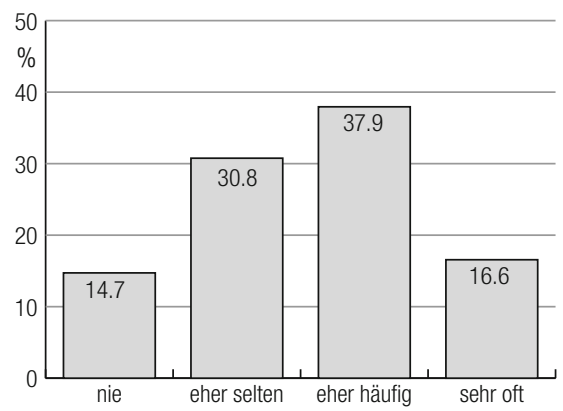


Die Antworten für die Nutzungsarten und für die allgemeine Nutzung hängen deutlich zusammen (vgl. Tabelle 4). Alle vier Nutzungsarten korrelieren paarweise signifikant positiv. Die Rangkorrelation ist bei den beiden Fragen zur politischen respektive zur analytischen Nutzung am höchsten, wobei sie aufgrund ihrer Werte als stark interpretiert werden kann. Diese vergleichsweise hohen Werte für die beiden Arten einer Nutzungsform unterstützen zwar die in der Theorie postulierte Annahme, dass grundsätzlich zwei Formen der Nutzung unterscheidbar sind. Gleichzeitig legt aber die Analyse nahe, dass die Frage zur Nutzung für die Gesetzgebung stärker mit den analytischen als mit den politischen Nutzungsarten zusammenhängt.

Tabelle 4: Zusammenhang der Nutzungsarten

\begin{tabular}{|c|c|c|c|c|c|}
\hline \multirow[t]{2}{*}{ Nutzungsform } & \multirow[t]{2}{*}{ Nutzungsart } & \multicolumn{3}{|c|}{ Nutzungsart $(\mathrm{N}=1125)$} & \multirow{2}{*}{$\begin{array}{r}\text { Nutzung } \\
\text { Gesetzgebung } \\
(\mathrm{N}=1099)\end{array}$} \\
\hline & & konzeptionell & instrumentell & legitimierend & \\
\hline \multirow[t]{2}{*}{ Analytische Nutzung } & konzeptionell & & & & $0.55^{\star \star \star}$ \\
\hline & instrumentell & $0.55^{\star \star \star}$ & & & $0.58^{\star \star \star}$ \\
\hline \multirow[t]{2}{*}{ Politische Nutzung } & legitimierend & $0.45^{\star \star \star}$ & $0.51^{\star \star \star}$ & & $0.46^{\star \star \star}$ \\
\hline & persuasiv & $0.42^{\star \star \star}$ & $0.51^{\star \star \star}$ & $0.60^{\star \star \star}$ & $0.45^{\star \star \star}$ \\
\hline
\end{tabular}

Kendalls $\tau_{\mathrm{b}}$, Zusammenhangsmasse nur für Befragte, die sämtliche Fragen zu den Nutzungsarten beantwortet haben. Signfikanzniveau ${ }^{\star \star \star} p<0.001$. Farblich hinterlegt nach Effektstärke.

Die Häufigkeit der Nutzung in den vier verschiedenen Arten variiert insgesamt kaum zwischen den Schweizer Parlamenten. Die in Abbildung 5 illustrierten parlamentsspezifischen Mittelwerte der Nutzungsarten weisen auf geringe Unterschiede zwischen den Maximal- und Minimalwerten: Während ein Parlamentsmitglied im Glarner Landrat im Schnitt Evaluationen «eher selten» legitimierend sowie persuasiv nutzt, tut dies ein Parlamentsmitglied im Genfer Kantonsrat oder im Nidwaldner Landrat durchschnittlich «eher häufig». Bei der analytischen Nutzung streuen die Werte hingegen weniger und die maximalen Unterschiede zwischen den Parlamenten betragen etwa den Wert einer halben Kategorie. Die Bundesversammlung $(\mathrm{CH})$ nimmt weiter keine spezifische Stellung in ihrem Nutzungsverhalten inne. Insgesamt ist daher die Varianz gering (vgl. 4.3.2). Dies gilt nicht nur für die Nutzung in den verschiedenen Arten, sondern auch für die Nutzung für die Gesetzgebung generell: Die parlamentsspezifische, durchschnittliche Verwendung für die Gesetzgebung liegt zwischen «eher selten» bis «eher häufig». So nutzen die Mitglieder des Glarner Landrats Evaluationen durchschnittlich am seltensten für die Gesetzgebung, während dies Mitglieder des Obwaldner Landrates am häufigsten tun. Damit lassen sich insgesamt weniger parlamentsspezifische Unterschiede bei der Nutzung feststellen als etwa bei der Einstellung gegenüber Evaluation, wie die Analysen der Befragungsdaten in Eberli et al. (2014) oder Eberli und Bundi (2017, 265) zeigen. 


\section{Abbildung 5: Durchschnittliche Nutzung in den einzelnen Schweizer Parlamenten}

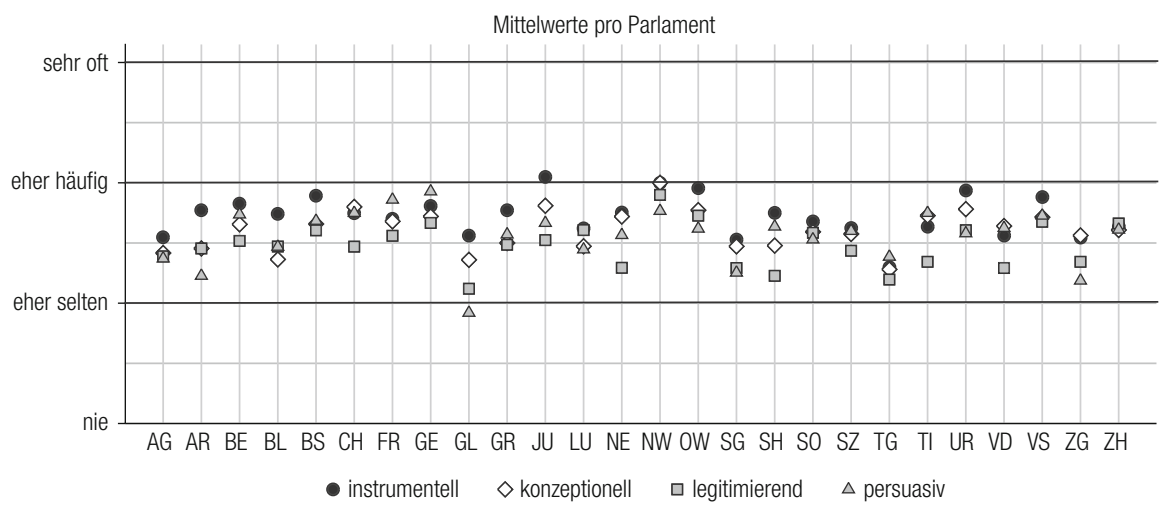

\subsubsection{Individuelle Erklärungsfaktoren: Messung und einzelne Zusammenhänge}

Zu den interessierenden unabhängigen Variablen dieser Untersuchung zählen die Erfahrung, die Parteizugehörigkeit, die Professionalisierung sowie der Bildungsgrad eines Parlamentsmitglieds (vgl. 3.1). Tabelle 5 präsentiert die Messung dieser Variablen für die quantitative Analyse sowie zentrale statistische Kennzahlen.

\section{Tabelle 5: $\quad$ Messung der individuellen Erklärungsfaktoren}

Erfahrung (in vollen Jahren in aktuellem Parlament)

Minimum: 0, Maximum: 35, Mittelwert: 6.9, Standardabweichung: 5.1, Median: 6

Parteizugehörigkeit (CVP, FDP, Grüne, SP oder SVP, inkl. Jung- oder Seniorenparteien)

CVP: $19.9 \%$, FDP: $23.4 \%$, Grüne: $9.6 \%$, SP: $26.5 \%$, SVP: $20.7 \%$

Professionalisierung (Für das parlamentarische Mandat aufgewendete Zeit in Prozent eines Vollzeitpensums)

Minimum: 0, Maximum: 100, Mittelwert: 24.1, Standardabweichung: 16.1, Median: 20.0

Bildungsgrad (Höchste abgeschlossene Ausbildung)

Sekundarstufe II (obligatorische Ausbildung, Berufs-/Maturitätsschulen): 23.1\%, höhere Berufsbildung (höhere Berufsbildung mit eidg. Fachausweis, Meisterdiplom/höhere Fachschule): 23.9\%, Fachhochschule (inkl. pädagogische Hochschule): 13.3\%, Universität: $39.8 \%$

$\mathrm{N}=1191$

Diese interessierenden unabhängigen Variablen hängen teilweise signifikant miteinander zusammen: Die Erfahrung und der Bildungsgrad korrelieren beide jeweils schwach positiv mit der Professionalisierung. Weiter sind FDP-Befragte im Vergleich mit allen anderen Befragten erfahrener, aber weniger professionalisiert. Befragte der Grünen hingegen sind verhältnismässig unerfahrener, aber sie wenden mehr Zeit für das Amt auf und haben 
eine relativ höhere Ausbildung abgeschlossen. Auch befragte SP-Parlamentsmitglieder sind professionalisierter und verfügen über einen verhältnismässig höheren Bildungsgrad. SVP-Befragte weisen dagegen einen geringeren Bildungsgrad aus. CVP-Befragte schliesslich sind weniger professionalisiert als Parlamentsmitglieder der anderen Parteien. ${ }^{43}$

Signifikante bivariate Zusammenhänge sind teilweise auch zwischen den vier individuellen Erklärungsfaktoren und der Evaluationsnutzung feststellbar. Die generelle Verwendung von Evaluationen für die Gesetzgebung korreliert signifikant positiv mit der Professionalisierung sowie dem Bildungsgrad. ${ }^{44}$ Wie Tabelle 6 zu den Evaluationsnutzungsarten zeigt, lassen sich ansonsten signifikante Zusammenhänge überwiegend bei der politischen Nutzung beobachten: Die Erfahrung korreliert negativ mit beiden politischen Nutzungsarten, die Professionalisierung demgegenüber korreliert positiv mit beiden politischen Nutzungsarten. Der Bildungsgrad korreliert nur signifikant positiv mit der persuasiven Verwendung. Befragte der SP verwenden Evaluationen häufiger persuasiv, während dies Befragte der CVP und SVP seltener tun. Auch die legitimierende Nutzung ist bei Befragten der SP ausgeprägter. Bei den analytischen Nutzungsarten hängt einzig die Professionalisierung signifikant positiv mit der konzeptionellen Verwendung zusammen.

Tabelle 6: Zusammenhänge Nutzungsarten und individuelle Erklärungsfaktoren

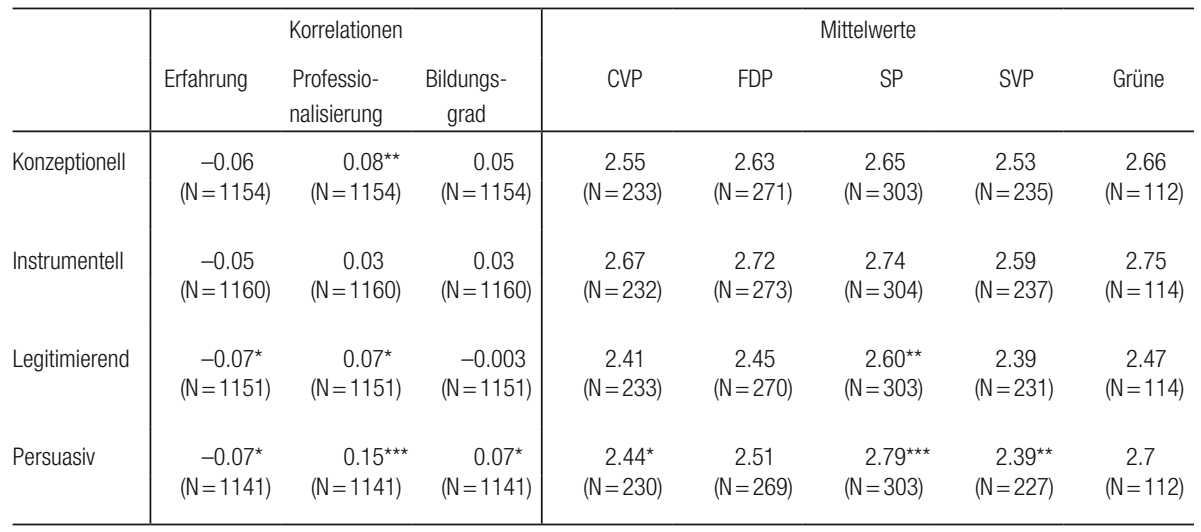

Spearman-Rangkorrelation, Mittelwerte: Mann-Whitney-U-Tests ${ }^{*} p<0.05,{ }^{* \star} p<0.01,{ }^{\star \star *} p<0.001$.

\subsection{Quantitative Analyse der individuellen Erklärungsfaktoren}

In der folgenden, multivariaten Analyse der individuellen Erklärungsfaktoren der Evaluationsnutzung werden alle individuellen Erklärungsfaktoren sowie weitere Variablen zusammen untersucht. Zunächst werden dazu die Variablen und das statistische Modell erläutert. Danach folgt die ausführliche Darstellung der Resultate. Dazu werden zuerst

43 Angaben zu sämtlichen Zusammenhängen befinden sich im Anhang.

44 Siehe Anhang. 
die Ergebnisse der Modelle präsentiert. Mittels Wahrscheinlichkeiten wird die Evaluationsnutzung dann in Abhängigkeit der einzelnen individuellen Erklärungsfaktoren vertieft diskutiert. Abschliessend werden kurz zusätzliche Modelle zur Überprüfung der Aussagekraft und Robustheit der Ergebnisse dargelegt. Weitere, detaillierte Ergebnisse befinden sich im Anhang.

\subsubsection{Variablen: Nutzungsarten, individuelle Erklärungsfaktoren, weitere Variablen}

Gegenstand der multivariaten Analyse sind die vier einzelnen Nutzungsarten. Jeweils die zwei Nutzungsarten in die analytische respektive politische Form zusammenzufassen, erscheint wenig lohnenswert, da zwei Items nur beschränkt eine aussagekräftige Skala bilden können. Entsprechend begrenzt sind auch die Möglichkeiten, um zu überprüfen, ob die zwei Nutzungsarten wirklich eine einzige zugrundeliegende Form betreffen (Eisinga et al. 2013). Die separate Analyse der einzelnen Nutzungsarten soll daher zusätzliche Informationen über die möglichen zugrundeliegenden Konzepte liefern. Ebenso erlaubt sie, vertiefte Informationen zu den Zusammenhängen zwischen unabhängigen Variablen und analytischer oder politischer Verwendung zu gewinnen. Schliesslich sprechen auch die in 4.2.1 vorgestellten Zusammenhangsmasse nur bedingt dafür, die einzelnen Nutzungsarten zu analytischer oder politischer Nutzung zusammenzufassen.

Zu den unabhängigen Variablen der multivariaten Analyse zählen die interessierenden Variablen der Erfahrung, der Parteizugehörigkeit, der Professionalisierung und des Bildungsgrads sowie Kontrollvariablen. Zur Kontrolle in die multivariate Analyse integriert werden Variablen, für die bisherigen Studien einen Zusammenhang mit der Evaluationsnutzung ausweisen und die auch mit interessierenden, unabhängigen Variablen im Zusammenhang stehen könnten. Aufgrund dieser Kriterien werden das Geschlecht und das Alter eines Parlamentsmitglieds sowie dessen politische Ambitionen in die multivariate Analyse integriert. Mehrere Untersuchungen weisen darauf hin, dass Parlamentarierinnen Evaluationen öfter nutzen (Bogenschneider und Corbett 2010, 144; Hird 2005b, 250). ${ }^{45}$ Zudem wenden Parlamentarierinnen gemäss Studien zu Schweizer Parlamenten in der Regel mehr Zeit für ihr Mandat auf (Bundi et al. 2017, 2018; Bütikofer 2014, 81 f.; Eberli et al. in press). Auch sind Parlamentarierinnen deutlich häufiger in linken Parteien vertreten. Das Alter - in Jahren gemessen - wird als Kontrollvariable eingeschlossen, da es für den Effekt der Erfahrung verantwortlich sein könnte (Askim 2009, 36). Zudem stellt Hird (2005b, 147) für ältere Parlamentsmitglieder eine geringere Nutzungshäufigkeit fest. ${ }^{46}$ Die politischen Ambitionen werden in der bisherigen Literatur dagegen seltener diskutiert. Gemäss Webber $(1986,255,286)$ gehört die Karriereorientierung zu den wichtigsten Einflussfaktoren der Nutzung von Policy-Information, da sie den Fokus der politischen Arbeit eines Parlamentsmitglieds bestimmt. Zur Messung der politischen Ambition dient die Kandidatur für die Nationalrats- und Ständeratswahlen 2015, die ein

45 Für politische Entscheidungsträgerinnen und träger stellt Oliver et al. $(2014,6)$ auch häufigere Nutzung durch Frauen fest, Lester $(1993,278)$ aber das Gegenteil.

46 Lester $(1993,278)$ beobachtet bei politischen Entscheidungsträgerinnen und trägern einen negativen Effekt des Alters. 
Jahr nach der Befragung stattfanden (vgl. Bundi et al. 2017). In die multivariate Analyse integriert wird die Kandidatur als eigenständige Variable sowie als Interaktion mit dem Alter. Dieser Interaktionsterm soll erlauben, die Zusammenhänge zwischen Kandidatur, Alter, Erfahrung und Professionalisierung besser zu entbündeln: Gemäss Bundi et al. (2017) hängen politische Ambitionen gerade bei jungen Parlamentsmitgliedern mit deren Professionalisierung zusammen, obwohl ältere Parlamentsmitglieder in der Regel mehr Zeit in ihr Amt investieren. Eine Erklärung dafür könnte in einer Gruppe älterer Neulinge begründet sein, die aufgrund ihrer arrivierten beruflichen Position oder ihrem Status als Pensionierte mehr Zeit aufwenden können.

Neben diesen ausgewählten Kontrollvariablen weist die bisherige Forschung auf Unterschiede in der Evaluationsnutzung hin, die sich aufgrund der Zugehörigkeit zur Regierungs- beziehungsweise Oppositionspartei oder aufgrund der geschäftsspezifischen Stellung eines Parlamentsmitglieds ergeben (Askim 2009; Florio et al. 1979; McDavid und Huse 2012; Mooney 2012; Raudla 2012; Speer et al. 2015). Diese Variablen sind aber weniger relevant. Einerseits ist die Zugehörigkeit zur Opposition in den schweizerischen Konkordanzdemokratien wenig aussagekräftig, da im vorliegenden Datensatz die Zugehörigkeit zur Opposition häufig gleichbedeutend wäre mit der Parteizugehörigkeit der Grünen. Die geschäftsspezifische Stellung eines Parlamentsmitglieds ist schliesslich nicht erfassbar, weil sich die Befragung auf die allgemeine Nutzung bezieht.

Die Aufnahme der unabhängigen Variablen ist zwar theoretisch und im Falle der Kontrollvariablen auch empirisch begründet, aber sie kann zu Multikollinearität führen. Neben den bereits erläuterten signifikanten Zusammenhängen zwischen der Erfahrung, der Parteizugehörigkeit, der Professionalisierung und des Bildungsgrads weist die deskriptive Analyse auf weitere signifikante Korrelationen bezüglich der Kontrollvariablen hin. So korreliert das Alter positiv mit der Erfahrung und der Professionalisierung, aber negativ mit dem Bildungsgrad. Auch sind Kandidierende sowie weibliche Befragte überdurchschnittlich jung. Zudem sind die befragten Parlamentarierinnen professionalisierter als ihre männlichen Pendants. Parlamentsmitglieder der Grünen und SP haben weiter einen höheren Anteil an Frauen als Parlamentsmitglieder aller anderen Parteien, SVP-Parlamentsmitglieder weisen hingegen einen vergleichsweise geringeren Frauenanteil aus. Der Anteil an Kandidierenden ist bei den Grünen schliesslich grösser als bei Parlamentsmitgliedern aller anderen Parteien, bei der FDP ist es umgekehrt. Alle Koeffizienten zeigen aber schwach bis mittlere Korrelationen an. Am höchsten ist der Effekt bei der Erfahrung und dem Alter mit einem Wert von 0.39. ${ }^{47}$ Die generell tiefen Werte für den Varianzinflationsfaktor deuten jedoch auf keine spezifischen Probleme der Multikollinearität. ${ }^{48}$

47 Die Zusammenhangsmasse enthält der Anhang.

48 Die VIF-Werte liegen unter 2, mit Ausnahme des Interaktionsterms Alter und Kandidatur (VIF = 24). Dies erklärt sich durch die Interaktion. Weitere Hinweise, inwiefern die Multikollinearität problematisch ist, wird der Vergleich der Modelle ohne sowie mit Interaktionsterm zeigen. 


\subsubsection{Modell: Varianten der ordinalen logistischen Regression}

Der Zusammenhang zwischen den einzelnen Arten der Evaluationsnutzung und den unabhängigen Variablen wird anhand ordinaler Regressionsmodelle untersucht (Fullerton 2009; Long und Freese 2014; Williams 2006). Die ordinale Regression bezweckt generell, die Wahrscheinlichkeiten für das Auftreten der geordneten Antwortkategorien der abhängigen Variable durch die unabhängigen Variablen hervorzusagen. Die verwendete abhängige Variable der Nutzungsart von Evaluationen ist geordnet: Sie kann in den Häufigkeiten bzw. Antwortkategorien «nie» (1), «eher selten» (2), «eher häufig» (3) sowie «sehr oft» (4) auftreten.

Im Folgenden werden verschiedene Varianten der ordinalen Regression eingesetzt: Das ordinale logistische Regressionsmodell (OLM), das generalisierte ordinale logistischen Regressionsmodell (GOLM) sowie das Partial Proportional Odds Modell (PPOM) (Fullerton 2009; Williams 2006). Dies ist angebracht, da die Variablen im ordinalen logistischen Regressionsmodell die sogenannte Proportional Odds Annahme erfüllen sollen. Diese Annahme besagt, dass der Effekt einer unabhängigen Variable für sämtliche Antwortkategorien gleich ist. Im vorliegenden Fall würde dies bedeuten, dass etwa die Erfahrung denselben Effekt hat auf Evaluationsnutzung von «nie» bis «sehr oft». Diese Annahme wird jedoch häufig verletzt, gerade bei grossen Fallzahlen (Long und Freese 2014, 331; Williams 2006, 2016). Auch beim vorliegenden Fall kann es plausibel sein, dass Erfahrung beispielsweise vor allem einen Effekt hat, ob ein Parlamentsmitglied Evaluationen überhaupt - d.h. häufiger als «nie» - nutzt, während die Erfahrung dann für die restlichen Antwortkategorien weniger relevant ist. Gilt die Proportional Odds Annahme nicht, kann das generalisierte ordinale logistische Regressionsmodell oder das Partial Proportional Odds Model verwendet werden. Im generalisierten ordinalen logistischen Regressionsmodell wird für jede Antwortkategorie je ein Effekt berechnet. Im Partial Proportional Odds Model werden hingegen nur unterschiedliche Effekte für die unabhängigen Variablen berechnet, welche die Proportional Odds Annahme nicht erfüllen. Dieses Modell ist sinnvoller, da es nur die Effekte aufzeigt, die sich tatsächlich unterscheiden. Im Folgenden werden daher die Effekte mit Wald-Tests auf ihre Gleichheit überprüft und je nach dem, ob sich die Koeffizienten für sämtliche, einige oder keine der Variablen signifikant voneinander unterscheiden, das generalisierte, das Partial Proportional Odds oder das ordinale logistische Regressionsmodell gerechnet (vgl. Fullerton 2009; Long und Freese 2014; Williams 2006). ${ }^{49}$

Die verwendeten Regressionsmodelle beziehen sich auf die individuelle Ebene der Parlamentsmitglieder, systematische Unterschiede zwischen den einzelnen Parlamenten werden aber ebenfalls kontrolliert. Dies ist notwendig, weil die Beobachtungen in den einzelnen Parlamenten möglicherweise nicht unabhängig voneinander sind, zumal die Mitglieder in ihren Parlamenten Gemeinsamkeiten haben, wie etwa organisatorische Strukturen oder eine ähnliche politische Kultur. In der folgenden Analyse werden die

49 Sämtliche Modelle werden mit Hilfe von Stata SE 13 geschätzt. Die Befehle lauten «ologit» (OLM) und "gologit2» (PPOM, GOLM, Williams 2006), für die Berechnung der Wahrscheinlichkeiten dient das Paket «SPost13» (Long und Freese 2014). 
Parlamente daher als Gruppen (Cluster) modelliert. In diesen Gruppen wird die Unabhängigkeit der Beobachtungen nicht vorausgesetzt. Zudem werden robuste Standardfehler verwendet (Long und Freese 2014, 104).

Da die vorliegende Datenstichprobe die Voraussetzungen für Regressionsmodelle mit mehreren Ebenen (Mehrebenenmodelle) nicht erfüllt, wird hingegen darauf verzichtet. Anhand von Mehrebenenmodellen würde die Varianz der Kantone modelliert, während die Varianz mit dem hier gewählten Vorgehen der Cluster nur kontrolliert wird (Hosmer et al. 2013; Rabe-Hesketh und Skrondal 2012). Wie die Ausführungen zu Abbildung 5 in 4.2.1 aufzeigen, unterscheidet sich die durchschnittliche Häufigkeit der Nutzung aber kaum für die 25 Kantonsparlamente und die Bundesversammlung. Dies bestätigt die Schätzung von Leermodellen zur Bestimmung der parlamentsspezifischen Varianz. Demgemäss wäre ein Mehrebenenmodell einzig bei der persuasiven Evaluationsnutzung überhaupt geeignet, da nur diese Nutzungsart signifikant zwischen den Parlamenten variiert. Auch bei der persuasiven Nutzungsart können aber die parlamentsspezifischen Variablen gemäss diesen Leermodellen nur maximal drei Prozent der gesamten Unterschiede in der Evaluationsnutzung erklären. Entsprechend sollten mögliche Fehler, die sich durch einen Verzicht auf ein Mehrebenenmodell ergeben, unbedeutend sein (vgl. Hosmer et al. 2013, 334). ${ }^{50}$ Folglich fokussiert die multivariate Analyse auf die individuellen Erklärungsfaktoren. Die parlamentsspezifischen Erklärungsfaktoren, namentlich das Kommissionensystem, werden mittels der Fallstudien untersucht. Dies scheint umso geeigneter, da der Effekt des Kommissionensystems auf die Evaluationsnutzung noch unerforscht ist. ${ }^{51}$

\subsubsection{Ergebnisse: Regressionsmodelle zur Evaluationsnutzung}

Beginnend mit den analytischen Nutzungsarten illustrieren die folgenden vier Tabellen die Ergebnisse der Regressionsmodelle, wobei die erste Tabelle zum besseren Verständnis ausführlicher erläutert wird. Die folgenden Tabellen mit den Ergebnissen listen die Koeffizienten der unabhängigen Variablen auf. Ein negativer Koeffizient weist dabei auf einen negativen Zusammenhang zwischen der unabhängigen Variablen und der Evaluationsnutzung hin, während ein positiver Koeffizient einen positiven Zusammenhang anzeigt. Neben den Koeffizienten und den robusten Standardfehlern in Klammern enthalten die Tabellen die Schwellenwerte ${ }^{52} \tau$ der ordinalen Regression sowie Statistiken zur Güte des Modells.

50 Diese sogenannte Intraklassenkorrelation liegt für die anderen Nutzungsarten zwischen 0.77 Prozent und 1.1 Prozent.

51 Ein weiterer Vorteil eines Verzichts auf Mehrebenenmodelle ist, dass die Lockerung der Proportional Odds Annahme zu relativ komplexen Mehrebenenmodellen führen würde. Bei diesen Modellen ist aber die Darstellung der Ergebnisse limitiert (vgl. Hedeker und Mermelstein 1998; Rabe-Hesketh und Skrondal 2012).

52 Mit den Schwellenwerten werden im ordinalen Regressionsmodell die Antwortkategorien bestimmt. Da es vier verschiedene Antwortkategorien gibt, sind drei Schwellenwerte vorhanden. 
Übereinstimmend mit den theoretischen Ausführungen in 3.1 werden für die individuellen Erklärungsfaktoren verschiedene Effekte erwartet: Bezüglich der Erfahrung eines Parlamentsmitglieds besagen $H_{1 a}$ und $H_{1 p}$, dass die analytische und die politische Evaluationsnutzung mit der Erfahrung sinkt. Somit werden für die Erfahrung Koeffizienten mit negativem Vorzeichen erwartet. Gemäss $H_{2 a}$ und $H_{2 p}$ sollten Parlamentsmitglieder moderater Parteien Evaluationen öfter analytisch und politisch verwenden. Folglich sollten sich die FDP und die CVP signifikant von den Grünen, SP und SVP unterscheiden. $H_{3 a}$ und $H_{3 p}$ für die Professionalisierung sagen hingegen nur einen positiven Effekt auf die analytische Verwendung voraus, was positive Koeffizienten bei den analytischen Nutzungsarten anzeigen würden. Der Bildungsgrad eines Parlamentsmitglieds sollte laut $H_{4 a}$ und $H_{4 p}$ zu häufigerer analytischer und politischer Nutzung führen.

\section{Analytisch: Instrumentelle Nutzung - Entscheide treffen}

Tabelle 7 präsentiert die Ergebnisse der Regressionsmodelle für die instrumentelle Verwendung von Evaluationen. Bei dieser Art erfüllen gemäss den Wald-Tests nicht alle Variablen die Proportional Odds Annahme, weshalb sich ein Partial Proportional Odds Modell am besten eignet. Für die Dummy-Variablen der SP-Parteizugehörigkeit, der höheren Berufsbildung, des universitären Abschlusses sowie des Geschlechts weist Tabelle 7 daher beispielsweise im vollständigen Modell drei Koeffizienten aus. Diese drei Koeffizienten stammen aus untergliederten Schätzungen: das erste Modell vergleicht Evaluationsnutzung in der Häufigkeit von «nie» mit allen drei höheren Antwortkategorien zusammen, das zweite Modell vergleicht Evaluationsnutzung in der Häufigkeit bis «eher selten» mit den zwei höheren Antwortkategorien zusammen, das dritte Modell vergleicht Evaluationsnutzung in der Häufigkeit bis «eher häufig» mit der höchsten Antwortkategorie «sehr oft». Für alle anderen Variablen gilt der Koeffizient über alle Antwortkategorien. Insgesamt weisen die Statistiken auf eine geringe Modellgüte hin. Das sogenannte Pseudo- $\mathrm{R}^{2}$ der Modelle liegt maximal bei $0.038 .{ }^{53}$

Bei den interessierenden Variablen zeigt Tabelle 7 signifikante Effekte für die Erfahrung, teilweise auch für die Parteizugehörigkeit sowie die Professionalisierung an. Der negative Koeffizient weist auf einen negativen Zusammenhang zwischen der Erfahrung und der instrumentellen Verwendung von Evaluationen hin. Bei der Parteizugehörigkeit bestehen nur im vollständigen Modell signifikante Unterschiede für die SP. Evaluationsnutzung in einer höheren Antwortkategorie als «nie» lässt sich demgemäss bei SP-Befragten signifikant häufiger beobachten als bei SVP-Befragten. Dieser Unterschied trifft auch für SP- und CVP-Befragte zu, wie ein zusätzliches Modell mit der CVP als Referenzpartei anzeigt (siehe Anhang). Andere zusätzliche Modelle mit der SP, der FDP und den Grünen als Referenzkategorie weisen hingegen auf keine weiteren signifikanten Parteienunterschiede hin. Die Professionalisierung hängt nur im vollständigen Modell mit

53 Inwiefern die Verwendung des Pseudo- $\mathrm{R}^{2}$ als Kennzahl für die Güte logistischer Regressionsmodelle sinnvoll ist, wird kontrovers diskutiert (Lacy 2006; Veall und Zimmermann 1992). Es liegt in der Regel deutlich tiefer als R2-Werte in verbreiteten, linearen Regressionsmodellen (Kohler und Kreuter 2012; Long und Freese 2014, 325). Hier soll das Pseudo-R2 daher hauptsächlich zum Vergleich der Modelle dienen. 
Tabelle 7: $\quad$ Resultate der Regressionsmodelle zur instrumentellen Nutzung

\begin{tabular}{|c|c|c|c|c|c|c|c|c|c|}
\hline & \multicolumn{3}{|c|}{$\begin{array}{c}\text { Modell } 1 \\
\text { Kontrollvariablen }\end{array}$} & \multicolumn{3}{|c|}{$\begin{array}{c}\text { Modell } 2 \\
\text { Vollständig ohne Interaktion }\end{array}$} & \multicolumn{3}{|c|}{$\begin{array}{c}\text { Modell } 3 \\
\text { Vollständig mit Interaktion }\end{array}$} \\
\hline & nie & $\begin{array}{r}\text { eher } \\
\text { selten }\end{array}$ & $\begin{array}{r}\text { eher } \\
\text { häufig }\end{array}$ & nie & $\begin{array}{r}\text { eher } \\
\text { selten } \\
\end{array}$ & $\begin{array}{r}\text { eher } \\
\text { häufig }\end{array}$ & nie & $\begin{array}{r}\text { eher } \\
\text { selten } \\
\end{array}$ & $\begin{array}{r}\text { eher } \\
\text { häufig }\end{array}$ \\
\hline Erfahrung & & & & $\begin{array}{r}-0.026^{\star} \\
(0.011)\end{array}$ & & & $\begin{array}{r}-0.029^{*} \\
(0.011)\end{array}$ & & \\
\hline $\mathrm{SP}$ & & & & $\begin{array}{l}0.713^{\star \star} \\
(0.271)\end{array}$ & $\begin{array}{c}0.305 \\
(0.179)\end{array}$ & $\begin{array}{c}0.096 \\
(0.220)\end{array}$ & $\begin{array}{c}0.289 \\
(0.178)\end{array}$ & & \\
\hline FDP & & & & $\begin{array}{l}0.265 \\
(0.157)\end{array}$ & & & $\begin{array}{c}0.250 \\
(0.162)\end{array}$ & & \\
\hline CVP & & & & $\begin{array}{c}0.176 \\
(0.162)\end{array}$ & & & $\begin{array}{c}0.185 \\
(0.166)\end{array}$ & & \\
\hline Grüne & & & & $\begin{array}{l}0.263 \\
(0.200)\end{array}$ & & & $\begin{array}{c}0.274 \\
(0.211)\end{array}$ & & \\
\hline Professionalisierung & & & & $\begin{array}{c}0.006 \\
(0.003)\end{array}$ & & & $\begin{array}{l}0.018^{\star \star} \\
(0.006)\end{array}$ & $\begin{array}{l}0.006^{\star} \\
(0.003)\end{array}$ & $\begin{array}{l}0.001 \\
(0.004)\end{array}$ \\
\hline Höhere Berufsbildung & & & & $\begin{array}{l}0.298 \\
(0.266)\end{array}$ & $\begin{array}{c}-0.229 \\
(0.210)\end{array}$ & $\begin{array}{c}-0.118 \\
(0.192)\end{array}$ & $\begin{array}{c}0.258 \\
(0.265)\end{array}$ & $\begin{array}{r}-0.218 \\
(0.209)\end{array}$ & $\begin{array}{r}-0.090 \\
(0.189)\end{array}$ \\
\hline Fachhochschule & & & & $\begin{array}{c}0.064 \\
(0.236)\end{array}$ & & & $\begin{array}{c}0.069 \\
(0.235)\end{array}$ & & \\
\hline Universität & & & & $\begin{array}{l}0.147 \\
(0.222)\end{array}$ & $\begin{array}{c}-0.164 \\
(0.210)\end{array}$ & $\begin{array}{c}0.243 \\
(0.168)\end{array}$ & $\begin{array}{c}0.162 \\
(0.233)\end{array}$ & $\begin{array}{r}-0.169 \\
(0.213)\end{array}$ & $\begin{array}{l}0.266 \\
(0.167)\end{array}$ \\
\hline Weiblich & $\begin{array}{c}0.164 \\
(0.256)\end{array}$ & $\begin{array}{c}0.173 \\
(0.155)\end{array}$ & $\begin{array}{c}-0.302 \\
(0.208)\end{array}$ & $\begin{array}{c}0.017 \\
(0.248)\end{array}$ & $\begin{array}{c}0.057 \\
(0.163)\end{array}$ & $\begin{array}{c}-0.375 \\
(0.202)\end{array}$ & $\begin{array}{c}0.007 \\
(0.266)\end{array}$ & $\begin{array}{c}0.046 \\
(0.163)\end{array}$ & $\begin{array}{r}-0.385^{\star} \\
(0.189)\end{array}$ \\
\hline Alter & $\begin{array}{c}-0.014 \\
(0.009)\end{array}$ & $\begin{array}{c}0.006 \\
(0.007)\end{array}$ & $\begin{array}{c}-0.008 \\
(0.008)\end{array}$ & $\begin{array}{c}0.004 \\
(0.006)\end{array}$ & & & $\begin{array}{c}0.012^{\star} \\
(0.006)\end{array}$ & & \\
\hline Kandidierend & $\begin{array}{c}-0.050 \\
(0.120)\end{array}$ & & & $\begin{array}{c}-0.132 \\
(0.135)\end{array}$ & & & $\begin{array}{l}1.431^{\star \star} \\
(0.538)\end{array}$ & & \\
\hline Kandidierend\#Alter & & & & & & & $\begin{array}{c}-0.032^{\star \star \star} \\
(0.010)\end{array}$ & & \\
\hline$\tau_{1}$ & & $\begin{array}{l}2.744^{\star \star} \\
(0.492)\end{array}$ & & & $\begin{array}{l}1.526^{\star \star \star} \\
(0.408)\end{array}$ & & & $\begin{array}{l}0.926^{*} \\
(0.399)\end{array}$ & \\
\hline$\tau_{2}$ & & $\begin{array}{c}0.215 \\
(0.337)\end{array}$ & & & $\begin{array}{c}0.349 \\
(0.380)\end{array}$ & & & $\begin{array}{r}-0.096 \\
(0.387)\end{array}$ & \\
\hline$\tau_{3}$ & & $\begin{array}{r}-1.086^{*} \\
(0.466)\end{array}$ & & & $\begin{array}{c}-1.839^{\text {** }} \\
(0.437)\end{array}$ & & & $\begin{array}{c}-2.246^{\star \star \star} \\
(0.478)\end{array}$ & \\
\hline $\mathrm{N}$ & & 1160 & & & 1160 & & & 1160 & \\
\hline $\mathrm{R}_{C U}^{2}$ & & 0.014 & & & 0.032 & & & 0.038 & \\
\hline LogLikelihood & & -1441 & & & -1432 & & & -1428 & \\
\hline Prob $>\chi^{2}$ & & 0.001 & & & 0.000 & & & 0.000 & \\
\hline
\end{tabular}

Partial Proportional Odds Modell, Proportional Odds Annahme erfüllt falls $p<0.05$ (Wald-Tests). Robuste Standardfehler in Klammern.

Bei Modellen mit nur einem Koeffizient für die drei Katgorien der abhängigen Variable «instrumentelle Nutzung» ist die Proportional Odds Annahme erfüllt, d.h., der Koeffizient ist über sämtliche Teilmodelle gleich. $p<0.001,{ }^{* \star} p<0.01,{ }^{\star} p<0.05$. 
Interaktionsterm signifikant positiv mit der instrumentellen Nutzung zusammen. Dies betrifft aber nur die Modelle zur häufigeren Evaluationsnutzung als «nie» sowie «eher selten». Sobald also Parlamentsmitglieder Evaluationen häufiger verwenden, scheint die Professionalisierung keinen bedeutenden Unterschied zu machen. Für den Bildungsgrad als vierte interessierende Variable sind keine Signifikanzen beobachtbar in Tabelle 7. Dies gilt auch für die im Anhang präsentierten zusätzlichen Modelle, bei denen je ein anderer Bildungsgrad als Referenzkategorie dient.

Die Kontrollvariablen erreichen gemäss Tabelle 7 nur Signifikanzen im vollständigen Modell mit Interaktionsterm. Kandidiert ein Parlamentsmitglied für die eidgenössischen Wahlen, verwendet es Evaluationen signifikant häufiger instrumentell. Der Interaktionsterm weist aber daraufhin, dass dieser positive Effekt der Kandidatur mit steigendem Alter abnimmt. Bei Nicht-Kandidierenden wirkt sich das Alter positiv auf die instrumentelle Evaluationsnutzung aus. Schliesslich geben Frauen seltener an, Evaluationen «sehr oft» instrumentell zu verwenden.

\section{Analytisch: Konzeptionelle Nutzung - ein Thema besser verstehen}

Tabelle 8 illustriert die Resultate für die Modelle zur konzeptionellen Evaluationsnutzung als zweite analytische Nutzungsart. Da alle Variablen die Proportional Odds Annahme erfüllen, basieren diese Resultate auf einem ordinalen logistischen Regressionsmodell. Insgesamt offenbaren die Statistiken eine ähnlich geringe Modellgüte wie bei der instrumentellen Nutzungsart.

Signifikante Effekte der interessierenden Variablen weist Tabelle 8 für die Erfahrung und die Professionalisierung aus. Die Erfahrung hängt analog zur instrumentellen Nutzungsart auch mit der konzeptionellen Nutzungsart signifikant negativ zusammen. Weiter hängt die Professionalisierung signifikant positiv mit der konzeptionellen Evaluationsnutzung zusammen. Neben diesen in Tabelle 8 ersichtlichen Effekten offenbaren die zusätzlichen Modelle mit anderen Parteien oder Bildungsabschlüssen als Referenzkategorien einen weiteren signifikanten Effekt für den Bildungsgrad: Bei Befragten mit Universitätsabschluss lässt sich signifikant häufiger konzeptionelle Nutzung in der Antwortkategorie "sehr oft» beobachten als bei Befragten mit Abschluss bis zur Sekundarstufe II. ${ }^{54}$ Schliesslich hängt gemäss Tabelle 8 keine der Kontrollvariablen signifikant mit der konzeptionellen Nutzungsart zusammen.

\section{Politisch: Legitimierende Nutzung - Entscheide begründen}

Nach diesen Ergebnissen für die analytischen Nutzungsarten illustriert Tabelle 9 die Resultate zur politischen Art der legitimierenden Evaluationsnutzung. Da die Proportional Odds Annahme nicht überall erfüllt ist, wird ein Partial Proportional Odds Modell

54 Vollständiges Modell mit Interaktion und SP- oder Grüne als Referenz für die Parteizugehörigkeit, $\mathrm{p}<0.05$. Ergebnisse zu sämtlichen Modellen mit anderen Referenzkategorien für die Parteizugehörigkeit und den Bildungsabschluss sind im Anhang aufgeführt. 
Tabelle 8: Resultate der Regressionsmodelle zur konzeptionellen Nutzung

\begin{tabular}{|c|c|c|c|}
\hline & $\begin{array}{c}\text { Modell } 1 \\
\text { Kontrollvariablen }\end{array}$ & $\begin{array}{c}\text { Modell } 2 \\
\text { Vollständig ohne Interaktion }\end{array}$ & $\begin{array}{c}\text { Modell } 3 \\
\text { Vollständig mit Interaktion }\end{array}$ \\
\hline Erfahrung & & $\begin{array}{l}-0.029^{\star \star} \\
(0.011)\end{array}$ & $\begin{array}{l}-0.030^{\star \star} \\
(0.011)\end{array}$ \\
\hline $\mathrm{SP}$ & & $\begin{array}{c}0.146 \\
(0.180)\end{array}$ & $\begin{array}{c}0.146 \\
(0.181)\end{array}$ \\
\hline FDP & & $\begin{array}{c}0.164 \\
(0.171)\end{array}$ & $\begin{array}{c}0.161 \\
(0.174)\end{array}$ \\
\hline CVP & & $\begin{array}{c}0.015 \\
(0.175)\end{array}$ & $\begin{array}{c}0.014 \\
(0.175)\end{array}$ \\
\hline Grüne & & $\begin{array}{c}0.123 \\
(0.189)\end{array}$ & $\begin{array}{c}0.125 \\
(0.188)\end{array}$ \\
\hline Professionalisierung & & $\begin{array}{l}0.011^{\star \star \star} \\
(0.003)\end{array}$ & $\begin{array}{l}0.011^{\star \star \star} \\
(0.003)\end{array}$ \\
\hline Höhere Berufsbildung & & $\begin{array}{c}0.031 \\
(0.131)\end{array}$ & $\begin{array}{r}0.0317 \\
(0.131)\end{array}$ \\
\hline Fachhochschule & & $\begin{array}{r}-0.112 \\
(0.185)\end{array}$ & $\begin{array}{r}-0.112 \\
(0.185)\end{array}$ \\
\hline Universität & & $\begin{array}{c}0.171 \\
(0.162)\end{array}$ & $\begin{array}{c}0.170 \\
(0.163)\end{array}$ \\
\hline Weiblich & $\begin{array}{c}0.147 \\
(0.153)\end{array}$ & $\begin{array}{c}0.059 \\
(0.140)\end{array}$ & $\begin{array}{c}0.056 \\
(0.139)\end{array}$ \\
\hline Alter & $\begin{array}{c}0.002 \\
(0.006)\end{array}$ & $\begin{array}{c}0.006 \\
(0.006)\end{array}$ & $\begin{array}{c}0.007 \\
(0.007)\end{array}$ \\
\hline Kandidierend & $\begin{array}{c}-0.031 \\
(0.120)\end{array}$ & $\begin{array}{r}-0.210 \\
(0.126)\end{array}$ & $\begin{array}{c}0.043 \\
(0.421)\end{array}$ \\
\hline Kandidierend\#Alter & & & $\begin{array}{r}-0.005 \\
(0.009)\end{array}$ \\
\hline$\tau_{1}$ & $\begin{array}{l}1.707^{\star \star \star} \\
(0.348)\end{array}$ & $\begin{array}{l}1.394^{\star \star \star} \\
(0.363)\end{array}$ & $\begin{array}{l}1.319^{\star \star \star} \\
(0.377)\end{array}$ \\
\hline$\tau_{2}$ & $\begin{array}{c}0.158 \\
(0.360)\end{array}$ & $\begin{array}{c}-0.174 \\
(0.365)\end{array}$ & $\begin{array}{c}-0.248 \\
(0.392)\end{array}$ \\
\hline$\tau_{3}$ & $\begin{array}{l}-1.782^{\star \star \star} \\
(0.345)\end{array}$ & $\begin{array}{l}-2.139^{\star \star \star} \\
(0.378)\end{array}$ & $\begin{array}{l}-2.214^{\star \star \star} \\
(0.398)\end{array}$ \\
\hline N & 1154 & 1154 & 1154 \\
\hline $\mathrm{R}_{\mathrm{Cu}}^{2}$ & 0.002 & 0.022 & 0.022 \\
\hline LogLikelihood & -1489 & -1478 & -1478 \\
\hline Prob $>\chi^{2}$ & 0.775 & 0.000 & 0.000 \\
\hline
\end{tabular}

Ordinales logistisches Regressionsmodell. Robuste Standardfehler in Klammern. Alle Koeffizienten erfüllen die Proportional Odds Annahme. ${ }^{* \star *} p<0.001,{ }^{* \star} p<0.01,{ }^{*} p<0.05$. 
Tabelle 9: $\quad$ Resultate der Regressionsmodelle zur legitimierenden Nutzung

\begin{tabular}{|c|c|c|c|c|c|c|c|c|c|}
\hline & \multicolumn{3}{|c|}{$\begin{array}{c}\text { Modell } 1 \\
\text { Kontrollvariablen }\end{array}$} & \multicolumn{3}{|c|}{$\begin{array}{c}\text { Modell } 2 \\
\text { Vollständig ohne Interaktion }\end{array}$} & \multicolumn{3}{|c|}{$\begin{array}{c}\text { Modell } 3 \\
\text { Vollständig mit Interaktion }\end{array}$} \\
\hline & nie & $\begin{array}{r}\text { eher } \\
\text { selten }\end{array}$ & $\begin{array}{r}\text { eher } \\
\text { häufig }\end{array}$ & nie & $\begin{array}{r}\text { eher } \\
\text { selten }\end{array}$ & $\begin{array}{r}\text { eher } \\
\text { häufig }\end{array}$ & nie & $\begin{array}{r}\text { eher } \\
\text { selten }\end{array}$ & $\begin{array}{r}\text { eher } \\
\text { häufig }\end{array}$ \\
\hline Erfahrung & & & & $\begin{array}{r}-0.026^{\star} \\
(0.012)\end{array}$ & & & $\begin{array}{r}-0.030^{\star} \\
(0.013)\end{array}$ & & \\
\hline SP & & & & $\begin{array}{l}0.261^{*} \\
(0.133)\end{array}$ & & & $\begin{array}{c}0.267^{*} \\
(0.134)\end{array}$ & & \\
\hline FDP & & & & $\begin{array}{c}0.103 \\
(0.125)\end{array}$ & & & $\begin{array}{c}0.088 \\
(0.132)\end{array}$ & & \\
\hline CVP & & & & $\begin{array}{c}-0.028 \\
(0.132)\end{array}$ & & & $\begin{array}{r}-0.019 \\
(0.132)\end{array}$ & & \\
\hline Grüne & & & & $\begin{array}{r}-0.022 \\
(0.213)\end{array}$ & & & $\begin{array}{r}-0.004 \\
(0.210)\end{array}$ & & \\
\hline Professionalisierung & & & & $\begin{array}{l}0.0100 \\
(0.006)\end{array}$ & $\begin{array}{l}0.001 \\
(0.004)\end{array}$ & $\begin{array}{l}0.007 \\
(0.004)\end{array}$ & $\begin{array}{l}0.011^{*} \\
(0.005)\end{array}$ & $\begin{array}{c}0.002 \\
(0.004)\end{array}$ & $\begin{array}{l}0.008^{*} \\
(0.004)\end{array}$ \\
\hline Höhere Berufsbildung & & & & $\begin{array}{r}-0.110 \\
(0.176)\end{array}$ & & & $\begin{array}{c}-0.104 \\
(0.179)\end{array}$ & & \\
\hline Fachhochschule & & & & $\begin{array}{c}-0.023 \\
(0.215)\end{array}$ & & & $\begin{array}{c}-0.022 \\
(0.215)\end{array}$ & & \\
\hline Universität & & & & $\begin{array}{r}-0.110 \\
(0.201)\end{array}$ & & & $\begin{array}{c}-0.115 \\
(0.207)\end{array}$ & & \\
\hline Weiblich & $\begin{array}{l}0.481^{\star \star} \\
(0.174)\end{array}$ & & & $\begin{array}{l}0.408^{*} \\
(0.177)\end{array}$ & & & $\begin{array}{c}0.384^{\star} \\
(0.174)\end{array}$ & & \\
\hline Alter & $\begin{array}{c}0.002 \\
(0.008)\end{array}$ & $\begin{array}{r}-0.016^{*} \\
(0.007)\end{array}$ & $\begin{array}{r}-0.013 \\
(0.011)\end{array}$ & $\begin{array}{l}0.006 \\
(0.009)\end{array}$ & $\begin{array}{c}-0.011 \\
(0.007)\end{array}$ & $\begin{array}{r}-0.009 \\
(0.010)\end{array}$ & $\begin{array}{c}0.016 \\
(0.010)\end{array}$ & $\begin{array}{c}-0.001 \\
(0.007)\end{array}$ & $\begin{array}{c}0.002 \\
(0.011)\end{array}$ \\
\hline Kandidierend & $\begin{array}{c}-0.046 \\
(0.109)\end{array}$ & & & $\begin{array}{c}-0.010 \\
(0.120)\end{array}$ & & & $\begin{array}{l}1.694^{\star \star \star} \\
(0.493)\end{array}$ & & \\
\hline Kandidierend\#Alter & & & & & & & $\begin{array}{c}-0.036^{\text {***}} \\
(0.008)\end{array}$ & & \\
\hline$\tau_{1}$ & $\begin{array}{l}1.401^{\star \star} \\
(0.450)\end{array}$ & & & $\begin{array}{l}1.212^{\star \star} \\
(0.462)\end{array}$ & & & $\begin{array}{c}0.690 \\
(0.498)\end{array}$ & & \\
\hline$\tau_{2}$ & $\begin{array}{c}0.596 \\
(0.351)\end{array}$ & & & $\begin{array}{c}0.538 \\
(0.376)\end{array}$ & & & $\begin{array}{c}0.021 \\
(0.371)\end{array}$ & & \\
\hline$\tau_{3}$ & $\begin{array}{r}-1.260^{\star} \\
(0.527)\end{array}$ & & & $\begin{array}{c}-1.448^{\star \star} \\
(0.547)\end{array}$ & & & $\begin{array}{c}-2.005^{\star \star \star} \\
(0.561)\end{array}$ & & \\
\hline $\mathrm{N}$ & & 1151 & & & 1151 & & & 1151 & \\
\hline $\mathrm{R}_{\mathrm{CU}}^{2}$ & & 0.025 & & & 0.039 & & & 0.047 & \\
\hline LogLikelihood & & -1497 & & & -1490 & & & -1485 & \\
\hline Prob $>\chi^{2}$ & & 0.006 & & & 0.000 & & & 0.000 & \\
\hline
\end{tabular}

Partial Proportional Odds Modell, Proportional Odds Annahme erfüllt falls $p<0.05$ (Wald-Tests). Robuste Standardfehler in Klammern.

Bei Modellen mit nur einem Koeffizient für die drei Katgorien der abhängigen Variable «legitimierende Nutzung» ist die Proportional Odds Annahme erfüllt, d. h., der Koeffizient ist über sämtliche Teilmodelle gleich. $p<0.001,{ }^{\star \star} p<0.01,{ }^{\star} p<0.05$. 
eingesetzt. Die Statistiken zur Modellgüte sind ähnlich oder besser als in den vorherigen Modellen für die analytische Verwendung.

Von den interessierenden Variablen zeigt Tabelle 9 für die Erfahrung, die SP-Parteizugehörigkeit sowie teilweise für die Professionalisierung signifikante Effekte. Die Erfahrung und die legitimierende Evaluationsnutzung hängen negativ zusammen. SP-Parlamentsmitglieder verwenden Evaluationen signifikant häufiger legitimierend als ihre Pendants der SVP. Andere signifikante Parteienunterschiede bestehen aber gemäss den Ergebnissen zusätzlicher Modelle nicht. ${ }^{55}$ Die Professionalisierung hat wie bei den analytischen Nutzungsarten einen positiven Effekt auf die legitimierende Nutzung, ist aber nur teilweise signifikant. Mit steigender Professionalisierung lässt sich so häufiger legitimierende Evaluationsnutzung in einer anderen Antwortkategorie als «nie» und besonders häufig in der Antwortkategorie "sehr oft» beobachten. Der Bildungsgrad als vierte interessierende Variable hängt wiederum nicht signifikant mit der legitimierenden Nutzung zusammen. ${ }^{56}$

Bei den Kontrollvariablen zeigen die Modelle signifikante Effekte für das Geschlecht und die Kandidatur sowie den Interaktionsterm von Kandidatur und Alter an. Parlamentarierinnen verwenden demgemäss Evaluationen häufiger legitimierend als Parlamentarier. Im Modell mit Interaktionsterm verwenden Kandidierende Evaluationen wiederum deutlich häufiger legitimierend als Nicht-Kandidierende. Dieser positive Effekt der Kandidatur ist jedoch wie bei der instrumentellen Nutzung schwächer für ältere Kandidierende.

\section{Politisch: Persuasive Nutzung - andere überzeugen}

Tabelle 10 präsentiert schliesslich die Ergebnisse der Regressionsmodelle zur persuasiven Verwendung als zweite politische Nutzungsart von Evaluationen. Auch bei dieser Nutzungsart erfüllen nicht alle Variablen die Proportional Odds Annahme und es werden wiederum Partial Proportional Odds Modelle angewendet. Die Güte der vollständigen Modelle zu dieser Nutzungsart ist verglichen mit den anderen drei Nutzungsarten klar am besten.

Die persuasive Evaluationsnutzung hängt signifikant mit der Erfahrung, der Parteizugehörigkeit sowie der Professionalisierung zusammen (vgl. Tabelle 10). Wie bei allen anderen Nutzungsarten wirkt sich die Erfahrung negativ auf die persuasive Verwendung aus. Die Professionalisierung hingegen hat wiederum einen positiven Effekt auf die persuasive Nutzung von Evaluationen. Bezüglich der Parteizugehörigkeit sind signifikante Unterschiede zwischen der SVP und der SP, der FDP sowie teilweise den Grünen feststellbar. Im Vergleich zu SVP-Parlamentsmitgliedern verwenden SP- sowie FDP-Parlamentsmitglieder Evaluationen häufiger persuasiv, also um andere zu überzeugen. Zusätzliche Modelle legen nahe, dass Parlamentsmitgliedern der SP eine besondere Rolle zukommt: Sie verwenden Evaluationen häufiger zur Überzeugung anderer als Parlamentsmitglieder

55 Ergebnisse der Modelle mit SP, FDP, CVP und Grünen als Referenz für Parteizugehörigkeit im Anhang.

56 Siehe Ergebnisse der Modelle mit anderen Referenzen für den Bildungsabschluss im Anhang. 
Tabelle 10: $\quad$ Resultate der Regressionsmodelle zur persuasiven Nutzung

\begin{tabular}{|c|c|c|c|c|c|c|c|c|c|}
\hline & \multicolumn{3}{|c|}{$\begin{array}{c}\text { Modell } 1 \\
\text { Kontrollvariablen }\end{array}$} & \multicolumn{3}{|c|}{$\begin{array}{c}\text { Modell } 2 \\
\text { Vollständig ohne Interaktion }\end{array}$} & \multicolumn{3}{|c|}{$\begin{array}{c}\text { Modell } 3 \\
\text { Vollständig mit Interaktion }\end{array}$} \\
\hline & nie & $\begin{array}{r}\text { eher } \\
\text { selten }\end{array}$ & $\begin{array}{r}\text { eher } \\
\text { häufig }\end{array}$ & nie & $\begin{array}{r}\text { eher } \\
\text { selten }\end{array}$ & $\begin{array}{r}\text { eher } \\
\text { häufig }\end{array}$ & nie & $\begin{array}{r}\text { eher } \\
\text { selten }\end{array}$ & $\begin{array}{r}\text { eher } \\
\text { häufig }\end{array}$ \\
\hline Erfahrung & & & & $\begin{array}{c}-0.021^{\star} \\
(0.009)\end{array}$ & & & $\begin{array}{l}-0.023^{\star \star} \\
(0.009)\end{array}$ & & \\
\hline SP & & & & $\begin{array}{l}0.644^{\star \star \star} \\
(0.167)\end{array}$ & & & $\begin{array}{l}0.641^{\star \star \star} \\
(0.168)\end{array}$ & & \\
\hline FDP & & & & $\begin{array}{l}0.259^{\star \star} \\
(0.101)\end{array}$ & & & $\begin{array}{l}0.241^{*} \\
(0.104)\end{array}$ & & \\
\hline CVP & & & & $\begin{array}{c}0.138 \\
(0.156)\end{array}$ & & & $\begin{array}{c}0.137 \\
(0.155)\end{array}$ & & \\
\hline Grüne & & & & $\begin{array}{c}0.757 \\
(0.419)\end{array}$ & $\begin{array}{l}0.664^{\star \star \star} \\
(0.196)\end{array}$ & $\begin{array}{r}-0.105 \\
(0.254)\end{array}$ & $\begin{array}{c}0.767 \\
(0.418)\end{array}$ & $\begin{array}{l}0.670^{\star \star \star} \\
(0.196)\end{array}$ & $\begin{array}{r}-0.105 \\
(0.259)\end{array}$ \\
\hline Professionalisierung & & & & $\begin{array}{l}0.017^{\star \star \star} \\
(0.004)\end{array}$ & & & $\begin{array}{l}0.017^{\star \star \star} \\
(0.004)\end{array}$ & & \\
\hline Höhere Berufsbildung & & & & $\begin{array}{r}-0.186 \\
(0.187)\end{array}$ & & & $\begin{array}{r}-0.183 \\
(0.188)\end{array}$ & & \\
\hline Fachhochschule & & & & $\begin{array}{c}0.129 \\
(0.289)\end{array}$ & $\begin{array}{c}0.306 \\
(0.253)\end{array}$ & $\begin{array}{r}-0.332 \\
(0.217)\end{array}$ & $\begin{array}{c}0.131 \\
(0.287)\end{array}$ & $\begin{array}{c}0.307 \\
(0.254)\end{array}$ & $\begin{array}{c}-0.331 \\
(0.217)\end{array}$ \\
\hline Universität & & & & $\begin{array}{r}-0.016 \\
(0.216)\end{array}$ & & & $\begin{array}{r}-0.018 \\
(0.219)\end{array}$ & & \\
\hline Weiblich & $\begin{array}{l}0.368^{\star \star} \\
(0.132)\end{array}$ & & & $\begin{array}{c}0.121 \\
(0.140)\end{array}$ & & & $\begin{array}{c}0.109 \\
(0.141)\end{array}$ & & \\
\hline Alter & $\begin{array}{c}-0.005 \\
(0.008)\end{array}$ & $\begin{array}{c}-0.017^{\star} \\
(0.007)\end{array}$ & $\begin{array}{c}-0.029^{\star \star \star} \\
(0.008)\end{array}$ & $\begin{array}{c}-0.005 \\
(0.008)\end{array}$ & $\begin{array}{c}-0.017^{\star} \\
(0.007)\end{array}$ & $\begin{array}{c}-0.029^{\star \star} \\
(0.009)\end{array}$ & $\begin{array}{c}0.002 \\
(0.008)\end{array}$ & $\begin{array}{c}-0.0104 \\
(0.008)\end{array}$ & $\begin{array}{r}-0.022^{\star} \\
(0.010)\end{array}$ \\
\hline Kandidierend & $\begin{array}{c}0.056 \\
(0.155)\end{array}$ & & & $\begin{array}{c}-0.177 \\
(0.115)\end{array}$ & & & $\begin{array}{c}0.931 \\
(0.485)\end{array}$ & & \\
\hline Kandidierend\#Alter & & & & & & & $\begin{array}{r}-0.023^{\star} \\
(0.009)\end{array}$ & & \\
\hline$\overline{\tau_{1}}$ & & $\begin{array}{l}1.894^{\star \star \star} \\
(0.455)\end{array}$ & & & $\begin{array}{l}1.512^{\star \star \star} \\
(0.426)\end{array}$ & & & $\begin{array}{l}1.196^{\star \star} \\
(0.431)\end{array}$ & \\
\hline$\tau_{2}$ & & $\begin{array}{c}0.932^{*} \\
(0.414)\end{array}$ & & & $\begin{array}{c}0.503 \\
(0.431)\end{array}$ & & & $\begin{array}{c}0.176 \\
(0.437)\end{array}$ & \\
\hline$\tau_{3}$ & & $\begin{array}{c}-0.289 \\
(0.481)\end{array}$ & & & $\begin{array}{c}-0.635 \\
(0.509)\end{array}$ & & & $\begin{array}{c}-0.984 \\
(0.539)\end{array}$ & \\
\hline N & & 1141 & & & 1141 & & & 1141 & \\
\hline $\mathrm{R}_{\mathrm{CU}}^{2}$ & & 0.027 & & & 0.085 & & & 0.088 & \\
\hline LogLikelihood & & -1480 & & & -1448 & & & -1447 & \\
\hline Prob $>\chi^{2}$ & & 0.000 & & & 0.000 & & & 0.000 & \\
\hline
\end{tabular}

Partial Proportional Odds Modell, Proportional Odds Annahme erfüllt falls $p<0.05$ (Wald-Tests). Robuste Standardfehler in Klammern.

Bei Modellen mit nur einem Koeffizient für die drei Katgorien der abhängigen Variable «persuasive Nutzung» ist die Proportional Odds Annahme erfüllt, d. h., der Koeffizient ist über sämtliche Teilmodelle gleich. $p<0.001,{ }^{\star \star} p<0.01,{ }^{\star} p<0.05$. 
von allen anderen Parteien. ${ }^{57}$ Weitere Unterschiede offenbaren die zusätzlichen Modelle für die Grünen. Bei Parlamentsmitgliedern der Grünen lässt sich im Gegensatz zu Parlamentsmitgliedern der SVP, der FDP sowie der CVP häufiger persuasive Nutzung in einer höheren Antwortkategorie als «eher selten» beobachten. ${ }^{58}$ Beim Bildungsgrad schliesslich sind in Tabelle 10 keine signifikanten Effekte erkennbar, die zusätzlichen Modelle im Anhang weisen aber auf einen signifikanten Unterschied hin: Parlamentsmitglieder mit Fachhochschulabschluss verwenden Evaluationen signifikant häufiger als «eher selten» persuasiv als Parlamentsmitglieder mit einem Abschluss der höheren Berufsbildung. ${ }^{59}$

Zusätzlich haben das Alter sowie der Interaktionsterm von Alter und Kandidatur einen signifikanten Effekt auf die persuasive Nutzung. Der Koeffizient des Alters zeigt zumeist einen negativen Zusammenhang an, ist aber nur teilweise signifikant: Je älter die Befragten sind, desto seltener lässt sich persuasive Evaluationsnutzung in einer höheren Antwortkategorie als «eher selten» oder «eher häufig» beobachten. Der Interaktionsterm von Alter und Kandidatur weist wiederum darauf hin, dass die persuasive Evaluationsnutzung bei Kandidierenden mit zunehmendem Alter sinkt.

\subsubsection{Ergebnisse: Wahrscheinlichkeiten der Evaluationsnutzung}

Basierend auf den zuvor dargestellten Modellen präsentiert dieser Abschnitt Wahrscheinlichkeiten der Evaluationsnutzung. Der Fokus liegt auf den im letzten Abschnitt diskutierten signifikanten Zusammenhängen. Neben durchschnittlichen Wahrscheinlichkeiten werden die Zusammenhänge anhand marginaler Effekte illustriert. Marginale Effekte zeigen die Veränderung der Wahrscheinlichkeit der Evaluationsnutzung auf, die entsteht, wenn sich eine unabhängige Variable unter der Konstanthaltung aller anderen unabhängigen Variablen ändert. Diese konkrete Illustration erlaubt, die praktische Bedeutung der unabhängigen Variablen besser einzuschätzen. Ergänzende Tabellen zu den marginalen Effekten befinden sich im Anhang.

\section{Erfahrung}

Die Erfahrung hängt mit allen vier Nutzungsarten signifikant negativ zusammen. Um diese Effekte besser zu veranschaulichen, illustriert Abbildung 6 die durchschnittlichen Wahrscheinlichkeiten eines Parlamentsmitglieds für die instrumentelle sowie die legitimierende Nutzung. Bezüglich der instrumentellen Nutzung zeigt die Abbildung, dass ein Parlamentsmitglied bei steigender Erfahrung bis 16 Jahre immer die höchste Wahrscheinlichkeit hat, Evaluationen eher häufig zu nutzen. Demgegenüber nähern sich mit zunehmender Erfahrung die Antwortkategorien «nie» und «sehr oft» an. Konkret hat ein Neumitglied mit 0 Jahren Erfahrung eine Wahrscheinlichkeit von 22.5 Prozent, Evaluati-

57 Vollständiges Modell mit/ohne Interaktion und SP als Referenz, CVP/SVP vs SP: $\mathrm{p}<0.001$, FDP vs SP: $\mathrm{p}<0.05$, Grüne vs SP nur für grösser als «eher häufig»: $\mathrm{p}<0.05$, Tabelle siehe Anhang.

58 Vollständiges Modell mit/ohne Interaktion und CVP bzw. FDP als Referenz, $\mathrm{p}<0.05$, Tabelle siehe Anhang.

59 Vollständiges Modell mit/ohne Interaktion, $\mathrm{p}<0.05$, Tabelle siehe Anhang. 
onen eher selten instrumentell zu verwenden. Nach einer ganzen Legislatur von üblicherweise vier Jahren steigt dies auf 24 Prozent. Bei zwei abgeschlossenen Legislaturen nimmt die Wahrscheinlichkeit der eher seltenen Verwendung weiter zu auf 25.4 Prozent. Zwei Legislaturen entsprechen gemäss Feh Widmer $(2015,95)$ etwa der durchschnittlichen Verweildauer eines Mitglieds in einem Kantonsparlament. Nach 16 Jahren $^{60}$ wächst die Wahrscheinlichkeit der eher seltenen instrumentellen Evaluationsnutzung nochmals an und beträgt 28.3 Prozent. Die Differenzen zwischen diesen verschiedenen Wahrscheinlichkeiten scheinen zwar gering, sie sind aber alle signifikant. ${ }^{61}$ Dies gilt ebenso für die anderen Antwortkategorien. Für die konzeptionelle Verwendung als zweite analytische Nutzungsart sind die Wahrscheinlichkeiten ähnlich, die Wahrscheinlichkeit der eher häufigen Evaluationsnutzung ist aber auf etwas tieferem Niveau. Wieder sind die Differenzen zwischen $0,4,8$ und 16 Jahren signifikant.

Bezüglich der legitimierenden Nutzung zeigt Abbildung 6 deutlichere Veränderungen. Während die eher häufige Verwendung für Neumitglieder die höchste Wahrscheinlichkeit aufweist, ist bereits nach etwas mehr als einer Legislatur die eher seltene Nutzung am wahrscheinlichsten. Demzufolge hat ein Neumitglied eine Wahrscheinlichkeit von 33.2 Prozent, Evaluationen eher selten legitimierend zu verwenden. Für ein Parlamentsmitglied mit 8 Jahren Erfahrung beträgt diese Wahrscheinlichkeit 36 Prozent. Auch diese Unterschiede bewegen sich auf einem kleinen Niveau, sind aber signifikant. Dies gilt wiederum für alle Antwortkategorien. Die Erfahrung hängt weiter ebenso mit der anderen politischen Nutzungsart der persuasiven Verwendung signifikant zusammen. Die

\section{Abbildung 6: Wahrscheinlichkeiten der Nutzung nach Erfahrung}

Instrumentell

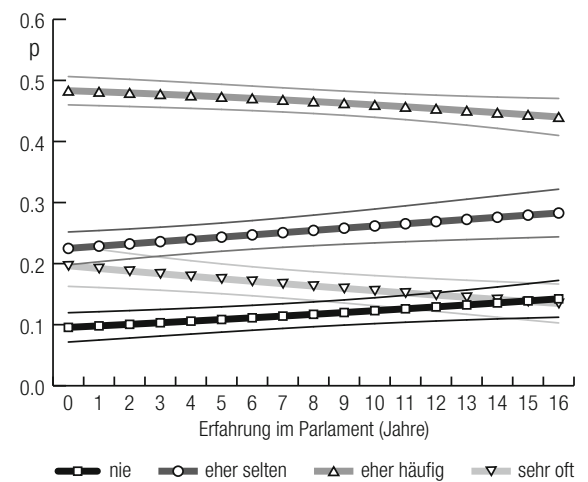

Legitimierend

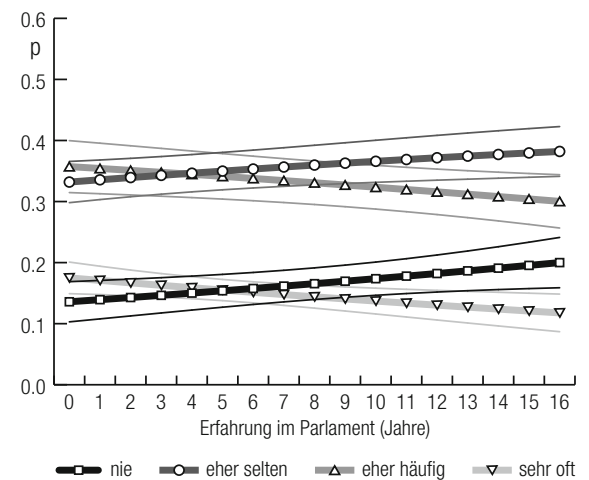

Durchschnittliche Wahrscheinlichkeiten mit 95\%-Konfidenzintervallen, vollständige Modelle mit Interaktion.

6095 Prozent der Parlamentsmitglieder haben 16 oder weniger Jahre Erfahrung.

61 Für die im Folgenden präsentierten, signifikanten Effekte liegt das Signifikanzniveau jeweils bei mindestens $\mathrm{p}<0.05$, siehe Resultate im Anhang. Es wurden auch marginale Effekte berechnet für ein typisches Parlamentsmitglied aller fünf Parteien, dessen Eigenschaften den Medianen und Mittelwerten der Stichprobe entsprechen. Die Signifikanz dieser marginalen Effekte eines typischen Parlamentsmitglieds deckt sich mit einigen Ausnahmen mit den präsentierten durchschnittlichen marginalen Effekten. 
Wahrscheinlichkeiten sind ähnlich wie bei der legitimierenden Verwendung, wobei Parlamentsmitglieder über den gesamten Zeitraum von 16 Jahren Erfahrung stets Evaluationen am wahrscheinlichsten eher häufig persuasiv verwenden. Die Differenzen zwischen $0,4,8$ und 16 Jahren sind jedoch auch für die persuasive Nutzung stets signifikant.

\section{Parteizugehörigkeit}

Signifikante Differenzen für die Parteizugehörigkeit sind vereinzelt bei der analytischen instrumentellen Verwendungsart, vor allem aber bei den politischen Verwendungsarten beobachtbar. Abbildung 7 illustriert daher die durchschnittlichen Differenzen in der Wahrscheinlichkeit im paarweisen Vergleich der Parteien. Diese paarweisen Vergleiche veranschaulichen, dass sich befragte Parlamentsmitglieder der SP am meisten in ihrer persuasiven Verwendung abheben. Gehört ein Parlamentsmitglied der SP an, ist die Wahrscheinlichkeit, Evaluationen persuasiv zu nutzen, signifikant höher als wenn es einer anderen Partei angehören würde. ${ }^{62}$ Beispielsweise hat ein Parlamentsmitglied der SP durchschnittlich eine Wahrscheinlichkeit von 22 Prozent, Evaluationen sehr oft persuasiv $\mathrm{zu}$ verwenden. Bei den anderen Parteien beträgt diese Wahrscheinlichkeit zwischen 12 Prozent (Grüne) und 16 Prozent (FDP). Dieser Effekt ist zudem nicht auf die höhere Quote an Parlamentarierinnen bei der SP zurückzuführen, da für die persuasive Verwendung bei der SP - und bei den restlichen Parteien - keine signifikanten Unterschiede zwischen den Geschlechtern feststellbar sind. Die Abbildung offenbart weiter keine

\section{Abbildung 7: Unterschiede der persuasiven Nutzung nach Parteizugehörigkeit}

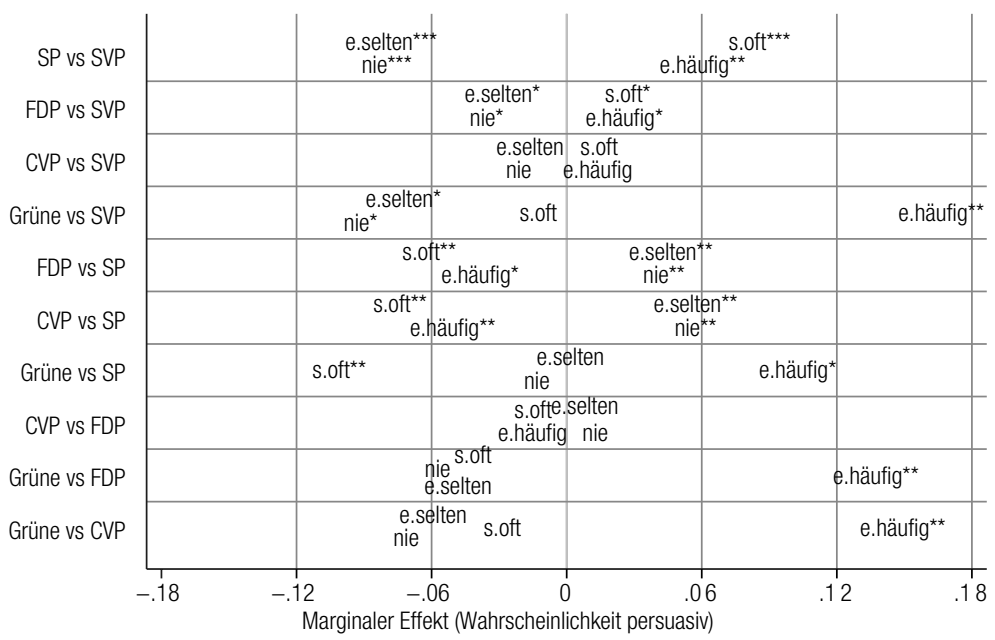

Durchschnittliche marginale Effekte, vollständiges Modell mit Interaktionsterm, ${ }^{\star \star \star} p<0.001,{ }^{\star \star} p<0.01,{ }^{\star} p<0.05$.

62 Zwischen den Grünen und der SP sind nur die Unterschiede in den Antwortkategorien «eher häufig» und «sehr oft» signifikant, wobei die häufigere Verwendung der SP-Parlamentsmitglieder aus der höheren Wahrscheinlichkeit der Antwortkategorie «sehr oft» resultiert. 
signifikanten Unterschiede für die CVP und die FDP beziehungsweise die SVP. Auch die Wahrscheinlichkeiten der Grünen unterscheiden sich nur punktuell: Sie haben eine deutlich höhere Wahrscheinlichkeit als alle anderen Parlamentsmitglieder, Evaluationen eher häufig persuasiv zu verwenden. Diese Differenzen sind beachtlich, da sie bis zu 17 Prozent ausmachen. Insgesamt legen schliesslich die unterschiedlichen Effekte für Grüne und SP, aber auch für FDP und SVP nahe, dass die Effekte auf der Parteizugehörigkeit gründen und nicht auf der ideologischen Positionierung (links-rechts).

\section{Abbildung 8: Unterschiede der legitimierenden Nutzung nach Parteizugehörigkeit (Differenz zu SP)}

Wahrscheinlichkeiten für «nie»

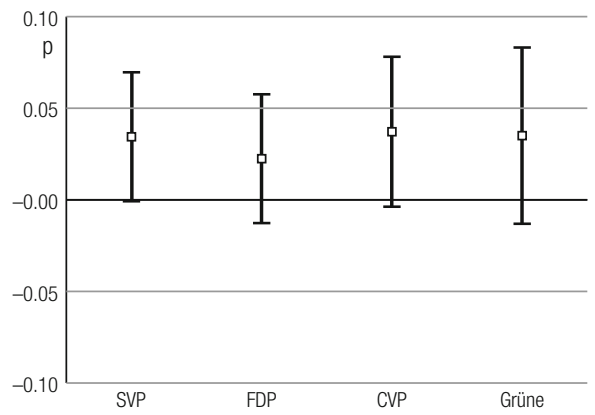

Wahrscheinlichkeiten für «eher häufig»

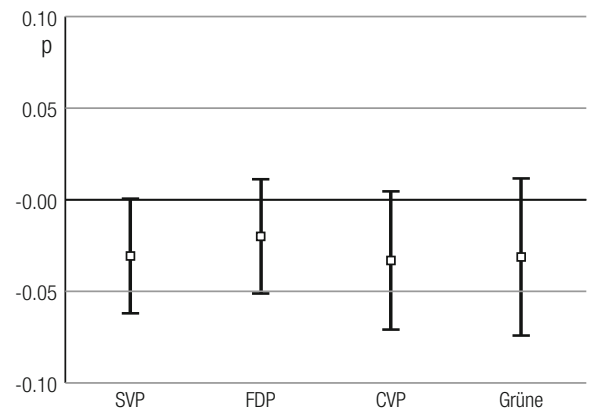

Wahrscheinlichkeiten für «eher selten»

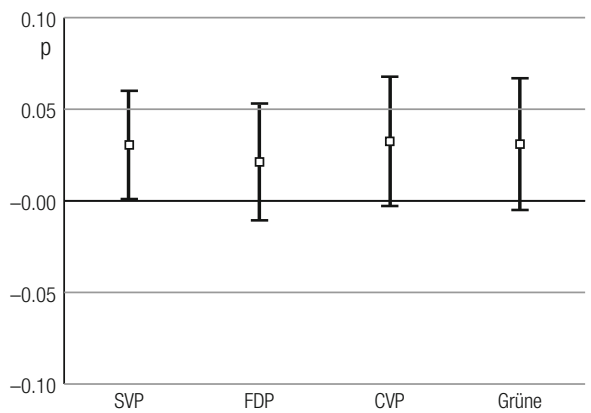

Wahrscheinlichkeiten für «sehr oft»

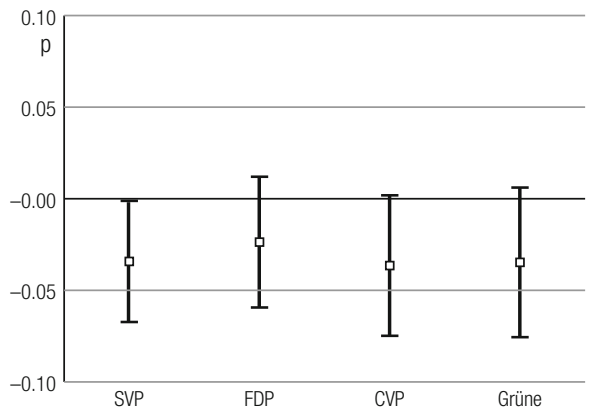

Durchschnittliche marginale Effekte mit 95\%-Konfidenzintervallen, vollständiges Modell mit Interaktionsterm.

Im Gegensatz zur persuasiven Verwendung sind die Unterschiede in den Wahrscheinlichkeiten für die instrumentelle und die legitimierende Verwendung weniger deutlich. Ersichtlich wird dies in Abbildung 8. Bei der legitimierenden Verwendung sind so nur die Differenzen zwischen SP und SVP für die Antwortkategorien «eher selten» und «sehr oft» signifikant. Die Abbildung illustriert aber, dass Parlamentsmitglieder der SP auch für die 
zweite politische Nutzungsart generell eine höhere Verwendung ausweisen. Keine signifikanten Unterschiede nach Parteizugehörigkeit sind dagegen für die Wahrscheinlichkeiten der instrumentellen Nutzung feststellbar.

\section{Professionalisierung}

Für die Professionalisierung sind bei allen vier Nutzungsarten signifikante Zusammenhänge beobachtbar, vor allem aber bei der analytischen konzeptionellen sowie der politischen persuasiven Verwendung. Abbildung 9 illustriert die kumulierte Wahrscheinlichkeit für die konzeptionelle sowie die legitimierende Evaluationsnutzung in Abhängigkeit des Zeitaufwands für das parlamentarische Mandat. Unabhängig des Pensums hat ein Parlamentsmitglied die höchste Wahrscheinlichkeit, Evaluationen eher häufig konzeptionell zu verwenden. Der positive Zusammenhang äussert sich dabei besonders in den Antwortkategorien «sehr oft» und «eher selten». Konkret verdoppelt sich die Wahrscheinlichkeit, Evaluationen sehr oft konzeptionell zu verwenden, von einem 10-prozentigen Zeitaufwand zu einem 100-prozentigen Zeitaufwand, während sich die eher seltene Nutzung fast halbiert (14.2 bis 30.7 Prozent, 30.9 bis 18 Prozent). Ein 10-prozentiges Pensum kann zwar in kleinen Kantonen der Realität entsprechen, ein kantonales Parlamentsmitglied investiert im Schnitt aber etwa 20 Prozent für das Mandat. ${ }^{63}$ Mit diesem Pensum nutzt ein Parlamentsmitglied Evaluationen mit einer durchschnittlichen Wahrscheinlichkeit von 15.5 Prozent sehr oft konzeptionell. Die Mitglieder der Kantonsparlamente gelten folglich klar als Milizpolitiker und Milizpolitikerinnen, die gemäss Literatur weniger als ein Drittel ihrer Zeit für das Mandat aufwenden (Bütikofer 2014, 69; Kerr 1981, 240). Berufspolitikerinnen und politiker investieren hingegen über zwei Drittel eines vollen Arbeitspensums für das Mandat. Mit einem Pensum von 66.6 Prozent nutzt ein Parlamentsmitglied Evaluationen mit einer Wahrscheinlichkeit von 22.8 Prozent sehr oft konzeptionell. Dies entspricht etwa den Mitgliedern der Bundesversammlung, die bei der Befragung durchschnittlich ein Pensum von 64.9 Prozent angeben. Die Differenzen zwischen diesen verschiedenen Zeitaufwänden sind alle für sämtliche Antwortkategorien signifikant. ${ }^{64}$ Für die instrumentelle Verwendung als zweite analytische Nutzungsart unterscheiden sich hingegen die Wahrscheinlichkeiten nach Grad der Professionalisierung deutlich weniger. Signifikant sind nur die Differenzen für die Antwortkategorie «nie»: Die durchschnittliche Wahrscheinlichkeit, Evaluationen nie instrumentell zu nutzen, beträgt bei einem 10-prozentigen Pensum 13.9 Prozent und bei einem 100-prozentigen Pensum 3.2 Prozent.

Für die legitimierende Verwendung fallen die Unterschiede gemäss Abbildung 9 weniger deutlich aus. Signifikant unterscheiden sich die Wahrscheinlichkeiten bei unterschiedlichem Professionalisierungsgrad nur bei den Antworten «nie» und «sehr oft». Ein

63 Zum Beispiel liegt das durchschnittliche Pensum der Befragten in der Stichprobe aus dem Landrat Glarus bei 7.4 Prozent, aus dem Kantonsrat Appenzell Ausserrhoden bei 12.9 Prozent. Der Mittelwert für alle kantonalen Parlamentsmitglieder beträgt 20.9 Prozent.

64 Nicht signifikant ist einzig die durchschnittliche Differenz in der Antwortkategorie «eher häufig» für ein 66-6-prozentiges Pensum verglichen mit einem Vollzeitpensum, Tabellen befinden sich im Anhang. 


\section{Abbildung 9: Wahrscheinlichkeiten der Nutzung nach Professionalisierung}

Konzeptionell

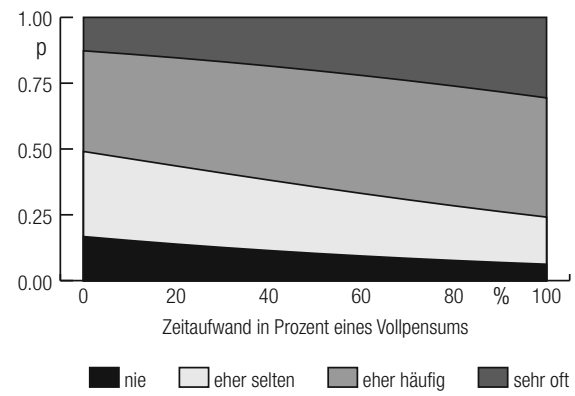

Legitimierend

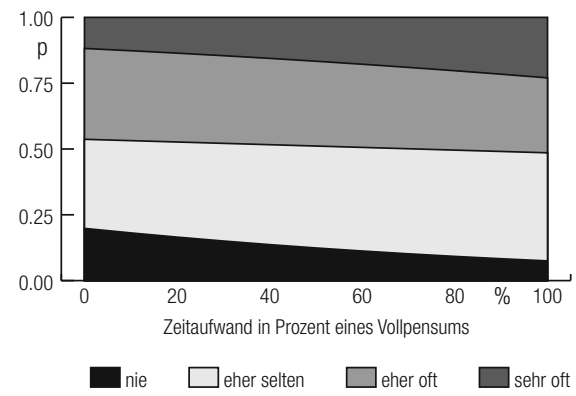

Durchschnittliche Wahrscheinlichkeiten, vollständige Modelle, konzeptionell: mit Interaktionsterm, legitimierend: ohne Interaktionsterm.

kantonales Parlamentsmitglied mit einem Pensum von 20 Prozent verwendet demgemäss Evaluationen mit einer Wahrscheinlichkeit von 14.2 Prozent nie legitimierend, während die Wahrscheinlichkeit bei einem 66.6-prozentigen Pensum 6.8 Prozent beträgt. Für die zweite politische Nutzungsart sind dagegen ähnlich prägnante Effekte wie bei der konzeptionellen Verwendung beobachtbar. Die durchschnittliche Wahrscheinlichkeit, Evaluationen sehr oft persuasiv zu nutzen, verdreifacht sich gar im Vergleich von einem 10- zu einem 100-prozentigen Pensum, während sich die Wahrscheinlichkeit bei eher seltener Evaluationsnutzung mehr als halbiert (13.3 zu 39.3 Prozent; $33.6 \mathrm{zu} 15$ Prozent). Bis auf einzelne Ausnahmen sind wieder alle Differenzen zwischen einem 10-, 20-, 66.6- und 100-prozentigem Pensum signifikant. Schliesslich haben die Parlamentsmitglieder trotz dieser Unterschiede stets die höchste durchschnittliche Wahrscheinlichkeit, Evaluationen eher häufig persuasiv zu verwenden.

Die Professionalisierung korreliert zwar signifikant positiv mit der Erfahrung, allerdings sind die Effekte dieser beiden individuellen Erklärungsfaktoren gegensätzlich. Wie sich dies auf die Wahrscheinlichkeiten der Nutzung auswirkt, illustriert Abbildung 10 für die persuasive Verwendung. Übereinstimmend mit dem negativen Effekt der Erfahrung zeigt die Abbildung auf, dass die durchschnittlichen Wahrscheinlichkeiten für die Antwortkategorien «nie» und «eher selten» mit zunehmender Erfahrung steigen, während sie für die Antwortkategorie «sehr oft» sinken. Je nach Grad der Professionalisierung geschieht dies aber auf einem klar unterschiedlichen Niveau: Während ein Parlamentsmitglied mit 8 Jahren Erfahrung und 20-prozentigem Pensum Evaluationen nie persuasiv mit einer durchschnittlichen Wahrscheinlichkeit von 15.5 Prozent nutzt, beträgt diese Wahrscheinlichkeit für ein Parlamentsmitglied mit derselben Erfahrung aber einem 66.6-prozentigen Pensum 7.8 Prozent. Die Konfidenzintervalle zeigen an, dass diese Niveauunterschiede zwischen einem Pensum von 20 Prozent und Pensen von 66.6 oder 100 Prozent in allen drei Antwortkategorien deutlich sind. Für die eher häufige persuasive Verwendung ist dagegen kein deutliches Muster erkennbar. 


\section{Abbildung 10: Wahrscheinlichkeiten der persuasiven Nutzung nach Erfahrung und Professionalisierung}

Wahrscheinlichkeit für «nie»

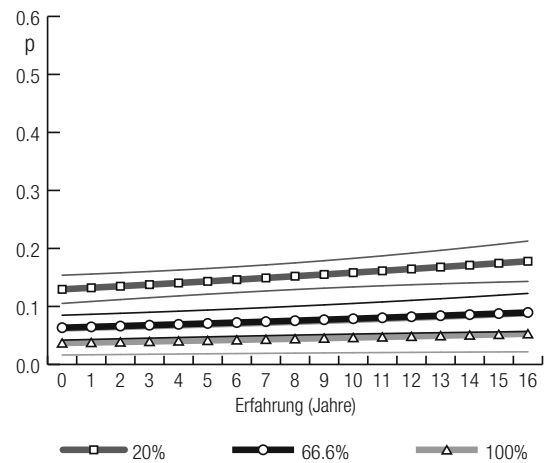

Wahrscheinlichkeit für «eher häufig»

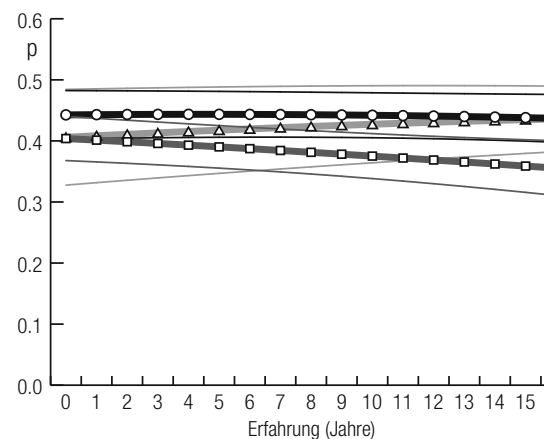

Wahrscheinlichkeit für «eher selten»

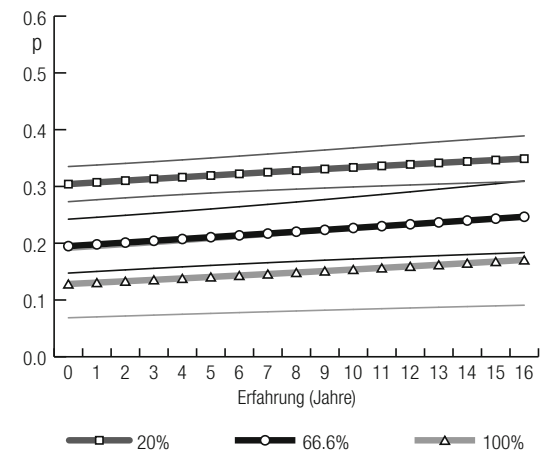

Wahrscheinlichkeit für «sehr oft»

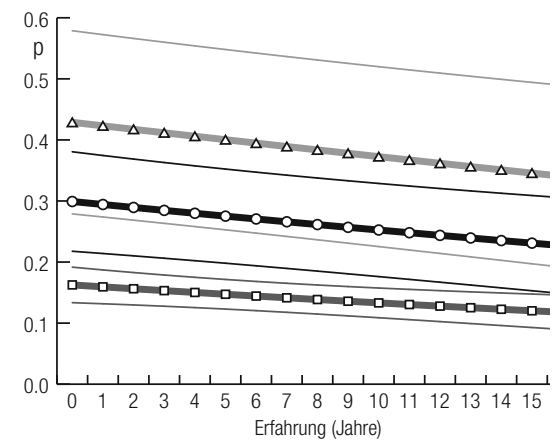

Durchschnittliche Wahrscheinlichkeiten bei einem Pensum von 20.0\%, 66.6\% und 100.0\% (Professionalisierung) mit 95\%-Konfidenzintervallen, vollständiges Modell mit Interaktionsterm.

\section{Bildungsgrad}

Die Ergebnisse der multivariaten Analyse verweisen nur auf einzelne signifikante Unterschiede zwischen den Bildungsabschlüssen bei der analytischen konzeptionellen sowie bei der politischen persuasiven Verwendung. Abbildung 11 präsentiert daher die durchschnittlichen marginalen Effekte der konzeptionellen Verwendung, wobei die Bildungsabschlüsse paarweise verglichen werden. Die Abbildung zeigt, dass die Unterschiede nach Bildungsgrad relativ klein und die marginalen Effekte nur für Befragte mit Universitätsabschluss signifikant sind. Diese Befragten haben eine signifikant höhere Wahrscheinlichkeit, Evaluationen sehr oft konzeptionell zu verwenden als alle anderen Befragten. ${ }^{65}$

65 Die Antwortkategorie «eher häufig» ist unabhängig des Bildungsgrads am wahrscheinlichsten. 
Maximal beträgt diese Differenz 6.5 Prozentpunkte. Da die Befragten mit Universitätsabschluss damit zur höchsten Antwortkategorie neigen, erfüllt diese Variable die Proportional Odds Annahme nicht. ${ }^{66}$ Zusätzlich legt die Abbildung nahe, dass der Zusammenhang zwischen dem Bildungsgrad und der konzeptionellen Evaluationsnutzung nicht linear ist. Befragte mit einem Fachhochschulabschluss weisen eine geringere Wahrscheinlichkeit auf, Evaluationen eher häufig oder sehr oft konzeptionell zu verwenden als Befragte mit einem Abschluss bis zur Sekundarstufe II oder Befragte mit einem Diplom der höheren Berufsbildung. Die marginalen Effekte für die politische persuasive Evaluationsnutzung sind im Anhang tabelliert. Für diese Nutzungsart sind nur einzelne signifikante Differenzen zwischen Befragten mit einem Fachhochschulabschluss sowie allen anderen Befragten feststellbar, wobei dies nur die Antwortkategorie «eher häufig» betrifft.

\section{Abbildung 11: Unterschiede der konzeptionellen Nutzung nach Bildungsgrad}

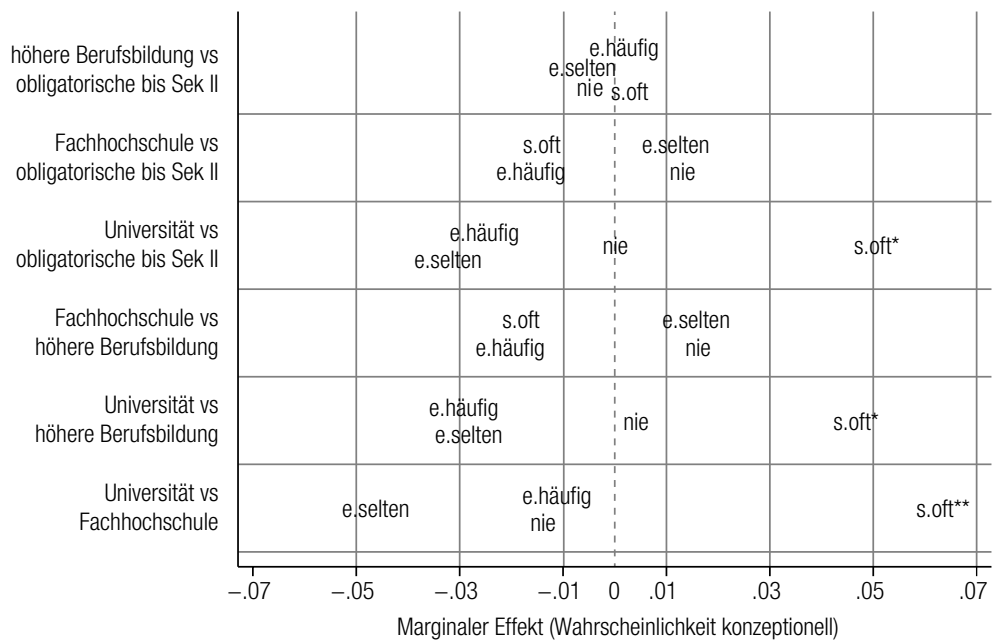

Durchschnittliche marginale Effekte, vollständiges Modell mit Interaktionsterm und SP als Referenz, ${ }^{* \star} p<0.01,{ }^{*} p<0.05$.

\section{Weitere Variablen: Geschlecht, Alter und Kandidatur für nationale Wahlen}

Gemäss den Regressionsmodellen hängen die Kontrollvariablen des Geschlechts, des Alters sowie der Kandidatur für die Nationalrats- und Ständeratswahlen teilweise signifikant mit der Evaluationsnutzung zusammen. Systematische geschlechtsspezifische Unterschiede offenbaren die Modelle nur bei der politischen legitimierenden Nutzung. Wie Abbildung 12 verdeutlicht, haben Parlamentarierinnen eine höhere durchschnittliche Wahrscheinlichkeit, Evaluationen eher häufig oder sehr oft legitimierend zu verwenden, als Parlamentarier. Keine oder eher seltene legitimierende Nutzung ist bei Parlamentari-

66 Bleibt der Zusammenhang über alle Antwortkategorien gleich, sollten die Antwortkategorien «nie» und "eher selten» respektive "eher häufig» und «sehr oft» auf je einer Seite zu beobachten sein. 
erinnen dagegen weniger wahrscheinlich als bei ihren männlichen Pendants. Alle diese geschlechtsspezifischen marginalen Effekte machen etwa 4.5 bis 5 Prozentpunkte aus und sind signifikant. Obschon ein relativ grosser Anteil der Frauen in der Stichprobe der SP angehört, sind die marginalen Effekte nicht parteigetrieben, da die Unterschiede zwischen Parlamentarierinnen und Parlamentarier für alle Parteien signifikant sind. ${ }^{67}$

\section{Abbildung 12: Unterschiede der legitimierenden Nutzung nach Geschlecht}

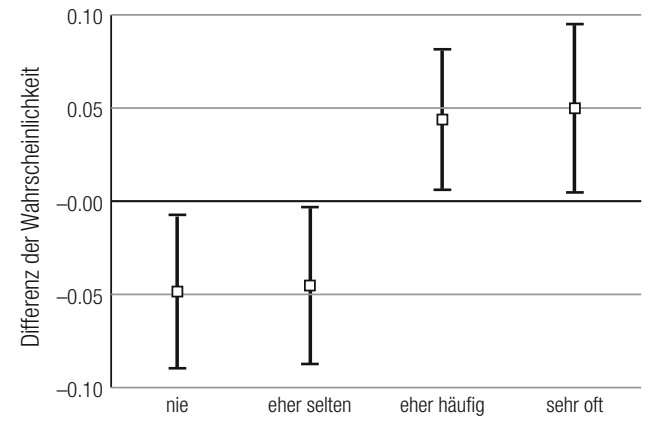

Durchschnittliche marginale Effekte mit 95\%-Konfidenzintervallen, vollständiges Modell mit Interaktionsterm.

Die Kandidatur für die Nationalrats- und Ständeratswahlen sowie deren Interaktion mit dem Alter hängt signifikant mit der analytischen instrumentellen sowie mit den beiden politischen Nutzungsarten zusammen. Befragte Kandidierende verwenden demgemäss Evaluationen eher, wobei dieser Effekt mit dem Alter abnimmt. Für die legitimierende Verwendung veranschaulicht wird dies in Abbildung 13, welche die durchschnittlichen Differenzen in den Wahrscheinlichkeiten von Kandidierenden und Nicht-Kandidierenden zwischen 20 und 70 Jahren aufzeigt. Diese Differenzen sind für Parlamentsmitglieder bis 33 respektive 35 Jahre signifikant, da die Konfidenzintervalle den Nullpunkt unterschreiten. Danach sind die Differenzen ab 53 Jahren wieder signifikant. So ist die Wahrscheinlichkeit eines 53-jährigen kandidierenden Parlamentsmitglieds, Evaluationen eher häufig legitimierend zu verwenden, um 2.5 Prozent geringer als bei einem nicht-kandidierenden Parlamentsmitglied. Bei einem Alter von 70 Jahren beträgt die Differenz 11.3 Prozentpunkte. Der signifikante Effekt betrifft folglich jene 54.6 Prozent der Kandidierenden in der Stichprobe, deren Alter entweder unter 35 Jahren oder über 53 Jahren liegt. ${ }^{68}$ Einen ähnlichen Effekt der Kandidatur wie in Abbildung 13 ist auch für die instrumentelle Evaluationsnutzung feststellbar. Für die persuasive Evaluationsnutzung sind dagegen die Differenzen deutlich geringer und erreichen daher keine Signifikanz über ein weiteres Altersspektrum. ${ }^{69}$

67 Für die Differenzen nach Geschlecht und Partei siehe Anhang.

68161 der 268 Kandidierenden in der Stichprobe für die legitimierende Evaluationsnutzung. Durchschnittsalter Nicht-Kandidierende: 52.4 Jahre. Durchschnittsalter Kandidierende: 48.7 Jahre. 


\section{Abbildung 13: Unterschiede der legitimierenden Nutzung nach Kandidatur und Alter}

Differenz der Wahrscheinlichkeit für «nie»

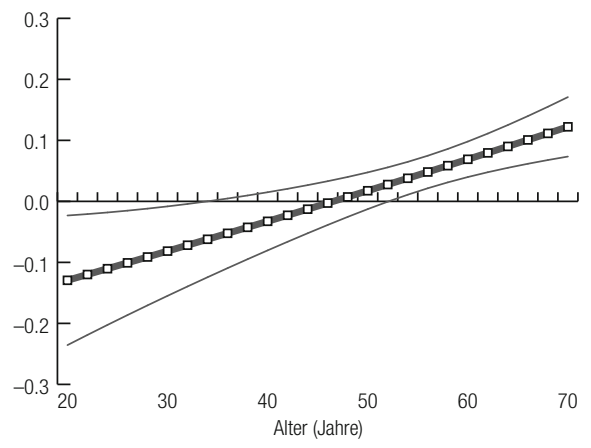

Differenz der Wahrscheinlichkeit für «eher häufig»

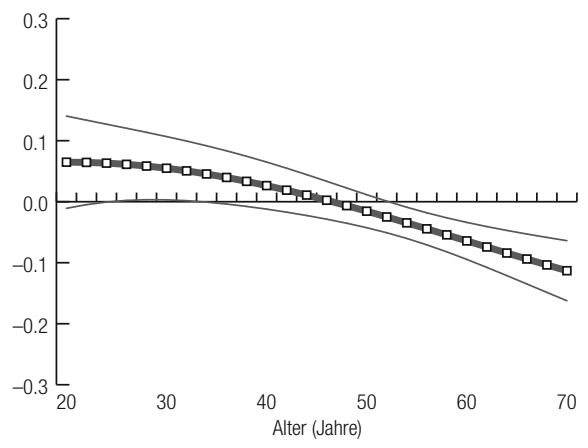

Differenz der Wahrscheinlichkeit für «eher selten»

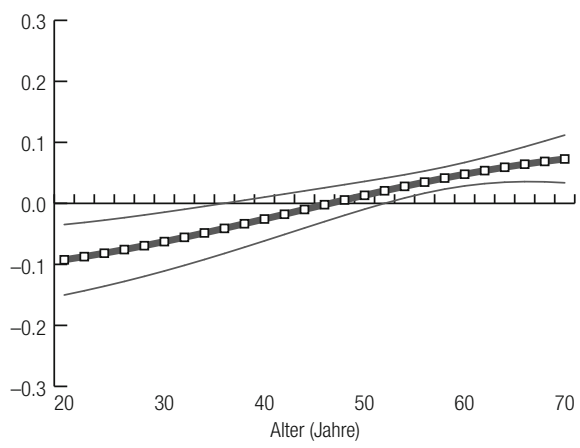

Differenz der Wahrscheinlichkeit für «sehr oft»

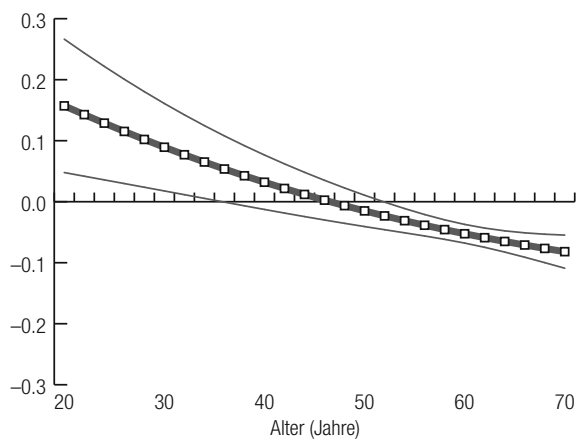

Durchschnittliche marginale Effekte mit 95\%-Konfidenzintervallen, vollständiges Modell mit Interaktion.

\subsubsection{Ergebnisse: Robustheit des verwendeten statistischen Vorgehens}

Um die Robustheit der Ergebnisse zu prüfen und deren Aussagekraft besser einzuschätzen, werden die zuvor präsentierten statistischen Modelle mit zusätzlichen Modellen verglichen. Im Fokus dieser Prüfung stehen Auswirkungen der Operationalisierung der abhängigen Variable der Evaluationsnutzung. Die Operationalisierung basiert auf zwei Teilen: Einerseits stützt sie sich auf die Frage zur Häufigkeit der Verwendung in der instrumentellen, konzeptionellen, legitimierenden sowie persuasiven Art. Anderseits beruht sie auf der Frage zur Nutzung von Evaluationen für die Gesetzgebung allgemein. Antworteten die Befragten, Evaluationen nie für die Aufgabe der Gesetzgebung zu verwenden, wurde ihnen die Frage nach den Arten der Nutzung gar nicht gestellt. Entsprechend wurde für diese Befragten auch «nie» als Antwort bei den vier Nutzungsarten eingefügt. Diese Codierung ermöglicht, Aussagen für die gesamte Stichprobe zu treffen (vgl. Manewitsch 2013). Zudem gaben nur sechs Befragte an, Evaluationen für die Gesetzgebung 
zu verwenden, aber dies nie in allen vier Arten der Nutzung zu tun. Es gilt aber sorgfältig abzuklären, inwiefern sich diese Codierung auswirkt, auch wenn sie nur 119 von 1191 Befragten betrifft. Sämtliche Ergebnisse zu den folgenden Ausführungen sind im Anhang aufgelistet.

Eine Möglichkeit zur Abklärung möglicher Auswirkungen der Operationalisierung stellt die vertiefte Analyse des Selektionsprozesses dar. ${ }^{70}$ Grundlage für diese Analyse bildet die Frage nach der Evaluationsnutzung für die Aufgabe der Gesetzgebung. Aus den Antworten zu dieser Frage kann eine Selektionsvariable gebildet werden, welche den Wert 0 annimmt, wenn ein Parlamentsmitglied antwortete, Evaluationen nie für die Gesetzgebung zu verwenden und somit die Frage zu den Arten der Nutzung nicht erhielt. Für alle anderen Befragten zeigt die Selektionsvariable den Wert 1 an. Gemäss einer logistischen Regression für diese Selektionsvariable wirken sich zwei individuelle Erklärungsfaktoren auf die Selektion aus: die Parteizugehörigkeit und die Professionalisierung. Parlamentsmitglieder der SP werden signifikant häufiger nach den Nutzungsarten gefragt als Parlamentsmitglieder der SVP. Zusätzliche Modelle mit anderen Parteien als Referenzkategorie zeigen keine weiteren parteispezifischen Unterschiede an. Weiter steigt mit dem Grad der Professionalisierung die Wahrscheinlichkeit signifikant an, die Frage nach den Nutzungsarten zu erhalten.

Eine andere Möglichkeit zur Untersuchung möglicher Verzerrungen aufgrund der Operationalisierung bilden multinomiale logistische Regressionsmodelle. Multinomiale logistische Regressionsmodelle nehmen keine Ordinalität an und vergleichen sämtliche Antwortkategorien miteinander. ${ }^{71}$ Folglich kann den 119 Befragten, die Evaluationen nie für die Gesetzgebung nutzen, eine eigene Antwortkategorie zugeschrieben werden, so dass das Modell insgesamt fünf Antwortkategorien enthält. ${ }^{72}$ Insgesamt bestätigen die Ergebnisse der multinomialen logistischen Regressionsmodelle den zuvor festgestellten grössten Selektionseffekt für die SP-Parteizugehörigkeit und die Professionalisierung. Die Resultate der multinomialen logistischen Regression bestätigen weiter die Resultate der in 4.3.3 präsentierten Modelle. Es sind aber einzelne zusätzliche signifikante Effekte für die Parteizugehörigkeit und den Bildungsgrad feststellbar.

Obwohl die multinomiale logistische Regression einen höheren Detailgrad bietet, eignen sich die gewählten ordinalen logistischen Regressionsmodelle besser für die multivariate Analyse. Multinomiale logistische Regressionsmodelle setzen zwar die Proportional Odds Annahme nicht voraus (Long und Freese 2014). Da sie sämtliche Antwortkategorien vergleichen, werden aber mehr Koeffizienten geschätzt als in ordinalen

70 Anstatt des folgenden Vorgehens könnten Selektionsmodelle (Heckman-Modelle) mittels eines zweistufigen Verfahrens gleichzeitig den Selektionsprozess und die Ausprägung der Nutzungsarten als abhängige Variable modellieren. Diese Selektionsmodelle sind zwar intuitiv, aber auch instabil und daher unsicher (Roderick und Schenker 1995, 57; Schafer und Graham 2002, 171 f.). Dies zeigt sich auch in einem Test mit den vorliegenden Daten.

71 Long und Freese (2014) empfehlen daher ordinale Modelle stets mit einem Modell zu vergleichen, das keine Ordinalität voraussetzt.

72 Am Beispiel der instrumentellen Nutzung: nie für die Aufgabe der Gesetzgebung [0], nie instrumentell [1], eher selten instrumentell [2], eher häufig instrumentell [3], sehr oft instrumentell [4]. 
logistischen Regressionsmodellen. Gerade Partial Proportional Odds Modelle bilden daher eine deutlich schlankere Herangehensweise. Sie erlauben, die Proportional Odds Annahme - wenn notwendig - für einzelne unabhängige Variablen zu lockern und liefern damit vertiefte Informationen zu diesen Variablen. Dies ist in den präsentierten Modellen nur für eine Minderheit der unabhängigen Variablen erforderlich, obwohl der Test für die Proportional Odds Annahme bei hohen Fallzahlen sensibel ist (Williams 2016, 18). Zudem ist das ordinale logistische Regressionsmodell auch einfacher zu interpretieren (Kühnel und Krebs 2010). Schliesslich spricht der Vergleich der Modellgüte ebenso für die verwendeten Modelle: Die Informationskriterien AIC und BIC liegen in sämtlichen ordinalen logistischen Regressionsmodellen unter den entsprechenden Werten im jeweiligen multinomialen Regressionsmodell. Zudem erhöht sich das Pseudo- $\mathrm{R}_{2}$, das tiefe Werte annimmt, nur gering im multinomialen Regressionsmodell.

Insgesamt deutet die Prüfung der Robustheit darauf hin, dass die Effekte zur Parteizugehörigkeit eher sensibel auf die Spezifizierung der abhängigen Variable reagieren, während die deutlichen Effekte für die Erfahrung und die Professionalisierung sowie die nicht bestehenden Unterschiede nach Bildungsgrad robust sind. Da sich die Ergebnisse vor allem bei wenig systematischen oder bei schwachen Effekten unterscheiden, ist die gewählte Operationalisierung durchaus gangbar. Eine zusätzliche Transformation der abhängigen Variable scheint aber aufgrund der beobachteten Auswirkungen und eines Informationsverlusts durch die Änderung der Antwortkategorien nicht angezeigt.

\subsection{Diskussion der Ergebnisse der quantitativen Analyse}

Zum Abschluss der Analyse der individuellen Erklärungsfaktoren werden nun die zentralen Ergebnisse des gesamten Kapitels zusammengefasst und mit der bestehenden Literatur verglichen sowie Grenzen der vorliegenden Analyse aufgezeigt. Zusammen mit dem Vergleich und der Diskussion der Fallstudien am Ende des nächsten Kapitels zu den kontextuellen Erklärungsfaktoren bilden diese Ausführungen die Basis für die Synthese im sechsten Kapitel des Buchs.

Grundlage der quantitativen Analyse der individuellen Erklärungsfaktoren bildet die Stichprobe der 1191 Mitglieder von 25 Kantonsparlamenten und der Bundesversammlung, die der CVP, FDP, SP, SVP und den Grünen angehören und an der Befragung «Parlamente und Evaluationen» teilnahmen. Beteiligt haben sich insgesamt verhältnismässig mehr Parlamentarierinnen, mehr Parlamentsmitglieder der Grünen sowie der SP und weniger Parlamentsmitglieder der SVP. Verglichen mit anderen Befragungen in Parlamenten ist die Antwortquote von 50.1 Prozent zufriedenstellend bis hoch und die Stichprobe repräsentativ. Neben beschreibenden Analysen werden hauptsächlich ordinale logistische Regressionsmodelle verwendet, um die Evaluationsnutzung zu untersuchen. Vier verschiedene Arten der Evaluationsnutzung sind Gegenstand der Untersuchung: Die instrumentelle und konzeptionelle Nutzung als Arten der analytischen Nutzung sowie die persuasive und legitimierende Nutzung als Arten der politischen Nutzung. Die Regressionsmodelle berücksichtigen mögliche parlamentsspezifische Effekte, es werden 
aber keine Mehrebenenmodelle angewendet, da die Nutzungsarten nicht wesentlich zwischen den Parlamenten variieren.

\subsubsection{Evaluationsnutzung: Relativ rege, häufiger analytisch als politisch}

Gemäss der Befragung verwendet etwa die Hälfte der Parlamentsmitglieder Evaluationen in verschiedenen Arten im Rahmen ihrer gesetzgeberischen Tätigkeiten. 49.5 Prozent der befragten Parlamentsmitglieder nutzen Evaluationen eher häufig bis sehr oft für die Gesetzgebung. Dabei verwenden 63.6 Prozent der Befragten Evaluationen eher häufig bis sehr oft in der instrumentellen Art, um Entscheide zu treffen. 57.5 Prozent der Befragten tun dies in der konzeptionellen Art, um ein Thema besser zu verstehen. Im Gegensatz zu dieser analytischen Nutzung berichten die befragten Parlamentsmitglieder über weniger umfangreiche politische Nutzung: 54.5 Prozent der Befragten verwenden Evaluationen eher häufig bis sehr oft in der persuasiven Art, um andere von ihrer Haltung zu überzeugen, während 48.3 Prozent der Befragten Evaluationen legitimierend nutzen, um Entscheide zu begründen. Die Bundesversammlung und die untersuchten 25 Kantonsparlamente unterscheiden sich aber nicht wesentlich in ihrer analytischen und politischen Nutzung.

Angesichts des politischen Charakters der Parlamente scheint die in der Befragung festgestellte Evaluationsnutzung rege, sie deckt sich aber mit dem grundsätzlich evaluationsfreundlichen Bild, das die Literatur zeichnet. Die bisherige Forschung zur Schweiz und andernorts zeigt, dass Parlamentsmitglieder systematisch generiertes Wissen nützlich finden und es auch als relevant ansehen (Balthasar 2009a; Bättig und Schwab 2015; Boyer und Langbein 1991, 528; Demaj et al. 2012, 38; Guston et al. 1997, 454; Hird 2009, 527; Janett 2001, 2009; Webber 1987, 617). Auch gemäss anderen Auswertungen der verwendeten Befragung sind mehr als zwei Drittel der Befragten positiv gegenüber Evaluationen eingestellt und lesen eher häufig bis sehr oft Zusammenfassungen von Evaluationen (Eberli und Bundi 2017; Eberli et al. 2014). Weiter weisen frühere Arbeiten darauf hin, dass grundsätzlich verschiedene Formen der Nutzung im Parlament vorkommen (vgl. Boyer und Langbein 1991, 529; Tabuga 2017, 9; Whiteman 1985a, 299). Dass Parlamentsmitglieder gemäss der vorliegenden Ergebnisse Evaluationen häufiger analytisch als politisch nutzen, stellen zwar andere Forschende ebenfalls fest (vgl. Hird 2005b, 144). Es spricht aber entgegen der in der Literatur verbreiteten - und in diesem Buch postulierten - Erwartung, dass die politische Nutzung im Parlament überwiegt (vgl. Frey 2012; Shulock 1998; Weiss 1989). Daher ist nicht auszuschliessen, dass die Parlamentsmitglieder sozial erwünscht antworteten und ihre analytische Nutzung überschätzt und, beziehungsweise oder, die politische Nutzung unterschätzt haben. Gleichzeitig gibt es aber wenig Studien, welche die verschiedenen Nutzungsformen im Parlament und generell in politischen Entscheidungsprozessen direkt gegenüberstellen (vgl. Amara et al. 2004; Nutley et al. 2007, 67). 


\subsubsection{Individuelle Erklärungsfaktoren: Bedeutsame Effekte der Erfahrung und Professionalisierung}

\section{Erfahrung: Hemmend für die analytische und politische Nutzung}

Gemäss den Hypothesen soll die Erfahrung negativ mit analytischer $\left(H_{l a}\right)$ und politischer Nutzung $\left(H_{1 p}\right)$ zusammenhängen. Erfahrene Parlamentsmitglieder sollen vermehrt auf informelle Informationsquellen sowie auf ihre geschaffene Expertise zurückgreifen und daher Evaluationen seltener analytisch verwenden. Ihre Expertise sollte ihnen zugleich Legitimität verschaffen, während unerfahrene Parlamentsmitglieder Evaluationen sowohl als Informationsgrundlage als auch zur Rechtfertigung ihrer Standpunkte verwenden können. Daher wird auch ein negativer Zusammenhang für die politische Nutzung erwartet.

Die Ergebnisse der multivariaten Analyse unterstützen diese Hypothesen eines negativen Zusammenhangs: Je erfahrener ein Parlamentsmitglied, desto geringer ist die Wahrscheinlichkeit der Verwendung von Evaluationen in allen Nutzungsarten. Ein Neumitglied verwendet beispielsweise Evaluationen eher selten konzeptionell mit einer Wahrscheinlichkeit von 22.5 Prozent, nach einer gesamten Legislatur von vier Jahren Erfahrung beträgt diese Wahrscheinlichkeit 24 Prozent. Diese Effekte zusätzlicher Jahre Erfahrung scheinen gering, sie sind aber dennoch für alle Nutzungsarten signifikant. Da die Erfahrung mit sämtlichen Nutzungsarten zusammenhängt, scheint die Stellung eines Mitglieds im Parlament generell bedeutsam für die Evaluationsnutzung zu sein, zumal mit zunehmender Erfahrung in der Regel eine einflussreichere Position im Parlament einhergeht.

Die Ergebnisse zur Erfahrung bekräftigen die bisherige - und überwiegende Evidenz eines negativen Effekts der Erfahrung auf die Nutzung von Evaluationen und anderen systematisch generierten Informationen (Raudla 2012; für pol. Entscheidungsträgerinnen und träger: Askim 2008; Lester 1993; Oliver 2014). Für die Argumentation für einen negativen Effekt zwischen der Erfahrung und der analytischen Verwendung war besonders der Zugang zu Informationen und das Erlangen von Erfahrungswissen zentral, welche sich mit der Amtsdauer ändern. Entsprechend muss ein negativer Effekt der Erfahrung auf die Nutzung nicht zwingend bedeuten, dass erfahrene Parlamentsmitglieder Evaluationen grundsätzlich als weniger relevant einstufen. Dafür spricht auch die Feststellung von Demaj et al. (2012), dass kantonale Parlamentsmitglieder Aufgabenund Finanzpläne mit zunehmender Erfahrung als nützlicher befinden.

\section{Parteizugehörigkeit: Häufigere politische Nutzung durch SP-Parlamentsmitglieder}

In den Hypothesen $H_{2 a}$ und $H_{2 p}$ wird erwartet, dass Parlamentsmitglieder moderater Parteien Evaluationen häufiger analytisch sowie politisch nutzen als Parlamentsmitglieder anderer Parteien. Die moderate Ausrichtung sollte den Parlamentsmitgliedern eine Flexibilität in der Position und eine Offenheit gegenüber Evaluationen erlauben, die für die analytische Verwendung notwendig sind. Zugleich sollten Parlamentsmitglieder moderater Parteien Evaluationen bedürfen, um zu rechtfertigen, wieso sie sich für einen - und 
nicht einen anderen - Standpunkt entscheiden. Folglich wird häufigere analytische und politische Nutzung von Parlamentsmitgliedern der FDP und der CVP erwartet.

Die Ergebnisse der multivariaten Analyse unterstützen $H_{2 a}$ und $H_{2 p}$ nicht. Zwar sind für die analytische instrumentelle und die beiden politischen Nutzungsarten signifikante Parteienunterschiede feststellbar, die Effekte sind aber nur deutlich bei der persuasiven Verwendung. Zudem verwenden CVP- und FDP-Parlamentsmitglieder Evaluationen nicht häufiger als Parlamentsmitglieder anderer Parteien. Vielmehr deuten die Ergebnisse auf einen SP-spezifischen Effekt hin. Befragte der SP nutzen Evaluationen signifikant häufiger persuasiv als alle anderen Befragten. Zusätzlich verwenden sie Evaluationen häufiger legitimierend als SVP-Parlamentsmitglieder. Für die instrumentelle Nutzung sind die marginalen Effekte nicht signifikant. Die weiteren Analysen bestärken diesen Eindruck einer besonderen Rolle der SP: Im Gegensatz zur SVP haben Befragte der SP eine höhere Wahrscheinlichkeit, Evaluationen häufiger als nie für die Gesetzgebung zu verwenden und daher überhaupt die Fragen zu den Nutzungsarten zu erhalten. Die restlichen Parteien unterscheiden sich dagegen nur vereinzelt in der persuasiven Nutzungsart: FDP-Parlamentsmitglieder nutzen Evaluationen häufiger persuasiv als SVP-Parlamentsmitglieder, Grüne tun dies wiederum häufiger als FDP-, SVP- und CVP-Parlamentsmitglieder.

Die präsentierten Resultate fügen sich in die bisher unklare Evidenzlage zu parteispezifischen Unterschieden in der Nutzung von systematischem Wissen ein. Die festgestellten Parteienunterschiede decken sich einzig mit dem Bild der vergleichsweise weniger evaluationsfreundlichen SVP-Parlamentsmitglieder (Demaj et al. 2012, 48; Eberli und Bundi 2017, 264f.; Eberli et al. 2014). Die häufigere politische Nutzung durch SP-Parlamentsmitglieder weist weiter auf keine besondere Rolle der Mitte-Parteien. Die Stärke der Ideologie scheint daher kein Filter zu bilden, wie Frey $(2012,279)$ dies vermutet. Gleichzeitig sprechen die Ergebnisse begrenzt für einen Filter für linke Parteien (vgl. Hird 2005a, 98). Die Grünen verwenden zwar Evaluationen häufiger persuasiv als Parlamentsmitglieder der CVP, FDP und SVP, aber ansonsten unterscheidet sich ihr Nutzungsverhalten nicht. Nur eine Erklärung, die geschichtlich am Fall Grossbritanniens begründet ist, würde den sozialdemokratischen Parteien eine besondere Bedeutung zuschreiben, da Labour dort als Förderin der EBP gesehen wird (vgl. 2.1.3; Sanderson 2002). Inwiefern dies aber auf die SP in der Schweiz wirkte, bleibt fraglich. Übereinstimmend mit den Schlussfolgerungen von Speer et al. (2015) legen die vorliegenden Ergebnisse daher nahe, dass die Zusammenhänge zwischen Partei und Nutzung weiter ohne erkennbares Muster erscheinen.

\section{Professionalisierung: Fördernd für Arten der analytischen und politischen Nutzung}

Für die Professionalisierung wird nur ein positiver Zusammenhang mit der analytischen Evaluationsnutzung erwartet $\left(H_{3 a}\right)$. Parlamentsmitglieder, die ihrem Mandat mehr Zeit widmen (können), sollten auch mehr Zeit für vertiefte Analysen oder das Konsultieren verschiedener Informationsquellen haben. Zudem könnte der Anspruch, informiert zu sein, mit der Professionalisierung steigen. Verschiedentlich wurde im Diskurs über die 
Schweizer Milizparlamente aber darauf hingewiesen, dass professionelle Parlamentsmitglieder nicht unbedingt so erscheinen möchten. Folglich wird kein Zusammenhang mit der politischen Nutzung erwartet $\left(H_{3 p}\right)$.

Die Ergebnisse der multivariaten Analyse unterstützen $H_{3 a}$ und $H_{3 p}$ nur bedingt: Zwar ist der Effekt der Professionalisierung auf die Nutzung positiv, betrifft aber beide Nutzungsformen. Signifikante Zusammenhänge sind für alle Nutzungsarten beobachtbar, besonders deutlich trifft dies für die konzeptionelle und die persuasive Nutzung zu. Der Effekt der Professionalisierung ist bei diesen zwei Nutzungsarten stark, sodass sich ein Parlamentsmitglied mit einem durchschnittlichen kantonalen Pensum und sein oder ihr Pendant auf Bundesebene signifikant voneinander unterscheiden. Dass die Professionalisierung sich besonders auf die konzeptionelle Evaluationsnutzung auswirkt, deckt sich mit der Herleitung von $\mathrm{H}_{3 a}$, wonach professionalisierte Parlamentsmitglieder vertieften Analysen mehr Zeit widmen können. Für den klaren Effekt auf die persuasive Verwendung fehlt hingegen eine theoretische Begründung. Darüber hinaus steigt mit der Professionalisierung die Wahrscheinlichkeit, Evaluationen häufiger als nie für die Gesetzgebung zu verwenden und damit die Fragen zu den Nutzungsarten überhaupt gestellt zu erhalten.

Die bisherige, spärliche Evidenz stimmt mit den vorliegenden Ergebnissen überein. Auch für die US-Bundesstaaten stellt Hird (2005b, 149) fest, dass professionalisierte Parlamentsmitglieder Policy-Analysen häufiger verwenden, um zu verstehen, wie eine politische Massnahme funktioniert. Dies deckt sich fast wörtlich mit der vorliegenden Formulierung der konzeptionellen Nutzung, die ebenso mit der Professionalisierung ansteigt. Keine Forschungsergebnisse existieren hingegen zur politischen Verwendung. Die Literatur weist aber auf eine mögliche Abhängigkeit von individueller und institutioneller Professionalisierung hin (Hird 2005b; Mooney 1993). In der Analyse der vorliegenden Befragung berichten etwa Eberli und Bundi $(2017,262)$ über Unterschiede zwischen der professionalisierteren Bundesversammlung und den Kantonsparlamenten. Mitglieder der Bundesversammlung erfahren demgemäss deutlich häufiger von Evaluationen durch die Parlamentsdienste und durch die parlamentarischen Kommissionen. Folglich stellt sich die Frage, ob die Zeit, die ein Parlamentsmitglied in das Mandat und damit in die Verwendung von Evaluationen einsetzen kann, zusätzlich begünstigt wird durch ein professionalisiertes Parlament mit gut ausgestatteten Parlamentsdiensten.

\section{Bildungsgrad: Einzelne, diffuse Effekte}

Gemäss $H_{4 a}$ und $H_{4 p}$ wird ein positiver Zusammenhang zwischen dem Bildungsgrad und analytischer sowie politischer Nutzung erwartet. Je gebildeter ein Parlamentsmitglied, desto besser geschult ist es darin, analytische Informationen aufzunehmen. Ebenso sollte mit der Bildung auch das Vertrauen in analytische Informationen steigen. Da sie damit selbst empfänglich für analytische Argumente sind, sollte dies dazu führen, dass Parlamentsmitglieder mit steigendem Bildungsgrad Evaluationen auch häufiger politisch nutzen. 
Der in $H_{4 a}$ und $H_{4 p}$ postulierte Zusammenhang widerspiegelt sich nicht in den Ergebnissen. Die Analyse zeigt nur einzelne signifikante Unterschiede in der analytischen konzeptionellen sowie der politischen persuasiven Verwendung. Diese Unterschiede sind aber nicht systematisch für einzelne Bildungsabschlüsse. Da die Signifikanzen ausserdem einzelne Antwortkategorien betreffen, stellt sich die Frage, ob sie nicht eher Antworteffekte statt inhaltliche Effekte darstellen.

Diese Ergebnisse tragen damit kaum zur bereits unschlüssigen Evidenz bezüglich des Effekts der Bildung bei (Hird 2005; für pol. Entscheidungsträger: Askim 2009; Lester 1993; Oliver 2014). Die festgestellten, unsystematischen Unterschiede könnten einerseits darauf hinweisen, dass der Bildungsgrad im Parlament weniger wichtig ist, da die Parlamentsmitglieder generell eine höhere Bildung aufweisen (Mooney 2012). So verfügen 39.8 Prozent der Befragten über einen universitären Abschluss. ${ }^{73}$ Anderseits könnten die Unterschiede auch weniger am Bildungsgrad und mehr am Gebiet der Ausbildung liegen. Etwa könnten Personen, die im Bildungs- oder Gesundheitsbereich tätig sind, eher mit Evaluationen vertraut sein, da die Evaluation respektive die evidenzbasierte Medizin sich in diesen Bereichen etabliert hat (vgl. Widmer und De Rocchi 2012, 35, 146).

\section{Weitere Variablen: Jüngere Kandidierende nutzen häufiger, Frauen öfter legitimierend}

Neben diesen interessierenden Variablen betrachtet die multivariate Analyse auch den Effekt des Geschlechts, des Alters, der Kandidatur für die Nationalrats- und Ständeratswahlen sowie deren Interaktion mit dem Alter. Signifikante geschlechtsspezifische Unterschiede offenbaren aber nur die Modelle zur politisch legitimierenden Nutzung: Parlamentarierinnen verwenden Evaluationen häufiger, um einen Entscheid zu begründen. Dies deckt sich mit bisherigen Ergebnissen aus den USA, die generell häufigere Nutzung bei Parlamentarierinnen feststellen (Bogenschneider und Corbett 2010; Hird 2005b). Das Alter hängt weiter zwar teilweise signifikant mit allen Nutzungsarten zusammen, diese Effekte sind aber jeweils nicht konsistent signifikant über die Antwortkategorien und zum Teil positiv, zum Teil negativ. Damit bleibt die Evidenzlage zum Zusammenhang zwischen dem Alter und der Evaluationsnutzung unschlüssig. Der Effekt der Kandidatur für Nationalrats- und Ständeratswahlen ist schliesslich mit dem Interaktionsterm der Kandidatur und des Alters ${ }^{74}$ verbunden. Beide Variablen hängen signifikant mit der legitimierenden und der instrumentellen Verwendung zusammen. Kandidierende verwenden Evaluationen häufiger legitimierend und instrumentell, mit zunehmendem Alter nimmt dieser Effekt jedoch ab. Dies deckt sich folglich mit dem negativen Effekt der Erfahrung. Die Annahme von Webber (1986), dass politische Ambitionen zu den wich-

73 Im Gegensatz dazu verfügen im Jahr 2013 38.9 Prozent der ständigen Wohnbevölkerung zwischen 25 und 64 Jahren in der Schweiz über einen Abschluss der Tertiärstufe, d.h. einen Abschluss der höheren Berufsbildung, einer Fachhochschule oder Universität (Bundesamt für Statistik 2014, 52).

74 Diese Interaktion sollte abbilden, dass ältere Kandidierende oft erfahrener sind als ältere Nicht-Kandidierende, während sich diese beiden Gruppen in der Professionalisierung vermutlich weniger deutlich unterscheiden. 
tigsten Einflussfaktoren der Verwendung von Policy-Information gehören, bestätigen die Ergebnisse aber nicht, da die Kandidatur alleine keinen signifikanten Effekt ausübt.

\subsubsection{Aussagekraft der Ergebnisse und Diskussion der quantitativen Analyse}

Am Ende dieses Kapitels zur quantitativen Herangehensweise ermöglichen die festgestellten Zusammenhänge, die Datengrundlage, die Messung sowie die Erklärungskraft der Modelle kritisch zu diskutieren. Die Datengrundlage besteht aus einer Online-Befragung mit Parlamentsmitgliedern, bei der sozial erwünschtes Antworten und die Selbstselektion von Befragungsteilnehmenden häufige Probleme sind (Bundi et al. 2016). Wie bereits erwähnt, legt die entgegen den Erwartungen häufigere Nutzung in den analytischen als in den politischen Arten nahe, dass die Befragten teilweise sozial erwünscht antworteten. Folglich haben die Parlamentsmitglieder möglicherweise die analytische Nutzung überschätzt und - oder - die politische Nutzung unterschätzt. Auf eine mögliche Selbstselektion deuten weiter die Ergebnisse zum Verhältnis der Parteizugehörigkeit und der Evaluationsnutzung. ${ }^{75}$ SP-Befragte, haben sich häufiger an der Befragung beteiligt, während SVP-Befragte seltener daran teilgenommen haben. Gleichzeitig nutzen SP-Befragte Evaluationen häufiger politisch als SVP-Befragte und haben auch eine höhere Wahrscheinlichkeit als SVP-Befragte, überhaupt die Frage zu den Nutzungsarten gestellt zu erhalten. Andere Auswertungen der vorliegenden Befragung zeigen zudem auf, dass SP-Befragte deutlich positiver gegenüber Evaluationen eingestellt sind und Evaluationsberichte häufiger lesen, während sich die befragten SVP-Parlamentsmitglieder zurückhaltender gegenüber Evaluationen äussern (Eberli und Bundi 2017, 264 f; Eberli et al. 2014). Das Ausmass und die Auswirkungen der möglichen Selbstselektion sind aber schwierig abzuschätzen, da die Verwendung in der Grundgesamtheit aller Schweizer Parlamentsmitglieder nicht bekannt ist. Die Häufigkeit der Verwendung kann aber angesichts der Übervertretung der SP als obere Grenze gesehen werden. Dieser Schluss gilt umso mehr, wenn sich sozial erwünschtes Antworten besonders bei positiv eingestellten Befragten äussert, wie Bundi et al. (2016) vermuten.

Ein weiterer, theoretisch bedeutender Aspekt der Analyse stellt die Messung der Evaluationsnutzung dar. Während theoretisch nur grundsätzlich zwischen analytischer und politischer Evaluationsnutzung unterschieden wird, befasst sich die Analyse differenzierter mit je zwei Arten dieser Nutzungsformen. Ein Kombinieren der beiden Arten scheint wenig sinnvoll, da die Variablen zu den Nutzungsarten bereits auf zwei Fragen beruhen, namentlich zur Evaluationsnutzung für die Aufgabe der Gesetzgebung generell sowie in den einzelnen Nutzungsarten. Noch deutlicher gegen eine Kombination der Nutzungsarten spricht aber, dass dies einen Informationsverlust bedeuten würde. Gerade weil die verschiedenen Nutzungsformen und ihre Beziehung generell selten und im Parlament kaum erforscht wurden, ist es lohnenswert, die Nutzungsarten einzeln zu betrachten (Amara et al. 2004; Nutley et al. 2007). Dies ermöglicht, Gemeinsamkeiten

75 Bundi et al. (2016) schliessen aus ihrer Analyse, dass sich die Parlamentsmitglieder aufgrund ihrer Evaluationsnachfrage nicht häufiger an der Befragung beteiligten. 
und Unterschiede der Nutzungsarten zu identifizieren und damit die Differenzierung in Nutzungsformen beleuchten. Gemäss den vorliegenden Ergebnissen sind verschiedene Herangehensweisen zur Unterscheidung der Evaluationsnutzung möglich. Für eine Unterteilung in zwei Nutzungsformen spricht, dass alle Nutzungsarten zwar stark miteinander korrelieren, die beiden Arten der analytischen und politischen Nutzung aber die höchsten Korrelationen erreichen. Für gar keine Unterteilung in Nutzugsformen spricht gleichzeitig, dass die individuellen Erklärungsfaktoren nicht zur Unterscheidung der analytischen und politischen Verwendung beitragen. Für eine differenziertere Unterscheidung in Nutzungsarten statt Nutzungsformen wiederum spricht, dass Zusammenhänge zwischen einer analytischen und einer politischen Art teilweise ähnlicher sind als zwischen den beiden Arten einer Nutzungsform. Ein Beispiel dafür sind etwa die starken Effekte der Professionalisierung auf die konzeptionelle sowie die persuasive Verwendung. Angesichts der geringen Modellgüte und den wenigen systematischen, signifikanten Zusammenhängen stellt sich weiter die Frage nach der Erklärungskraft der individuellen Erklärungsfaktoren. Insgesamt legen die Ergebnisse nahe, dass die gewählten, individuellen Variablen mehr zur Erklärung der politischen als der analytischen Nutzung beitragen. Aus einer methodischen Perspektive kann ausserdem die tatsächliche Aussagekraft der Kennzahlen für die Modellgüte für ordinale logistische Regressionsmodelle kritisch diskutiert werden, da sich ihre Werte nur wenig ändern, auch wenn viele der verwendeten Variablen signifikant sind und einen erheblichen Effekt haben (vgl. Kohler und Kreuter 2012; Lacy 2006; Long 1997; Veall und Zimmermann 1992). Ersichtlich ist dies etwa bei den Modellen zur persuasiven Nutzungsart. Weiter erreichen auch die zur Überprüfung der Robustheit geschätzten alternativen Modellierungen keine bessere Güte.

Insgesamt bezieht sich die vorliegende Analyse der individuellen Erklärungsfaktoren der Evaluationsnutzung auf die generelle, gewissermassen durchschnittliche Nutzung der Parlamentsmitglieder, die anhand der Online-Befragung erfasst wurde. Parlamentsmitglieder nehmen jedoch je nach Geschäft unterschiedliche Rollen ein und haben andere Interessen. Ebenso sind die Gegenstände der behandelten Geschäfte und damit verbunden etwa ihr Konfliktpotential oder ihre wissenschaftliche Erforschung sehr unterschiedlich. Das folgende Kapitel befasst sich anhand Fallstudien von realen Gesetzgebungsprozessen mit solchen kontextuellen Erklärungsfaktoren, namentlich dem Kommissionensystem als institutionellen Kontext, der Konfliktivität und Wissenschaftsnähe einer Massnahme als politischen Kontext sowie der Rechtzeitigkeit und Aktualität der Evaluationen als auch der Übereinstimmung der Evaluationsergebnisse als evaluationsspezifischen Kontext. 


\section{Analyse der kontextuellen Erklärungsfaktoren der Evaluationsnutzung}

Nachdem sich das vorangehende Kapitel mit den individuellen Erklärungsfaktoren der Evaluationsnutzung befasst hat, widmet sich dieses Kapitel den gemäss der Theorie identifizierten kontextuellen Erklärungsfaktoren: Dem Kommissionensystem eines Parlaments, der Wissenschaftsnähe und Konfliktivität einer Massnahme, der Aktualität und Rechtzeitigkeit von Evaluationen sowie der Übereinstimmung der Evaluationsergebnisse. Diese Erklärungsfaktoren sind Gegenstand der folgenden qualitativen Analyse, die vergleichende Fallstudien von acht Gesetzgebungsprozessen in den Kantonsparlamenten Bern und Zürich umfasst. Vergleichende Fallstudien erlauben, die Evaluationsnutzung im Verlauf parlamentarischer Gesetzgebungsprozesse zu verfolgen und den Einfluss der kontextuellen Erklärungsfaktoren zu untersuchen, die in den verschiedenen Gesetzgebungsprozessen variieren (George und Bennett 2005, 19-22; Yin 2014, 16). Auch wenn es relativ beständig bleibt, wird das Kommissionensystem ebenso als Kontextfaktor untersucht. Der Einfluss dieses Erklärungsfaktors blieb bisher unerforscht. Eine qualitative Herangehensweise bietet sich daher an, um nicht nur die Hypothesen zu überprüfen, sondern auch Erkenntnisse über die Funktionsweise eines möglichen Zusammenhangs zu gewinnen. Ziel der qualitativen Analyse ist eine vertiefte "real-world perspective" (Yin 2014, 4) auf die Evaluationsnutzung. Damit kann sie die vorangehende, schweizweite generelle Betrachtung in der quantitativen Analyse ergänzen und zur Einordnung der Befunde sowie zur Validierung der Messung der Evaluationsnutzung beitragen.

Das Kapitel beginnt mit einer Beschreibung des Vorgehens. Die vier darauffolgenden Unterkapitel präsentieren die Fallstudien zu je einer Massnahme. Ein Vergleich der Fallstudien und eine Diskussion der Hypothesen schliesst dieses zweite empirische Kapitel ab.

\subsection{Vorgehen: Fallstudien von Gesetzgebungsprozessen}

Im Folgenden wird das Vorgehen der qualitativen Analyse dargelegt. Nach Erläuterungen zum Design der Fallstudien und der Auswahl der Fälle folgen Ausführungen zur Datenerhebung, zur Messung der Evaluationsnutzung sowie zur Analysemethode. Abschliessend wird der Aufbau der Fallstudien skizziert. Detaillierte Informationen zur Erhebung, Messung und Analyse der Daten enthält auch das Fallstudienprotokoll im Anhang.

\subsubsection{Design: Acht Fallstudien, ausgewählt nach Kommissionensystem, Wissenschaftsnähe und Konfliktivität}

Ein kantonaler Gesetzgebungsprozess zu einer politischen Massnahme stellt den Gegenstand einer Fallstudie dar. Da Gesetzgebungsprozesse oft mehrere Massnahmen umfassen, kann ein Fall Bestandteil eines grösseren Gesetzgebungsprojekts sein. Während sich ein Fall eindeutig auf einen Kanton bezieht, ist der zeitliche Rahmen schwieriger abzu- 
grenzen. Bedarf, ein Gesetz zu revidieren oder neu zu erlassen, kann aus diversen Gründen (gleichzeitig) entstehen. Beispielsweise können Vorschriften auf der Bundesebene, interkantonale Vereinbarungen, aber auch Initiativen des Parlaments oder der Stimmberechtigten dazu führen. Folglich beginnt ein Gesetzgebungsprozess in den Fallstudien mit dem ersten dafür relevanten Ereignis. Ein Fall endet mit der abschliessenden Abstimmung im Parlament. ${ }^{76}$ Den Fall chronologisch zu betrachten und damit die vorparlamentarische Phase neben der parlamentarischen Phase in die Analyse einzuschliessen, ist sinnvoll für das Verständnis des Gesetzgebungsprozesses sowie zur Identifikation von Zusammenhängen zwischen Kontextfaktoren und Evaluationsnutzung.

Die Fallstudien befassen sich hauptsächlich mit der Evaluationsnutzung der Parlamentsmitglieder. Um diese zu analysieren, wird auch die Verwendung von Evaluationen durch die Verwaltung und die Regierung betrachtet. Die bisherige Forschung zeigt, dass Parlamentsmitglieder der Arbeit der Exekutive grosse Aufmerksamkeit schenken (vgl. Frey 2012, 288; Himmelsbach 2014; Shulock 1999, 237). Gerade für die kantonalen Milizparlamente kann angenommen werden, dass sie Vorarbeiten der Verwaltung bedürfen, um Evaluationen zu berücksichtigen (vgl. Frey 2012, 307). Folglich sollten Parlamentsmitglieder Evaluationen eher verwenden, wenn dies Verwaltung und Regierung bereits tun. Die Evaluationsnutzung der Parteien, Interessengruppen, Fachpersonen oder anderer Dritten wird schliesslich in den Fallstudien beobachtbar sein, aber nicht vertieft analysiert.

Um den Erklärungsbeitrag der kontextuellen Faktoren zu testen, erfolgt die Fallauswahl theoriegeleitet, sodass die Fälle möglichst most similar cases bilden sollen (George und Bennett 2005, 81). Demzufolge sollen die Fälle bis auf eine der drei Variablen zum institutionellen und politischen Kontext vergleichbar sein. Dies betrifft das Kommissionensystem eines Parlaments $\left(H_{5 a}, H_{5 p}\right)$ sowie die Wissenschaftsnähe $\left(H_{6 a}, H_{6 p}\right)$ und Konfliktivität einer Massnahme $\left(H_{7 a}, H_{7 p}\right)$. Die evaluationsspezifischen Variablen der Aktualität und Rechtzeitigkeit der Evaluationen und der Übereinstimmung der Evaluationsergebnisse zählen ebenso zu den untersuchten kontextuellen Erklärungsfaktoren, bilden aber keine Auswahlkriterien für die Fälle. Um die Ausprägung und Varianz dieser beiden Variablen festzustellen, wären bereits vertiefte Abklärungen notwendig.

Insgesamt werden in zwei Kantonsparlamenten je vier Gesetzgebungsprozesse ausgewählt, die sich in Wissenschaftsnähe und Konfliktivität unterscheiden. Die Zahl von insgesamt acht Fällen ermöglicht es, die einzelnen Fälle detailliert zu betrachten. Es wird ein embedded multiple case study design verfolgt, da auf verschiedene Akteure (Parlamentsmitglieder, Verwaltung, Regierung) und mehrere Phasen des Gesetzgebungsprozesses fokussiert wird (vgl. Yin 2014, 53).

Um die Fälle vergleichen, Evaluationsnutzung beobachten und die Hypothesen überprüfen zu können, müssen die ausgewählten Fälle grundlegende Bedingungen erfüllen (vgl. Collier und Mahoney 1996, 69, 88). Als erste Grundbedingung sollte der Gesetzgebungsprozess abgeschlossen sein. Um die Sammlung der Dokumente zu vereinfachen

76 Weitere Informationen zum Ablauf eines Gesetzgebungsprozesses und dessen Analyse befinden sich in 5.1.2. 
und Erinnerungslücken der Befragten in den Interviews zu reduzieren, sollte zweitens die parlamentarische Beratung im Jahr 2010 oder später abgeschlossen worden sein. Drittens sollten mindestens zwei oder mehr Evaluationen zur untersuchten Massnahme verfügbar sein, wobei eine der Evaluationen zeitnah zum Gesetzgebungsprozess erschienen sein sollte. ${ }^{77}$ Zur Betrachtung der Übereinstimmung der Evaluationsergebnisse $\left(H_{9 a}, H_{9 p}\right)$ bedarf es mehr als eine Evaluation. Viertens sollten sich die Parlamentsmitglieder auf die Vorarbeit der Verwaltung stützen können, was parlamentarische Initiativen ausschliesst, da eine parlamentarische Kommission diese Geschäfte vorbereitet. Fünftens sollten die Massnahmen in den ausgewählten Kantonen in einem ähnlichen Zeitraum diskutiert worden sein.

\section{Erstes Auswahlkriterium: Institutioneller Kontext - Kommissionensystem}

Das Kommissionensystem für die gesetzgeberisch tätigen Legislativkommissionen dient als Kriterium für die Auswahl von zwei Kantonsparlamenten. Grundsätzlich bestehen in den kantonalen Parlamenten drei Typen von Kommissionssystemen: (1) Systeme mit ständigen Legislativkommissionen, (2) gemischte Systeme und (3) Systeme mit nichtständigen Legislativkommissionen (Trippolini 2007, 28; vgl. 2.4.2). Um die Hypothesen $H_{5 a}$ und $H_{5 p}$ zu den Kommissionensystemen zu untersuchen, wird je ein Kantonsparlament ausgewählt, in dem ständige respektive nichtständige Legislativkommissionen die betrachteten Massnahmen beraten haben.

Konkret werden die Kantonsparlamente Bern und Zürich ausgewählt. Ihre Kommissionensysteme unterscheiden $\operatorname{sich}^{78}$, die beiden Kantone und ihre Parlamente sind sich aber sonst ähnlich. Gemäss Tabelle 11 unterscheiden sich die beiden Kantonsparlamente nur betreffend ihre personellen Ressourcen, die in Zürich umfangreicher sind. Dies lässt sich aber auch darauf zurückführen, dass die nichtständigen Kommissionen keine eigenen Sekretariate hatten (Trees et al. 2013, 49, 55). Neben den in der Tabelle aufgeführten Eigenschaften sind sich die Kantone Bern und Zürich auch in Bezug auf die Evaluation ähnlich. Bisherige Auswertungen attestieren den beiden Kantonen eine stetige und verhältnismässig rege Evaluationstätigkeit (Balthasar und Rieder 2009; Frey et al. 2016). Auch gesetzlich ist die Evaluation in beiden Kantonen verankert (Wirths und Horber-Papazian 2016). ${ }^{79}$ Zudem gab es in beiden Kantonen Reformen zur wirkungsorientierten Verwaltungsführung (vgl. Arnold 2008; Rieder und Widmer 2007). Darüber hinaus sind die Fälle in den beiden einwohnerstärksten Kantonen auch von praktischer Relevanz, da ihre Gesetzgebung sowohl innerhalb der Schweiz als auch international von Interesse ist.

77 Es wird als zeitnah aufgefasst, wenn die Evaluation weniger als zwei Jahre vor der Vernehmlassung respektive drei Jahre vor dem Regierungsratsantrag erschienen ist.

78 Der Grosse Rat des Kantons Bern wechselte mit der Legislatur ab 2014 auf ein ständiges Kommissionensystem (Trees et al. 2013). Die betrachteten Fälle waren zu diesem Zeitpunkt abgeschlossen.

79 Weiterführende Informationen zur gesetzlichen Verankerung siehe (Arnold 2008, Nuspliger 2005, Wirths 2017). 
Tabelle 11: Eigenschaften der Kantone Bern und Zürich sowie deren Parlamente

\begin{tabular}{lll}
\hline & $\begin{array}{l}\text { Zürich } \\
\text { Kantonsrat Zürich }\end{array}$ & $\begin{array}{l}\text { Bern } \\
\text { Grosser Rat des Kantons Bern }\end{array}$ \\
\hline Grösse Parlament & 180 Mitglieder & 160 Mitglieder \\
Kommissionensystem & ständig & nichtständig (ab Juni 2014: ständig) \\
Parlamentsdienste (2013) & 14.50 Vollzeitstellen & 9.15 Vollzeitstellen \\
Zeitaufwand Mandat & $30.95 \%$ eines Vollzeitpensums & $27.89 \%$ eines Vollzeitpensums \\
Parteiensystem & gemässigtes Mehrparteiensystem & gemässigtes Mehrparteiensystem \\
Effektive Anzahl Parteien & 5.79 (Wahljahr 2011) & 5.71 (Wahljahr 2010) \\
Bevölkerung Kanton & 1'446'354 Einwohnerlnnen & 1'009'418 Einwohnerlnnen \\
Amtssprache(n) & Deutsch (83.5\% Erstsprache) & Deutsch (85.0\% Erstsprache), \\
& & Französisch (10.6\% Erstsprache) \\
Kantonsverwaltung (2012) & 24'406.30 Vollzeitstellen & 25'053.60 Vollzeitstellen \\
\hline
\end{tabular}

Stand 2014 bei der Fallauswahl, Ausnahmen mit Jahresangabe in Klammern. Quellen: Parteiensystem: Vatter (2014), effektive Anzahl Parteien nach Sitzanteilen, Berechnung gemäss Laakso und Taagepera (1979), Parlamentsdienste: Regierungsrat des Kantons Zürich (2014); Staatskanzlei des Kantons Bern (2014), Zeitaufwand: Bundi et al. (2017), Bevö|kerung und Amtssprache: Bundesamt für Statistik (2016), Kantonsverwaltung: BADAC (2016).

\section{Zweites und drittes Auswahlkriterium: Politischer Kontext - Wissenschaftsnähe und Konfliktivität einer Massnahme}

Anhand des zweiten und des dritten Auswahlkriteriums werden die zu untersuchenden, politischen Massnahmen identifiziert. Das Kriterium der Wissenschaftsnähe dient dabei zunächst zur Eingrenzung bestimmter Politikbereiche. ${ }^{80}$ Die Wissenschaftsnähe ist ein schwer fassbares Konzept. Grundsätzlich beinhaltet sie die wissenschaftliche Erfassbarkeit des Gegenstands, der Ziele und Wirkungen einer Massnahme (vgl. 3.3.2.1; Balthasar und Müller 2014, 76; Boswell 2009; Jenkins-Smith und Sabatier 1993). Sie bezieht sich daher einerseits auf die Frage, ob die Zielgrössen einer Massnahme direkt und einfach gemessen werden können oder ob sie nicht direkt messbar sind und einer Definition bedürfen. Damit zusammenhängend bezieht sich die Wissenschaftsnähe anderseits auf die Frage, inwiefern der Wirkungszusammenhang einer Massnahme isoliert und festgestellt werden kann. Die Wissenschaftsnähe kann sich beispielsweise aufgrund von Weiterentwicklungen der Forschung ändern (Head 2008b, 104; Jenkins-Smith und Sabatier 1993, 52). Im Zeitfenster von wenigen Jahren, die ein Gesetzgebungsprozess dauert, sollte dies jedoch relativ unverändert bleiben.

Das Kriterium der Konfliktivität dient der weiteren Identifikation der Massnahmen. Dabei wird bezweckt, verhältnismässig konfliktive und weniger konfliktive Fälle sowohl bei den Massnahmen mit relativ geringer als auch bei den Massnahmen mit relativ grosser Wissenschaftsnähe auszuwählen. Es muss jedoch generell von einer gewissen Konfliktivität ausgegangen werden, wenn das Parlament eine Massnahme diskutiert (Frey 2012, 73).

80 Eine Eingrenzung auf bestimmte Politikbereiche ist zudem sinnvoll aufgrund der unterschiedlichen kantonalen Kompetenzen und Evaluationsaktivität. 
Zur Einschätzung der Konfliktivität dienen einerseits die folgenden Indikatoren: (1) Ob das Parlament über die Massnahme abgestimmt hat und mit welchem Ergebnis, (2) mit welchem Ergebnis die Schlussabstimmung ausfiel sowie (3) ob mit dem Ergreifen eines Referendums gedroht wurde und ein solches stattfand (vgl. dazu Klöti und Schneider 1989, 33). Bei der Einschätzung der Konfliktivität berücksichtigt wird anderseits auch der Gegenstand der Massnahme.

Die zwei Massnahmen, die eine relativ grosse Wissenschaftsnähe aufweisen, werden aus der Energie- und Transportpolitik ausgewählt. In diesen Bereichen geht es häufig um technisch-naturwissenschaftliche Fragen. Daher sollten die Zielgrössen quantifizierbar sein und Wirkungszusammenhänge sollten verhältnismässig einfach isoliert werden können. ${ }^{81}$

(1) Die verbrauchsabhängige Heiz- und Warmwasserkostenabrechnung (VHKA) ist exemplarisch für eine sehr wissenschaftsnahe Massnahme. Das Ziel, den Energieverbrauch für Heizung und Warmwasser zu senken, kann direkt z. B. in Kilowattstunden erfasst werden und ist durch Zähler in Gebäuden einfach zu erheben. Auch intervenierende Faktoren des Wirkungszusammenhangs sind relativ einfach zu begrenzen und quantifizieren. Dies sind etwa die Preise für Heizstoffe, die Kaufkraft, das Heizverhalten in der benachbarten Wohnung, die Aussentemperatur oder die Anzahl Personen in der Wohnung.

(2) Das Steuersystem für energieeffiziente Fahrzenge betrifft naturwissenschaftliche sowie (verhaltens-)ökonomische Fragestellungen. Das Ziel, den Energieverbrauch der neuimmatrikulierten Fahrzeuge zu reduzieren, kann relativ direkt erhoben werden. Das Fahrverhalten wirkt sich zwar auf den effektiven Treibstoffverbrauch aus, der grundsätzliche Energiebedarf und die Energieeffizienz eines Fahrzeugs können aber als bedeutendere, langfristigere Einflussgrössen gesehen werden (de Haan et al. 2007a; Keller 2007, 81, 147). Der durchschnittliche Treibstoffverbrauch kann anhand der Typengenehmigung der Fahrzeuge lückenlos erfasst werden. Der Wirkungszusammenhang scheint schwieriger identifizierbar zu sein als bei der VHKA, da eine Vielzahl von Entscheidungskriterien den Fahrzeugkauf bestimmt (de Haan et al. 2007b). Viele dieser Kriterien sind aber objektiv erfassbar, etwa die Preise für Treibstoffe, die Kaufkraft oder die wirtschaftliche Situation.

Die Konfliktivität war bei der VHKA deutlich geringer als beim Steuersystem für energieeffiziente Fahrzeuge. In beiden Kantonen gab es kein Referendum im Zusammenhang mit der VHKA. Da der Kantonsrat Zürich die Änderungen an der VHKA aber vergleichsweise knapp beschloss, wird die Konfliktivität als «eher tief» eingeordnet, während sie mangels Diskussion im Grossen Rat des Kantons Bern als «tief» gesehen werden kann. Das Steuersystem für energieeffiziente Fahrzeuge hingegen betrifft nicht nur energie- und transportpolitische, sondern auch steuerliche Fragen. Solche Fragen zu Motorfahrzeug-

81 Das in der Energiepolitik zentrale Problem des Klimawandels wird auch als wicked problem (Head 2008b, 107) gesehen und ist daher schwierig erfassbar. Im Folgenden wird aber auf Massnahmen fokussiert. 
steuern können stark polarisieren, wie die vergleichsweise häufigen Volksabstimmungen dazu zeigen (vgl. Vatter 2002, 284). Auch in beiden Kantonen fand ein Referendum zum Steuersystem für energieeffiziente Fahrzeuge statt. Da die Abstimmungen im Parlament im Kanton Zürich aber deutlich knapper ausfielen, wird die Konfliktivität als «hoch» eingestuft, während sie für den Grossen Rat in Bern als «eher hoch» gesehen werden kann.

Die zwei Massnahmen, die eine relativ geringe Wissenschaftsnähe aufweisen, werden aus der Bildungs- und Sozialpolitik ausgewählt. In diesen Bereichen stehen komplexe menschliche Interaktionen im Vordergrund und die Zielgrössen sind schwierig zu erfassen oder gar zu definieren (Boswell 2009, 243-244). Dies erschwert die Identifizierung von Wirkungszusammenhängen (Böttcher et al. 2010, 9).

(1) Die Schulsozialarbeit (SSA) verdeutlicht die Problematik schwer definier- und erfassbarer Zielgrössen. Es bestehen mehrere Lesarten und folglich vielfältige, zuweilen ungenau definierte Ziele der SSA (Drilling und Fabian 2010; Speck und Olk 2010b). Mehrheitlich sind dies die frühe Erkennung und Betreuung von psychosozialen Problemlagen der Lernenden sowie die Entlastung der Lehrpersonen. Zur Erfassung dieser Ziele und Wirkungen bedarf es Befragungen oder sonstige subjektive Einschätzungen. Da meist mehrere Massnahmen auf die betroffenen Lernenden abzielen, ist ein kausaler Wirkungszusammenhang begrenzt identifizierbar (Fabian et al. 2010, 206-207). Weiter betreffen die Wirkungen der SSA verschiedene Adressatinnen und Adressaten wie z. B. Lernende, Lehrpersonen, Erziehende, Schulen oder Fachstellen (Baier 2008; Speck und Olk 2010a, 315-323).

(2) Auch bei der Basis-/Grundstufe ${ }^{82}$ ist die Zielgrösse nicht direkt erfassbar. Generell wird in der Grund- oder Basisstufe das Ziel verfolgt, den Kindern eine individuelle Passage vom spielerischen Kindergarten zur schulischen Primarstufe zu ermöglichen. Die individuelle Entwicklung als Zielgrösse kann nur mit Tests der Kompetenzen, Befragungen oder Beobachtungen gemessen werden. Zudem wird diskutiert, inwiefern bildungspolitische Massnahmen (nur) mittels Kompetenzen untersucht werden sollen (Biesta 2010, 42). Weiter erschwert eine Vielzahl intervenierender Faktoren die Feststellung eines Wirkungszusammenhangs, namentlich die Klassendynamik, die Lehrperson sowie generell die Erziehenden und das weitere Umfeld des Kindes (Mabry 2010, 20).

Bei der SSA war wiederum die Konfliktivität geringer als bei der Basis-/Grundstufe. Bei der SSA gab es in beiden Kantonsparlamenten nur vereinzelt knappe Abstimmungen im Parlament, ein Referendum wurde nicht ergriffen. Daher kann die Konfliktivität der SSA in beiden Kantonen als «eher tief» eingeordnet werden. Die Basis-/Grundstufe, welche das bisher bekannte System von Kindergarten und Primarschule auflösen sollte, zählt hingegen zu einer der umfassenden Reformen der schweizerischen Bildungspolitik. In mehreren Kantonen wurde sie mittels Referenden bekämpft (Bieber 2014, 185-187; Quesel 2012; Schlaufer 2018). Auch im Kanton Zürich fand eine Volksabstimmung

Die Basis- und die Grundstufe sind verwandte Modelle. 
statt, weswegen die Konfliktivität als «hoch» eingestuft wird, während sie in Bern nur der Grosse Rat diskutierte und die Konfliktivität daher als «eher hoch» gesehen werden kann.

\section{Übersicht der acht untersuchten Gesetzgebungsprozesse}

Die obigen Ausführungen zu den acht ausgewählten Fällen illustriert Abbildung 14. In Kombination der Hypothesen $H_{5 a}$ bis $H_{7 p}$ zu den institutionellen und politischen Kontextfaktoren verfügt ein Fall mit ständigen Kommissionen, einer hohen Wissenschaftsnähe und mittlerer Konfliktivität über die beste Ausgangslage für eine hohe analytische Evaluationsnutzung. Entsprechend wäre die VHKA im Zürcher Kantonsrat most likely für die analytische Evaluationsnutzung. Analog würde der wenig wissenschaftsnahe, aber konfliktive Fall der Grundstufe in Zürich die beste Ausgangslage für eine hohe politische Evaluationsnutzung ausweisen. Diese Erwartungen müssen aber relativiert werden, da die Theorie offenlässt, wie sich die Variablen in der Interaktion insgesamt auf die Evaluationsnutzung auswirken.

\section{Abbildung 14: Übersicht der ausgewählten Gesetzgebungsprozesse nach Auswahlkriterien}
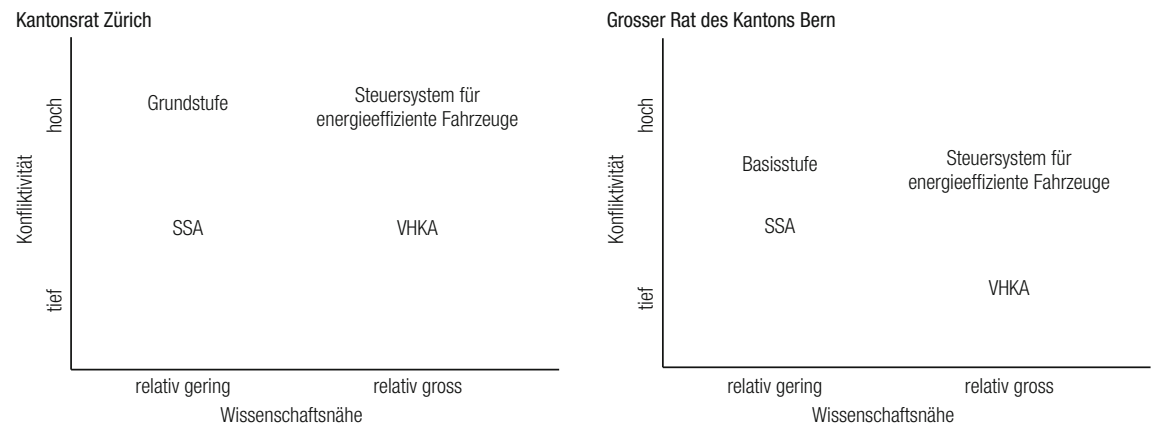

Die Auswahl der Fälle ist entscheidend für die Befunde der Fallstudien und deren Interpretation (Collier und Mahoney 1996; George und Bennett 2005; Gerring 2007; Mahoney und Goertz 2004; Seawright und Gerring 2008). Gerade bei most similar cases stellt sich aber die Frage, ob übereinstimmende, vergleichbare Fälle gefunden und ob sämtliche relevanten Variablen in die Fallauswahl einbezogen werden können (George und Bennett 2005, 156, 165). Grundsätzlich erleichtert jedoch die föderale Struktur der Schweiz, vergleichbare Fälle zu finden, da die Kantone in die gemeinsame Struktur des Bundes eingebettet und mit ähnlichen Fragestellungen oder Problemen konfrontiert sind. Ähnlichkeiten herstellen, die Vielzahl an auswählbaren Fällen begrenzen und die Möglichkeit der Evaluationsnutzung sicherstellen, können zudem die grundlegenden Rahmenbedingungen. Schliesslich erlauben die detaillierte Betrachtung und Analyse der einzelnen Fälle, den Erklärungsbeitrag der kontextuellen Faktoren besser einzuschätzen und zusätzliche Erklärungsfaktoren zu identifizieren (Collier 1993; George und Bennett 2005, 81, 
178-179). Dadurch lässt sich letztlich besser abschätzen, inwiefern die zuvor skizzierten politischen Kontextfaktoren zutreffen und die Fälle vergleichbar sind.

\subsubsection{Grundlagen: Dokumentation der Gesetzgebungsprozesse, Evaluationen und Interviews}

Die Fallstudien basieren auf drei Quellen: Der Dokumentation des Gesetzgebungsprozesses, den verfügbaren Evaluationen sowie Interviews. Durch unterschiedliche, sich ergänzende Quellen sollen die Fälle möglichst umfassend untersucht und gleichzeitig die Resultate validiert werden (vgl. Yin 2014, 120-121).

Zur Illustration der vorliegenden Dokumentation gibt Tabelle 12 eine vereinfachte Übersicht eines üblichen Gesetzgebungsprozesses in den Kantonen Bern und Zürich. ${ }^{83}$ Der Gesetzgebungsprozess kann grundsätzlich in drei Phasen unterteilt werden: In der vorparlamentarischen Phase arbeiten Regierung und Verwaltung den Gesetzesentwurf aus. Nachdem der Regierungsrat seinen Antrag verabschiedet hat, beginnt die parlamentarische Phase. Die Kommission und die Fraktionen beraten zunächst die Vorlage vor. Das Plenum entscheidet dann zuerst, ob es auf die Vorlage eintritt. Ist dies der Fall, folgt unmittelbar danach die erste Lesung, in der das Plenum die Vorlage paragraphenweise berät. In der Beratung behandelt das Plenum Anträge, welche die Kommission, Kommissions- oder Parlamentsmitglieder stellen. Nach einem ähnlichen Verfahren laufen die Beratungen in den Kommissionen ab, wobei die Kommissionen zusätzlich Anhörungen durchführen können. Nach der ersten Plenumssitzung geht die Vorlage zurück in die Kommissionen und Fraktionen. Ab diesem Zeitpunkt unterscheidet sich das Verfahren in den beiden betrachteten Kantonsparlamenten: Im Kantonsrat Zürich geht die Vorlage an die Redaktionskommission, die sich lediglich formalen, gesetzestechnischen Fragen widmet. Daher berät der Kantonsrat in der zweiten so genannten Redaktionslesung nur formale Änderungen; für die Beratung inhaltlicher Änderung bedarf es eines Antrags auf Rückkommen. In Bern dient hingegen die zweite Lesung in der Kommission und im Plenum zur Bereinigung offener Punkte der ersten Lesung. Auch können Kommissionsoder Parlamentsmitglieder Anträge der ersten Lesung wiederholen oder neue Anträge stellen. Beide Parlamente beenden die zweite Lesung mit der Schlussabstimmung. Dies läutet die nachparlamentarische Phase ein. Die Vorlage kann nach der Schlussabstimmung jedoch nochmals ins Parlament gelangen, falls die Stimmberechtigten das konstruktive Referendum ergreifen. Im Kanton Zürich galt das konstruktive Referendum während der untersuchten Gesetzgebungsprozesse, es wurde aber 2013 abgeschafft (Regierungsrat des Kantons Zürich 2013).

Die relevantesten Dokumente zur Analyse der Evaluationsnutzung der Parlamentsmitglieder sind die Protokolle der Kommissions- und Plenumsberatungen. Die Kommissionsprotokolle sind vertraulich, können aber für Forschungszwecke zugänglich

83 Die Ausführungen stützen sich auf die folgenden Quellen: Bern: Verfassung des Kantons Bern (KV) vom 6. Juni 1993 (Stand 01.01.2011), Gesetz über den Grossen Rat (aGrossratsgesetz; GRG) vom 8. November 1988, Geschäftsordnung für den Grossen Rat (aGO) vom 9. Mai 1989; Zürich: Verfassung des Kantons Zürichs vom 27. Februar 2005 (KV) (Stand 01.01.2011), Kantonsratsgesetz (KRG) vom 5. April 1981 (Stand 01.05.2011), Geschäftsreglement des Kantonsrates vom 15. März 1999 (Stand 01.05.2011). 


\section{Tabelle 12: Phasen eines Gesetzgebungsprozesses und dazugehörige Dokumentengrundlage}

\begin{tabular}{|c|c|c|c|}
\hline Verfahrensschritt & Beteiligte Akteure & Dokumentengrundlage $^{a}$ & \\
\hline \multicolumn{4}{|l|}{ Vorparlamentarische Phase } \\
\hline $\begin{array}{l}\text { Auslösung des Gesetz- } \\
\text { gebungsverfahrens }\end{array}$ & $\begin{array}{l}\text { Regierung, Verwaltung, } \\
\text { Parlament, Gemeinden, } \\
\text { Stimmberechtigte }\end{array}$ & $\begin{array}{l}\text { Initiativen, Parl. Vorstösse, Anträge, } \\
\text { Plenumsprotokolle, Antwort oder } \\
\text { Beschlüsse von Regierungsrat }\end{array}$ & \multirow{3}{*}{$\begin{array}{l}\text { Medienmitteilungen } \\
\text { Parteien, } \\
\text { - Interviews Parla- } \\
\text { mentsmitglieder } \\
(\mathrm{N}=20), \\
\text { - Interviews Verwal- } \\
\text { tung }(\mathrm{N}=7)\end{array}$} \\
\hline $\begin{array}{l}\text { Ausarbeitung Vorlage } \\
\text { (evtl. Vernehmlassung) }\end{array}$ & & $\begin{array}{l}\text { Regierungsratsbeschluss, Bericht, } \\
\text { Entwurf Vernehmlassung, Stellung- } \\
\text { nahmen Parteien }\end{array}$ & \\
\hline Antrag des Regierungsrats & Regierung & Antrag des Regierungsrats & \\
\hline \multicolumn{4}{|l|}{ Parlamentarische Phase } \\
\hline Zuweisung an Kommission & $\begin{array}{l}\text { Büro (BE), Geschäfts- } \\
\text { leitung (ZH) }\end{array}$ & Liste Kommissionsmitglieder (BE) & \\
\hline Kommissionsberatung & $\begin{array}{l}\text { Kommission, Regie- } \\
\text { rungsrat, Verwaltung }\end{array}$ & $\begin{array}{l}\text { Protokolle, Anträge, } \\
\text { Abstimmungsresultate }\end{array}$ & \multirow{3}{*}{$\begin{array}{l}\text { Medienmitteilungen } \\
\text { Parteien, } \\
\text { - Interviews Parla- } \\
\text { mentsmitglieder } \\
(\mathrm{N}=20) \text {, }\end{array}$} \\
\hline Fraktionsberatung & Fraktionen & & \\
\hline $\begin{array}{l}\text { Eintreten } \\
\text { Erste Lesung }\end{array}$ & Parlament, Regierung & $\begin{array}{l}\text { Protokolle, Anträge, } \\
\text { Abstimmungsresultate }\end{array}$ & \\
\hline $\begin{array}{l}\text { Kommissionsberatung } \\
\text { Fraktionsberatung }\end{array}$ & $\begin{array}{l}\text { Kommission, Regie- } \\
\text { rungsrat, Verwaltung } \\
\text { Fraktionen }\end{array}$ & $\begin{array}{l}\text { Protokollec }{ }^{c} \text { Anträge, } \\
\text { Abstimmungsresultate }\end{array}$ & \multirow[t]{3}{*}{$\begin{array}{l}\text { Interviews Ver- } \\
\text { waltung }(\mathrm{N}=7)\end{array}$} \\
\hline Zweite Lesung & Parlament, Regierung & $\begin{array}{l}\text { Protokolle, Anträge, } \\
\text { Abstimmungsresultate }\end{array}$ & \\
\hline Schlussabstimmung & & & \\
\hline \multicolumn{4}{|l|}{ Nachparlamentarische Phase } \\
\hline Publikation des Gesetzes & $\begin{array}{l}\text { Staatskanzlei (BE)/ } \\
\text { Geschäftsleitung (ZH) }\end{array}$ & Referendumsvorlage & \\
\hline $\begin{array}{l}\text { (konstruktives }{ }^{\text {b) Referendum/ }} \\
\text { Volksabstimmung }\end{array}$ & $\begin{array}{l}\text { Stimmberechtigte, Kan- } \\
\text { tonsratsmitglieder (ZH), } \\
\text { Gemeinden (ZH) }\end{array}$ & $\begin{array}{l}\text { Regierungsratsbeschluss, Initiative, } \\
\text { Abstimmungszeitung und -resultat }\end{array}$ & \\
\hline Inkrafttreten & Regierung & Gesetzestext & \\
\hline
\end{tabular}

a Kursiv gesetzt sind direkt beobachtbare Quellen, welche die Grundlage für die Analyse der Evaluationsnutzung im Parlament bilden. Kommission, Fraktion und Parlament beraten nochmals über den Volks- (BE) bzw. Gegenvorschlag (ZH).

Zürich: keine Protokolle der Redaktionskommission gesammelt. 
gemacht werden. Sie geben den wesentlichen Inhalt der Beratungen wieder, aber sind keine Wortprotokolle. Im Gegensatz dazu sind die Wortprotokolle der Beratungen im Plenum öffentlich. Neben den Protokollen wird die Evaluationsnutzung im Parlament auch anhand der parlamentarischen Vorstösse ${ }^{84}$ sowie der Medienmitteilungen der Parteien analysiert. Die Medienmitteilungen dienen jedoch nur als Ergänzung, da sie nicht vollständig online verfügbar sind. Weiter relevant sind die Dokumente der Regierung und Verwaltung. Fokussiert wird dabei auf Dokumente, die für die Parlamentsmitglieder zentral sind, etwa der Antrag des Regierungsrats. Diese Dokumente ermöglichen ein Verständnis des untersuchten Gesetzgebungsprozesses, bilden aber auch die Grundlage zur Analyse der Evaluationsnutzung von Regierung und Verwaltung. Berichte von Zeitungen, die eine weitere Informationsquelle für Parlamentsmitglieder sein können, werden hingegen nicht berücksichtigt. Aufgrund bisheriger Forschungsergebnisse scheint das aufwändige Sammeln der Zeitungsberichte kaum gewinnbringend. Gemäss Frey (2012) leisteten die Medien in den untersuchten schweizerischen Gesetzesrevisionen keinen zentralen Beitrag zur Erklärung des Einflusses von Evidenz in die Politik (vgl. Horber-Papazian und Bützer 2011).

Die zweite Quelle für die Fallstudien bilden die verfügbaren Evaluationen zur untersuchten Massnahme. Die Definition von Evaluation in den Fallstudien beruht auf dem Wirkungsmodell der untersuchten politischen Massnahme, das diese in fünf Stufen unterteilt: Input, Prozess, Output (Leistungen), Outcome (Wirkungen bei direkten Adressatinnen und Adressaten) und Impact (Wirkungen über Adressatinnen und Adressaten hinaus). ${ }^{85}$ Evaluationen befassen sich mit einer der Stufen des Wirkungsmodells und beurteilen diese anhand eines systematischen und transparenten Vorgehens. Sie können als Bericht oder auch als Präsentation zuhanden der Parlamentsmitglieder vorhanden sein. Neben Evaluationen werden weiter Zusammenfassungen erfasst, wenn sie die zentralen Ergebnisse der Evaluationen wiedergeben. Für Parlamentsmitglieder sollten Zusammenfassungen besonders relevante, kompakt aufbereitete Informationen bieten. Andere Studien werden weder systematisch erfasst noch analysiert. Insgesamt dienen die Evaluationen und Zusammenfassungen dazu, die Funktionsweise der Massnahme besser zu verstehen, einen Überblick über den Forschungsstand zu gewinnen und damit die evaluationsspezifischen Erklärungsfaktoren zu erfassen.

Zentral für die Identifikation einer Evaluation ist demgemäss das Wirkungsmodell der untersuchten Massnahme. Die gesammelten Studien oder relevanten Präsentationen werden auf dieser Grundlage klassifiziert: Machen die Studien Aussagen zu einer der Stufen des Wirkungsmodells, zählen sie als Evaluationen. Wenn die Studien keine eigene empirische Basis haben, sondern relevante Ergebnisse bisheriger Evaluationen wiedergeben, werden sie als Zusammenfassungen bezeichnet. Die Wirkungsmodelle selbst werden basierend auf den vorhandenen Studien sowie der Dokumentation des Gesetzgebungsprozesses erstellt. Folglich sind die Wirkungsmodelle als Interpretationsleistungen im

84 Parlamentarische Initiativen, Motionen, Postulate und Interpellationen.

85 Aufgrund des schweizerischen Vollzugsföderalismus kann das Wirkungsmodell neben den Kantonen auch die Gemeinden umfassen (vgl. Sager und Rüefli 2005). 
Rahmen der Fallstudie zu verstehen. Indem sich die Wirkungsmodelle auf die bestehenden Arbeiten stützen, geben sie daher vor allem die bisherige Betrachtungsweise einer Massnahme wieder. Wie in 2.1.1 erläutert, scheint schliesslich generell ein relativ weit gefasstes Verständnis von Evaluationen zweckmässig, da Parlamentsmitglieder gemäss bisherigen Studien kaum zwischen verschiedenen Formen systematischen Wissens unterscheiden (vgl. Cairney 2016, 22; Fornerod 2001, 72; Lomas und Brown 2009, 914; Webber 1987, 612; Whiteman 1995, 158).

Interviews sind die dritte Quelle für die Fallstudien. Für jeden Fall wurden drei Interviews mit aktuellen oder ehemaligen Mitgliedern der jeweils vorberatenden parlamentarischen Kommission angestrebt: Mit einem Mitglied des Präsidiums der Kommis$\operatorname{sion}^{86}$ sowie mit je einem Kommissionsmitglied der befürwortenden und der gegnerischen Seite. Bei der Auswahl wurde weiter darauf geachtet, dass die wesentlichen Standpunkte abgedeckt sind und die Parteizugehörigkeit relativ ausgewogen verteilt ist. Ein Interview sollte zusätzlich mit der Person stattfinden, die das Geschäft seitens der Verwaltung federführend betreute. Als eine der wenigen Personen vonseiten der Verwaltung nahm diese jeweils an allen Kommissionssitzungen teil und kann daher Aussagen zur Nutzung der Parlamentsmitglieder sowie der Verwaltung und Regierung machen.

Die Fallstudien basieren auf insgesamt 27 Interviews. Zwischen zwei und sechs Interviews beinhalten Aussagen zu einem Fall. Nur Fall 1 zur VHKA im Kantonsrat Zürich liegen weniger als vier Interviews zugrunde, was den Absagen geschuldet ist, die sich bei diesem Fall häuften. Zu den vier Fällen zur SSA sowie zur Basis- und Grundstufe können indes mehr als vier Interviews verwendet werden. In beiden Parlamenten diskutierte jeweils dieselbe Kommission diese beiden Massnahmen. Interviews mit weiteren Personen, etwa Evaluierenden, wurden nicht geführt, da der Bezug zum Parlament nicht immer vorausgesetzt werden kann.

Die Interviews dienen vorrangig dazu, die Evaluationsnutzung zu untersuchen und den Gesetzgebungsprozess besser zu verstehen. Thematisiert wird auch der generelle Umgang mit Evaluationen. ${ }^{87}$ Dies ist auch wertvoll zur Interpretation und Validierung der Aussagen. Die Interviews wurden mit Erlaubnis der Befragten aufgezeichnet und transkribiert. In den Fallstudien werden daher Zitate verwendet, wobei lediglich die Funktion der Person ${ }^{88}$ angegeben wird, was kein Rückschluss auf die einzelne Person erlauben soll.

\subsubsection{Evaluationsnutzung: Erfassung in Interviews und Dokumenten}

Trotz der viel erforschten Frage der Evaluationsnutzung gibt es keine etablierte Herangehensweise, Evaluationsnutzung und ihre Formen zu messen (2.1.4; Henry und Mark 2003, 310; vgl. Leviton 2003).$^{89}$ Ein Problem, das sich bei der Erfassung der Nutzung

86 Dieses befasst sich vermutlich vertieft mit der politischen Massnahme (Mooney 2012, 12). Zudem obliegt es dem Präsidium, Informationen an die Kommissionsmitglieder weiterzuleiten.

87 Die Gesprächsleitfäden befinden sich im Fallstudienprotokoll im Anhang.

88 Dabei wird zwischen Parlamentsmitgliedern (PM) und Verwaltung (Verw.) unterschieden.

89 Zur Messung der unabhängigen Variablen siehe 2.1.1 sowie das Fallstudienprotokoll im Anhang. 
grundsätzlich stellt, ist die Frage, wann Nutzung beginnt (Knott und Wildavsky 1980, 546). Ein weiteres Problem betrifft die zuweilen fehlende Sichtbarkeit der Nutzung. So kann die Nutzung von Evaluationen auch zum Entscheid führen, nichts zu tun oder einfach die eigene Position bestärken (Patton 1985; Whiteman 1985a, 303). Zudem ist es im Nachhinein schwierig, die Nutzung einer konkreten Evaluation von anderen Informationsquellen zu trennen (Whiteman 1985b, 220).

Neben diesen generellen Schwierigkeiten stellt sich die Frage, wie sich die Formen der analytischen und politischen Nutzung praktisch voneinander unterscheiden lassen. Während sich analytische Verwendung von der politischen Verwendung theoretisch durch die Offenheit gegenüber der Evidenz und die Bereitschaft zum Überdenken der eigenen Position abgrenzt, scheint dies in der Praxis zu verschwimmen (Frey 2012, 316; Weiss 1990, 183; vgl. auch Tabuga 2016, 8-11). Interviews können zwar Einblicke in Hintergründe und Absichten der Evaluationsnutzung geben. Allerdings kann es sein, dass sich die Befragten nicht mehr an die Verwendung erinnern oder sich dieser nicht bewusst sind (Balthasar 2009b, 238; Weiss 1980, 381-382; Whiteman 1995, 173). Weiter können Befragte ihre Absichten nicht offenlegen wollen oder ihre Handlungen im Nachhinein rationalisieren (Frey 2012, 116). Zudem können Befragte dazu neigen, in ihren Angaben zur Nutzung zu übertreiben (Leviton 2003, 533).

Die analytische Nutzung wird massgeblich anhand der Interviews erfasst, da diese am meisten Hinweise zu den Hintergründen der Verwendung geben. Die analytische Nutzung bedarf theoretisch einer Auseinandersetzung mit Evaluationen, bei der Parlamentsmitglieder offen gegenüber den Ergebnissen oder bereit zum Überdenken der eigenen Position sind. Da Letzteres begrenzt erfassbar ist, konzentrieren sich die Fallstudien besonders auf die Frage, ob und wie sich Parlamentsmitglieder mit Evaluationen auseinandersetzten. Ein grundlegender Anhaltspunkt dafür bildet die Erinnerung an Evaluationen oder Präsentationen. Anhaltspunkte für eine differenziertere Auseinandersetzung stellen weiter das Wiedergeben oder die Diskussion von Evaluationsergebnissen dar. Dies knüpft an das Konzept des differentiated thinking an, das Mooney (2012) bei seinen Interviews mit Parlamentsmitgliedern der US-Staaten verwendete. Zusätzliche mögliche Anhaltspunkte für eine Auseinandersetzung mit Evaluationen sind, wenn Befragte selbst nach Evaluationen recherchierten oder Fachpersonen fragten. Schliesslich werden die Aussagen zur Auseinandersetzung mit Evaluationen soweit wie möglich im Vergleich mit den anderen Interviews und der Dokumentation validiert.

Die politische Nutzung wird hingegen hauptsächlich auf Grundlage der Dokumentation untersucht, da sie darin beobachtbar ist. Als politische Verwendung wird einerseits verstanden, wenn jemand aus Evaluationen zitiert oder darauf verweist. Anderseits wird auch zu politischer Nutzung gezählt, wenn jemand Evaluationen kritisiert, nach Evaluationen fragt oder diese fordert. Gerade bei der Forderung von Evaluationen stellt sich jedoch die Frage, ob dies nicht auch mit einer Evidenzoffenheit geschehen und daher analytische Nutzung darstellen kann. Da dies eher wahrscheinlich scheint, je früher der Gesetzgebungsprozess und je offener die Positionen sind, werden solche Äusserungen mit Hilfe des Zeitpunkts interpretiert (Frey 2012, 117; Whiteman 1985a, 308). Die Aussagen in den Interviews werden ergänzend miteinbezogen, da sie Einblicke zur Nut- 
zung etwa zur Überzeugung eines anderen Parlamentsmitglieds geben und daneben zur Validierung der Befunde dienen können.

\subsubsection{Analysemethode: Wer nutzte wann was und in welchem Zusammenhang?}

Die Interviews und die Dokumente des Gesetzgebungsprozesses werden anhand der strukturierenden qualitativen Inhaltsanalyse untersucht (Kuckartz 2010; Mayring 2010; Schreier 2014). Die Textpassagen zur Evaluationsnutzung, zu den untersuchten Kontextfaktoren und zu weiteren Hintergrundinformationen werden dabei mit thematischen Codes erfasst (Kuckartz 2010, 61; Schreier 2014). Diese thematischen Codes werden zunächst theoriengeleitet gebildet und danach an das empirische Material angepasst. Gleichzeitig werden die Textpassagen mit Faktencodes zu ihren Urheberinnen und Urhebern sowie ihrem Gegenstand versehen (Kuckartz 2010, 61). Die Inhaltsanalyse erfolgt in MAXQDA.

Um die Evaluationsnutzung zu erfassen, werden die Dokumente analog zur Vorgehensweise in verschiedenen Studien gestützt auf die folgenden Leitfragen analysiert: Wer nutzte wann was und in welchem Zusammenhang (Cousins und Leithwood 1986, 341; Weiss 1981, 24-25)? Wenn möglich werden dazu die einzelnen Studien identifiziert, so dass am Ende eine Übersicht über die Beobachtungen der Nutzung nach Phase, Form, Akteur und Quellen verfügbar ist. Als Evaluationsnutzung wird aber auch betrachtet, wenn aus der Textpassage hervorgeht, dass die erwähnte Studie oder deren Befunde auf einem systematischen und transparenten Vorgehen beruhen. Dies gilt ebenso bei einem Verweis auf mehrere Studien. Neben Evaluationen wird zusätzlich erfasst, wenn Zusammenfassungen oder andere systematisch und transparent aufbereitete Daten zur untersuchten Massnahme verwendet werden. Letzteres betrifft beispielsweise das Zitieren von Statistiken. Die Analyse der Nutzung fokussiert jedoch nur auf jene Abschnitte, in denen die Vorlage allgemein oder die Massnahme thematisiert werden. ${ }^{90}$ Die restlichen Passagen werden mit Stichworten durchsucht. Auf eine vertiefte Analyse der Argumentation in den parlamentarischen Debatten wird aufgrund der Erfahrungen in einer ersten Analyse in Eberli (2015) verzichtet.

Zur Identifikation eines Zusammenhangs zwischen den unabhängigen Variablen und der Evaluationsnutzung dienen Varietäten des process tracing ${ }^{91}$ gemäss George und Bennett (2005, 210-211). Demgemäss bildet die detaillierte, chronologische Betrachtung des Gesetzgebungsprozesses die Grundlage für das weitere analytische Vorgehen. Sie kann die theoretischen Erwartungen stützen oder Hinweise zu alternativen Erklärungen geben (Collier 2011, 828-829; George und Bennett 2005, 210). Nach der Analyse der einzelnen Fälle folgt der Fallstudienvergleich. Auf der Grundlage der Beobachtungen zu sämtlichen Fällen wird darin das (relative) Ausmass der Evaluationsnutzung festgestellt.

90 Bei Protokollen sind dies konkret die Debatten über Eintreten oder Rückweisung, Schlussvoten sowie die Detailberatung zu den entsprechenden Gesetzesartikeln.

91 Forschende vertreten unterschiedliche Auslegungen (Beach und Pedersen 2013; Collier 2011). Process tracing in der striktesten Auslegung anzuwenden, scheint nur bei klar weniger als acht Fällen möglich. 
Zudem wird überprüft, inwiefern die anderen Fälle mit den festgestellten Erklärungen eines Falls vergleichbar sind oder damit kontrastieren (Yin 2014, 166-167).

\subsubsection{Präsentation der Fallstudien: Paarweise nach ausgewählter Massnahme}

In den folgenden Unterkapiteln werden die Fallstudien als Paare gebündelt nach den vier politischen Massnahmen präsentiert, wobei die Fallstudien anhand der theoretisch erwarteten Häufigkeit der Nutzung sortiert sind. Die Unterkapitel beginnen jeweils mit einer Präsentation des Hintergrunds und der Wirkungsweise der Massnahme sowie der verfügbaren Evaluationen. Danach folgen die Studien des jeweiligen Falls im Kanton Zürich und im Kanton Bern, die je eine Chronik des Gesetzgebungsprozesses und die Analyse der Evaluationsnutzung umfassen. Eine Zusammenfassung schliesst jedes Unterkapitel ab. Da die Massnahmen unterschiedlich komplex sind, die Gesetzgebungsprozesse anders abliefen oder Parlamentsmitglieder Evaluationen verschieden häufig verwendeten, unterscheidet sich die Länge der Unterkapitel. Nach den Unterkapiteln folgt der Fallstudienvergleich zur gesamthaften Analyse der kontextuellen Erklärungsfaktoren.

\subsection{Verbrauchsabhängige Heiz- und Warmwasserkostenabrechnung (VHKA)}

\subsubsection{Hintergrund und Funktionsweise der verbrauchsabhängigen Heiz- und Warmwasserkostenabrechnung}

Für die gesetzliche Regelung von energiepolitischen Massnahmen im Gebäudebereich sind in der Schweiz hauptsächlich die Kantone zuständig. Auf Bundesebene ist aber spezifiziert, zu welchen Gegenständen die Kantone Vorschriften zu erlassen haben. Je nach Kanton vollziehen weiter die Gemeinden diese Vorschriften (EnDK und EnergieSchweiz 2010).

Auf Bundesebene waren die Vorschriften zur VHKA bisher wechselhaft. Der Bund führte die VHKA 1990 ein. ${ }^{92}$ Seither gilt bei allen Neubauten die VHKA. Bei bestehenden Gebäuden sah die Bundesregelung hingegen eine Nachrüstung von Geräten zur Erfassung und Regelung des Heizverbrauchs vor, welche bis Ende April 1998 stattfinden sollte (Ökozentrum Langenbruck 1995, 2-3). Im Juni 1998 verabschiedete die Bundesversammlung aber erstmals das Energiegesetz ${ }^{93}$, in dem sie die Pflicht zur Nachrüstung in bestehenden Gebäuden aufgrund des Widerstands der Hauseigentümerverbände wieder abschaffte (Rieder et al. 2008, 6). Die letzte Anpassung der Bundesvorschriften zur VHKA erfolgte 2007. Im Rahmen des neu erlassenen Stromversorgungsgesetzes ${ }^{94}$ weitete die Bundesversammlung die Pflicht zur VHKA von Neubauten auf bestehende Bauten aus, sofern diese wesentlich erneuert werden.

92 Bundesbeschluss vom 14. Dezember 1990 für eine sparsame und rationelle Energienutzung, AS 1991 1018.

93 Energiegesetz (EnG) vom 26. Juni 1998, AS 1999197.

94 Bundesgesetz über die Stromversorgung vom 23. März 2007, AS 20073425. 
Im Zuge erster Energiesparbestrebungen erliessen einige Kantone noch vor dem Bund Vorschriften zur VHKA. Zuerst führte der Kanton Basel-Landschaft die VHKA im Jahr 1980 ein (Rieder et al. 2008, 42). Der Kanton Zürich folgte 1982, der Kanton Bern im Jahr 1989 (Linder et al. 1990, 67; Rieder et al. 2008, 6, 40).95 Die wechselhafte Gesetzgebung auf Bundesebene betreffend bestehender Gebäude hatte aber Auswirkungen auf die kantonalen Richtlinien. Die Kantone vollzogen die 1990 beschlossene Regelung für die VHKA in bestehenden Gebäuden nicht überall. Nachdem die Bundesversammlung 1998 die Regelungen für bestehende Gebäude wieder aufhob, schafften verschiedene Kantone diese Bestimmungen ebenfalls wieder ab (EnDK 2008, 99, 127).

Seit den ersten Bundesregelungen gibt es auch Bestrebungen, die kantonalen Vorschriften zur VHKA und anderen energiepolitischen Massnahmen zu harmonisieren. 2000 verabschiedete die Konferenz Kantonaler Energiedirektoren (EnDK) 2000 erstmals die Mustervorschriften der Kantone im Energiebereich (MuKEn). ${ }^{96}$ Die MuKEn sind in zwei Teile gegliedert: Den ersten Teil bilden die Basisbestimmungen, die auf den Anforderungen des Bundes gründen und die möglichst alle Kantone detailgemäss übernehmen sollen. Der zweite Teil umfasst freiwillige Module (EnDK 2000). 2008 und zuletzt 2014 passte die EnDK die MuKEn an (EnDK 2008, 2014). Die VHKA in Neubauten gehört seit der Einführung der MuKEn zum Basismodul, 2008 kam die Regelung für die VHKA in bestehenden Gebäuden nach wesentlicher Erneuerung hinzu (EnDK 2008, 23). Die VHKA in bestehenden Bauten bildete hingegen stets ein freiwilliges Modul (Rieder et al. 2008, 6-7).

Die Funktionsweise der VHKA illustriert das Wirkungsmodell in Abbildung 15, das im Rahmen der Fallstudien erstellt wurde. Da die Gebäudevorschriften im Energiebereich und auch das Baurecht kantonal unterschiedlich gehandhabt und vollzogen werden, kann dieses Wirkungsmodell nur teilweise für alle Kantone gelten (EnDK 2010, 73 f.). ${ }^{97}$ Es ist daher auf die beiden untersuchten Kantone Bern und Zürich ausgerichtet, in denen die Gemeinden die Vorschriften massgeblich implementieren. Demzufolge gliedert sich das Wirkungsmodell in die zwei Ebenen der Kantone und Gemeinden (Sager und Rüefli 2005).

Das Erlassen von Vorschriften zur VHKA und die fachliche Unterstützung der Gemeinden bilden gemäss dem Wirkungsmodell die Leistung des Kantons. Diese Vorschriften regeln hauptsächlich, für welche Gebäude die VHKA gilt. Weiter verfassen die kantonalen Fachstellen etwa Informationsbroschüren oder stellen den Gemeinden Unterlagen im so genannten Vollzugsordner zusammen. Um diese Leistungen zu erbringen, benötigt der Kanton personelle und finanzielle Ressourcen und tauscht sich mit den Gemeinden aus. Im Fall des Kantons Zürich soll zudem die Private Kontrolle die Gemeinden entlasten. Demgemäss bestätigen private Kontrolleure den Gemeinden, dass

95 Für detaillierte Informationen zur Gesetzgebung der beiden Kantone siehe 5.2.3.1 und 5.2.4.1.

961992 erarbeiteten das Bundesamt für Energie und die Konferenz kantonaler Energiefachstellen (EnFK) die Musterverordnung zur rationellen Energienutzung in Hochbauten (EnDK 2008, 4).

97 Siehe auch die Webseite der Bau-, Planungs- und Umweltdirektorenkonferenz, http://www.dtap.ch/bpuk [Stand 17.03.17]. 


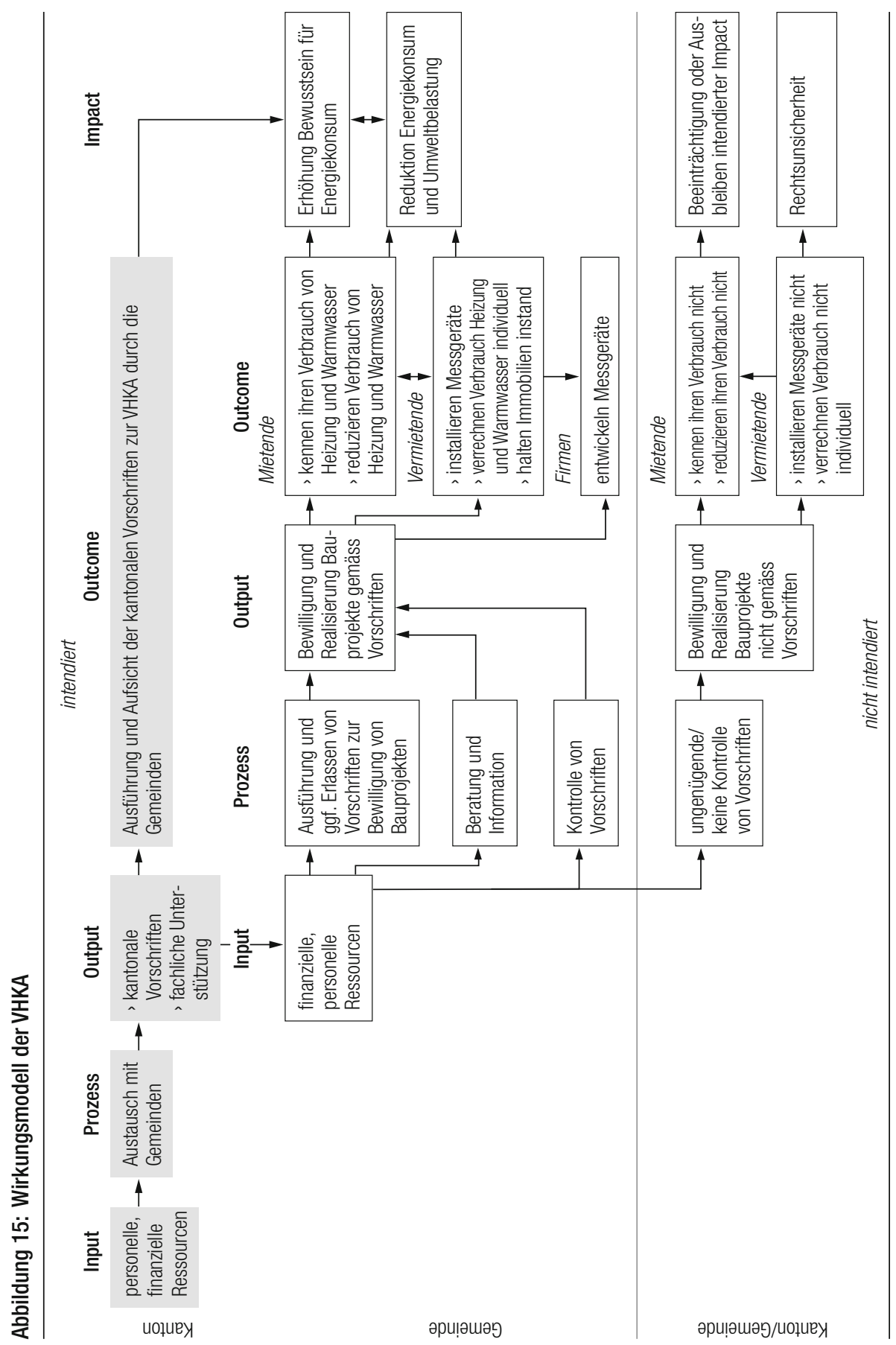


ein Bauprojekt den Vorschriften entspricht (Linder et al. 1990, 75).$^{98}$ Die Anerkennung der privaten Kontrolleure kann daher auch zum Prozess gezählt werden.

Die Leistungen des Kantons stellen dann neben personellen und finanziellen Ressourcen den Input der Gemeinden dar. Darauf aufbauend erlassen die Gemeinden selbst im Rahmen ihrer Möglichkeiten (Ausführungs-)Vorschriften zur Baubewilligung und zum Verfahren. Weiter beraten sie die Vermietenden und kontrollieren das Einhalten der Vorschriften. ${ }^{99}$ Diese Prozesse ermöglichen, dass Bauprojekte in den Gemeinden vorschriftsgemäss erfolgen und Geräte zur Messung des individuellen Verbrauchs von Heizung und Warmwasser installiert werden. Die Kontrollen sollen sichern, dass die Hausbesitzenden den Verbrauch auch individuell abrechnen.

Die Wirkungsweise bei den Mietenden als hauptsächliche Adressatinnen und Adressaten der VHKA ist einfach: Indem sie ihren Energieverbrauch für Heizen und Warmwasser kennen und bezahlen, wächst ihr Bewusstsein für ihren Energiekonsum und sie haben einen Anreiz, diesen zu senken (Rieder et al. 2008, 20). Die Vermietenden als zweite Adressatinnen und Adressaten installieren die Messgeräte und verrechnen den Verbrauch individuell. Da Mietende bei einem hohen Energieverbrauch Druck auf die Vermietenden machen, sorgen die Vermietenden daneben auch dafür, die Liegenschaft instand zu halten (Linder et al. 1990, 70; Rieder et al. 2008, 20). Als dritte Adressatinnen installieren und entwickeln die Firmen Messgeräte (Rieder et al. 2008, 20). Insgesamt soll die VHKA so zu einem verbesserten Bewusstsein für den Energiekonsum für Heizen und Warmwasser führen und den Energiekonsum reduzieren. Dies sollte sich auch in einer geringeren Umweltbelastung äussern (Linder et al. 1990; Rieder et al. 2008).

Die Wirkungen bei den Adressatinnen und Adressaten und der gesamthafte Impact hängen wesentlich von einem funktionierenden Vollzug ab. Ist einerseits das Verfahren mangelhaft, erfolgen Bauprojekte nicht gemäss den Vorschriften und die Messgeräte werden eventuell gar nicht installiert. Anderseits bedarf ein funktionierender Vollzug auch einer greifenden Kontrolle. Während die Gemeinde fertiggestellte Bauprojekte überprüft, können die Mietenden die Umsetzung kontrollieren, indem sie fehlerhafte Vermietende melden. In einer ähnlichen Weise können auch die Firmen die Installation der Messgeräte melden und so zur Kontrolle beitragen (Rieder et al. 2008, 41). Während Neubauten ohnehin Baubewilligungen benötigen, sind viele dieser Kontrollmechanismen besonders beim Vollzug der VHKA in bestehenden Gebäuden relevant. Ein Vollzugsdefizit bedeutet schliesslich nicht nur, dass die Mietenden ihr Verhalten nicht anpassen (können) und die intendierten gesamthaften Wirkungen beeinträchtigt sind oder ausfallen, sondern es führt auch zu Rechtsunsicherheit.

98 Siehe auch Planungs- und Baugesetz (PBG) vom 7. September 1975, LS 700.1.

99 Für die Zuständigkeiten der Baubewilligungsverfahren im Kanton Zürich siehe PBG, Art. 2c, im Kanton Bern siehe Baugesetz (BauG) vom 9. Juni 1985, Art. 33. 


\subsubsection{Verfügbare Evaluationen zur verbrauchsabhängigen Heiz- und Warmwasser- kostenabrechnung}

In den Gesetzgebungsprozessen der Fälle 1 und 2 waren insgesamt sechs Evaluationen und eine Zusammenfassung der wesentlichen Evaluationsergebnisse vorhanden. Gemäss den in den Fallstudien angewendeten Kriterien werden nur Evaluationen oder Zusammenfassungen berücksichtigt, welche nach der letzten Gesetzesänderung im Juni 2000 im Grossen Rat des Kantons Bern respektive im Juli 2001 im Kantonsrat Zürich erschienen sind. ${ }^{100}$ Während den untersuchten Gesetzgebungsprozessen fand weder in Zürich noch in Bern eine Präsentation von Evaluationen oder allgemein den Befunden zur VHKA statt.

Die sechs Evaluationen entstanden im Rahmen von insgesamt vier Evaluationsprojekten. Je ein Projekt in Auftrag gaben die kantonalen Behörden in Zürich beziehungsweise in Bern. Die VHKA war aber nicht alleiniger Gegenstand dieser beiden Projekte. So wurde die VHKA im Kanton Zürich im Rahmen der regelmässigen Kontrolle der Umsetzung der Energievorschriften untersucht, das Projekt im Kanton Bern widmete sich der kantonalen Energiepolitik der 1990er-Jahre (Achtnich 2009; Achtnich und Schwyn 2006; Iten et al. 2000). Die anderen zwei Evaluationsprojekte und die Zusammenfassung beauftragte das Bundesamt für Energie (BFE). Dazu zählt die umfassende Untersuchung der VHKA im Jahr 2008, die auch eine detaillierte Fallstudie des Kantons Bern enthält (Rieder et al. 2008). Auftragnehmer aller sechs Evaluationen sind private Evaluationsoder Ingenieurbüros, die Autorenschaft beschränkt sich auf einen kleinen Kreis. Sämtliche Evaluationen sind in Berichtsform erschienen und enthalten eine Zusammenfassung. Separate Kurzberichte sind nicht vorhanden, was auch die vergleichsweise geringe Anzahl an Evaluationen erklärt.

Zusätzlich zu diesen sechs Evaluationen sind mehrere Evaluationen bereits vor den 2000er-Jahren erschienen. Die Stadt Zürich beauftragte etwa bereits Mitte der 1980er-Jahre Evaluationen im Rahmen der Einführung der VHKA (IPSO 1986, 1989). Weiter führte der Kanton Bern 1991 eine Umsetzungsevaluation durch (Rieder et al. 2008, 17). Auch der Bund förderte diese frühe Evaluationstätigkeit. So gab die AGEVAL den Auftrag, den Prozess der Einführung der VHKA und deren Wirkungen zu untersuchen (Linder et al. 1990; siehe auch Widmer 1996). Im Rahmen des Programms «Energie 2000» erschienen schliesslich zwei weitere umfassende Evaluationen (COPLAN ibe 1997; Ökozentrum Langenbruck 1995).

Die zentralen Ergebnisse der sechs Evaluationen und der Zusammenfassung fasst Tabelle 13 zusammen, wobei sich nur ein Teil der Evaluationen mit den Wirkungsstufen des Outcome und Impact der VHKA befasst. Die meisten Erkenntnisse dazu stammen aus der umfassenden Evaluation von Rieder et al. (2008). Nur diese Studie liefert Hinweise zur Wirkung bei den Mietenden, zum Bewusstsein für Energiekonsum und zur Rechtsunsicherheit. Die Wirkung der VHKA auf die Firmen, die im entworfenen Wirkungsmodell ebenfalls ein Outcome bildet, war kein Gegenstand der sechs verfügbaren

100 Eine Evaluation zählt nur zu den verfügbaren Evaluationen des Falls 2 in Bern, da sie im Juli 2000 erschien. Genauere Informationen zu den kantonalen Gesetzgebungsprozessen enthalten 5.2.3 und 5.2.4. 


\section{Tabelle 13: Evaluationsergebnisse zur Wirkung der VHKA}

\section{Outcome (Evaluationen und Zusammenfassungen, $\mathrm{N}=3$ )}

\section{Mietende}

Gemäss der Befragung in Rieder et al. (2008, 58-66) akzeptiert die Mehrheit der Mietenden die VHKA. Allerdings erreicht die VHKA nur einen Viertel der Mietenden in Gebäuden mit VHKA-Pflicht, weil teilweise nicht nach individuellem Verbrauch abgerechnet wird oder die Mietenden die VHKA oder ihre Abrechnung nicht kennen. Etwa 60 Prozent der Mietenden, welche die VHKA erreicht, ergreifen sinnvolle Sparmassnahmen. Andere Mietende bemühen sich aber auch, Energie zu sparen. Die restlichen Evaluationen machen keine Aussagen zu den Mietenden.

\section{Vermietende}

Die Ergebnisse zu den Vermietenden sind nicht schlüssig. Während gemäss Iten et al. (2000) die Vermietenden im Kanton Bern der VHKA skeptisch gegenüberstehen, stellen Rieder et al. (2008) in Befragungen von Vermietenden aus sechs Kantonen fest, dass diese die VHKA grundsätzlich akzeptieren und als sinnvoll betrachten. Indessen zeigt diese Befragung, dass die Vermietenden in etwa vier von zehn Fällen die entsprechenden Geräte nicht installieren oder den Verbrauch nicht abrechnen. Dieses Vollzugsdefizit besteht bei bestehenden und bei neuen Bauten. Auch findet etwa die Hälfte der befragten Vermietenden den Aufwand für die VHKA als hoch bis sehr hoch.

\section{Firmen}

Zu diesem Aspekt liegen keine Ergebnisse vor.

\section{Impact (Evaluationen und Zusammenfassungen, $\mathrm{N}=5$ )}

\section{Erhöhung Bewusstsein Energiekonsum}

Rieder et al. (2008) schliessen aus der Befragung, dass die VHKA für den Energieverbrauch sensibilisiert und die Mietenden sinnvolle Massnahmen zu dessen Reduktion ergreifen. Andere Evaluationen geben keine Hinweise auf diesen Zusammenhang.

\section{Reduktion Energiekonsum und Umweltbelastung}

Die Ergebnisse aus zwei Evaluationen zeigen, dass die VHKA den Verbrauch für Heizung und Warmwasser durchschnittlich um bis zu 15 Prozent reduzieren kann. Gemäss den Auswertungen des tatsächlichen Energieverbrauchs in Rieder et al. $(2008,8)$ scheint diese Reduktion in Neubauten eher möglich, aber die absolute Einsparung in bestehenden Bauten ist deutlich höher. Der Anteil der VHKA an der gesamten Reduktion des Energieverbrauchs, den die kantonalen Gebäudevorschriften erzielen, beträgt 2.5 bis 4 Prozent. Dies beschränkt sich im Wesentlichen auf neue Bauten, da die Nachrüstungspflicht bei bestehenden Gebäuden aufgrund mangelhaftem Vollzug nicht eingehalten wird (Kessler und Iten 2003; Kessler und Kasser 2008).

\section{Rechtsunsicherheit}

Gemäss Rieder et al. (2008, 87-89) kennen über 80 Prozent der befragten Vermietenden die Vorschriften zur VHKA, nur ein Viertel der Befragten war aber mit den kantonalen Behörden diesbezüglich in Kontakt. Der mangelnde Vollzug der Vorschriften ist daher nicht mit fehlender Information, sondern vielmehr mit ungenügender Kontrolle zu erklären. Andere Evaluationen oder Zusammenfassungen befassen sich nicht mit diesem Aspekt.

Die Tabelle enthält nur Literaturangaben, wenn Ergebnisse auf einzelnen Evaluationen beruhen oder widersprüchlich sind. Bei übereinstimmenden Ergebnissen wird auf Literaturangaben verzichtet. 
Evaluationen. Hingegen geben die Evaluationen zusätzlich Aufschluss über andere Wirkungsstufen der VHKA, besonders über deren Vollzug (vgl. Achtnich 2009; Achtnich und Schwyn 2006; Rieder et al. 2008). Da die meisten Wirkungszusammenhänge in Tabelle 13 aber nur von einer Evaluation untersucht wurden und sich die Erkenntnisse zu den übrigen Zusammenhängen teilweise widersprechen, kann die Übereinstimmung der Ergebnisse insgesamt als tief bezeichnet werden.

Da die VHKA auch Gegenstand von früheren Evaluationen war, ergibt sich dennoch eine breite Wissensbasis. In Übereinstimmung mit Rieder et al. (2008) weisen mehrere frühere Evaluationen einen durchschnittlichen Energiespareffekt von bis zu 15 Prozent nach (Linder et al. 1990, 89, 95; Ökozentrum Langenbruck 1996, 33). Eine andere Evaluation zeigt zudem, dass die VHKA vor allem in geschäftlich genutzten Räumen Einsparungen bringt, während Familien mit Kindern sowie fremdsprachige Personen tiefere Sparpotentiale haben (COPLAN ibe 1997). Weiter wird deutlich, dass die VHKA auch einen Effekt hat, da dadurch in bestehenden Bauten erst Thermostatventile eingebaut worden sind, die eine individuelle Regulierung der Heizung ermöglichen (COPLAN ibe 1997, 9). Schliesslich scheint die VHKA bei bestehenden Bauten nur nach einer Sanierung sinnvoll, da eine Reduktion des Heizens teilweise zu Schäden und rascherem Sanierungsbedarf führt (Linder et al. 1990, 92).

\subsubsection{Verbrauchsabhängige Heiz- und Warmwasserkostenabrechnung im Kantonsrat Zürich} (Fall 1)

\section{Ablauf und Inhalt des Gesetzgebungsprozesses}

Die VHKA wurde im Kanton Zürich im Rahmen der Revision des kantonalen Energiegesetzes diskutiert, mit der sich der Kantonsrat massgeblich zwischen April 2010 und Juli 2011 befasste. Ziel dieser wenig umfassenden Revision war es, die Vorschriften zur Energienutzung in Gebäuden an das 2007 geänderte Energiegesetz des Bundes und an die 2008 verabschiedeten MuKEn anzupassen. Die Anpassungen betrafen neben der VHKA drei weitere Massnahmen im Gebäudebereich sowie eine Änderung der Strafbestimmungen bei Verletzung dieser Vorschriften.

Bereits vor dem untersuchten Gesetzgebungsprozess diskutierte der Kantonsrat die VHKA mehrfach kontrovers. ${ }^{101}$ Der Kantonsrat führte die VHKA in Neubauten bei der erstmaligen Verabschiedung des kantonalen Energiegesetzes im Jahr 1982 ein (Linder et al. 1990, 68). Anlässlich der Revision des Energiegesetzes Mitte der 1990er-Jahre beschloss er dann, die VHKA auf bestehende Bauten auszuweiten. Diese Vorschrift war allerdings einer der Hauptgründe, wieso sich die CVP, FDP und SVP in einer Volksabstimmung erfolglos gegen diese Revision des Energiegesetzes einsetzten (Neuenschwander 1995; Regierungsrat des Kantons Zürich 2008b, 4). Diese Ausweitung machte der Kantonsrat im Jahr 2001 wieder rückgängig. Anstoss dazu gab die parlamentarische Initiative 303/1998 von Kantonsratsmitgliedern der CVP, FDP und SVP. Wie bereits in den

101 Dieser Absatz widmet sich nur den zentralen früheren Diskussionen zur VHKA. 
vorherigen Diskussionen, sprachen sich die EVP, die Grünen und die SP für die VHKA aus. Der Regierungsrat schlug weiter als Kompromiss vor, die VHKA bei Sanierungen von bestehenden Gebäuden vorzuschreiben. Diesen Kompromiss lehnte der Kantonsrat aber ab (Kantonsrat Zürich 2001a, 2001c). Dies war die letzte Änderung der VHKA vor dem untersuchten Gesetzgebungsprozess.

Anpassungsbedarf an den Bestimmungen der VHKA ergab sich nicht nur aufgrund der Änderungen der MuKEn von 2008, sondern auch anlässlich einer Einzelinitiative ${ }^{102}$ aus dem Jahr 2006. Zwei Stimmberechtigte des Kantons Zürich, davon ein ehemaliger Kantonsrat der Grünen, reichten die Einzelinitiative mit folgendem Wortlaut ein: "Auf dem Gebiet des Kantons Zürich ist in allen Liegenschaften mit vier oder mehr Mietobjekten die individuelle Heizkostenabrechnung einzuführen.» Bei der ersten Diskussion im Kantonsrat lehnten die CVP, FDP und SVP die Einzelinitiative ab, während sich die SP und Grünen dafür aussprachen. Dies reichte zur vorläufigen Unterstützung der Einzelinitiative. Daraufhin erstellte der Regierungsrat im September 2008 einen Bericht und beantragte, die Einzelinitiative abzulehnen. Die Kommission für Energie, Verkehr und Umwelt (KEVU) hörte die beiden Initianten im Februar 2009 an (F1-PM1). Im Folgenden berieten die KEVU und der Kantonsrat aber die Einzelinitiative zusammen mit dem Energiegesetz.

Anfang März 2010 verabschiedete der Regierungsrat seinen Antrag zur Änderung des Energiegesetzes. Eine Vernehmlassung dieser Änderungen hatte davor nicht stattgefunden. In seinem Antrag zum Energiegesetz schlug der Regierungsrat vor, den Wortlaut der Bestimmungen zur VHKA in Neubauten an denjenigen aus den MuKEn anzupassen. Weiter beantragte er eine Erweiterung der VHKA-Pflicht bei Gesamterneuerungen des Heizungs- und Warmwassersystems bestehender Bauten sowie bei einer wärmetechnischen Erneuerung eines Gebäudes in einem Kleinwärmeverbund. Zudem sah der Regierungsrat vor, die gesetzlichen Grundlagen für eine Befreiung der VHKA auf Verordnungsebene einzuführen und die Strafbestimmungen bei einem Verstoss gegen die VHKA-Pflicht ausführlicher zu formulieren. Eine Übernahme des freiwilligen Moduls der MuKEn zur VHKA in bestehenden Gebäuden lehnte er ab, da er angesichts der Vorgeschichte dieser Massnahme Vollzugsschwierigkeiten erwartete (Regierungsrat des Kantons Zürich 2010c). Zuständig für die Vorlage vonseiten des Regierungsrats war Baudirektor Kägi (SVP). Die verfügbaren Medienmitteilungen der Parteien enthalten keine Reaktionen auf die vom Regierungsrat vorgeschlagene Regelung der VHKA.

Die KEVU beriet die Revision des Energiegesetzes danach von Mitte April bis Mitte Dezember 2010 an insgesamt 14 Sitzungen, wobei sie sich an 2 Sitzungen vertieft mit der VHKA befasste. ${ }^{103}$ Die KEVU zählte 15 Mitglieder, das Präsidium hatte die SVP inne.

102 Im Kanton Zürich können einzelne Stimmberechtigte eine Einzelinitiative einreichen. Genaueres zu den Bestimmungen und dem Verfahren siehe Verfassung des Kantons Zürich vom 27. Februar 2005, LS 101, Art. 24, Art. 31; Gesetz über die politischen Rechte vom 1. September 2003 (GPR), LS 161, Art. 119b und Art. 139.

103 Im Plenum spricht der Kommissionspräsident von 15 Sitzungen (Kantonsrat Zürich 2011f, 13998). Gemäss den Kommissionsprotokollen und Verantwortlichen des Kantonsrats scheint aber die Anzahl von 14 Sitzungen plausibel. 
Nach einer Präsentation der gesamten Vorlage durch Regierung und Verwaltung befasste sich die Kommission paragraphenweise mit den verschiedenen Massnahmen der Vorlage und klärte offene Punkte. Dazu führte die KEVU Anhörungen mit einer Fachperson zu Biogasanlagen sowie mit den Initiantinnen und Initianten der parlamentarischen Vorstösse durch. Danach folgten die erste und zweite Lesung sowie die abschliessende Abstimmung. Die Anträge zur Änderung der VHKA lehnte die KEVU ab und übernahm den Antrag des Regierungsrats.

Der Kantonsrat begann mit der ersten Lesung des Energiegesetzes im März 2011. Eintreten auf die Vorlage war unumstritten, die VHKA erwähnten nur der Kommissionspräsident sowie die Kantonsräte der CVP und der EVP in ihren Reden zum Eintreten. In der Detailberatung diskutierte der Kantonsrat die VHKA danach aufgrund von zwei Minderheitsanträgen der Grünliberalen und der Grünen. Während ein Minderheitsantrag vorsah, die VHKA-Pflicht in Neubauten ab zwei statt fünf Wohneinheiten einzuführen, umfasste der andere Minderheitsantrag die Nachrüstung der VHKA in bestehenden Gebäuden innerhalb von fünf Jahren. Die Antragsteller argumentierten, dass die VHKA eine technisch einfache und kostengünstige, aber wirksame Massnahme sei. Die SP befürwortete die Anträge der Grünen und Grünliberalen, CVP, EDU, FDP und SVP sprachen sich dagegen aus. ${ }^{104}$ Der Kantonsrat lehnte beide Anträge mit 63.3 Prozent respektive 63.4 Prozent der Stimmen ab (Kantonsrat Zürich 2011f, 14018-14026).

Den zweiten Teil der ersten Lesung des Energiegesetzes und die Beratung der Einzelinitiative 278/2006 zur VHKA führte der Kantonsrat einen Monat nach den Regierungs- und Kantonsratswahlen vom April 2011 durch. Da die KEVU die Vorlage in der alten Legislatur beraten hatte, blieben die Kommissionsanträge unverändert. Allerdings änderte sich die Zusammensetzung des Kantonsrats. Die Grünliberalen legten von 10 auf 19 Sitze zu und die BDP war erstmals mit 6 Sitzen im Parlament vertreten. ${ }^{105}$ Dies geschah mehrheitlich auf Kosten der CVP und FDP, in geringerem Ausmass der SVP. Die linken Parteien SP, Grüne und AL konnten insgesamt ihre Anzahl Sitze halten. Die Zuständigkeiten bei der Regierung änderten sich nach den Wahlen nicht.

Unmittelbar nach dem zweiten Teil der ersten Lesung des Energiegesetzes befasste sich der Kantonsrat anlässlich der Beratung der Einzelinitiative noch einmal mit der VHKA. Die Grünliberalen, Grünen, SP und EVP stellten den Minderheitsantrag, eine Vorlage zur Einzelinitiative auszuarbeiten. Wiederum argumentierten sie, dass die VHKA eine technisch einfach Massnahme sei, die eine Energiereduktion bewirke. Auch würde die VHKA Gerechtigkeit für die Mietenden bringen. CVP, FDP und SVP hingegen lehnten die Einzelinitiative ab, da sie die bisherige Regelung für zweckmässig erachteten (Kantonsrat Zürich 2011a). Der Kantonsrat lehnte den Minderheitsantrag mit 52.5 Prozent der Stimmen knapp ab. In der darauffolgenden Schlussabstimmung im Juli 2011 sprach

104 Die Position der EVP ist unklar. Der Sprecher der EVP befürwortete zwar die VHKA in der Eintretensdebatte, nachher nahm er dazu keine Stellung (Kantonsrat Zürich 2011f, 14009). Die Abstimmungsergebnisse sind rechnerisch nur erklärbar, wenn zumindest Teile der EVP gegen restriktivere Regeln stimmten.

105 Erwähnenswert im Zusammenhang mit diesem Fall und den Wahlen scheint, dass sich die Nuklearkatastrophe von Fukushima zwei Monate vor der Wahl ereignete. Dieses Ereignis erwähnten die Kantonsratsmitglieder auch mehrfach in der Debatte (vgl. Kantonsrat Zürich 2011f, 14001-14005). 
sich der Kantonsrat mit 79.4 Prozent für die Revision des Energiegesetzes aus. Dagegen stimmte nur die Mehrheit der SVP. Eine Minderheit der SVP nahm die Gesetzesrevision aber an. Die Kantonsratsmitglieder aller anderen Parteien taten dies ebenso. Das Gesetz dürfte eine so grosse Mehrheit erreicht haben, da die Grünliberalen, Grünen und die SP noch in der Redaktionslesung einige der in der alten Legislatur getroffenen Entscheide zu ihren Gunsten ändern konnten (Kantonsrat Zürich 2011b, 739, 744). Es wurde kein Referendum gegen das Energiegesetz ergriffen.

\section{Evaluationsnutzung}

Noch vor der Diskussion des Energiegesetzes befasste sich der Kantonsrat mit der Einzelinitiative 278/2006, Evaluationen verwendete in diesem Zusammenhang aber nur die Regierung. Die Initianten formulierten ihre Forderung in einem Satz und reichten keine weitergehende Begründung ein. Als der Kantonsrat danach über die vorläufige Unterstützung der Einzelinitiative beriet, verwiesen die Kantonsratsmitglieder nicht auf Evaluationen. Die Sprecherin der SP, welche die Einzelinitiative unterstützte, erwähnte zwar, dass die VHKA «nachweislich zu einer klaren Reduktion des Verbrauchs» führt, nannte aber keine Quellen (Kantonsrat Zürich 2007, 14656). Aufgrund der vorläufigen Unterstützung des Kantonsrats erstellte der Regierungsrat einen Antrag und erläuterte darin die Wirkung der VHKA anhand von Evaluationen:

Mit der Einführung der VHKA wird der Heizenergieverbrauch um rund 10 bis $15 \%$ gesenkt, wie verschiedene Studien von Bund und anderen Kantonen zeigen. Dieser Wert wurde auch durch die Erfahrungen der Stadt Zürich mit ihren eigenen Bauten bestätigt. (Regierungsrat des Kantons Zürich 2008b, 2)

Weiter verwies der Regierungsrat im Antrag auf seinen Energieplanungsbericht 2006, um allgemein den Energiebedarf in Gebäuden zu illustrieren (Regierungsrat des Kantons Zürich 2006). Später hörte die KEVU die Initianten an. Nur die Folienpräsentation der Initianten, nicht aber das Protokoll dieser Sitzung der KEVU ist verfügbar. ${ }^{106}$ Die Präsentation der Initianten enthielt zwar Angaben zur Reduktion des Energieverbrauchs, zur technischen Umsetzung der VHKA sowie Möglichkeiten der Befreiung der VHKA, aber keine Verweise auf Evaluationen. Die Plenumsdiskussion der Einzelinitiative fand danach mit dem Energiegesetz statt.

Bei der Ausarbeitung der Regelung zur VHKA stützte sich die Verwaltung nicht auf Evaluationen. Die verschiedenen Studien, die im Laufe der vergangenen Jahrzehnte erschienen, waren aber durchaus bekannt. Auch habe das Amt für Abfall, Wasser, Energie und Luft (AWEL) selbst Messungen gemacht zur VHKA, diese aber teilweise nicht publiziert. Bei der VHKA als etablierte Massnahme habe man bereits auf viele praktische Erfahrungen zählen können, die wichtiger als Studien gewesen seien. «Evaluation in dem Sinne ist die tägliche Vollzugserfahrung.» Zudem sei die Verwaltung mit ihrer Exper-

106 Die Folienpräsentation war dank des befragten Parlamentsmitglieds verfügbar (F1-PM1). 
tise in der Kommission anerkannt und müsse keine Quellenangaben liefern (F1-Verw.). Übereinstimmend mit diesen Ausführungen enthält der Regierungsratsantrag zum Energiegesetz keine Verweise auf Evaluationen der VHKA. Die Regierung weist aber auf die ausführlicheren Informationen in ihrem Antrag zur Einzelinitiative hin (Regierungsrat des Kantons Zürich 2010c). In der einzigen verfügbaren Medienmitteilung der Parteien sprechen die Grünen die VHKA gar nicht an (Grüne Kanton Zürich 2010b).

Die KEVU befasste sich in zwei ihrer Sitzungen vertieft mit der VHKA, die Interviews und Dokumente deuten aber auf praktisch keine Evaluationsnutzung. Das befragte Kommissionsmitglied, das eine Ausweitung der VHKA befürwortete, kennt keine Evaluationen zur VHKA und hatte daher auch keine gelesen. Informationen zur VHKA hätte vielmehr die Absprache mit einem der Initianten der Einzelinitiative geliefert: «Man versucht vielleicht weniger auf Studien zu gehen als auf Experten, die man aus einem Bereich kennt, in der Hoffnung, dass die dann auch einmal eine Studie gelesen haben» (F1-PM1). Auch würden die Zuständigen aus der Verwaltung fundiert informieren. Zudem sei das Informationsbedürfnis bei einer so einfachen Massnahme nicht besonders gross gewesen (F1-PM1). Entsprechend enthalten auch die Kommissionsprotokolle keine Hinweise auf Evaluationen durch die Kommissionsmitglieder. Die VHKA war zwar Thema während der Beratung der KEVU, aber im Zentrum der Diskussion stand die politische Machbarkeit und technische Umsetzung der VHKA. Regierung und Verwaltung erläuterten zudem die Geschichte, Vollzug und Wirkungen der VHKA, bezogen sich dabei aber nicht auf Evaluationen.

In der darauffolgenden Debatte des Energiegesetzes diskutierten die Kantonsratsmitglieder verschiedene Aspekte der VHKA ohne auf Evaluationen einzugehen. Während sich die Kantonsratsmitglieder der Grünen, Grünliberalen und SP aufgrund der technisch einfachen Umsetzung, der Wirksamkeit sowie des Verursacherprinzips für die VHKA aussprachen, begründeten die Kantonsratsmitglieder der FDP, CVP und SVP ihre Position etwa damit, dass eine restriktivere Regelung der VHKA unverhältnismässig, nicht kosteneffizient und für die Hauseigentümer sehr aufwändig sei. Auf Evaluationen verwiesen die Kantonsratsmitglieder aber nicht. Ein grüner Kantonsrat erläuterte explizit, dass die VHKA «bei Weitem die kostengünstigste Massnahme zur Senkung des Heizenergieverbrauchs um 10 bis 15 Prozent» sei, nannte jedoch keine Quellen für diese Zahlen (Kantonsrat Zürich 2011f, 14020-14021).

Ähnlich wie die erste Lesung der VHKA im Energiegesetz verlief die Kantonsratsdebatte zur Einzelinitiative, die drei Monate später stattfand. Die Kantonsratsmitglieder führten hauptsächlich dieselben Argumente an. Ein Kantonsrat der Grünliberalen, der die VHKA befürwortete, nannte wiederum explizit die 10 bis 15 Prozent Einsparung des Heizverbrauchs, die eine VHKA in Altbauten zur Folge hätte (Kantonsrat Zürich 2011a, 356). Er bezog sich aber auf keine Quelle. Hingegen verwies eine Kantonsrätin der SP auf einen Bericht des BFE, um die Konsequenzen der VHKA auf die kleinen und mittleren Unternehmen (KMU) zu erläutern:

Ich habe letzte Woche vom Bundesamt für Energie eine Studie bekommen, die klar ausweist, dass 60 Prozent der KMU, die durch eine breite 
Studie des BFE befragt worden sind, nicht wissen, wie viel genau sie an Energie und Wärme benutzen und hauptsächlich, wie sie motiviert werden können in der Richtung, energieeffiziente Massnahmen im Gebäudebereich umzusetzen, gerade weil sie Mieterinnen oder Mieter sind. (Kantonsrat Zürich 2011a, 359)

Die Kantonsrätin argumentierte bereits in der vorherigen Debatte zur VHKA ähnlich, ging aber nicht auf diesen Bericht ein. Die Quelle dieses Berichts war nicht zu eruieren und es ist daher nicht eindeutig abschätzbar, ob dieser Bericht der Definition von Evaluation entspricht, die den Fallstudien zugrunde liegt. ${ }^{107}$ In der darauffolgenden Redaktionslesung erwähnten schliesslich die Kantonsratsmitglieder die VHKA nicht. In der gesamten parlamentarischen Phase war nur eine Medienmitteilung der Grünen verfügbar, in der sie die VHKA aber nicht thematisierten (Grüne Kanton Zürich 2011a).

\section{Evaluationsnutzung: Zeitpunkt und Quellen}

Die wichtigsten Ereignisse des Gesetzgebungsprozesses und die Publikationsdaten der Evaluationen sowie Zusammenfassungen illustriert Abbildung 16. Bis zur ersten Sitzung der KEVU am 20. April 2010 waren sämtliche fünf Evaluationen und eine Zusammenfassung erschienen, darunter auch die umfassende Evaluation der VHKA aus dem Jahr 2008 (Rieder et al. 2008). Neben dieser Evaluation scheinen zwei weitere Berichte aus 2006 und 2009 relevant, da sie sich dem Kanton Zürich widmen (Achtnich 2009; Achtnich und Schwyn 2006). ${ }^{108}$ Zusätzlich zu den abgebildeten Evaluationen und Zusammenfassungen erschienen mehrere umfassende Evaluationen der VHKA bereits vor der letzten Gesetzesänderung im Jahr 2001 (COPLAN ibe 1997; Linder et al. 1990; Ökozentrum Langenbruck 1995).

Die vereinzelte Evaluationsnutzung in Fall 1 lässt sich nicht auf spezifische Quellen zurückführen. ${ }^{109}$ So sind dem befragten Kommissionsmitglied keine Evaluationen bekannt, da es sich über einen der Urheber der Einzelinitiative informiert hatte (F1PM1). Politische Evaluationsnutzung der Kantonsratsmitglieder ist nur einmal beobachtbar, als eine Kantonsrätin der SP in der Plenumsdebatte der Einzelinitiative einen Bericht des BFE zitierte (Kantonsrat Zürich 2011a, 359). Wie bereits ausgeführt, konnte die Quelle dieses Berichts aber nicht eruiert werden. Mehrere Kantonsratsmitglieder verwendeten zwar gewissermassen Evaluationsergebnisse, indem sie erwähnten, dass die VHKA den Energieverbrauch um 10 bis 15 Prozent reduziert (Kantonsrat Zürich 2011a, 356; 2011f., 14021). Allerdings nannten sie keine Quellen und ihre Ausführungen implizieren

107 Der Bericht war weder in den BFE-Datenbanken noch in einer allgemeinen Internetrecherche auffindbar. Der Bericht macht gemäss dem Zitat Aussagen zum Outcome (Mietende kennen Heizverbrauch), aber es ist unklar, ob dies in Bezug zum Output und generell zur VHKA geschieht.

108 In Fall 1 ist eine Evaluation weniger als in Fall 2 verfügbar, da die letzte Gesetzesänderung zur VHKA in Zürich erst 2001 stattfand. Diese Evaluation befasst sich aber ohnehin mit der Berner Energiepolitik.

109 Die folgenden Fallstudien enthalten teilweise eine tabellarische Übersicht der politischen Evaluationsnutzung. In Fall 1 wird aufgrund der geringen (politischen) Nutzung darauf verzichtet. 
kein systematisches und transparentes Vorgehen. Ansonsten verwiesen die Kantonsratsmitglieder weder allgemein auf beispielsweise Studien, noch forderten sie Evaluationen oder kritisierten diese. Die Regierung verwies einzig auf «verschiedene Studien von Bund und anderen Kantonen» sowie auf Ergebnisse einer Studie, die nicht publiziert worden ist (vgl. F1-Verw., Regierungsrat des Kantons Zürich 2008b, 2). Gemäss den Interviewaussagen waren die verfügbaren Evaluationen aber durchaus bekannt.

\section{Abbildung 16: Zeitliche Verfügbarkeit der Evaluationen zur VHKA im Kantonsrat Zürich}

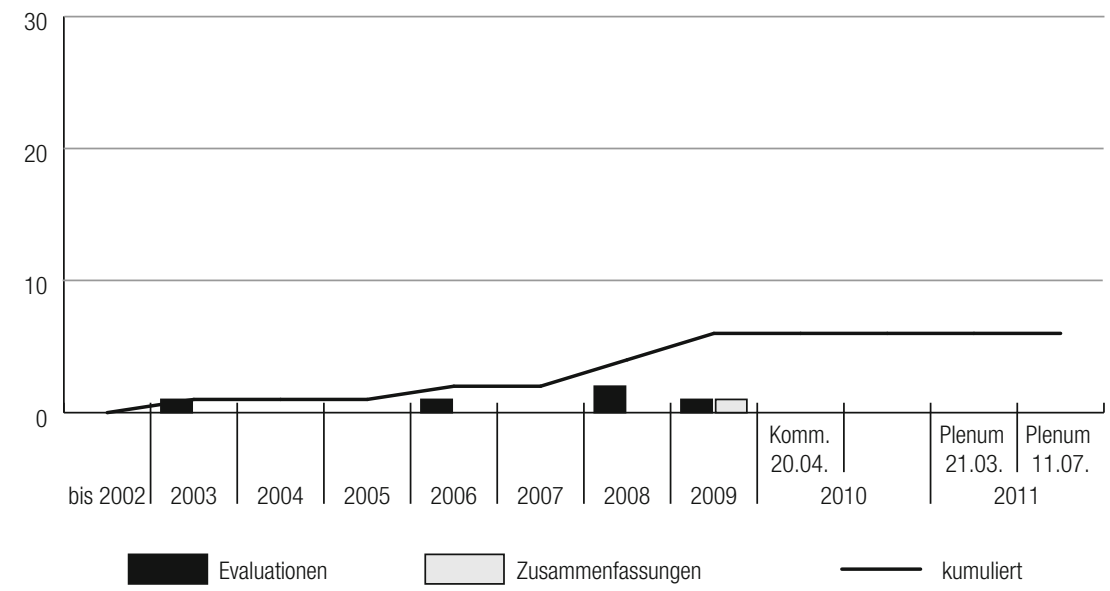

Die Anzahl bezieht sich auf die in einem Jahr beziehungsweise bis zum gegebenen Zeitpunkt erschienenen Evaluationen.

Evaluationsnutzung: Positionen und Argumente

Die Ergebnisse der Evaluationen unterstützen gewissermassen die Befürworterinnen und Befürworter einer restriktiveren VHKA-Regelung als auch deren Gegnerinnen und Gegner. Die Position der Befürworterinnen und Befürworter wird gestützt, da die neuste umfassende Evaluation von 2008 attestiert, dass die VHKA wirkt und auf Akzeptanz stosst. Anderseits unterstützt diese Evaluation auch die Gegnerinnen und Gegner, indem sie Schwierigkeiten beim Vollzug aufzeigt (Rieder et al. 2008). Diese Evaluation sowie allgemein die Aussagekraft der Ergebnisse diskutiert die befragte Person der Verwaltung aber kritisch. Die Evaluation sei vom BFE in Auftrag gegeben worden und daher - wie andere solche Studien auch - politisch gefärbt. Die Studie selbst sei zu «abgehoben», effektiv brauche man Messprogramme (F1-Verw.). Generell mögen die Ergebnisse zur VHKA zwar rechnerisch stimmen, in der Praxis sähe es aber anders aus. Schwierigkeiten beim Vollzug und die mangelnde Akzeptanz von Vermietenden und Mietenden seien auch zu berücksichtigen. Weiter seien Ergebnisse im Energiebereich nach etwa zwei Jahren bereits nicht mehr aktuell (F1-Verw.). 
Nur Parlamentsmitglieder der Befürworterinnen und Befürworter einer restriktiven Regelung der VHKA verwendeten Evaluationen in Fall 1, wobei die äusserst geringe Evaluationsnutzung und die kleine Stichprobe der Befragten diese Beobachtung klar relativieren. Es wurde überhaupt nur ein Kommissionsmitglied befragt. Dieses Kommissionsmitglied, das eine strengere VHKA-Regelung befürwortete, informierte sich über einen der Urheber der Einzelinitiative, der eine Fachperson für solche Fragen sei (F1PM1). Zudem verwies eine Kantonsrätin der SP auf einen Bericht des BFE in einer Plenumsdebatte. Auch wenn dies die einzige beobachtbare Nutzung in Fall 1 darstellt, bezogen sich die Kantonsratsmitglieder beider Seiten häufig auf Aspekte zur Umsetzung, Wirkung und Wirtschaftlichkeit der VHKA. Dies sind folglich Aspekte, zu denen die Evaluationen Aussagen machen. Da die Ergebnisse der Evaluationen verschiedene Standpunkte zur VHKA stützen, hätten sie Befürworterinnen und Befürworter sowie Gegnerinnen und Gegner einer restriktiveren Regelung der VHKA in ihre Argumentation integrieren können.

\section{Evaluationsnutzung: Bedeutung}

Im Gesetzgebungsprozess zur VHKA waren Evaluationen gemäss den Interviews nicht bedeutsam. Das befragte Kommissionsmitglied kennt selber keine Evaluation. Wie bei anderen Gesetzgebungsprozessen auch, seien Evaluationen höchstens möglicherweise indirekt eingeflossen, indem die Urheber der Einzelinitiative oder Verbände die Ergebnisse der Evaluationen aufnahmen und dann ein «Kondensat» davon weitergaben (F1-PM1). Auch die befragte Person aus der Verwaltung schätzt Evaluationen als nicht bedeutsam ein im Gesetzgebungsprozess. Die VHKA sei generell nur ein kleiner Baustein der Anpassungen an die MuKEn gewesen. Zur Zeit der Einführung der VHKA seien hingegen Evaluationen durchaus von Interesse gewesen:

Als man 1985 angefangen hat, über die VHKA zu diskutieren, da sind natürlich schon erste Berichte, hatten eine Bedeutung. [...] Wir sind hier in einer Phase, in der alles schon passiert ist und Erfahrungen da sind und wir täglich beobachten. [...] Dann wandert das wirklich in den praktischen Teil ab. (F1-Verw.)

\subsubsection{Verbrauchsabhängige Heiz- und Warmwasserkostenabrechnung im Berner Grossen Rat} (Fall 2)

\section{Ablauf und Inhalt des Gesetzgebungsprozesses}

Die VHKA wurde in Bern im Rahmen der Totalrevision des kantonalen Energiegesetzes (KEnG) diskutiert, mit der sich der Grosse Rat zwischen September 2009 und März 2010 sowie aufgrund des Volksvorschlags nochmals im Herbst 2010 befasste. Die Totalrevision bezweckte hauptsächlich, die Energiestrategie des Regierungsrats von 2006 umzusetzen. 
Daneben beinhaltete sie Anpassungen an die geänderte Gesetzgebung auf Bundesebene und an die MuKEn von 2008. Die VHKA war Bestandteil dieser Anpassungen, gehörte aber nicht zu den wichtigsten Massnahmen der Totalrevision. Zentrale Inhalte waren vielmehr die Rechte und Pflichten der Gemeinden in der Energieplanung und nutzung, die Einführung einer Sanierungspflicht für bestehende Gebäude, des obligatorischen Gebäudeenergieausweises der Kantone (GEAK) sowie einer Lenkungsabgabe auf Strom.

Obwohl die VHKA im Kanton Bern bis zur Totalrevision im Wesentlichen auf Verordnungsebene geregelt war, befasste sich der Grosse Rat bereits vor dem untersuchten Gesetzgebungsprozess mehrfach mit ihr. 1981 legte er im Energiegesetz die Grundlage, damit der Regierungsrat die VHKA für neue und bestehende Bauten einführen kann. Dies tat der Regierungsrat 1989, wobei bestehende Bauten je nach Baujahr bis Ende 1992 beziehungsweise bis Ende 1995 nachzurüsten waren. Aufgrund des Energienutzungsbeschlusses verschärfte der Regierungsrat die VHKA-Pflicht bei bestehenden Bauten auf fünf Wohneinheiten und verlängerte die Frist zur Nachrüstung bis Ende April 1998 (Grosser Rat des Kantons Bern 2000a; Staatskanzlei des Kantons Bern 1993, 92-94). ${ }^{110}$ Die letzte Gesetzesänderung vor dem untersuchten Gesetzgebungsprozess fand im Jahr 2000 statt. Infolge der parlamentarischen Initiative 220/1998 eines FDP-Grossrats schaffte der Grosse Rat die Pflicht zur Nachrüstung der VHKA in bestehenden Bauten ab. Gleichzeitig führte er die VHKA im Fall von Gesamterneuerungen bestehender Bauten ein. Von den grossen Parteien befürworteten die SVP und die FDP diese Änderung, während sich die SP und das grüne Bündnis dagegen aussprachen (Grosser Rat des Kantons Bern 2000b).

Die Änderung der VHKA war kein Gegenstand der parlamentarischen Vorstösse zur Revision des KEnG. Nur ein Vorstoss, den der Grosse Rat im Rahmen des KEnG behandelte, zielte auf die VHKA (Regierungsrat des Kantons Bern 2009d, 5-6). Dieser Vorstoss von Grossratsmitgliedern der Grünen, CVP, EVP und SP bezog sich aber generell auf Energiesparmassnahmen. Die VHKA selbst erwähnten die Grossratsmitglieder im Vorstosstext nicht.

Die Vernehmlassung zur Totalrevision des KEnG eröffnete der Regierungsrat im Dezember 2008. Regierungsrätin Egger-Jenzer von der SP als Vorsteherin der BVE leitete das Geschäft. Der Vernehmlassungsentwurf sah vor, dass die VHKA an die MuKEn angepasst wird und neu ab fünf Wohneinheiten bei Neubauten sowie bestehenden Bauten nach Gesamterneuerung gilt. Dies bedeutete eine Lockerung der VHKA-Pflicht, da sie bisher für neue und bestehende Bauten nach Gesamterneuerung ab vier Wohneinheiten galt. Zudem sah der Vernehmlassungsentwurf wie die frühere Gesetzgebung eine Bestrafung bei Verstoss gegen die VHKA-Pflicht vor. Diese in der Vernehmlassung vorgeschlagene Gesetzesänderung der VHKA vollzog der Regierungsrat bereits vier Monate zuvor im August 2008 in der Kantonalen Energieverordnung (KEnV). Anlässlich der Totalrevision sollte diese Regelung aber auf Gesetzesebene verankert werden (Regierungsrat des Kantons Bern 2008a, 40). Gemäss den verfügbaren Vernehmlassungsantworten

110 Die VHKA war in diversen Verordnungen geregelt. 1989 bis 1993: Verordnung über die verbrauchsabhängige Heiz- und Warmwasserkostenabrechnung (VVHKA), 1993 bis 2003: Allgemeine Energieverordnung (AEV), ab 2003: Kantonale Energieverordnung (KEnV). 
begrüssten die Grünen und die SP diese Regelung (Grüne Kanton Bern 2009b). Die SP wendete aber ein, dass der Vernehmlassungsentwurf nicht zur eigentlichen Abrechnung nach Verbrauch verpflichte (SP Kanton Bern 2009, 5). Die SVP vertrat eine skeptische Position (SVP Kanton Bern 2009c). Die EVP und die FDP bezogen sich in ihren Vernehmlassungsantworten nicht auf die VHKA (EVP Kanton Bern 2009; FDP Kanton Bern 2009b).

Im Juli 2009 verabschiedete der Regierungsrat dann seinen Antrag zur Totalrevision des KEnG. Die Bestimmungen zur VHKA übernahm der Regierungsrat aus der Vernehmlassungsvorlage, er fügte aber hinzu, dass bei Installation der Messgeräte auch der Verbrauch entsprechend individuell abgerechnet werden muss (Regierungsrat des Kantons Bern 2009d). Die verfügbaren Medienmitteilungen, in denen die Parteien auf den Antrag des Regierungsrats reagieren, enthalten keinen Hinweis auf die VHKA. Generell zeichnete sich ein konfliktiver Gesetzgebungsprozess ab. Während die Grünen mit ihrer Initiative «Bern erneuerbar» noch mehr Druck auf die Energiegesetzgebung machen wollten, wies die SVP in ihrer Medienmitteilung bereits auf ein allfälliges Referendum hin (Grüne Kanton Bern 2009a; SVP Kanton Bern 2009b).

Im September 2009 beriet danach die dafür gegründete Kommission Energiegesetz die Vorlage zur Totalrevision des KEnG in drei ganztägigen Sitzungen. Die Kommission zählte wie üblich 17 Mitglieder, das Präsidium hatte die FDP inne. Die Kommissionsberatung begann mit einem Referat einer Fachperson der Klimaforschung und einer Präsentation der Regierung. Danach folgte die Debatte über Eintreten, das unbestritten war. In der späteren paragraphenweisen Detailberatung gab es nochmals drei Referate, zu denen Fachpersonen aus der Praxis sowie Vertretungen von Interessengruppen eingeladen waren. In der Detailberatung diskutierte die Kommission auch kurz die VHKA: Die SP stellte erfolglos den Antrag, die VHKA-Pflicht auf sämtliche bestehenden Bauten auszuweiten, die Kommission folgte aber dem Antrag der Regierung.

Die erste Lesung im Plenum erfolgte in der Novembersession von 2009. Bis auf die SVP sprachen sich alle Parteien für Eintreten auf die Vorlage aus. Die Grossratsmitglieder und auch die Regierungsrätin erwähnten die VHKA in ihren Reden der Eintretensdebatte nicht. In der folgenden Detailberatung wiederholte die SP ihren Antrag nicht und der Grosse Rat verabschiedete die Regelung ohne Beratung (Grosser Rat des Kantons Bern 2009a, 1213).

Die VHKA war kein Traktandum an den Kommissionssitzungen für die zweite Lesung und auch der Grosse Rat diskutierte sie im Rahmen seiner zweiten Lesung nicht. In den Reden vor der Schlussabstimmung erwähnten ebenso weder die Grossratsmitglieder noch die Regierungsrätin die VHKA. In der Schlussabstimmung nahm der Grosse Rat die Gesetzesänderung mit 56.3 Prozent an. Neben der SVP stimmten die BDP, die EDU, eine Mehrheit der FDP, das Mitglied der Freiheits-Partei (FPS) sowie ein Parteiunabhängiger und ein Mitglied der SP gegen die Gesetzesänderung. Die CVP, die EVP, eine Minderheit der FDP, Grünliberale, Grüne und die SP stimmten der Totalrevision zu. Einzelne Grossratsmitglieder der EDU sowie der FDP enthielten sich.

Nach der grossrätlichen Beratung initiierten der Hauseigentümerverband des Kantons Bern (HEV), weitere Interessengruppen sowie Mitglieder der BDP, FDP und SVP 
wie mehrfach angekündigt das (konstruktive) Referendum. Der Volksvorschlag «Für eine Energiepolitik ohne Bürokratie und neue Stromsteuer» entsprach weitgehend der Grossratsvorlage des KEnG, enthielt aber den obligatorischen GEAK für ältere Bauten sowie die Förderabgabe auf Strom nicht. Bezüglich der VHKA sah der Volksvorschlag keine Änderungen vor.

Da die Initianten keine Änderungen der VHKA vorschlugen, war sie nicht Gegenstand der Beratungen des Volksvorschlags in der Kommission und im Grossen Rat. Die Regierungsrätin erwähnte die VHKA aber kurz in ihrem Schlussvotum im Grossen Rat, da sie sich in ihrer Argumentation für die GEAK-Pflicht auf die Funktionsweise der VHKA bezog (Grosser Rat des Kantons Bern 2010a, 969). In der Schlussabstimmung empfahl der Grosse Rat, den Volksvorschlag anzunehmen. Die Ablehnung der ursprünglichen Grossratsvorlage kam vor allem zustande, da sich die Mehrheiten im Grossen Rat in den Wahlen zuvor deutlich zugunsten des bürgerlichen Blocks aus BDP, EDU, FDP und SVP verschoben. Übereinstimmend mit dem Grossen Rat nahmen die Stimmberechtigten den Volksvorschlag an.

\section{Evaluationsnutzung}

Zur Ausarbeitung der Regelung der VHKA hat sich die Verwaltung nicht auf Evaluationen gestützt. Die VHKA habe generell eine sehr geringe Bedeutung im Energiegesetz gehabt und man habe «eigentlich 1:1 kopiert von MuKEn» (F2-Verw.). Bei der Einführung der VHKA gab die Bau-, Energie- und Verkehrsdirektion (BVE) aber zwei Evaluationen in Auftrag. ${ }^{111}$ Generell seien zur VHKA sicher viele Studien erstellt worden, die aber vor der Revision im Jahr 2009 relevant gewesen seien. Auch die neuste Evaluation, die das BFE in Auftrag gab und 2008 publizierte, habe Beachtung gefunden und man habe geprüft, ob die Einschätzungen mit den eigenen Erfahrungen übereinstimmen. Letztlich sei aber eine wiederholte Ausdehnung der VHKA auf bestehende Gebäude nicht als sinnvoll angesehen worden und daher sei es in der Totalrevision «einzig und alleine darum gegangen, diese den MuKEn anzupassen» (F2-Verw.). Im Vortrag zur Vernehmlassung sowie im Regierungsratsantrag widmeten sich folglich nur kurze, spezifische Abschnitte der VHKA, in denen der Regierungsrat aber nicht auf Evaluationen verwies (Regierungsrat des Kantons Bern 2008b, 2009d).

Bezüglich der Parlamentsmitglieder und Parteien legt die Dokumentenanalyse nahe, dass weder die VHKA noch Evaluationen ein Thema in der vorparlamentarischen Phase waren. Der Grosse Rat diskutierte die VHKA nicht während der Beratung der parlamentarischen Vorstösse zur Revision des Energiegesetzes und verwendete daher auch keine Evaluationen. In den verfügbaren Stellungnahmen zur Vernehmlassung bezogen sich nur die Grünen, die SP und SVP überhaupt auf die Regelung zur VHKA, sie verwiesen in diesem Zusammenhang aber nicht auf Evaluationen (Grüne Kanton Bern 2009b; SP Kanton Bern 2009; SVP Kanton Bern 2009c). Die Parteien erwähnten die VHKA

111 Sommer (1989), die zweite Evaluation des BVE ist nicht zugänglich (vgl. Rieder et al. 2008, 17). 
schliesslich nicht in den verfügbaren Medienmitteilungen während der vorparlamentarischen Phase.

In der späteren Beratung der Totalrevision des Energiegesetzes befasste sich die grossrätliche Kommission nur kurz mit der VHKA, Evaluationen fanden aber auch in dieser Phase keine Verwendung. Die befragten Kommissionsmitglieder erinnern sich alle nicht an Evaluationen im Zusammenhang mit der VHKA. Vielleicht habe die Regierung das in die Vorlage «eingepflanzt», das wisse man nicht (F2-PM1). Ein anderes befragtes Kommissionsmitglied, das sich gegen eine Ausweitung der VHKA-Pflicht aussprach, hatte weiter den Eindruck, dass es früher ein Gutachten des HEV des Kantons Bern zur VHKA gegeben habe, konnte aber keine konkretere Information dazu geben (F2PM2). Eine solche Quelle war der befragten Person in der Verwaltung jedoch nicht bekannt, ebenfalls war eine Recherche im Internet ergebnislos (F2-Verw.). Die Gründe für die Nicht-Nutzung von Evaluationen hängen gemäss den Gesprächen mit den Kommissionsmitgliedern mit der Bedeutung der VHKA in der Totalrevision sowie mit ihrer Geschichte zusammen. Das Energiegesetz sei ein grosses Unterfangen gewesen und habe noch viel wichtigere Punkte als die VHKA enthalten. Die VHKA habe man nur kurz diskutiert (F2-PM3). "Der Hauptentscheid ist ein paar Jahre vorher gefallen» (F2-PM2) und die VHKA sei wenig umstritten gewesen (F2-PM1). Entsprechend sei der Bedarf nach Evaluationen relativ gering gewesen: «Da man natürlich gesagt hat, das ist eh' etwas, was man machen möchte, hat man ja nicht mehr gross weiter nach Belegdokumenten geforscht» (F2-PM1). Die Kommissionsprotokolle stimmen mit diesen Ausführungen überein: Der Abschnitt zur Detailberatung der VHKA enthält keine Verweise auf Evaluationen von Mitgliedern der Kommission oder Regierung und Verwaltung.

In der restlichen parlamentarischen Phase bis zur Schlussabstimmung war die VHKA kein Diskussionsthema, was auch bedeutet, dass keine Evaluationsnutzung feststellbar ist. Weder die Grossratsmitglieder noch die Regierungsrätin erwähnten die VHKA in der ersten Lesung und der Grosse Rat übernahm die Regelung zur VHKA ohne Diskussion. Auch in der zweiten Lesung der Kommission sowie des Grossen Rats fand die VHKA keine Erwähnung. Zudem beinhalten sämtliche verfügbaren Medienmitteilungen der gesamten parlamentarischen Phase bis zur Schlussabstimmung keinen Verweis auf die VHKA und folglich auch nicht auf deren Evaluationen.

Nach der Einreichung des Volksvorschlags ist eine einzige Erwähnung der VHKA beobachtbar: Die Regierungsrätin bezog sich in ihrer Schlussrede bei der Debatte des Grossratsbeschlusses zum Volksvorschlag kurz auf die VHKA und deren Funktionsweise, auf Evaluationen verwies sie aber nicht (Grosser Rat des Kantons Bern 2010a). Wiederum thematisierten die Parteien in den verfügbaren Medienmitteilungen weder die VHKA noch deren Evaluationen.

\section{Evaluationsnutzung: Zeitpunkt und Quellen}

Abbildung 17 stellt die Erscheinungsdaten der Evaluationen und der Zusammenfassung sowie wichtige Zeitpunkte des Gesetzgebungsprozesses dar. Sämtliche sechs Evaluationen und die eine Zusammenfassung waren bis zur ersten Kommissionssitzung am 11. Sep- 
tember 2009 vorhanden. Von diesen Quellen dürfte die Evaluation zur VHKA, die das BFE in Auftrag gab, besonders relevant sein (Rieder et al. 2008). Diese Evaluation ist sehr umfassend, zudem befasst sie sich vertieft mit dem Kanton Bern. Allerdings erschien die Evaluation im November 2008 und damit sehr knapp vor der Eröffnung der Vernehmlassung im Dezember 2008. Eine weitere relevante Quelle dürfte die vergleichsweise älteste Evaluation aus dem Jahr 2000 sein, da sich diese der Berner Energiepolitik widmet (Iten et al. 2000). Schliesslich erschienen bereits vor der letzten Gesetzesänderung umfassende Evaluationen zur VHKA in der Schweiz und im Kanton Bern (COPLAN ibe 1997; Linder et al. 1990; Ökozentrum Langenbruck 1995; Sommer 1989; vgl. auch Rieder 2008, 17).

\section{Abbildung 17: Zeitliche Verfügbarkeit der Evaluationen zur VHKA im Berner Grossen Rat}

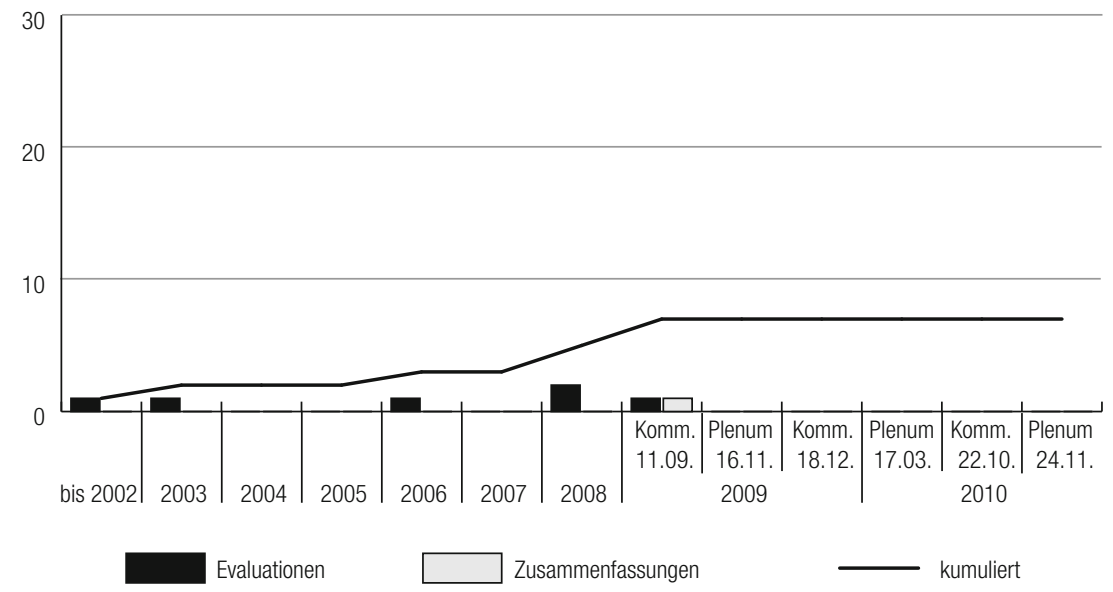

Die Anzahl bezieht sich auf die in einem Jahr beziehungsweise bis zum gegebenen Zeitpunkt erschienenen Evaluationen.

Da sich in Fall 2 generell keine Evaluationsnutzung der Parlamentsmitglieder beobachten lässt, ist auch eine Analyse der verwendeten Quellen nicht möglich. Zwei der befragten Kommissionsmitglieder sind keine Evaluationen zur VHKA bekannt (F2-PM1; F2-PM3). Ein befragtes Kommissionsmitglied erwähnt im Gespräch zwar ein Gutachten des HEV Kanton Bern. Dieses habe gezeigt, dass die Einführung der VHKA bei Altbauten nicht sinnvoll sei. An genauere Informationen zum Gutachten, wie zum Beispiel das Erscheinungsdatum, erinnert sich dieses Kommissionsmitglied aber nicht (F2-PM2). Ein solches Gutachten ist aber weder mit Hilfe der befragten Person in der Verwaltung noch einer Internetrecherche auffindbar. Politisch verwendeten die Parlamentsmitglieder Evaluationen gar nicht: Weder verwiesen sie auf spezifische Quellen oder allgemein Evaluationen, noch forderten sie Evaluationen oder kritisierten diese. Auch die Regierung und Verwaltung bezog sich in ihren Dokumenten nicht auf Evaluationen. Das Gespräch zeigte aber, dass die neuste Evaluation von 2008 durchaus bekannt war (F2-Verw.). 
Evaluationsnutzung: Positionen und Argumente

In Fall 2 verwendeten die Grossratsmitglieder unabhängig von ihrer Position nie Evaluationen. Wie bereits im vorherigen Abschnitt zu Fall 1 erläutert, unterstützen die Evaluationsergebnisse verschiedene Positionen und Argumente. Die Interviews und die Dokumentenanalyse deuten aber darauf hin, dass es in Fall 2 gar keine klar unterschiedlichen Positionen gab. So beantragte die SP zwar in der Kommission, die VHKA-Pflicht wieder auf bestehende Bauten auszuweiten, zuvor in der Vernehmlassung und danach im Plenum forderte sie dies aber nicht. Gemäss dem befragten Kommissionsmitglied, das eine restriktivere Regelung befürwortet hätte, war die VHKA auch sonst nie Thema: «Im Energiegesetz wurde wieder etwas kurz diskutiert. Das war das einzige Mal, wo ich das Wort VHKA wieder einmal gehört habe, aber ansonsten war völliges Stillschweigen» (F2-PM3). Auf der Seite der Bürgerlichen war es ähnlich: «Man hatte einfach gefunden, das ist jetzt etwas, das schon läuft, das kann man machen» (F2-PM1).

\section{Evaluationsnutzung: Bedeutung}

Die fehlende Evaluationsnutzung und die Interviewaussagen lassen auf keine Bedeutung der Evaluationen im Gesetzgebungsprozess zur VHKA in Fall 2 schliessen. Die befragten Kommissionsmitglieder können sich alle nicht daran erinnern, dass Evaluationen zur VHKA ein Thema waren. Die VHKA habe bei dieser Totalrevision des KEnG generell «mehr als Selbstverständlichkeit gegolten» (F2-PM1). Auch die befragte Person aus der Verwaltung schreibt Evaluationen zur VHKA in diesem Gesetzgebungsprozess keine Bedeutung zu (F2-Verw.).

\subsubsection{Fazit: Keine oder vereinzelte Nutzung zur verbrauchsabhängigen Heiz- und Warmwasserkostenabrechnung}

Die vorangehenden Abschnitte präsentierten die Fallstudien zur Nutzung von Evaluationen bei der Diskussion der VHKA. Die Anpassung der VHKA stand jedoch nicht im Zentrum der beiden untersuchten Gesetzgebungsprozesse. Während die Mitglieder des Zürcher Kantonsrats Evaluationen dennoch vereinzelt nutzten, fanden sie im Berner Grossen Rat gar keine Verwendung.

Nachdem die VHKA in früheren Gesetzgebungsprozessen ein umstrittenes Thema war, befasste sich der Kantonsrat Zürich in Fall 1 anhand der Revision des kantonalen Energiegesetzes sowie anlässlich einer im Jahr 2006 eingereichten Einzelinitiative erneut damit. Ziel der Revision des Energiegesetzes war, die kantonale Gesetzgebung an die MuKEn 2008 anzupassen. Neben anderen energiepolitischen Massnahmen im Gebäudebereich sollte daher die bisherige VHKA-Pflicht für Neubauten ab fünf Wohneinheiten auf bestehende gesamterneuerte Bauten ausgeweitet werden. Die Einzelinitiative forderte hingegen eine noch restriktivere VHKA-Pflicht. Die Anpassung an die MuKEn 2008 war unter den grossen Parteien gar nicht umstritten. Restriktivere Regelungen der VHKA lehnten aber die CVP, FDP und SVP ab, während sich die Grünliberalen, Grünen, die SP dafür aussprachen. Die Abstimmungsergebnisse zur VHKA im Kantonsrat fielen zwar 
teilweise knapp aus, die VHKA war aber gemäss den Dokumenten und Interviews keine zentrale Massnahme der Gesetzesrevision. Generell war das Energiegesetz kaum umstritten im Kantonsrat und ein Referendum wurde daher nicht ergriffen. Zusammenfassende Ausführungen über die Evaluationsnutzung in Fall 1 gibt Tabelle 14 wieder.

\title{
Tabelle 14: VHKA im Kantonsrat Zürich - Übersicht der Nutzung
}

\begin{abstract}
Parlament Analytisch: keine Auseinandersetzung, Information über Fachperson
Das befragte Kommissionsmitglied, das eine restriktivere VHKA-Pflicht befürwortete, hat sich gemäss dem Interview nicht mit Evaluationen zur VHKA befasst. Vielmehr informierte es sich im Austausch mit einem der Urheber der Einzelinitiative, der in Energiefragen eine Fachperson sei (F1-PM1).

Politisch: einzelner Verweis auf Evaluationen, keine Nutzung in anderen Arten

Eine Kantonsrätin der SP, die eine restriktive VHKA-Pflicht befürwortete, verwies in der parlamentarischen Phase während der Plenumsdebatte der Einzelinitiative auf einen Bericht des BFE, dessen Quelle nicht ermittelbar ist. Die Parlamentsmitglieder verwiesen auf keine «Evaluationen» allgemein, sie forderten oder kritisierten Evaluationen auch nicht.
\end{abstract}

\begin{abstract}
Regierung Analytisch: Evaluationen bekannt, nicht darauf gestützt
und

Verwaltung Politisch: einzelner Verweis auf Evaluationen

Das AWEL führte bei der Einführung der VHKA selbst Messungen durch und die Evaluationen waren durchaus bekannt, es stützte sich aber nicht auf Evaluationen zur Ausarbeitung der Regelung der VHKA im untersuchten Gesetzgebungsprozess (F1-Verw.). Die Dokumente enthalten einen einzigen Hinweis auf politische Nutzung: Die Regierung verwies im Antrag zur Einzelinitiative auf verschiedene Evaluationen, nannte aber keine konkrete Quelle.
\end{abstract}

In Fall 2 diskutierte der Grosse Rat des Kantons Bern die VHKA im Rahmen der Totalrevision des KEnG. Hauptsächliches Ziel dieser Totalrevision war, die Energiestrategie 2006 des Regierungsrats umzusetzen. Daneben bezweckte sie, die Berner Gesetzgebung an diejenige des Bundes und an die MuKEn anzupassen, wobei die VHKA zu diesen Anpassungen zählte. Nachdem die VHKA bei früheren Diskussionen durchaus umstritten war, blieb sie aber im untersuchten Gesetzgebungsprozess praktisch unerwähnt. Keine der Parteien sprach sich gegen die Anpassung an die MuKEn aus. Die SP forderte in der Kommissionsphase noch die (restriktivere) Ausweitung der VHKA-Pflicht auf bestehende Gebäude, im Plenum besprach der Grosse Rat die VHKA danach aber nicht. Andere Massnahmen der Totalrevision waren dagegen sehr umstritten, der Grosse Rat nahm das KEnG aber knapp in der Schlussabstimmung an. Bürgerliche Parteien, der HEV Kanton Bern und weitere Interessengruppen reichten danach ein konstruktives Referendum ein, in dem sie die zwei umstrittensten Massnahmen aus der Vorlage strichen. Ein neugewählter, bürgerlicherer Grosser Rat unterstützte diesen Volksvorschlag, den letztlich auch die Stimmberechtigten in der Abstimmung bevorzugten. Evaluationen 
fanden im gesamten Gesetzgebungsprozess keine Verwendung, weitere Informationen dazu enthält Tabelle 15.

\title{
Tabelle 15: VHKA im Berner Grossen Rat - Übersicht der Nutzung
}

\begin{abstract}
Parlament Analytisch: keine
Die Interviewaussagen legen nahe, dass die befragten Kommissionsmitglieder Evaluationen der VHKA nicht berücksichtigten. Zwei Befragte kennen gar keine Evaluationen (F2-PM1, F2-PM3). Ein Kommissionsmitglied, das eine Ausweitung der VHKA auf bestehende Bauten ablehnte, erwähnte ein Gutachten zur VHKA, das aber nicht auffindbar ist (F2-PM2).

Politisch: keine

Die Parlamentsmitglieder verwiesen weder auf spezifische Evaluationen noch auf «Evaluationen» oder «Studien» allgemein. Auch forderten sie keine Evaluationen oder kritisierten diese.

Regierung Analytisch: Evaluationen bekannt, nicht darauf gestützt

und

Verwaltung Politisch: keine

Die BVE gab zu früheren Zeitpunkten zwar Evaluationen in Auftrag und die Evaluationen waren durchaus bekannt, Evaluationen fanden aber keine Verwendung zur Ausarbeitung der Regelung der VHKA im untersuchten Gesetzgebungsprozess (F2-Verw.). Auch verwiesen Regierung und Verwaltung nie auf Evaluationen.
\end{abstract}

\subsection{Steuersystem für energieeffiziente Fahrzeuge}

\subsubsection{Hintergrund und Funktionsweise des Steuersystems für energieeffiziente Fahrzeuge}

Beim Erwerb, Halten und Nutzen von Fahrzeugen fallen in der Schweiz verschiedene Steuern an. Einerseits fordert der Bund die einmalige Automobilsteuer beim Import oder der Herstellung eines Fahrzeugs. Weiter in seiner Kompetenz sind die Autobahngebühren und die Mineralölsteuer. Anderseits erheben die Kantone jährlich Motorfahrzeugsteuern ${ }^{112}$ für das Halten eines Fahrzeugs (Bundesamt für Strassen ASTRA 2016).

Steuersysteme für energieeffiziente Fahrzeuge waren einerseits ab den 2000er-Jahren auf der politischen Agenda des Bundes. Infolge der Standesinitiative des Kantons Bern vom 30. November 2005 diskutierte die Bundesversammlung über ein Bonus-MalusSystem für die Steuern beim Import von Automobilen. Der Entwurf zur Initiative wurde aber in der Vernehmlassung im Jahr 2009 stark kritisiert. Nach einer zweimaligen Erstreckung schrieb die Bundesversammlung 2013 dann die Standesinitiative ab, da sich die

112 Die Motorfahrzeugsteuern werden im Kanton Zürich «Verkehrsabgaben» genannt. 
Politik des Bundes und der Kantone mittlerweile in die vorgeschlagene Richtung entwickelt habe (Ständerat und Kommission für Umwelt 2012).

Basierend auf den Motorfahrzeugsteuern waren anderseits Steuersysteme für energieeffiziente Fahrzeuge bereits um die Jahrtausendwende ein Thema in den Kantonen. Etwa hat Luzern ab 1996 ein Bonus-Malus-System für sparsame, emissionsarme Fahrzeuge versuchsweise eingeführt, das dann ab 2002 von einem Bonussystem für alternative Antriebe abgelöst wurde (Keller et al. 2002, 74). Auch in anderen Kantonen existierten bereits Mitte der 2000er-Jahre Steuerermässigungen für Elektro-, Gas- oder Hybridfahrzeuge, es wurden aber auch Steuersysteme gefordert, die energieeffiziente Fahrzeuge unabhängig von ihrer Antriebsart begünstigen (de Haan und Peters 2005, 9; Ernst Basler Partner 2010, 6). Als Reaktion darauf arbeitete die Vereinigung der Strassenverkehrsämter der Schweiz (asa) Empfehlungen in Form eines Muster-Rabattmodells aus. Dieses asa-Modell wurde in Zusammenarbeit von Strassenverkehrsämtern aus 18 Kantonen, dem BFE, dem Bundesamt für Umwelt (BAFU) und dem Touring Club Schweiz (TCS) unter Begleitung von Forschenden der Eidgenössischen Technischen Hochschule Zürich (ETHZ) und der privaten Firma INFRAS erstellt (asa 2007). Darauf abgestützt empfahl die Konferenz der Kantonalen Justiz- und Polizeidirektorinnen und direktoren (KKJPD) 2007, ein solches Rabattmodell einzuführen (KKJPD 2007).

Abbildung 18 illustriert das für die Fallstudien ausgearbeitete Wirkungsmodell des Steuersystems für energieeffiziente Fahrzeuge. Das grundlegende Ziel des Steuersystems ist, über finanzielle Anreize einen energie- und umweltfreundlicheren Fahrzeugbestand zu erreichen. Dazu werden die Motorfahrzeugsteuern für neu immatrikulierte Fahrzeuge mit besonders wenig Treibstoffverbrauch und geringen Emissionen mittels eines Bonus gesenkt. Ebenso kann ein Malus auf die Motorfahrzeugsteuern für besonders treibstoffund schadstoffintensive Fahrzeuge gegeben werden. Der Malus kann gleichzeitig dazu dienen, den Ertragsausfall aufgrund des Bonus zu kompensieren. ${ }^{113}$ Das Kriterium für den Bonus oder Malus bildet die Energieetikette. Seit 2003 sind die Händler verpflichtet, die Energieetikette beim Verkauf neuer Personenwagen sichtbar anzubringen. Die Energieetikette informiert über den Treibstoffverbrauch, die $\mathrm{CO}_{2}$-Emissionen und die relative Energieeffizienz von Personenwagen. Dabei werden die Fahrzeugmodelle in sieben Kategorien von A mit der besten bis G mit der schlechtesten Energieeffizienz eingeteilt (UVEK 2002). ${ }^{114}$ Ein absolutes Kriterium in Form eines Grenzwerts des $\mathrm{CO}_{2}$-Ausstosses kann dieses relative Kriterium der Energieetikette ergänzen. In dieser Ausgestaltung entspricht die Massnahme grundsätzlich dem Modell der asa (2007), wobei diese zusätzlich empfiehlt, den Bonus und Malus auf drei bis vier Jahre zu beschränken.

Das Steuersystem richtet sich primär an Käuferinnen und Käufer von Neuwagen. Die Käuferinnen und Käufer sollen aufgrund des Bonus respektive Malus energieeffi-

113 Bei einem System ohne Malus könnte dies mit einer generellen Steuererhöhung kompensiert werden.

114 Der Bundesrat revidierte 2006 und 2011 die Energieetikette, darüber hinaus wird sie regelmässig aktualisiert. Ursprünglich plante der Bundesrat, die Energieetikette durch eine Umweltetikette zu ersetzen. 2010 entschied er aber, die Energieetikette beizubehalten (UVEK Bundesrat 2011; Eidgenössisches Departement für Umwelt 2006; 2010). 
zientere Fahrzeuge erwerben. Dies soll auch nichtfinanzielle Wirkungen auf die Käuferinnen und Käufer haben, indem sie etwa ihr Image von energieeffizienten Fahrzeugen verbessern. Der Bonus könnte aber unerwünschte Wirkungen haben, so dass die Käuferinnen und Käufer den Bonus verwenden, um etwa ein grösseres Fahrzeug zu erwerben. Das Steuersystem richtet sich zweitens an die Produzenten und Händler, die ihre Entwicklungs- und Marketingstrategien mehr auf energieeffiziente Fahrzeuge konzentrieren sollen. Insgesamt soll das Steuersystem so den Energiekonsum und die Emissionen von $\mathrm{CO}_{2}$ und anderen Schadstoffen reduzieren. Diese Reduktion ist langfristig, da Neuwagen

\section{Abbildung 18: Wirkungsmodell des Steuersystems für energieeffiziente Fahrzeuge}

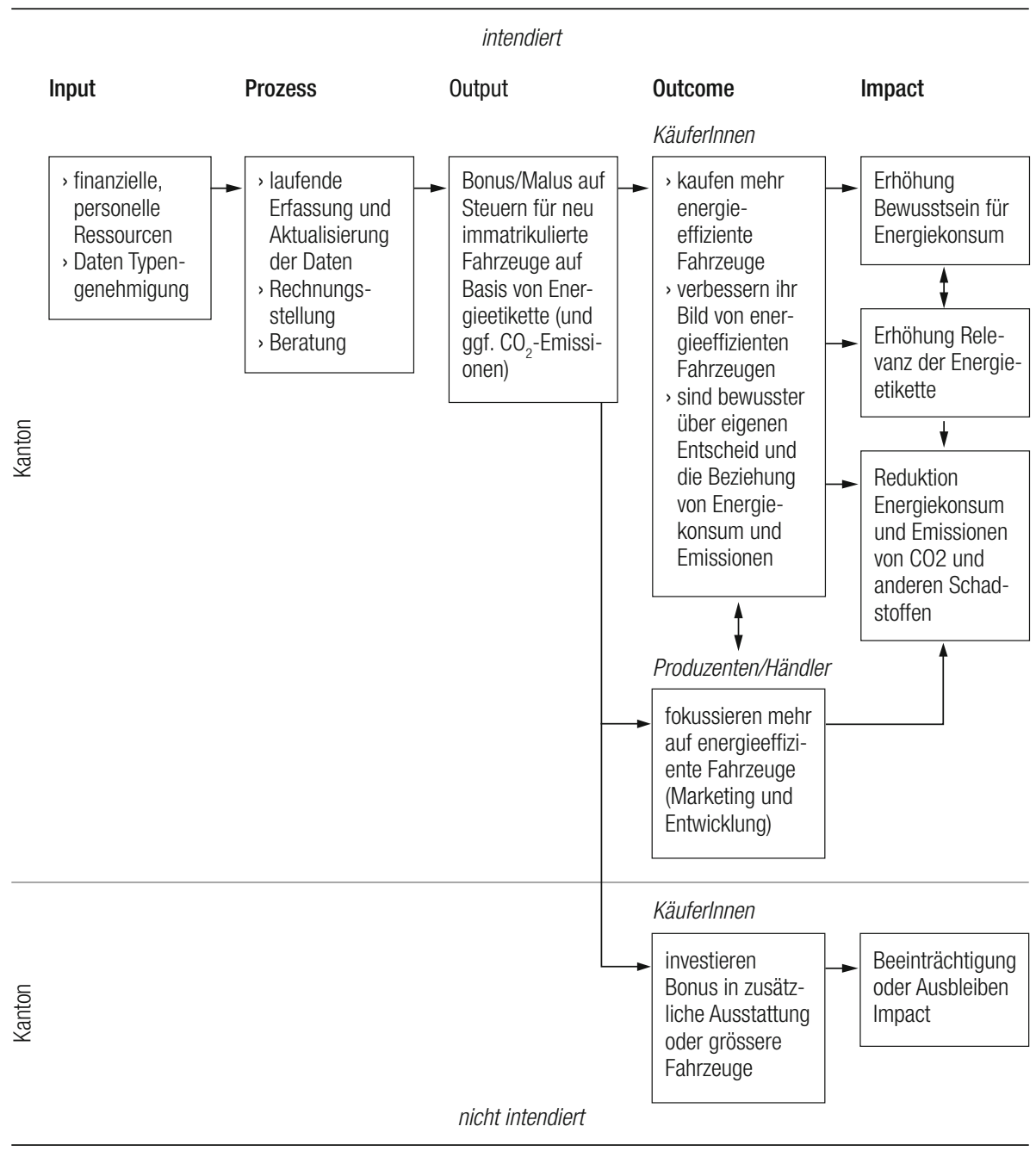


weiterverkauft werden. Aufgrund der nicht rein finanziellen Wirkung soll das Steuersystem auch generell zu einem erhöhten Bewusstsein für den Energiekonsum führen. Überwiegen die unerwünschten Wirkungen bei den Käuferinnen und Käufer, dürften diese Gesamtwirkungen beeinträchtigt werden oder gar nicht eintreten (asa 2007; de Haan et al. 2007a; de Haan et al. 2007b). Für die Wirkung des Steuersystems ist schliesslich die Energieetikette wesentlich. Sie ist bereits bekannt und ein einfaches Kriterium für Käuferinnen und Käufer sowie die Händler. Auch kann die Energieetikette einfach und zuverlässig in das bestehende Steuersystem der Kantone eingebaut werden (Regierungsrat des Kantons Bern 2009a, 5). Dank der Aktualisierung der Energieetikette passt sich das Steuersystem zudem laufend dem technischen Stand an (asa 2007).

\subsubsection{Verfügbare Evaluationen zum Steuersystem für energieeffiziente Fahrzeuge}

Insgesamt konnten 16 Evaluationen identifiziert werden, die sich mit Steuersystemen für energieeffiziente Fahrzeuge befassen. Dazu zählen auch sieben Evaluationen, welche die Funktionsweise des Steuersystems für energieeffiziente Fahrzeuge am Beispiel der Bundesebene untersuchen. Neben den Evaluationen gibt es drei Zusammenfassungen, die zentrale Ergebnisse der Evaluationen wiedergeben. Dies betrifft die Dokumentation der asa (2007). Weiter zählen dazu je eine Präsentation in den Kantonen Bern und Zürich, die im Laufe der untersuchten Gesetzgebungsprozesse stattfanden.

Die ermittelten Evaluationen entstanden fast zur Hälfte im Rahmen eines Forschungsprojekts der ETHZ, das hauptsächlich von der Vereinigung Schweizer AutomobilImporteure (auto-schweiz) und dem Forschungsfonds der schweizerischen Erdöl-Vereinigung (FEV) unterstützt wurde. Hintergrund dieser Zusammenarbeit ist die 2002 geschlossene Zielvereinbarung zwischen dem Eidgenössischen Departement für Umwelt, Verkehr, Energie und Kommunikation (UVEK) und auto-schweiz, den Treibstoffverbrauch der Personenwagen-Neuwagenflotte von 2000 bis 2008 pro Jahr um durchschnittlich drei Prozent zu senken (Godet und de Haan 2007, 6). Weiter unterstützten das BFE, die asa oder einzelne Autohersteller punktuell Studien des Projekts. Bei den restlichen ermittelten Evaluationen handelt es sich um Auftragsstudien des BFE oder in einem Fall des Autogewerbeverbands Schweiz (AGVS). Zudem gab das Strassenverkehrs- und Schifffahrtsamt des Kantons Bern (SVSA) zwei Evaluationen in Auftrag. Meist erstellten private Forschungsbüros diese Auftragsstudien, teilweise waren auch Forschende der ETHZ beteiligt. Die Autorenschaft der Evaluationen ist daher auf einen kleinen Kreis konzentriert. Ein Teil dieser Forschenden wirkte auch an der Erstellung des Modells von asa (2007) mit.

Tabelle 16 gibt einen Überblick der wichtigsten Ergebnisse zur Wirkung von Steuersystemen. Insgesamt befassen sich die Evaluationen mit den meisten der Zusammenhänge, die das entworfene Wirkungsmodell enthält (vgl. auch Abbildung 18). Die Evaluationen stellen zudem weitgehend übereinstimmende Wirkungen fest. Da die Evaluationen Steuersysteme für energieeffiziente Fahrzeuge aber fast ausschliesslich aus einer Ex-Ante-Perspektive betrachten, gehen sie von verschiedenen Konzeptionen aus. ${ }^{115}$

115 Nur zwei kurze Berichte beinhalten eine Ex-Post-Perspektive, untersuchen den Zusammenhang von Steuersystem und Kaufverhalten aber nicht auf der individuellen Ebene (de Haan et al. 2006; de Haan und Peters 2005). 
Einige Grundzüge, wie die Basierung auf der Energieetikette und die Besteuerung von Neuwagen sind zwar unbestritten, die Gestaltung der Steuersysteme ist aber unterschiedlich (Bonus-Malus, Bonussystem, Differenzierung der Kategorien). ${ }^{16}$ Entsprechend beziehen sich die Aussagen für die Wirkungen jeweils auf den in der Evaluation skizzierten Entwurf, was die Vergleichbarkeit der Ergebnisse erschwert. Zudem wurden verschiedene Zusammenhänge überwiegend oder nur von einem Forschungsprojekt ${ }^{117}$ mit denselben oder ergänzten Daten analysiert. Weiter fokussieren die Evaluationen hauptsächlich auf die Frage, wie das Verhalten der Neuwagenkäuferinnen und -käufer mit dem Energieverbrauch und den $\mathrm{CO}_{2}$-Emissionen zusammenhängt. $\mathrm{Zu}$ schwerer greifbaren Zusammenhängen wie der Verbesserung des Images energieeffizienter Fahrzeuge gibt es wenige Erkenntnisse. Die kaufpsychologischen Effekte untersuchten Forschende der ETHZ zwar mittels einer Befragung. Sie publizierten diese Ergebnisse aber nur in Fachzeitschriften (vgl. Peters et al. 2006; Peters et al. 2011). Insgesamt kann daher die Übereinstimmung der Evaluationsergebnisse als moderat bezeichnet werden.

\subsubsection{Bonussystem im Kantonsrat Zürich (Fall 3)}

\section{Ablauf und Inhalt des Gesetzgebungsprozesses}

Das Steuersystem für energieeffiziente Fahrzeuge wurde in Zürich im Rahmen der Revision des Gesetzes über die Verkehrsabgaben und den Vollzug des Strassenverkehrsrechts des Bundes (Verkehrsabgabengesetz) diskutiert, die der Kantonsrat hauptsächlich zwischen Juni 2010 und November 2011 beriet. Die Ziele dieser Gesetzesrevision waren, die Verkehrsabgaben verursachergerechter auszugestalten sowie Anreize zu ökologischem Verhalten zu schaffen, wobei diese Änderungen haushaltsneutral bleiben sollten (Regierungsrat des Kantons Zürich 2010d). Um diese Ziele zu erreichen, beinhaltete die Gesetzesrevision neben dem Steuersystem als zentralen Bestandteil auch eine Anpassung der Bemessungsgrundlage. Die Verkehrsabgaben sollten damit erstmals seit 1972 geändert werden, da frühere Revisionsversuche in fünf Volksabstimmungen oder einmal bereits früher im Parlament scheiterten.

Vier parlamentarische Vorstösse, die Kantonsratsmitglieder der CVP, EVP, FDP, SP und der Grünen in den Jahren 2005 bis 2007 einreichten, lösten diese Gesetzesänderung aus. ${ }^{118}$ Diese Kantonsratsmitglieder der verschiedenen Parteien forderten, das Verkehrsabgabengesetz zu revidieren und Anreizsysteme einzuführen. Während einige Vorstösse eine generelle Revidierung des Verkehrsabgabengesetzes bezweckten, zielten andere auf Ermässigungen für spezifische Fahrzeuge und Antriebe ab. Übereinstimmend mit dieser breiten parteilichen Unterstützung überwies der Kantonsrat sämtliche Vorstösse an den

116 Nur eine Evaluation vergleicht verschiedene Entwürfe eines möglichen Steuersystems auf Bundesebene. Darin wird ein Bonussystem für Fahrzeuge der Kategorie A mit genereller Steuererhöhung als am effizientesten bezeichnet, wobei zur Akzeptanz der Massnahme auch ein Bonus für die Kategorie B erwägt werden könne (de Haan et al. 2007b, 119).

117 Unerwünschte Wirkungen untersuchte zum Beispiel nur das ETH-Forschungsprojekt.

118 Motionen 119/2005, 176/2005, 15/2007 sowie 78/2007. 


\section{Tabelle 16: Evaluationsergebnisse zur Wirkung des Steuersystems für energieeffiziente Fahrzeuge}

\section{Outcome (Evaluationen und Zusammenfassungen, $\mathrm{N}=14$ )}

\section{Käuferinnen und Käufer}

Die Käuferinnen und Käufer sprechen auf die Energieetikette an und akzeptieren Steuersysteme, wobei sie die Finanzierung des Bonus durch einen Malus vorziehen. Gemäss den Befragungen ziehen sie den Bonus in den Kaufentscheid ein, etwa würden sie deswegen häufiger Fahrzeuge mit Diesel kaufen. Der Bonus würde aber auch für den Kauf von grösseren Fahrzeugen oder zusätzlicher Ausstattung eingesetzt. Zudem würde er weniger wichtig, je höher das Einkommen oder je grösser das bevorzugte Fahrzeug einer Käuferin oder eines Käufers ist. In den Literaturreviews wird zwar auf die symbolisch-normative Wirkung von Steuersystemen aufmerksam gemacht, dies untersucht aber keine Evaluation empirisch.

\section{Produzenten/Händler}

Aussagen zu den Reaktionen der Händler enthält nur eine Evaluation (de Haan et al. 2010). Demgemäss sehen die befragten Händler einen Bonus zwar als Chance, um mehr Fahrzeuge zu verkaufen, aber sie befürchten auch eine Umsatzeinbusse, da kleinere Autos bevorzugt werden würden. Weiter weisen sie darauf hin, dass die Energieetikette einfach bleiben soll und die Kantone harmonisierte Systeme haben sollten, um dies verständlich an die Käuferinnen und Käufer kommunizieren zu können. Zu den Reaktionen der Produzenten liegen keine Ergebnisse vor.

\section{Impact (Evaluationen und Zusammenfassungen, $\mathrm{N}=11$ )}

\section{Erhöhung des Bewusstseins für Energiekonsum generell und der Relevanz der Energieetikette generell}

Zu diesem Aspekt liegen keine Ergebnisse vor.

\section{Reduktion Energieverbrauch und $\mathrm{CO}_{2}$-Emissionen}

Gemäss allen Simulationen führen Steuersysteme für energieeffiziente Fahrzeuge zu einer Reduktion der $\mathrm{CO}_{2}$-Emissionen der Neuwagenflotte, wobei dieser Effekt langfristig wächst. Die errechneten Angaben zur Kosteneffizienz sind aber sehr unterschiedlich gross. So wird für ein Bonus auf Hybridwagen von 250 Franken pro vermiedene Tonne $\mathrm{CO}_{2}$ berechnet, während beim Bonus-Malus-System, wie es im Kanton Bern vorgeschlagen wurde, eine Kosteneffizienz von 20 bis 35 Franken pro vermiedene Tonne $\mathrm{CO}_{2}$ geschätzt wird (de Haan et al. 2006; ETH 2008).

\section{Reduktion andere Luftschadstoffe}

Die Auswirkungen auf den Ausstoss der restlichen Schadstoffe thematisieren nur zwei Evaluationen (Hammer und Maibach 1999; Hammer et al. 2001). Die Ergebnisse dieser Berechnungen sind widersprüchlich und deuten auf eine knappe Zunahme respektive Abnahme.

Die Tabelle enthält nur Literaturangaben, wenn Ergebnisse auf einzelnen Evaluationen beruhen oder widersprüchlich sind. Bei übereinstimmenden Ergebnissen wird auf Literaturangaben verzichtet.

Regierungsrat. Bei der Beratung wiesen aber Mitglieder der SVP in ihren ablehnenden Voten gegen die Vorstösse darauf hin, später gegebenenfalls das Referendum zu ergreifen (Kantonsrat Zürich 2006, 10954; 2008, 5296).

Der Regierungsrat eröffnete die Vernehmlassung im April 2009. Da der Regierungsrat beabsichtigte, das Verkehrsabgabengesetz gleichzeitig mit dem Strassengesetz 
zu revidieren, schickte er beide Vorlagen zusammen in die Vernehmlassung. Das Verkehrsabgabengesetz arbeitete die Sicherheitsdirektion unter CVP-Regierungsrat Hollenstein aus (Regierungsrat des Kantons Zürich 2009a). In der Vernehmlassung schlug der Regierungsrat vor, die Verkehrsabgaben für Fahrzeuge der Kategorie A um 100 Prozent respektive für Fahrzeuge der Kategorie B um 50 Prozent für die ersten zwei Jahre nach Inverkehrsetzung zu ermässigen (Volkswirtschaftsdirektion des Kantons Zürich 2009). Die verfügbaren Stellungnahmen der Parteien sind gespalten: Die FDP begrüsste das Bonussystem grundsätzlich. Die SP und die Grünen hielten den Schadstoffausstoss und die Fahrleistung als zu wenig berücksichtigt. Die SVP lehnte das Bonussystem vollständig ab (FDP Kanton Zürich 2009; Grüne Kanton Zürich 2009; SP Kanton Zürich 2009b; SVP Kanton Zürich 2009).

Nach der Trennung der beiden Gesetzesprojekte verabschiedete der Regierungsrat seinen Antrag zum Verkehrsabgabengesetz im April 2010. Als Reaktion auf die Vernehmlassung koppelte er den Bonus an den Schadstoffgrenzwert von $140 \mathrm{Gramm} \mathrm{CO}_{2}$ pro Kilometer. Ebenso senkte er den Bonus für Fahrzeuge der Kategorie A auf 80 Prozent. Weiter verlängerte der Regierungsrat die Dauer des Bonus auf drei Jahre und schuf Übergangsbestimmungen für jene Fahrzeuge, die kurz vor Inkrafttreten des Gesetzes in Verkehr gesetzt wurden (Regierungsrat des Kantons Zürich 2010d). Die verfügbaren Medienmitteilungen zeigen, dass die Grünen diese Änderungen begrüssten, sie ihnen aber noch zu wenig weit reichten. Die SVP vertrat weiter eine klar ablehnende Haltung (Grüne Kanton Zürich 2010a; SVP Kanton Zürich 2010).

Zwei Monate später nahm die Kommission für Wirtschaft und Abgaben (WAK) die Beratung der Vorlage auf, wobei die Vorlage in insgesamt neun Sitzungen über acht Monate Traktandum war. Nach der Präsentation der Vorlage hörte die WAK Vertretungen des Gewerbes, der Automobilverbände sowie externe Fachpersonen zu Anreizsystemen und zu gesundheitlichen Auswirkungen des Automobilverkehrs an. Danach beriet sie die Vorlage in zwei Lesungen und stimmte darüber ab. Das Bonussystem stand während der Kommissionsberatungen im Zentrum und die WAK regte zwei Änderungen

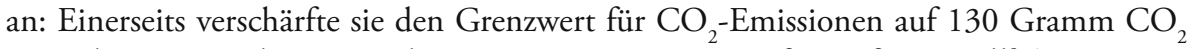
pro Kilometer. Anderseits erarbeitete sie ein Bonussystem für Lieferwagen ${ }^{119}$ (Kantonsrat Zürich 2011g, 14487-14490).

Vor der Behandlung im Kantonsrat forderte dann der Kantonale Gewerbeverband Zürich (KGV), dass die Vorlage zurückgezogen werden müsse. Dies begründete er damit, dass gemäss dem Gesetz zur administrativen Entlastung der Unternehmen (Entlastungsgesetz) Aussagen über die Auswirkungen auf die Unternehmen fehlten. Ebenso liege keine Regulierungsfolgeabschätzung vor (Kantonaler Gewerbeverband Zürich 2011). Dieses Anliegen des KGV vertrat die SVP in der ersten Lesung des Kantonsrats im April 2011. Folglich besprachen die Kantonsratsmitglieder bei dieser Lesung den Rückweisungsantrag der SVP und die Frage des Eintretens. Die SVP beantragte zusätzlich Nichteintreten, da sie das Bonussystem und die Verkehrsabgabenrevision im Gesamten ablehnten. In ihrem Antrag auf Nichteintreten wies die Sprecherin der SVP darauf hin, dass die SVP

119 Die Energieetikette gilt nur für Personenwagen (5.3.1). 
das Referendum ergreifen werde, wenn der Kantonsrat diese Gesetzesrevision verabschieden würde. Der Kantonsrat beschloss, auf die Vorlage einzutreten, wies sie aber an den Regierungsrat zurück (Kantonsrat Zürich 2011g). Der Regierungsrat prüfte das Anliegen und hielt in seinem Beschluss fest, dass das Entlastungsgesetz nicht für die Vorlage gelte und keine Regulierungsfolgeabschätzung notwendig sei (Regierungsrat des Kantons Zürich 2011a).

Der Kantonsrat behandelte darauffolgend das Verkehrsabgabengesetz in einer zweiten Lesung im September 2011. Da im April 2011 Kantons- und Regierungsratswahlen stattgefunden hatten, war nun der neugewählte Regierungsrat Fehr (SP) zuständig. Im Kantonsrat kam es vor allem zu Verschiebungen bei den Grünliberalen, die ihre Sitzzahl von 10 auf 19 beinahe verdoppelten. In der neuen Legislatur wechselten 10 der 15 WAK-Mitglieder. ${ }^{120}$ Das Präsidium ging von der SP zur SVP. ${ }^{121}$ Da die WAK die ganze Vorlage in der alten Legislatur beraten hatte, übernahm sie aber die Kommissionsanträge für die zweite Lesung unverändert (vgl. auch F3-PM2; F3-PM3).

In dieser zweiten Lesung im Plenum kam es zu einer nochmaligen Eintretensdebatte, da die SVP erneut einen Rückweisungsantrag stellte. Ihre ablehnende Haltung begründete sie mit denselben Argumenten wie in der ersten Lesung. Erneut drohte ein Mitglied der SVP, ein Referendum zu lancieren. Der Kantonsrat lehnte den Rückweisungsantrag der SVP aber klar ab. In der darauffolgenden Detailberatung stand das Bonussystem als Grundlage von neun der zwölf Kommissions- oder Minderheitsanträgen eindeutig im Zentrum. ${ }^{122}$ Der Kantonsrat lehnte die meisten der Minderheitsanträge knapp mit 55.8 bis 59.4 Prozent der Stimmen ab. Nur zwei Anträge, darunter der Antrag der SVP, das Bonussystem für Personenwagen ganz zu streichen, lehnten mehr als zwei Drittel des Kantonsrats ab.

In der folgenden Redaktionslesung im November 2009 beantragte die Redaktionskommission keine Änderungen. Hingegen schlug die EDU vor, einen neuen Paragrafen einzuführen, der einen Bonus für Grossfamilien vorsah, was der Kantonsrat klar ablehnte. In der Schlussabstimmung nahm der Kantonsrat das Verkehrsabgabengesetz äusserst knapp mit 80 zu 76 Stimmen an. Die gegnerischen Stimmen stammten von den linken Parteien AL und Grüne sowie von den beiden rechten Parteien EDU und SVP. Die restlichen Parteien stimmten für das Verkehrsabgabengesetz. Die Fraktionen der SVP und der EDU reichten nach der Redaktionslesung das Behördenreferendum ein. In der Volksabstimmung nahmen die Stimmberechtigten das Verkehrsabgabengesetz an.

120 Gemäss Vergleich der Anträge der Kommission erste Lesung und zweite Lesung.

121 Das Präsidium wechselte zuvor während den Beratungen einmal parteiintern in der SP.

122 Sieben Minderheitsanträge für das Bonussystem für Personenwagen: Vier Minderheitsanträge (Grüne, Grünliberale, EVP, Grüne/SP) betrafen die Höhe und die Anpassung der $\mathrm{CO}_{2}$-Ausstoss-Grenze, ein Minderheitsantrag (SP) sah mit dem Emissionscode ein weiteres Kriterium vor, ein bzw. zwei Minderheitsanträge (SVP) sahen das Streichen des Bonussystems und der Übergangsregelungen vor. Zwei Minderheitsanträge für das Bonussystem für Lieferwagen zur Höhe der $\mathrm{CO}_{2}$-Ausstoss-Grenze (Grüne, SP), wobei diese in einem Antrag zusätzlich von der Fahrleistung abhängig gemacht werden sollte (SP). 


\section{Evaluationsnutzung}

In der Diskussion der Vorstösse zum Bonussystem erwähnten die Kantonsratsmitglieder nur vereinzelt Zusammenfassungen. Die Motionärinnen und Motionäre der vier Vorstösse begründeten diese mit der Luftverschmutzung sowie mit einer verursachergerechten Besteuerung, bezogen sich aber nicht auf Evaluationen. In der Plenumsdiskussion der zwei Motionen aus 2007 wiesen jedoch zwei Kantonsräte auf die Empfehlungen der asa (2007) hin: Während der grünliberale Kantonsrat dies tat, um zu zeigen, dass die notwendigen Grundlagen vorhanden sind, verwendete der SVP-Kantonsrat dies, um die Bedingungen des Bonussystems zu präzisieren: «Die ASA-Studie, Empfehlung der Vereinigung der Strassenverkehrsämter der Schweiz, spricht von einer Steuerbefreiung von drei Jahren» (Kantonsrat Zürich 2008, 5282). Auch die Regierungsrätin wies auf die Empfehlungen der asa hin, um den aktuellen Stand der Arbeiten zu erläutern. Ansonsten erwähnten die Kantonsratsmitglieder keine Evaluationen. Sie verwiesen aber in allen Plenumsdiskussionen zu den Vorstössen auf Statistiken zur Verwendung von Fahrzeugen sowie zu den gesundheitlichen Auswirkungen von Feinstaub und diskutierten Letztere kritisch (Kantonsrat Zürich 2006, 2008).

Bei der Ausarbeitung der Vorlage stützte sich die Verwaltung eher indirekt auf Evaluationen. Gemäss dem Interview beteiligte sich das Strassenverkehrsamt zwar bei der Erarbeitung des asa-Modells und das Modell sowie das dahinterstehende Fachwissen waren bekannt, man hätte es aber eher als Leitlinien eingebunden. Im Vordergrund seien die Entwürfe und Erfahrungen aus anderen Kantonen gestanden und auf einen stärkeren Einbezug von Fachpersonen oder Evaluationen sei daher verzichtet worden. Vielmehr hätte man sich an den Ergebnissen der Vernehmlassung orientiert, um eine politisch mehrheitsfähige Vorlage zu entwerfen (F3-Verw.). Entsprechend hat auch der Regierungsrat im Bericht zur Vernehmlassung und später im Antrag lediglich darauf verwiesen, dass das Bonussystem im Wesentlichen dem Modell entspreche, «das unter Mitwirkung des Bundes, der ETH, von Vertreterinnen und Vertretern der kantonalen Umwelt- und Energiefachstellen sowie der Vereinigung der Strassenverkehrsämter im Juli 2007 verabschiedet wurde» (Regierungsrat des Kantons Zürich 2010d, 9).

Die weiteren Dokumente enthalten kaum Hinweise auf eine Evaluationsnutzung der Parlamentsmitglieder oder Parteien in der vorparlamentarischen Phase. In den verfügbaren Stellungnahmen verwies einzig die FDP auf das «asa-Rabattmodell» (FDP Kanton Zürich 2009). Ohne spezifische Quellen zu nennen, machten daneben die Grünen allgemein auf die Auswirkungen des Verkehrs aufmerksam: «Die Auswirkungen auf die Gesundheit von Mensch und Natur sind gravierend. Dies alles belegt der Kanton in eigenen Studien und Berichten» (Grüne Kanton Zürich 2009).

Die WAK beschäftigte sich besonders während der Hearings mit Evaluationen. Sie lud zwei externe Fachpersonen ein, wobei nur das erste Referat in Bezug auf das Bonussystem relevant war. ${ }^{123}$ Gestützt auf das ETH-Forschungsprojekt präsentierte die Fachperson Ergebnisse zu Steuersystemen und deren Wirkungsweise. Um die Relevanz des Bonussys-

123 Zur Vertretung der Interessengruppen waren zusätzlich drei Personen eingeladen. 
tems zu illustrieren, enthielt das Referat auch Hintergrundinformationen zur Entwicklung des Fahrzeugmarkts und zum Energiesparpotential des Verkehrs. ${ }^{124}$ Die zweite Präsentation befasste sich mit den gesundheitlichen Auswirkungen der verkehrsbedingten Luft- sowie Lärmbelastung. Um diese Auswirkungen darzustellen, zitierte diese zweite Fachperson verschiedene Studien, die sich aber nicht auf das Bonussystem bezogen. Die Präsentation zum Bonussystem beurteilt die Mehrheit der Befragten äusserst positiv: «Es war sehr überzeugend und wirklich ausgezeichnet, ja, also vom Fachlichen her» (F3-PM1; vgl. auch F3-PM3). Nur ein Kommissionsmitglied, das sich für das Bonussystem aussprach, kann sich aber an spezifische Inhalte der Präsentation erinnern. Besonders die Ergebnisse zur psychologischen Wirkung seien wichtig gewesen (F3-PM3). Es war auch dieses Kommissionsmitglied, das die externe Fachperson der Kommission vorschlug. ${ }^{125}$ Bei eigenen Recherchen sei es auf eine Präsentation der externen Fachperson gestossen und habe gesehen, dass diese konkret etwas zum Thema zu sagen habe. Das befragte Kommissionsmitglied auf der gegnerischen Seite erinnert sich hingegen nicht an die Präsentation zum Bonussystem (F3-PM2). Es gäbe sehr viele Hearings. Eigene Recherchen hatte dieses Kommissionsmitglied nur gemacht, um Steuertarife zu berechnen.

Gemäss den Protokollen verwendeten die Kommissionsmitglieder Evaluationen während des Hearings meist in einer Weise, die als analytisch oder politisch interpretiert werden kann. Drei verschiedene Kommissionsmitglieder, die das Bonussystem befürworteten, fragten so eine eingeladene Person einer Interessengruppe auf der gegnerischen Seite, welche Daten ihre Äusserungen belegten. Aus den Protokollen erschliesst sich jedoch nicht, inwiefern diese Fragen ergebnisoffen gestellt waren. In einem weiteren Beispiel fragte ein anderes Kommissionsmitglied die eingeladene Fachperson, ob der psychologische Lenkungseffekt vorhanden sei. Auch hier ist es schwierig abzuschätzen, ob diese Frage dem besseren Verständnis oder eher als Bestätigung dienen sollte. Eindeutig der politischen Nutzung zuschreiben lassen sich aber zwei Beobachtungen während der Kommissionsphase: In der Eintretensdebatte nach den Hearings begründete ein Kommissionsmitglied, das sich für das Bonussystem aussprach, seine Position mit dem in der Präsentation dargelegten Lenkungseffekt. Weiter verwies die FDP in einer weiteren Medienmitteilung erneut auf das asa-Modell (FDP Kanton Zürich 2010b).

In der ersten Lesung des Kantonsrats fanden Evaluationen vereinzelt Verwendung. Die Kommissionspräsidentin erwähnte die Hearings in ihrer Einführungsrede. Die externe Fachperson habe darin darauf verwiesen, dass «viele Käuferinnen und Käufer beim Autokauf nicht wirklich rechneten, sondern irrational handelten» (Kantonsrat Zürich 2011g, 14488). In ihrem Antrag auf Rückweisung in der ersten Lesung forderte die SVP weiter eine Regulierungsfolgenabschätzung (Kantonsrat Zürich 2011g, 14491). ${ }^{126} \mathrm{Da}$ die SVP diese Forderung zu einem späten Zeitpunkt stellte, scheint sie weniger mit einer ergebnisoffenen als mit einer taktisch-verzögernden Absicht verbunden gewesen zu sein.

124 Dabei verwies die Fachperson auf verschiedene Studien (z. B. McKinsey \& Company 2009).

125 Die Einladung der zweiten externen Fachperson erfolgte auf Antrag eines anderen Kommissionsmitglieds, das sich für das Bonussystem aussprach.

126 Die SVP vertrat das Anliegen des KGV. 
In der weiteren parlamentarischen Beratung bezogen sich weder Parlamentsmitglieder noch der Regierungsrat auf Evaluationen. In der zweiten Lesung sprach aber ein Kantonsrat der Grünen Berechnungen zur $\mathrm{CO}_{2}$-Zielerreichung an, während zwei Kantonsräte der SVP den durch den Menschen verursachten Klimawandel teilweise oder generell in Frage stellten. Ein SVP-Kantonsrat erläuterte dazu ein Experiment der European Organization for Nuclear Research (CERN) (Kantonsrat Zürich 2011c, 1040). Die Dokumente und Interviews weisen schliesslich auf keine Verwendung von Evaluationen in der restlichen parlamentarischen Phase hin. Niemand hätte «irgendein Gutachten gebracht oder noch etwas in Auftrag gegeben» (F3-PM1). Auch in den weiteren verfügbaren Medienmitteilungen der Parteien gibt es keine Hinweise auf Evaluationen.

\section{Evaluationsnutzung: Zeitpunkt und Quellen}

Abbildung 19 illustriert die Publikationsdaten der Evaluationen und Zusammenfassungen im Zeitverlauf und zeigt die wichtigsten Ereignisse im Gesetzgebungsprozess. Demgemäss lagen in Fall 3 sämtliche Evaluationen bis zur ersten Plenumssitzung vor. Noch während der Kommissionsphase erschien eine Evaluation (Ernst Basler Partner 2010) und die Präsentation der externen Fachperson fand statt. Die Empfehlungen der asa erschienen bereits 2007.

\section{Abbildung 19: Zeitliche Verfügbarkeit der Evaluationen zum Bonussystem im Kantonsrat Zürich}

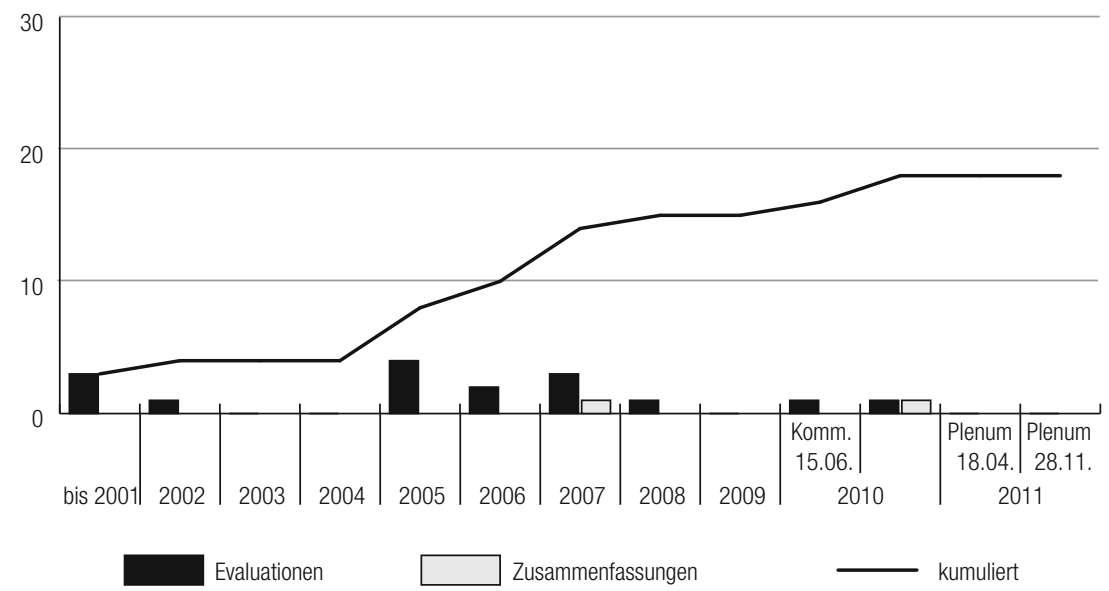

Die Anzahl bezieht sich auf die in einem Jahr beziehungsweise bis zum gegebenen Zeitpunkt erschienenen Evaluationen.

Von diesen verfügbaren Evaluationen fanden in Fall 3 zwei Evaluationen Verwendung: Mehr oder weniger vertieft setzten sich zwei der befragten Parlamentsmitglieder mit der Präsentation auseinander, während sich das dritte Parlamentsmitglied gar nicht daran erinnert. Neben der Präsentation verwiesen die Kantonsratsmitglieder zusätzlich auf die 
Dokumentation der asa (2007), wie die Tabelle 17 zeigt. Zudem verwendeten die Kantonsratsmitglieder auch Evaluationen politisch, indem namentlich die SVP diese forderte. Weiter fragten die Kantonsratsmitglieder nach Fachpersonen oder nach dem Vorhandensein von Evaluationen. Der Regierungsrat verwies schliesslich nur auf die Empfehlungen der asa (2007).

Die Parlamentsmitglieder verwendeten asa (2007) erstmals relativ zeitnah nach ihrem Erscheinen, auch griffen sie die Präsentation der externen Fachperson rasch auf. Ein grünliberaler Kantonsrat bezog sich aber im November 2008 gerade nicht aufgrund

\section{Tabelle 17: Bonussystem im Kantonsrat Zürich - politische Nutzung durch Parlament und Exekutive}

\begin{tabular}{|c|c|c|c|c|c|}
\hline & & & $\begin{array}{l}\text { Verweise } \\
\text { oder Zitate }\end{array}$ & $\begin{array}{l}\text { ment } \\
\text { andere Arten } \\
\text { politischer } \\
\text { Nutzung }\end{array}$ & $\begin{array}{l}\text { Regierung und } \\
\text { Verwaltung } \\
\text { Verweise } \\
\text { oder Zitate }\end{array}$ \\
\hline \multicolumn{3}{|c|}{ Anstoss - vorparlamentarische Phase } & & & \\
\hline $\begin{array}{l}\text { Parlamentsmitglied: } \\
\text { Grüne }\end{array}$ & Motion 119/2005 & 25.04 .2005 & & & \\
\hline $\begin{array}{l}\text { Parlamentsmitglied: } \\
\text { Grüne, CVP, EVP }\end{array}$ & Motion 176/2005 & 20.06 .2005 & & & \\
\hline Plenum & $\begin{array}{l}\text { Überweisung 119/2005, } \\
\text { 176/2005 }\end{array}$ & 27.03 .2006 & & & \\
\hline $\begin{array}{l}\text { Parlamentsmitglied: } \\
\text { FDP }\end{array}$ & Motion 15/2007 & 22.01.2007 & & & \\
\hline $\begin{array}{l}\text { Parlamentsmitglied: } \\
\text { SP, EVP }\end{array}$ & Motion 78/2007 & 12.03.2007 & & & \\
\hline Plenum & $\begin{array}{l}\text { Überweisung 15/2007, } \\
78 / 2007\end{array}$ & 24.11 .2008 & asa (2007) & & asa (2007) \\
\hline Regierungsrat & Vernehmlassung & 16.04.2009 & asa (2007) & & asa (2007) \\
\hline Regierungsrat & Antrag Regierungsrat & 14.04 .2010 & & & asa (2007) \\
\hline \multicolumn{6}{|c|}{ Parlamentarische Phase } \\
\hline Kommission & Beratung (Hearing, Antrag) & $\begin{array}{l}15.06 .2010- \\
15.03 .2011\end{array}$ & $\begin{array}{l}\text { asa (2007), } \\
\text { Präsentation WAK }\end{array}$ & $\begin{array}{l}\text { Experten/ } \\
\text { nach Evaluationen } \\
\text { fragen }\end{array}$ & \\
\hline Plenum & Beratung und Rückweisung & 18.04 .2011 & Präsentation WAK & Evaluation fordern & \\
\hline Plenum & Beratung & 12.09.2011 & & & \\
\hline Plenum & $\begin{array}{l}\text { Redaktionslesung, } \\
\text { Schlussabstimmung }\end{array}$ & 28.11.2011 & & & \\
\hline
\end{tabular}

Eingeschlossen sind nur Verweise, die auf eine Quelle schliessen lassen. Allgemeine Formulierungen wie «Evaluationen zeigen» usw. sind nicht in der Tabelle enthalten. 
ihrer Aktualität auf die Zusammenfassung von asa (2007): «Seit Sommer 2007 liegt diese Broschüre (‘Rabattmodell für die kantonale Motorfahrzeugsteuer zur Förderung energie- und umwelteffizienter Personenwagen) vor und dennoch ist der Kanton, trotz Versprechen, uns das im vierten Quartal 2008 vorzulegen, noch nicht so weit» (Kantonsrat Zürich 2008, 5293). Zudem verwiesen Parlamentsmitglieder und Parteien noch bis zu drei Jahre später auf asa (2007), als aktuellere Evaluationen verfügbar gewesen wären.

\section{Evaluationsnutzung: Positionen und Argumente}

Während Befürworterinnen und Befürworter wie auch Gegnerinnen und Gegner des Bonussystems Evaluationen politisch verwendeten, war die analytische Evaluationsnutzung einseitig. Nur die Befragten, die sich für das Bonussystem aussprachen, können sich an Evaluationen erinnern und setzten sich teilweise damit auseinander (F3-PM1; F3-PM3). Das Kommissionsmitglied, das sich gegen das Bonussystem aussprach, kann sich hingegen nicht an die Präsentation der externen Fachperson erinnern (F3-PM2). Befürworterinnen und Befürworter des Bonussystems verwendeten zudem Evaluationen öfters politisch, indem sie auf Evaluationen verwiesen. Die gegnerische Seite erwähnte in der Diskussion der Vorstösse nur einmal asa (2007), um Bedingungen des Bonussystems zu präzisieren. Demgegenüber forderte die SVP als Gegnerin des Bonussystems eine Evaluation in Form einer Regulierungsfolgeabschätzung. In der Kommissionsberatung fragten weiter die Mitglieder, die das Bonussystem befürworteten, die eingeladenen Personen der gegnerischen Interessengruppen mehrfach, ob sie ihre Argumente mit Daten belegen könnten. Wie bereits erläutert, könnten diese Fragen auch als politische Nutzung interpretiert werden, indem gegnerische Argumente durch Fragen nach der Evidenzbasierung entkräftet werden sollen.

Insgesamt unterstützen Evaluationen grösstenteils die Befürworterinnen und Befürworter der Bonussysteme, da ihre Ergebnisse zeigen, dass Bonussysteme den Energieverbrauch und die $\mathrm{CO} 2-$ Emmissionen senken können. Einzelne Aspekte, wie das unerwünschte Verhalten der Käuferinnen und Käufer, stützen hingegen die gegnerische Position. Das einzige Kommissionsmitglied, das sich zu den Ergebnissen äusserte, schätzt die Ergebnislage einseitig ein. Vielleicht seien Evaluationen mit gegenteiligen Ergebnissen nicht berücksichtigt worden, da sie fehlten. So sei auf der einen Seite die Präsentation einer Fachperson gewesen, die seit Jahren dazu forsche, anerkennt sei und gesagt habe, die Massnahme wirke. Demgegenüber sei das Bauchgefühl gestanden: "Man konnte nicht sagen: «man hat es schon dort eingeführt und es zeigte sich, dass es keine Wirkung entfalten hat » (F3-PM3).

\section{Evaluationsnutzung: Bedeutung}

Inwiefern Evaluationen insgesamt bedeutsam für den Gesetzgebungsprozess waren, beurteilen die befragten Kommissionsmitglieder unterschiedlich. Das Kommissionsmitglied, das sich differenziert mit der Präsentation auseinandersetzte und diese anregte, schreibt ihr auch eine Schlüsselrolle im Gesetzgebungsprozess zu: 
Die entscheidende Aussage aus meiner Sicht war, [...] dass das Bonussystem gerade auf der psychologischen Ebene wirkt. Also, dass es gar nicht so darum geht, was jetzt, wie viel jetzt das auf die Gesamtlebensdauer, Gesamtfahrleistung des Autos betrachtet ist, wie viele Rappen man pro Kilometer spart, was ja dann in einem vernachlässigbaren Bereich ist. (F3-PM3)

Diese Aussage sei notwendig gewesen, um die grundlegende Befürchtung zu entkräften, dass man etwas Neues, Kompliziertes einführe, das nichts bringe. Im Gegensatz dazu betrachten die anderen zwei Befragten die Bedeutung von Evaluationen als gering (F3PM1; F3-PM2). Auch die befragte Person aus der Verwaltung teilt diesen Eindruck der Kommissionsmitglieder.

\subsubsection{Bonus-Malus-System im Berner Grossen Rat (Fall 4)}

\section{Ablauf und Inhalt des Gesetzgebungsprozesses}

Das Steuersystem für energieeffiziente Fahrzeuge wurde im Kanton Bern im Rahmen der Revision des Gesetzes über die Besteuerung der Strassenfahrzeuge (BSFG) diskutiert, mit der sich der Grosse Rat mehrfach in den Jahren 2009, 2010 sowie kurz 2011 befasste. Gemäss der Bezeichnung «Ecotax» war das hauptsächliche Ziel der Revision, die Motorfahrzeugsteuern ökologischer auszugestalten. Dazu sollte neu ein Bonus-Malus-System eingeführt werden. Als zweiten Bestandteil enthielt die Revision des BSFG eine leichte Senkung der Motorfahrzeugsteuern, um diese interkantonal attraktiver zu positionieren (Regierungsrat des Kantons Bern 2009a). Diese Revision war Gegenstand eines insgesamt langen Gesetzgebungsprozesses, da ein Volksvorschlag zustande kam und dessen Abstimmung wiederholt werden musste.

Zwischen 2004 und 2006 regten vier parlamentarische Vorstösse an, das BSFG ökologischer auszugestalten. ${ }^{127}$ Diese Vorstösse beinhalteten alle die Forderung, ein Anreizsystem bei der Besteuerung der Fahrzeuge einzuführen, wobei einzelne Vorstösse lediglich die steuerliche Begünstigung einzelner Fahrzeuge oder Antriebsarten forderten. Der Grosse Rat überwies sämtliche dieser Vorstösse. Zumal die Vorstösse von links bis rechts von Grossräten der SP, FDP und SVP stammten, fanden sie grösstenteils breite Unterstützung im Grossen Rat.

Im Januar 2008 eröffnete der Regierungsrat die Vernehmlassung unter Führung der Polizei- und Militärdirektion (POM) des FDP-Regierungsrats Käser. Um den Entwurf zu erarbeiten, hatte der Regierungsrat davor eine Expertenkommission einberufen. Diese bestand aus je einem Grossratsmitglied der FDP, Grünen, SP und SVP, der Verkehrsverbände sowie der Verwaltung und zeitweise externen Fachpersonen. ${ }^{128} \mathrm{Im}$ Vernehmlassungsentwurf schlug der Regierungsrat vor, dass die Motorfahrzeugsteuern zeitlich

127 Motionen 255/2004, 130/2006, 165/2006 sowie 170/2006.

128 Zusammensetzung der Expertenkommission gemäss Interviewaussagen (F3-Verw.). 
befristet bis zu 80 Prozent vergünstigt oder bis zu 70 Prozent verteuert werden können. Genaueres sollte die Verordnung festlegen. Gemäss dem Entwurf der Verordnung sollten die Motorfahrzeugsteuern neu immatrikulierter Fahrzeuge der Kategorie A während vier Jahren um 80 Prozent ermässigt werden, während sie für neuimmatrikulierte Fahrzeuge der Kategorie F und G unbefristet um 30 respektive 50 Prozent verteuert werden sollten. Dieser Zuschlag zog der Regierungsrat auch für Fahrzeuge der Kategorie E in Erwägung. Ebenso schlug er vor, die Motorfahrzeugsteuern von über 20-jährigen Fahrzeugen um 30 Prozent zu verteuern. Schliesslich beabsichtigte der Regierungsrat, die Motorfahrzeugsteuern für Elektro-Fahrzeuge um 80 Prozent zu ermässigen. Um die Finanzierung zu sichern, sollte die generelle Steuersenkung zudem erst drei Jahre nach dem Bonus-MalusSystem in Kraft treten (Regierungsrat des Kantons Bern 2008c). In ihren Stellungnahmen zur Vernehmlassung begrüssten die Parteien das Bonus-Malus-System grundsätzlich, wobei sich die Grünen für eine Ausdehnung des Malus auf Fahrzeuge der Kategorie E aussprachen, während die SVP den Malus generell ablehnten (Grüne Kanton Bern 2008; SVP Kanton Bern 2008).

Im April 2009 verabschiedete der Regierungsrat dann seinen Antrag zur Änderung des BSFG. Im Gegensatz zur Vernehmlassungsvorlage konkretisierte er die Höhe der Vergünstigungen und Zuschläge, indem er Bandbreiten von 20 Prozentpunkten angab. ${ }^{129}$ Diese Änderung erfolgte aufgrund der Vernehmlassung sowie eines Rechtsgutachtens (Tschannen und Wyss 2008). Die geplante Höhe der Vergünstigungen und Zuschläge in der Verordnung blieb aber unverändert. Nur schlug der Regierungsrat als zweite Variante vor, zusätzlich Fahrzeugen der Kategorie B einen Bonus von 20 Prozent zu geben (Regierungsrat des Kantons Bern 2009a). In den verfügbaren Medienmitteilungen äusserte sich die SVP wiederum kritisch zum Malus (SVP Kanton Bern 2009a). Die FDP schlug vor, eine Übergangsregelung zu schaffen, um einem Abwarten von Fahrzeugkäufen entgegenzuwirken (FDP Kanton Bern 2009a).

Vor den Kommissionsberatungen stellten die POM und eine eingeladene, externe Fachperson das Bonus-Malus-System den Grossratsmitgliedern im Rahmen einer Mittagsveranstaltung vor. Die Sitzung der einberufenen Kommission Gesetz über die Besteuerung der Strassenfahrzeuge (ECOTAX) fand zwei Wochen danach statt. Die Kommission zählte wie üblich 17 Mitglieder, das Präsidium hatte die SVP inne. Die Kommission diskutierte an dieser eintägigen Sitzung über Eintreten und beriet die Vorlage im Detail. Die SP stellte einen Rückweisungsantrag, da die Wirkungen des Bonus-Malus-Systems zu gering seien und die Motorfahrzeugsteuern grundsätzlich geändert werden sollten. Die Kommission lehnte diesen Antrag ab und folgte dem Vorschlag des Regierungsrats zum Bonus-Malus-System.

In der ersten Lesung im Grossen Rat war Eintreten auf die Revision nicht bestritten. Der Grosse Rat diskutierte das Bonus-Malus-System anhand von zwei Minderheitsanträgen der SVP, die verlangten, den Bonus um ein Jahr zu verlängern. Diese Minderheitsanträge lehnte der Grosse Rat klar mit 78.6 beziehungsweise 76.6 Prozent ab. Die SVP stellte keine Minderheitsanträge zur Abschaffung des Malus; sie war diesbezüglich

129 Für die Fahrzeuge der Kategorie A legte er etwa die Höhe der Vergünstigung auf 60 bis 80 Prozent fest. 
aber gespalten. So kritisierte der SVP-Redner den Malus, aber der Kommissionspräsident - ebenfalls ein SVP-Grossrat - setzte sich stark für das Bonus-Malus-System ein und bezeichnete sich als "grosser Fan des Gesetzes» (Grosser Rat des Kantons Bern 2009b, 1289; 1298). ${ }^{130}$ Zusätzlich beschloss der Grosse Rat in der ersten Lesung, eine Übergangsregelung für das Bonus-Malus-System für die zweite Lesung vorzubereiten (Regierungsrat des Kantons Bern 2009b).

Nach diesen Beratungen schlug die Kommission in ihrem Antrag zur zweiten Lesung vor, Fahrzeugen, die in der zweiten Jahreshälfte von 2010 erstmals immatrikuliert wurden, einen Bonus zu gewähren (Regierungsrat des Kantons Bern 2009c). Diesem Antrag stimmte der Grosse Rat in der zweiten Lesung im November 2009 mit 67.4 Prozent der Stimmen zu. In der Schlussabstimmung stimmte eine deutliche Mehrheit von 82.7 Prozent für die Gesetzesänderung. Dagegen stimmten ein fraktionsloses Grossratsmitglied, einzelne Grossratsmitglieder der SVP sowie die Mehrheit der SP. Einzelne Mitglieder der EVP, SP und SVP enthielten sich. Während die SVP-Grossratsmitglieder ihr Votum mit dem Malus begründeten, stimmten die SP-Grossratsmitglieder wegen der Begünstigung der schweren Fahrzeuge dagegen (Grosser Rat des Kantons Bern 2009b, 1298-1299).

Auf diese erste, konsens-geprägte Phase des Gesetzgebungsprozesses folgte mit dem Einreichen eines Volksvorschlags im April 2010 eine zweite, konfliktgeprägte Phase. Dieser Volksvorschlag kam besonders aufgrund der Initiative einer Einzelperson aus dem Automobilgewerbe zustande (Grosser Rat des Kantons Bern 2010b, 1004). Er sah vor, den Bonus für Fahrzeuge der Kategorie A auf 40 Prozent, denjenigen der Fahrzeuge der Kategorie B auf 20 Prozent festzulegen. Der Malus wurde gestrichen. Allerdings zielten der Volksvorschlag und die spätere Diskussion vor allem auf die Steuerhöhe und die Verwendung der Steuergelder ab, da die Steuersenkung von den 5.6 Prozent in der Grossratsvorlage auf 33.3 Prozent im Volksvorschlag erhöht wurde (Regierungsrat des Kantons Bern 2010a).

Die Einreichung des Volksvorschlags führte zu mehrfachen Beratungen im Grossen Rat. Erstens verschob der Grosse Rat die Übergangsregelung und das Inkrafttreten, da die im Gesetz geschriebenen Daten nicht mehr eingehalten werden konnten. Diese Verschiebung bereitete die ständige Justizkommission des Grossen Rats vor, da eine Bestellung einer nichtständigen Kommission zu lange gedauert hätte. Zweitens beriet die wiederum dafür ernannte Kommission Volksvorschlag Motorfahrzeugsteuer und Änderung Gesetz über die Besteuerung der Strassenfahrzeuge den Grossratsbeschluss zum Volksvorschlag. 7 der 17 Kommissionsmitglieder aus der ersten Kommission zur Grossratsvorlage waren auch in dieser zweiten Kommission beteiligt, das Präsidium wechselte aber zur SP. Dies erschien nötig, da der SVP-Grossrat, der die erste Kommission präsidierte, sich zuerst für die Grossratsvorlage und danach für den Volksvorschlag einsetzte (vgl. F4-PM1). Die Kommission beriet den Volksvorschlag im Oktober 2010 in einer zweistündigen Sitzung. Nach Präsentation der Vorlagen, Anhörung des Initiativkomitees und der Beratung stellte

130 Diese Aussage wurde später im Gesetzgebungsprozess und den Interviews oft aufgegriffen (Grosser Rat des Kantons Bern 2010b, 1001). 
sie den Antrag, die Ablehnung des Volksvorschlags zu empfehlen. Der Grosse Rat diskutierte den Grossratsbeschluss in der darauffolgenden Novembersession. Mit 73.9 Prozent der Stimmen sprach er sich für eine Empfehlung auf Ablehnung aus. Nur die SVP stimmte geschlossen dagegen.

Diese nochmalige Beratung des Gesetzes erfolgte durch einen neugewählten Grossen Rat. Nach diesen Wahlen im März 2010 wurde der Grosse Rat bürgerlicher, da die BDP in ihren ersten Wahlen auf 25 Sitze kam, während die SVP nur drei Sitze verlor. Die restlichen grossen Parteien FDP, SP und Grüne verloren Sitze. Auch führten die Wahlen dazu, dass vier Mitglieder der (ersten) Kommission zur Grossratsvorlage nicht mehr im Grossen Rat vertreten waren. Die Zuständigkeit bei der POM hingegen blieb gleich.

Auch nach der Empfehlung des Grossen Rats zur Ablehnung des Volksvorschlags dauerte es noch lange bis zum definitiven politischen Entscheid. In der ersten Volksabstimmung ergab sich sowohl eine Mehrheit für den Volksvorschlag als auch für die Grossratsvorlage, wobei der Volksvorschlag in der Stichfrage 363 Stimmen mehr erlangte. Aufgrund einer Beschwerde verlangte das Verwaltungsgericht Bern die Nachzählung der Stimmen. Weil 29 Gemeinden ihre Stimmzettel nicht mehr auffinden konnten, war eine solche Nachzählung aber nicht möglich und der Regierungsrat beschloss die Wiederholung der Abstimmung. Diese Verzögerungen führten dazu, dass der Grosse Rat das Inkrafttreten und die Übergangsregelung erneut verschieben musste. Gegen den Beschluss zur Wiederholung und diese Verschiebung reichten das Initiativkomitee und die SVP Beschwerde beim Bundesgericht ein. Dieses wies die Beschwerden ab (Staatskanzlei des Kantons Bern 2012, 48). In der Wiederholung der Abstimmung sprachen sich die Stimmberechtigten letztlich für den Volksvorschlag und gegen die Grossratsvorlage aus.

\section{Evaluationsnutzung}

Bei der Diskussion der Vorstösse bezogen sich die Grossratsmitglieder nicht auf Evaluationen zum Steuersystem. Ihre Vorstösse begründeten sie generell mit der Notwendigkeit, den Erdölverbrauch und den Ausstoss von Luftschadstoffen zu verringern, sie verwiesen aber nicht auf Evaluationen. Eine Ausnahme war der Motionär von der SP, der einen Evaluationsbericht ${ }^{131}$ erwähnte, aber sich auf die Gewichtsbesteuerung allgemein und nicht auf das Steuersystem bezog (Grosser Rat des Kantons Bern 2006, 1075). Der Regierungsrat wies hingegen in seiner Antwort zu den vier Motionen von 2006 darauf hin, dass die Motorfahrzeugsteuer gemäss dem Forschungsprojekt der ETH kein wichtiges Kriterium für den Fahrzeugkauf bilde und ein Steuersystem eher als Sensibilisierung wirken könne (Grosser Rat des Kantons Bern 2006, 1080).

Zur Ausarbeitung der Vorlage bezog die Verwaltung Evaluationen, besonders Studien des ETH-Forschungsprojekts, stark ein. Gemäss den Interviewaussagen waren das ETH-Forschungsprojekt und die entsprechenden Fachpersonen durch die Zusammenarbeit mit dem Bund sowie der asa im SVSA bekannt. Seit Beginn der Gesetzgebungs-

131 Hammer und Maibach (1999), der Motionär nannte Autoren aber nicht. 
arbeit in der Expertenkommission hätte das SVSA die Studien berücksichtigt und eine der Fachpersonen beigezogen. ${ }^{132}$ Als die Vorlage für die Vernehmlassung ausgearbeitet worden sei, hätten Regierung und Verwaltung zusätzlich ein Gutachten in Auftrag gegeben (ETH 2008). Die Studien des ETH-Projekts und das Gutachten habe man etwa verwendet, um die Dauer des Bonus und Mitnahmeeffekte abzuschätzen. Zentral gewesen sei demgemäss «vor allem der Ansatz Käuferverhalten, Beeinflussbarkeit von dem, Grenzen der Beeinflussbarkeit und das Quantifizieren der Wirkungen» (F4-Verw.). Dies habe auch dazu geführt, dass man auf eine Evaluation des $\mathrm{CO}_{2}$-Wirkungsziels verzichten wollte. Schliesslich hat das SVSA daneben allgemein Fachpersonen des Bundes in die Expertenkommission eingeladen.

Übereinstimmend mit dieser geschilderten Nutzung enthalten der Bericht zur Vernehmlassung und der Regierungsratsantrag mehrere Verweise auf Evaluationen (Regierungsrat des Kantons Bern 2008c, 2009a). ${ }^{133}$ Mit diesen Verweisen betonte der Regierungsrat einerseits, dass sich die Änderungen auf wissenschaftliche Erkenntnisse sowie Empfehlungen der asa stützen. Anderseits lieferte er damit detailliere Informationen:

Die Eidgenössische Technische Hochschule Zürich (ETH) untersucht seit einigen Jahren mit interdisziplinären, wissenschaftlichen Methoden das Autokaufverhalten in der Schweiz. [...] Dabei wird die Wirksamkeit von fiskalischen Anreizsystemen unter bestimmten Bedingungen bestätigt. Unter anderem wird festgehalten, dass bei der Motorfahrzeugsteuer ein allfälliger Rabatt mindestens 50 Prozent betragen sollte und der Anreiz sofort oder in den ersten drei Jahren nach Neuwagenkauf anfallen und kumulativ CHF 1000.- übersteigen müsste. (Regierungsrat des Kantons Bern 2009a, 5)

Im Gegensatz zum Bericht zur Vernehmlassung enthält der Regierungsantrag zusätzlich Verweise auf das dann verfügbare Gutachten. ${ }^{134}$ Der Regierungsrat verwendete es vor allem, um zu erklären, welche Fahrzeugkategorien einen Bonus respektive Malus erhalten und was die zu erwartenden Wirkungen sind. Weiter begründete er damit, wieso er den Vernehmlassungsentwurf fast nicht veränderte:

Die zeitliche Befristung von Ermässigungen ist im Hinblick auf die mit der Revision vorgesehene Zielsetzung durch verschiedene Studien der Forschungsstelle der ETH treffend begründet. An der Befristung der Vergünstigungen für Fahrzeuge, die dem Effizienzbewertungssystem des Bundes

132 Die befragte Person sprach im Interview allgemein von den «ETH-Studien», besonders erwähnte sie de Haan et al. (2007a), die beiden Gutachten (Ernst Basler Partner 2010; ETH 2008) sowie asa (2007).

133 asa (2007); de Haan et al. (2007a) sowie generelle Hinweise auf das ETH-Forschungsprojekt. Im Regierungsantrag zusätzlich ETH (2008).

134 Auch wurde auf ein Rechtsgutachten verwiesen (Tschannen und Wyss 2008). 
unterliegen, wird festgehalten. (Regierungsrat des Kantons Bern 2009a, 17-18)

Die Dokumente und die Interviews geben nur bedingt Auskunft darüber, inwiefern Grossratsmitglieder und Parteien Evaluationen in dieser vorparlamentarischen Phase berücksichtigten. In der Expertenkommission waren vier Grossratsmitglieder beteiligt, darunter auch ein befragtes Kommissionsmitglied (F4-PM3). Dieses erinnert sich noch an die Präsentation der externen Fachperson sowie die Unterlagen, die sie erhalten hätten. Dies lässt den Schluss zu, dass zumindest diese Parlamentsmitglieder sich bereits in der vorparlamentarischen Phase mit Evaluationen befassten. In den verfügbaren Stellungnahmen zur Vernehmlassung und sonstigen Medienmitteilungen verwies einzig die SP auf die vom ETH-Forschungsprojekt aufgezeigte Wirkung: «Ab einem Bonus ab rund 1000 Franken kann ein solches Anreizsystem durchaus Wirkung entfalten» (SP Kanton Bern 2008, 2). Die Grünen wiesen weiter allgemein auf Statistiken des Erdölverbrauchs für den motorisierten Individualverkehr hin, um die Notwendigkeit von Anreizsystemen aufzuzeigen (Grüne Kanton Bern 2008).

Über das Bonus-Malus-System informierten sich die Kommissionsmitglieder gemäss den Interviewaussagen vor allem an einer Veranstaltung des Grossen Rats und während ihrer Sitzung zur ersten Lesung. Vor der Kommissionssitzung organisierte die POM eine Mittags-veranstaltung, an der sich etwa 60 Grossratsmitglieder beteiligten (F4-Verw.). Zu dieser Mittagsveranstaltung lud sie die beigezogene, externe Fachperson ein, die das Gutachten präsentierte sowie allgemein darlegte, "wie der Käufer tickt» (F4-Verw.). An der Kommissionssitzung zwei Wochen später stellten daher Regierung und Verwaltung das Gutachten nicht mehr vor, Beteiligte aus der Verwaltung stützten sich aber im Laufe der Sitzung bei verschiedenen Fragen auf Evaluationen. ${ }^{135}$

Die Interviews legen nahe, dass sich die Befragten mit diesen Informationen auseinandersetzten. Da sich das eine befragte Kommissionsmitglied auch in der Expertenkommission beteiligt hatte, war es schwierig, zwischen diesen Gremien und Phasen zu trennen. Die Präsentation sowie die "psychologischen Verhaltensstudien» blieben aber in Erinnerung, ebenfalls auch, dass man diese dazu nutzte, um etwa die Dauer des Bonus festzulegen (F4-PM3). Das zweite befragte Kommissionsmitglied, das skeptisch gegenüber dem Bonus-Malus-System war, erinnert sich gut an die Präsentation und das Gutachten, nahm Letzteres aber kritisch auf und befand, es hätte nicht nur eine fachliche, sondern auch eine politische Komponente (F4-PM2). Dies betraf vor allem die Frage der Weiterentwicklung der Energieetikette und der zukünftigen Zuordnung der Fahrzeuge in die Effizienzkategorien. Deswegen setzte sich dieses Kommissionsmitglied selbst mit dem BFE in Kontakt, was die kritische Haltung bestätigt habe. Auch im Plenum wiesen Parlamentsmitglieder auf die Auseinandersetzung mit Evaluationen in der Kommissionsarbeit hin:

135 Sie nahmen Bezug auf de Haan et al. (2007a); ETH (2008), sowie generell auf die ETH. Weiter verwiesen sie auf das Rechtsgutachten von Tschannen und Wyss (2008). 
Die beiden Mitglieder der BDP hatten zunächst eine gewisse Sympathie für diesen Antrag. Aber wir mussten uns von den Experten belehren lassen, dass wir einen Überlegungsfehler machen: Mit einer Ausdehnung der Geltungsdauer der Vergünstigung von drei auf vier Jahre kann man nicht die schadstoffärmeren Autos im Occasionsmarkt entsprechend positionieren. (Grosser Rat des Kantons Bern 2009d, 809)

In den Kommissionsberatungen verwiesen die Grossratsmitglieder vereinzelt auf Evaluationen oder forderten diese. Ein Mitglied stellte die Expertise der ETH in einem Punkt in Frage, wobei dies als politische oder analytische Nutzung interpretiert werden kann. Ein anderes Mitglied forderte, dass die Regierung zur Berichterstattung über die Wirkungen verpflichtet werde. Dieses Kommissionsmitglied reichte aber keinen Antrag für eine Klausel im Gesetz ein, sondern erwähnte die Forderung nochmals zuhanden des Kommissions- sowie später des Plenumsprotokolls (Grosser Rat des Kantons Bern 2009d, 800).

In der ersten Lesung des BSFG im Plenum verwiesen die Grossratsmitglieder mehrfach auf Evaluationen. Ein FDP-Grossrat verdeutlichte die Wirkung des Steuersystems anhand Informationen aus dem Gutachten und dem Regierungsantrag:

Die ETH geht von einer Steuerungswirkung von lediglich 5 Prozent auf die Neuwagenkäufe aus. [...] Es geht also primär darum, ein politisches und psychologisches Zeichen zu setzen, damit beim Kaufentscheid die verbesserte Energieeffizienz eines Fahrzeugs einen kleinen Beitrag leisten kann, um der Umwelt etwas weniger zu schaden. (Grosser Rat des Kantons Bern 2009d, 800)

Weiter begründeten die Grossratsmitglieder ihre Positionen in der Detailberatung mit Evaluationen oder "den Fachleuten» (Grosser Rat des Kantons Bern 2009d, 809). Die Parlamentsmitglieder wiesen neben Evaluationen auf anderes Fachwissen hin, etwa zum Energieverbrauch und Energiesparpotential des Verkehrs. ${ }^{136}$ Schliesslich betonte der Regierungsrat die Evidenzbasierung der Vorlage: «Mehrere Forschungsberichte und ein Gutachten der ETH bestätigen uns, dass dieser Vorschlag nach heutigem Wissensstand die erwünschte Wirkung erzielen wird» (Grosser Rat des Kantons Bern 2009d, 803).

In der zweiten Lesung in der Kommission sowie im Plenum diskutierte der Grosse Rat das Bonus-Malus-System nur am Rande, auch Evaluationen fanden nur vereinzelt Verwendung. Einzig ein Grossratsmitglied verwies auf Evaluationen und eigene Recherchen in den Schlussvoten der zweiten Lesung im Plenum (Grosser Rat des Kantons Bern 2009b, 1293, 1298). Das Protokoll der Kommissionssitzung sowie die verfügbaren

136 Ein SP-Grossratsmitglied verwies etwa auf McKinsey \& Company (2009), der Kommissionspräsident verwies auf die Präsentationen von Fachpersonen im Rahmen des neunten Berner Verkehrstags 2009 (Grosser Rat des Kantons Bern 2009d, 798, 805). 
Medienmitteilungen der Kommissions- und Plenumsphase enthalten keine Verweise auf Evaluationen.

Ob Evaluationen in der ersten nachparlamentarischen Phase Verwendung fanden, ist aufgrund mangelnder Informationen unklar. In dieser Phase sind keine Medienmitteilungen der Parteien oder Informationen aus den Interviews verfügbar. Die Evaluationsnutzung des Referendumskomitees war kein Gegenstand der Fallstudien, es begründete den Volksvorschlag aber vor allem mit Kostenaspekten (Staatskanzlei des Kantons Bern 2011).

Zur Ausarbeitung ihres Antrags zum Grossratsbeschluss zum Volksvorschlag stützten sich Regierung und Verwaltung wieder auf Evaluationen. Sie gaben erneut ein Gutachten in Auftrag, das die Besteuerung und die ökologische Wirkung des Volksvorschlags und der Grossratsvorlage vergleicht (Ernst Basler Partner 2010). Inwiefern dieses Gutachten dem SVSA als Informations- oder Entscheidungsgrundlage diente, lässt sich aus den Dokumenten und Interviews nicht eindeutig schliessen. ${ }^{137}$ Verschiedene Anhaltspunkte sprechen dafür, dass dieses vergleichende Gutachten auch als Überzeugungsmittel dienen sollte. Einerseits kann aufgrund der Ausgestaltung des Steuersystems in den beiden Vorlagen eindeutig ein geringerer ökologischer Effekt angenommen werden. Das vergleichende Gutachten attestierte so der Grossratsvorlage eine vierfach grössere $\mathrm{CO}_{2}$-Reduktion (Ernst Basler Partner 2010,12). Anderseits stützten sich Regierung und Verwaltung im Parlament und in der Abstimmungskampagne stark auf das Gutachten. Im Regierungsratsantrag zum Grossratsbeschluss war dem Gutachten etwa eine Seite gewidmet (Regierungsrat des Kantons Bern 2010a, 9). ${ }^{138}$ Ebenso stellte es die Verwaltung in der Kommission vor. Nach der parlamentarischen Beratung präsentierte dann die beigezogene, externe Fachperson das vergleichende Gutachten an den Medienkonferenzen zur Abstimmung sowie zu deren Wiederholung. Es sei am Ende auch eine finanzpolitisch wichtige Vorlage geworden. Zudem habe die beigezogene Fachperson «auf eine sehr gute Art erklären können» und man habe dies auch für die Diskussion im Kanton nutzen wollen (F4-Verw.).

Die Interviews und die Dokumente weisen darauf hin, dass die Parlaments- und Kommissionsmitglieder Evaluationen in der Beratung des Volksvorschlags wenig berücksichtigten. Die Kommissionsmitglieder erhielten zwar das vergleichende Gutachten und die Verwaltung präsentierte es in der Sitzung, aber nur zwei Kommissionsmitglieder sprachen es kurz an. ${ }^{139}$ Auch kann sich ein befragtes Kommissionsmitglied nicht mehr explizit an Evaluationen in dieser Phase erinnern (F4-PM3). Das andere befragte Kommissionsmitglied gibt dagegen an, das Gutachten gelesen zu haben. Es habe aber der Kommission nicht dazu gedient, die Wirkungen zu erfassen (F4-PM1). Die Kommissionsmitglieder diskutierten in der Sitzung vielmehr über die Verwendung des Ertrags der Motorfahr-

137 Das Interview mit der Verwaltung fokussierte auf die Grossratsvorlage.

138 Daneben enthält der Regierungsratsantrag Verweise zum ersten Gutachten (ETH 2008) sowie zum asaModell.

139 Diese Kommissionsmitglieder verwiesen auf das vergleichende Gutachten, um die Entwicklung auf dem Fahrzeugmarkt zu veranschaulichen und um Steuertarife zu vergleichen. 
zeugsteuer. In der darauffolgenden Beratung des Grossratsbeschlusses im Plenum thematisierten die Grossratsmitglieder daher vor allem die finanzpolitischen Auswirkungen des Volksvorschlags. Der Kommissionspräsident sowie der Regierungsrat erwähnten das vergleichende Gutachten, ohne inhaltlich darauf einzugehen (Grosser Rat des Kantons Bern 2010b, 998, 1004). Weiter sprachen der Kommissionspräsident und Grossratsmitglieder etwa der EVP, FDP, Grünen oder der SP auch die geringe ökologische Komponente des Volksvorschlags an, aber erwähnten das vergleichende Gutachten nicht. In beiden Beratungen zur Verschiebung des Inkrafttretens und der Übergangsbestimmungen verwiesen die Grossratsmitglieder, die Regierung und die Verwaltung zudem weder in der Justizkommission noch im Plenum auf Evaluationen. Schliesslich enthalten auch die verfügbaren Medienmitteilungen der Parteien während dieser zweiten und dritten parlamentarischen Phase keine Hinweise auf Evaluationen.

\section{Evaluationsnutzung: Zeitpunkt und Quellen}

Die Verfügbarkeit der Evaluationen sowie die wichtigsten Ereignisse des Gesetzgebungsprozesses stellt Abbildung 20 dar. Demgemäss waren fast sämtliche Evaluationen und Zusammenfassungen bis zur ersten Sitzung der Kommission vorhanden. Dazu zählt auch die Präsentation der Vorlage, die im Rahmen einer Mittagsveranstaltung stattfand. Vor der Kommissionsberatung des Volksvorschlags am 13. Oktober 2010 erschienen noch zwei weitere Evaluationen, darunter auch das zweite vergleichende Gutachten von Volksvorschlag und Grossratsvorlage. ${ }^{140}$

Von allen verfügbaren Evaluationen verwendeten die Parlamentsmitglieder vor allem die beiden Gutachten (Ernst Basler Partner 2010; ETH 2008) sowie die Präsentation. Die befragten Mitglieder der ersten Kommission zur Gesetzesvorlage erinnern sich an die Präsentation sowie die Fachperson und setzten sich damit auseinander (F4PM2; F4-PM3). Vereinzelt machten sie zusätzlich eigene Recherchen (F4-PM2). Auch das befragte Mitglied, das nur in der zweiten Kommission zum Volksvorschlag beteiligt war, erinnert sich an die Gutachten. Wie Tabelle 18 zeigt, beschränken sich auch die Verweise der Parlamentsmitglieder auf diese drei Quellen. Neben diesen spezifischen Quellen erwähnten die Grossratsmitglieder mehrfach das ETH-Projekt und deren Fachpersonen sowie ihre eigenen Recherchen. Weiter kritisierten sie einzelne Punkte der Evaluationen oder forderten die Regierung auf, die Wirkungen des Steuersystems nach Inkrafttreten $\mathrm{zu}$ evaluieren. Regierung und Verwaltung wiesen dagegen auf diverse Quellen hin und bezogen sich mehrfach allgemein auf die ETH oder das Forschungsprojekt.

Die Parlamentsmitglieder verwendeten diese oben genannten Quellen alle relativ zeitnah nach ihrem Erscheinen. Allerdings zeichnen sich sämtliche der genutzten Informationen dadurch aus, dass sie nicht nur aktuell, sondern spezifisch für den Kanton Bern erstellt wurden. Weder andere Evaluationen, die auch zeitnah erschienen sind, noch Evaluationen, auf welche die Regierung zusätzlich verwiesen hatte, fanden Verwendung im Parlament.

140 Neben dem vergleichenden Gutachten erschien de Haan et al. (2010) in diesem Zeitraum. 


\section{Abbildung 20: Zeitliche Verfügbarkeit der Evaluationen zum Bonus-Malus-System im Berner Grossen Rat}

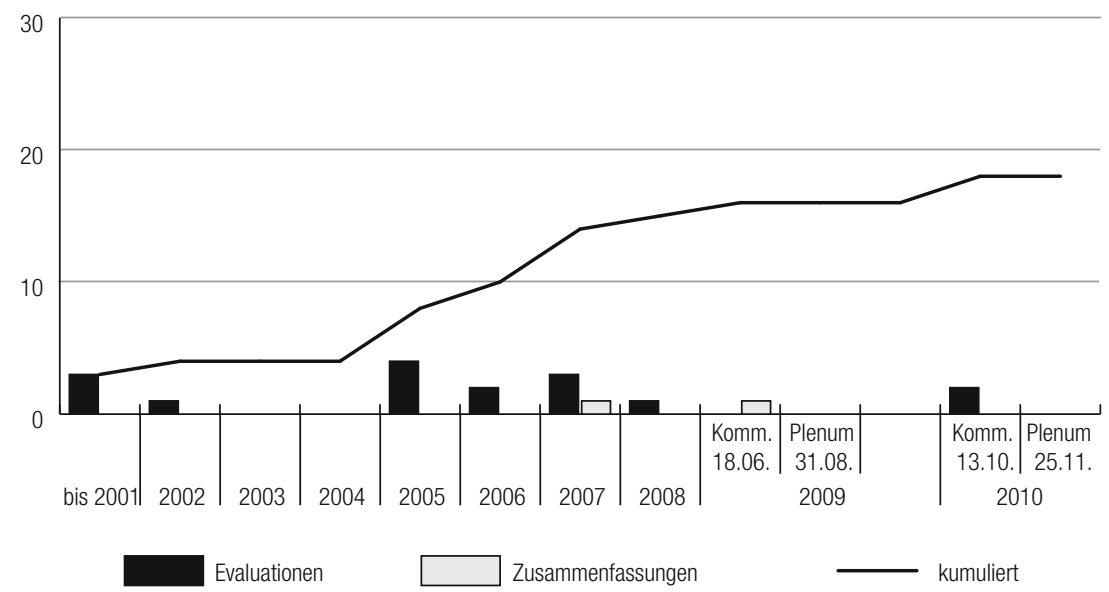

Die Anzahl bezieht sich auf die in einem Jahr beziehungsweise bis zum gegebenen Zeitpunkt erschienenen Evaluationen.

\section{Evaluationsnutzung: Positionen und Argumente}

Gemäss den Evaluationen lassen sich mit Bonus-Malus-Systemen der Energiekonsum und die $\mathrm{CO}_{2}$-Emissionen von Fahrzeugen reduzieren, was insgesamt die Befürworterinnen und Befürworter dieser Massnahme unterstützt. Übereinstimmend mit dieser tendenziell einseitigen Ergebnislage befinden die Befragten die Evaluationen als unterschiedlich überzeugend. Einerseits beurteilt ein befragtes Kommissionsmitglied, das die Massnahme befürwortete, die Grundlagen als gut und sehr wichtig (F4-PM3). Anderseits schätzt das befragte Kommissionsmitglied, das der Massnahme skeptisch gegenüberstand, dass Regierung und Verwaltung sowie das Gutachten die Ergebnisse einseitig darstellten. Die Aussagen zu den Entwicklungen bezüglich der Energieetikette hätten sich nicht mit denjenigen des Bundes gedeckt, zudem habe das Gutachten die negativen Auswirkungen auf das Gewerbe und weniger vermögende Personen nicht erwähnt. Ebenso seien die finanziellen Aspekte widersprüchlich gewesen (F4-PM2). Im Gespräch wurde aber deutlich, dass dieses Kommissionsmitglied den zugrundeliegenden Wirkungszusammenhang nicht in Frage stellte. Vielmehr betrachtete es folglich Rahmenbedingungen zum BonusMalus-System kritisch.

Evaluationen nutzten in Fall 4 sowohl Gegnerinnen und Gegner als auch Befürworterinnen und Befürworter des Bonus-Malus-Systems. Die befragten Kommissionsmitglieder befassten sich unabhängig von ihrer Position mit Evaluationen, auch wenn sie deren Ergebnisse unterschiedlich beurteilten (F4-PM2; F4-PM3, vgl. Absatz zuvor). Weiter hat sich das befragte Kommissionsmitglied, das nur in der zweiten Phase des Gesetzgebungsprozesses in der Kommission sass, weniger differenziert damit auseinandergesetzt (F4PM1). Unabhängig von ihrer Position verwendeten die Grossratsmitglieder auch Evalua- 
Tabelle 18: Bonus-Malus-System im Berner Grossen Rat - politische Nutzung durch Parlament und Exekutive

\begin{tabular}{|c|c|c|c|c|c|}
\hline & & & \begin{tabular}{|l} 
\\
Verweise \\
oder Zitate
\end{tabular} & $\begin{array}{l}\text { ament } \\
\text { andere Arten politi- } \\
\text { scher Nutzung }\end{array}$ & $\begin{array}{l}\text { Regierung/ } \\
\text { Verwaltung } \\
\text { Verweise } \\
\text { oder Zitate }\end{array}$ \\
\hline \multicolumn{6}{|c|}{ Anstoss - vorparlamentarische Phase } \\
\hline $\begin{array}{l}\text { Parlamentsmitglied: } \\
\text { SVP }\end{array}$ & Motion 255/2004 & 19.10.2004 & & & \\
\hline Plenum & Überweisung 255/2004 & 15.06 .2005 & & & \\
\hline $\begin{array}{l}\text { Parlamentsmitglied: } \\
\text { SVP, SP }\end{array}$ & Motion 130, 143/2006 & 06.06 .2006 & & & \\
\hline $\begin{array}{l}\text { Parlamentsmitglied: } \\
\text { FDP, SVP }\end{array}$ & Motion $165,170 / 2006$ & 13.06.2006 & & & \\
\hline Plenum & $\begin{array}{l}\text { Überweisung 130, 143, } \\
165,170 / 2006\end{array}$ & 28.11 .2006 & & & \\
\hline Regierungsrat & Vernehmlassung & 18.01 .2008 & & & $\begin{array}{l}\text { asa (2007), de } \\
\text { Haan et al. (2007a) }\end{array}$ \\
\hline Regierungsrat & Antrag Regierungsrat & 28.04 .2009 & & & $\begin{array}{l}\text { asa (2007), } \\
\text { de Haan et al. } \\
\text { (2007a), } \\
\text { ETH (2008) }\end{array}$ \\
\hline \multicolumn{6}{|c|}{ Parlamentarische Phase I } \\
\hline Kommission & $\begin{array}{l}\text { Beratung (erste Lesung, } \\
\text { Antrag) }\end{array}$ & 18.06.2009 & Präsentation & $\begin{array}{l}\text { Evidenz fordern, } \\
\text { kritisieren }\end{array}$ & $\begin{array}{l}\text { de Haan et al. } \\
\text { (2007a), ETH } \\
(2008)\end{array}$ \\
\hline Plenum & Erste Lesung & 31.08 .2009 & $\begin{array}{l}\text { Präsentation, } \\
\text { ETH (2008) }\end{array}$ & $\begin{array}{l}\text { Evidenz fordern, } \\
\text { Experten fragen }\end{array}$ & $\begin{array}{l}\text { asa (2007), ETH } \\
\text { (2008) }\end{array}$ \\
\hline Kommission & $\begin{array}{l}\text { Beratung (zweite } \\
\text { Lesung, Antrag) }\end{array}$ & 15.10 .2009 & & & \\
\hline Plenum & $\begin{array}{l}\text { Zweite Lesung, } \\
\text { Schlussabstimmung }\end{array}$ & $\begin{array}{l}18.11 .2009- \\
19.11 .2009\end{array}$ & Präsentation & & \\
\hline \multicolumn{6}{|c|}{ (Vor-)Parlamentarische Phase II } \\
\hline Regierungsrat & Antrag Regierungsrat & 11.08 .2010 & & & $\begin{array}{l}\text { asa (2007), Ernst } \\
\text { Basler Partner } \\
\text { (2010) }\end{array}$ \\
\hline Kommission & $\begin{array}{l}\text { Beratung (Antrag Grossrats- } \\
\text { beschluss Volksvorschlag) }\end{array}$ & 13.10 .2010 & $\begin{array}{l}\text { Ernst Basler Partner } \\
\text { (2010) }\end{array}$ & & $\begin{array}{l}\text { Ernst Basler } \\
\text { Partner (2010) }\end{array}$ \\
\hline Plenum & $\begin{array}{l}\text { Lesung Grossratsbeschluss } \\
\text { Volksvorschlag }\end{array}$ & 25.11 .2010 & & & $\begin{array}{l}\text { Ernst Basler } \\
\text { Partner (2010) }\end{array}$ \\
\hline
\end{tabular}

Eingeschlossen sind nur Verweise, die auf eine Quelle schliessen lassen. Allgemeine Formulierungen wie «Evaluationen zeigen» usw. sind nicht in der Tabelle enthalten. 
tionen politisch. In der ersten Phase der Grossratsvorlage bezogen sich die Grossratsmitglieder, die das Bonus-Malus-System befürworteten, meist auf das Gutachten. Zudem forderten Befürworterinnen und Befürworter eine zukünftige Evaluation und kritisierten einzelne Ergebnisse. Die wenigen Mitglieder, die dem Bonus-Malus-System skeptisch gegenüberstanden, verwendeten Evaluationen ebenfalls politisch. So erklärte ein Parlamentsmitglied die Ergebnisse eigener Recherchen und diskutierte die Einflussnahme durch Evaluationen kritisch. In der zweiten Phase des Gesetzgebungsprozesses nach dem Ergreifen des konstruktiven Referendums nutzten die Grossratsmitglieder Evaluationen zwar seltener politisch, es bezogen sich aber beide Seiten auf das dann erschienene vergleichende Gutachten.

\section{Evaluationsnutzung: Bedeutung}

Die befragten Kommissionsmitglieder bewerten die Bedeutung der Evaluationen unterschiedlich für die Phasen vor und nach dem Einreichen des Volksvorschlags. Die befragten Mitglieder der Kommission der Grossratsvorlage beurteilen die Bedeutung als hoch (F4-PM2; F4-PM3). Solche Grundlagen seien sehr stark berücksichtigt worden. Es habe sie gebraucht, um eine Meinung zu bilden (F4-PM2). Äusserungen in der Plenumsdebatte bestätigen dies: ${ }^{141}$

Es ist ein Glücksfall, wenn solche Kräfte an der Quelle einer Gesetzesrevision sind, denn so kann man auch wirklich wissenschaftlich untermauerte Beschlüsse fassen. Es läuft also nicht so, wie es in der Politik oft der Fall ist, nämlich «Handgelenk mal Pi». Vielmehr entsteht hier nun wirklich einmal etwas, das sehr gut begründet und fundiert ist. (Grosser Rat des Kantons Bern 2009d, 801)

Auch aus der Sicht der befragten Person aus der Verwaltung sind das Gutachten und die Präsentation gut bei den Grossratsmitgliedern angekommen. In der zweiten Phase zum Volksvorschlag hingegen sei es nicht mehr um die Wirkung gegangen. Die Befragten schreiben den Evaluationen in dieser Phase daher keine bis kaum Bedeutung zu (F4PM1; F4-PM3). Gerade als die Abstimmung wiederholt werden musste, "ging es gar nicht mehr um die Sache» (F4-PM1).

\subsubsection{Fazit: Zumeist politisch in Zürich, analytisch und politisch bei Beratungsbeginn des Bonus-Malus-Systems in Bern}

Die vorangehenden Abschnitte präsentierten die Fallstudien zum Steuersystem für energieeffiziente Fahrzeuge. Während die Zürcher Kantonsratsmitglieder Evaluationen vereinzelt analytisch und mehrfach politisch nutzten, setzten sich die Berner Grossratsmitglieder besonders in der ersten Phase des Gesetzgebungsprozesses mit Evaluationen auseinander und verwiesen auch darauf.

141 Daneben siehe auch Grosser Rat des Kantons Bern (2009b, 1298). 
In Fall 3 war das Bonussystem Teil der Revision des Verkehrsabgabengesetzes, bei der sich von Anfang an ein konfliktiver Gesetzgebungsprozess abzeichnete. Mehrere Revisionen des Verkehrsabgabengesetzes waren zuvor gescheitert und die SVP kündete früh ihre Bereitschaft an, das Referendum zu ergreifen. Im Vordergrund bei diesem Gesetzgebungsprozess stand daher, eine mehrheitsfähige Vorlage zu finden. Von den grossen Parteien positionierte sich nur die SVP komplett gegen die Gesetzesrevision und das Bonussystem (SVP Kanton Zürich 2009, 2010, 2011). Die Grünen sprachen sich gewissermassen dagegen aus, da das Bonussystem ihnen zu wenig auf Ökologie ausgerichtet war. CVP, FDP und SP befürworteten das Bonussystem und befanden sich daher in einer Mitteposition. Die Mehrheiten für die einzelnen Anträge fielen knapp aus, die Revision nahm der Kantonsrat letztlich an, obschon eine «unheilige Allianz» der linken Parteien AL und Grüne und der rechten Parteien EDU und SVP dagegen stimmten. Letztere ergriffen danach das Behördenreferendum, die Stimmbevölkerung nahm aber die Gesetzesrevision an. Die Befunde zur Evaluationsnutzung in Fall 3 resümiert Tabelle 19.

Das Bonus-Malus-System in Fall 4 diskutierte der Berner Grosse Rat anlässlich der Revision des BSFG, wobei die Diskussion zunächst konsensorientiert und dann konfliktiv geprägt war. In der ersten, konsensorientierten Phase befürworteten sämtliche Parteien einen Bonus und nur Teile der SVP sprachen sich gegen einen Malus aus. Bereits

\section{Tabelle 19: Bonussystem im Kantonsrat Zürich - Übersicht der Nutzung}

Parlament Analytisch: unterschiedlich, von differenzierter Auseinandersetzung zu keiner Erinnerung

Das Kommissionsmitglied, das sich gegen das Bonussystem aussprach, kann sich nicht an die Präsentation der Fachperson erinnern (F3-PM2). Die anderen Kommissionsmitglieder, die das Bonussystem grundsätzlich befürworteten, beurteilen die Präsentation positiv (F3-PM1, F3-PM3). Nur ein Kommissionsmitglied setzte sich aber gemäss den Interviewaussagen vertieft mit der Präsentation auseinander und machte eigene Recherchen (F3-PM3).

Politisch: Verweise auf Evaluationen in allen Phasen, fordern und in Frage stellen von Evaluationen Die Dokumente enthalten zumindest einzelne Verweise auf Evaluationen in allen Phasen des Gesetzgebungsprozesses (asa 2007, Präsentation). Mehrere Kommissionsmitglieder, die das Bonussystem befürworteten, fragten zudem nach Evaluationen oder anderen Daten als Grundlage für die Äusserungen auf der gegnerischen Seite. Weiter forderte die SVP eine Regulierungsfolgeabschätzung zu Beginn der Plenumsdiskussion.

Verwaltung Analytisch: Evaluationen bekannt, nicht darauf gestützt

Politisch: einzelne Verweise auf Evaluationen

Das Interview und die Dokumentenanalyse legen nahe, dass das Strassenverkehrsamt bei der Ausarbeitung des Bonussystems Evaluationen (asa 2007) nur als Leitlinien verwendete. Bezüglich des Bonussystems fragte es keine externen Fachpersonen an. Die Plenumsprotokolle, der Bericht zur Vernehmlassung und der Regierungsantrag enthalten je einen Verweis auf Evaluationen (asa 2007). 
in dieser ersten Phase diskutierte der Grosse Rat in den Beratungen daher intensiver über finanzpolitische Aspekte der Gesetzesrevision des BSFG. Die Minderheitsanträge, die auf eine Lockerung der Bonuskriterien abzielten, lehnte der Grosse Rat klar ab. Er sprach sich auch deutlich - und mit der mehrheitlichen Unterstützung der SVP-Fraktion - für die Gesetzesrevision aus. Das Einreichen eines konstruktiven Referendums markierte den Beginn der zweiten Phase, die deutlich konfliktiver und noch stärker auf Steuerfragen fokussiert war. Der Volksvorschlag sah so vor, die Steuersenkung von den vorgeschlagenen 5.6 Prozent auf 33.3 Prozent zu erhöhen, den Malus zu streichen und den Bonus permanent statt befristet zu vergeben. Die SVP unterstützte als einzige Partei den Volksvorschlag. Der Grosse Rat empfahl relativ deutlich, den Volksvorschlag abzulehnen und die Grossratsvorlage mit dem Bonus-Malus-System anzunehmen. Die Stimmbevölkerung sprach sich aber in der wiederholten Abstimmung letztlich für den Volksvorschlag aus. Die beobachtete Evaluationsnutzung präsentiert Tabelle 20.

\section{Tabelle 20: Bonus-Malus-System im Berner Grossen Rat - Übersicht der Nutzung}

Parlament Analytisch: differenzierte Auseinandersetzung mit Evaluationen bei der Diskussion der Grossratsvorlage, danach kaum relevant

Bei der Diskussion der Grossratsvorlage haben sich die befragten Kommissionsmitglieder (F4-PM2; F4-PM3) gemäss den Interviews differenziert mit dem Gutachten (ETH 2008) und der Präsentation auseinandergesetzt und teilweise eigene Recherchen getätigt. Die Plenumsprotokolle legen nahe, dass sich andere Kommissionsmitglieder ebenso vertieft mit dem Gutachten befassten. Das Gutachten (Ernst Basler Partner 2010), das Volksvorschlag und Grossratsvorlage vergleicht, hat ein befragtes Kommissionsmitglied zwar gelesen, aber es schien wenig relevant (F4-PM1). Das andere befragte Kommissionsmitglied kann sich an diese Phase nicht mehr erinnern (F4-PM3).

Politisch: Verweise auf Evaluationen, vereinzeltes Kritisieren oder Fordern von Evaluationen bei der Diskussion der Grossratsvorlage, danach nur einzelne Verweise auf Evaluationen

In der ersten und zweiten Lesung der Grossratsvorlage verwiesen die Grossratsmitglieder mehrfach auf Evaluationen (Gutachten, ETH 2008, Präsentation, «ETH-Projekt»), vereinzelt taten dies die Parteien in den verfügbaren Medienmitteilungen. Bei der Diskussion der Grossratsvorlage in Kommission und Plenum kritisierten oder forderten die Grossratsmitglieder zudem vereinzelt Evaluationen. In der Kommissions- und Plenumsberatung des Volksvorschlags verwiesen die Grossratsmitglieder nur sporadisch auf Evaluationen (Ernst Basler Partner 2010).

Regierung Analytisch: wissenschaftliche Begleitung während des gesamten Prozesses

und

Verwaltung Politisch: Verweise auf diverse Evaluationen in Unterlagen und Kommissionsberatungen

Gemäss dem Interview und den Dokumenten hat sich das SVSA bei der Ausarbeitung der Grossratsvorlage sehr elaboriert mit Evaluationen auseinandergesetzt und bezog eine externe Fachperson stets mit ein (asa 2007; de Haan et al. 2007a; Ernst Basler Partner 2010; ETH 2008). Weiter enthalten der Bericht zur Vernehmlassung, die beiden Regierungsanträge und die Kommissionsprotokolle viele Verweise auf diese Evaluationen und das «ETH-Forschungsprojekt» generell. 


\subsection{Schulsozialarbeit}

\subsubsection{Hintergrund und Funktionsweise der Schulsozialarbeit}

Die Schulsozialarbeit (SSA) verfolgt gemäss Drilling $(2009,95)$ das Ziel, «Kinder und Jugendliche im Prozess des Erwachsenwerdens zu begleiten, sie bei einer für sie befriedigenden Lebensbewältigung zu unterstützen und ihre Kompetenzen zur Lösung von persönlichen und/oder sozialen Problemen zu fördern». Sie verbreitete sich ausgehend von städtischen Gebieten ab Mitte der 1990er-Jahre in sämtlichen Kantonen der Deutschschweiz. ${ }^{142}$ Das Angebot der SSA konzentriert sich überwiegend auf die Volksschule, wobei die SSA zunächst oft auf Sekundarstufe I eingeführt wurde (Baier 2008; Drilling und Fabian 2010). Eine zentrale Rolle bei der Einführung und Umsetzung der SSA nehmen die Gemeinden ${ }^{143}$ als Trägerinnen der Volksschulen ein. Aus einer Vielzahl von Gründen initiierten meist die Schulen selbst die SSA (Drilling und Fabian 2010; Neuenschwander et al. 2007). Sie hatten etwa Bedarf nach professioneller Unterstützung zur Bewältigung von Problemlagen wie Mobbing, Gewalt, Suchtverhalten sowie familiäre oder leistungsbezogene, schulische Probleme (Müller und Gurny 2004). Darüber hinaus trug auch die Einführung von operativen Schulleitungen, generell präventive Überlegungen sowie der Erfolg der SSA selbst zu deren Verbreitung bei (Baier 2008; Müller 2005; Neuenschwander et al. 2007).

Mit dem Erfolg und der Verbreitung der SSA in den Gemeinden stellte sich in den 2000er-Jahren zunehmend die Frage nach der Rolle des Kantons. Die Gemeinden äusserten so Bedarf an fachlicher Unterstützung beim Aufbau und Bereitstellen der SSA. Weiter sahen sie Bedarf in den Bereichen der Finanzierung sowie der Koordination der SSA mit anderen Fachstellen und Organisationen. Zuweilen wurde auch die rechtliche Verankerung der SSA gefordert, um eine Harmonisierung und Gleichstellung zwischen den Gemeinden zu erreichen (Baier 2008, 100-102; Drilling und Fabian 2010, 156). Diese Entwicklungen fanden vorwiegend in den einzelnen Kantonen statt: Auf interkantonaler Ebene ist nur eine Initiative der Nordwestschweizerischen Erziehungsdirektorenkonferenz (NW EDK) in Form der Erstellung einer Prospektivstudie bekannt (NW EDK 2004). ${ }^{144}$ Zudem erstellte der Schweizerische Berufsverband Soziale Arbeit 2004 erstmals Richtlinien zur SSA (Drilling und Fabian 2010; Müller 2005). ${ }^{145}$

142 In der Romandie und dem Tessin wurde die SSA früher eingeführt, ist aber anders konzipiert (Baier 2008; Drilling und Fabian 2010).

143 Im Kanton Zürich sind die Schulgemeinden, die politischen Gemeinden entsprechen können, für die Volksschule zuständig, Volksschulgesetz des Kantons Zürich vom 7. Februar 2005, Art. 77. Im Kanton Bern sind es die politischen, sog. Einwohnergemeinden oder die gemischten Gemeinden (Einwohnergemeinde und Bürgergemeinde), Volksschulgesetz des Kantons Bern vom 19. März 1992, Art. 5.

144 Im Jahr 2003 waren Bern und Zürich Mitglied der NW EDK (Nordwestschweizerische Erziehungsdirektorenkonferenz 2003). Der Kanton Zürich trat per 2011 aus.

1452010 aktualisierten der 2005 gegründete Verband avenir social sowie der 2006 gegründete SchulsozialarbeiterInnen-Verband (SSAV) diese Richtlinien (avenir social und SSAV 2010). 
Das für die Fallstudie entworfene Wirkungsmodell der SSA illustriert Abbildung 21. Da die Gemeinden die SSA realisieren, enthält das Wirkungsmodell die Gemeindeund die Kantonsebene (vgl. Sager und Rüefli 2005). Die SSA umfasst diverse Leistungen, die sich vier Teilbereichen zuordnen lassen (Müller 2004, 23). Erstens kann die SSA Lernende, Erziehende, Lehrpersonen oder Schulleitungen beraten. Zweitens kann die SSA bei Problemsituationen vermitteln. Zu diesem zweiten Teilbereich der interdisziplinären Fallarbeit gehört auch die Aufgabe der Triage, d.h. den Kontakt zu ausserschulischen Stellen herzustellen, die Fälle zu begleiten und sie an die entsprechenden Stellen zu übergeben. Drittens kann die SSA zur Schulentwicklung beitragen, indem sie Schulprojekte oder Anlässe etwa zum Thema der Gesundheitsförderung gestaltet. Viertens kann die SSA schulbezogene soziokulturelle Animation leisten. Dazu zählt beispielsweise die Organisation von Freizeitangeboten. Die Ausgestaltung bezüglich der vier Teilbereiche ist in der Praxis sehr unterschiedlich. Die Schulen definieren in der Regel die Aufgaben und Strategie der SSA nach Bedarf, wobei dieser auch von der Organisation und Stufe der Schule abhängt. In der Deutschschweiz liegt der Schwerpunkt der SSA generell bei der Beratung und interdisziplinären Fallarbeit (Baier 2008; Drilling und Fabian 2010; Müller 2004; Neuenschwander et al. 2007).

Die SSA richtet sich an diverse Adressatinnen und Adressaten. Hauptsächliche Adressatinnen und Adressaten sind die Lernenden, deren Selbstwahrnehmung, Problemlösekompetenz, Sozialverhalten oder auch Schulkompetenzen die SSA stärken soll (Baier 2008, 108-109; Fabian et al. 2010, 199). Die Erziehenden sind gerade bei Beratungen, der Fallarbeit oder Anlässen wichtige Adressatinnen und Adressaten. Dritte, zentrale Adressatinnen und Adressaten bilden die Lehrpersonen, welche die SSA unterstützen und entlasten soll (Speck und Olk 2010b, 321). Weitere Adressatinnen sind die Schulleitung und ausserschulische Behörden. Für die Schule soll die SSA zur Schulentwicklung und qualität beitragen, während sie für die ausserschulischen Behörden eine Ansprechpartnerin bildet und mit ihnen fallweise zusammenarbeitet (Amt für Jugend und Berufsberatung 2011, 6). Insgesamt sollen diese Wirkungen bei den Adressatinnen und Adressaten zu verschiedenen langfristigen, miteinander zusammenhängenden Effekten führen. Reduzieren sollen sich etwa Konflikte zwischen den Lernenden, Suchtprobleme, Schulabbrüche oder Gefährdungsmeldungen. Damit verbunden sind eine bessere Früherkennung von Problemen und ein angenehmeres Schulklima. Ebenso kann die SSA an der Schnittstelle von Schule und ausserschulischen Behörden die interinstitutionelle Zusammenarbeit verbessern.

Um die Leistungen zu erbringen und Wirkung zu entfalten, benötigt die SSA Ressourcen und muss bei den Adressatinnen und Adressaten bekannt, akzeptiert und in der Schule vernetzt sein. Die Ressourcen können diese letztgenannten Aspekte beeinflussen. So kann die Präsenz und Vernetzung der SSA davon abhängen, wie sie an die Schule angegliedert ist (Baier 2008). Auch bedarf es personeller Ressourcen, um die Qualität der SSA zu sichern und die regionale Zusammenarbeit verschiedener SSA-Stellen zu ermöglichen. Eine mangelnde Präsenz oder Vernetzung der SSA kann etwa dazu führen, dass sich weniger informelle und damit niederschwellige Gelegenheiten zu Austausch und Beratung ergeben und letztlich weniger Anmeldungen erfolgen. Auch kann dies die Wahrneh- 


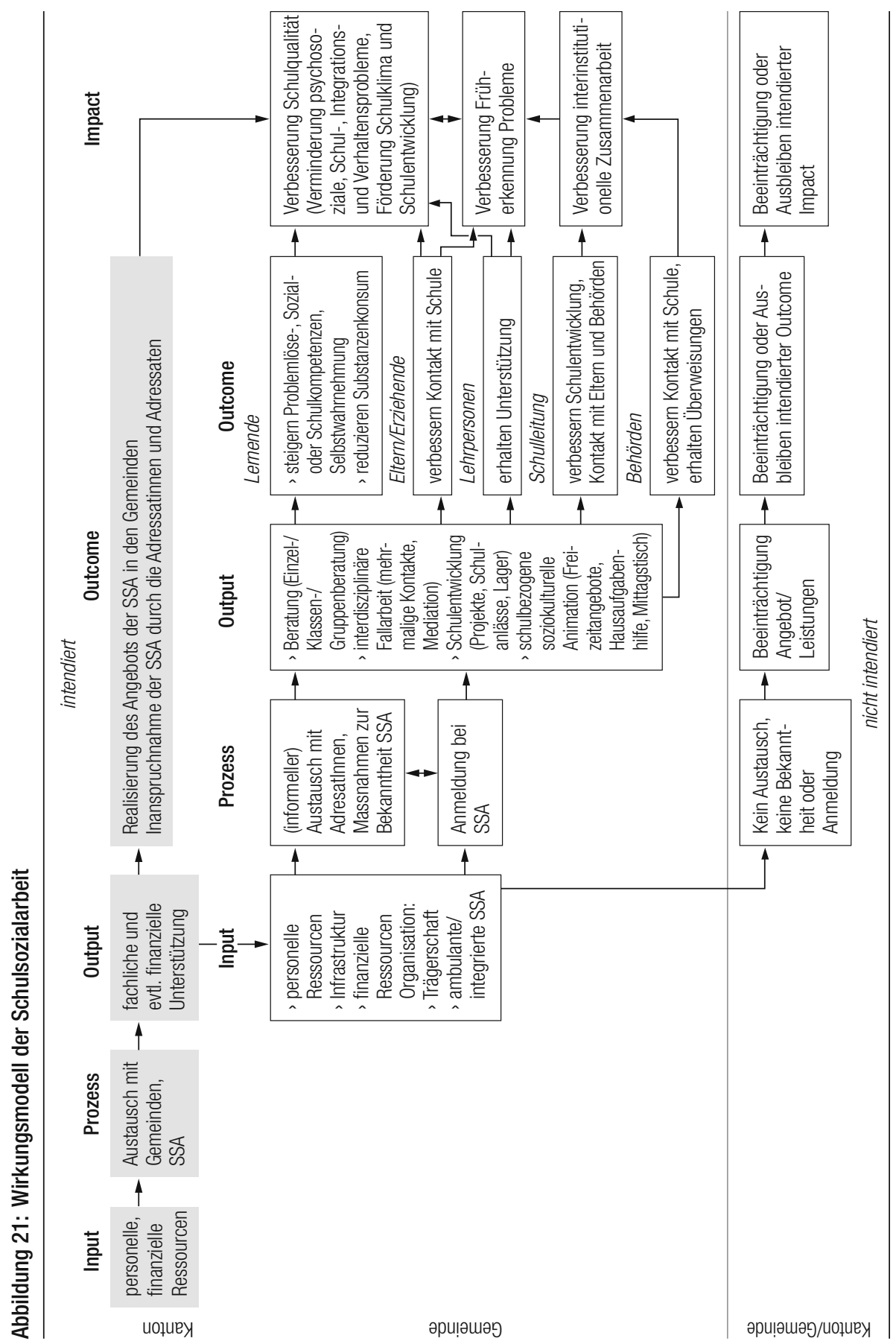


mung der Probleme in der Schule erschweren oder einen Beitrag zur Schulentwicklung hindern (vgl. Baier 2008; Drilling und Fabian 2010).

Der Beitrag der Kantone betrifft diese Bereitstellung der Ressourcen ${ }^{146}$ und Expertise. Wie im Wirkungsmodell illustriert, kann dies für den Kanton als Leistung und demgemäss als Output betrachtet werden, während dies für die Gemeinden den Input darstellt. Indem dadurch möglichst wirksame Leistungen auf Gemeindeebene realisiert werden, profitiert auch der Kanton von den langfristigen Wirkungen.

\subsubsection{Verfügbare Evaluationen zur Schulsozialarbeit}

Während der untersuchten Gesetzgebungsprozesse in Zürich und Bern waren insgesamt 17 Evaluationen zur SSA verfügbar. Zu den Evaluationen kommen acht Zusammenfassungen, die meist über SSA-Projekte in der Deutschschweiz informieren und die zentralen Ergebnisse der Evaluationen dieser Projekte ausführen. ${ }^{147}$ Sämtliche Evaluationen und Zusammenfassungen liegen in Berichtsform vor. Präsentationen fanden in den untersuchten Gesetzgebungsprozessen keine statt. Da der Gesetzgebungsprozess in Zürich rund ein Jahr vor demjenigen in Bern beendet war, standen zudem für Fall 5 nur 13 Evaluationen zur Verfügung.

Die 17 Evaluationen stammen aus insgesamt 11 Evaluationsprojekten. Die Mehrheit dieser Evaluationsprojekte widmet sich der Untersuchung der SSA in einer Gemeinde oder Schule und nehmen dabei ausschliesslich eine begleitende oder Ex-post-Perspektive ein. Die Gemeinden führten die SSA in der Regel für eine Probezeit ein, die Evaluationen sollten diese Einführung begleiten und Entscheidungsgrundlagen für den definitiven Entscheid geben. Ein solches Vorgehen war in der Deutschschweiz sehr verbreitet (Drilling und Fabian 2010, 156; vgl. Drilling 2009). Deshalb existieren weitere Evaluationen von SSA-Projekten in den Kantonen Bern und Zürich, die aber nicht öffentlich zugänglich sind und folglich die vorliegenden Suchkriterien nicht erfüllen. ${ }^{148}$ Eine Minderheit von drei Evaluationsprojekten hat dagegen einen kantonalen Hintergrund. So bestehen je eine Bestandesaufnahme des Angebots der SSA für die Kantone Bern und Zürich. Solche Bestandesaufnahmen fehlten zumindest zu dem Zeitpunkt in anderen Kantonen (Neuenschwander et al. 2007, 36). Ein drittes Evaluationsprojekt widmet sich der Aufgabenteilung von SSA und der offenen Jugendarbeit (Iseli und Pfiffner 2011a, 2011b). Die Evaluationsprojekte in Auftrag gaben oft die Gemeinden oder Kantone. Vereinzelt förderten der Schweizerische Nationalfonds (SNF) oder die Kommission für Technologie und Innovation (KTI) Evaluationsprojekte (vgl. Fabian et al. 2007; Müller 2004). Die Autorenschaft stammt ausschliesslich aus den Fachhochschulen und hatte teilweise wei-

146 In den Fällen 5 und 6 unterscheiden sich die Regelungen zum Beitrag der Kantone, siehe 5.4.3 und 5.4.4.

147 Die SSA war zudem häufig Gegenstand von Abschlussarbeiten, die aber hier nicht eingeschlossen sind (Drilling 2009).

148 Z.B. die Evaluation der SSA in Thun (vgl. Fabian et al. 2010), für einen Überblick zu ersten Evaluationen der SSA siehe Baier 2008. Baier hat nicht namentlich genannt, welche Gemeinden Evaluationen gemacht haben, er gibt aber einen guten Überblick über die Anfänge der Evaluationstätigkeit zur Schulsozialarbeit. Inwiefern diese Evaluationen für die Parlamentsmitglieder zugänglich waren, bleibt offen. 
tere beratende Mandate zur SSA inne (vgl. Erziehungsdirektion des Kantons Bern und Amt für Kindergarten 2008; Fabian et al. 2007).

Die zentralen Ergebnisse zu den Wirkungen der SSA fasst Tabelle 21 zusammen. Ein Grossteil der Evaluationen und Zusammenfassungen beinhalten Informationen zu den Wirkungen der SSA. Auch werden alle in Abbildung 21 postulierten Wirkungszusammenhänge von mehreren Evaluationen oder Zusammenfassungen thematisiert. Dieser umfassende Kenntnisstand wird besonders durch den ähnlichen Aufbau der Evaluationen zu den SSA-Projekten in Gemeinden oder Schulen sowie gleiche Autorenschaft erreicht. Die Evaluationen, die auf der Kantonsebene ansetzen, widmen sich hingegen nur vereinzelt den Wirkungen. Insgesamt verdeutlichen die Ausführungen in Tabelle 21, dass die Evaluationen weitgehend übereinstimmende Ergebnisse zu den Wirkungszusammenhängen feststellen. Nur einzelne Studien widersprechen selten den Resultaten aus allen anderen Evaluationen. ${ }^{149}$ Folglich kann die Übereinstimmung der Ergebnisse als hoch bezeichnet werden.

Im Gegensatz zur hohen Übereinstimmung der Ergebnisse der verfügbaren Evaluationen wird der Kenntnisstand in der Forschung über die SSA kontrovers diskutiert (vgl. Speck und Olk 2010b, 7). Diese Diskussion betrifft besonders die Frage, inwiefern Wirkungen der SSA tatsächlich und mit Indikatoren wie etwa der Gewaltverminderung feststellbar sind (Speck und Olk 2010a, 321). Auch in den für die Fallstudien untersuchten Evaluationen basieren die Aussagen zur Wirkung der SSA meist auf der Einschätzung der beteiligten Personen, die aber die Frage nach Wirkungen nicht oder nur zögerlich beantworten (vgl. Winkelmann und Neuenschwander 2010). Zudem verwenden die Forschenden den Begriff der Wirkungen nur in Anführungszeichen oder sprechen von Wirkpotential (Müller und Willimann 2008; Speck und Olk 2010a). Der Mangel an Erkenntnissen zu objektiven, feststellbaren Wirkungen wird einerseits in der Entwicklung der Evaluation der SSA begründet, die sich zuerst auf die Einführungsphase der SSA fokussierte (Drilling und Fabian 2010). Anderseits sind die Wirkungen der SSA generell schwierig feststellbar, da sie kaum zu isolieren sind. Stellvertretend dafür etwa sind die Ergebnisse zu den relativ beschränkten Wirkungen der SSA bei Lernenden. Wie die Evaluierenden aber ausführen, ist ein solches Ergebnis nicht unbedingt als negativ zu interpretieren, sondern zeigt vielmehr, dass die Zielgruppe der Lernenden mit Belastungen und Problemen erreicht werden und die SSA auch latente Problemsituationen erkennen kann (Fabian et al. 2008, 2010).

\subsubsection{Schulsozialarbeit im Kantonsrat Zürich (Fall 5)}

\section{Ablauf und Inhalt des Gesetzgebungsprozesses}

Die SSA wurde im Kanton Zürich im Rahmen der Totalrevision des Gesetzes über die Jugendhilfe ${ }^{150}$ (Jugendhilfegesetz) diskutiert, mit der sich der Kantonsrat massgeblich zwischen Februar 2010 und März 2011 befasste. Das Gesetz regelt die ambulanten Leis-

149 Ein Beispiel ist die Bewertung der Präsenz oder Erreichbarkeit der SSA durch die Lehrpersonen.

150 Nach der Revision änderte die Bezeichnung zu Kinder- und Jugendhilfegesetz (KJHG). 


\section{Tabelle 21: Evaluationsergebnisse zur Wirkung der Schulsozialarbeit}

\section{Outcome (Evaluationen und Zusammenfassungen, $\mathrm{N}=23$ )}

\section{Lernende}

Besonders psychosozial belastete Lernende nutzen die SSA. Einzelne Befragungen zeigen aber, dass sich ein Drittel aller Lernenden bei Problemen nicht an die SSA wenden würde (Stohler et al. 2009). Lernende, welche die SSA nutzen, betrachten sie als hilfreich. Eine Evaluation weist darauf hin, dass die SSA vor allem hilfreich ist, wenn die Belastung der Lernenden gering und ihre Problemlösefähigkeiten ausgebaut sind. Bei komplexen Problemlagen ist der Beitrag der SSA dagegen beschränkt (Fabian et al. 2010). So reduzieren Lernende, welche die SSA nutzen, gemäss einer anderen Evaluation weder ihren Alkoholkonsum noch berichten sie über ein besseres Wohlbefinden (Fabian et al. 2008).

\section{Erziehende}

Nur wenige Evaluationen widmen sich den Erziehenden. Die befragten Erziehenden kommen nur fallbezogen mit der SSA in Kontakt, sind aber mit dem Angebot zufrieden. Die Auswirkungen auf das Verhältnis zur Schule sind unbekannt.

\section{Lehrpersonen}

Die Lehrpersonen sind zufrieden mit der Zusammenarbeit mit der SSA. Für sie ist die SSA hilfreich und gut erreichbar - in einzelnen Evaluationen bewerten sie die geringe Präsenz jedoch kritisch (Müller und Willimann 2008). Mehrere Evaluationen weisen die zeitlich und besonders die emotionale Entlastung als deutlichste Wirkung der SSA aus. Laut einzelnen Quellen ändern die Lehrpersonen mit der SSA auch ihre Sichtweise auf die Lernenden (Speck und Olk 2010).

\section{Schulleitung}

Die Schulleitungen sind mit der Zusammenarbeit mit der SSA zufrieden. Die SSA entlastet sie, die Art der Entlastung (emotional, zeitlich) bleibt aber unklar. Die Präsenz bewerten die Schulleitungen unterschiedlich. Sie sehen die SSA als vernetzt mit den Behörden an. Inwiefern die SSA auf die Schulentwicklung wirkt, ist nicht bekannt.

\section{Behörden}

Gemäss den Interviews arbeitet die SSA generell gut mit ausserschulischen Stellen oder der Heilpädagogik zusammen und die betreffenden Akteure sehen die SSA als Bereicherung oder Entlastung. In einzelnen Projekten werden aber Schwierigkeiten bei der Absprache mit den Sozialdiensten bei Gefährdungsmeldungen festgestellt.

\section{Impact (Evaluationen und Zusammenfassungen, $\mathrm{N}=15$ )}

Verbesserung Schulqualität (Verminderung psychosoziale, Schul-, Integrations- und Verhaltensprobleme, Förderung Schulklima und Schulentwicklung)

Die SSA trägt gemäss den Lehrpersonen dazu bei, Konflikte zwischen Lernenden frühzeitig und professionell zu entschärfen. Weitreichendere Wirkungen sind nur schwer feststellbar und nicht eindeutig auf die SSA zurückzuführen. Negative Wirkungen der SSA stellen die Evaluationen keine fest.

\section{Verbesserung Früherkennung Probleme}

Nur wenige Evaluationen machen Aussagen zum Effekt der SSA auf die Früherkennung. Die gute Erreichung problembelasteter Lernender, die Sensibilisierung der Lehrpersonen oder die Erkennung latenter Probleme werden aber als Indikatoren für eine verbesserte Früherkennung gesehen.

\section{Verbesserung interinstitutionelle Zusammenarbeit}

Die verschiedenen Stellen arbeiten grundsätzlich gut zusammen. Verschiedene Evaluationen der SSA in einzelnen Gemeinden weisen aber auf Probleme spezifisch bei der Zusammenarbeit mit den Sozialdiensten hin.

Die Tabelle enthält nur Literaturangaben, wenn Ergebnisse auf einzelnen Evaluationen beruhen oder widersprüchlich sind. Bei übereinstimmenden Ergebnissen wird auf Literaturangaben verzichtet. 
tungen der Jugendhilfe, «die der Verbesserung der Entwicklungschancen von Kindern und Jugendlichen und der Entfaltung ihrer sozialen Verhaltensweisen dienen sollen» (Regierungsrat des Kantons Zürich 2008a, 1). Ziel der Totalrevision des Jugendhilfegesetzes war einerseits, neue Leistungen der ambulanten Jugendhilfe - namentlich die SSA und die familienergänzende Betreuung im Vorschulbereich - gesetzlich zu verankern. Anderseits bezweckte die Totalrevision, die Organisation, Strukturen und Finanzierung der ambulanten Jugendhilfe zu klären.

Der Kantonsrat befasste sich vor der in Fall 5 betrachteten Totalrevision des Jugendhilfegesetzes nur am Rande mit der SSA. Er diskutierte die SSA im Jahr 2001 anlässlich des Postulats 324/2000 zweier Kantonsrätinnen der SP. Sie forderten, eine gesetzliche Grundlage zu schaffen, um die Koordinierung von Jugendhilfe und Schule zu klären und bezogen sich dabei im Wesentlichen auf die SSA. Der Kantonsrat lehnte das Postulat aber ab. Explizit für das Postulat sprach sich neben der SP nur die EVP aus. Die Grünen waren gespalten, die FDP und die SVP lehnten das Postulat ab. Im Rahmen der untersuchten Totalrevision behandelte der Kantonsrat danach keine weiteren Vorstösse zur SSA.

Die Jugendhilfe neu zu organisieren und die SSA zu regeln, stand aber ausserhalb des Kantonsrats mehrfach zur Diskussion. Die Reform der Kinder- und Jugendhilfe war bereits ab 1995 Teil eines Projekts der Wirkungsorientierten Verwaltungsführung (wif!). Einen ersten Entwurf einer solchen Reform präsentierte die Bildungsdirektion im Jahr 2000. Dieser Entwurf scheiterte aber am Widerstand der Gemeinden. 2003 ging ein weiterer Entwurf eines umfassenderen Rahmengesetzes zur stationären und ambulanten Kinder- und Jugendhilfe in die Vernehmlassung, fand aber ebenso keine Mehrheit (Amt für Jugend und Berufsberatung 2008; Regierungsrat des Kantons Zürich 2008a). Im Hinblick auf die Umsetzung der Neugestaltung des Finanzausgleichs und der Aufgabenteilung zwischen Bund und Kantonen (NFA) sollte dann das Gesetz über die Finanzierung der Jugendhilfe und Sonderschulung ( $\mathrm{FiG}$ ) ausschliesslich finanzielle Fragen regeln. In der Vernehmlassung dieses Gesetzes im Jahr 2006 übten die Gemeinden wiederum deutlich Kritik. Der Regierungsrat verzichtete in der Folge auf das FiG und gab später der Bildungsdirektion den Auftrag für die Totalrevision (Regierungsrat des Kantons Zürich 2007a).

Geleitet von Bildungsdirektorin Aeppli (SP) ging der Entwurf zur Totalrevision des Jugendhilfegesetzes im Januar 2009 in die Vernehmlassung. Der Vernehmlassungsentwurf sah vor, dass die Gemeinden für ein bedarfsgerechtes Angebot der SSA sorgen, wobei sie die SSA auch mittels Leistungsvereinbarungen über die regionale Jugendhilfestelle des Kantons beziehen können. Weiter schlug der Regierungsrat vor, dass die Bildungsdirektion für die Koordination sowie die Qualitätssicherung zuständig ist, Ausbildungsvoraussetzungen setzt und Bestimmungen zur Erhebung des Bedarfs erlässt (Regierungsrat des Kantons Zürich 2008a, 7). ${ }^{151}$ Mit diesem Entwurf beabsichtigte der Regierungsrat, die bestehende Praxis im Gesetz zu verankern. So gab es bereits ab 2006 eine Projektstelle für SSA im Amt für Jugend und Berufsberatung (AJB) und die Mehrheit der Schulgemein-

151 Diese Zuständigkeiten der Bildungsdirektion betreffen alle Leistungen der ambulanten Jugendhilfe. 
den $^{152}$ hatten die SSA schon eingeführt (Regierungsrat des Kantons Zürich 2008a). Von den Stellungnahmen der Parteien zur Vernehmlassung ist nur jene der SP verfügbar. Sie sprach sich darin für die SSA aus, forderte aber, dass sich der Kanton an der Finanzierung der SSA beteiligt (SP Kanton Zürich 2009a). Gemäss dem Regierungsratsantrag lehnte zudem nur die SVP eine Regelung der SSA ab, während alle anderen grossen Parteien dies befürworteten (Regierungsrat des Kantons Zürich 2009b, 18).

Noch im selben Jahr beschloss der Regierungsrat seinen Antrag zur Totalrevision des Jugendhilfegesetzes. Er hielt bezüglich der SSA grundsätzlich an seinem Vorschlag aus dem Vernehmlassungsentwurf fest. Dies begründete er mit «rechts- und finanzpolitischen Überlegungen» (Regierungsrat des Kantons Zürich 2009b, 22). Der Regierungsrat verzichtete aber darauf, dass die Bildungsdirektion Bestimmungen zur Erhebung des Bedarfs machen kann. Weiter fügte er Übergangsregelungen hinzu, gemäss denen die Gemeinden drei Jahre nach Inkrafttreten der Gesetzesänderungen Angebote der SSA zur Verfügung stellen müssen. Die verfügbaren Medienmitteilungen enthalten keine Informationen zu den Reaktionen der Parteien auf diese Änderungen zur SSA.

$\mathrm{Ab}$ dem 2. Februar 2010 nahm dann die kantonsrätliche Kommission für Bildung und Kultur (KBIK) ihre Beratungen zur Totalrevision des Jugendhilfegesetzes auf. Die KBIK bestand aus 15 Mitgliedern, das Präsidium hatte die SVP inne. Sie beriet die Vorlage in neun Sitzungen. Nachdem die Regierung und Verwaltung die Vorlage in der ersten Sitzung präsentierten und eine klare Mehrheit der KBIK Eintreten beschloss, widmete sich die KBIK während zwei Sitzungen der ersten Lesung der Vorlage. Die drei folgenden Sitzungen befasste sich die KBIK mit den Anhörungen. Eingeladen waren Vertretungen der Bezirksjugendkommissionen, der Bezirkspräsidien, der Gemeindepräsidien, der offenen Jugendarbeit, der Sozialkonferenz, der Schulleitungen und Schulpräsidien sowie auf der operativen Ebene tätige Personen. In den weiteren fünf Sitzungen folgten die zweite Lesung der Vorlage, die Abstimmungen über die Anträge und die Schlussabstimmung. Die SSA war eines der Hauptthemen in der Kommissionsberatung, die KBIK schlug jedoch keine Änderungen zur Regelung der SSA vor.

Nach unbestrittenem Eintreten führte der Kantonsrat die erste Lesung der Vorlage in zwei Sitzungen im Februar 2011 durch. Er diskutierte die SSA anhand von zwei Minderheitsanträgen. Die SVP beantragte, den Gesetzesartikel zur SSA zu streichen. Sie befand, dass es keiner solcher Vorschrift im Gesetz bedürfe, da die Gemeinden die SSA ohnehin selbst bei Bedarf einführen würden. Die EDU stellte als Kompromiss einen Antrag auf eine Kann-Formulierung. Dies sollte den Gemeinden freistellen, SSA zu führen, während sie gemäss dem Kommissionsantrag SSA bei Bedarf zur Verfügung stellen müssen. Die CVP, FDP, Grünliberale, Grüne und SP sprachen sich aber für eine gesetzliche Verankerung der SSA in Form des Kommissionsantrags aus, da die SSA eine zentrale Unterstützung der Schulen bilde. Der Kantonsrat lehnte die Minderheitsanträge daher

1522006 bot ein Drittel aller Schulgemeinden (exkl. Stadt Zürich) SSA an (Hartmann Grass 2007, 2). Im Herbst 2010 waren es 85 Prozent der Sekundar- und 58 Prozent der Primarschulgemeinden, wobei dies auch Projekte in frühen Phasen einschliesst (Amt für Jugend und Berufsberatung 2011, 14). 
mit 64.3 Prozent (SVP) beziehungsweise mit 69.2 Prozent (EDU) ab (Kantonsrat Zürich 2011d, 13577-13583).

Im April 2011 beriet der Kantonsrat die Vorlage in der Redaktionslesung. Zur SSA gab es weder Anträge der Redaktionskommission, noch stellte ein Kantonsratsmitglied Antrag auf Rückkommen. In der Schlussabstimmung sprachen sich 68.1 Prozent der Kantonsratsmitglieder für die Totalrevision des Jugendhilfegesetzes aus. Nur Kantonsratsmitglieder der SVP und der EDU lehnten die Vorlage ab. Die SVP sprach sich gegen die Vorlage aus, da verschiedene Massnahmen - darunter die SSA - die Gemeindeautonomie einschränken würden. Im Gegensatz zu vorherigen Bemerkungen fügte der Redner der SVP an, angesichts der ansonsten breiten Unterstützung auf ein (hoffnungsloses) Referendum zu verzichten. Alle anderen Parteien nahmen das Jugendhilfegesetz an (Kantonsrat Zürich 2011e, 13882-13888). Es wurde kein Referendum gegen die Vorlage ergriffen.

\section{Evaluationsnutzung}

Im Vorfeld der Totalrevision des Jugendhilfegesetzes diskutierte der Kantonsrat die SSA nur anhand eines Vorstosses, Evaluationen flossen dazu aber nicht ein. Ihr Postulat zur Regelung der Kooperation zwischen Schule und Sozialer Arbeit begründeten die Antragstellerinnen mit zunehmenden Unklarheiten betreffend der Zuständigkeiten der verschiedenen Stellen in den Bereichen Familie, Jugendhilfe und Schule. Weder im Vorstosstext noch in der Plenardebatte nutzten sie Evaluationen in ihrer Argumentation. Eine der Antragstellerinnen wies einzig darauf hin, dass Projekte erprobt und Resultate vorliegen würden (Kantonsrat Zürich 2001b, 8669). Da diese Formulierung keine systematische und transparente Vorgehensweise impliziert, zählt sie gemäss dem hier gewählten Analyseverfahren nicht als Evaluationsnutzung. Andere Kantonsratsmitglieder verwiesen ebenso nicht auf Evaluationen während der Plenumsdebatte des Postulats.

Bei der Ausarbeitung der Vorlage stützten sich das AJB und die Bildungsdirektion gemäss den Interviews nicht auf Evaluationen. Die Befragten sehen die SSA generell als weniger relevantes Thema des Gesetzgebungsprozesses an. Es sei bei der SSA lediglich um organisatorische Fragen zu den Zuständigkeiten von Gemeinden und Kanton gegangen (F5/7-Verw.). Auch sei die Wirksamkeit der SSA gar nicht diskutiert worden, da diese «evident» gewesen sei. Alle Akteure hätten gesagt, "die Schulsozialarbeit sei das einzige Entlastende, das die Bildungsdirektion in den letzten 20 Jahren gemacht hat. Es hat damals schon so ein super «Standing» gehabt» (F5-Verw.). Die Befragten weisen gleichzeitig darauf hin, dass sich die Verwaltung zum früheren Zeitpunkt des Aufbaus der SSA mit Evaluationen auseinandersetzte. So entwickelte die Verwaltung das Projekt der SSA mit Hilfe eines Fachbeirates, in dem auch die damalige Hochschule für Soziale Arbeit Zürich und die Pädagogische Hochschule Zürich (PHZH) vertreten waren (vgl. F5-Verw.; Hartmann Grass 2007). Übereinstimmend mit den Aussagen zur Ausarbeitung der Vorlage enthalten die Erläuterungen zur Vernehmlassung keine Verweise auf Evaluationen und auch gar keinen Beschrieb der Wirkungen der SSA (Regierungsrat des Kantons Zürich 2008a, 7). Auch im darauffolgenden Antrag bezog sich der Regierungsrat nicht auf Evaluationen (Regierungsrat des Kantons Zürich 2009b, 16). 
Inwiefern sich die Parteien in ihren Stellungnahmen zur Vernehmlassung oder anderen Medienmitteilungen auf Evaluationen bezogen, kann anhand der Dokumentenanalyse nur bedingt abgeschätzt werden. Es ist einzig eine Stellungnahme zur Vernehmlassung der SP verfügbar sowie eine Medienmitteilung der FDP nach dem Erscheinen des Regierungsratsantrags. In ihrer Stellungnahme verwies die SP nicht auf Evaluationen, die FDP erwähnte die SSA in ihrer Medienmitteilung nicht.

Die KBIK befasste sich in elf Kommissionssitzungen mit der Vorlage und der SSA, die Interviews deuten aber auf nahezu keine Evaluationsnutzung der Kommissionsmitglieder. Die Befragten geben alle an, keine Evaluationen beigezogen zu haben (F5/7PM2-PM5). Zwei Befragten ist aber bekannt, dass Evaluationen auf Gemeindeebene existierten und dass das AJB die Gemeinden in der Anfangsphase mit fachlicher und wissenschaftlicher Begleitung unterstützte (F5/7-PM3; F5/7-PM4). Die Interviewaussagen weisen auf mehrere Gründe, wieso die Befragten Evaluationen nicht nutzten: Erstens hatten die Befragten keinen Bedarf danach, da sie als Lehrperson oder Mitglied der Schulpflege bereits Erfahrungen mit der SSA gemacht hatten: «Ich kenne die Schulsozialarbeit bis ins letzte Detail, weil ich sie [Anm.: als Mitglied der Schulpflege] ja selbst 12 Jahre lang hatte, ich musste mich nicht informieren über irgendeine Evaluation zur SSA» (F5/7-PM3). Zweitens sei es in der Diskussion nicht über «die SSA als Solches gegangen» (F5/7-PM2). Vielmehr sei es darum gegangen, wie viel der Kanton zu Aufgaben der Gemeinden vorschreiben könne. "Welche Wirkung dass es hat, war zu dem Zeitpunkt wie nicht mehr besprochen worden, oder wenig besprochen worden, weil die meisten Gemeinden das eh' schon hatten» (F5/7-PM4). Drittens fügt ein befragtes Kommissionsmitglied an, sich weniger mit der SSA befasst zu haben, da dies vor allem Thema eines anderen Fraktionsmitglieds war (F5/7-PM5).

Die Kommissionsprotokolle enthalten weiter keine Hinweise darauf, dass die Kommissionsmitglieder Evaluationen forderten, diese kritisierten oder darauf verwiesen. Bei der Präsentation der Vorlage und später bei der ersten und zweiten Lesung diskutierte die KBIK vor allem über die Formulierung im Gesetzestext. Anlass zur Diskussion gab dabei vor allem der Begriff «bedarfsgerecht». Bei den Anhörungen fragte die KBIK dann die Vertretungen der Gemeindepräsidien, der Lehrpersonen, Schulleitungen, Schulpflegen sowie die operativ in der Jugendhilfe tätigen Personen zu ihrer Haltung zur SSA. Sämtliche dieser eingeladenen Personen sprachen sich grundsätzlich für die SSA aus. Sie bezogen sich in ihren Stellungnahmen hauptsächlich auf ihre Erfahrungen in der Praxis, auf Evaluationen verwiesen sie nicht. Die Protokolle lassen weiter darauf schliessen, dass einige der eingeladenen Personen bei der Anhörung oder danach zusätzlich Informationsblätter verteilten, diese sind aber nicht in den Kommissionsprotokollen enthalten. Zur Phase der Kommissionsberatungen sind schliesslich keine Medienmitteilungen verfügbar. In der darauffolgenden Debatte im Kantonsrat bezogen sich die Parlamentsmitglieder ebenfalls nicht auf Evaluationen. Wiederum war die SSA durchaus eines der zentralen Themen, auf die Wirkungen der SSA wiesen in der Detailberatung nur Mitglieder der Grünen, der SP und in geringerem Ausmass der EVP hin. Eine Kantonsrätin der SP, die auch das Postulat 324/2000 beantragte, präsentierte Zahlen zur aktuellen Verbreitung der SSA, ohne allerdings einen Hinweis auf den Hintergrund dieser Statistik zu 
geben: «Schulsozialarbeit boomt, boomt sehr. Über zwei Drittel der Gemeinden im Kanton Zürich führen heute Schulsozialarbeit auf mindestens einer Stufe. Genau gesagt sind es 23 Prozent auf der Oberstufe und 50 Prozent auf der Primarschulstufe» (Kantonsrat Zürich 2011d, 13579). Es stellt sich aber die Frage, woher diese Zahlen stammen oder ob sie richtig protokolliert worden sind. Gemäss den Empfehlungen des AJB, die zur selben Zeit wie die Kantonsratsdebatte im Februar 2011 erschienen, boten damals 85 Prozent der Sekundarschulgemeinden und 58 Prozent der Primarschulgemeinden SSA an (Amt für Jugend und Berufsberatung 2011, 14).

In der später stattfindenden Redaktionslesung ist ebenso keine Evaluationsnutzung feststellbar. Die Kantonsratsmitglieder der EVP, FDP, Grünen, der SP und der SVP erwähnten die SSA in ihren Schlussreden, ohne auf Evaluationen zu verweisen. In dieser Phase der Plenumsdiskussion ist zudem nur eine Medienmitteilung der FDP verfügbar. Darin erwähnte sie die SSA, ging aber nicht auf Evaluationen ein.

\section{Evaluationsnutzung: Zeitpunkt und Quellen}

Abbildung 22 illustriert die wichtigsten Daten des Gesetzgebungsprozesses sowie die Erscheinungsdaten der Evaluationen und Zusammenfassungen im Zeitverlauf. Für den Fall 5 sind insgesamt 13 Evaluationen und 8 Zusammenfassungen verfügbar. Dies sind vier Evaluationen weniger als im Fall 6 im Kanton Bern, wobei sich diese vier Evaluationen alle mit dem Kanton Bern befassen. Wie die Abbildung zeigt, war bei der Einreichung des Postulats im Jahr 2000 noch keine Evaluation verfügbar respektive öffentlich zugänglich. ${ }^{153}$ Eine Bestandesaufnahme des Kantons Zürich erschien aber bereits 2004. Bis zur ersten Kommissionssitzung waren dann elf Evaluationen und fünf Zusammenfassungen vorhanden, bis zur ersten Debatte im Plenum standen sämtliche Evaluationen und Zusammenfassungen zur Verfügung. Während der parlamentarischen Beratung erschienen sind auch die Empfehlungen des AJB. ${ }^{154}$

Obwohl verschiedene Evaluationen und Zusammenfassungen zur Verfügung standen und auch im Zeitverlauf neue Quellen dazukamen, haben die Parlamentsmitglieder in Fall 5 keine dieser Quellen verwendet. Die Interviews zeigen, dass zwei befragten Kommissionsmitgliedern zwar Evaluations- oder Beratungstätigkeiten bekannt sind, spezifische Quellen nennen die Befragten aber keine (F5/7-PM3; F5/7-PM4). Die Protokolle, Stellungnahmen und Medienmitteilungen enthalten zudem keine Hinweise auf politische Nutzung in irgendeiner Art: Die Parlamentsmitglieder oder Parteien verwiesen weder auf spezifische Evaluationen oder Zusammenfassungen, noch argumentierten sie allgemein mit Evaluationen oder Studien. Nur zweimal verwies eine Kantonsrätin der SP auf Resultate oder Zahlen zur Verbreitung der SSA, ohne allerdings ein systematisches und transparentes Vorgehen zu implizieren. Zudem forderten keine Parlamentsmitglieder Evaluationen oder kritisierten diese. Die Regierung und Verwaltung verwiesen ebenso nie

153 Es gab etwa die unveröffentlichte Evaluation der SSA Zürich-Limmattal (Drilling 2009, 79).

154 Zwischen Kommissionsberatung und Plenumsdebatte sind daneben die folgenden Evaluationen und Zusammenfassungen erschienen: Drilling und Fabian (2010); Fabian et al. (2010); Speck und Olk (2010a) Winkelmann und Neuenschwander (2010). 


\section{Abbildung 22: Zeitliche Verfügbarkeit der Evaluationen zur Schulsozialarbeit im Kantonsrat Zürich}

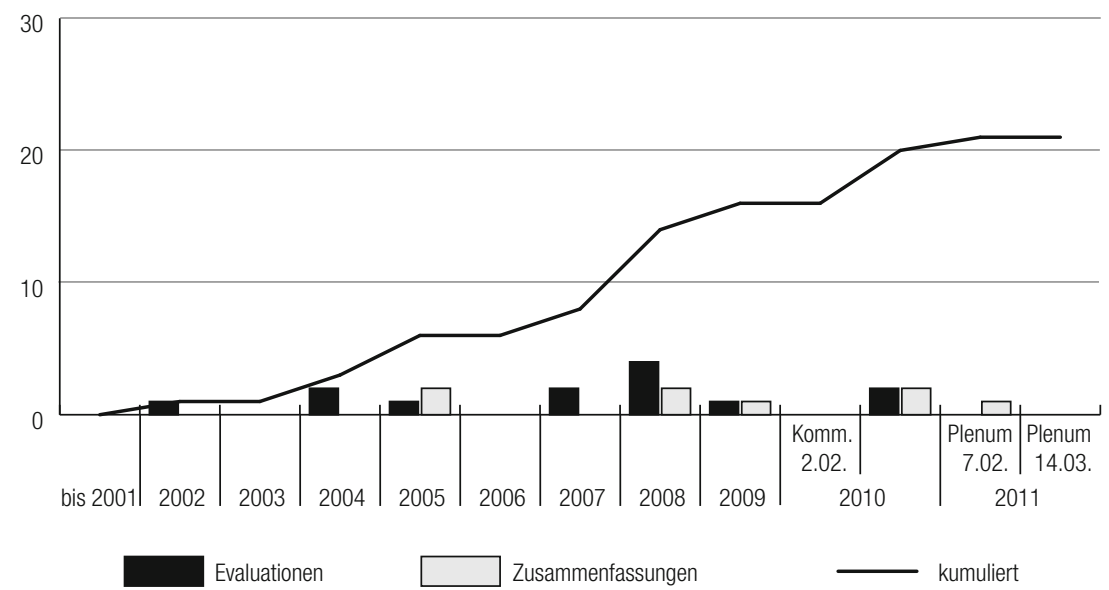

Die Anzahl bezieht sich auf die in einem Jahr beziehungsweise bis zum gegebenen Zeitpunkt erschienenen Evaluationen.

auf Evaluationen. Eine Tabelle zur politischen Nutzung, wie sie in den Studien der Fälle 3 und 4 enthalten ist, wird daher nicht präsentiert.

\section{Evaluationsnutzung: Positionen und Argumente}

Die Parlamentsmitglieder und Parteien nutzten unabhängig von ihrer Position keine Evaluationen. Im Gesetzgebungsprozess selbst setzten sich sämtliche Kommissionsmitglieder nicht mit Evaluationen auseinander und können keine Ergebnisse wiedergeben. Zwei Befragte wissen aber von Evaluationen auf Gemeindeebene oder von wissenschaftlicher Unterstützung für das AJB (F5/7-PM3; F5/7-PM4). Diese beiden Befragten sprachen sich für die SSA aus. Sie waren aber auch beide in ihren Gemeinden für die Volksschule zuständig. Evaluationen politisch verwendet haben weder Befürworterinnen und Befürworter der SSA noch deren Gegnerinnen und Gegner.

Insgesamt unterstützen die übereinstimmenden Evaluationsergebnisse zur SSA deren Befürworter und Befürworterinnen. Die Evaluationen zeigen, dass die SSA ihre Adressatinnen und Adressaten erreicht und diese sie schätzen. Weiter wird aus den Evaluationen deutlich, dass die SSA besonders für die Lehrpersonen, aber auch für die Schulleitungen entlastend wirkt. Die Dokumentenanalyse und die Interviewaussagen legen nahe, dass auch jene Akteure, die sich gegen eine gesetzliche Regelung der SSA aussprachen, nicht an der Wirkung der SSA zweifelten. So fügte der Redner der SVP in seinem Schlussvotum zum Jugendhilfegesetz an: 
Die SVP teilt übrigens bezüglich des Sinns von Kinderkrippen und Schulsozialarbeit die Haltung der meisten anderen Parteien. Wir haben aber offenbar als einzige bürgerliche Partei das Vertrauen, dass es deswegen kein kantonales Gesetz braucht und die Bürgerinnen und Bürger eigenverantwortlich und marktwirtschaftlich handeln. (Kantonsrat Zürich 2011e, 13883)

\section{Evaluationsnutzung: Bedeutung}

Gemäss den Interviews waren Evaluationen im gesamten Gesetzgebungsprozess nicht bedeutsam. Die befragten Parlamentsmitglieder befinden einstimmig, dass Evaluationen kein Thema waren. Auch machen mehrere Befragte darauf aufmerksam, dass die Wirkung der SSA gar nicht zur Diskussion stand (F5/7-PM2; F5/7-PM4). Gleichzeitig bemerkt ein befragtes Kommissionsmitglied, dass der Aufbau der SSA vor allem im bilateralen Austausch zwischen den Gemeinden und dem AJB geschah. Darin eingeschlossen sei auch die wissenschaftliche Begleitung und Unterstützung (F5/7-PM4). Diesen Eindruck bestätigten die Interviews mit Personen in der Verwaltung. Evaluationen und der Einbezug von Fachpersonen habe durchaus eine Bedeutung gehabt beim Aufbau des Projekts SSA. Sie seien wichtig gewesen, «auch als Legitimation, dass das AJB so eine starke Rolle dann übernimmt: Man hat es auch fachlich begründet» (F5-Verw.). Diese Bestrebungen der Fachpersonen und des AJB seien aber «unter dem Radar der Politik gelaufen» (F5/7-Verw.).

\subsubsection{Schulsozialarbeit im Berner Grossen Rat (Fall 6)}

\section{Ablauf und Inhalt des Gesetzgebungsprozesses}

Im Kanton Bern war die SSA ein Element der Teilrevision des Volksschulgesetzes (VSG-BE), die der Grosse Rat zwischen September 2011 und März 2012 diskutierte. Ziel der Revision - auch REVOS 2012 genannt - war es, das VSG-BE an die interkantonale Vereinbarung über die Harmonisierung der obligatorischen Schule (HarmoS) anzupassen und die Bildungsstrategie 2009 umzusetzen. Die SSA zählte zu einer dieser Massnahmen aus der Bildungsstrategie. Als weitere Massnahme aus der Bildungsstrategie umfasste REVOS 2012 die Basisstufe, die Gegenstand des Falls 8 ist (vgl. 5.5). Zur Anpassung an HarmoS beinhaltete REVOS 2012 zudem den obligatorischen zweijährigen Kindergarten, den organisatorischen Beitrag zum Unterricht in heimatlicher Sprache und Kultur, die Grundlage für sprachregionale Lehrpläne sowie die flächendeckende Einführung von Schulsekretariaten.

Die SSA war vor und während des Gesetzgebungsprozesses Gegenstand von insgesamt fünf Vorstössen. ${ }^{155}$ Drei Vorstösse reichten SP-Grossratsmitglieder zwischen 2003 und 2007 ein. Sie sprachen sich für die SSA aus und forderten, die SSA einzuführen. Eine 
befürwortende Position nahm grundsätzlich auch die Interpellation des FDP-Grossrats im Jahr 2007 ein. Er fragte darin unter anderem nach dem weiteren Vorgehen zur SSA, da der Regierungsrat die Regelung der SSA von der Teilrevision des VSG-BE im Jahr 2008 ins Jahr 2012 verschob. Nur die Motion aus dem Jahr 2011 vonseiten der SVP sprach sich gegen die SSA aus. Zwei SVP-Grossratsmitglieder forderten während der Vernehmlassung von REVOS 2012, auf eine flächendeckende Einführung der SSA, deren Finanzierung durch den Kanton sowie deren Regelung im VSG-BE zu verzichten. Diese Motion besprach der Grosse Rat erst nach der ersten Lesung des VSG-BE, weswegen die Antragstellenden sie zurückzogen.

Anfang November 2010 eröffnete der Regierungsrat die Vernehmlassung zu REVOS 2012, die der grüne Regierungsrat Pulver als Vorsteher der Erziehungsdirektion (ERZ) leitete. Der Regierungsrat schlug im Vernehmlassungsentwurf vor, eine Rechtsgrundlage zu schaffen, damit der Kanton Beiträge an die Kosten der Gemeinden für die SSA leisten kann, jedoch nicht muss. Weiter sah der Vernehmlassungsentwurf vor, dass sich der Kanton an bis zu 30 Prozent der Lohnkosten beteiligt, wobei der Regierungsrat über die Mittel beschliesst. Die Verordnung sollte daher weitere Beitragskriterien regeln. Den Bedarf für die Regelung der SSA begründete der Regierungsrat einerseits mit der Zunahme von sozialen Problemlagen und der Entlastung der Lehrpersonen. So hatten 2007 bereits insgesamt 25 meist grössere Gemeinden in Bern die SSA zumindest als Pilotprojekt eingeführt und weitere 23 Gemeinden planten die Einführung der SSA. Anderseits sah der Regierungsrat die SSA als klares Anliegen des Grossen Rats (Regierungsrat des Kantons Bern 2010b, 10 f.). Gemäss den verfügbaren Stellungnahmen begrüsste die FDP den Vernehmlassungsentwurf (FDP Kanton Bern 2011). Die SVP forderte dagegen eine maximale Beteiligung von 20 Prozent der Lohnkosten, während die Grünen und die SP eine verpflichtende und keine Kann-Formulierung verlangten (Grüne Kanton Bern 2011; SP Kanton Bern 2011; SVP Kanton Bern 2011).

Der Regierungsrat verabschiedete seinen Antrag zu REVOS 2012 ein halbes Jahr nach der Vernehmlassung. Darin beantragte er neu einen verpflichtenden Beitrag des Kantons an die Kosten der Gemeinden für die SSA. Dies begründete er mit den Forderungen der SP und den Grünen, aber vor allem auch mit den Forderungen der Gemeinden, die eine gewisse Planungssicherheit benötigten (Regierungsrat des Kantons Bern 2011a, 12, 31). Andere inhaltliche Änderungen nahm der Regierungsrat nicht vor.

Im September 2011 traf sich dann die dafür gegründete Kommission Volksschulgesetz an einer ganztägigen Sitzung zur ersten Lesung der Vorlage. Die Kommission bestand aus 17 Mitgliedern, das Präsidium oblag der EVP. In dieser Sitzung bestritt keine der Parteien Eintreten auf die Vorlage. Die SSA war eines der zentralen Themen dieser Lesung, die gestellten Anträge zur SSA erhielten aber keine Mehrheit und die Kommission nahm keine Änderungen an den Regelungen zur SSA vor.

In der darauffolgenden ersten Lesung im November 2011 war Eintreten auch im Plenum nicht bestritten. Der Grosse Rat diskutierte die SSA auf der Grundlage von drei Anträgen, die alle die Verringerung des Kantonsbeitrags bezweckten. Die SVP stellte zwei Anträge: den Gesetzesartikel zur SSA zu streichen oder, falls dies keine Mehrheit findet, nur höchstens 20 Prozent der Lohnkosten zu bezahlen. Grossräte der FDP und der 
BDP beantragten drittens, die Kann-Formulierung des Vernehmlassungsentwurfs wieder aufzunehmen. Die Antragsteller sahen diese Formulierung als transparenter für die Gemeinden an. So hätten die Gemeinden auch mit einer verpflichtenden Formulierung nur bedingt Planungssicherheit, wenn das Gesetz einen Höchstbeitrag beinhalte (Grosser Rat des Kantons Bern 2011c, 1051). Dieser Antrag vonseiten der FDP erstaunte, da sie sich zuvor in der Kommission gegen eine Kann-Formulierung aussprach. Während der Grosse Rat die beiden Anträge der SVP mit 75.4 Prozent respektive mit 69.7 Prozent deutlich abwies, lehnte er den Antrag der FDP denkbar knapp mit 72 zu 71 Stimmen ab. Diese unterschiedlichen Resultate entstanden aufgrund der Haltung der BDP und der FDP: Sie stimmten grösstenteils für ihren Antrag zur Kann-Formulierung, lehnten aber die Anträge der SVP ab. Die Grünen, EVP, SP und die Fraktion CVP-Grünliberale hingegen sprachen sich gegen sämtliche Anträge aus. Die SVP stimmte sämtlichen Anträgen zu, wobei einzelne Mitglieder gegen die Kann-Formulierung votierten.

Während die SSA dann in der Kommission kein Thema mehr war, diskutierte sie der Grosse Rat in der zweiten Lesung noch einmal anhand der gleichen drei Anträge aus der ersten Lesung. Die Ergebnisse zu den Anträgen der SVP zur Streichung der SSA und der Begrenzung des Kantonsbeitrags an die Lohnkosten fielen mit 76.5 Prozent respektive 68.3 Prozent Ablehnung wieder ähnlich aus. Den Antrag von FDP und BDP zur Kann-Formulierung lehnte der Grosse Rat in der zweiten Lesung aber deutlicher mit 58.6 Prozent ab. Dieses veränderte Ergebnis kam zustande, da die Mehrheit der BDP-Mitglieder dieses Mal gegen die Kann-Formulierung stimmte. Ansonsten blieben die Positionen gleich. In der Schlussabstimmung nahm der Grosse Rat dann die Teilrevision des VSG-BE klar mit 136 zu 4 Stimmen an. Nur einzelne SVP-Grossratsmitglieder stimmten dagegen. Aus dem Schlussvotum einer SVP-Grossrätin wird deutlich, dass Reformen wie die Basisstufe oder die SSA und deren Kostenfolgen der Grund für diese Opposition waren (Grosser Rat des Kantons Bern 2012, 365). Der Stimme enthielten sich Grossratsmitglieder der SVP sowie der SP. Die SP hatte mehrfach betont, dass ihr die Änderungen zu wenig weit reichten (Grosser Rat des Kantons Bern 2012; SP Kanton Bern 2011). Das Referendum wurde nicht ergriffen.

\section{Evaluationsnutzung}

Insgesamt vier parlamentarische Vorstösse sprachen sich vor der Vernehmlassung von REVOS 2012 für die SSA aus, Evaluationen wurden bei drei dieser Vorstösse thematisiert. Die Antragstellenden begründeten ihre Vorstösse mit der Zunahme von neuen, vielfältigen und komplexen sozialen Problemlagen der Lernenden sowie mit der Etablierung der SSA in einigen Berner Gemeinden. In der Plenumsdiskussion der ersten Motion 206/2003 griff die Sprecherin der SP, welche die Motion befürwortete, die «Auswertungen von Pilotprojekten» auf und verwies auf die demnach eindeutigen Wirkungen der SSA (Grosser Rat des Kantons Bern 2004, 402). Der Sprecher der SVP, der die SSA im Gegensatz zur SP als keine kantonale Aufgabe sah, wies zudem auf ein Evaluationsprojekt der SSA in einem Schulkreis der Stadt Bern hin (Grosser Rat des Kantons Bern 2004, 403; von Matt 2002). Mehrfache Evaluationsnutzung lässt sich weiter bei der Motion 
87/2007 feststellen. So wies die Antragstellerin in ihrer Begründung darauf hin, dass Evaluationen vorliegen würden und die SSA daher rasch eingeführt werden könne. Dies bestätigte sie nochmals in ihrer Rede im Plenum:

Gesamtschweizerisch liegen inzwischen genügend wissenschaftliche Studien und Evaluationen vor, welche die Wirksamkeit und den Nutzen der Schulsozialarbeit bestätigen. Wir müssen nicht schon wieder alles selber aufrollen und prüfen, sondern dürfen auf diese Erfahrungen zurückgreifen. (Grosser Rat des Kantons Bern 2007, 1087)

Zusätzlich erläuterte die Antragstellerin die Ergebnisse der Bestandesaufnahme zur SSA in den Gemeinden des Kantons Bern (Neuenschwander et al. 2007). Daraus schloss sie, dass angesichts der in der Studie nachgewiesenen Angebotsvielfalt rasch gehandelt werden soll (Grosser Rat des Kantons Bern 2007, 1087-1088). Bereits vor der Plenumsdiskussion hatte der Regierungsrat die Ergebnisse der Bestandesaufnahme in seiner Antwort zur Motion 87/2007 präsentiert. Umgekehrt schloss er aber aus der Angebotsvielfalt, dass mit einer kantonalen Regelung abgewartet werden müsse. Auf diese Bestandesaufnahme und eine weitere Zusammenarbeit mit dem Bereich der Sozialen Arbeit der Berner Fachhochschule (BFH) wies der Regierungsrat auch schon in seiner Antwort zur Interpellation 30/2007 zwei Monate zuvor hin. Weitere Verweise auf Evaluationen sind während der Diskussion der Vorstösse vor REVOS 2012 nicht beobachtbar. In der Diskussion von 206/2003 verwies die Motionärin aber zusätzlich auf Statistiken der Fachleute (Grosser Rat des Kantons Bern 2004, 401).

Um die Vorlage vorzubereiten, stützte sich die ERZ nur bedingt auf Evaluationen. Zwar hatte sie die Bestandesaufnahme von 2007 in Auftrag gegeben und 2008 zusammen mit den Fachpersonen an der BFH sowie der PH Bern einen Leitfaden erarbeitet, aber die befragte Person aus der Verwaltung thematisiert diese Arbeiten nur am Rande. Es habe eigentlich keine Belege zur Wirksamkeit der SSA gegeben, sondern nur die Evaluationen in den Gemeinden und die kantonale Übersicht zum Stand der Einführung der SSA (F6/8-Verw.). Diese kantonale Übersicht in Form der Bestandesaufnahme hat der Regierungsrat dann auch in der Vernehmlassung erläutert und als Grundlage verwendet, um die finanziellen Auswirkungen der SSA zu berechnen (Regierungsrat des Kantons Bern 2010b, 11, 24). Zudem hat der Regierungsrat (2010b, 11) im Zusammenhang mit der SSA auf zusätzliche Informationen verwiesen. Namentlich verwies er auf den oben genannten Leitfaden zur SSA sowie auf den Bericht zur Strategie "Jugend und Gewalt» (Erziehungsdirektion des Kantons Bern und Amt für Kindergarten 2008; Felber und Erziehungsdirektion des Kantons Bern 2009). Auf die Bestandesaufnahme und diese zusätzlichen Informationen verwies der Regierungsrat (2011a, 11-12) später auch in seinem Regierungsratsantrag.

Weitere Evaluationsverwendung in der Phase bis zu den Kommissionsberatungen ist nur vereinzelt beobachtbar. Die politischen Parteien äusserten sich in den verfügbaren Stellungnahmen zur Vernehmlassung zwar alle zur SSA, bezogen sich aber nicht auf Evaluationen. In den verfügbaren Medienmitteilungen der vorparlamentarischen Phase 
thematisierten nur die Grünen die SSA, ohne auf Evaluationen zu verweisen. Noch während der Vernehmlassung reichte zudem eine SVP-Grossrätin die Motion 4/2011 zum Verzicht der Integration der SSA ins VSG-BE ein. Dies begründete die Motionärin unter anderem mit der Bestandesaufnahme:

Da bei einer Bestandesaufnahme der Schulsozialarbeit im Kanton Bern vom Mai 2007 (von Neuenschwander, Iseli und Stohler) als Hauptgründe der Einführung der Schulsozialarbeit Verhaltensprobleme der Schülerinnen und Schüler sowie die Prävention angegeben wurden, muss das bestehende Beratungs- und Unterstützungsangebot für die Mehrheit der Gemeinden als genügend beurteilt werden Grosser Rat des Kantons Bern. (Grosser Rat des Kantons Bern, 1)

$\mathrm{Zu}$ dieser Motion fand keine Plenumsdiskussion statt, da der Grosse Rat sie erst nach der ersten Lesung der Revision des VSG behandelt hätte.

Gemäss den Interviewaussagen setzte sich die Kommission in ihrer ersten Lesung kaum mit Evaluationen auseinander. Zwei befragte Kommissionsmitglieder, die sich gegen die SSA aussprachen, kennen keine Evaluationen zur SSA (F6/8-PM2; F6/8-PM3). Den anderen Befragten sind Evaluationen zumindest bekannt. So erinnert sich ein Kommissionsmitglied an die Beratungs- und Informationstätigkeiten der BFH in einzelnen Gemeinden und gemäss einem Beschrieb auch an die Bestandesaufnahme (F6/8-PM1). Ein anderes Kommissionsmitglied kennt eine Evaluation in einem Schulhaus (F6/8-PM4). Differenziert damit auseinandergesetzt oder gelesen haben die befragten Kommissionsmitglieder die Evaluationen gemäss ihren Angaben allerdings nicht. Andere Massnahmen, besonders die Basisstufe (vgl. 5.5.1), seien stärker im persönlichen Fokus gestanden (F6/8-PM5). Zudem haben sich die Kommissionsmitglieder bereits informiert gefühlt. Die meisten Leute würden «irgendwie jemanden kennen, der in einer Schule arbeitet, der über die Schulsozialarbeit berichtet hat, oder selbst eine Schulsozialarbeiterin oder einen Schulsozialarbeiter kennen» (F6/8-PM4; vgl. F6/8-PM1). Diese untergeordnete Rolle von Evaluationen spiegelt sich auch im Protokoll der Kommission wider. Bei der Diskussion in der Kommission ging es mehrheitlich um die Kosten und weniger die Wirkungen der SSA. Nur ein Kommissionsmitglied der SVP, das der SSA skeptisch gegenüberstand, verwies auf die Bestandesaufnahme, um die Gründe für eine Einführung der SSA zu illustrieren. Regierung und Verwaltung bezogen sich in der Kommissionssitzung nicht auf Evaluationen.

In der darauffolgenden ersten Lesung im Grossen Rat lässt sich nur ein Verweis auf Evaluationen feststellen, da ein SP-Grossrat aus der Evaluation seiner Gemeinde Uetendorf zitierte:

[Wir hatten] doch den Eindruck, es handle sich wieder um eine neue Aufgabe, die auf uns zukommt, aber nicht sehr viel bringt. Wir haben verlangt, dass nach drei Jahren eine Evaluation durchgeführt wird. [...] Ich lese Ihnen einen kurzen Abschnitt aus dem Evaluationsbericht vor. Dieser 
ist 63 Seiten lang, wer ihn lesen will, dem stelle ich ihn gerne zur Verfügung. "Die Stellungnahmen bei den Lehrpersonen zeigen eine deutliche Entlastung bei den sozialen Fragestellungen und Brennpunkten. Wo Probleme auftreten, können diese kompetent bearbeitet und begleitet werden. Die verbesserte Vernetzung der Schule mit Behörden und Fachstellen zeigt Wirkung. Insbesondere die intensivere Zusammenarbeit mit dem regionalen Sozialdienst und der örtlichen Vormundschaftsbehörde». (Grosser Rat des Kantons Bern 2011c, 1054)

In der Grossratsdiskussion waren generell vor allem die Kosten der SSA, die Aufgabenteilung zwischen Kanton und Gemeinden und die verschiedenen Angebote der Jugendhilfe ein Thema. Der Regierungsrat verwies schliesslich nicht auf Evaluationen.

In den Protokollen der zweiten Lesung in der Kommission sowie im Grossen Rat ist keine Evaluationsnutzung mehr feststellbar. Während sich die Kommission in der zweiten Lesung nicht mehr mit der SSA auseinandersetzte, diskutierte der Grosse Rat in der zweiten Lesung nochmals dieselben Anträge zur SSA wie in der ersten Lesung. Ihre grundsätzliche Haltung zur SSA oder die Wirkung der SSA erwähnten die Grossratsmitglieder aber noch seltener als in der ersten Lesung (Grosser Rat des Kantons Bern 2012, 354f.). Schliesslich thematisiert nur eine Medienmitteilung der SP aus der parlamentarischen Phase die SSA, aber sie enthält keine Verweise auf Evaluationen.

\section{Evaluationsnutzung: Zeitpunkt und Quellen}

Gemäss Abbildung 23 waren bis zur ersten Kommissionssitzung zu REVOS 2012 am 22. September 201116 Evaluationen und sämtliche 8 Zusammenfassungen verfügbar. Nur eine Evaluation, die sich mit der SSA in der Berner Gemeinde Münsingen befasste, wurde noch bis zur ersten Plenumssitzung veröffentlicht. Inwiefern die Evaluationen und Zusammenfassungen, die 2011 erschienen, aber relevant für die Evaluationsnutzung sind, ist fraglich. Der Regierungsrat hatte seinen Antrag bereits Anfang Juni 2011 verabschiedet. Schon deutlich länger verfügbar war zu diesem Zeitpunkt die Bestandesaufnahme für den Kanton Bern, die 2007 erschien (Neuenschwander et al. 2007). Zudem existierte bereits eine Evaluation zu einem Berner Schulkreis, bevor eine Grossrätin der SP 2003 den ersten Vorstoss zur Einführung der SSA einreichte (von Matt 2002).

Diese zwei letztgenannten Berichte - die Bestandesaufnahme der SSA im Kanton Bern und die Evaluation der SSA im Berner Schulkreis Brunnmatt - waren insgesamt die relevantesten Quellen in Fall 6. Die Gespräche lassen einerseits darauf schliessen, dass diese beiden Berichte als einzige Quellen je einem befragten Kommissionsmitglied bekannt sind (F6/8-PM1; F6/8-PM4). Da die Befragten gleichzeitig deutlich machen, dass sie sich nicht mit diesen Quellen auseinandergesetzt haben, sind sie dennoch kaum relevant für die ohnehin begrenzte analytische Nutzung. Anderseits zeigt Tabelle $22 \mathrm{klar}$, dass sich die politische Nutzung ebenso fast ausschliesslich auf diese beiden Quellen konzentriert, wobei die Bestandesaufnahme von Neuenschwander et al. (2007) klar dominiert. Nachdem die Regierung erstmals auf die Bestandesaufnahme verwies, griffen sie die 
Grossratsmitglieder mehrfach auf. Neben der Bestandesaufnahme und der Evaluation der SSA im Berner Schulkreis Brunnmatt durch von Matt (2002) verwies ein Grossrat zudem auf den nicht veröffentlichten Evaluationsbericht der Gemeinde Uetendorf. Zusätzlich haben die Grossratsmitglieder Evaluationen in allgemeinerer Form in ihre Argumentation eingeschlossen. Sie erwähnten etwa "Auswertungen von Pilotprojekten» oder «erste wissenschaftliche Studien und Evaluationen» in der Begründung und Diskussion der parlamentarischen Vorstösse (vgl. Grosser Rat des Kantons Bern 2004, 2007). Da diese allgemeinen Formulierungen aber auf keine spezifische Quelle schliessen lassen, sind sie in der Tabelle nicht aufgeführt. Evaluationen gefordert oder kritisiert haben schliesslich die Grossratsmitglieder nie während des gesamten Gesetzgebungsprozesses.

\section{Abbildung 23: Zeitliche Verfügbarkeit der Evaluationen zur Schulsozialarbeit im Berner Grossen Rat}

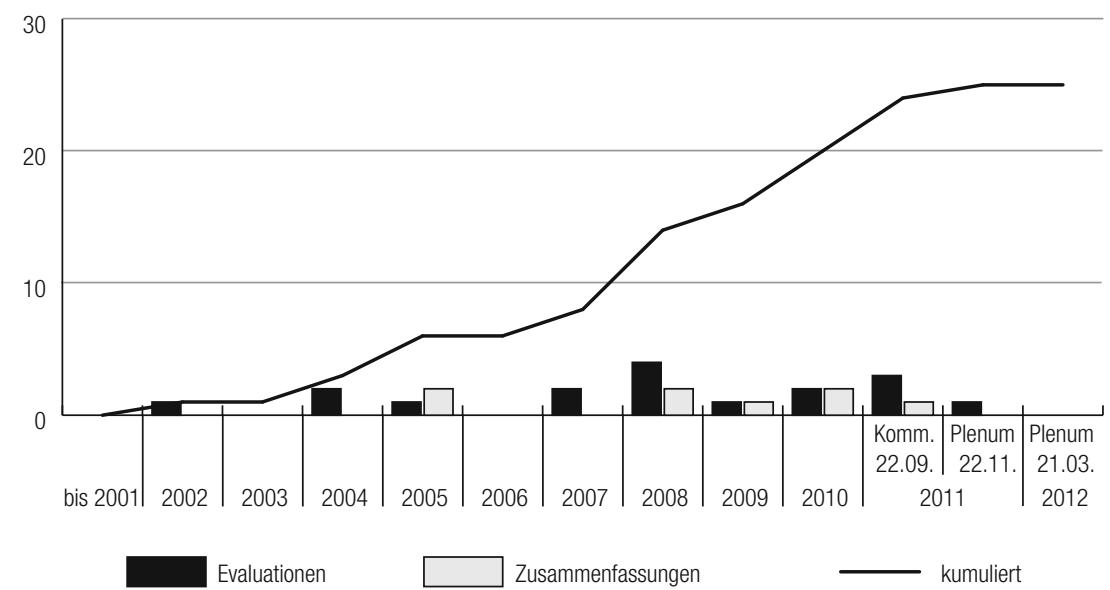

Die Anzahl bezieht sich auf die in einem Jahr beziehungsweise bis zum gegebenen Zeitpunkt erschienenen Evaluationen.

Die politische Nutzung in Fall 6 bezog sich folglich zunächst auf aktuelle Quellen (siehe Tabelle 22). Die Evaluation im Berner Schulkreis Brunnmatt griff ein Grossrat weniger als zwei Jahre nach Erscheinen auf. Die Grossratsmitglieder erwähnten die Bestandesaufnahme ebenfalls erstmals noch in ihrem Erscheinungsjahr 2007, sie verwiesen aber auch noch vier Jahre danach im September 2011 darauf. Zu diesem Zeitpunkt wären aktuellere Evaluationen oder Zusammenfassungen zur Verfügung gestanden, die aber alle keine Verwendung fanden (siehe Abbildung 23). Allerdings fokussierten diese Evaluationen auf einzelne Gemeinden; eine neuere, umfassende kantonale Übersicht analog zu Neuenschwander et al. (2007) gab es nicht. Wie aktuell weiter die Evaluation der Gemeinde Uetendorf zum Zeitpunkt der Nutzung war, kann nur geschätzt werden. Gemäss den Informationen aus der Rede des Grossratsmitglieds ist die Evaluation vermutlich 2010 
und damit ein Jahr vor deren Nutzung erschienen (vgl. Grosser Rat des Kantons Bern 2011c, 1054).

\section{Tabelle 22: $\quad$ Schulsozialarbeit im Berner Grossen Rat - politische Nutzung durch Parlament und Exekutive}

\begin{tabular}{|c|c|c|c|c|}
\hline & & & $\begin{array}{ll} & \text { Parlament } \\
& \\
\text { Verweise } & \text { andere Art politi- } \\
\text { oder Zitate } & \text { scher Nutzung } \\
\end{array}$ & $\begin{array}{l}\text { Regierung/ } \\
\text { Verwaltung } \\
\text { Verweise } \\
\text { oder Zitate }\end{array}$ \\
\hline \multicolumn{3}{|c|}{ Anstoss - vorparlamentarische Phase } & & \\
\hline $\begin{array}{l}\text { Parlamentsmitglied: } \\
\text { SP }\end{array}$ & Motion 206/2003 & 08.09 .2003 & & \\
\hline Plenum & Überweisung 206/2003 & 21.04 .2004 & von Matt (2002) & \\
\hline $\begin{array}{l}\text { Parlamentsmitglied: } \\
\text { FDP }\end{array}$ & Interpellation 030/2007 & 22.01 .2007 & & $\begin{array}{l}\text { Neuenschwander } \\
\text { et al. (2007) }\end{array}$ \\
\hline $\begin{array}{l}\text { Parlamentsmitglied: } \\
\text { SP }\end{array}$ & Motion 13/2007 & 22.01 .2007 & & \\
\hline $\begin{array}{l}\text { Parlamentsmitglied: } \\
\text { SP }\end{array}$ & Motion 87/2007 & 19.03.2007 & & $\begin{array}{l}\text { Neuenschwander } \\
\text { et al. (2007) }\end{array}$ \\
\hline Plenum & $\begin{array}{l}\text { Überweisung und } \\
\text { Abschreibung 13/2007 }\end{array}$ & 13.06.2007 & & \\
\hline Plenum & Überweisung 87/2007 & 13.09.2007 & $\begin{array}{l}\text { Neuenschwander et } \\
\text { al. (2007) }\end{array}$ & \\
\hline Regierungsrat & Vernehmlassung & 01.11 .2010 & & $\begin{array}{l}\text { Neuenschwander } \\
\text { et al. (2007) }\end{array}$ \\
\hline $\begin{array}{l}\text { Parlamentsmitglied: } \\
\text { SVP }\end{array}$ & Motion 4/2011 & 03.01 .2011 & $\begin{array}{l}\text { Neuenschwander et } \\
\text { al. (2007) }\end{array}$ & \\
\hline Regierungsrat & Antrag Regierungsrat & 08.06.2011 & & $\begin{array}{l}\text { Neuenschwander } \\
\text { et al. (2007) }\end{array}$ \\
\hline \multicolumn{5}{|c|}{ Parlamentarische Phase } \\
\hline Kommission & $\begin{array}{l}\text { Beratung (Erste Lesung, } \\
\text { Antrag) }\end{array}$ & 22.09 .2011 & $\begin{array}{l}\text { Neuenschwander et } \\
\text { al. (2007) }\end{array}$ & \\
\hline Plenum & Erste Lesung & $\begin{array}{l}22.11 .2011- \\
23.11 .2011\end{array}$ & Evaluation Uetendorf & \\
\hline Parlamentsmitglied & Rückzug 4/2011 & & & \\
\hline Kommission & $\begin{array}{l}\text { Beratung (Zweite Lesung, } \\
\text { Antrag) }\end{array}$ & 16.02 .2012 & & \\
\hline Plenum & $\begin{array}{l}\text { Zweite Lesung, Schluss- } \\
\text { abstimmung }\end{array}$ & 21.03.2012 & & \\
\hline
\end{tabular}

Eingeschlossen sind nur Verweise, die auf eine Quelle schliessen lassen. Allgemeine Formulierungen wie «Evaluationen zeigen» usw. sind nicht in der Tabelle enthalten. Bei der Evaluation der Schulsozialarbeit in der Gemeinde Uetendorf sind keine näheren Angaben verfügbar. 


\section{Evaluationsnutzung: Positionen und Argumente}

Indem die Evaluationen zeigen, dass die SSA die Erwartungen erfüllt, unterstützen sie insgesamt die Befürworterinnen und Befürworter einer Einführung der SSA. Auch ein befragtes Kommissionsmitglied ${ }^{156}$ meint, die Evaluationen hätten gezeigt, dass die SSA nicht unendlich viel koste, aber etwas bringe und die Lehrkräfte entlaste (F6/8-PM1). Gemäss den Protokollen diskutierten SP-Vertreterinnen gleichzeitig die Messbarkeit der Wirkung der SSA mehrfach kritisch, um das Gegenargument der hohen Kosten der SSA zu entkräften: «Wie bei allen Präventionsmassnahmen sind die positiven Folgen manchmal nicht unmittelbar, und insbesondere nicht sofort in "Cash» messbar» (Grosser Rat des Kantons Bern 2011c, 1052). Weiter relativiert die befragte Person in der Verwaltung die Aussagekraft der Evaluationen. Die Studien attestierten der SSA einfach «eine gewisse Plausibilität, dass sie in gewissen Situationen die Schule in ihrer Arbeit unterstützt» (F6/8-Verw.).

Verwendet haben Evaluationen zumeist Grossratsmitglieder, die eine Einführung der SSA befürworteten. Nur Kommissionsmitgliedern auf dieser Seite waren Evaluationen überhaupt bekannt. Die beiden befragten Kommissionsmitglieder, die sich gegen die SSA aussprachen, haben zwar zumindest teilweise einen Hintergrund im Bildungsbereich, aber sie erinnern sich an keine Evaluationen zur SSA (F6/8-PM2; F6/8-PM3). Politische Nutzung lässt sich zwar auf beiden Seiten, aber deutlich häufiger bei den Befürworterinnen und Befürwortern beobachten. Grossratsmitglieder, die sich für eine Einführung der SSA aussprachen, verwiesen auf Evaluationen im Zusammenhang mit dem Nutzen der SSA. Obwohl verschiedene Parteien die SSA befürworteten und die Evaluationen gemäss den Gesprächen auch Mitgliedern mehrerer Parteien bekannt waren, verwiesen nur Grossratsmitglieder der SP auf Evaluationen. Diese Verwendung fand besonders während der Diskussion der von SP-Grossratsmitgliedern eingereichten Vorstösse statt. Grossratsmitglieder, die eine Einführung der SSA ablehnten, verwiesen hingegen nur vereinzelt und wenig systematisch auf Evaluationen.

\section{Evaluationsnutzung: Bedeutung}

Inwiefern Evaluationen und deren Ergebnisse im Gesetzgebungsprozess bedeutsam waren, beurteilen die Akteure in den Interviews unterschiedlich. Da zwei befragte Kommissionsmitglieder keine Evaluationen kennen, können sie diesen auch keine Bedeutung zuschreiben (F6/8-PM2; F6/8-PM3). Die anderen Befragten wissen von Evaluationen, aber schätzen deren Bedeutung unterschiedlich ein. Zwei Befragte befinden, Evaluationen hätten keine bis wenig Bedeutung gehabt. So sei die SSA zu einem relativ späten Zeitpunkt in das VSG-BE integriert worden. Es sei eigentlich klar gewesen, dass man die SSA «braucht und dass das etwas Gutes ist» (F6/8-PM4). Die Evaluationen zur SSA seien nur «in dem Sinne ein Thema gewesen, als dass die einzelnen Gemeinden, die das hatten, evaluierten und es dort zum Teil Sprecher gab, die dann sagen konnten, bei uns hat es sich bewährt» (F6/8-PM5). Im Gegensatz zu diesen Einschätzungen schreibt ein anderes Kommissionsmitglied den Evaluationen und der Mitwirkung der BFH an den

156 Die anderen Kommissionsmitglieder konnten keine Aussagen zu den Evaluationsergebnissen machen. 
SSA-Projekten eine hohe Bedeutung zu: «Bei der Schulsozialarbeit glaube ich hat eben die BFH [...] eine sehr wichtige Rolle gespielt. Dass man wirklich Beispiele gesehen hat, und ausgewertet hat, es kostet nicht unendlich viel, es bringt etwas, es entlastet die Lehrkräfte» (F6/8-PM1). Die befragte Person in der Verwaltung schätzt die Bedeutung wie die meisten Kommissionsmitglieder hingegen als gering ein. Bei der SSA sei es weniger um die Wirksamkeit, als um die Verteilung der Kosten auf Gemeinden und Kanton gegangen (F6/8-Verw.).

\section{Fazit: Evaluationen zur Schulsozialarbeit bekannt, politische Nutzung in Bern}

Die vorangehenden Abschnitte befassten sich mit den Fallstudien zur Nutzung von Evaluationen im Rahmen der Diskussion der SSA. Die Wirksamkeit der SSA war jedoch in beiden Fällen nicht umstritten. Während Evaluationen lediglich einzelnen Mitgliedern des Zürcher Kantonsrats bekannt waren, nutzen die Berner Grossratsmitglieder Evaluationen vor allem politisch. Dies taten sie besonders bei der Diskussion von Vorstössen, die den Gesetzgebungsprozess der SSA angestossen haben.

In Fall 5 befasste sich der Kantonsrat Zürich während der Totalrevision des Jugendhilfegesetzes mit der SSA. Nachdem mehrere Revisionsversuche in der Vernehmlassung gescheitert waren, sollte eine Totalrevision die Zuständigkeiten im Jugendhilfebereich klären und neue Leistungen der Jugendhilfe - darunter die SSA - aufnehmen. Die SSA gesetzlich zu verankern, war ein Anliegen, das hauptsächlich die Regierung und Verwal-

\section{Tabelle 23: Schulsozialarbeit im Kantonsrat Zürich - Übersicht der Nutzung}

\section{Parlament Analytisch: keine Auseinandersetzung mit Evaluationen, Evaluationstätigkeit teilweise bekannt}

Alle befragten Kommissionsmitglieder geben an, sich bei der Beratung der SSA nicht mit Evaluationen auseinandergesetzt zu haben (F5/7-PM2 bis F5/7-PM5). Zwei Befragte, die sich für die SSA aussprachen, wissen aber, dass es Evaluationen in Gemeinden gab und die SSA-Projekte wissenschaftlich begleitet wurden (F5/7-PM3; F5/7-PM4). Sie kennen jedoch keine spezifische Evaluation.

Politisch: keine Verweise auf Evaluationen oder Nutzung in anderen politischen Arten

Die Parlamentsmitglieder verwiesen weder auf «Evaluationen» oder «Studien» in allgemeiner Form, noch bezogen sie sich auf spezifische Quellen in allen Phasen des Gesetzgebungsprozesses. Auch forderten die Parlamentsmitglieder keine Evaluationen und kritisierten sie auch nicht.

Regierung Analytisch: wissenschaftliche Unterstützung in der Anfangsphase der SSA

und

Verwaltung Politisch: keine Verweise auf Evaluationen

Das AJB stützte sich zwar in der Anfangsphase des SSA-Projekts auf Evaluationen und besonders auf die Expertise verschiedener Fachpersonen, für die Ausarbeitung des revidierten Jugendhilfegesetzes waren Evaluationen gemäss den Interviews aber nicht relevant (F5-Verw.; vgl. F5/7-Verw.). Regierung und Verwaltung verwiesen nie auf Evaluationen in allen untersuchten Dokumenten. 
tung vorantrieben. Die Mehrheit der Parteien begrüsste dennoch diese gesetzliche Verankerung der SSA. Als einzige grosse Partei lehnte die SVP die gesetzliche Regelung ab. Sie sprach sich aber nicht gegen die Wirkung der SSA aus, sondern sah die Autonomie der Gemeinden als gefährdet. Der Kantonsrat beriet die SSA anhand zwei Minderheitsanträge, die er aber beide relativ klar ablehnte. Das Jugendhilfegesetz nahm er ebenso relativ deutlich an. In den Kantonsratsdebatten drohte die SVP zwar mit einem Referendum, sie sah aber letztlich davon ab. Informationen zur Evaluationsnutzung in Fall 5 gibt Tabelle 23.

In Fall 6 behandelte der Berner Grosse Rat die SSA im Rahmen der Revision des VSG-BE. Die SSA war neben der Basisstufe eines der Kernthemen dieser Gesetzesrevision (siehe Fall 8). Die Verankerung der SSA im kantonalen Gesetz initiierte im Wesentlichen der Grosse Rat: Mehrere parlamentarische Vorstösse seit 2003 forderten, die SSA einzuführen. Mit Ausnahme der SVP befürworteten sämtliche Parteien grundsätzlich die SSA. Die Minderheitsanträge, die der Grosse Rat in beiden Lesungen behandelte, waren dennoch teilweise sehr umstritten. Die Grossratsmitglieder betonten in ihren Reden jedoch, dass es weniger um die Wirksamkeit der SSA als um Kostenfragen ging. Letztlich

\section{Tabelle 24: Schulsozialarbeit im Berner Grossen Rat - Übersicht der Nutzung}

Parlament Analytisch: keine Auseinandersetzung, Evaluationen teilweise bekannt

Die zwei befragten Kommissionsmitglieder, die sich gegen die SSA aussprachen, kennen gemäss ihren Angaben keine Evaluationen zur SSA (F6/8-PM2; F6/8-PM3). Die anderen drei befragten Kommissionsmitgliedern, welche die SSA befürworteten, wissen hingegen, dass Evaluationen existierten (F6/8-PM1; F6/8-PM4; F6-8/PM5). Explizit erwähnen sie von Matt (2002) und Neuenschwander et al. (2007). Alle befragten Kommissionsmitglieder haben jedoch Evaluationen während des Gesetzgebungsprozesses nicht berücksichtigt.

Politisch: Verweise auf Evaluationen besonders während der Diskussion der Vorstösse, keine Nutzung in anderen Arten

Die Parlamentsmitglieder verwiesen in allen Phasen auf Evaluationen. Dies taten sie besonders häufig im Rahmen der Diskussion der Vorstösse, die noch vor der Beratung des VSG-BE stattfand. Neben Neuenschwander et al. (2007) verwiesen sie allgemein auf «wissenschaftliche Studien» oder «Auswertungen». Besonders oft verwiesen SP-Grossratsmitglieder, die sich für die SSA aussprachen, auf Evaluationen. In einzelnen Fällen taten dies auch Grossratsmitglieder der SVP auf der gegnerischen Seite. Ein Fordern oder Kritisieren von Evaluationen ist dagegen nicht beobachtbar.

Regierung Analytisch: wissenschaftliche Begleitung vor dem Gesetzgebungsprozess

und

Verwaltung Politisch: Verweise auf Evaluationen in vorparlamentarischer Phase

Die ERZ gab Evaluationen in Auftrag (Neuenschwander et al. 2007) und arbeitete mit der BFH und PH Bern, gemäss dem Interview waren aber Evaluationen kaum relevant bei der Ausarbeitung des VSG-BE (F6/8Verw.). In Antworten auf die parlamentarischen Vorstösse, in den Vernehmlassungsunterlagen und in ihrem Antrag verwies die Regierung aber mehrfach auf Neuenschwander et al. (2007). 
lehnte der Grosse Rat sämtliche Minderheitsanträge teilweise knapp ab. Die Revision des VSG-BE nahm der Grosse Rat dagegen fast einstimmig an, ein Referendum wurde nicht eingereicht. Einen Überblick über die Evaluationsnutzung in Fall 6 gibt Tabelle 24.

\subsection{Basis- respektive Grundstufe}

\subsubsection{Hintergrund und Funktionsweise der Basis- respektive Grundstufe}

Eine Neugestaltung des Kindergartens und der ersten Schuljahre wurde in den Kantonen ab Ende der 1990er-Jahre diskutiert. Anlass dazu gab die Beobachtung, dass der Übergang vom Kindergarten in die Schule oftmals einen Einschnitt für die Kinder bedeutet. Eingeflossen in die Diskussion sind ebenso die Forschungserkenntnisse zur Relevanz der frühen Förderung sowie die Befunde der PISA-Studien, die eine hohe Chancenungleichheit offenbarten. Auch wurde festgestellt, dass sich die Kinder bezüglich ihres Lern- und Entwicklungsstands bei Schulbeginn zunehmend stärker unterscheiden (EDK-Ost 2005, 2010). Angesichts dieser Beobachtungen gab die Schweizerische Konferenz der kantonalen Erziehungsdirektoren (EDK) Ende der 1990er-Jahre zwei Studien in Auftrag, die eine mögliche pädagogische und organisatorische Neugestaltung der Eintrittsstufe zum Gegenstand hatten (EDK 1997; EDK 1999). Basierend auf diesen beiden Studien präsentierte sie im Jahr 2000 Rahmenanforderungen der Basisstufe als solche Neugestaltung und empfahl den Kantonen, sich damit auseinanderzusetzen (EDK 2000, 2-3).

Parallel zu diesen gesamtschweizerischen Bemühungen wurde die Basisstufe auch in einzelnen Regionen und Kantonen diskutiert. Einerseits beabsichtigte der Kanton Zürich, das mit der Basisstufe verwandte Modell der Grundstufe flächendeckend einzuführen, die Stimmberechtigten lehnten dies im November 2002 aber knapp ab (Regierungsrat des Kantons Zürich 2003). ${ }^{157}$ Anderseits lancierte die Erziehungsdirektoren-Konferenz der Ostschweizer Kantone und des Fürstentums Liechtenstein (EDK-Ost) im Mai 2002 das Entwicklungsprojekt «Erziehung und Bildung in Kindergarten und Unterstufe im Rahmen der EDK-Ost». Im Rahmen dieses so genannten Projekts EDK-Ost 4bis8 sollten pädagogische Grundlagen für die Basis- und Grundstufe geschaffen und diese in Schulversuchen erprobt werden. Weiter sollte das Projekt von einer Evaluation begleitet werden. Kurz nach Beginn öffnete die EDK-Ost das Projekt für andere Kantone, so dass ab 2006 sämtliche deutsch- und mehrsprachigen Kantone sowie das Fürstentum Liechtenstein daran teilnahmen. Schulversuche starteten ab dem Schuljahr 2003/04. Insgesamt beteiligten sich 400 Lehrpersonen in 106 Grundstufen- und 64 Basisstufenklassen mit circa 3000 Kindern am Projekt (EDK-Ost 2010, 17-23). Diese Dimensionen machten die bildungspolitische Zusammenarbeit zwischen den Kantonen im Rahmen von EDK-Ost 4bis8 einzigartig (vgl. auch Grossenbacher 2008, 7; Stamm 2009, 79). EDKOst 4 bis8 erfolgte aber auch verbunden mit anderen Entwicklungen der schweizerischen Bildungspolitik, wie dem Leitsatz der integrativen Förderung sowie der Einführung des

157 Siehe Kapitel 5.5.3. 
obligatorischen, zweijährigen Kindergartens durch das HarmoS-Konkordat ${ }^{158}$ (Grossenbacher 2008, 3-4; Stamm 2009).

Abbildung 24 zeigt das für die Fallstudien entworfene Wirkungsmodell der Basis- respektive Grundstufe. Auch dieses Wirkungsmodell umfasst die Kantons- und die Gemeindeebene, da die Gemeinden als Trägerinnen der Volksschulen die Basis- oder Grundstufe anbieten. Das Modell der Grundstufe fasst die ersten beiden Kindergartenjahre und das erste Primarschuljahr zusammen, während die Basisstufe zusätzlich das zweite Primarschuljahr umfasst. Um diese Modelle zu realisieren, müssen jahrgangsgemischte Klassen gebildet werden. Der Schuleintritt erfolgt ab dem vollendeten vierten Altersjahr. Die Grundstufe kann gemäss dem individuellen Lerntempo in zwei bis vier Jahren durchlaufen werden; in der Basisstufe sind dies drei bis fünf Jahre. Da nach der Basis- oder Grundstufe die ersten Entscheide der Schullaufbahn folgen, ist auch der Einbezug der Erziehenden wesentlich (Projektkommission 4bis8 der EDK-Ost und Partnerkantone 2007, 43-44). Die Basisoder Grundstufe wird im Teamteaching von einer Lehrperson des Kindergartens sowie einer Lehrperson der Unterstufe unterrichtet. Daneben erhalten die Lehrpersonen zusätzliche heilpädagogische Unterstützung (EDK-Ost 2010, 22). Neben den personellen Ressourcen bedarf es Räumlichkeiten, die sich eignen, um homogene Lern- und Entwicklungsgruppen zu bilden (Projektkommission 4bis8 der EDK-Ost und Partnerkantone 2007, 10).

Als Bildungsangebot soll die Basis- oder Grundstufe primär auf die Adressatinnen und Adressaten der Kinder wirken. Die Basis- oder Grundstufe soll den Kindern erlauben, die Kulturtechniken intrinsisch und nach ihrem individuellen Entwicklungsstand zu erlernen. So können die Kinder fliessend vom spielerischen Lernen des Kindergartens zum aufgabenorientierten Lernen der Unterstufe übergehen (EDK-Ost 2010, 20). Als nicht beabsichtigte Wirkung könnte die Bildung altersdurchmischter Gruppen aber dazu führen, dass gerade jüngere oder kleinere Kinder negative soziale Interaktionen erleben, z. B. unter Druck gesetzt oder geschlagen werden (Vogt et al. 2010, 69). Als zweite Adressatinnen und Adressaten sollen die Erziehenden die Zusammenarbeit mit den Lehrpersonen als respektvoll empfinden und die Förderung ihres Kindes als zufriedenstellend erleben (Projektkommission 4bis8 der EDK-Ost und Partnerkantone 2007, 43-33). Die Lehrpersonen als dritte Adressatinnen und Adressaten sollen besonders von den Kenntnissen der anderen Lehrperson oder der heilpädagogischen Unterstützung profitieren und eine anregende Zusammenarbeit erfahren (Vogt et al. 2008, 89; Vogt et al. 2010). Da das Teamteaching kein volles Pensum erlaubt, müssen die Lehrpersonen eventuell eine weitere Stelle suchen. Dies und das Teamteaching selbst können zu einer Mehrbelastung führen, was eine unerwünschte Wirkung darstellt (Projektkommission 4bis8 der EDK-Ost und Partnerkantone 2007, 40-41). Treten die erwünschten Wirkungen ein, verbessert die Basis- oder Grundstufe die Schulqualität. Die Unterrichtspraxis ist individualisiert, entwicklungsfördernd und kann alle Kinder integrieren. Dies führt zu einer Kontinuität der Lernform und der Bezugspersonen in den ersten Schuljahren (Projektkommission 4bis8 der EDK-Ost und Partnerkantone 2007, 12-15).

158 Die Kantone Zürich und Bern sind HarmoS nach einer Volksabstimmung im November 2008 respektive im September 2009 beigetreten (EDK 2016). 


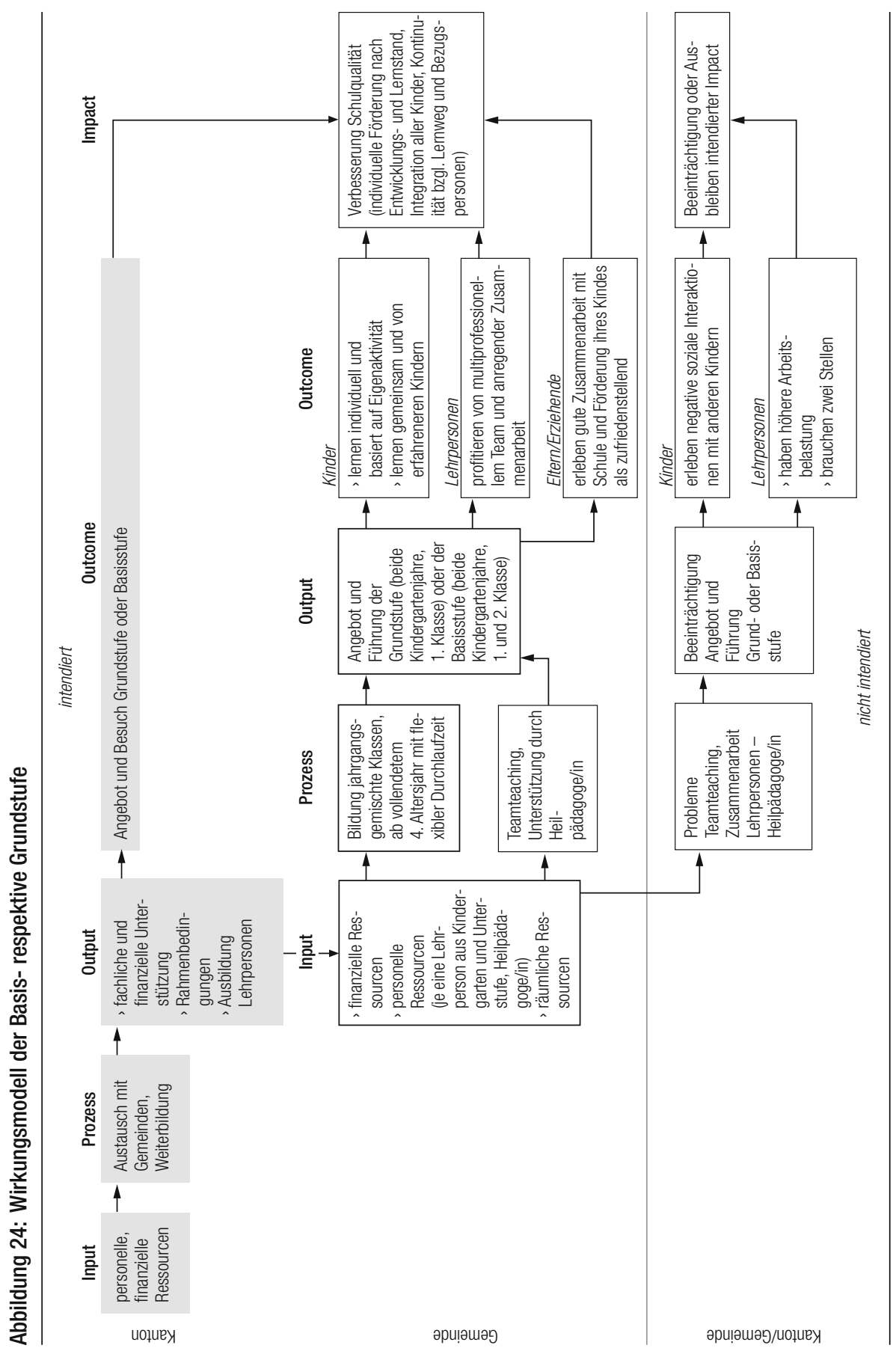


Die Realisierung der Basis- oder Grundstufe und deren Wirkungen sind das Resultat der Umsetzung der kantonalen Politik durch die Gemeinden. Der Kanton setzt Rahmenbedingungen für die Basis- oder Grundstufe, stellt die Ausbildung der Lehrpersonen bereit und unterstützt die Gemeinden fachlich und eventuell auch finanziell. ${ }^{159}$

\subsubsection{Verfügbare Evaluationen zur Basis- respektive Grundstufe}

Zum Zeitpunkt der betrachteten Gesetzgebungsprozesse in Bern und Zürich waren insgesamt 24 Evaluationen verfügbar, die sich mit der Basis- oder der Grundstufe befassen. Dazu zählen auch zwei Präsentationen, die im Rahmen von Hearings während des Gesetzgebungsprozesses zu Fall 7 stattfanden. Zu diesen Evaluationen kommen drei Zusammenfassungen, die wesentliche Inhalte der Evaluationen wiedergeben.

Die 24 Evaluationen lassen sich insgesamt 8 Evaluationsprojekten zuordnen. Sechs Evaluationsprojekte fanden im Rahmen von EDK-Ost 4bis8 statt. Darin eingeschlossen sind die zwei umfassenden Evaluationen, welche die Projektkantone von EDK-Ost 4 bis8 in Auftrag gaben. Folglich waren auch Klassen aus den Kantonen Bern und Zürich Gegenstand der beiden Evaluationen. Während die summative Evaluation den Lernstand der Kinder untersuchte, befasst sich die formative Evaluation mit der Einführung sowie Weiterentwicklung der Basis- respektive Grundstufe und deren Einschätzung aus Sicht der Beteiligten (EDK-Ost 2010; Moser et al. 2010; Vogt et al. 2010). Die Ergebnisse der Evaluationen sind in insgesamt elf Berichten und in vier Präsentationen dargestellt. Zwei Präsentationen fanden während den Beratungen des untersuchten Gesetzgebungsprozesses im Kanton Zürich statt und sind daher nur für Fall 7 relevant. Kernstück dieser verschiedenen Berichte bildet der Projektschlussbericht der EDK-Ost (2010), der einen Überblick über das gesamte Projekt und die Evaluationsergebnisse gibt. Das Projekt EDKOst 4bis8 wurde zudem mit weiteren Materialien umfassend dokumentiert, die hier aber nicht als Evaluationen verstanden werden (EDK-Ost 2010). ${ }^{160}$ Zusätzlich vier Evaluationsprojekte gaben die Bildungsdirektion des Kantons Zürich und die ERZ des Kantons Bern im Rahmen von EDK-Ost 4bis8 in Auftrag (Fankhauser et al. 2011; Stöckli und Stebler 2009; Trachsler 2009; Wagner-Willi und Widmer-Wolf 2009). Diese Evaluationsprojekte sollten spezifische Fragen beleuchten oder die Einführung der Grundstufe in Zürich sowie des "Cycle élémentaire» in französischsprachigen Gemeinden des Kantons Bern $^{161}$ allgemein evaluieren. Erstellt haben die Evaluationen universitäre Institute sowie in je einem Fall ein privates Forschungsbüro beziehungsweise die ERZ selbst. Neben diesen sechs Evaluationsprojekten im Rahmen von EDK-Ost 4bis8 befassen sich zwei ältere Evaluationsprojekte mit der Einführung der Grundstufe in Zürich (Bildungsdirektion des Kantons Zürich 2003; Stamm 2002, 2003).

159 Die Regelung zur finanziellen Unterstützung unterscheidet sich in den Fällen 7 und 8, siehe 5.5.3, 5.5.4.

$160 \mathrm{Zu}$ diesen Materialen zählen interne Befragungen der kantonalen Projektleitungen, Tagungen, eine Videodokumentation sowie mehrere Positionspapiere.

161 Die ERZ erstellte zudem vor dem Schulversuch zwei Berichte zur Basisstufe (EDK-Ost 2010, 47). Diese Berichte waren nicht zugänglich und wurden in den Interviews oder Dokumenten nicht thematisiert. 


\title{
Tabelle 25: Evaluationsergebnisse zur Wirkung der Basis- respektive Grundstufe
}

\author{
Outcome (Evaluationen und Zusammenfassungen, $\mathrm{N}=23$ ) \\ Kinder \\ Die videobasierte Unterrichtsbeobachtung zeigt, dass die Lehrpersonen individualisierten Unterricht und Förderung ein- \\ setzen, aber eher altershomogene Gruppen bilden. Die Kinder bilden altersheterogene Gruppen und lernen von älteren \\ Kindern. Kinder, die in einer Basis-/Grundstufe sind, haben besonders ausgeprägte soziale Kompetenzen und erleben \\ gemäss den Befragungen nie bis selten negative Interaktionen mit anderen Kindern. Während in der Basis-/Grundstufe \\ eine Verunsicherung bei Eintritt festgestellt wird, gilt dies im Kindergarten beim Übertritt in die 1. Klasse. Die Fallstudien \\ zu Kindern mit besonderem Förderbedarf weisen auf Stigmatisierungspotential durch separierende Unterrichtsformen in \\ der Grundstufe und den Übertritt in die Unterstufe (Wagner-Willi und Widmer-Wolf 2009).
}

\section{Lehrpersonen}

Die befragten Lehrpersonen schätzen generell das Teamteaching sowie die Zusammenarbeit mit der Heilpädagogin oder dem Heilpädagogen, der Erfolg des Teamteachings ist aber personenabhängig. Das Teamteaching und die Umstellung auf ein neues Modell führen zu einer höheren Belastung, die über die Zeit abnimmt. Belastend wirkt gemäss Evaluationen aus Zürich auch die Unklarheit über die Zukunft des Schulversuchs (Trachsler 2009; Stöckli und Stebler 2009). Das Pensum der Kindergarten- und Unterstufenlehrpersonen ist höher als jenes der Basis-/Grundstufenlehrpersonen, wobei sich ein Viertel der Basis-/Grundstufenlehrpersonen ein höheres Pensum wünscht.

\section{Erziehende}

Die befragten Erziehenden sind mit der Basis-/Grundstufe zufrieden. Sie schätzen die Zusammenstellung in altersheterogene Gruppen und das Teamteaching. Ebenso sehen sie ihr Kind als richtig gefordert an.

\section{Impact (Evaluationen und Zusammenfassungen, $\mathrm{N}=23$ )}

\section{Individuelle Förderung nach Entwicklungs- und Lernstand}

Die Kinder verweilen unterschiedlich lange in der Eingangsstufe, die Regeldauer bleibt aber drei Jahre für die Grundund vier Jahre für die Basisstufe. Die Lernstandserhebung der summativen Evaluation von EDK-0st 4bis8 zeigt, dass die Kinder in der Basis-/Grundstufe zunächst einen statistisch signifikant grösseren Lernfortschritt als im Kindergarten erreichen: «Dieser Vorsprung wurde für die Kompetenzbereiche phonologische Bewusstheit, Lesen, Schreiben und Mathematik nachgewiesen. Die Kindergartenkinder holen die Rückstände bis zum Ende der 1. Klasse weitgehend, bis zum Ende der 2. Klasse vollständig auf» (EDK-Ost 2010: 95). Kinder aus benachteiligten Familien haben denselben Lernfortschritt, können aber ihren anfänglichen Rückstand nicht ausgleichen. Laut der videobasierten Unterrichtsbeobachtung in der formativen Evaluation gibt es zudem noch Potential bei der individuellen und der spielerischen Förderung der Kulturtechniken.

\section{Integration aller Kinder}

Im Schulversuch EDK-0st 4bis8 mussten nahezu keine Sonderklassen gebildet werden. Eine frühere Evaluation stellt aber fest, dass die Integrationsfähigkeit je nach Rahmenbedingungen beschränkt ist (Stamm 2002, 2003). Zudem stellt sich bei diesen Kindern die Frage nach dem Übertritt in die reguläre Unterstufe (Wagner-Willi und Widmer-Wolf 2009).

\section{Kontinuität bzgl. Lernweg und Bezugspersonen}

Das von den Eltern wahrgenommene Wohlbefinden oder die Motivation der Kinder ändert sich vom Kindergarten in die Unterstufe, während es in der Basis-/Grundstufe gleich bleibt. Offen bleibt aber der Übertritt nach der Basis-/Grundstufe, besonders für Kinder mit besonderem Förderbedarf (Wagner-Willi und Widmer-Wolf 2009).

Die Tabelle enthält nur Literaturangaben, wenn Ergebnisse auf einzelnen Evaluationen beruhen oder widersprüchlich sind.

Bei übereinstimmenden Ergebnissen wird auf Literaturangaben verzichtet 
Die meisten Evaluationen wurden begleitend zum oder nach dem Schulversuch durchgeführt. Die summative und die formative Evaluation von EDK-Ost 4bis8 dauerten von 2004 bis 2010. Solche Längsschnittstudien sind relativ selten und aufwändig. Die Evaluation von EDK-Ost 4bis8 bildet daher schweizweit «die erste Erhebung des Lernstandes von rund 1000 Kindern (Versuchs- und Kontrollgruppe) ab 1. Kindergartenjahr bis 3. Primarklasse und die erste umfangreiche Befragung der Eltern und Lehrpersonen» (EDK-Ost 2010, 91).

Tabelle 25 erläutert die wichtigsten Ergebnisse zur Wirkung der Basis- oder Grundstufe. Wie die Erläuterungen zeigen, untersuchten Evaluationen sämtliche der zuvor im Modell dargestellten Wirkungen (vgl. Abbildung 24). Die Evaluationsergebnisse zu diesen Wirkungen stimmen überein. Allerdings basieren diese Ergebnisse in der Regel auf höchstens drei bis vier Evaluationsprojekten, die teilweise mit Klassen aus demselben Versuch der EDK-Ost arbeiten. Die Fallstudien von Kindern mit besonderem Förderbedarf weisen zudem darauf hin, dass diese Kinder zwar ebenso vom Grundstufenmodell profitieren, die allgemeinen Ergebnisse von EDK-Ost 4bis8 aber bedingt auf sie übertragbar sind (Wagner-Willi und Widmer-Wolf 2009). Insgesamt kann daher die Übereinstimmung der Ergebnisse als moderat interpretiert werden.

In ihren Arbeiten diskutieren die Evaluierenden von EDK-Ost 4bis8 die eigenen Ergebnisse kritisch. Sie sehen die Ziele der Basis- oder Grundstufe als erreicht an. Sie zeigen aber auch andere mögliche Lesarten auf: Wer sich an den expliziten Zielen orientiert, die mit den Modellen verfolgt
werden, wird eher von einem Erfolg sprechen. [...] Wer von der Ausrich-
tung des Lernens am Lern- und Entwicklungsstand der Kinder zugleich
grössere Lernfortschritte erwartet hat, wird eher enttäuscht sein. Allerdings
werden solche Erwartungen den neuen Modellen deshalb nicht gerecht,
weil die Forderung nach grösseren Lernfortschritten gar nie gestellt wurde
und für alle drei Modelle der Lehrplan der Volksschule massgebend ist.
(EDK-Ost 2010,115)

\subsubsection{Grundstufe im Kantonsrat Zürich (Fall 7)}

\section{Ablauf und Inhalt des Gesetzgebungsprozesses}

Die Grundstufe wurde im Kanton Zürich im Rahmen der «prima-Initiative - Kantonale Volksinitiative für die Weiterentwicklung der Kindergartenstufe» diskutiert, die der Kantonsrat zwischen September 2010 und Juli 2012 behandelte. Das Komitee rund um den überparteilichen Verein Chance Volksschule reichte die Volksinitiative am 15. März 2010 in der Form der allgemeinen Anregung ein. ${ }^{162}$ Neben Vertreterinnen und Vertreter

162 Zum Verfahren bei Volksinitiativen in der Form der allgemeinen Anregung siehe Gesetz über die politischen Rechte (GPR) vom 1. September 2003 LS 161, Art. 120, Art. 138. 
von Berufsverbänden im Schulbereich, Forschenden und Lehrpersonen beteiligten sich Politiker und Politikerinnen der CVP, FDP, Grüne und SP im Initiativ- und Unterstützungskomitee (Regierungsrat des Kantons Zürich 2009c). Die Initiative verlangte, das Volksschulgesetz (VSG-ZH) so zu ändern, dass die Kindergartenstufe ohne gleichzeitige Änderung des Eintrittsalters um mindestens ein Jahr verlängert wird. Weiter sollen altersdurchmischte Klassen geführt werden und in mindestens der Hälfte der Lektionen zwei Lehrpersonen unterrichten. Ebenso soll die Kindergartenstufe eine unterschiedliche Verweildauer ermöglichen (Regierungsrat des Kantons Zürich 2010b). Da sich die prima-Initiative nur auf die Einführung einer Basis- oder Grundstufe bezog, umfasst der Gesetzgebungsprozess keine weiteren Massnahmen.

Bereits vor der prima-Initiative stand die Grundstufe im Kanton Zürich mehrfach zur Diskussion. Das erste Mal befasste sich der Kantonsrat im Rahmen der Totalrevision des VSG-ZH im Jahr 2002 mit der Grundstufe, die in der entsprechenden Vernehmlassung anstelle der Basisstufe präferiert wurde. Die CVP, FDP, SP und Teile der Grünen sprachen sich für die Grundstufe aus, während die EDU, EVP, SVP und ein anderer Teil der Grünen sie ablehnten. Ihre Ablehnung begründeten sie mit der mangelnden Erprobung der Grundstufe (Kantonsrat Zürich 2002). Die Bildungsdirektion hatte aufgrund der damaligen Rechtslage nur zusammen mit einer Privatschule einen Versuch durchgeführt. Während der Kantonsrat der flächendeckenden Einführung der Grundstufe zustimmte, lehnten die Stimmberechtigten die Totalrevision knapp ab (Regierungsrat des Kantons Zürich 2003). Kurz danach beriet der Kantonsrat erneut über eine ähnliche Totalrevision des VSG-ZH, die aber die Einführung der Grundstufe nicht vorsah. Die Stimmberechtigten befürworteten diese Totalrevision 2005 dann deutlich (Statistisches Amt des Kantons Zürich 2016). Ein zweites Mal diskutierte der Kantonsrat über die Grundstufe anlässlich der Schulversuche. Die KBIK reichte die Leistungsmotion 33/2003 ein, die forderte, Schulversuche mit der Grundstufe ab Schuljahr 2004/05 ins Budget zu integrieren. ${ }^{163}$ Der Kantonsrat überwies die Leistungsmotion, woraufhin der Regierungsrat beschloss, Schulversuche ab dem Schuljahr 2004/05 gestaffelt zu starten und bis Ende Schuljahr 2008/09 durchzuführen (Regierungsrat des Kantons Zürich 2003).

In Bezug auf die prima-Initiative beantragte der Regierungsrat im Juli 2010, die Volksinitiative in der allgemeinen Form den Stimmberechtigten zu unterbreiten und ihnen zu empfehlen, die Initiative abzulehnen. Die Grundstufe erreiche zwar ihre Ziele, sei aber nicht besser als das herkömmliche Modell des Kindergartens und der Primarschule. Wegen ihrer Mehrkosten lehnte daher der Regierungsrat die Einführung der Grundstufe ab (Regierungsrat des Kantons Zürich 2010b). Zeitgleich mit dem Antrag verlängerte der Regierungsrat die Schulversuche mit der Grundstufe bis Ende Schuljahr 2013/14 (Regierungsrat des Kantons Zürich 2010a). ${ }^{164}$

163 Seit 2002 ermöglicht das Bildungsgesetz (LS 410.1) des Kantons Zürich Schulversuche auf allen Stufen, wobei diese evaluiert werden müssen.

164 Um den Projektschlussbericht von EDK-Ost 4bis8 abzuwarten, verlängerte der Regierungsrat bereits 2007 die Schulversuche bis Ende Schuljahr 2011/12 (Regierungsrat des Kantons Zürich 2007b). 
Die KBIK befasste sich darauffolgend an drei Sitzungen mit der prima-Initiative. Da das Komitee die Initiative als allgemeine Anregung formulierte, beriet die KBIK darüber, ob entsprechend dem Regierungsratsantrag direkt über die Vorlage abgestimmt und was für eine Abstimmungsempfehlung gegeben werden soll, oder ob eine Umsetzungsvorlage und gegebenenfalls ein Gegenvorschlag ausgearbeitet werden soll. ${ }^{165}$ Dazu hörte die KBIK das Initiativkomitee an und lud Fachpersonen der Projektevaluation von EDK-Ost 4 bis8 ein. Die KBIK beantragte danach, den Regierungsrat zu beauftragen, eine Umsetzungsvorlage mit Gegenvorschlag auszuarbeiten. Der Kantonsrat und die Stimmberechtigten bedürften eine genauere, ausformulierte Vorlage, um einen Entscheid zu treffen. Da die Umsetzungsvorlage laut der prima-Initiative eine flächendeckende Einführung vorsehen muss, schlug die KBIK vor, im Gegenvorschlag ein Modell mit Wahlmöglichkeit zwischen Kindergarten/Primarstufe und Grundstufe auszuarbeiten (Kantonsrat Zürich 2010b). Damit griff sie im Wesentlichen die parlamentarische Initiative 65/2010 auf, die Kantonsrätinnen der FDP zeitgleich mit der prima-Initiative einreichten, aber keine Unterstützung im Kantonsrat fand (Kantonsrat Zürich 2010a).

Im Dezember 2010 folgte der Kantonsrat mit 57.1 Prozent dem Antrag der KBIK. AL, CVP, FDP, Grüne, SP sowie einzelne Mitglieder der EVP und Grünliberalen stimmten für die Ausarbeitung einer Umsetzungsvorlage und eines Gegenvorschlags. Die EDU, EVP, SVP und die Mehrheit der grünliberalen Mitglieder lehnten die Ausarbeitung einer Umsetzungsvorlage und eines Gegenvorschlags aus unterschiedlichen Gründen ab. Während die Grünliberalen vor allem eine Wahlmöglichkeit von zwei Modellen ablehnten, sprachen sich die anderen Parteien generell gegen die Grundstufe aus.

Seinen Antrag zur Umsetzungsvorlage und zum Gegenvorschlag verabschiedete der Regierungsrat im September 2011. Beide Vorlagen sahen vor, dass die ersten drei Jahrgänge in der Grundstufe zusammen geführt werden, wobei die Kinder die Grundstufe in zwei bis vier Jahren durchlaufen können. Weitere Rahmenbedingungen wie die Klassengrösse, Anzahl Wochenlektionen, das Pensum der Lehrpersonen sowie deren Entlohnung würden ausserhalb des VSG-ZH geregelt. ${ }^{166}$ Der Kanton beteiligt sich an der Grundstufe mit koordinativer und fachlicher Unterstützung sowie im Rahmen der Weiterbildung für Lehrpersonen, die wiederkehrenden Mehrkosten der Grundstufe tragen jedoch zu 80 Prozent die Gemeinden. Aufgrund dieser Kosten lehnte der Regierungsrat in seinem Antrag eine flächendeckende Einführung der Grundstufe ab, befürwortete aber die im Gegenvorschlag vorgesehene Wahlmöglichkeit (Kantonsrat Zürich 2012a; Regierungsrat des Kantons Zürich 2011b).

Auf Grundlage dieses zweiten Regierungsratsantrags beriet die KBIK in weiteren drei Sitzungen Anfang 2012 die Umsetzungsvorlage und den Gegenvorschlag. Die Zusammensetzung der KBIK hatte sich seit ihrer letzten Beratung der prima-Initiative 2010 geändert. In der neuen Legislatur blieben 6 der 15 Mitglieder in der KBIK, das Präsidium wechselte von der SVP zu den Grünen. Die Zuständigkeit bei der Regierung änderte sich dagegen nicht. Nach einer Präsentation der Vorlage durch Regie-

165 Das Verfahren bei allgemein formulierten Volksinitiativen führte mehrfach zu Unklarheiten in der KBIK. 166 Diese Rahmenbedingungen entsprachen denjenigen des Schulversuchs. 
rung und Verwaltung beschloss die KBIK, von weiteren Anhörungen abzusehen und führte zwei Lesungen der Vorlage durch. Die KBIK sprach sich für den Gegenvorschlag aus, inhaltliche Änderungen an der Umsetzungsvorlage oder dem Gegenvorschlag beantragte sie nicht.

Der Kantonsrat folgte im April 2012 wiederum dem Antrag der KBIK. Der Antrag der SVP, auf die Umsetzungsvorlage nicht einzutreten, lehnte er knapp mit 85 zu 75 Stimmen ab. Wie bereits in der ersten Kantonsratssitzung zur prima-Initiative 2010 stimmte dieselbe Koalition von EDU, Grünliberalen, SVP und einer Mehrheit der EVP für den Antrag auf Nichteintreten. Das im Vergleich zur ersten Kantonsratssitzung knappere Ergebnis kam zustande, da die Grünliberalen in den Wahlen 2011 ihre Sitze von 10 auf 19 erhöhten. Wiederum sprachen sich die Grünliberalen gegen das gleichzeitige Bestehen zweier Modelle aus, während die EDU, eine Mehrheit der EVP und die SVP die Grundstufe gänzlich ablehnten.

Die Abstimmungsempfehlung beschloss der Kantonsrat erst anlässlich der Schlussabstimmung in der Redaktionslesung. Er stimmte beiden Vorlagen relativ knapp mit 53.3 respektive 58.7 Prozent zu, wobei er den Stimmberechtigten von Gesetzes wegen den Gegenvorschlag zur Annahme empfahl. Die AL, BDP, CVP, FDP, Grüne, SP sowie einzelne Mitglieder der EVP befürworteten sowohl Gegenvorschlag als auch Umsetzungsvorlage. Die AL, BDP, Grüne und SP sprachen sich für beide Vorlagen aus, um die Grundstufe zumindest als Wahlmöglichkeit zu erhalten (Kantonsrat Zürich 2012b). Die FDP und die CVP unterstützten die flächendeckende Einführung in der Abstimmung dagegen nur, da ohne die Umsetzungsvorlage auch kein Gegenvorschlag unterbreitet werden kann, wobei sie Letzteren klar bevorzugten. Teile der Grünliberalen befürworteten weiter lediglich die Umsetzungsvorlage. Andere Teile der Grünliberalen, die Mehrheit der EVP, die EDU und SVP lehnten beide Vorlagen ab. Die Stimmberechtigten lehnten letztlich beide Vorlagen ab.

\section{Evaluationsnutzung}

Das Komitee der prima-Initiative erwähnte zwar im Initiativtext, dass die laufenden Schulversuche berücksichtigt werden, verwies aber nicht auf Evaluationen (Regierungsrat des Kantons Zürich 2010b, 1-2). Die Initiative begründete es mit den Vorteilen der individuellen Förderung und Verweildauer in der Eintrittsstufe, der pädagogischen Kontinuität sowie altersdurchmischtem Lernen. Ebenso argumentierte das Initiativkomitee, dass die Kindergartenpädagogik weiter ausgebaut werde und sich die investierten Kosten der Eingangsstufe in der späteren Schullaufbahn auszahlen werden.

Verwendung fanden Evaluationen dagegen bei der Diskussion der parlamentarischen Initiative 65/2010 zur wahlweisen Einführung der Grundstufe, die drei FDP-Kantonsrätinnen gleichzeitig wie die prima-Initiative einreichten. Die Kantonsratsmitglieder griffen die Evaluation von EDK-Ost 4 bis8 in der Plenumsdiskussion auf, die kurz nach dem Erscheinen des Projektschlussberichts stattfand. Die Parlamentsmitglieder aus sechs verschiedenen Parteien (CVP, EVP, FDP, Grüne, SP, SVP) verwiesen auf den Projektschlussbericht oder die Ergebnisse des Schulversuchs. Die EVP und die SVP, die der 
Grundstufe skeptisch gegenüberstanden, betonten die gleichen Lernstände der Kinder im Kindergarten und in der Grundstufe bei höheren Kosten der Grundstufe. Ein EVP-Kantonsrat folgerte daraus:

Aufgrund des Schlussberichts muss man sich ernsthaft fragen, ob die Grundstufe noch ihre Berechtigung hat. Hier sind die Befürworter gefordert, Argumente zu liefern, die allenfalls für eine Weiterführung sprechen würden. (Kantonsrat Zürich 2010a, 11578)

Diese Resultate zum gleichen Lernerfolg der Modelle griffen auch die anderen Parlamentsmitglieder auf, zusätzliche Ergebnisse erwähnten die Sprecherinnen der CVP und der SP. Stellvertretend dafür die Kantonsrätin der SP:

Wir wollen, dass die positiven Aspekte aus den Versuchen in allen Schulen umgesetzt werden. Und positive Aspekte wurden einige erkannt und im Schlussbericht auch beschrieben. Die Versuche zeigen klar, dass sich das altersdurchmischte Lernen sehr bewährt. [...] Ebenfalls konnte aufgezeigt werden, dass die Integration von Kindern mit Defiziten in einer Grundbeziehungsweise Basisstufe besser bewerkstelligt werden kann als im Kindergarten. (Kantonsrat Zürich 2010a, 11580)

Generell waren die Resultate ein prägendes Thema dieser Kantonsratsdebatte, auch wenn die Kantonsratsmitglieder den Zusammenhang mit der Evaluation von EDK-Ost 4bis8 nicht immer herstellten. Neben diesem Schlussbericht verwies ein Kantonsrat der SVP auf die PISA-Studien, zudem forderte die Sprecherin der CVP eine Evaluation, um die weitere Schullaufbahn der Grundstufenkinder zu beobachten (Kantonsrat Zürich 2010a, 11574, 11576). Auf eine weitere mögliche Evaluation verwies auch die FDP in ihrer Pressemitteilung zur parlamentarischen Initiative (FDP Kanton Zürich 2010a).

Bei der Ausarbeitung der Vorlage stützte sich die Verwaltung am stärksten auf die gemeinsame Evaluation von EDK-Ost 4bis8. Die Bildungsdirektion bezog sie von Anfang an ein, da "wir an sich noch wenig Erfahrung hatten und wirklich der Meinung waren, wir wollen wissen, ob diese Grundstufe die Ergebnisse bringt» (F5/7-Verw.). Zudem sei eine Evaluation bei einem Schulversuch ohnehin gesetzlich vorgeschrieben. Wie üblich hat die Abteilung Bildungsplanung diese Evaluation wissenschaftlich begleitet (F5/7-Verw.). Neben dieser Evaluation hat die Bildungsdirektion während des Schulversuchs drei weitere Evaluationen in Auftrag gegeben. Diese bezweckten, (1) das Unterstützungsangebot vonseiten des Kantons zu untersuchen, (2) die Umsetzung und Wirkung der Grundstufe für Kinder mit besonderem Förderbedarf vertieft zu analysieren sowie (3) den Schulversuch in jenen Klassen, die nicht an der Evaluation von EDK-Ost 4 bis8 beteiligt waren, begleitend zu evaluieren. Zusätzlich konnte die Bildungsdirektion auf zwei Berichte zurückgreifen, die im Rahmen der Revision des VSG-ZH Anfang der 2000er-Jahre erstellt worden waren (Bildungsdirektion des Kantons Zürich 2003; Stamm 2002, 2003). Diese Berichte - die sehr wohlwollend waren und die Kosten etwas entkräf- 
teten - seien aber in der Diskussion der prima-Initiative nicht mehr aufgekommen (F5/7Verw.). Auch der Regierungsratsantrag enthält vor allem Verweise auf die Evaluation im Rahmen von EDK-Ost 4bis8. Auf insgesamt zwei Seiten erläuterte der Regierungsrat den Hintergrund und die wichtigsten Ergebnisse der formativen und summativen Evaluation. In einem Abschnitt thematisierte er zudem die Kosten, die eine Anpassung der Räume an die Grundstufe gemäss einem älteren Bericht auslösen würde (Regierungsrat des Kantons Zürich 2010b, 5-8). Obwohl der Regierungsrat Evaluationen folglich im Antrag durchaus diskutierte, seien sie in der Argumentation nicht im Vordergrund gestanden: «Wir haben mehr politisch argumentiert, also weniger mit der Evaluation. Aus dem einfachen Grund, Sie müssen letztlich einfach eine politische Mehrheit gewinnen» (F5/7-Verw.).

Unklar blieb in den Gesprächen, inwiefern sich die Kommissionsmitglieder bereits vor der Kommissionsberatung mit Evaluationen beschäftigten. Die befragten Mitglieder der Kommission geben an, dass sie sich bereits zuvor mit der Grundstufe auseinandersetzten. Zum einen war diese bei der VSG-ZH 2002 diskutiert worden, zum anderen waren die Befragten als Lehrpersonen oder Mitglieder der Schulbehörden mit der Grundstufe konfrontiert. Bis auf die zuvor erwähnte Pressemitteilung der FDP sind bis zur Plenumsdiskussion schliesslich keine Medienmitteilungen der Parteien verfügbar.

Die KBIK befasste sich in der parlamentarischen Phase zweimal mit der Grundstufe, wobei sie sich mit Evaluationen besonders während der ersten Kommissionsberatung auseinandersetzte. In dieser ersten Beratung diskutierte die KBIK über die Frage, ob der Regierungsrat eine Umsetzungsvorlage und einen Gegenvorschlag ausarbeiten soll. Dazu lud sie Personen aus dem Initiativkomitee ein. Diese bezogen sich auf die Evaluation von EDK-Ost 4 bis8 und schlossen daraus, dass die Grundstufe weiterverfolgt werden solle. Im Verlauf derselben Sitzung verwiesen mehrere Kommissionsmitglieder auch auf die Evaluation von EDK-Ost 4bis8. Zudem beschloss die KBIK auf Anregung mehrerer Kommissionsmitglieder, Fachpersonen zur Präsentation der Evaluationsergebnisse einzuladen. Die Präsentationen der Fachpersonen beurteilen die befragten Parlamentsmitglieder sowie die Person aus der Verwaltung sehr positiv. Die Präsentationen seien «sehr wissenschaftlich» (F5/7-PM3) gewesen und die Kommissionsmitglieder hätten sich vertieft damit auseinandergesetzt (F5/7-PM3, F5/7-PM4). Auch das befragte Kommissionsmitglied, das die Grundstufe ablehnte, befindet die Präsentation als «sehr, sehr neutral» (F5/7-PM2). Hingegen waren Mitglieder, die eine flächendeckende Einführung der Grundstufe befürworteten, gemäss dem Kommissionsprotokoll kritischer: In ihren Augen wertete eine der Fachpersonen die Initiative. Auf diesen Eindruck angesprochen, erklärte diese Fachperson ihre Interpretationen ausführlicher und gab ihre persönliche Meinung preis. Auch im Interview befindet das befragte Kommissionsmitglied, diese Person habe «eine schwierige Rolle gespielt» (F5/7-PM5). Diese Fachperson war jedoch in der KBIK bekannt, da sie auch an anderen Hearings beteiligt war. Mehrere Befragte nehmen sie generell als sehr professionell wahr (F5/7-PM2, F5/7-PM3). Schliesslich erhielten die Kommissionsmitglieder gemäss den Interviews auch den Projektschlussbericht (F5/7-PM4).

Die Interviews legen folglich nahe, dass sich sämtliche der befragten Kommissionsmitglieder differenziert mit der Evaluation von EDK-Ost 4 bis8 auseinandersetzten. Sie erinnern sich an die Präsentation und können zentrale Ergebnisse ausführen. Diese 
beziehen sich vor allem auf den Lernstand, der gemäss der summativen Evaluation am Ende der Grundstufe und der ersten Klasse gleich ist. Aus diesen Ergebnissen schliessen die Befragten einerseits, dass es relativ kleine Unterschiede gäbe zwischen Kindergarten und Grundstufe (F5/7-PM1 bis PM4). Anderseits weisen zwei Befragte darauf hin, dass das Modell auf die nächsten Schulstufen ausgedehnt werden müsse, sollte sich die individuelle Förderung entfalten und der Lernstand nicht angleichen (F5/7-PM3; F5/7-PM4). Diese zentralen Ergebnisse haben die Befragten gemäss ihren Angaben auch in ihrer Argumentation verwendet (F5/7-PM2 bis PM5). Zudem betonen mehrere befragte Kommissionsmitglieder den «stark legitimatorischen Charakter» (F5/7-PM1) dieser Evaluation für die Schulversuche, die von Anfang an gefordert worden sei und zum Gesetzgebungsprozess gehört habe (vgl. F5/7-PM2; F5/7-PM5). Andere Evaluationen erwähnen die Befragten schliesslich nicht. Bis auf ein Kommissionsmitglied (F5/7-PM5) besuchten sie auch die Grundstufe nicht, kein Kommissionsmitglied sah sich die Videodokumentation an.

In der zweiten Phase der Kommissionsberatung zur mittlerweile ausgearbeiteten Umsetzungsvorlage und dem Gegenvorschlag fanden Evaluationen nur selten Erwähnung. Ein Kommissionsmitglied, das erst bei dieser zweiten Phase in der KBIK sass, forderte eine separate Evaluation des Lernstands der Grundstufenkinder für den Kanton Zürich. Daraufhin erklärten Regierung und Verwaltung den Aufbau des Schulversuchs, fassten Ergebnisse der Evaluation von EDK-Ost 4bis8 in Bezug auf den Lernfortschritt zusammen und diskutierten Schwierigkeiten bei der Interpretation. Dabei verwiesen sie auch auf Lernstandserhebungen ${ }^{167}$, welche die Bildungsdirektion bereits vor den Versuchen mit der Grundstufe durchführen liess. Weiter zitierte ein Mitglied der Verwaltung aus dem Evaluationsbericht zu baulichen Massnahmen der Grundstufe, den die Bildungsdirektion im Rahmen der Revision des VSG-ZH 2002 erstellte (Bildungsdirektion des Kantons Zürich 2003). Folglich konzentriert sich die Evaluationsnutzung in dieser zweiten Phase vor allem auf die Verwaltung und Regierung.

Im Plenum besprach der Kantonsrat die prima-Initiative dreimal, wobei die Evaluation von EDK-Ost 4 bis8 besonders an zwei Sitzungen thematisiert wurde. Diese beiden Sitzungen widmeten sich der Frage der Ausarbeitung einer Umsetzungsvorlage und eines Gegenvorschlags sowie diesen zwischenzeitlich vom Regierungsrat erarbeiteten Vorlagen. Die Inhalte der beiden Debatten sind relativ ähnlich und die Evaluation von EDK-Ost 4bis8 sehr präsent: In beiden Debatten haben die Regierungsrätin, die Kommissionspräsidenten und Kantonsratsmitglieder der CVP, EVP, EDU, FDP, Grüne, SP, SVP sowie teilweise der AL auf Evaluationen verwiesen. ${ }^{168}$ Die Kantonsratsmitglieder fokussierten auf die Ergebnisse zu den Lernstandserhebungen aus der summativen Evaluation. Nur Kantonsrätinnen der SP sowie der Grünen, die eine flächendeckende Einführung der Grundstufe befürworteten, verwiesen zusätzlich auf Ergebnisse der formativen Evaluation zur Zufriedenheit der Eltern und Belastung der Lehrpersonen. Neben den Evaluationen erwähnten die Kantonsratsmitglieder in der ersten Debatte keine, in der zweiten

167 Siehe Moser et al. (2011); Moser und Hollenweger (2008); Moser et al. (2004).

168 AL nur in der zweiten Sitzung. 
Debatte vereinzelt andere Studien. So erwähnte eine Kantonsrätin der CVP PISA und die sich daraus ableitende Wichtigkeit der Frühförderung, die BDP sowie FDP verwiesen allgemein auf die Bildungsforschung und die Regierungsrätin erwähnte den Jahresbericht der Fachstelle Schulbeurteilung der Bildungsdirektion (2012).

Die Ergebnisse der Lernstandserhebungen im Rahmen von EDK-Ost 4 bis8 führten auch zu Kritik an der Versuchsanlage der Evaluation, wie in der Rede eines Kantonsrats der SVP:

Am Ende der zweiten Klasse lässt sich kein Unterschied zwischen Grundstufenkindern und ehemaligen Kindergärtlerinnen und Kindergärtlern mehr feststellen, und dies trotz eineinhalb so vielen Lehrkräften in der Grundstufe. Eine hohe Investition mit keinem Ertrag! Bei derart wenig Lernvorsprung der Grundstufe könnte man sogar den Schluss ziehen, dass bei gleich vielen Lehrkräften pro Klasse vermutlich die Kindergartenschülerinnen und -schüler erfolgreicher abschneiden würden. Aber Sie werden es kaum glauben, trotz teurer Evaluation wurde dieser Umstand gar nicht untersucht. (Kantonsrat Zürich 2010b, 12884)

Folglich bemängelten die Parlamentsmitglieder, die eine Einführung der Grundstufe ablehnten, die Vergleichbarkeit von Kindergarten und Grundstufe angesichts unterschiedlicher Stellenprozente der Lehrpersonen. Auf befürwortender Seite hingegen wendeten die Parlamentsmitglieder ein, dass ein höherer Lernfortschritt kein Ziel des Schulversuchs war und dieser nicht möglich ist bei gleichen Lernzielen. Dazu etwa das Votum einer grünen Kantonsrätin:

Leider haben sie, die Gegner, diese ursprünglichen Projektziele aus den Augen verloren und vergessen, dass auch in der Grundstufe nur mit Wasser gekocht wird. Grundlage ist nämlich auch dort der Lehrplan der Kindergartenstufe beziehungsweise der ersten Klasse. (Kantonsrat Zürich 2012a, 3339)

Zudem kritisierte diese Kantonsrätin der Grünen wiederholt die Kommunikation der Evaluationsergebnisse durch die Regierung (Kantonsrat Zürich 2010b, 12889; 2012a, 3345).

Deutlich geringere Evaluationsnutzung ist dann bei der dritten Beratung im Kantonsrat anlässlich der Redaktionslesung und Schlussabstimmung zu beobachten. Nur je ein Kantonsratsmitglied der Grünliberalen sowie der Grünen bezog sich auf die Evaluation von EDK-Ost 4bis8. Die Kantonsratsmitglieder der anderen grossen Parteien erwähnten die Evaluation nicht explizit, thematisierten aber dennoch die Ergebnisse. Auch enthalten die verfügbaren Medienmitteilungen während der parlamentarischen Phase nur vereinzelt Hinweise auf Evaluationen. So weisen die Grünen darauf hin, dass sich die Grundstufe gemäss dem Evaluationsbericht von EDK-Ost 4 bis 8 «als Antwort auf die erweiterten Anforderungen» bewährt habe (Grüne Kanton Zürich 2011b). 
Evaluationsnutzung: Zeitpunkt und Quellen

Abbildung 25 illustriert die Anzahl Evaluationen nach Erscheinungsdatum und zeigt, dass bis zur ersten Kommissionssitzung am 21. September 2010 insgesamt 22 Evaluationen und Zusammenfassungen vorlagen. Dazu zählt auch der Projektschlussbericht von EDK-Ost 4bis8, der Ende Juni 2010 erschienen war. Bis zur ersten Sitzung im Plenum des Kantonsrats am 6. Dezember 2010 kamen noch vier Evaluationen und Zusammenfassungen dazu. In der Zwischenzeit verfügbar waren die Schlussberichte der formativen und summativen Evaluation von EDK-Ost 4bis8. Bis zum Abschluss des Geschäfts im Kantonsrat erschien dann nur noch eine weitere Evaluation, diese befasste sich aber mit dem Kanton Bern.

\section{Abbildung 25: Zeitliche Verfügbarkeit der Evaluationen zur Grundstufe im Kantonsrat Zürich}

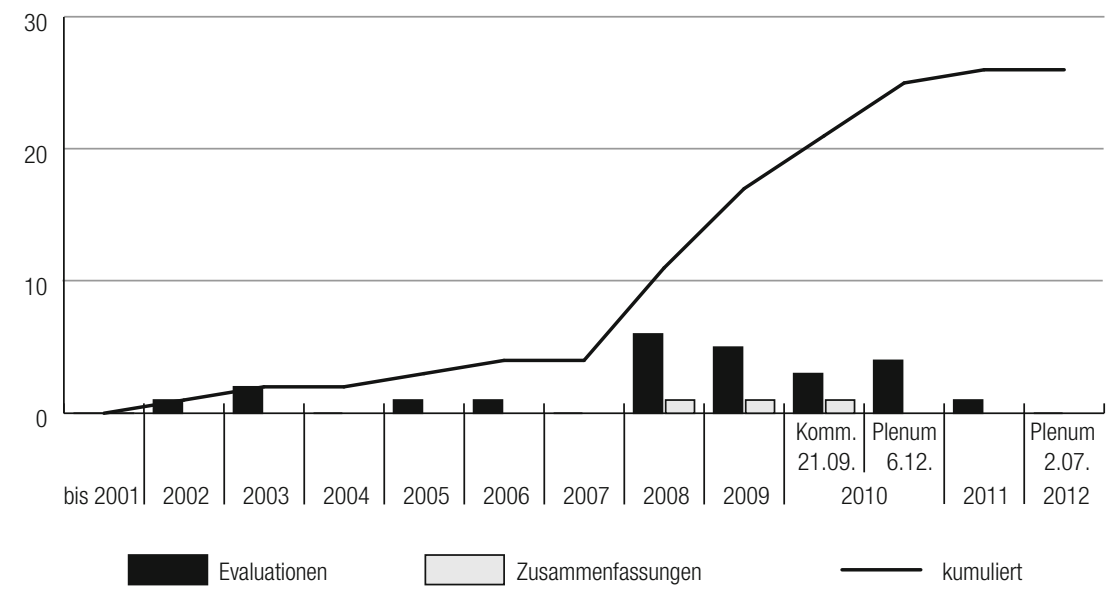

Die Anzahl bezieht sich auf die in einem Jahr beziehungsweise bis zum gegebenen Zeitpunkt erschienenen Evaluationen.

Von diesen Evaluationen und Zusammenfassungen waren zwei Quellen zentral für die Evaluationsnutzung: Der Projektschlussbericht der EDK-Ost (2010) sowie die Präsentationen in der KBIK. Dies trifft für die analytische Nutzung zu, da sich die befragten Parlamentsmitglieder besonders mit diesen beiden Quellen auseinandersetzten (vgl. F5/7-PM3). Auch die Verwaltung stützte sich gemäss den Interviewaussagen bei der Ausarbeitung und Begleitung der Grundstufe vor allem auf die Evaluation der EDKOst (F5/7-Verw.). Gemäss der Auflistung in Tabelle 26 konzentrierte sich die politische Nutzung ebenfalls auf diese beiden Quellen. Die Parlamentsmitglieder verwiesen fast nur auf die Evaluation von EDK-Ost 4bis8, wobei sie die Resultate der summativen Evaluation häufiger ansprachen. Bei der Analyse der Voten ist aber nicht immer eruierbar, auf welches der verschiedenen Dokumente zur Evaluation von EDK-Ost 4bis8 sich die 
Parlamentsmitglieder beziehen. Die Parlamentsmitglieder sprachen generell vom Schlussbericht oder der Evaluation (vgl. Kantonsrat Zürich 2010b, 12884, 12891). Besonders in der ersten Sitzung stellten zudem einige Parlamentsmitglieder den Hintergrund der Evaluation und deren Autorenschaft ungenau dar. Daher stellte die Regierungsrätin in ihrer Rede klar, dass diese Studie von einem interkantonalen Team von Fachleuten erarbeitet wurde und nicht von der Bildungsdirektion (Kantonsrat Zürich 2010b, 12897). Es ist aber anzunehmen, dass sich die Parlamentsmitglieder meist auf den Projektschlussbericht der EDK-Ost (2010) bezogen. Die Kommissionsmitglieder erhielten diesen Bericht (F5/7-PM4), zudem verwiesen die Parlamentsmitglieder teilweise explizit darauf. Ebenso erschien der Projektschlussbericht früher als die Schlussberichte der Evaluationen. Neben dem Projektschlussbericht haben einzelne Parlamentsmitglieder auf die Präsentationen im Rahmen des Hearings der KBIK sowie auf eine der dort anwesenden Fachpersonen verwiesen. Daneben haben sie Evaluationen in anderen Arten politisch genutzt. Ein Parlamentsmitglied der CVP forderte eine Evaluation, um die langfristige Entwicklung der Grundstufenkinder zu beobachten. Später forderte ein Parlamentsmitglied der SVP eine Lernstandserhebung der Grundstufenkinder für den Kanton Zürich. Kritisiert haben die Parlamentsmitglieder die Evaluationen vor allem in der parlamentarischen Phase. Auch Regierung und Verwaltung verwiesen meist auf den Projektschlussbericht sowie auf den Bericht der Bildungsdirektion des Kantons Zürich (2003) zu Kosten der Anpassung der Schulräume an die Grundstufe.

Die Parlamentsmitglieder verwiesen insgesamt auf aktuelle Quellen, wobei sie im Verlauf des Gesetzgebungsprozesses keine (noch) neueren Quellen aufgriffen (vgl. Tabelle 26). So wiesen die Parlamentsmitglieder weniger als zwei Wochen nach dessen Erscheinen erstmals auf den Bericht der EDK-Ost (2010) hin, als sie die parlamentarische Initiative 65/2010 im Plenum debattierten. Danach verwiesen sie weiter darauf. Die einzigen neueren Quellen, die sie noch aufnahmen, waren die Präsentationen in der KBIK.

\section{Evaluationsnutzung: Positionen und Argumente}

Die Evaluationsergebnisse zur Basis- und Grundstufe stimmen zwar überein, es sind aber mehrere Lesarten zugunsten unterschiedlicher Gruppen der Parlamentsmitglieder möglich. Einerseits zeigte die Evaluation, dass sich die Grundstufe klar bewährt. Anderseits illustrierte gerade die summative Evaluation, dass die Grund- oder Basisstufe sowie der Kindergarten gleichwertig sind (EDK-Ost 2010, 100, 115). Diese ambivalenten Ergebnisse sprechen die Kommissionsmitglieder auch in den Interviews an. Die Ergebnisse hätten eigentlich für die Grundstufe gesprochen, aber die Unterschiede zum Kindergarten seien sehr klein gewesen. Dies sei zwar logisch bei einem Vergleich von zwei guten Systemen, es erschwere aber gerade angesichts der höheren Kosten eine Argumentation für die Grundstufe. Auch aus diesen Gründen hat dieses Kommissionsmitglied die freiwillige Einführung befürwortet (F5/7-PM4). Für ein zweites Kommissionsmitglied, das ebenfalls die freiwillige Einführung befürwortete, ist zentral, dass «nicht bewiesen wurde, dass der Kindergarten total daneben ist gegenüber der Grundstufe. Es ist einfach eine andere Schulungsform» (F5/7-PM3). Auch die befragte Person in der Verwaltung schätzt, dass 
dieser Vergleich von Kindergarten und Grundstufe, besonders auf den Lernfortschritt bezogen, «die Diskussion am meisten beeinflusst und auch den Befürwortern geschadet hat» (F5/7-Verw.).

Übereinstimmend mit den verschiedenen Lesarten der Ergebnisse haben Parlamentsmitglieder mit unterschiedlichen Positionen Evaluationen analytisch und politisch genutzt. Alle Kommissionsmitglieder setzten sich unabhängig von ihrer Position differenziert mit Evaluationen auseinander und können sich noch an deren zentrale Ergebnisse erinnern. Auch nutzten die Parlamentsmitglieder Evaluationen unabhängig von ihrer Position in ihren Argumentationen. Generell haben sie aber seltener auf die formative Evaluation verwiesen. Dies trifft besonders für die Parlamentsmitglieder zu, die eine Einführung der Grundstufe ablehnten. Unterschiedlich nutzten die

Tabelle 26: Grundstufe im Kantonsrat Zürich - politische Nutzung durch Parlament und Exekutive

\begin{tabular}{|c|c|c|c|c|c|}
\hline & & & \begin{tabular}{|l} 
Parlament \\
Verweise \\
oder Zitate \\
\end{tabular} & $\begin{array}{l}\text { andere Arten poli- } \\
\text { tischer Nutzung }\end{array}$ & \begin{tabular}{|l} 
Regierung/ \\
Verwaltung \\
Verweise \\
oder Zitate \\
\end{tabular} \\
\hline \multicolumn{3}{|c|}{ Anstoss - vorparlamentarische Phase } & & & \\
\hline Parlamentsmitglied: & Parl. Initiative 65/2010 & 15.03 .2010 & & & \\
\hline FDP & & & & & \\
\hline Plenum & Beratung 65/2010 & 05.07 .2010 & EDK-0st (2010) & Evaluation fordern & \\
\hline Regierung & Antrag Regierungsrat & 07.07 .2010 & & & $\begin{array}{l}\text { EDK-Ost (2010), } \\
\text { Bildungsdirektion } \\
\text { (2003) }\end{array}$ \\
\hline \multicolumn{6}{|c|}{ Parlamentarische Phase } \\
\hline Kommission & Beratung (Hearing, Antrag) & $\begin{array}{l}21.09 .2010- \\
02.11 .2010\end{array}$ & EDK-Ost (2010) & $\begin{array}{l}\text { Evaluationen kri- } \\
\text { tisieren, Experten } \\
\text { fragen }\end{array}$ & \\
\hline Plenum & Beratung und Rückweisung & 06.12 .2010 & $\begin{array}{l}\text { EDK-Ost (2010), } \\
\text { Präsentation KBIK }\end{array}$ & $\begin{array}{l}\text { Evaluationen } \\
\text { kritisieren }\end{array}$ & EDK-Ost (2010) \\
\hline Regierung & Antrag Regierungsrat & 28.09 .2011 & & & $\begin{array}{l}\text { EDK-Ost (2010), } \\
\text { Bildungsdirektion } \\
\text { (2003) }\end{array}$ \\
\hline Kommission & Beratung & $\begin{array}{l}10.01 .2012 \\
07.02 .2012\end{array}$ & EDK-Ost (2010) & $\begin{array}{l}\text { Evaluationen } \\
\text { fordern }\end{array}$ & $\begin{array}{l}\text { EDK-Ost (2010), } \\
\text { Bildungsdirektion } \\
\text { (2003) }\end{array}$ \\
\hline Plenum & Erste Lesung & 16.04 .2012 & EDK-Ost (2010) & $\begin{array}{l}\text { Evaluationen } \\
\text { kritisieren }\end{array}$ & \\
\hline Plenum & $\begin{array}{l}\text { Redaktionslesung, Schluss- } \\
\text { abstimmung }\end{array}$ & 02.07 .2012 & EDK-0st (2010) & & \\
\hline
\end{tabular}

Eingeschlossen sind nur Verweise, die auf eine Quelle schliessen lassen. Allgemeine Formulierungen wie «Evaluationen zeigen» usw. sind nicht in der Tabelle enthalten. 
Parlamentsmitglieder aber Evaluationen in anderen politischen Arten. Etwa forderten nur Parlamentsmitglieder in der parlamentarischen Phase Evaluationen, die entweder die freiwillige Einführung befürworteten oder die Einführung ablehnten. Kritisiert haben die Evaluationen nur jene Parlamentsmitglieder, die eine flächendeckende Einführung forderten oder die Einführung ganz ablehnten. Während Erstere vor allem die Messung des Lernstands ein Jahr nach der Grundstufe aufgriffen, kritisierten Letztere die mangelnde Vergleichbarkeit des Kindergartens und der Grundstufe.

\section{Evaluationsnutzung: Bedeutung}

Die Analyse der Interviews und der Dokumente legt nahe, dass Evaluationen im Gesetzgebungsprozess durchaus bedeutsam waren. Alle befragten Kommissionsmitglieder befinden die Durchführung und die Präsentation der Evaluation als sehr sinnvoll. Auch können sie sich an die Verwendung der Evaluationen in den Argumentationen erinnern. Obwohl sie eine solche Präsenz der Evaluationen in der Debatte zuweilen als unüblich befinden (vgl. F5/7-PM4), diskutieren die Befragten aber die substanzielle Bedeutung der Evaluationen kritisch:

Es ist wirklich ausgiebig über diese Evaluation diskutiert worden. Man hat diese Berichte bekommen, man konnte alles lesen, man hat sich gegenseitig mit den Argumenten beworfen, aber eben, jeder hat dann das rausgelesen, was er wollte. (F5/7-PM4)

Entsprechend habe die Evaluation die eigene Meinung bestärkt, aber zu keinem Meinungsumschwung geführt. Im Kantonsrat würden andere Faktoren zählen (F5/7-PM3, vgl. auch F5/7-PM4). Besondere Bedeutung kam etwa den Kosten zu, die für die Grundstufe deutlich höher ausfallen ohne sich in deutlichen Ergebnissen der Evaluation niederzuschlagen (F5/7-PM3, F5/7-PM4). Dagegen spricht das befragte Parlamentsmitglied, das die flächendeckende Grundstufe befürwortete, den Evaluationsergebnissen eine grosse Bedeutung zu. Für die eigene Position war dies jedoch negativ:

Der Killer war, nach drei Jahren sind die Kinder eh' wieder am gleichen Ort, [...] ich habe $\mathrm{x}$-mal versucht zu erklären, dass es ja gar nicht darum gegangen ist, dass die Kinder schneller vorwärts kommen, [...] man hat es nicht mehr umkehren können. (F5/7-PM5)

Folglich sind andere Aspekte wenig bedeutsam gewesen, obwohl diese für die Grundstufe gesprochen hätten. Etwa meint das Kommissionsmitglied, das die Grundstufe ablehnte, dass vor allem die Zufriedenheit ein ausschlaggebendes Argument hätte sein können (F5/7-PM2). Schliesslich teilt die befragte Person aus der Verwaltung den Eindruck der Parlamentsmitglieder: Die Parlamentsmitglieder hätten die Evaluationen zwar verhältnismässig oft und unabhängig von ihrer Position erwähnt, aber letztlich habe dies nicht viel verändert (F5/7-Verw.). 


\subsubsection{Basisstufe im Berner Grossen Rat (Fall 8)}

\section{Ablauf und Inhalt des Gesetzgebungsprozesses}

Die Basisstufe wurde in Bern wie die SSA in Fall 6 im Rahmen von REVOS 2012 diskutiert, die zwischen September 2011 und März 2012 Gegenstand der Beratungen des Grossen Rats war. Auch für die Basisstufe sah die Bildungsstrategie vor, einen politischen Entscheid in REVOS 2012 zu fällen (Erziehungsdirektion des Kantons Bern 2009, 30; Grosser Rat des Kantons Bern 2009c). Die Basisstufe war jedoch auch Bestandteil des Ziels, die Schulein-gangsstufe im Rahmen der Anpassungen an HarmoS neu zu regeln. ${ }^{169}$

Der Grosse Rat befasste sich bereits vor REVOS 2012 mehrfach mit der Basisstufe. Noch vor der Jahrtausendwende diskutierte er die Basisstufe aufgrund zweier Motionen einer Grossrätin der Grünen Freien Liste ${ }^{170}$. Beide Motionen forderten, die Einführung der Basisstufe vorzubereiten. Nachdem der Grosse Rat die erste Motion ablehnte, überwies er die zweite Motion 234/1999 mit einer knappen Mehrheit als Postulat. Darauffolgend beschloss der Regierungsrat 2004 unter Führung des damaligen Erziehungsdirektors Annoni (FDP), die Basisstufe in Schulversuchen von Schuljahr 2005/06 bis Ende Schuljahr 2009/10 zu erproben (EDK-Ost 2010, 47). Für den französischsprachigen Teil des Kantons konzipierte die ERZ mit dem Cycle élémentaire ein eigenes Modell, das sich an der Basisstufe und an der Schulentwicklung in der Romandie orientiert. Im Cycle élémentaire werden die beiden Jahrgänge der Kindergartenklasse und die ersten beiden Jahrgänge der Unterstufe je gemischt geführt. Diese gemischten Klassen des Kindergartens und der Unterstufe haben jedoch gemeinsame Projekte sowie Lerngruppen und die Lernziele sind wie bei der Basisstufe auf das Ende der zweiten Klasse ausgelegt (Fankhauser et al. 2011; Regierungsrat des Kantons Bern 2011b, 9). Aufgrund der Konzipierungsarbeiten startete der Versuch des Cycle élémentaire ein Jahr später. ${ }^{171}$

Weitere Male diskutierte der Grosse Rat die Basisstufe noch vor REVOS anhand fünf Vorstössen. ${ }^{172}$ Sämtliche dieser Vorstösse stammten von Grossratsmitgliedern der SVP oder der BDP. Während zwei Vorstösse die Basisstufe indirekt betrafen, äusserten sich die Grossratsmitglieder in den anderen drei Vorstössen skeptisch gegenüber der Basisstufe, bisweilen forderten sie gar, die Einführung der Basisstufe zu sistieren. Sämtliche dieser Vorstösse - in einem Fall nur einzelne Punkte davon - überwies der Grosse Rat an den Regierungsrat.

In der Vernehmlassung im November 2010 beantragte der Regierungsrat die freiwillige Einführung der Basisstufe oder des Cycle élémentaire. Da sich der Kanton finanziell an den Schulkosten beteiligt, sah der Regierungsrat für die Einführung der Basisstufe ein Bewilligungsverfahren durch die ERZ vor. Für diese Bewilligungen sah er eine Obergrenze vor sowie eine Reihe von Bedingungen, namentlich zu genügenden Kinderzahlen

169 Informationen zu weiteren Massnahmen von REVOS gibt Kapitel 5.4.3 zu Fall 6.

170 Die Grüne Freie Liste war eine eigene Partei und Fraktion im Grossen Rat. Sie schloss sich 2006 mit dem Grünen Bündnis zur Grünen Partei des Kantons Bern zusammen (Grüne Partei der Schweiz 2014, 13).

171 Der Regierungsrat verlängerte beide Schulversuche während des Gesetzgebungsprozesses je um zwei Jahre. 172 Motion 199/2008, 121/2010, 182/2010, dringliche Motion 308/2011 und Interpellation 230/2009. 
und Infrastruktur, zur Sicherung pädagogischer Massnahmen sowie zur Zustimmung der Beteiligten. Von einer flächendeckenden Einführung sah der Regierungsrat ab, da die Gemeinden im Kanton Bern je nach Bedarf und Akzeptanz ihr Modell wählen können sollen. Weiter sprachen auch Kostengründe dagegen. Zudem fügte der Regierungsrat an, dass sich der Kindergarten auch bewähre und es bereits eine Vielzahl an Reformen gäbe (Regierungsrat des Kantons Bern 2010b, 8-9). Die Parteien äusserten sich unterschiedlich zu diesem Vorschlag. Die SVP lehnte auch eine freiwillige Basisstufe komplett ab (SVP Kanton Bern 2011). Bei der EVP, FDP, den Grünen und der SP fand die Basisstufe Zustimmung (EVP Kanton Bern 2011; FDP Kanton Bern 2011; Grüne Kanton Bern 2011; SP Kanton Bern 2011). Allerdings betonte die FDP, dass sie die Basisstufe vornehmlich als bedürfnisgerechte Lösung für einzelne Gemeinden sehe. Die SP und die Grünen hingegen hätten auch eine flächendeckende Einführung begrüsst.

Im Juni 2011 legte der Regierungsrat seinen Antrag zur Revision des VSG-BE vor. Bezüglich der Basisstufe nahm der Regierungsrat im Vergleich zur Vernehmlassung nur Änderungen betreffend die Voraussetzungen für die Bewilligung vor. So strich er die Zustimmung der Lehrpersonen und zuständigen Gemeindeorgane als Bedingung für die Bewilligung (Regierungsrat des Kantons Bern 2011a, 31).

In einer eintägigen Sitzung im September 2011 beriet die dafür gegründete Kommission Volksschulgesetz den Regierungsratsantrag in der ersten Lesung. Die Kommission bestand aus 17 Mitgliedern, das Präsidium hatte die EVP inne. Regierung und Verwaltung präsentierten zunächst die Vorlage, Eintreten auf die Vorlage war unbestritten. Die Basisstufe war zwar ein zentrales Thema in der folgenden Detailberatung der Kommission, Anträge zur Basisstufe lehnte die Kommission aber ab und schlug daher keine inhaltlichen Änderungen vor.

Auch in der darauffolgenden ersten Lesung zur Basisstufe im Grossen Rat im November 2011 war Eintreten unbestritten. Der Grosse Rat diskutierte die Basisstufe auf der Grundlage von zwei Anträgen. ${ }^{173}$ Ein Antrag der SVP sah die Streichung der Basisstufe vor, während der zweite Antrag der CVP die Finanzierung der Basisstufe den Gemeinden überlassen wollte. Beide Anträge lehnte der Grosse Rat deutlich mit 84.7 Prozent respektive 70.3 Prozent ab. Während die CVP, FDP, Grünliberale und Teile der SVP den zweiten Antrag unterstützten, stimmte nur eine Minderheit der SVP für den Antrag zur Streichung der Basisstufe, der von einer Grossrätin aus ihren Reihen kam. Diese Spaltung der SVP zur Frage der Basisstufe zeigte sich bereits während der Debatte. Mehrere SVP-Grossratsmitglieder sprachen sich für die Basisstufe aus. Sie begründeten dies vor allem damit, dass so Gemeinden die untersten Stufen der Volksschule selbst führen könnten, was angesichts kleiner und generell sinkender Schülerzahlen ansonsten nicht möglich wäre (Grosser Rat des Kantons Bern 2011c, 1059). Zusätzlich zu diesen Anträgen zur Basisstufe beantragte die FDP, Mehrjahrgangsklassen von Kindergarten und Primarstufe oder Primar- und Realstufe zu erlauben, falls die örtlichen Gegebenheiten dies erfordern. Da dieser Antrag den gemeinsamen Unterricht von Kindergarten und

173 Einen dritten Antrag zog die Antragstellerin der SVP zu Beginn der Beratungen zurück. Sie beantragte damit dieselben Rahmenbedingungen der Basisstufe für konventionelle Kindergartenklassen. 
Primarstufe ohne die Pädagogik und Rahmenbedingungen der Basisstufe ermöglichen würde, bezeichneten ihn einige Grossratsmitglieder als "Basisstufe light» (Grosser Rat des Kantons Bern 2012, 359). Die befürwortende Seite sah in diesem Antrag hingegen die Möglichkeit, dass Gemeinden Kindergartenklassen mit der Primarstufe zusammen führen können, auch wenn sie die Bedingungen für die Basisstufe, z. B. betreffend Schülerzahlen, nicht erfüllen. Da die FDP diesen Antrag erstmals in der ersten Lesung im Grossen Rat stellte, beschloss der Grosse Rat, den Antrag zur Vorberatung in die Kommission zu geben.

In der zweiten, halbtägigen Lesung der Kommission sowie in der zweiten Lesung im Grossen Rat war die Basisstufe nur noch Diskussionsthema in Verbindung mit dem Antrag der FDP zur so genannten Basisstufe light. Entgegen dem Antrag der SP, den gemeinsamen Unterricht von Kindergartenklassen und der ersten Jahre der Primarstufe an die sinngemässe Erfüllung der Bedingungen zu knüpfen, sprach sich der Grosse Rat für den Regierungsratsantrag aus. Dieser sah vor, Mehrjahrgangsklassen aus Kindergarten und den ersten Jahren der Primarstufe zu erlauben, um eine wohnortsnahe Schulung zu ermöglichen. In der Schlussabstimmung nahm der Grosse Rat das revidierte VSG-BE mit $136 \mathrm{zu} 4$ Stimmen bei 6 Enthaltungen an. Wie bereits in 5.4 erläutert, stimmten lediglich einzelne Grossratsmitglieder der SVP dagegen. Der Stimme enthielten sich Mitglieder der SVP und der SP. Es wurde kein Referendum gegen das VSG-BE ergriffen.

\section{Evaluationsnutzung}

Bereits vor der Vernehmlassung von REVOS 2012 thematisierten die Grossratsmitglieder Evaluationen im Rahmen der Diskussion der parlamentarischen Vorstösse. Die Interpellation 230/2009 bezog sich hauptsächlich auf die Evaluation von EDK-Ost 4bis8. Die Antragstellerin kritisierte darin Evaluationen, indem sie fragte, inwiefern die Ergebnisse zwischen dem Kindergarten und der Grund- oder Basisstufe angesichts der unterschiedlichen Ressourcen vergleichbar seien. Weiter fragte sie, ob der Regierungsrat die bisherigen Ergebnisse auch «zum Teil ernüchternd» einschätzt und ob es weitere Ergebnisse aus anderen Kantonen gäbe. In seiner Antwort bezog sich der Regierungsrat auf die Zwischenberichte der Evaluation (Moser et al. 2008; Vogt et al. 2008) sowie eine Zusammenfassung (Grossenbacher 2008), die ein Jahr vor Einreichung der Interpellation erschienen waren. Weiter verwiesen die Grossratsmitglieder in den Vorstössen 121/2010 und 182/2010 auf den Projektschlussbericht der EDK-Ost (2010), der einige Monate vor der Einreichung der Vorstösse publiziert worden war. Sie forderten die Sistierung der Basisstufe, da die Kinder in diesem Modell keine besseren Lernfortschritte erreichen würden, aber die Basisstufe viel teurer sei als der Kindergarten.

Die Evaluation ${ }^{174}$ von EDK-Ost 4bis8 diente auch der ERZ bei der Ausarbeitung der Vorlage. Die ERZ sei mit dem Bericht relativ sachlich und unaufgeregt umgegangen, der Bericht lasse aber verschiedene Interpretationen zu: «Wenn Sie knallhart auf die Ergebnisse gehen, was können die Schüler, ja, dann ist er relativ ernüchternd» (F6/8-Verw.).

174 Im Interview konkret genannt wurden: Projektschlussbericht, Zwischen- und Schlussberichte der (formativen und summativen) Evaluation (F6/8-Verw.). 
Daher habe die ERZ «relativ offen gesagt: ja, die Leistungen sind nicht besser, aber das sei auch nicht das Ziel gewesen» (F6/8-Verw.). In seinem Vortrag zur Vernehmlassung fasste der Regierungsrat die wichtigsten Ergebnisse auf einer halben Seite zusammen, wobei er Ergebnisse aus der summativen und der formativen Evaluation integrierte. In «der Überzeugung, dass die Basisstufe ein zukunftsgerichtetes Modell ist und gestützt auf die Erfahrungen im Schulversuch und die Ergebnisse der Evaluation schlug» der Regierungsrat des Kantons Bern (2010b, 7) daher vor, die Basisstufe einzuführen. Ebenfalls nannte er Ergebnisse der Evaluation des Cycle élémentaire, die aber zu diesem Zeitpunkt noch nicht abgeschlossen war. Dies betraf wieder summative Fragen wie den Lernfortschritt, aber auch formative Fragen wie die Zufriedenheit der Lehrpersonen. Auch verwies der Regierungsrat auf eine Zusammenfassung, allerdings allgemeiner im Zusammenhang mit der frühen Förderung (Stamm 2009). Neben diesen Evaluationen oder Zusammenfassungen enthielt der Vortrag einen Verweis auf den Bericht der EDK (1997) zur Eingangsstufe. Die gleichen Verweise enthält auch der spätere Regierungsratsantrag.

In Stellungnahmen und Medienmitteilungen als Reaktionen auf die Vernehmlassung verwiesen auch die Parteien auf Evaluationen. Dabei beschränkten sie sich auf die Ergebnisse zum Lernstand. Die FDP nahm eine skeptische Position ein, da «die heute bekannten Studien zeigen, dass die beiden Modelle (Basisstufe und «ordentliche` Klassenzüge) mit Blick auf den massgebenden Schulerfolg gleichwertig sind (FDP Kanton Bern 2011, 2). Die SVP begründete ihre Ablehnung der Basisstufe mit den «Auswertungen des Schulversuches» (SVP Kanton Bern 2011, 2). Bereits kurz nach Erscheinen des Projektschlussberichts hatte die SVP eine Medienmitteilung publiziert, in der sie das Beenden des Schulversuchs forderte. Darin sprach sie zwar von den Ergebnissen der Evaluation, die Ausführungen lassen aber weder auf eine Quelle noch auf ein systematisches und transparentes Vorgehen schliessen. Die verfügbaren Stellungnahmen der EVP, Grünen sowie der SP enthalten dagegen keine Hinweise auf Evaluationen.

Anlässlich der Diskussion von Vorstössen befasste sich der Grosse Rat auch nach der Vernehmlassung und vor den Kommissionsberatungen zweimal mit der Basisstufe und der Evaluation von EDK-Ost 4bis8. Angesichts der kommenden Beratungen des VSG-BE verzichtete der Grosse Rat auf eine Diskussion der Motion 121/2010. Daher sprach nur der Antragsteller der BDP, der seine Forderung wie bereits im Vorstosstext mit den Ergebnissen der Evaluation begründete. Er machte aber deutlich, dass es um die Mehrkosten der Basisstufe gehe:

Zum Pädagogischen äussere ich mich hier nicht; das ist nicht der Kernpunkt. Liest man jedoch den Bericht und spricht man mit Leuten, die sich nicht so laut äussern wie die Befürworter der Basisstufe, so muss man ehrlicherweise sagen, dass der Versuch nicht ganz das gebracht hat, was er hätte bringen sollen. (Grosser Rat des Kantons Bern 2011b, 313)

Dagegen diskutierte der Grosse Rat später ausführlicher die Motion 182/2010 der SVP. Eine der Antragstellerinnen dieser Motion, die auch die Interpellation 230/2009 einreichte, forderte, «dass man jetzt einfach diese Ergebnisse als Facts nimmt und daraus die 
entsprechenden Konsequenzen zieht» (Grosser Rat des Kantons Bern 2011d, 778). Diese Grossrätin verwies ausführlich auf die Evaluation von EDK-Ost 4bis8 und integrierte Zitate der Autorenschaft der Evaluationsberichte in ihre Rede, wobei diese Zitate aus Zeitungsartikeln stammten. ${ }^{175}$ Zwei Grossrätinnen der Grünen und der SP, die sich gegen den Vorstoss aussprachen, diskutierten diese Verweise auf Evaluationen danach kritisch:

Mir ist schon oft aufgefallen, dass sie sehr gerne aus Studien zitiert. Aber wenn man selber dieselben Studien gelesen hat, stellt man fest, dass sie sehr ausgewählt zitiert. Es war nämlich nicht das Ziel oder die Idee, die der Basisstufe zugrunde liegt, die Leistung der vier- bis achtjährigen Kinder zu steigern. (Grosser Rat des Kantons Bern 2011d, 776)

Diese Hinweise in den Reaktionen zur Vernehmlassung sowie in der Diskussion der Vorstösse lassen insgesamt darauf schliessen, dass sich einige Grossratsmitglieder bereits vor der Kommissionsberatung - wenn auch mit divergierenden Interpretationen - vertieft mit der Evaluation von EDK-Ost 4 bis8 respektive dem Projektschlussbericht befassten. ${ }^{176}$

Die Grossratsmitglieder setzten sich danach auch in der Kommissionsberatung mit dem Projektschlussbericht auseinander, den alle Kommissionsmitglieder erhielten (F6/8-PM1). Vier der fünf befragten Kommissionsmitglieder ${ }^{177}$ können sich an den Bericht erinnern und hatten ihn zumindest «relativ diagonal gelesen» (F6/8-PM4). Für die befragten Kommissionsmitglieder, die sich für die Basisstufe aussprachen, sind das soziale Lernen, das Auffangen der heterogenen Entwicklungsstände, die pädagogische Kontinuität sowie die Haltung der Lehrpersonen wesentliche Ergebnisse des Berichts (F6/8-PM1, F6/8-PM5). Das befragte Kommissionsmitglied, das die Basisstufe klar ablehnte, betont hingegen die Ergebnisse zum Lernstand, zum Wohlbefinden und zum Selbstvertrauen (F6/8-PM2). An diese Ergebnisse erinnert sich auch das vierte Mitglied, das der Basisstufe aus Kostengründen eher skeptisch gegenüberstand. Für eine solche Argumentation seien die Kenntnisse über die Evaluation ausreichend gewesen, meinte dieses Mitglied, «aber wenn ich jetzt gewollt hätte, das [die Basisstufe] qualitativ in Frage zu stellen, [...] dann hätte ich mich viel, viel intensiver damit beschäftigen müssen» (F6/8-PM4). Den Projektschlussbericht finden die befragten Kommissionsmitglieder sehr hilfreich und übersichtlich: «Nur schon wer die Einleitung hatte, hat eigentlich einen guten ersten Eindruck gehabt. Also mich hat das eine sehr wertvolle Studie gedünkt» (F6/8-PM5). Teilweise relativieren die Befragten aber, dass sie sich bereits lange mit der Basisstufe befassten (F6/8-PM2; F6/8-PM5). Auch hatten sie sich anderweitig über den Dokumentarfilm oder Schulbesuch über die Basisstufe informiert (F6/8-PM4, F6/8-PM5).

175 Verwendete Quellen: EDK-Ost (2010); Sommer (2011) sowie ein Artikel aus der Neuen Luzerner Zeitung.

176 In den Interviews kann die analytische Nutzung der Kommissionsmitglieder kaum zeitlich eingeordnet werden.

177 F6/8-PM1, F6/8-PM2, F6/8-PM4, F6/8-PM5. 
Übereinstimmend mit den Informationen aus den Interviews enthalten die Kommissionsprotokolle mehrere Verweise auf die Evaluation von EDK-Ost 4bis8. Während der Detailberatung der Basisstufe gab die Regierung und Verwaltung Ergebnisse der formativen und summativen Evaluation wieder. Auch stellte sie klar, dass eine bessere Leistung der Kinder nicht das Ziel gewesen sei, sondern eine Steigerung des Wohlbefindens. Auch Kommissionsmitglieder der befürwortenden und gegnerischen Seite verwiesen auf die Evaluation, sie nannten die Quelle aber selten explizit. Wiederum hoben die Kommissionsmitglieder der gegnerischen Seite die Ergebnisse der summativen Evaluation hervor, während die Kommissionsmitglieder der befürwortenden Seite eher die Ergebnisse der formativen Evaluation thematisierten. Daneben zitierte ein Kommissionsmitglied Statistiken zur Anzahl der Unterrichtslektionen (Schweizerische Koordinationsstelle für Bildungsforschung 2010).

Trotz dieser vertieften Auseinandersetzung der befragten Kommissionsmitglieder mit dem Projektschlussbericht stellt sich die Frage, wie bekannt er über den Kreis der Kommission hinaus war. Ein befragtes Kommissionsmitglied sprach auch Personen aus anderen Fraktionen auf den Projektschlussbericht an und schätzt, dass er bei den Kommissions- und auch weiteren Grossratsmitgliedern bekannt war (F6/8-PM5). Auch ein anderes befragtes Kommissionsmitglied sprach besonders die eigenen Parteimitglieder darauf an, aber kommt gegensätzlich zum Schluss, dass der Bericht schlecht zur Kenntnis genommen worden sei (F6/8-PM2). Auf eine geringe Bekanntheit weist auch das Interview mit einem stellvertretenden Kommissionsmitglied, das sich erst bei der zweiten Lesung an den Beratungen beteiligte. Dieses stellvertretende Kommissionsmitglied hatte den Projektschlussbericht nicht erhalten. Es kennt zwar die Ergebnisse zum Lernstand, irrtümlicherweise nimmt es aber an, dass diese bei der Beratung des VSG-BE noch nicht bekannt waren (F6/8-PM3).

In der ersten Lesung im Plenum war die Evaluation dann einer von mehreren Gesichtspunkten. Ein - teilweise zentraler - Bestandteil war sie nur in einer Minderheit der Reden der Grossratsmitglieder. "Bildungsqualität kann auch ohne Basisstufe erreicht werden. Das steht so auch im Evaluationsbericht der Schweizerischen Konferenz der kantonalen Erziehungsdirektoren (EDK)", sagte etwa ein Grossrat aus der Fraktion der CVP-Grünliberalen und beantragte, dass die Gemeinden die (freiwillige) Basisstufe finanzieren sollten (Grosser Rat des Kantons Bern 2011c, 1040). Grossratsmitglieder, die eine Einführung der Basisstufe begrüssten, wendeten dagegen ein, dass die Kosten auch gemäss dem Bericht der EDK-Ost unklar seien und zudem die Zielsetzung der Basisstufe erreicht worden sei (Grosser Rat des Kantons Bern 2011c, 1040, 1058). Auch der Kommissionspräsident sowie eine SVP-Grossrätin, die gegen die Basisstufe opponierte, erwähnten den Bericht (Grosser Rat des Kantons Bern 2011c, 1038, 1058). Demgegenüber war die Evaluation kein Bestandteil der Mehrheit der Reden der Grossratsmitglieder, der Regierungsrat bezog sich ebenfalls nicht darauf. Vielmehr sprachen die Grossratsmitglieder über die Finanzierung der Basisstufe sowie deren Attraktivität für ländliche Gemeinden. Zudem begründeten die Grossratsmitglieder ihre Position aus persönlicher Erfahrung, etwa ein Grossrat der SVP: 
Meine Tochter ist Schulleiterin in Matten, in einer Projektschule für die Basisstufe. Ich habe ebenfalls zwei Grosskinder, welche die Basisstufe besuchen. Gemessen an den Kenntnissen, die ich an der Front im Namen der Familie gewinnen durfte, bin ich ein überzeugter Vertreter der Basisstufe (Grosser Rat des Kantons Bern 2011c, 1060).

In der darauffolgenden Kommissionsberatung für die zweite Lesung verwiesen die Mitglieder nur vereinzelt auf Evaluationen. Am Rande der Kommissionssitzung griff ein Mitglied zweimal auf, dass die Kinder gemäss dem Projektschlussbericht bereits früher lesen, rechnen und schreiben können und dies bei einem Umzug in eine andere Gemeinde Probleme bereiten könne. Die Kommission ging aber nicht weiter darauf ein. Auch die Regierung und Verwaltung erwähnten Evaluationen nicht.

Die Grossratsmitglieder verwiesen in der zweiten Lesung im Plenum ebenfalls nur im Einzelfall auf Evaluationen. Die Basisstufe war indirekt ein Thema bei der Beratung des Artikels, der Mehrjahrgangsklassen von Kindergarten und den ersten Primarstufen ausnahmsweise ermöglichen sollte. Um ihre Ablehnung dieses Artikels zu begründen, erwähnten zwei Grossratsmitglieder der SP Evaluationen. Eine Grossrätin las aus dem Projektschlussbericht vor:

Im letzten Kapitel des Schlussberichts kann man lesen: Allfällige Hoffnungen, die innovativen Elemente und die Vorteile, welche die erprobten Eingangsstufenmodelle auszeichnen, seien ohne Weiteres auch in traditionellen Modellen realisierbar, dürften enttäuscht werden. [...] (Grosser Rat des Kantons Bern 2012, 362).

Die Schlussreden, die allerdings nur zwei Grossratsmitglieder, der Kommissionspräsident sowie der Regierungsrat hielten, enthalten dagegen keine Verweise auf Evaluationen. Auch die wenigen verfügbaren Medienmitteilungen der Parteien in der gesamten parlamentarischen Phase enthalten keine solchen Verweise.

\section{Evaluationsnutzung: Zeitpunkt und Quellen}

Gemäss Abbildung 26 waren sämtliche 22 Evaluationen und 3 Zusammenfassungen bis zur ersten Lesung in der Kommission am 22. September 2011 und damit auch bis zur ersten Lesung im Grossen Rat verfügbar. Die Zwischenberichte der Evaluation von EDKOst 4bis8 lagen bereits bei den ersten eingereichten Vorstössen im September 2008 vor. Der Projektschlussbericht der EDK erschien im Juni 2010 und entsprechend vor der Diskussion der Vorstösse, die vor der Beratung des VSG-BE stattfand. Da im Gegensatz zu Fall 7 in Zürich keine Präsentation stattfand, sind in Fall 8 zwei Evaluationen weniger verfügbar. 


\section{Abbildung 26: Zeitliche Verfügbarkeit der Evaluationen zur Basisstufe im Berner Grossen Rat}

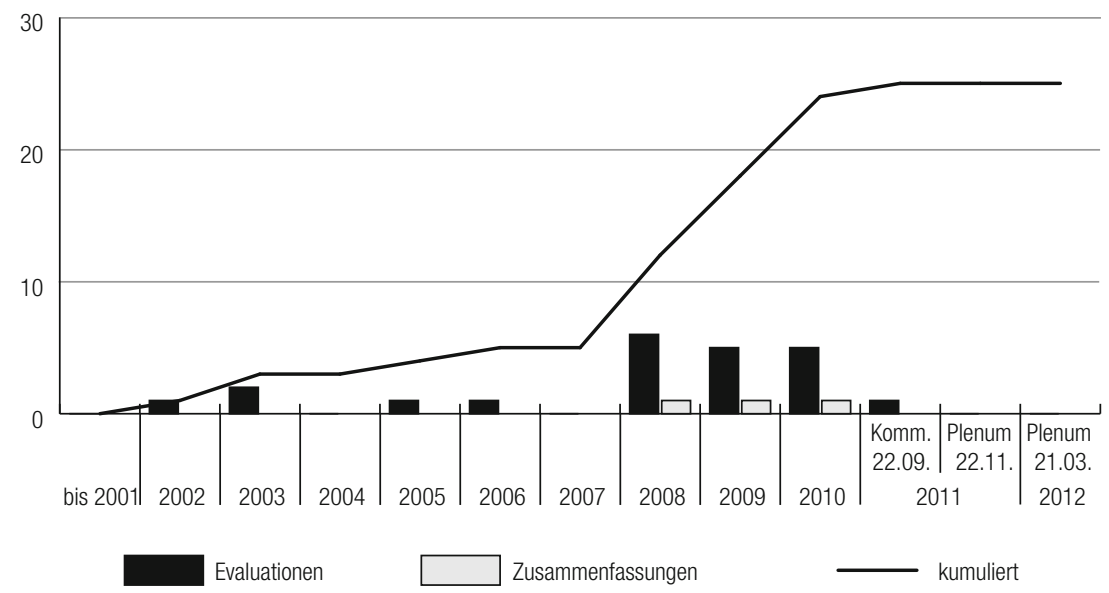

Die Anzahl bezieht sich auf die in einem Jahr beziehungsweise bis zum gegebenen Zeitpunkt erschienenen Evaluationen.

Von allen diesen Quellen konzentrierte sich die Evaluationsnutzung klar auf den Projektschlussbericht der EDK-Ost (2010). Die befragten Kommissionsmitglieder beziehen sich stets und nur auf diesen Bericht. Mehrere Befragte haben sich ausführlich damit auseinandergesetzt und können sich an diverse Inhalte erinnern (F6/8-PM2; F6/8-PM5). Wie die Übersicht in Tabelle 27 zeigt, verwiesen die Parlamentsmitglieder auch stets auf den Projektschlussbericht. In ihren Argumentationen nannten sie die Quelle nicht immer klar. Vielmehr sprachen sie von «signifikant besseren Lernleistungen», von "Auswertungen» oder "Untersuchungen» (Grosser Rat des Kantons Bern 2011d; SVP Kanton Bern 2011). Dennoch kann vermutet werden, dass es sich dabei um Hinweise auf den Projektschlussbericht der EDK-Ost (2010) handelt: Sämtliche Kommissionsmitglieder erhielten den Projektschlussbericht und auch in den Interviews thematisierten sie nur ihn. Andere Arten der politischen Nutzung lassen sich hingegen seltener beobachten. Eine Grossrätin stellte in der Interpellation 230/2009 die Vorgehensweise der Evaluation in Frage und erkundigte sich nach Ergebnissen aus weiteren Kantonen. In der Beratung des Vorstosses 182/2010 kritisierten zwei Grossrätinnen weniger Evaluationen als den selektiven Umgang damit durch eine andere Grossrätin. Die Verwaltung und Regierung stützte sich ebenso auf den Projektschlussbericht und allgemein auf die Evaluation EDKOst 4bis8. Die verschiedenen Verweise in Tabelle 27 deuten aber auf einen Einbezug von zusätzlichen Quellen.

Die Grossratsmitglieder bezogen sich mit dem Projektschlussbericht zwar auf eine aktuelle Quelle, sie aktualisierten die Quellen danach aber nicht. So bezog sich die Interpellation 230/2009 auf die Zwischenberichte der Evaluation von EDK-Ost 4bis8, die ein Jahr zuvor erschienen. Auch den Projektschlussbericht griffen die Parlamentsmitglieder 


\section{Tabelle 27: Basisstufe im Berner Grossen Rat - politische Nutzung durch Parlament und Exekutive}

\begin{tabular}{|c|c|c|c|c|c|}
\hline & & & $\begin{array}{l}\text { Verweise } \\
\text { oder Zitate }\end{array}$ & $\begin{array}{l}\text { ament } \\
\text { andere Arten poli- } \\
\text { tischer Nutzung }\end{array}$ & $\begin{array}{l}\text { Regierung/ } \\
\text { Verwaltung } \\
\text { Verweise } \\
\text { oder Zitate }\end{array}$ \\
\hline \multicolumn{3}{|c|}{ Anstoss - vorparlamentarische Phase } & & & \\
\hline $\begin{array}{l}\text { Parlamentsmitglied: } \\
\text { SVP }\end{array}$ & Motion 199/2008 & 01.09 .2008 & & & \\
\hline Plenum & Überweisung 199/2008 & 08.09.2008 & & & \\
\hline $\begin{array}{l}\text { Parlamentsmitglied: } \\
\text { SVP }\end{array}$ & Interpellation 230/2009 & 04.06 .2009 & & $\begin{array}{l}\text { Evaluation kriti- } \\
\text { sieren; Experten } \\
\text { fragen }\end{array}$ & $\begin{array}{l}\text { Moser et al. } \\
\text { (2008); } \\
\text { Vogt et al. (2008); } \\
\text { Grossenbacher } \\
\text { (2008) }\end{array}$ \\
\hline $\begin{array}{l}\text { Parlamentsmitglied: } \\
\text { BDP }\end{array}$ & Motion 121/2010 & 25.08.2010 & EDK-0st (2010) & & \\
\hline $\begin{array}{l}\text { Parlamentsmitglied: } \\
\text { SVP }\end{array}$ & Motion 182/2010 & 15.10 .2010 & EDK-0st (2010) & & EDK-Ost (2010) \\
\hline Regierungsrat & Vernehmlassung & 01.11 .2010 & EDK-Ost (2010) & & $\begin{array}{l}\text { EDK-Ost (2010); } \\
\text { Fankhauser (2011, } \\
\text { [im Erscheinen]) }\end{array}$ \\
\hline Plenum & Überweisung 121/2010 & 28.03.2011 & EDK-0st (2010) & & \\
\hline Regierung & Antrag Regierungsrat & 08.06.2011 & & & $\begin{array}{l}\text { EDK-Ost (2010), } \\
\text { Fankhauser (2011) }\end{array}$ \\
\hline Plenum & Teil-Überweisung 182/2010 & 05.09.2011 & EDK-Ost (2010) & $\begin{array}{l}\text { Evaluation kriti- } \\
\text { sieren; Experten } \\
\text { fragen }\end{array}$ & \\
\hline \multicolumn{6}{|c|}{ Parlamentarische Phase } \\
\hline Kommission & $\begin{array}{l}\text { Beratung (Erste Lesung, } \\
\text { Antrag) }\end{array}$ & 22.09.2011 & EDK-Ost (2010) & & EDK-Ost (2010) \\
\hline Plenum & Erste Lesung & $\begin{array}{l}22.11 .2011- \\
23.11 .2011\end{array}$ & EDK-0st (2010) & & \\
\hline $\begin{array}{l}\text { Parlamentsmitglied: } \\
\text { SVP }\end{array}$ & Dringliche Motion 308/2011 & & & & \\
\hline Kommission & $\begin{array}{l}\text { Beratung (Zweite Lesung, } \\
\text { Antrag) }\end{array}$ & 16.01.2012 & EDK-0st (2010) & & \\
\hline Plenum & Überweisung 308/2011 & 19.03.2012 & & & \\
\hline Plenum & $\begin{array}{l}\text { Zweite Lesung, } \\
\text { Schlussabstimmung }\end{array}$ & 21.03 .2012 & EDK-0st (2010) & & \\
\hline
\end{tabular}

Eingeschlossen sind nur Verweise, die auf eine Quelle schliessen lassen. Allgemeine Formulierungen wie «Evaluationen zeigen» usw. sind nicht in der Tabelle enthalten. 
zwei Monate nach Erscheinen in der Motion 121/2010 auf. Danach blieb es bei Verweisen darauf. Die Schlussberichte der formativen und summativen Evaluation sowie die Evaluation des Cycle élémentaire haben die Grossratsmitglieder nicht verwendet. Allerdings hielt etwa bei der Detailberatung der Basisstufe nur eine Grossrätin ihre Rede auf Französisch.

\section{Evaluationsnutzung: Positionen und Argumente}

In Fall 8 haben Parlamentsmitglieder mit unterschiedlichen Positionen zur Basisstufe Evaluationen genutzt, aber unterschiedliche Aspekte betont. Die SVP auf der gegnerischen Seite der Basisstufe verwies auf die Ergebnisse der summativen Evaluation zu den Lernfortschritten. Auch kritisierte sie die mangelnde Vergleichbarkeit mit dem Kindergarten. Sie schrieb etwa in einer Medienmitteilung, dass «die Basisstufe trotz mehr eingesetzten Ressourcen keine signifikant besseren Testergebnisse für die Kinder erbrachte als das bisherige Modell» (SVP Kanton Bern 2010). Den Kindergarten als gleichwertiges Angebot betonten auch jene Parlamentsmitglieder, die sich für eine freiwillige Einführung der Basisstufe mit der vollständigen Finanzierung durch die Gemeinden aussprachen. Die befürwortende Seite der freiwilligen Einführung der Basisstufe ging zwar auch auf den Lernstand ein, aber wendete ein, dass eine Leistungssteigerung nicht Ziel des Projekts gewesen sei. Die Verweise von dieser Seite fokussierten daher auf Ergebnisse der formativen Evaluation. So erwähnen diese Parlaments- und Kommissionsmitglieder die Zufriedenheit der Lehrpersonen, Behörden sowie der Erziehenden und die Konzipierung der Basisstufe als kindgerechtes Modell. Ähnlich argumentierte die Regierung und Verwaltung, etwa in der Antwort zur Motion 182/2010: «Die Basisstufe ist ein Modell, welches den Kindern unbestritten einen kindgerechten Übertritt vom Kindergarten in die Unterstufe ermöglicht. Dies gelingt - wie die Evaluation des interkantonalen Schulversuchs gezeigt hat - ohne dass die schulische Leistung der Kinder darunter leidet» (Grosser Rat des Kantons Bern 2011d, 774). Diese unterschiedliche Gewichtung der Ergebnisse zeigte sich auch in den Interviews. So sind für das befragte Kommissionsmitglied auf der gegnerischen Seite die Ergebnisse aus der summativen Evaluation zentral (F6/8-PM2), während die befürwortenden Kommissionsmitglieder eher das soziale Lernen, die pädagogische Kontinuität oder die Haltung der Lehrpersonen hervorheben (F6/8-PM1, F6/8-PM5).

Diese Ausführungen zeigen, wie bereits in Fall 7, dass trotz übereinstimmenden Ergebnissen aus wenigen Evaluationen mehrere Lesarten möglich sind:

Es ist nicht klar, die Basisstufe ist gut, die Basisstufe bringt mindestens die gleichen Resultate, es ist sicher für schwächere Kinder eher gut, und nachher erschöpft es sich dann schon schnell und dann wird es wirklich von beiden Seiten instrumentalisiert (F6/8-PM1).

Die Befragten erinnern sich daher, dass sich die Grossratsmitglieder gegenseitig einen einseitigen Umgang mit der Evaluation vorgeworfen haben (vgl. F6/8-PM2, F6/8-PM5). In einer Medienmitteilung sowie in der Plenumsdebatte der Motion 182/2010 hat etwa 
die SVP argumentiert, der Regierungsrat beachte die Forschungsergebnisse nicht und wolle die Basisstufe aus ideologischen Gründen einführen. Gleichzeitig haben Grossrätinnen der Grünen und der SP die einseitige Darstellung der Evaluationsergebnisse namentlich durch eine Grossrätin der SVP kritisiert. Schliesslich relativeren mehrere Befragte auch, dass es in der Bildungsforschung kaum eindeutige Resultate gäbe und besonders die Lehrperson und weniger die Schulorganisation entscheidend sei (F6/8-PM1, F6/8-PM4).

\section{Evaluationsnutzung: Bedeutung}

Die Mehrheit der Kommissionsmitglieder schreibt den Evaluationen in den Interviews eine gewisse Bedeutung für den Gesetzgebungsprozess zu. Es sei wichtig gewesen, dass es den Projektschlussbericht gegeben habe, da wenig Erfahrungswissen vorhanden gewesen sei (F6/8-PM4). Gleichzeitig relativieren die Befragten die Bedeutung der Evaluationen. Einerseits erklären sie sich dies durch die zu wenig deutlichen Resultate:

wenn es jetzt irgendwo heisst, die Selbständigkeit ist ganz ein wenig grösser bei den Basisstufenschülern, dann ist das durchaus in einem PH-Bereich [...], beschäftigt man sich damit, aber in der Politik nicht. (F6/8-PM4).

Anderseits erachten die befragten Kommissionsmitglieder andere Aspekte als relevanter. So hätten Evaluationen bei den Einen vielleicht eine Rolle in Form einer Bestätigung gespielt. Andere Aspekte der Basisstufe seien aber bedeutsam gewesen, wie etwa die Organisation und das Erhalten von kleinen Schulen oder das Einsparen von Kosten durch die Integration von Kindern (F6/8-PM5). Solche Aspekte hätten die Position der Basisstufe gestärkt:

[Anm.: Bei der Basisstufe,] dort bin ich überzeugt, haben nicht Berichte eine Rolle gespielt, sondern dort hat wirklich der Erfolg von der Basisstufe auf dem Land eine Rolle gespielt, [...] dass nachher SVP-Vertreter und zwar namhafte SVP-Vertreter ans Rednerpult gestanden sind und gesagt haben, ich war selbst in einer Basisstufe und ich finde es gut. (F6/8-PM1).

Auch die befragte Person in der Verwaltung befindet andere Faktoren für den Erfolg der Basisstufe als ausschlaggebend. Die Evaluation sei aber für das gesamte Bildungsumfeld und gerade für die Entwicklung des Kindergartens wirkungsvoll gewesen:

Wenn man den Versuch, die Ergebnisse rein nur auf die Basisstufe fokussiert und ihn zum Beispiel nur daran misst, ob das jetzt in einem Kanton eingeführt werden konnte oder nicht, dann greift das zu kurz für mich. Die gesamte Debatte, der Versuch, die Diskussion über den Bericht hat in den Köpfen viel mehr ausgelöst. [...] Wir haben nachher auch die gesamte Gesetzesrevision unter diesen roten Faden der Flexibilisierung gestellt; Flexibilisierung der Übergänge (F6/8-Verw.). 
Das befragte Kommissionsmitglied, das sich gegen die Basisstufe aussprach, findet hingegen, dass die Ergebnisse nicht umgesetzt wurden und schreibt den Evaluationen keine Bedeutung zu (F6/8-PM2).

\subsubsection{Fazit: Präsente Evaluation zur Basis- respektive Grundstufe - analytische und politische Nutzung in Zürich und Bern}

Die vorangehenden Abschnitte stellten die Fallstudien zur Evaluationsnutzung bei der Diskussion der Basis- respektive Grundstufe vor. In beiden Fällen nutzten die Parlamentsmitglieder Evaluationen durchaus analytisch und auch politisch, wobei sich die Art der politischen Nutzung jedoch unterscheidet.

Der Gesetzgebungsprozess zur Einführung der Grundstufe in Fall 7 im Kanton Zürich war von Anfang an konfliktgeprägt. Die Grundstufe wurde bereits anlässlich der Totalrevision des VSG-ZH im Jahr 2002 intensiv diskutiert, die Stimmberechtigten lehnten die Einführung der Grundstufe aber ab (Kantonsrat Zürich 2004). Den Gesetzgebungsprozess zu Fall 7 löste dann eine Initiative des überparteilichen Vereins Chance Volksschule aus. Auf Antrag des Kantonsrats arbeitete die Regierung eine Umsetzungsvorlage zur flächendeckenden Einführung der Grundstufe sowie einen Gegenvorschlag

\section{Tabelle 28: $\quad$ Grundstufe im Kantonsrat Zürich - Übersicht der Nutzung}

\begin{abstract}
Parlament Analytisch: Differenzierte Auseinandersetzung mit Evaluationen
Alle befragten Kommissionsmitglieder haben den Projektschlussbericht von EDK-Ost 4bis8 erhalten. Vertieft damit auseinandergesetzt haben sie sich gemäss ihren Angaben im Rahmen der Präsentation in der KBIK (F5/7-PM1 bis PM5). Besonders kritisch diskutieren die Befragten das Ergebnis zum ähnlichen Lernstand. Auf vertiefte Auseinandersetzung mit Evaluationen deuten zudem mehrere Hinweise in den Kommissionsprotokollen.
\end{abstract}

Politisch: Verweise auf Evaluationen in allen Phasen des Prozesses, andere Arten der politischen Nutzung in der parlamentarischen Phase

In allen Phasen verwiesen Parlamentsmitglieder unabhängig von ihrer Position auf Evaluationen (EDK-Ost 2010; Präsentation KBIK). Parlamentsmitglieder forderten auch Evaluationen in allen Phasen. Parlamentsmitglieder, die eine flächendeckende Grundstufe befürworteten oder die Grundstufe komplett ablehnten, kritisierten zudem Evaluationen in der parlamentarischen Phase.

Regierung Analytisch: Erstellung mehrerer Evaluationen, Fokus auf Evaluation von EDK-0st 4bis8

und

Verwaltung Politisch: Verweise auf Evaluationen in allen Phasen des Prozesses

Gemäss dem Interview und der Dokumentenanalyse stützte sich die Bildungsdirektion vor allem auf die gemeinsame Evaluation von EDK-0st 4bis8, sie gab aber noch weitere Evaluationen in Auftrag (Stöckli und Stebler 2009; Trachsler 2009; Wagner-Willi und Widmer-Wolf 2009). Im Regierungsratsantrag, während den Beratungen in der KBIK sowie im Plenum haben Regierung und Verwaltung nur auf EDK-Ost (2010) und auf eine frühere Studie der Bildungsdirektion (2003) verwiesen. 
zur freiwilligen Einführung aus. Von den grossen Parteien sprachen sich die SP und die Grünen für die flächendeckende Einführung aus, die CVP und FDP unterstützten die freiwillige Einführung der Grundstufe, die SVP lehnte eine Einführung der Grundstufe ganz ab. In der Schlussabstimmung nahm der Kantonsrat die flächendeckende und die freiwillige Einführung eher knapp an. Er empfahl von Gesetzes wegen den Gegenvorschlag, die Zürcher Stimmberechtigten lehnten aber beide Vorlagen ab. Einen Überblick über die Evaluationsnutzung in Fall 7 liefert Tabelle 28.

Deutlich weniger konfliktiv war der Gesetzgebungsprozess zur Einführung der Basisstufe in Fall 8 in Bern. Die Basisstufe war eines der zentralen Themen der Revision des VSG-BE. Der Grosse Rat diskutierte die Basisstufe aber bereits zuvor, als er im Jahr 2000 den Grundstein für die Schulversuche legte. Nach diesen teilweise kontroversen Diskussionen war die Basisstufe im untersuchten Gesetzgebungsprozess weniger umstritten. Nur die SVP sprach sich offiziell gegen die Basisstufe aus. Einige SVP-Mitglieder befürworteten sie aber, um den ländlichen Gemeinden den Erhalt ihrer Schulen zu ermöglichen. Die restlichen Parteien sprachen sich für die freiwillige Einführung der Basisstufe aus, wobei die CVP, FDP, Grünliberalen und Teile der SVP die Finanzierung

\section{Tabelle 29: Basisstufe im Berner Grossen Rat - Übersicht der Nutzung}

Parlament Analytisch: Mehrheitlich differenzierte Auseinandersetzung mit Evaluationen

Vier der fünf befragten Kommissionsmitglieder haben den Projektschlussbericht der EDK-Ost gelesen. Befragte, die sich skeptisch zur Basisstufe oder deren Finanzierung äusserten, betonen die Ergebnisse zum ähnlichen Lernstand (F6/8-PM2, F6/8-PM4). Für die Befragten, welche die Basisstufe befürworteten, sind dagegen etwa soziales Lernen oder pädagogische Kontinuität zentral (F6/8-PM1, F6/8-PM5). Das fünfte befragte, stellvertretende Kommissionsmitglied kennt die Ergebnisse zum Lernstand, erinnert sich aber nicht, dass diese während der Revision des VSG-BE bereits bekannt waren (F6/8-PM3).

Politisch: Verweise auf Evaluationen in allen Phasen, selten andere Arten der politischen Nutzung

Die Parlamentsmitglieder verwiesen in allen Phasen auf Evaluationen (EDK-Ost 2010). Parlamentsmitglieder, die sich für die Basisstufe aussprachen, betonten die Zufriedenheit der Erziehenden und Lehrpersonen, während die gegnerische Seite auf die Testergebnisse zu den Lernfortschritten fokussierte. Parlamentsmitglieder kritisierten auch vereinzelt Evaluationen, wobei sich diese Kritik an die Vorgehensweise der Evaluation und an den selektiven Umgang mit Evaluationen einzelner anderer Parlamentsmitglieder richtete.

Regierung Analytisch: Erstellung eigener Evaluation, Fokus auf Evaluation von EDK-Ost 4bis8

und

Verwaltung Politisch: Verweise auf verschiedene Quellen in allen Phasen des Prozesses

Das Interview und die Dokumentenanalyse legen nahe, dass sich die ERZ hauptsächlich auf die Evaluation von EDK-Ost 4bis8 stützte, aber einen sachlichen, unaufgeregten Umgang damit anstrebte. Während die Regierung und Verwaltung in den Beratungen selten und nur auf diese Evaluation verwiesen, enthielten die schriftlichen Dokumente verschiedene Quellen. Die Regierung und Verwaltung bezogen sich auch auf die Evaluation des Cycle élémentaire, welche die Abteilung Bildungsplanung und Evaluation der ERZ erstellte, sowie auf weitere Berichte im Rahmen von EDK-Ost 4bis8 (Zwischenberichte der Evaluationen, Grossenbacher 2008). 
vollständig den Gemeinden überlassen wollten. Ein solcher Änderungsantrag sowie ein Streichungsantrag der Basisstufe lehnte der Grosse Rat klar ab. Fast einstimmig nahm er dann die Revision des VSG-BE an, ein Referendum wurde nicht eingereicht. Die Evaluationsnutzung in Fall 8 ist in Tabelle 29 zusammengefasst.

\subsection{Vergleich und Diskussion der Beobachtungen in den Fallstudien}

Nach der Präsentation der einzelnen Fallstudien werden nun die Befunde verglichen, um den Zusammenhang zwischen der Evaluationsnutzung und den kontextuellen Erklärungsfaktoren gesamthaft zu analysieren und zu diskutieren. Dazu werden zuerst die Befunde der Fallstudien zur Evaluationsnutzung der Parlamentsmitglieder erläutert. Danach folgt die Diskussion der kontextuellen Erklärungsfaktoren, in der die beobachtete Evaluationsnutzung mit den theoretischen Erwartungen verglichen und anhand der bestehenden Literatur erörtert wird. Für diese Diskussion werden einerseits die Befunde zur Evaluationsnutzung mit der Ausprägung des jeweiligen kontextuellen Erklärungsfaktors verglichen, anderseits werden die Befunde zu den politischen Massnahmen jeweils paarweise verglichen. Ausführungen zur Aussagekraft der Fallstudienbefunde schliessen den Vergleich und die Diskussion ab.

Grundlage für die folgenden Ausführungen bilden je eine Fallstudie zu den Massnahmen VHKA (Fall 1, Fall 2), Steuersystem für energieeffiziente Fahrzeuge (Fall 3, Fall 4), SSA (Fall 5, Fall 6) und Basis-/Grundstufe (Fall 7, Fall 8) im Kantonsrat Zürich und im Grossen Rat des Kantons Bern. Die Fallstudien basieren einerseits auf insgesamt 27 Interviews mit Mitgliedern der parlamentarischen Kommissionen und der Verwaltung. Anderseits gründen sie auf der Analyse der verfügbaren Evaluationen, der Kommissions- und Plenumsprotokolle, der parlamentarischen Vorstösse, den verfügbaren Medienmitteilungen der Parteien sowie Dokumente der Regierung und Verwaltung. Eine Zusammenfassung der einzelnen Fallstudien befindet sich jeweils im letzten Abschnitt der Unterkapitel zu den einzelnen Massnahmen (5.2.5, 5.3.5, 5.4.5 und 5.5.5).

\subsubsection{Evaluationsnutzung: Ähnlich häufig je nach Massnahme}

Tabelle 30 präsentiert das Ausmass der in den Fallstudien beobachteten analytischen Evaluationsnutzung, die vorrangig durch die Interviews mit Kommissionsmitgliedern erfasst wird (vgl. 5.1). Das Ausmass der analytischen Nutzung wird in Tabelle 30 danach eingeordnet, in welcher Weise und wie viele befragte Parlamentsmitglieder sich mit Evaluationen auseinandersetzten. Diese Einordnung erfolgt stets relativ im Vergleich der acht Fallstudien und ist daher nicht direkt auf andere Studien der Evaluationsnutzung übertragbar. Um einem Fall (eher) häufige analytische Nutzung zuzuschreiben, müssen die Ausführungen in den meisten Interviews darauf schliessen lassen, dass sich die Befragten differenziert mit Evaluationen auseinandersetzten. Bei Fällen mit (eher) seltener analytischer Nutzung setzte sich gemäss den Interviews zumindest eines der befragten Kommissionsmitglieder überhaupt mit Evaluationen auseinander oder eine Berücksichtigung von Evaluationen ist aufgrund der Beobachtungen nicht ganz auszuschliessen. 
Keine analytische Nutzung liegt hingegen vor, wenn alle Befragten Evaluationen nicht berücksichtigten und auch die Dokumentenanalyse keine Hinweise auf eine Auseinandersetzung mit Evaluationen liefert.

\section{Tabelle 30: Analytische Nutzung - Einordnung der Beobachtungen aus den Fallstudien}

\begin{tabular}{|c|c|c|}
\hline Häufig & $\begin{array}{l}\text { Differenzierte Auseinandersetzung mit Evaluationen (fast) aller } \\
\text { befragten Kommissionsmitglieder. }\end{array}$ & $\begin{array}{l}\text { Fall 7: Grundstufe Zürich } \\
\text { Fall 8: Basisstufe Bern }\end{array}$ \\
\hline Eher häufig & $\begin{array}{l}\text { Differenzierte Auseinandersetzung mit Evaluationen der Mehrheit } \\
\text { der befragten Kommissionsmitglieder. }\end{array}$ & $\begin{array}{l}\text { Fall 4: Steuersystem für energie- } \\
\text { effiziente Fahrzeuge Bern }\end{array}$ \\
\hline Eher selten & $\begin{array}{l}\text { Auseinandersetzung mit Evaluationen der Mehrheit der befragten } \\
\text { Kommissionsmitglieder, differenziert höchstens vereinzelt. }\end{array}$ & $\begin{array}{l}\text { Fall 3: Steuersystem für energie- } \\
\text { effiziente Fahrzeuge Zürich }\end{array}$ \\
\hline Selten & $\begin{array}{l}\text { Auseinandersetzung mit Evaluationen von mindestens einem } \\
\text { befragten Kommissionsmitglied oder nicht ausschliessbar. }\end{array}$ & $\begin{array}{l}\text { Fall 1: VHKA Zürich } \\
\text { Fall 5: SSA Zürich } \\
\text { Fall 6: SSA Bern }\end{array}$ \\
\hline Keine & Keine Hinweise auf eine Auseinandersetzung mit Evaluationen. & Fall 2: VHKA Bern \\
\hline
\end{tabular}

In derselben Kategorie sind die Fälle nach Nummer aufsteigend aufgelistet. Zusammenfassungen werden gleich wie Evaluationen behandelt.

Insgesamt variiert die analytische Evaluationsnutzung in den acht Fallstudien deutlich, keine bis eher seltene analytische Nutzung überwiegt jedoch. Fünf Fälle können zu dieser Kategorie eingeordnet werden. In der Hälfte der Fälle legen die Interviews aber gleichzeitig nahe, dass sich mindestens ein befragtes Kommissionsmitglied differenziert mit Evaluationen auseinandersetzte. Die drei Fälle mit eher häufiger oder häufiger analytischer Evaluationsnutzung betreffen zwei Massnahmen: Die Grund- oder Basisstufe (Fälle 7 und 8) sowie das Steuersystem für energieeffiziente Fahrzeuge (Fall 4). Generell ist das beobachtete Ausmass der analytischen Evaluationsnutzung im Kantonsrat Zürich und im Grossen Rat des Kantons Bern für die Fälle zur gleichen Massnahme jeweils ähnlich.

Analog zur analytischen Nutzung beschreibt Tabelle 31 die Einordnung der beobachteten politischen Nutzung. Zur Erfassung der politischen Nutzung dienen vornehmlich die Dokumente der Gesetzgebungsprozesse, namentlich Kommissions- und Plenumsprotokolle oder Vorstosstexte (vgl. 5.1). ${ }^{178}$ Eingeordnet wird das Ausmass der politischen Verwendung nach ihren verschiedenen Arten und deren Verbreitung im Gesetzgebungsprozess, wobei sich diese Einordnung wiederum auf die relative Ausprägung der Nutzung in den acht Fallstudien bezieht. (Eher) häufige politische Nutzung setzt voraus, dass sowohl ein Verweisen auf Evaluationen in einer argumentativen Art als auch etwa ein Fordern oder Kritisieren in einer taktischen Art beobachtet wird, wobei

178 Medienmitteilungen und Stellungnahmen der Parteien bilden nur ergänzende Dokumente, da sie nicht vollständig online verfügbar sind (vgl. 5.1). 
mindestens eine der Nutzungsarten ausgeprägt ist. ${ }^{179}$ Bei (eher) seltener politischer Nutzung sind dagegen beide Nutzungsarten nicht ausgeprägt oder es lässt sich nur eine der Nutzungsarten beobachten. Bei keiner politischen Nutzung sind weder ein Verweis auf Evaluationen noch eine Forderung, Kritik oder ein Fragen nach Evidenz feststellbar.

Wie bei der analytischen Nutzung unterscheidet sich das Ausmass der politischen Nutzung in den acht Fallstudien, bei der Hälfte der Fälle kann jedoch von einer eher häufigen bis häufigen politischen Verwendung gesprochen werden. Die Ausführungen in Tabelle 31 zeigen, dass Verweise auf Evaluationen am weitesten verbreitet waren. In allen sechs Fällen mit politischer Nutzung ist mindestens ein Verweis in den Plenumsprotokollen beobachtbar. Politische Verwendung taktischer Art ist hingegen weniger verbreitet, obwohl es verhältnismässig einfacher erscheint, da Parlamentsmitglieder Evaluationen nicht kennen müssen, um danach zu fragen oder diese zu fordern. Hinweise auf Letzteres enthalten die Dokumente in vier Fällen, wobei die Parlamentsmitglieder nicht immer formell eine Evaluation forderten, indem sie etwa eine Regulierungsfolgeabschätzung verlangten. In drei Fällen fragten die Parlamentsmitglieder hingegen nach Evaluationen oder kritisierten diese.

\section{Tabelle 31: Politische Nutzung - Einordnung der Beobachtungen aus den Fallstudien}

\begin{tabular}{|c|c|c|}
\hline Häufig & $\begin{array}{l}\text { Mehrfaches Verweisen auf Evaluationen und Kritik an Evaluatio- } \\
\text { nen in allen Phasen. Vereinzeltes Fordern von Evaluationen. }\end{array}$ & Fall 7: Grundstufe Zürich \\
\hline Eher häufig & $\begin{array}{l}\text { (Zum Teil mehrfaches) Verweisen auf Evaluationen in allen } \\
\text { Phasen. Andere Arten politischer Nutzung in mindestens einer } \\
\text { Phase. }\end{array}$ & $\begin{array}{l}\text { Fall 3: Steuersystem für energie- } \\
\text { effiziente Fahrzeuge Zürich } \\
\text { Fall 4: Steuersystem für energie- } \\
\text { effiziente Fahrzeuge Bern } \\
\text { Fall 8: Basisstufe Bern }\end{array}$ \\
\hline Eher selten & $\begin{array}{l}\text { (Zum Teil vereinzelte) Verweise auf Evaluationen in allen Phasen. } \\
\text { Keine anderen Arten politischer Nutzung. }\end{array}$ & Fall 6: SSA Bern \\
\hline Selten & $\begin{array}{l}\text { Vereinzeltes Verweisen auf Evaluationen. Keine anderen Arten } \\
\text { politischer Nutzung. }\end{array}$ & Fall 1: VHKA Zürich \\
\hline Keine & $\begin{array}{l}\text { Kein Verweisen auf Evaluationen. Keine anderen Arten politi- } \\
\text { scher Nutzung. }\end{array}$ & $\begin{array}{l}\text { Fall 5: SSA Zürich } \\
\text { Fall 2: VHKA Bern }\end{array}$ \\
\hline
\end{tabular}

In derselben Kategorie sind die Fälle nach Nummer aufsteigend aufgelistet.

Tabelle 32 zeigt eine Gesamtübersicht des Ausmasses an analytischer und politischer Nutzung pro Fall, allerdings sind aufgrund der unterschiedlichen Datengrundlagen (Interviews, Dokumente) nur bedingt Aussagen zum Verhältnis der beiden Nutzungsfor men möglich. Gemäss den theoretischen Überlegungen wird erstens erwartet, dass poli-

179 Die beiden Arten der politischen Nutzung sind in der vorliegenden Einordnung gleichberechtigt. Dies erklärt, warum die Einstufung der Fälle 3 und 4 von Eberli $(2015,2018)$ abweicht. 
Tabelle 32: Übersicht der Beobachtungen der Nutzung aus den Fallstudien

\begin{tabular}{|c|c|c|c|c|c|c|c|c|}
\hline & \multicolumn{2}{|c|}{ VHKA } & \multicolumn{2}{|c|}{$\begin{array}{c}\text { Steuersystem für } \\
\text { energieeffiziente } \\
\text { Fahrzeuge }\end{array}$} & \multicolumn{2}{|c|}{ SSA } & \multicolumn{2}{|c|}{ Basis-/Grundstufe } \\
\hline & Zürich & Bern & Zürich & Bern & Zürich & Bern & Zürich & Bern \\
\hline & Fall 1 & Fall 2 & Fall 3 & Fall 4 & Fall 5 & Fall 6 & Fall 7 & Fall 8 \\
\hline $\begin{array}{l}\text { Analytische } \\
\text { Nutzung }\end{array}$ & selten & keine & \begin{tabular}{|l} 
eher \\
selten
\end{tabular} & $\begin{array}{l}\text { eher } \\
\text { häufig }\end{array}$ & selten & selten & häufig & häufig \\
\hline $\begin{array}{l}\text { Politische } \\
\text { Nutzung }\end{array}$ & selten & keine & $\begin{array}{l}\text { eher } \\
\text { häufig }\end{array}$ & $\begin{array}{l}\text { eher } \\
\text { häufig }\end{array}$ & keine & $\begin{array}{l}\text { eher } \\
\text { selten }\end{array}$ & häufig & $\begin{array}{l}\text { eher } \\
\text { häufig }\end{array}$ \\
\hline
\end{tabular}

tische Nutzung öfter als analytische Nutzung vorkommt (vgl. 3.1). In parlamentarischen Entscheidungsprozessen ist die Vertretung von Ideologien und Interessen ausgeprägter. Bei solchen politischen Entscheidungen sollten Parlamentsmitglieder Evaluationen primär verwenden, um ihre Positionen zu verteidigen und Mehrheiten dafür zu gewinnen. Anhand der Fallstudien kann diese Erwartung aber nicht abschliessend diskutiert werden, da die Beobachtungen der Nutzungsformen kaum vergleichbar sind. Für eine häufigere politische statt analytische Nutzung spricht, dass nicht nur Kommissionsmitglieder Evaluationen politisch nutzten, sondern anlässlich der Plenumsdebatten auch andere Parlamentsmitglieder, wie dies etwa in den Fällen 7 und 8 zur Basis-/Grundstufe geschah. Die analytische Nutzung konzentriert sich hingegen auf Kommissionsmitglieder (vgl. 5.6.2). Da folglich gewisse analytische Aktivitäten - wenn auch bei einem kleinen Kreis - beobachtbar sind, beschränkt sich aber die Evaluationsnutzung auch im Parlament nicht nur auf die politische Verwendung. Zweitens wird gemäss den theoretischen Überlegungen erwartet, dass die analytische Nutzung die politische Nutzung fördert (vgl. 3.1). Selbst wenn ein Parlamentsmitglied Evaluationen ergebnisoffen und mit kaum gefestigten Positionen analytisch nutzt, um sich etwa zu informieren, ist fraglich, ob dies nicht stets auch mit einer Absicht zur (möglichen) politischen Nutzung verbunden ist. Diese zweite Erwartung wird unterstützt, da beide Nutzungsformen in der Regel gemeinsam vorkommen: In keinem Fall wurde vergleichsweise häufige analytische Nutzung und vergleichsweise seltene politische Nutzung (oder umgekehrt) beobachtet.

Diese Beobachtungen zur analytischen und politischen Verwendung in den acht Fallstudien schliessen generell an den bisherigen Forschungsstand an. In Übereinstimmung mit früheren Arbeiten weisen die Fallstudien auf eine deutliche Variation zwischen den einzelnen Gesetzgebungsprozessen hin (Frey 2012; Whiteman 1985b, 1995). Auch bestätigen sie, dass verschiedene Formen der Verwendung im Parlament beobachtbar sind (Boyer und Langbein 1991; Frey 2012; Tabuga 2017; Whiteman 1985a). Wie bereits erwähnt, gibt es keine eindeutigen Schlüsse zur relativen Verbreitung der Formen. Daher geben die Fallstudien einerseits Anhaltspunkte für die verbreitete Beobachtung, dass die symbolische Nutzung im Parlament überwiegt (vgl. Shulock 1999; Weiss 1987, 1989). Anderseits scheint auch die Feststellung anderer Forschen- 
der plausibel, dass sich Parlamentsmitgliedern dank Evaluationen neue Betrachtungsweisen erschliessen (Bogenschneider et al. 2013; Hird 2005b; Morshed et al. 2017).

\subsubsection{Institutioneller Kontext: Keine systematische Variation in den Milizparlamenten}

In der vorliegenden Untersuchung interessiert das Kommissionensystem als institutioneller Faktor der Evaluationsnutzung. Dieser Faktor diente als Kriterium zur Auswahl der Kantonsparlamente Bern und Zürich. Die beiden Kantone und ihre Parlamente teilen viele ähnliche Eigenschaften, das Kommissionensystem ist aber unterschiedlich: Während sich im Kantonsrat Zürich ständige Fachkommissionen mit den untersuchten Massnahmen befassten, taten dies im Grossen Rat des Kantons Bern eigens dafür einberufene, nichtständige Fachkommissionen.

\section{Kommissionensystem: Kommissionen, aber nicht deren System relevant}

Das Kommissionensystem soll sich gemäss der Theorie vor allem auf die analytische Verwendung auswirken. Sind Kommissionen nichtständig, wissen die Parlamentsmitglieder nicht mit Sicherheit, ob sie bei der Beratung eines themenähnlichen Geschäfts wieder in der Kommission sitzen werden (Lüthi 1997, 148; Mattson und Strøm 1995, 270). Daher sollten Mitglieder von Parlamenten mit nichtständigen Kommissionen einen geringeren Anreiz haben, sich vertieft mit Evaluationen auseinanderzusetzen und einen themenspezifischen Sachverstand zu erarbeiten. Folglich sollten Mitglieder von Parlamenten mit ständigen Kommissionen Evaluationen häufiger verwenden $\left(H_{5 a}\right)$. Kein Zusammenhang wird hingegen für die politische Nutzung erwartet $\left(H_{5 p}\right)$. Da Mitgliedern von ständigen Kommissionen bereits ein hoher Sachverstand attestiert wird, ist fraglich, inwiefern sie Evaluationen benötigen, um sich Legitimität zu verschaffen.

Generell zeigen die Fallstudien, dass den Kommissionen wie angenommen eine zentrale Bedeutung bei der analytischen Nutzung von Evaluationen zukommt. Die Ausführungen in den Interviews bestätigen, dass sich die Kommissionen zumeist vertieft mit den Geschäften auseinandersetzen und diese sachlich diskutieren (vgl. F1-PM1; F2-Verw.; F3-Verw.). Parlamentsmitglieder, die nicht in der Kommission sitzen, seien in der Regel weniger oder gar nicht über die Geschäfte informiert. Auf Evaluationen in Form von Berichten oder Präsentationen durch Fachpersonen würden Parlamentsmitglieder daher am ehesten in der Kommissionsarbeit stossen (vgl. F4-PM3; F5/7-PM4; F5/7-PM5). Gemäss einigen befragten Kommissionsmitgliedern sind die Fronten in dieser Phase weniger verhärtet und Evidenz oder darauf basierte Argumente können am ehesten einfliessen (F3-PM1; F3-PM3). Folglich scheint die analytische Verwendung am wahrscheinlichsten während den Kommissionsberatungen. In Bezug auf die politische Nutzung nehmen die Kommissionen in den untersuchten Fällen dagegen eher eine indirekt zentrale Rolle ein. Parlamentsmitglieder verwendeten Evaluationen zwar auch in den vertraulichen Kommissionssitzungen politisch, sie tun dies aber weniger ausgeprägt als in den öffentlichen Plenumsdebatten. Da die Kommissionsmitglieder jedoch oft Reden im Plenum halten, sind sie ebenfalls für die politische Nutzung in der Plenumsphase verantwortlich. Schliesslich dürften sie ausserhalb des Parlaments zu analytischer und 
politischer Nutzung beitragen, da sie aufgrund ihrer Spezialisierung beispielsweise in Arbeitsgruppen der Partei vertreten sind (vgl. F5/7-PM4; F5/7-PM5).

In Bezug auf das Kommissionensystem deuten die paarweisen Vergleiche der Fallstudien aber auf keinen Zusammenhang mit der Evaluationsnutzung hin und unterstützen daher nur $H_{5 p}$. Die beiden Fälle einer Massnahme sollten sich vor allem aufgrund ihres Kommissionensystems unterscheiden, während sie jeweils ähnlich wissenschaftsnah und konfliktiv sein sollten. Entgegen den Erwartungen zeigen diese paarweisen Vergleiche der Fallstudienbefunde aber, dass die analytische Nutzung gesamthaft ausgeglichen ist. Gemäss Tabelle 32 im vorherigen Abschnitt ist das beobachtete Ausmass an analytischer Nutzung pro Massnahme zwar nicht immer gleich in beiden Parlamenten, aber es ist auch weder konstant höher in Fällen des Kantonsrats Zürich mit ständigen Kommissionen noch in Fällen des Grossen Rats des Kantons Bern mit nichtständigen Kommissionen. Dies trifft ebenso für die politische Nutzung zu.

Die Feststellung keines Zusammenhangs zwischen dem Kommissionensystem und der Evaluationsnutzung ist umso erstaunlicher, da weitere Unterschiede mit dem Kommissionensystem verbunden sind. Ein erster Unterschied betrifft die Anhörungen. Trotz der entsprechenden Rechte fanden Anhörungen im Berner Grossen Rat lediglich im Rahmen der Totalrevision des Energiegesetzes in Fall 2 statt, während die Kommissionen im Zürcher Kantonsrat in allen vier Fällen Anhörungen abhielten (Fälle 1, 3, 5, 7). Nur zur Diskussion des Steuersystems für energieeffiziente Fahrzeuge (Fall 3) und der Grundstufe (Fall 7) luden die Kommissionen aber Fachpersonen ein. ${ }^{180}$ Die Fallstudien zeigen, dass diese Präsentationen durch Fachpersonen zentral waren für die Evaluationsnutzung. Gemäss den Interviews setzten sich zumindest einige der Kommissionsmitglieder vertieft mit den Präsentationen auseinander, auch verwiesen die Parlamentsmitglieder später darauf. Im Generellen sind die Anhörungen aber relativ kurz (F3-PM1; F3-PM3). Mitunter stellen die Befragten daher deren Rolle in Frage: In den Anhörungen «kommt der eine und sagt was, dann der andere. Dann schaut man, ob es gerade noch Fragen gibt, die Unterlagen haben ja die Leute und es hat dann wie wenig Wirkung, oder?» (F3-PM1). Weiter weisen die Befragten darauf hin, dass bereits die Auswahl der Fachpersonen ein politischer Entscheid sei. Um sich nicht dem Vorwurf einer einseitigen Informationsbeschaffung auszusetzen, müssten die Einladungen zu Anhörungen ausgewogen sein (F1PM1; F3-PM1; F5/7-PM4; F6/8-PM1).

Zweitens führten die Kommissionensysteme zu Unterschieden in der Zeitspanne, in der sich die Kommissionen mit einer Vorlage beschäftigen. In den Zürcher Fällen war eine Vorlage in 6 bis 14 Sitzungen der ständigen Kommissionen ein Traktandum, während sich die nichtständigen Kommissionen im Kanton Bern in der Regel an zwei bis maximal acht Sitzungen ausschliesslich der Vorlage widmeten. Auch verstrich im Zürcher Kantonsrat von der ersten Kommissions- bis zur ersten Plenumsberatung jeweils fast ein Jahr, während die Plenumsberatungen im Berner Grossen Rat in der Regel zwei bis drei Monate nach den Kommissionsberatungen folgten. Diese Unterschiede legen nahe, dass Evaluationen im Zürcher System mehr Zeit und damit mehr Möglichkeiten haben, um

180 Teilweise luden die Kommissionen Fachpersonen ein, die nicht zur untersuchten Massnahme sprachen. 
Verwendung zu finden (vgl. Frey 2012; Mader 2009). Zum anderen ermöglicht das Berner System eher, sich auf eine Vorlage zu konzentrieren.

Ein dritter, aber für die Evaluationsnutzung kaum relevanter Unterschied betrifft die Sekretariate der Kommissionen. Nur die ständigen Kommissionen in Zürich hatten eigene Sekretariate, bei den nichtständigen Kommissionen in Bern erfüllte die Verwaltung diese Aufgabe (Art. 40 aGo; Trees et al. 2013, 49). Generell thematisierten die Befragten die Sekretariate in den Interviews aber am Rande oder nie. Es sei in der Verantwortung der einzelnen Kommissionsmitglieder, sich zu informieren (F3-PM1). Auch die Kommissionsprotokolle der Zürcher Fälle weisen auf keine aktive Rolle der Sekretariate in den Beratungen hin.

Erklärungsansätze für den nicht festgestellten Zusammenhang können die Interviews mit den Mitgliedern des Grossen Rats des Kantons Bern liefern, der im Jahr 2014 von nichtständigen auf ständige Fachkommissionen wechselte. Diese Ausführungen bestätigen zwar grösstenteils, dass die ständigen Fachkommissionen ermöglichen, einen themenspezifischen Sachverstand aufzubauen und die Berichterstattung über diese Themen - möglicherweise in Form von Evaluationen - besser zu verfolgen (F2-PM1; F2-PM3; F6/8-PM2). Ebenso weisen sie auf Unterschiede in der Selektion der Kommissionsmitglieder hin. Teilweise hätten geographische oder wahltaktische Überlegungen eine Rolle gespielt (F2-PM3; F4-PM1). Bisweilen hätten sich auch Parlamentsmitglieder mit einschlägigem Hintergrund aus terminlichen Gründen nicht an einer Kommission beteiligen können (F4-PM1). Gleichzeitig scheint die unterschiedliche Selektion der Kommissionsmitglieder letztlich wenig ins Gewicht zu fallen. Bei wichtigen Vorlagen, wie dies etwa die Teilrevision des Volksschulgesetzes in den Fällen 6 und 8 zur SSA und Basisstufe war, seien schon die Bildungsfachleute der Parteien und Mitglieder von Interessenvertretungen aus dem Bildungsbereich in der Kommission gesessen (vgl. F4-PM1). Deshalb schätzt ein befragtes Kommissionsmitglied ein, dass diese Vorlage nicht anders behandelt worden wäre in einem System mit ständigen Kommissionen (F6/8-PM1). Weiter befindet ein anderes befragtes Grossratsmitglied, dass sich die Mitglieder in nichtständigen Kommissionen teilweise besser vorbereitet hätten, gerade da sie fachfremd gewesen seien (F6/8-PM5). Ein anderer Erklärungsansatz für die nicht festgestellten Unterschiede ist schliesslich die unterschiedliche Kommissionsatmosphäre. Übereinstimmend mit den Befunden von Lüthi (1997) für die Bundesversammlung weisen die Befragten mehrfach darauf hin, dass selbst Kommissionen eines Parlaments unterschiedlich funktionieren (vgl. F1-PM1; F3-PM1; F3-PM2; F5/7-PM5; F6/8-PM3).

Zusammenfassend deuten die Beobachtungen in den Fallstudien auf keine Unterschiede in der Evaluationsnutzung aufgrund des Kommissionensystems hin, sie zeigen aber generell die wichtige Rolle der Kommissionen für die analytische und zu einem geringeren Grad für die politische Nutzung. Der Faktor des Kommissionensystems war bisher - nach bestem Wissen - noch kein Gegenstand der Nutzungsforschung. Die Fallstudien bestätigen jedoch die bisherigen Ergebnisse, wonach eine differenzierte Betrachtung der Vorlagen und analytische Nutzung in der Kommissionsphase und damit in früheren Phasen des Gesetzgebungsprozesses wahrscheinlich ist (Florio et al. 1979; Mooney 1993, 2012; Weiss 1987; Whiteman 1985a). Dass politische Nutzung auch in 
den Kommissionen beobachtbar ist, unterstützt die bisherige Forschung bis zu einem gewissen Grad. Gemäss den Fallstudien nutzen die Parlamentsmitglieder Evaluationen vergleichsweise seltener in den Kommissionen als im Plenum. Erzählungen zur Schweiz (Frey 2012, 109) und Studien zum US-amerikanischen Kongress legen hingegen keine solchen Unterschiede nahe (Esterling 2007, 2011; Shulock 1998, 1999). Der US-amerikanische Kontext ist aber nur bedingt vergleichbar, da Sitzungen dieser Kommissionen in der Regel öffentlich sind (Esterling 2011, 175).

\section{Anderer institutioneller Faktor: Hemmender Effekt des Milizsystems?}

Als weitere Eigenschaft der parlamentarischen Institutionen in der Schweiz sprachen die Kommissionsmitglieder in den Interviews teilweise das Milizsystem an, das sie insgesamt als eher hemmend für die Evaluationsnutzung wahrnehmen. Sie bewerten Evaluationen zwar grundsätzlich als hilfreich, um komplexe Inhalte auch als Milizparlamentsmitglied zu verstehen (F2-PM2). Gleichzeitig begrenze aber das Milizprinzip die Zeit, die man in Evaluationen investieren könne (F5/7-PM1). Neben den beruflichen Tätigkeiten und der Familie bleibe wenig Zeit, sich in politische Geschäfte zu vertiefen (F6/8-PM4; F5/7-PM5). Auch wenn bei Veranstaltungen von Firmen oder Interessenverbänden bisweilen Studien präsentiert würden, könne man daher nicht immer teilnehmen (F5/7-PM2). Oft seien Evaluationen zwar interessant, aber «dann fängt man an zu lesen und dann auf der Seite 4 denkt man, nein, ich habe keine Zeit, um 150 Seiten zu lesen» (F5/7-PM4). Anstatt Recherchearbeiten selbst zu übernehmen, müsste man deswegen eigentlich auf administrative und operative Unterstützung zählen können (F3-PM1). Die Rolle des Milizsystems wurde aber in den Interviews nicht systematisch thematisiert.

\subsubsection{Politischer Kontext: Konfliktivität zentral, bedeutende Stellung der Verwaltung}

Die Wissenschaftsnähe und die Konfliktivität bilden die untersuchten politischen Kontextfaktoren. Diese beiden Faktoren dienten zur Auswahl der untersuchten politischen Massnahmen und decken daher gemäss 5.1 verschiedene Ausprägungen ab: Einerseits weisen die VHKA und das Steuersystem für energieeffiziente Fahrzeuge im Bereich der Energie- und Transportpolitik eine relativ grosse Wissenschaftsnähe auf, die VHKA war aber wesentlich weniger konfliktiv als das Steuersystem für energieeffiziente Fahrzeuge. Anderseits verfügen die SSA und die Basis-/Grundstufe über eine relativ geringe Wissenschaftsnähe, wobei die SSA wiederum deutlich weniger konfliktiv als die Basis-/Grundstufe war.

\section{Wissenschaftsnähe: Keine systematischen Differenzen beobachtbar}

Gemäss den Hypothesen hängt die Wissenschaftsnähe auf unterschiedliche Weise mit den beiden Formen der Evaluationsnutzung zusammen. Mit wissenschaftsnahen Fragen, die eher naturwissenschaftlich und technisch geprägt sind, sollten Parlamentsmitglieder weniger vertraut sein. Zu diesen Fragen sollten sie daher weniger vorgefasste Meinungen 
haben und mehr Informationen bedürfen. Dies sollte die analytische Nutzung begünstigen $\left(H_{6 a}\right)$. Politisch sollten die Parlamentsmitglieder dagegen Evaluationen häufiger verwenden, je geringer die Wissenschaftsnähe ist $\left(H_{6 p}\right)$. Die komplexen Wirkungszusammenhänge und deren schwierige Erfassbarkeit sollten mehr Möglichkeiten zur politischen Nutzung bieten. Einerseits dürften die verwendeten Methoden und Zielgrössen einfacher zu kritisieren sein, anderseits sollte der grössere Interpretationsspielraum auch verschiedene Standpunkte unterstützen.

Der Vergleich der beobachteten analytischen und politischen Evaluationsnutzung nach Wissenschaftsnähe in Abbildung 27 zeigt auf, dass die Beobachtungen aus den Fallstudien die Hypothesen nicht unterstützen. Entgegen den Erwartungen ist das Ausmass der analytischen Nutzung nicht generell höher für die Fälle mit einer vergleichsweise grossen Wissenschaftsnähe. Gesamthaft ist die analytische Nutzung gar tendenziell häufiger in den Fällen mit relativ geringer Wissenschaftsnähe. Zudem lässt sich die analytische Verwendung einzig in den wenig wissenschaftsnahen Fällen 7 und 8 zur Basis-/ Grundstufe als häufig bezeichnen, während im vergleichsweise wissenschaftsnahen Fall 2 zur VHKA gar keine analytische Evaluationsnutzung beobachtbar ist. Insgesamt sind die Differenzen bezüglich der Wissenschaftsnähe und der Evaluationsnutzung aber gering.

\section{Abbildung 27: Häufigkeit der Nutzung nach Wissenschaftsnähe}

Analytische Nutzung

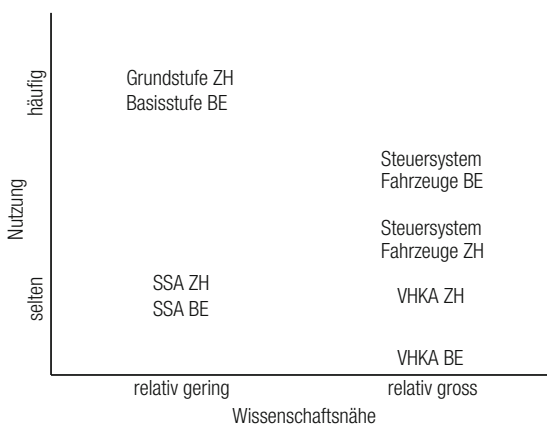

Politische Nutzung

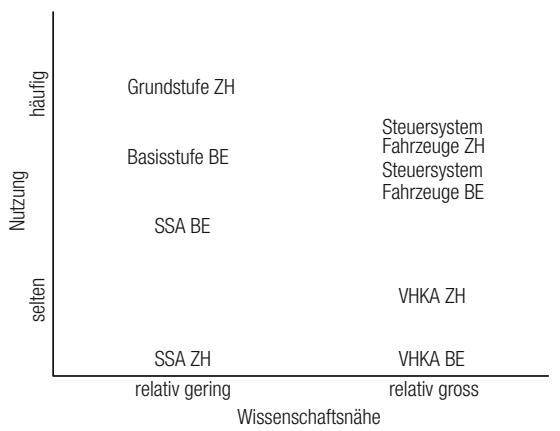

Bei der relativ wissenschaftsnahen Massnahme der VHKA weichen erwartete und beobachtete Evaluationsnutzung klar voneinander ab: Parlamentsmitglieder verwendeten Evaluationen zur VHKA nur vereinzelt bis gar nie analytisch und politisch. Eine mögliche Erklärung dafür könnte sein, dass Parlamentsmitglieder bei wissenschaftsnahen Fragen möglicherweise keine Evaluationen bedürfen, da gut erfassbare Gegenstände auch eine gewisse Verständlichkeit implizieren. Bei der VHKA als «einfaches Geschäft» (F1PM1) könnte dies zutreffen. Gegen diese Erklärung spricht aber, dass Evaluationen in früheren Diskussionen im Grossen Rat des Kantons Bern und im Kantonsrat Zürich 
durchaus Verwendung fanden. So enthalten die Protokolle der Plenumsdebatten über die Abschaffung der VHKA in Altbauten anfangs der 2000er-Jahre mehrere Verweise auf Evaluationen und vereinzelt auch Kritik daran. ${ }^{181}$ Folglich scheinen andere Erklärungen einleuchtender. Gemäss den Interviewaussagen und den Abstimmungsergebnissen war die VHKA etwa umstrittener in den 2000er-Jahren. Zudem stellt sie als eine der ersten Massnahmen der Energiepolitik mittlerweile eine verhältnismässig alte, wenig innovative Massnahme dar (vgl. Rieder et al. 2008).

Bei der zweiten relativ wissenschaftsnahen Massnahme des Steuersystems für energieeffiziente Fahrzeuge ist analytische Evaluationsnutzung beobachtbar, die Fallstudien weisen aber darauf hin, dass die Wissenschaftsnähe nicht die zentrale Eigenschaft dieser Massnahme war. Der naturwissenschaftliche, ökologische Hintergrund der Massnahme entspricht zwar einer relativ grossen Wissenschaftsnähe, die Massnahme betrifft indes auch steuerliche Fragen. Die Diskussion des Steuersystems für energieeffiziente Fahrzeuge im Kantonsrat Zürich in Fall 3 bezog sich so von Anfang an mehr auf Steuern. Die für Abgaben zuständige WAK beriet diese Vorlage und nicht die für energie- und umweltpolitische Fragen zuständige KEVU. Obwohl das Steuersystem für energieeffiziente Fahrzeuge für die WAK keine typische Vorlage darstellte, erachten mehrere Befragte Steuerfragen generell als wenig evaluationsfreundlich (vgl. auch F5/7-PM1; F3-PM1). Bei Steuern hätten die Parteien festgefahrene Positionen, «wo das Thema vielleicht nicht einem klassischen Links-Rechts-Schema folgt, dort sind Evaluationen wichtig für eine neutrale Meinungsbildung»(F2-PM1). Diese Dominanz von steuerlichen Fragen und deren Zusammenhang mit der Evaluationsnutzung veranschaulicht auch der Fall 4 in Bern. In den frühen Phasen des Gesetzgebungsprozesses stand der naturwissenschaftlich-ökologische Aspekt mehr im Vordergrund und die Parlamentsmitglieder verwendeten Evaluationen analytisch zur Erfassung des Gegenstands als auch politisch in den Plenumsdebatten (F4-PM2; F4-PM3; Grosser Rat des Kantons Bern 2009b, 1299). Die Diskussion verlagerte sich aber bereits nach der ersten Lesung vermehrt auf steuerliche Fragen, die nach der Einreichung des konstruktiven Referendums klar dominierten. In dieser zweiten konfliktiven Phase nutzten die Kommissionsmitglieder Evaluationen nur selten analytisch und politisch. Folglich waren die Gesetzgebungsprozesse auch mit einer hohen Konfliktivität verbunden, wenn steuerliche statt wissenschaftsnahe Aspekte die Diskussion dominierten (vgl. 5.6.3.2).

Bei den relativ wenig wissenschaftsnahen Massnahmen der SSA und der Grundstufe deuten die Ausführungen in den Evaluationen und teilweise auch in den Interviews darauf hin, dass beide bildungspolitische Massnahmen zwar über schwer isolierbare Wirkungen verfügen, deren Messbarkeit sich aber unterscheidet. Übereinstimmend mit dem Verständnis einer relativ geringen Wissenschaftsnähe konnten die verfügbaren Evaluationen kaum Wirkungen der SSA quantifizieren (vgl. F6/8-Verw.). Vielmehr beruhen die festgestellten Wirkungszusammenhänge der SSA auf «Vermutungen» und "plausibel

181 Grosser Rat des Kantons Bern (2000a, 367-369; 2000b, 537); Kantonsrat Zürich (1999, 518 f.; 2001a, 7025, 7028, 7031; 2001c, 8961). Mehrere Verweise betreffen die Evaluation des Ökozentrum Langenbruck (1995). 
wirkenden Kalkulationen» (Vögeli-Mantovani und Grossenbacher 2005, 125; vgl. auch Speck und Olk 2010b, 7). Bei der Basis-/Grundstufe hingegen erlaubte die summative Evaluation des Schulversuchs, die Wirkungen in Form von Kompetenzen zu messen. Auf diesen messbaren Aspekt des Lernstands war die Diskussion besonders in Fall 7 im Kantonsrat Zürich fokussiert. Zentrales Ergebnis der summativen Evaluation und der Diskussion im Kantonsrat war, dass die Kinder der Grundstufe und des Kindergartens nach der Schuleingangsphase wieder den gleichen Lernstand haben. Mit diesem Ergebnis habe die Evaluation den Befürworterinnen und Befürwortern der Grundstufe geschadet (F5/7-Verw.). Deutlicher für die Grundstufe gesprochen als die Testergebnisse hätte so die Zufriedenheit der Eltern (F5/7-PM2). Andere Zürcher Befragte relativierten, dass Unterschiede zwischen Schulformen im Generellen «Feinkorrekturen» (F5/7-PM4) betreffen und schwierig zu messen seien. Zudem habe im Fall der Grundstufe auch der Kindergarten als Kontrollgruppe reagiert (F5/7-Verw.; vgl. F6/8-Verw.). Die Bedeutung dieser Evaluationsergebnisse und damit der Messbarkeit können daher kritisch diskutiert werden.

Eine tendenziell seltenere Verwendung bei den relativ wissenschaftsnahen, naturwissenschaftlichen Fragen generell erklären könnte schliesslich, dass sich Evaluationen hier in einem Spannungsfeld zwischen der Politikwissenschaft und technisch orientierten Energiefragen befinden (vgl. F1-PM1 und Linder in Widmer 1996, 55). Zudem dominieren naturwissenschaftliche Bewertungsverfahren (Hirschi 2009). Folglich dürfte die Nützlichkeit von Evaluationen aus einer politikwissenschaftlichen Perspektive deutlich höher eingeschätzt werden. Umgekehrt könnte entsprechend die Nützlichkeit von Evaluationen bei den verwandteren sozialen Fragen mit relativ geringer Wissenschaftsnähe anerkannter sein.

Insgesamt offenbaren die Beobachtungen aus den Fallstudien keinen Zusammenhang zwischen der Wissenschaftsnähe und der Evaluationsnutzung der Parlamentsmitglieder. Die vorliegenden Ergebnisse wiedersprechen folglich den Fallstudien von Frey (2012, 294f., 308) zu schweizerischen Gesetzgebungsprozessen. Frey stellt fest, dass sich die Parlamentsmitglieder nur bei Revisionen in wissenschaftsnahen Politikbereichen von stichhaltiger Evidenz überzeugen liessen und diese folglich analytisch nutzten. Sie untersuchte aber keine bildungs- oder energiepolitischen Fragen. Eine Erklärung für die mangelnde Übereinstimmung mit bisherigen Ergebnissen könnte auch in der Notwendigkeit einer klareren Konzeption der Wissenschaftsnähe begründet sein, deren Bedarf auch die Fallstudien aufzeigen.

\section{Konfliktivität: Entscheidend, besonders für politische Nutzung}

Zwei Hypothesen drücken den erwarteten Zusammenhang zwischen der Konfliktivität und der Evaluationsnutzung aus: Einerseits sollten Parlamentsmitglieder gemäss $H_{7 a}$ Evaluationen bei einer moderaten Konfliktivität am häufigsten analytisch verwenden. In solchen Situationen sollten die Parlamentsmitglieder verhältnismässig offen gegenüber Evaluationen sein, ein gewisser Handlungsdruck dürfte aber auch bestehen. Bei einer geringen Konfliktivität ist dieser Handlungsdruck erwartungsgemäss zu gering, während 
die Parlamentsmitglieder bei einer hohen Konfliktivität hingegen an ihren vorgefassten Positionen festhalten sollten. Gerade im konsensorientierten schweizerischen System sollten zudem die Mechanismen zur Konfliktregelung die Rolle von Evaluationen einschränken. Je höher die Konfliktivität, desto häufiger sollten anderseits Parlamentsmitglieder Evaluationen politisch verwenden $\left(H_{7 p}\right)$. Bei stark umstrittenen Vorlagen sollten Parlamentsmitglieder Evaluationen als vielfältiges politisches Mittel einsetzen, um etwa ihre Position zu begründen, andere Positionen zu schwächen oder Entscheide zu verzögern.

Gemäss Abbildung 28 unterstützen die Ergebnisse der Fallstudien die Erwartungen zur analytischen Nutzung teilweise, während sie mit den Erwartungen zur politischen Nutzung übereinstimmen. Die Beobachtungen aus den Fallstudien legen nahe, dass eine relativ gesehen eher hohe Konfliktivität die analytische Verwendung fördert. In den Fällen mit einer eher tiefen oder tiefen Konfliktivität setzten sich die Parlamentsmitglieder deutlich seltener mit Evaluationen auseinander als in den Fällen mit eher hoher bis hoher Konfliktivität. Folglich scheint die analytische Nutzung einer gewissen Konfliktivität zu bedürfen. $\mathrm{Ob}$ auch eine sehr hohe Konfliktivität die analytische Nutzung begünstigt, ist unklar, da sich die beiden Fälle mit einer hohen Konfliktivität unterscheiden. In Übereinstimmung mit $H_{7 p}$ illustriert Abbildung 28 dagegen deutlich einen positiven Zusammenhang zwischen Konfliktgrad und politischer Verwendung. Sie zeigt zudem, dass die beobachtete politische Nutzung bei einer eher tiefen Konfliktivität stärker variierte. Obwohl die Ergebnisse zur politischen Nutzung den erwarteten positiven Zusammenhang klar unterstützen, dominierte diese aber nicht vollends in konfliktiven Fällen. Folglich implizieren die Fallstudien, dass mit der Konfliktivität nicht nur die politische Nutzung, sondern - zumindest bis zu einem gewissen Konfliktgrad - auch die analytische Nutzung steigt.

\section{Abbildung 28: Häufigkeit der Nutzung nach Grad der Konfliktivität}

Analytische Nutzung

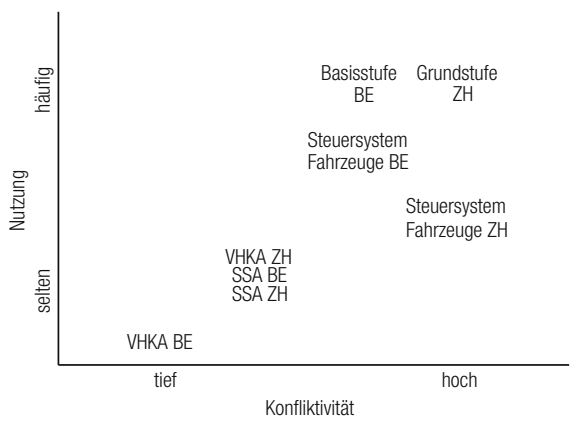

Politische Nutzung

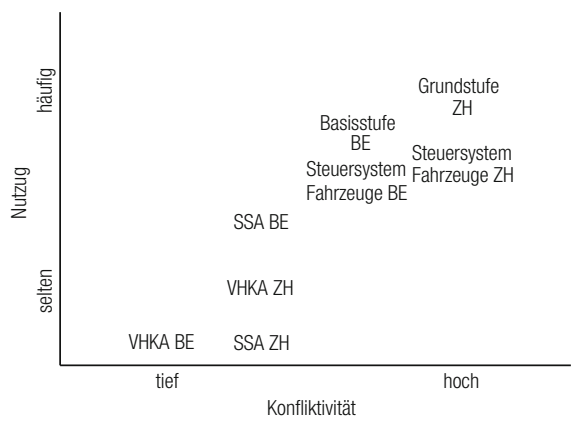

Bei Gesetzgebungsprozessen mit einer hohen Konfliktivität verwendeten die Parlamentsmitglieder Evaluationen weiter erwartungsgemäss in vielfältigen Arten politisch. In den beiden konfliktivsten Fällen des Steuersystems für energieeffiziente Fahrzeuge und der Grundstufe nutzten die Zürcher Parlamentsmitglieder Evaluationen politisch in ihrer 
Argumentation sowie auf andere Arten. Etwa forderten die Gegner des Steuersystems für energieeffiziente Fahrzeige eine Regulierungsfolgeabschätzung relativ spät im Gesetzgebungsprozess in Fall 3. Im Rahmen der Diskussion der Grundstufe (Fall 7) kritisierten die Parlamentsmitglieder dagegen vor allem Evaluationen. In den beiden eher konfliktiven Fällen der Basisstufe und des Steuersystems für energieeffiziente Fahrzeuge im Kanton Bern lässt sich ebenso ein Kritisieren, Fordern oder Fragen nach Evidenz beobachten, das aber weniger ausgeprägt war. Bei den restlichen Fällen ist keine politische Verwendung taktischer Art feststellbar. Da die konfliktreichsten Fälle im Kantonsrat Zürich waren, stellt sich weiter die Frage, inwiefern die Beobachtung verschiedener Nutzungsarten parlamentsspezifisch sein könnte. Diese Frage kann aber in der vorliegenden Untersuchung nicht abschliessend beantwortet werden.

Entgegen den Erwartungen ist hingegen die beobachtete, relativ rege analytische Nutzung bei den Fällen mit der vergleichsweise höchsten Konfliktivität (siehe Abbildung 28). Dies trifft besonders auf den Fall 7 zur Grundstufe im Kantonsrat Zürich zu, bei der sich sämtliche Parlamentsmitglieder differenziert mit Evaluationen auseinandersetzten. Diese wider Erwarten häufige analytische Verwendung könnte zum einen in massnahmenspezifischen Eigenschaften begründet sein, da sich die Parlamentsmitglieder auch im etwas weniger konfliktiven Fall 8 zur Basisstufe vertieft mit Evaluationen befassten. Eine andere mögliche Erklärung könnte in der Art der analytischen Verwendung liegen. Die Ausführungen in den Interviews legen nahe, dass sich die Parlamentsmitglieder zwar mit Evaluationen auseinandersetzten und sich so mit der Basis-/Grundstufe befassten, sie aber ihre Entscheide nicht darauf abstützen:

Es ist wirklich ausgiebig über diese Evaluation diskutiert worden. Man hat diese Berichte bekommen, man konnte alles lesen, man hat sich gegenseitig mit den Argumenten eben beworfen, aber eben [...], jeder hat dann das rausgelesen, was er wollte. (F5/7-PM4)

Die Beobachtungen im zweiten Fall mit der vergleichsweise höchsten Konfliktivität decken sich hingegen grösstenteils mit den Erwartungen. In diesem Gesetzgebungsprozess zum Steuersystem für energieeffiziente Fahrzeuge im Kantonsrat Zürich stand die Suche nach einer mehrheitsfähigen Vorlage im Vordergrund. Nur ein befragtes Parlamentsmitglied beschreibt die Evaluationen vertieft, während sich ein anderes Parlamentsmitglied gar nicht an Evaluationen erinnert (F3-PM3; F3-PM2). Folglich lassen die Beobachtungen aus den Fällen 3 und 7 den Schluss zu, dass analytische Verwendung bei einer hohen Konfliktivität möglich ist, aber eher in konzeptioneller Art oder in einzelnen Fällen.

Die Betrachtung der eher wenig umstrittenen Gesetzgebungsprozesse zur SSA weist zudem darauf hin, dass die Eigenschaften des Konflikts mit der Evaluationsnutzung in Beziehung stehen könnten. Die SSA wurde sowohl im Grossen Rat des Kantons Bern als auch im Kantonsrat Zürich relativ rege diskutiert und die Abstimmungen zu den Minderheitsanträgen fielen teilweise gar knapper aus als Abstimmungen in den ansonsten konfliktiveren Gesetzgebungsprozessen. Allerdings ging es in der Diskussion in beiden Kantonen nicht um das grundsätzliche Für und Wider der SSA. Die SSA war zum 
Zeitpunkt der Gesetzesberatungen bereits in der Mehrheit ${ }^{182}$ der grossen Gemeinden der beiden Kantone geplant oder etabliert. Die Wirksamkeit der SSA war unbestritten, wie die Ausführungen in den Protokollen und in den Interviews illustrieren (F5/7-PM4; F5/7-PM5; F6/8-PM3; F6/8-PM5; Grosser Rat des Kantons Bern 2011c, 1055). Der Konflikt und der gesamte Diskurs drehte sich daher um die Frage der Notwendigkeit einer kantonalen Vorschrift für die Gemeinden (Kantonsrat Zürich 2011d, 13539). Die geringe analytische und besonders die geringe politische Nutzung sind folglich auch damit zu erklären, dass es im Diskurs und damit in den Argumentationen kaum um die Wirkung ging.

Schliesslich veranschaulichen die Fallstudien zu den anderen beiden wenig konfliktiven Fällen der VHKA deutlich, dass damit eine geringe Aufmerksamkeit vonseiten des Parlaments und folglich wenig Möglichkeiten zur Evaluationsnutzung einhergehen. Am klarsten ist dieser Zusammenhang in Fall 2 zur VHKA im Grossen Rat Bern ersichtlich. Gemäss den Befragten aus Kommission und Verwaltung war die VHKA «wirklich einfach kein Thema mehr» (F2-Verw.). Zu ihr gab es keine Vorstösse, die Kommission diskutierte sie kurz, im Plenum stand sie dann gar nicht zur Diskussion. Dies schränkt nicht nur die Möglichkeiten ein, Evaluationsnutzung überhaupt zu beobachten, auch dürften die Parlamentsmitglieder kaum Zeit investiert haben, um sich mit der VHKA auseinanderzusetzen. In Zürich beschäftigte sich der Kantonsrat deutlich stärker mit der VHKA, aber auch dort war es «kein Thema, das bewegt hatte» (F1-Verw.). Die beiden Fallstudien illustrieren zudem auch, dass die Aufmerksamkeit, welche eine Massnahme erhält, auch von dem gesamten Gesetzesprojekt abhängt. Im Kanton Bern war die VHKA nur eine von vielen Änderungen im Rahmen der umfassenden Totalrevision des Energiegesetzes (F2-PM3; F1-Verw.). Der Kantonsrat in Zürich befasste sich dagegen mit der VHKA in einer relativ kleinen Teilrevision des Energiegesetzes sowie anhand einer Einzelinitiative, was verhältnismässig mehr Gelegenheiten zur Evaluationsnutzung bieten dürfte.

Zusammenfassend weisen die Resultate der Fallstudien darauf hin, dass eine gewisse Konfliktivität die analytische und die politische Nutzung fördert. Wie andere Studien nahelegen, bedarf es für die Auseinandersetzung im Parlament einer gewissen Konfliktivität (Frey 2012, 308; vgl. Tabuga 2017, 14). In Bezug auf die analytische Verwendung decken sich die Ergebnisse mit den Befunden von Frey $(2012,308)$, die in einigen konfliktiven Gesetzgebungsprozessen beobachtete, dass sich Parlamentsmitglieder von forschungsbasierten Informationen überzeugen liessen. Sie sprechen aber nicht für einen generell förderlichen Effekt der Konfliktivität auf die analytische Evaluationsnutzung, wie er etwa von Balthasar und Müller (2014) oder Mooney (1993) postuliert wird. Auch kann entgegen den Erwartungen von Whiteman (1985a, 305) nicht per se von «fluideren» Positionen bei wenig umstrittenen Fragen ausgegangen werden. Dies zeigen gerade die Fallstudien zur wenig konfliktiven VHKA, in der die Positionen bereits in früheren Gesetzgebungsprozessen ausgemacht wurden. In Bezug auf die politische Nutzung stimmen die vorliegenden Befunde mit den relativ weit verbreiteten Ergebnissen eines

182 Bern: Neuenschwander et al. (2007), Zürich: Amt für Jugend und Berufsberatung (2011). 
positiven Zusammenhangs mit der Konfliktivität überein (Frey 2012; Jenkins-Smith und Sabatier 1993; Whiteman 1985a; 1985b; vgl. Rissi und Sager 2013).

\section{Anderer politischer Kontextfaktor: Verwaltung mit bestimmender Stellung}

Wie die Verwaltung und die Regierung mit Evaluationen in einem Gesetzgebungsprozess umgehen, kann als weiterer, politischer Kontextfaktor bezeichnet werden. Die Ausführungen von Regierung und Verwaltung in den Antworten zu den Vorstössen, den Vernehmlassungsunterlagen sowie im Regierungsratsantrag bildeten jeweils eine wichtige Informationsquelle für die Parlamentsmitglieder (F2-Verw.; F4-PM2; F5/7-PM4; F6/8-PM2). Zudem präsentierten die Verwaltung und die Regierung die Vorlage in den Kommissionssitzungen, wobei sie in diesem Rahmen auf Evaluationen verwiesen und teilweise deren Befunde zusammenfassten. Ausführlicher und häufiger taten sie dies aber in schriftlichen Unterlagen. ${ }^{183}$

In den Fallstudien waren das Nutzungsverhalten von Regierung und Verwaltung und die damit verbundenen Auswirkungen auf das Nutzungsverhalten der Parlamentsmitglieder - sehr unterschiedlich. Die deutlich aktivste Rolle nahm die Exekutive in Fall 4 des Steuersystems für energieeffiziente Fahrzeuge im Kanton Bern ein. Während des gesamten Gesetzgebungsprozesses bezog sie eine Fachperson mit ein, weiter gab sie zwei Gutachten in Auftrag und verwies auf diese Gutachten sowie weitere Evaluationen und Studien in den Anträgen sowie während den Beratungen. Auch die Kommissionsmitglieder setzten sich bei der Diskussion der Grossratsvorlage vertieft mit Evaluationen auseinander, was auch auf einen überzeugenden Effekt der Präsentation zurückzuführen ist (vgl. F2-PM3; F4-PM1):

Vorab möchte ich dem Regierungspräsidenten und dem Strassenverkehrsamt ganz herzlich für die professionelle Betreuung und Aufbereitung dieser Gesetzesvorlage gratulieren. In vorbildlicher Art und Weise ging dies vor sich. Es begann damit - Sie können sich erinnern - dass der gesamte Grosse Rat zu einer Präsentation der Vorlage Ecotax durch Experten eingeladen wurde. Dort wurde der Grundstein für das heutige Gesetz gelegt. Es gelang ihnen so gut, dass andere Denkmodelle, die es auch gäbe, gar keine Chance hatten, weiterverfolgt zu werden. (Grosser Rat des Kantons Bern 2009b, 1298)

In der darauffolgenden Diskussion des Volksvorschlags lässt sich ein solcher Effekt auf den parlamentarischen Gesetzgebungsprozess nicht mehr identifizieren, auch wenn die Regierung und Verwaltung das zweite Gutachten in Auftrag gegeben hatten und dieses in der Kommissionssitzung ausführlich erläuterten. Der Grund für den relativ starken Einbezug von Evaluationen durch die Exekutive in diesem Fall scheint auch taktisch gewesen

183 Die von der Verwaltung und Regierung zitierten Evaluationen sind in den Tabellen im Abschnitt «Evaluationsnutzung nach Zeitpunkt und Quelle» aufgelistet. 
zu sein. Die hinzugezogene Fachperson könne «auf sehr gute Art erklären» (F4-Verw.), was man auch für die Diskussion im Kanton habe nutzen wollen. Gleichzeitig verdeutlichen die Ausführungen im Interview, dass die Grundlagen die Exekutive überzeugten und eine "fundierte, abgestützte, Wissens-Meinung» (F4-Verw.) erlaubten. Zudem kann der starke Einbezug damit erklärt werden, dass zu Beginn kein konfliktiver Gesetzgebungsprozess vorhersehbar war, sondern die Vorstösse vonseiten der FDP, SP und auch SVP einen breit abgestützten Konsens signalisierten.

Die Regierung und die Verwaltung verwendeten auch in den Fällen 7 und 8 der Basis-/Grundstufe Evaluationen, sie gaben ihnen aber ein anderes Gewicht. Sie standen vor der Herausforderung, wie mit den Ergebnissen der Evaluation von EDK-Ost 4bis8 umzugehen war, aus denen man "wirklich verschiedene Sachen rauslesen» (F6/8-Verw.) könne. In beiden Fällen waren die Ergebnisse der Evaluation von EDK-Ost 4bis8 ausführlich im Antrag des Regierungsrats zusammengefasst und auch Gegenstand der Kommissionsberatungen, die Regierung und die Verwaltung bezogen sich aber in ihrer Argumentation für die freiwillige Einführung der Grund- beziehungsweise Basisstufe nicht stark darauf. In Bern sei man bewusst sachlich und unaufgeregt mit der Evaluation von EDK-Ost 4bis8 umgegangen. Man habe «relativ offen gesagt: ja, die Leistungen sind nicht besser, aber das sei auch nicht das Ziel gewesen» (F6/8-Verw.). Auch in Zürich habe man «mehr politisch argumentiert, also weniger mit der Evaluation», um eine Mehrheit zu gewinnen (F5/7-Verw.).

Der zweite Fall zum Steuersystem für energieeffiziente Fahrzeuge im Kanton Zürich zeigt dagegen, dass die Evaluationsnutzung bereits bei der Aufbereitung des Geschäfts durch die Exekutive gehemmt sein kann. Evaluationen waren zwar bekannt, bei der Ausarbeitung des Gesetzes stützten sich die Regierung und Verwaltung aber kaum darauf (F3-Verw.). Einerseits hätten sie auf einen stärkeren Einbezug von Expertise verzichtet, da man Vorlagen zu den Verkehrsabgaben einfach halten müsse. Anderseits seien Informationen aus informellen Kontakten mit Autohändlern und vor allem aus dem Vernehmlassungsverfahren wichtiger gewesen (F2-Verw.). ${ }^{184}$ Nachdem Revisionen des Verkehrsabgabengesetzes zuvor fünf Mal an der Urne scheiterten, stand ganz klar im Fokus der Bemühungen von Regierung und Verwaltung, eine mehrheitsfähige Vorlage zu entwerfen.

Der Vergleich zwischen den Fallstudien der SSA legt schliesslich eine Bedeutung des Auslösers des Gesetzgebungsprozesses nahe. Im Kanton Bern kam die Initiative für die SSA vom Grossen Rat. Damit übereinstimmend sind besonders bei der Diskussion der parlamentarischen Vorstösse Verweise auf Evaluationen beobachtbar. In Zürich dagegen ging die Initiative zur Verankerung der SSA von der Verwaltung aus und Evaluationen fanden keine Verwendung im Parlament.

Aus den Ausführungen in den Interviews geht hervor, dass die befragten Kommissionsmitglieder die Informationen der Verwaltung als glaubwürdig wahrnehmen und sich in allen Gesetzgebungsprozessen gut informiert fühlten. Vor allem im Rahmen der Gesetzgebungsprozesse zu den Massnahmen mit relativ grosser Wissenschaftsnähe beto-

184 Auch in einem anderen Gespräch betonte ein Mitglied der Verwaltung die Bedeutung der Vernehmlassung, die ja "auch eine Art Evaluation» sei (F6/8-Verw.). 
nen die befragten Kommissionsmitglieder die Hilfestellung durch die Verwaltung. So hätte die Verwaltung allfällige Abklärungen für sie gemacht und sei mit Informationen zur Verfügung gestanden (F2-PM3; vgl. F1-PM1; F4-PM1; F4-PM3; F6/8-PM4). Zwar räumten die befragten Kommissionsmitglieder ein, dass die Exekutive auch ihre Interessen hätte und diskutierten ihren Einbezug in die Kommissionsberatungen mitunter kritisch (F3-PM1; F6/8-PM3; F6/8-PM2). Sie befanden aber die Informationen vonseiten der Exekutive als «fundiert» (F1-PM1). Die Ausführungen vonseiten der Beteiligten aus der Verwaltung stimmen damit überein. Man hätte ein "gutes Vertrauensverhältnis» (F2-Verw.) mit den Kommissionsmitgliedern und fühle sich «als Fachleute anerkannt» (F1-Verw.).

Insgesamt unterstützen die Ergebnisse aus den Fallstudien die bisherige Forschung zur Nutzung von Evaluationen in der Schweiz: Frey (2012) und Himmelsbach (2014) stellen in ihren Analysen auf Bundesebene fest, dass der Verwaltung eine zentrale Position in der Beschaffung, Vermittlung und Aufbereitung von Evidenz zukommt (vgl. Balthasar 2009a). In ihrer Untersuchung des Walliser Kantonsparlaments betrachtet auch Horber-Papazian (2011) die Verwaltung als wesentlich für den Umgang der Parlamentsmitglieder mit Wirkungsindikatoren. Gerade in den Milizparlamenten, wie dies der Kantonsrat Zürich und der Grosse Rat Bern sind, wird die Verwaltung als umso bedeutender für die Verarbeitung von Evaluationen gesehen (vgl. Frey 2012). Die Bedeutung der Verwaltung war zwar am bedeutendsten beim Bonus-Malus-System für energieeffiziente Fahrzeuge, das eine nichtständige Kommission ohne Sekretariat beriet, über die anderen Fälle scheint aber keine systematische Interaktion von Kommissionensystem und Rolle der Verwaltung ersichtlich. ${ }^{185}$ Übereinstimmend mit den Beobachtungen zu Fall 3 weisen schliesslich verschiedene Studien zur Schweiz daraufhin, dass Evidenz in konfliktiven Gesetzgebungsprozessen bereits bei der Ausarbeitung der Vorlage durch die Verwaltung wenig Einflussmöglichkeiten hat (vgl. Ledermann 2014; Sager und Rissi 2011).

\subsubsection{Evaluationsspezifischer Kontext: Nutzung einschlägiger Evaluationen}

Als evaluationsspezifische Kontextfaktoren umfasst der theoretische Rahmen weiter die Rechtzeitigkeit und Aktualität der Evaluationen sowie die Übereinstimmung der Evaluationsergebnisse zu einer Massnahme. Die Grundlage für die Diskussion dieser Erklärungsfaktoren bilden die Abschnitte in den Fallstudien, die sich der Evaluationsnutzung nach Zeitpunkt sowie nach Positionen der Parlamentsmitglieder widmen.

\section{Rechtzeitigkeit und Aktualität: Rechtzeitig verfügbar, Aktualität nicht wegweisend}

Die rechtzeitige Verfügbarkeit von Evaluationen kann als Voraussetzung für Evaluationsnutzung angesehen werden. Gemäss den theoretischen Erläuterungen als rechtzeitig für die analytische und politische Verwendung werden Evaluationen aufgefasst, wenn sie

185 Dies deckt sich mit der Einschätzung einer geringen Relevanz der Sekretariate der ständigen Kommissionen für die Evaluationsnutzung (vgl. 5.6.2.1), wobei die Rolle dieser Sekretariate nicht systematisch untersucht wurde. 
bis zur frühen Kommissionsphase erschienen sind. Bis zu diesem Zeitpunkt sollten sich die Parlamentsmitglieder über eine Massnahme informieren und daher verhältnismässig offen gegenüber Evaluationen sein. Idealerweise sollten die Evaluationen noch vor den Kommissionsberatungen erscheinen, da die Verwaltung diese dann in ihren Dokumenten aufbereiten kann. Politisch verwenden Parlamentsmitglieder Evaluationen zwar erwartungsgemäss besonders nach dem Bezug ihrer Positionen in späteren Phasen des Gesetzgebungsprozesses. Sie sollten aber vor allem in der Kommissionsphase von Evaluationen erfahren. Übereinstimmend mit der zentralen Bedeutung der Aktualität in der Politik wird ein positiver Zusammenhang mit der analytischen $\left(H_{8 a}\right)$ und der politischen Evaluationsnutzung $\left(H_{8 p}\right)$ erwartet. Informationen aus aktuellen Evaluationen haben eine hohe Überzeugungskraft, da sie sich eher auf die momentane Situation übertragen lassen. Diese hohe Überzeugungskraft sollte sie auch als politisches Mittel attraktiv machen.

In den untersuchten Gesetzgebungsprozessen erschienen die Evaluationen gemäss Tabelle 33 in der Regel rechtzeitig für die analytische und politische Verwendung, einige zentrale Quellen waren aber erst im Laufe der Kommissions- oder vereinzelt der Plenumsphase vorhanden. In drei Fällen erschienen alle Evaluationen bereits vor der ersten Kommissionssitzung und waren daher alle rechtzeitig verfügbar. Die beiden Fälle der VHKA zeigen aber, dass dies nicht zu analytischer oder politischer Nutzung führen muss. In den restlichen fünf Fällen war die Mehrheit der Evaluationen ebenfalls bis zur ersten Kommissionssitzung vorhanden, einzelne Quellen waren aber erst später verfügbar. Mit einigen dieser Quellen setzten sich die Parlamentsmitglieder durchaus vertieft auseinander und verwiesen mehrfach darauf in den Debatten. Namentlich betrifft dies die Präsentationen der Fachpersonen, die beim Steuersystem für energieeffiziente Fahrzeuge (Fall 3) und bei der Grundstufe (Fall 7) im Kantonsrat Zürich sowie beim Steuersystem für energieeffiziente Fahrzeuge im Grossen Rat des Kantons Bern (Fall 4) stattfanden. Folglich scheinen Informationen, die in Präsentationen direkt an die Parlamentsmitglieder vermittelt werden, auch in der Kommissionsphase noch rechtzeitig für die analytische und politische Verwendung.

Entgegen den Hypothesen $H_{8 a}$ und $H_{8 p}$ verwendeten die Parlamentsmitglieder aktuellere Evaluationen oder Zusammenfassungen nicht häufiger. Dies verdeutlichen besonders die Abschnitte in den Fallstudien zur Evaluationsnutzung nach Quelle und Zeitpunkt (siehe auch Tabelle 33). Die Parlamentsmitglieder verwendeten zwar aktuelle Quellen: Sie griffen Evaluationen oder Zusammenfassungen innert Monaten oder spätestens innerhalb zwei Jahren erstmals auf. Die Verwendung während des gesamten Gesetzgebungsprozesses konzentrierte sich jedoch in der Regel auf eine bis maximal drei Quellen. Auch wenn teilweise relevante Quellen erschienen, nahmen die Parlamentsmitglieder diese nicht auf. Ein Beispiel dafür sind die Schlussberichte der Evaluation von EDK-Ost 4bis8, die keine Verwendung fanden. Folglich ist im Nutzungsverhalten der Parlamentsmitglieder kein konsequenter Fokus auf Aktualität ersichtlich.

Die verwendeten Evaluationen oder Zusammenfassungen stellen zumeist einschlägige Quellen dar. Zu den genutzten Quellen zählen einerseits, wie zuvor erwähnt, durch Fachpersonen vermittelte Informationen. Anderseits sind auch einzelne Berichte einschlägig. In Fall 4 verwendeten etwa die Berner Grossratsmitglieder zwei Gutachten 
Tabelle 33: Verfügbarkeit der Evaluationen und identifizierbare, genutzte Quellen

\begin{tabular}{|c|c|c|c|c|c|c|c|c|}
\hline & \multicolumn{4}{|c|}{ VHKA } & \multicolumn{4}{|c|}{ Steuersystem für energieeffiziente Fahrzeuge } \\
\hline & \multicolumn{2}{|c|}{ Fall 1: Zürich } & \multicolumn{2}{|c|}{ Fall 2: Bern } & \multicolumn{2}{|c|}{ Fall 3: Zürich } & \multicolumn{2}{|c|}{ Fall 4: Bern } \\
\hline & Datum & $\begin{array}{l}\text { Anzahl } \\
\text { Eval./ } \\
\text { Zusf. }\end{array}$ & Datum & $\begin{array}{l}\text { Anzahl } \\
\text { Eval./ } \\
\text { Zusf. }\end{array}$ & Datum & $\begin{array}{l}\text { Anzahl } \\
\text { Eval./ } \\
\text { Zusf. }\end{array}$ & Datum & $\begin{array}{l}\text { Anzahl } \\
\text { Eval./ } \\
\text { Zusf. }\end{array}$ \\
\hline \multicolumn{9}{|l|}{ Verfügbar bis vor: } \\
\hline - Regierungsratsantrag & 03.03 .2010 & & 01.07 .2009 & 7 & 14.04 .2010 & 15 & 28.04 .2009 & 15 \\
\hline - 1. Kommissionssitzung & 20.04 .2010 & - & 11.09 .2009 & - & 15.06 .2010 & +1 & 18.06 .2009 & +1 \\
\hline - 1. Plenumssitzung & 21.03 .2011 & & 16.11 .2009 & - & 18.04 .2011 & +2 & 31.08 .2009 & - \\
\hline - Schlussabstimmung & 11.07 .2011 & & 24.11 .2010 & - & 28.11 .2011 & - & 25.11 .2010 & +2 \\
\hline Analytische Nutzung & \multicolumn{2}{|l|}{ selten } & \multicolumn{2}{|l|}{ keine } & \multicolumn{2}{|l|}{ eher selten } & \multicolumn{2}{|l|}{ eher häufig } \\
\hline Verwendete Quellen & \multicolumn{2}{|c|}{$\begin{array}{l}\text { bilateraler Austausch } \\
\text { mit Fachperson }\end{array}$} & \multicolumn{2}{|l|}{-} & \multicolumn{2}{|c|}{$\begin{array}{l}\text { Präsentation WAK } \\
(2010)\end{array}$} & \multicolumn{2}{|c|}{$\begin{array}{l}\text { Präsentation Plenum } \\
\text { (2009), ETH (2008), } \\
\text { Ernst Basler Partner } \\
\text { (2010) }\end{array}$} \\
\hline Politische Nutzung & \multicolumn{2}{|l|}{ selten } & \multicolumn{2}{|l|}{ keine } & \multicolumn{2}{|l|}{ eher häufig } & \multicolumn{2}{|l|}{ eher häufig } \\
\hline \multirow[t]{4}{*}{ Verwendete Quellen } & \multicolumn{2}{|c|}{ nicht identifizierbar } & \multicolumn{2}{|l|}{-} & \multicolumn{2}{|c|}{$\begin{array}{l}\text { asa (2007), Präsentation } \\
\text { WAK (2010) }\end{array}$} & \multicolumn{2}{|c|}{$\begin{array}{l}\text { Präsentation Plenum } \\
\text { (2009), ETH (2008), } \\
\text { Ernst Basler Partner } \\
(2010)\end{array}$} \\
\hline & \multicolumn{4}{|c|}{ SSA } & \multicolumn{4}{|c|}{ Basis-/Grundstufe } \\
\hline & \multicolumn{2}{|c|}{ Fall 5: Zürich } & \multicolumn{2}{|c|}{ Fall 6: Bern } & \multicolumn{2}{|c|}{ Fall 7: Zürich } & \multicolumn{2}{|c|}{ Fall 8: Bern } \\
\hline & Datum & $\begin{array}{l}\text { Anzahl } \\
\text { Eval./ } \\
\text { Zusf. }\end{array}$ & Datum & $\begin{array}{l}\text { Anzahl } \\
\text { Eval./ } \\
\text { Zusf. }\end{array}$ & Datum & $\begin{array}{l}\text { Anzahl } \\
\text { Eval./ } \\
\text { Zusf. }\end{array}$ & Datum & $\begin{array}{l}\text { Anzahl } \\
\text { Eval./ } \\
\text { Zusf. }\end{array}$ \\
\hline \multicolumn{9}{|l|}{ Verfügbar bis vor: } \\
\hline - Regierungsratsantrag & 16.12 .2009 & 16 & 08.06 .2011 & 23 & 07.07 .2010 & 22 & 08.06 .2011 & 25 \\
\hline -1. Kommissionssitzung & 02.02 .2010 & - & 22.09.2011 & +1 & 21.09 .2010 & - & 22.09 .2011 & - \\
\hline -1. Plenumssitzung & 07.02 .2011 & +5 & 22.11.2011 & +1 & 06.12 .2010 & +4 & 22.11 .2011 & - \\
\hline - Schlussabstimmung & 14.03 .2011 & - & 21.03 .2012 & - & 02.07 .2012 & +1 & 21.03 .2012 & - \\
\hline Analytische Nutzung & selten & & selten & & häufig & & häufig & \\
\hline Verwendete Quellen & - & & $\begin{array}{l}\text { Neuenschwar } \\
\text { (2007), von N }\end{array}$ & $\begin{array}{l}\text { ider et al. } \\
\text { latt (2002) }\end{array}$ & $\begin{array}{l}\text { EDK-Ost (201 } \\
\text { tationen KBIK }\end{array}$ & $\begin{array}{l}\text { 0), Präsen- } \\
\text { (2010) }\end{array}$ & EDK-Ost (2010 & \\
\hline Politische Nutzung & keine & & eher selten & & häufig & & eher häufig & \\
\hline Verwendete Quellen & - & & $\begin{array}{l}\text { Neuenschwal } \\
(2007)\end{array}$ & ider et al. & $\begin{array}{l}\text { EDK-Ost (201 } \\
\text { tationen KBIK }\end{array}$ & $\begin{array}{l}\text { 0), Präsen- } \\
(2010)\end{array}$ & EDK-Ost (2010 & \\
\hline
\end{tabular}

Die verwendeten Quellen beziehen sich auf die Nutzung der Parlamentsmitglieder, aufgeführt sind nur identifizierbare Quellen.

Hellgraue Schattierung: Rechtzeitig für analytische und politische Nutzung. 
analytisch und politisch, die sich spezifisch auf das Berner Bonus-Malus-System bezogen. Weiter verwendeten die Parlamentsmitglieder vor allem umfassende Evaluationen oder Berichte mit Überblickscharakter. Beispiele sind der Projektschlussbericht der EDK-Ost (2010) bei der Basis-/Grundstufe, Neuenschwander et al. (2007) bei der SSA im Kanton Bern oder asa (2007) beim Steuersystem für energieeffiziente Fahrzeuge im Kanton Zürich. Allerdings wären solche Berichte zu Massnahmen wie der VHKA (Rieder et al. 2008) oder zur SSA in Zürich (Müller 2004) ebenfalls verfügbar gewesen, fanden aber keine Verwendung.

Grundsätzlich legen also die Fallstudien nahe, dass Evaluationen in der Regel rechtzeitig zur Verfügung standen und Parlamentsmitglieder die Quellen erstmals relativ zeitnah nach deren Erscheinen verwendeten, aber ihre Nutzung nicht konsequent auf die Aktualität der Quellen ausrichteten. Die vorliegenden Ergebnisse weisen daher im Gegensatz zur Studie des US-Kongresses von Florio et al. $(1979,69)$ darauf hin, dass das Timing in den untersuchten Gesetzgebungsprozessen keine major barrier darstellte. Vielmehr decken sie sich mit einer anderen Studie zum US-Kongress von Boyer und Langbein (1991), die Evaluationen grösstenteils als rechtzeitig bezeichnen. Schliesslich sprechen die vorliegenden Ergebnisse übereinstimmend mit der bestehenden Literatur für eine gewisse Vorlaufzeit der Evaluationen (Ginsburg und Rhett 2003; Leviton und Hughes 1981). Sie illustrieren jedoch, dass Parlamentsmitglieder Evaluationen auch schon nach Wochen oder Monaten aufgreifen können.

\section{Übereinstimmung der Evaluationsergebnisse: Gewisser Konsens förderlich}

Übereinstimmende Ergebnisse aus Evaluationen sollten gemäss den Hypothesen unterschiedlich mit der analytischen und der politischen Nutzung zusammenhängen. Generell wird die Verfügbarkeit von mehreren Evaluationen als förderlich für die Nutzung angesehen, da eine breitere Evidenzbasis eine umfassendere Perspektive ermöglicht (vgl. Ginsburg und Rhett 2003; Leviton 2003). Besteht ein wissenschaftlicher Konsens aus übereinstimmenden Ergebnissen mehrerer Evaluationen, können sich Parlamentsmitglieder der Evidenz weniger verschliessen. Dies sollte die analytische Verwendung begünstigen $\left(H_{9 a}\right)$. Die politische Verwendung sollten übereinstimmende Ergebnisse hingegen einschränken $\left(H_{g_{p}}\right)$. Bei übereinstimmenden Ergebnissen sollten Parlamentsmitglieder vor allem Evaluationen in ihrer Argumentation verwenden, wenn sich ihre Position mit den Ergebnissen deckt. Sind die Ergebnisse widersprüchlich, können sich Parlamentsmitglieder unabhängig von ihrer Position auf Evaluationen abstützen. Evaluationen zu fordern oder zu kritisieren sollte schliesslich unabhängig der Evidenzlage möglich sein.

Die beobachtete Evaluationsnutzung in den Fallstudien unterstützt die Hypothesen nicht: Parlamentsmitglieder nutzten Evaluationen am häufigsten analytisch und politisch bei den beiden Massnahmen, zu denen die Übereinstimmung der Evaluationsergebnisse als relativ moderat angesehen werden kann (vgl. Tabelle 34). Mit Evaluationen zur SSA, deren Ergebnisse am meisten übereinstimmten, setzte sich kein befragtes Kommissionsmitglied differenziert auseinander. Nur ein Teil der Befragten weiss überhaupt von Evaluationstätigkeiten. Gleichzeitig lässt sich bei den Fällen zur VHKA, deren Evaluati- 
onsergebnisse relativ wenig übereinstimmten, politische Evaluationsnutzung nicht oder nur ganz vereinzelt beobachten.

\section{Tabelle 34: Häufigkeit der Nutzung und Übereinstimmung der Evaluationsergebnisse}

\begin{tabular}{|c|c|c|c|c|c|c|}
\hline \multicolumn{2}{|c|}{ Evaluationen und Zusammenfassungen } & \multirow[t]{3}{*}{$\mathrm{N}$} & \multirow[t]{3}{*}{ Massnahme } & \multirow[t]{3}{*}{ Fall } & \multirow[t]{3}{*}{$\begin{array}{l}\text { analytische } \\
\text { Nutzung }\end{array}$} & \multirow[t]{3}{*}{ politische Nutzung } \\
\hline \multicolumn{2}{|c|}{ Übereinstimmung } & & & & & \\
\hline Einordnung & Beschrieb & & & & & \\
\hline \multirow[t]{2}{*}{ tief } & $\begin{array}{l}\text { Wirkungszusammenhänge meist von einer } \\
\text { Evaluation/Zusammenfassung bzw. vereinzelt }\end{array}$ & 6 & VHKA & Fall 1 & $\begin{array}{l}\text { selten } \\
\text { (einseitig) }\end{array}$ & $\begin{array}{l}\text { selten } \\
\text { (einseitig) }\end{array}$ \\
\hline & $\begin{array}{l}\text { nicht untersucht, Ergebnisse ansonsten teilweise } \\
\text { widersprüchlich. }\end{array}$ & 7 & & Fall 2 & $\begin{array}{l}\text { keine } \\
(-)\end{array}$ & $\begin{array}{l}\text { keine } \\
(-)\end{array}$ \\
\hline \multirow[t]{2}{*}{ moderat } & $\begin{array}{l}\text { einzelne Wirkungszusammenhänge nicht oder in } \\
\text { einer Evaluation/Zusammenfassung untersucht, }\end{array}$ & 18 & Steuersystem & Fall 3 & $\begin{array}{l}\text { eher selten } \\
\text { (einseitig) }\end{array}$ & $\begin{array}{l}\text { eher häufig } \\
\text { (ausgewogen) }\end{array}$ \\
\hline & $\begin{array}{l}\text { Ergebnisse ansonsten weitgehend übereinstimmend, } \\
\text { oft basierend auf einem Projekt. }\end{array}$ & 18 & & Fall 4 & $\begin{array}{l}\text { eher häufig } \\
\text { (ausgewogen) }\end{array}$ & $\begin{array}{l}\text { eher häufig } \\
\text { (ausgewogen) }\end{array}$ \\
\hline \multirow[t]{2}{*}{ moderat } & $\begin{array}{l}\text { alle Wirkungszusammenhänge mehr als in zwei } \\
\text { Evaluationen/Zusammenfassungen untersucht, }\end{array}$ & 27 & $\begin{array}{l}\text { Basis-/ } \\
\text { Grundstufe }\end{array}$ & Fall 7 & $\begin{array}{l}\text { häufig } \\
\text { (ausgewogen) }\end{array}$ & $\begin{array}{l}\text { häufig } \\
\text { (ausgewogen) }\end{array}$ \\
\hline & $\begin{array}{l}\text { weitgehend übereinstimmend, oft basierend auf } \\
\text { einem Versuch (EDK-Ost 4bis8). }\end{array}$ & 25 & & Fall 8 & $\begin{array}{l}\text { häufig } \\
\text { (ausgewogen) }\end{array}$ & $\begin{array}{l}\text { eher häufig } \\
\text { (ausgewogen) }\end{array}$ \\
\hline \multirow[t]{2}{*}{ hoch } & $\begin{array}{l}\text { alle Wirkungszusammenhänge mehr als in zwei } \\
\text { Evaluationen/Zusammenfassungen untersucht, }\end{array}$ & 21 & SSA & Fall 5 & $\begin{array}{l}\text { selten } \\
\text { (einseitig) }\end{array}$ & $\begin{array}{l}\text { kein } \\
(-)\end{array}$ \\
\hline & $\begin{array}{l}\text { weitgehend übereinstimmend, basierend auf diverse } \\
\text { Projekte in Gemeinden. }\end{array}$ & 25 & & Fall 6 & $\begin{array}{l}\text { selten } \\
\text { (einseitig) }\end{array}$ & $\begin{array}{l}\text { eher selten } \\
\text { (ausgewogen) }\end{array}$ \\
\hline
\end{tabular}

Übereinstimmung bezieht sich auf Wirkungszusammenhänge zu Outcome bei direkten Adressatinnen und Adressaten sowie Impact gemäss den entworfenen Wirkungsmodellen. Ausgewogen bedeutet, dass die Parlamentsmitglieder Evaluationen unabhängig ihrer Position nutzten.

Weiter deckt sich die beobachtete politische Evaluationsnutzung auch nicht mit den Erwartungen eines unterschiedlichen Musters (vgl. Tabelle 34). Bei übereinstimmenden Ergebnissen verwendeten nicht nur Parlamentsmitglieder Evaluationen politisch, die von diesen Ergebnissen unterstützt werden. Die Fallstudien verdeutlichen vielmehr, dass Parlamentsmitglieder mit unterschiedlichen Positionen dieselben Evaluationen in ihrer Argumentation verwenden können. Etwa können Parlamentsmitglieder, deren Position den Ergebnissen der Evaluationen widerspricht, diese dennoch zur Erläuterung von Hintergrundinformationen verwenden (siehe Fall 3 oder Fall 6). Zusätzlich weisen die Fallstudien zur Basisstufe darauf hin, dass Parlamentsmitglieder Ergebnisse zu verschiedenen Fragestellungen aus einer Evaluation verwenden können. Die Gegnerinnen und Gegner der Basis-/Grundstufe beriefen sich so vor allem auf den festgestellten ähnlichen Lernstand, den die Kinder am Ende der verschiedenen Modelle der Eingangsstufe haben, während die Befürworterinnen und Befürworter der Basis-/Grundstufe hingegen die hohe Zufriedenheit der Erziehenden sowie die Erreichung der pädagogischen Ziele betonten. 
Die Analyse der Fallstudien weist darauf hin, dass das Bestehen eines grossen Evaluations- oder Forschungsprojekts die Verwendung von Evaluationen und gewissermassen die Übereinstimmung der Ergebnisse fördert. Die beiden Fälle mit einer moderaten Übereinstimmung zeichnen sich dadurch aus, dass die teilweise umfassenden Evaluationen je auf einem grossen Forschungsprojekt (Steuersystem für energieeffiziente Fahrzeuge) oder Schulversuch (Basis-/Grundstufe) basierten. Dies impliziert zwangsläufig eine gewisse Übereinstimmung der Ergebnisse, es sollte aber auch die Bekanntheit der Evaluationen oder Fachpersonen und damit deren Zugang zum Parlament begünstigen. Einzelne umfassende Evaluationen (VHKA) oder viele kleinere Evaluationsprojekte in den Gemeinden (SSA) haben vermutlich nicht die Strahlkraft solcher grossen Projekte. Folglich lassen die Fallstudien den Schluss zu, dass Evaluationsnutzung einer gewissen Übereinstimmung bedarf. Sind Evaluationen ein Thema, finden sie unabhängig der Position der Parlamentsmitglieder Verwendung. Im Gegensatz zu Esterling (2004) deuten die Fallstudien daher auf keine einseitige Nutzung bei übereinstimmenden Ergebnissen hin. Parlamentsmitglieder können Evaluationen gemäss den Fallstudien nicht nur unabhängig von ihrer Position fordern oder kritisieren, sondern sie auch damit argumentieren. Dies stützt die in der Literatur postulierte Annahme, dass eine selektive, politische Nutzung stets möglich ist (vgl. Boswell 2009; Frey 2012; Weiss 1990). Indem die Fallstudien schliesslich aufzeigen, dass übereinstimmende und umfassende Ergebnisse nicht immer entsprechend berücksichtigt werden, decken sie sich auch mit den Befunden von Frey $(2012,275)$.

\subsubsection{Aussagekraft der Ergebnisse und Rahmenbedingungen der Interpretation}

Die Interpretation und Aussagekraft der zuvor präsentierten Ergebnisse ist in vielerlei Hinsicht vom Vorgehen bei den Fallstudien abhängig. Generell ist zu beachten, in welchem Kontext sich die untersuchten Gesetzgebungsprozesse befinden. Ausgewählt wurden Fälle in den Bereichen der Energie-, Transport-, Bildungs- und Sozialpolitik in zwei grossen, mehrheitlich deutschsprachigen Kantonen. Verglichen mit anderen Politikbereichen ist die Evaluationstätigkeit in diesen vier Bereichen allgemein hoch. Besonders im Bildungsbereich sind Evaluationen auch auf kantonaler Ebene sehr verbreitet. Zudem findet ein grosser Teil der Evaluationstätigkeit auf kantonaler Ebene in den Kantonen Bern und Zürich statt (Balthasar und Rieder 2009, 407-409; Frey et al. 2016, 512). Diese zwei grössten Kantone haben zwar entsprechend ausgebaute Verwaltungen, aber für die Gesetzgebung zuständig sind dennoch Milizparlamente (Bundi et al. 2017; Vatter 2014). Im schweizerischen politischen System prägen zudem das Vernehmlassungsverfahren vor der parlamentarischen Phase sowie eine mögliche Volksabstimmung danach die Gesetzgebungsprozesse. Folglich begrenzen diese zentralen Merkmale die Übertragbarkeit der Ergebnisse auf andere Politikbereiche oder andere politische Systeme.

Die Auswahl der Fälle hat ebenso Folgen für die Interpretation der Ergebnisse. Neben den Rahmenbedingungen für sämtliche Fälle bildeten die kontextuellen Faktoren des Kommissionensystems, der Wissenschaftsnähe und der Konfliktivität die Kriterien zur Auswahl der acht Fälle. Gemäss der Logik der most similar cases sollten die Fälle bis auf 
einen der drei Faktoren vergleichbar sein. Da sich die Auswahl aber auf Fälle mit variierender Ausprägung der Faktoren beschränkt, können die Ergebnisse eines Falls nicht mit einem anderen Fall mit gleicher Ausprägung der Faktoren verglichen werden (Yin 2014, 57-61). Dies würde gerade bei Resultaten Klärung verschaffen, die nicht eindeutig sind oder nicht den Erwartungen entsprechen. Erschwerend kommt hinzu, dass die Evaluationsnutzung nicht nur von den drei untersuchten Faktoren abhängt. Dies bedeutet, dass der Fallstudienvergleich nicht erlaubt, einen einzigen zentralen Faktor zu identifizieren. Gleichzeitig ermöglicht die vertiefte, differenzierte Betrachtung der Evaluationsnutzung in den Fallstudien aber, Erklärungsansätze abzuleiten und auf zukünftige Forschungsfragen hinzuweisen.

Neben diesen generelleren Implikationen des Kontexts und des Fallstudiendesigns sind die Definitionen von Evaluation und Nutzung für die Interpretation des beobachteten Ausmasses der Evaluationsnutzung grundlegend. In den Fallstudien wurden beide Konzepte relativ offen definiert. Evaluationen beinhalten gemäss der Definition eine systematische und transparente Beurteilung einer der fünf Stufen des Wirkungsmodells, d.h. Input, Prozess, Output, Outcome und Impact. Dieses Wirkungsmodell wurde im Rahmen dieser Untersuchung für die vier untersuchten Massnahmen auf Basis bestehender Evaluationen und weiterer Fachliteratur erstellt. Aus dieser Definition folgt, dass teilweise auch deskriptive Studien zur Leistung (Output) einer Massnahme als Evaluationen zählten, die sich aber nur wenig auf die gemäss Klöti $(1997,46)$ bei Evaluationen so zentralen Wirkungen (Outcome, Impact) einer Massnahme beziehen. Da kompakt aufbereitete Informationen für Parlamentsmitglieder ebenfalls nützlich sein sollten, wurden neben Evaluationen auch Zusammenfassungen der zentralen Ergebnisse der Evaluationen erfasst. Bei der Definition der analytischen Nutzung stand die Frage im Zentrum, ob und wie differenziert sich die befragten Kommissionsmitglieder mit Evaluationen auseinandersetzten. In Übereinstimmung mit bisherigen Studien war das Verständnis der politischen Nutzung ebenfalls breit. Es umfasste auch taktische Arten dieser Nutzung, wie etwa das Fordern und Kritisieren von Evaluationen sowie das Fragen nach Evaluationen (vgl. Frey 2012). Um als Nutzung von Evaluationen oder Zusammenfassung zu qualifizieren, müssen zudem die spezifischen Quellen nicht zwingend ersichtlich sein, sondern die Aussagen müssen auf ein systematisches und transparentes Vorgehen schliessen lassen.

Solche breiten Definitionen der beiden zentralen Konzepte zu verwenden, ist angesichts der Beobachtungen in den Fallstudien durchaus gerechtfertigt. Zwar fanden deskriptiv ausgerichtete Evaluationen häufig Verwendung in einigen der untersuchten Gesetzgebungsprozessen, gerade beim Steuersystem für energieeffiziente Fahrzeuge in Zürich (Fall 3) und bei der SSA in Bern (Fall 6). 186 Zudem konzentrierte sich die politische Verwendung in Fall 3 zum Steuersystem für energieeffiziente Fahrzeuge massgeblich auf Nutzung taktischer Art. Allerdings lässt sich auch mit diesen lockeren Definitionen keine weitverbreitete analytische oder politische Nutzung in den schweizerischen Kantonsparlamenten beobachten. Zudem stimmt diese pragmatische Herangehensweise mit der bisherigen Forschung überein, die ein beschränktes Ausmass an Nutzung von

186 Namentlich: asa (2007) und Neuenschwander et al. (2007). 
Evidenz im Parlament und allgemein an systematischer Aufbereitung der Evidenz in den Kantonen erwarten lässt (vgl. die Studien zur Schweiz Balthasar und Müller 2014; Blatter et al. 2015; Fornerod 2001; Frey 2012; Horber-Papazian 2011). Schliesslich erlauben diese Definitionen ein umfassendes Bild der Evaluationsnutzung zu geben, was dieses Buch bezweckt.

Bei der Interpretation des Ausmasses an beobachteter Evaluationsnutzung zusätzlich relevant ist der Fokus auf Kommissionsmitglieder. Die Kommissionsmitglieder stehen einerseits im Zentrum der Untersuchung der analytischen Nutzung, da mit ihnen Interviews geführt wurden. Diese Samplingstrategie erfolgte, weil analytische Nutzung am ehesten bei Kommissionsmitgliedern überhaupt beobachtbar scheint. Generell nehmen Parlamentsmitglieder wenn möglich in Kommissionen Einsitz, in denen ihre zentralen Interessen liegen. Folglich sind die Kommissionsmitglieder vielleicht bereits auf andere Weise mit einer Massnahme konfrontiert, wie beispielsweise als Schulpräsident bei der SSA oder als Immobilienbesitzerin bei der VHKA. Bei der Wahl der Befragten wurden zudem Kommissionsmitglieder präferiert, die in den Kommissionsberatungen aktiv waren. Diese Samplingstrategie ist zwar anfällig für Verzerrungen, erscheint angesichts der Ergebnisse aber wiederum sinnvoll, da Evaluationen dennoch keineswegs allen befragten Kommissionsmitgliedern bekannt waren. Anderseits sind die Kommissionsmitglieder auch für die politische Nutzung zentral, da sie in der Regel die Position ihrer Fraktion im Plenum ausführen oder Anträge darlegen. Insgesamt führt dieser Fokus auf Kommissionsmitglieder daher zu einer Überschätzung der Evaluationsnutzung des gesamten Parlaments. Evaluationen verwenden nicht alle Parlamentsmitglieder, sondern hauptsächlich einzelne Parlamentsmitglieder mit Bezug zu einem Geschäft (Mooney 2012; Songer 1988; vgl. generell Newman und Head 2015).

Einschränkungen der Interpretation und Aussagekraft der Ergebnisse bestehen auch aufgrund der zugrundeliegenden Daten. Die Informationen aus den Interviews als erste Datenquelle basieren weitgehend auf der Offenheit und Erinnerungsfähigkeit der Parlamentsmitglieder. Diese Erinnerungsfähigkeit ist eingeschränkt, da die Interviews lange nach dem Gesetzgebungsprozess stattfanden (vgl. Herbert 2014; Leviton 2003). Zumal die untersuchten Massnahmen teilweise sehr zentrale Geschäfte waren, dürfte die Erinnerungsfähigkeit dennoch relativ intakt gewesen sein. Weiter kann angenommen werden, dass sich die befragten Parlamentsmitglieder noch an Evaluationen erinnern können, wenn diese eine zentrale Rolle spielten. Die Interviews können aber generell einen begrenzten Einblick in die vielen Geschehnisse geben, die während eines Gesetzgebungsprozesses in mündlicher Form im Parlament, in Sitzungen der Fraktionen oder in Gesprächen zwischen den Parlamentsmitgliedern ablaufen. Die Protokolle der Kommissions- und Plenumssitzung als zweite Datenquelle haben zwar den Vorteil, dass sie die Beratungen vollständig wiedergeben, sie sind aber nur begrenzt vergleichbar innerhalb sowie zwischen den Fällen. Differenzen in den Abläufen und Regeln bei den Kantonsparlamenten Bern und Zürich sowie die unterschiedliche Protokollierung der Kommissionsund Plenumsdebatten behindern Vergleiche der schriftlich dokumentierten Nutzung. Angesichts dieser Schwierigkeiten scheint es auch wenig sinnvoll, die Beobachtungen der politischen Nutzung zu quantifizieren. 
Trotz allen diesen erwähnten Punkten, die den Rahmen für die Interpretation der Ergebnisse der Fallstudien setzen, bieten die Fallstudien realer Gesetzgebungsprozesse ausführliche Einblicke in die Verwendung von Evaluationen in den schweizerischen Kantonsparlamenten. Das nächste Kapitel verbindet diese Einblicke zur Evaluationsnutzung und ihren kontextuellen Erklärungsfaktoren aus den Fallstudien mit den zentralen Ergebnissen zur Evaluationsnutzung und ihren individuellen Erklärungsfaktoren aus der Analyse der Befragungsdaten. Neben dieser Synthese der zentralen Erkenntnisse der vorliegenden Untersuchung werden im nächsten Kapitel zudem die Aussagekraft und die Tragweite dieser Erkenntnisse kritisch diskutiert. Ein kurzer Ausblick zu den Folgerungen, die sich aus diesen Erkenntnissen für die Rolle der Evaluation in den Parlamenten ergeben, folgt dann in einem separaten und letzten Kapitel des Buchs. 


\section{Synthese und Diskussion der Ergebnisse}

Wie nutzen die schweizerischen Parlamentsmitglieder Evaluationen und was sind Erklärungsfaktoren dieser Nutzung? Diese zwei zentralen Fragen des vorliegenden Buchs handeln vom Spannungsfeld zwischen systematisch generiertem Wissen und dem politischen Handeln der demokratisch gewählten Parlamentsmitglieder. Evaluationen beurteilen die Konzeption, die Umsetzung oder Wirkungen einer Politik anhand wissenschaftlicher Methoden und liefern damit den Parlamentsmitgliedern solch systematisch generiertes Wissen, das sie bei der Erfüllung ihrer Aufgaben unterstützen kann (Bättig und Schwab 2015; Speer et al. 2015). Für die Aufgabe der Gesetzgebung, die hier im Vordergrund steht, können Parlamentsmitglieder Evaluationen verwenden, um Änderungsbedarf bei bestehenden Regelungen zu erkennen oder neue Regelungen zu konzipieren. Im Einklang mit Forderungen nach einer evidenzbasierten Politikgestaltung tragen Evaluationen folglich dazu bei, dass sich das parlamentarische Handeln an den Leistungen und Wirkungen einer Politik orientieren kann. Die demokratisch gewählten Volksvertreterinnen und Volksvertreter im Parlament können bei ihren Entscheiden aber auch andere Gesichtspunkte als die Evidenz aus Evaluationen berücksichtigen, etwa die Interessen der Wählerschaft oder ideologische Grundhaltungen (Weiss 1999). Gerade im Fall von Parlamenten wird erwartet, dass solche ideologischen oder interessenbasierten Gesichtspunkte die politische Stossrichtung wesentlich festlegen. Folglich sollten Parlamentsmitglieder Evaluationen vor allem als politisches Mittel zur Unterstützung der eigenen Position verwenden und weniger als massgebliche Grundlage für ihre Entscheide (Balthasar 2005; Weiss 1989; Whiteman 1985b). Daraus folgt, dass Evaluationen kaum entscheidend sein sollten in der parlamentarischen Politikgestaltung. Ob und wie Parlamentsmitglieder Evaluationen tatsächlich verwenden, wurde bisher aber kaum empirisch untersucht, selbst wenn Evaluationen in der Schweiz klar etabliert sind und der übergeordneten Frage nach der Nutzung von systematisch generiertem Wissen in der Politik generell hohe Aufmerksamkeit zukommt.

Vor diesem Spannungsfeld wird im vorliegenden Buch grundsätzlich zwischen der analytischen und der politischen Nutzung von Evaluationen unterschieden, die durch individuelle und kontextuelle Faktoren beeinflusst wird (vgl. 3). Parlamentsmitglieder verwenden Evaluationen analytisch, um sich zu informieren und um zu ihren politischen Positionen zu gelangen. Dabei sind sie offen gegenüber Evaluationsergebnissen und bereit, ihre Positionen zu überdenken. Eine solche Offenheit bedarf es für die politische Nutzung nicht. Parlamentsmitglieder verwenden Evaluationen politisch als strategisches Mittel, um ihre eigene Position zu stützen. Die beiden Nutzungsformen repräsentieren folglich den grundlegenden Gegensatz zwischen einer analytisch-problemlösenden und einer politisch-strategischen Logik der Verwendung von Evaluationen (vgl. Boswell 2009; Frey 2012). Sie können in verschiedenen Nutzungsarten auftreten: Politisch nutzen kann ein Parlamentsmitglied Evaluationen beispielsweise persuasiv zur Überzeugung anderer oder legitimierend zur Bekräftigung eines bereits gefassten Entscheids. Aufgrund der konfrontativen Natur parlamentarischer Entscheidungsprozesse wird generell erwartet, dass 
Parlamentsmitglieder Evaluationen häufiger politisch als analytisch verwenden (Weiss 1987, 1989; Whiteman 1985b). Zumal die analytisch-problemlösende und die politisch-strategische Logik miteinander verbunden sind, wird weiter erwartet, dass die analytische Nutzung die politische Nutzung begünstigt (3.1; vgl. Boswell 2009; Frey 2012).

Die Evaluationsnutzung und ihre Erklärungsfaktoren werden anhand quantitativer und qualitativer Methoden untersucht. Eine schweizweite Online-Befragung bildet die Grundlage für die quantitative Analyse der individuellen Erklärungsfaktoren mittels ordinaler logistischer Regressionsmodelle. Die Stichprobe für die Analyse umfasst 1191 Parlamentsmitglieder der fünf grössten Parteien CVP, FDP, Grüne, SP und SVP aus 25 Kantonsparlamenten und der Bundesversammlung (vgl. 4.1). Die kontextuellen Faktoren werden anhand qualitativer Fallstudien von acht Gesetzgebungsprozessen in den Parlamenten der Kantone Bern und Zürich analysiert. Gegenstand der Fallstudien bilden je zwei Massnahmen der Energie- und Transportpolitik sowie der Bildungs- und Sozialpolitik pro Kantonsparlament. Die Fallstudien basieren einerseits auf einer Analyse der verfügbaren Evaluationen zur betrachteten Massnahme sowie von Dokumenten des untersuchten Gesetzgebungsprozesses, namentlich der Kommissions- und Plenumsprotokolle. Anderseits stützen sie sich auf Interviews mit 20 Parlamentsmitgliedern und 7 Personen aus den Kantonsverwaltungen (vgl. 5.1). Die beiden Methoden ergänzen sich: Während die Befragung die generelle Nutzung von Evaluationen in den schweizerischen Parlamenten umfassend darstellt, ermöglichen die Fallstudien einen vertieften Einblick in die Evaluationsnutzung, indem sie diese in echten Beispielen nachzeichnen. Dank dieser Kombination betrachtet die vorliegende Untersuchung das vielfältige Phänomen der Evaluationsnutzung zugleich umfassend und vertieft.

Strukturiert nach den zwei zentralen Fragen des vorliegenden Buchs präsentieren die folgenden Ausführungen die zentralen Erkenntnisse dieser Untersuchungen und stellen sie der bisherigen Literatur gegenüber. Im Lichte dieser Erkenntnisse wird danach diskutiert, inwiefern sich das gewählte Vorgehen zur Erforschung der Evaluationsnutzung eignet und welche Tragweite die Ergebnisse haben.

\subsection{Analytische und politische Evaluationsnutzung, meist durch Kommissionsmitglieder}

Wie nutzen die schweizerischen Parlamentsmitglieder Evaluationen? Die vorliegenden Untersuchungen zeigen, dass Parlamentsmitglieder Evaluationen sowohl analytisch als auch politisch verwenden. Gemäss der Befragung sind beide Formen der Nutzung relativ verbreitet, die analytische Nutzung ist aber ausgeprägter. 63.6 Prozent der befragten Parlamentsmitglieder verwenden Evaluationen eher häufig bis sehr oft in der instrumentellen Art, um einen Entscheid zu treffen, und 57.5 Prozent tun dies konzeptionell, um eine politische Massnahme besser zu verstehen. 48.3 Prozent der Befragten hingegen nutzen Evaluationen eher häufig bis sehr oft in der legitimierenden Art, um einen bereits getroffenen Entscheid zu begründen, und 54.5 Prozent verwenden Evaluationen persuasiv, um andere zu überzeugen. Die verschiedenen Kantonsparlamente und die Bundesversamm- 
lung unterscheiden sich kaum darin, wie häufig ihre Mitglieder Evaluationen verwenden (vgl. 4.2.1). Die Fallstudien relativieren das in der Befragung festgestellte Ergebnis einer verbreiteten und zudem ausgeprägt analytischen Verwendung: Evaluationsnutzung ist nicht in allen untersuchten Gesetzgebungsprozessen beobachtbar. Gemäss den Interviews setzte sich in vier der acht Fälle zumindest eines der befragten Kommissionsmitglieder differenziert mit Evaluationen auseinander. In diesen vier Fällen und einem weiteren Fall enthalten die analysierten Dokumente zudem Hinweise darauf, dass Parlamentsmitglieder auf Evaluationen verwiesen, diese gefordert oder kritisiert haben. Da die analytische Nutzung hauptsächlich anhand Interviews und die politische Nutzung vor allem anhand Dokumentenanalysen untersucht worden ist, lassen sich die Häufigkeiten der beiden Nutzungsformen schwer vergleichen. Generell kommen analytische und politische Nutzung wie erwartet zusammen vor, es gibt aber mehr Fälle mit einer vergleichsweise häufigen politischen Verwendung (vgl. 5.6.1). Insgesamt geben die Ergebnisse der beiden Analysen keine klare Antwort zur Annahme einer generell häufigeren politischen Verwendung in Parlamenten.

Diese Erkenntnisse zur Evaluationsnutzung in den schweizerischen Parlamenten stimmen grösstenteils mit bisherigen Befunden überein. Dass Evaluationsnutzung in parlamentarischen Gesetzgebungsprozessen variiert und in unterschiedlichen Formen vorkommt, stellen in ihren Fallstudien auch Frey (2012) für die schweizerische Bundesversammlung und Whiteman (1985a, 1995) für den US-Kongress fest (vgl. auch Boyer und Langbein 1991; Tabuga 2017). Diese Studien und die vorliegenden Ergebnisse zeigen folglich, dass Parlamentsmitglieder Evaluationen nicht ausschliesslich als politisches Mittel verwenden. Evaluationsnutzung im Parlament ist demzufolge politisch und analytisch.

Die vorliegenden Ergebnisse veranschaulichen weiter die besondere und weitreichende Bedeutung der parlamentarischen Kommissionen für die Evaluationsnutzung. Besonders zentral sind die Kommissionen für die analytische Verwendung: Die Parlamentsmitglieder setzten sich jeweils im Rahmen der Kommissionsberatungen mit Evaluationen oder Präsentationen von Fachpersonen auseinander. Verweise auf Evaluationen oder andere Arten der politischen Verwendung waren zwar in den Kommissionen vergleichsweise selten beobachtbar, die Kommissionsmitglieder waren aber häufig Sprecherinnen oder Sprecher ihrer Fraktionen in den Plenumsdebatten. Die Fallstudien zeigen, dass zumindest einige der Kommissionsmitglieder Evaluationen im Plenum erwähnten, wenn diese bereits in der Kommissionsberatung relevant waren. Folglich beschränkt sich die Evaluationsnutzung auf eine kleine Anzahl an Parlamentsmitgliedern mit bestimmten Rollen im Parlamentsbetrieb. Dies deckt sich mit der bisherigen Forschung zur Nutzung durch Parlamentsmitglieder und andere politische Entscheidungsträgerinnen und -träger (Mooney 1993, 2012; Newman und Head 2015; Songer 1988; vgl. Webber 1987; Weiss 1989; Whiteman 1995).

Die Fallstudien weisen nicht nur auf eine Konzentration der Evaluationsnutzung auf bestimmte Mitglieder und Rollen, sondern auch auf einen Fokus auf spezifische Quellen hin. Die Kommissionsmitglieder haben vor allem über externe Fachpersonen sowie die Exekutive von Evaluationen erfahren. Dies deckt sich mit Analysen der Protokolle der Kommissionen des US-Kongresses (Esterling 2011; Michaels und Gruszczynski 
2016). In den untersuchten Gesetzgebungsprozessen setzten sich die Parlamentsmitglieder besonders mit den Präsentationen von externen Fachpersonen auseinander und verwendeten diese politisch. Dies spricht für die Wichtigkeit direkt vermittelter sowie mündlicher Information, was mehrere Forschende betonen (Hird 2009; Raudla 2012, 1009; Weiss 1987, 98; 1989, 414). Neben den Präsentationen verwendeten die Parlamentsmitglieder zusammenfassende Berichte sowie im Berner Grossen Rat auch kantonsspezifische Evaluationen. Die analytische und politische Nutzung beschränkt sich daher auf zahlenmässig wenige, aber dafür einschlägige Quellen.

\subsection{Erfahrung, Professionalisierung und Konfliktivität bedeutsam für Nutzung}

Was sind Erklärungsfaktoren der Nutzung? Einen wesentlichen Effekt auf die individuelle Nutzung haben gemäss der quantitativen, multivariaten Analyse besonders die Erfahrung und die Professionalisierung eines Parlamentsmitglieds (vgl. 4.4). Je erfahrener ein befragtes Parlamentsmitglied, desto seltener verwendet es Evaluationen analytisch und politisch. Dieser Effekt ist nicht nur in den ersten Jahren nach der Wahl ins Parlament signifikant, sondern bleibt es über mehrere Amtsperioden hinweg. Eine negative Wirkung der Erfahrung stimmt auch mit den theoretischen Erwartungen überein. Erfahrene Parlamentsmitglieder sollten Evaluationen seltener analytisch und politisch verwenden, da sie mit zunehmender Erfahrung ihre Positionen festigen und Evaluationen weniger bedürfen, um sich Legitimität zu verschaffen. Die Professionalisierung als zweiter zentraler individueller Erklärungsfaktor hat hingegen einen positiven Effekt: Je mehr Zeit ein befragtes Parlamentsmitglied in das Amt investiert, desto häufiger nutzt es Evaluationen analytisch und politisch. Dies trifft besonders auf die konzeptionelle und die persuasive Verwendung zu. Investiert ein Parlamentsmitglied 66.6 Prozent eines Vollzeitpensums in das Mandat, verwendet es Evaluationen signifikant häufiger, um ein Thema besser zu verstehen oder um andere zu überzeugen als ein Parlamentsmitglied mit einem Pensum von 20 Prozent. So gesehen bestehen Unterschiede zwischen Bund und Kantone, zumal die Arbeitsbelastungen von 66.6 und 20 Prozent etwa dem Durchschnitt in der Bundesversammlung und in den Kantonsparlamenten entsprechen (vgl. Bundi et al. 2017; Bütikofer 2014). Ein positiver signifikanter Effekt besteht aber auch bei kleineren Unterschieden, etwa zwischen 10 und 20 Prozent Zeitaufwand. Die begünstigende Wirkung der Professionalisierung auf die konzeptionelle Nutzung deckt sich mit der theoretischen Erwartung, dass es für professionalisierte Parlamentsmitglieder eher möglich ist, Fragen vertieft zu betrachten. Übereinstimmend damit weisen einige Parlamentsmitglieder in den Interviews darauf hin, dass die Miliztätigkeit einschränke, wie detailliert man sich mit wie vielen Fragen auseinandersetzen könne (vgl. 5.6.2.2). Dass professionalisierte Parlamentsmitglieder Evaluationen auch häufiger politisch verwenden, wurde nicht erwartet, zumal die Mitglieder der Schweizer Milizparlamente wenig Anreiz haben dürften, ihre Professionalität mit Evaluationen zu unterstreichen.

Die weiteren untersuchten individuellen Erklärungsfaktoren der Parteizugehörigkeit und des Bildungsgrads sind gemäss der quantitativen Analyse dagegen weniger 
relevant für die Evaluationsnutzung. Die Parteizugehörigkeit hängt lediglich mit der politischen Verwendung zusammen; deutlich sind die Parteienunterschiede vor allem für die befragten Parlamentsmitglieder der SP. Sie verwenden Evaluationen häufiger zur Überzeugung anderer als alle anderen Befragten, ebenso nutzen sie Evaluationen häufiger zur Bekräftigung bisheriger Positionen als SVP-Befragte. Generell nutzen SP-Befragte Evaluationen signifikant häufiger für die Gesetzgebung als SVP-Befragte. Auch in den Fallstudien sind Unterschiede zwischen den Parteien beobachtbar, allerdings folgen diese keinem systematischen Muster, selbst wenn die Position der Partei zu den behandelten Massnahmen miteinbezogen wird. Vielmehr zeigen die Fallstudien, dass das Verhalten einzelner Parlamentsmitglieder die parteispezifischen Beobachtungen wesentlich beeinflussen kann. Zwischen dem Bildungsgrad und der Evaluationsnutzung sind schliesslich keine systematischen Zusammenhänge feststellbar, da die quantitative Analyse nur einzelne signifikante Unterschiede offenbart.

Gemäss der qualitativen Analyse der kantonalen Gesetzgebungsprozesse ist die Konfliktivität einer Massnahme der wesentliche kontextuelle Erklärungsfaktor der Evaluationsnutzung (vgl. 5.6.3.2). Die Kommissionsmitglieder nutzten Evaluationen besonders in den Fällen mit einer vergleichsweise hohen Konfliktivität analytisch, indem sie sich differenziert mit Evaluationen auseinandersetzten. Gleichzeitig verwendeten die Parlamentsmitglieder Evaluationen umso häufiger politisch, je konfliktiver der untersuchte Gesetzgebungsprozess war. Bei umstrittenen Massnahmen ist die politische Nutzung in diversen Arten beobachtbar, etwa indem Parlamentsmitglieder Evaluationen kritisierten, forderten, auf diese verwiesen oder gar daraus zitierten. Dies unterstützt die Hypothese, wonach Parlamentsmitglieder Evaluationen bei konfliktiven Situationen als vielfältiges politisches Mittel einsetzen. Entgegen der Hypothese verwendeten aber die Parlamentsmitglieder Evaluationen bei vergleichsweiser hoher Konfliktivität auch häufig analytisch. Eine Erklärung dafür ist, dass umstrittene Themen generell über eine hohe Brisanz verfügen und öffentliches Interesse generieren. Daher dürften Parlamentsmitglieder einerseits einen hohen Anreiz haben, sich damit zu beschäftigen. Anderseits dürfte aufgrund der längeren Zeit, die einem heiss diskutierten Thema gewidmet wird, Evaluationsnutzung wahrscheinlicher zu beobachten sein. In den spezifischen Fällen der Basis-/Grundstufe, die konfliktiv waren, dürfte schliesslich die von Anfang an geplante, umfassende Evaluation die analytische Nutzung begünstigt haben.

Wenig deutliche Muster offenbaren die Fallstudien für die untersuchten kontextuellen Faktoren des Kommissionensystems der Parlamente und der Wissenschaftsnähe einer Massnahme (vgl. 5.6). Zwar bestehen Unterschiede in den Beratungen der nichtständigen und ständigen Kommissionen, diese äussern sich jedoch nicht in einem divergierenden Nutzungsverhalten der Mitglieder des Berner Grossen Rats und des Zürcher Kantonsrats. Dies stimmt mit den geringen parlamentsspezifischen Unterschieden überein, die in den Befragungsdaten beobachtbar sind (vgl. 4.2.1). Ein Zusammenhang ist ebenso wenig ersichtlich zwischen beobachteter Evaluationsnutzung und der Wissenschaftsnähe einer Massnahme. Relativ häufige analytische und politische Nutzung - und im Gegensatz dazu keine bis seltene Nutzung - ist sowohl in den wissenschaftsnahen, energie- und transportpolitischen sowie in den weniger wissenschaftsnahen, bildungs- und sozialpo- 
litischen Massnahmen feststellbar. Folglich sind andere Eigenschaften der untersuchten Massnahmen bedeutsamer für die Evaluationsnutzung.

Die untersuchten evaluationsspezifischen Kontextfaktoren wirken sich gemäss den Fallstudien ebenso kaum auf die Evaluationsnutzung aus (vgl. 5.6). Fast sämtliche Evaluationen waren jeweils vor der parlamentarischen Beratung verfügbar, die Parlamentsmitglieder haben aber auch Quellen verwendet, die später erschienen sind. Bei diesen Quellen handelte es sich um direkt vermittelte Informationen etwa im Rahmen eines Hearings. Die Parlamentsmitglieder griffen die verwendeten Quellen weiter jeweils relativ kurz nach deren Erscheinen erstmals auf, wie bereits erläutert, fokussierten sie aber auf einschlägige Quellen. Inwiefern die Evaluationsergebnisse zu einer Massnahme übereinstimmen, scheint ebenso kaum mit der Evaluationsnutzung zusammenzuhängen. Parlamentsmitglieder verwiesen unabhängig von ihrer Position und der Evidenzlage auf Evaluationen, forderten oder kritisierten diese.

Die Fallstudien geben zusätzlich einen Einblick, wie die Exekutive Evaluationen verwendete und damit auch einen Beitrag zur parlamentarischen Nutzung leistete (vgl. 5.6.3.3). Gemäss den Interviews waren die zentralen, verfügbaren Evaluationen in der Verwaltung stets bekannt, zudem enthielt der für die Parlamentsmitglieder zentrale Regierungsratsantrag in sechs der acht Fälle Verweise auf Evaluationen. Da die oft zeitlich vorgelagerten Arbeiten der Regierung und Verwaltung eine Informationsgrundlage für die Parlamentsmitglieder bilden, können sie beeinflussen, ob Evaluationen Teil der Diskussion der Vorlage werden und welchen Stellenwert sie darin einnehmen. Die Fälle zur Basis/Grundstufe zeigen etwa auf, dass die Regierung und Verwaltung zwar Evaluationen thematisierten, diesen aber kein besonderes Gewicht gaben. Im Fall zum Steuersystem für energieeffiziente Fahrzeuge im Kanton Bern waren Evaluationen dagegen zentral in der Argumentation der Exekutive. Inwiefern sich Verwaltung und Regierung bei der Ausarbeitung der Vorlage überhaupt auf Evaluationen stützen, dürfte gemäss den Fallstudien auch von ihren Interessen und ihrer Einstellung gegenüber Evaluationen abhängen.

Übereinstimmend mit der bisherigen Literatur kann Evaluationsnutzung gemäss den vorliegenden Untersuchungen folglich als kontextabhängiges, multifaktorielles Phänomen bezeichnet werden (Nutley et al. 2007; Whiteman 1985a, 1985b, 1995). Frühere Arbeiten untersuchten eine Vielzahl von Faktoren der Nutzung und stellten teilweise widersprüchliche Ergebnisse fest. Die vorliegende Untersuchung bekräftigt aber die bisherige, überwiegende Evidenz zu einem negativen Zusammenhang zwischen der Erfahrung und der Evaluationsnutzung. Der zweite, wesentliche individuelle Erklärungsfaktor der Professionalisierung ist erst vereinzelt betrachtet worden (vgl. Hird 2005b, 149). Die vorliegenden Ergebnisse eines positiven Effekts stimmen aber damit überein. Der kontextuelle Faktor der Konfliktivität ist dagegen vieldiskutiert (vgl. Boswell 2009; Esterling 2011; Frey 2012; Jenkins-Smith und Sabatier 1993; Whiteman 1985a). Die vorliegenden Ergebnisse unterstreichen die Relevanz dieses Faktors weiter und stützen die verbreitete Feststellung, dass Evaluationen in konfliktiven Situationen häufiger politisch verwendet werden (Frey 2012; Tabuga 2017; Whiteman 1985a, 1985b). Bisherige Studien sind dagegen uneinig über den Zusammenhang zwischen analytischer Nutzung 
und Konfliktivität. Übereinstimmend mit den vorliegenden Beobachtungen zeigen aber auch andere Forschende, dass sich Parlamentsmitglieder auch bei umstrittenen Vorlagen vertieft informieren und sich von Evaluationen überzeugen lassen können (Frey 2012; Mooney 1993). Dass die Unterstützung der Verwaltung wesentlich ist für das Milizparlament, um Informationen aus Evaluationen zu berücksichtigen, bekräftigt dieses Buch erneut (vgl. Frey 2012; Horber-Papazian 2011).

\subsection{Diskussion des Forschungsansatzes und Tragweite der Ergebnisse}

Wie aussagekräftig sind nun die vorliegenden Ergebnisse? Um diese Frage zu erläutern, ist es notwendig, die konzeptionellen und empirischen Grundlagen der vorliegenden Untersuchung zu diskutieren, besonders in Bezug auf die zentrale Variable der Evaluationsnutzung. Gemäss dem theoretischen Rahmen lassen sich grundsätzlich die zwei Formen der analytischen und politischen Evaluationsnutzung unterscheiden, die in verschiedenen Arten auftreten können. In der empirischen Analyse wurden dann ebensolche Arten betrachtet. Obschon die vorliegenden Ergebnisse aufzeigen, dass auch eine feinkörnigere oder gar keine Unterteilung der Nutzungsformen möglich wären, sprechen verschiedene Gründe für das verwendete Vorgehen. Konzeptionell gesehen ist die Zweiteilung in analytische und politische Nutzung sinnvoll, da sie die grundlegende Unterscheidung zwischen einer analytisch-problemlösenden und einer politisch-strategischen Handlungslogik aufgreift. Diese Unterscheidung gilt auch für die verschiedenen Verwendungsarten. Die Nutzung feinkörniger auf Ebene dieser Verwendungsarten zu unterteilen, scheint hingegen wenig sinnvoll, da bereits die analytische und die politische Nutzung schwierig voneinander abgrenzbar sind. Die politische Nutzung ist gewissermassen inhärent mit der analytischen Nutzung und ihrer problemlösenden Logik verbunden. Beim Einsatz von Evaluationen als politisches Mittel wird gerade davon Gebrauch gemacht, dass solches systematisch generiertes Wissen Rationalität oder Verbesserungsorientierung verkörpert (Boswell 2009, 249). Empirisch gesehen ist die Zweiteilung in analytische und politische Nutzung auch sinnvoll, da sie einen gemeinsamen Nenner für die quantitative und qualitative Analyse ermöglicht. Dennoch verschiedene Verwendungsarten zu betrachten, ist angesichts der wenig erforschten Evaluationsnutzung im Parlament wertvoll. Ebenso erlaubt es erst, die Konzeptualisierung kritisch zu diskutieren. Zudem lassen sich so Zusammenhänge zwischen den verschiedenen Nutzungsarten und den Erklärungsfaktoren analysieren.

Gemessen wird die analytische und politische Evaluationsnutzung in der vorliegenden Untersuchung anhand einer Befragung, Interviews sowie Dokumentenanalysen. Folglich stützen sich die Ergebnisse auf Selbsteinschätzungen der Parlamentsmitglieder. Gerade in der Befragung können sozial erwünschtes Antworten oder eine unausgewogene Teilnahme der Befragten zu einer Überschätzung der Verwendung führen, während bei den Interviews Erinnerungslücken möglich sind, zumal die untersuchten Gesetzgebungsprozesse mehrere Jahre zurückliegen (Bailer 2014; vgl. Herbert 2014; Leviton 2003; Raudla 2012). Als relativ direkte Art, die Gedanken und Absichten von 
Politikerinnen und Politikern zu erfassen, sind Befragungen und Interviews gleichzeitig sehr wertvoll bei der Erforschung des Verhaltens von Parlamentsmitgliedern (Bailer 2014, 167). Mit dem direkten Kontakt schafft dieses Buch auch einen Mehrwert gegenüber früheren Arbeiten, welche die Nutzung von systematisch generiertem Wissen anhand Angaben der Mitarbeitenden im Parlament erfassen (z. B. Weiss 1989; Whiteman 1985a, 1985b). Die verschiedenen Datengrundlagen sollen zudem eine aussagekräftige Messung der Evaluationsnutzung gewährleisten, da die Dokumentenanalysen in den Fallstudien die Selbsteinschätzungen in den Interviews und der Befragung validieren können. Dies ist ein weiterer Mehrwert im Vergleich mit bisherigen Studien der Nutzungsforschung, die oft ausschliesslich auf Selbsteinschätzungen der (potenziell) Nutzenden beruhen (vgl. Leviton 2003, 526). Angesichts der theoretischen sowie empirisch schwierigen Erfassung und Differenzierung der Nutzung stellt sich schliesslich generell die Frage, ob statt von Verwendung analog zu Frey $(2012,59)$ von Verwendungsabsichten gesprochen werden soll.

Welchen Beitrag leistet das vorliegende Buch also zur Erklärung der Evaluationsnutzung in den Schweizer Parlamenten und generell zur Verwendung von systematisch generiertem Wissen durch politische Entscheidungsträgerinnen und -träger? Die vorliegende Untersuchung zeigt auf, dass die Evaluationsnutzung in den Schweizer Parlamenten mit mehreren Erklärungsfaktoren zusammenhängt, wobei lediglich ein Teil der untersuchten Erklärungsfaktoren klar bedeutsam ist. Diese bedeutsamen Erklärungsfaktoren der Erfahrung sowie der Konfliktivität, nicht aber der individuellen Professionalisierung, waren bereits mehrfach Gegenstand der Nutzungsforschung. Obschon die bisherigen Studien teilweise unterschiedliche Zusammenhänge feststellen, bestätigen die vorliegenden Ergebnisse die grundsätzlichen Befunde zum Effekt der Erfahrung sowie der Konfliktivität. Der Beitrag der vorliegenden Untersuchung liegt somit kaum in der Identifikation neuer Erklärungsfaktoren. Vielmehr leistet das vorliegende Buch einen Beitrag durch die profunde Analyse der Evaluationsnutzung im spezifischen, parlamentarischen Kontext. Die Verwendung von Evaluationen oder anderem systematisch generierten Wissen in Parlamenten ist generell selten sowie in der Schweiz bisher nicht so umfassend und vertieft untersucht worden. Im Gegensatz zur bisherigen Nutzungsforschung befasst sich dieses Buch differenziert mit dem Zusammenhang zwischen den Erklärungsfaktoren und den einzelnen Formen der Nutzung. Viele Studien der Nutzungsforschung gehen stattdessen von Nutzung aus, sodass nicht deutlich wird, wie die Erklärungsfaktoren zur Nutzung in unterschiedlichen Formen stehen (vgl. Amara et al. 2004; Cousins und Shulha 2006; Nutley et al. 2007). Damit zusammenhängend widmen sich viele Studien vorrangig der analytischen, aber nicht der politischen Nutzung, die gerade im parlamentarischen Kontext relevant ist (vgl. Johnson et al. 2009; Whiteman 1985b). Folglich liegt der Beitrag der Arbeit darin, die Nutzung in Parlamenten zu konzeptualisieren und danach ein differenzierteres, auf verschiedenen Quellen gestütztes Bild der Nutzung in den Parlamenten zu erfassen. Damit trägt das vorliegende Buch zur Weiterentwicklung der regen Nutzungsforschung, aber auch zur Parlamentsforschung bei.

$\mathrm{Zu}$ beachten bei der Diskussion der Aussagekraft der Ergebnisse und des Beitrags des Buchs ist der Fokus der vorliegenden Untersuchung: Dieses Buch widmet sich der 
Verwendung von Evaluationen der schweizerischen Parlamentsmitglieder im Rahmen ihrer gesetzgeberischen Tätigkeiten. Die quantitative Analyse konzentriert sich auf Parlamentsmitglieder der fünf grossen Parteien. Die qualitative Analyse befasst sich mit Gesetzgebungsprozessen in Politikbereichen mit einer vergleichsweise hohen Evaluationsaktivität. Dafür sorgt auch die Auswahl der beiden grössten Kantone der Schweiz, die für den Grossteil der Evaluationen auf kantonaler Ebene verantwortlich sind (Balthasar und Rieder 2009; Frey et al. 2016). Zudem ermöglicht die Untersuchung von acht Gesetzgebungsprozessen zu vier Massnahmen zwar vertiefte Einblicke in die Evaluationsnutzung, gleichzeitig ist es eine zu geringe Anzahl, um die diversen Zusammenhänge zu überprüfen (vgl. Collier und Mahoney 1996; George und Bennett 2005).

Trotz dieses Fokus' auf die Evaluationsnutzung der schweizerischen Parlamentsmitglieder im Rahmen der gesetzgeberischen Tätigkeiten dürfte die Tragweite der vorliegenden Erkenntnisse darüber hinausgehen. Einerseits sind die Erkenntnisse zur Nutzung für die Gesetzgebung auch für andere Tätigkeiten im Rahmen der Aufsicht oder der Budgetkompetenz relevant. So nehmen die Kommissionen für die Erledigung aller drei Aufgaben eine zentrale Position ein. Zudem beschäftigen sich die drei Aufgaben teilweise mit ähnlichen Fragen. Wie eine Politik umgesetzt wird, ist beispielsweise von Interesse bei Gesetzesrevisionen, bei der Aufsicht der Exekutive, aber auch bei der Verteilung des Budgets. Da Evaluationen eine Form systematisch generierten Wissens sind, können anderseits die Erkenntnisse auch generelle Hinweise auf die Verwendung systematisch generierten Wissens in Parlamenten geben. Schliesslich sollten die Ergebnisse auch auf politische Systeme mit einem ähnlichen Aufbau der Parlamente und vergleichbarer Etablierung der Evaluation, namentlich etwa in den US-Bundesstaaten, anwendbar sein. Auch für Parlamente in anderen politischen Systemen sind die Ergebnisse von Relevanz, wenn auch der Milizcharakter sowie die Eigenheiten des schweizerischen politischen Systems bei der Interpretation berücksichtigt werden sollten.

Der Fokus der vorliegenden Untersuchung weist gleichzeitig auf Fragen für die zukünftige Erforschung der parlamentarischen Verwendung von Evaluationen hin. Aufgrund der vorliegenden Erkenntnisse vielversprechend scheint die Untersuchung der Nutzung bei parlamentarischen Vorstössen. Bisherige Untersuchungen betrachteten Vorstösse vor allem als ein Instrument zur Nachfrage nach Evaluationen (vgl. Bundi 2016, 2018a, 2018b; Zwaan et al. 2016). Die Fallstudien zeigen allerdings auf, dass die Parlamentsmitglieder bereits in den Vorstössen und deren Diskussion Evaluationen oder anderes systematisch generiertes Wissen aufgreifen, um etwa die Grösse eines Problems oder mögliche Lösungen zu beschreiben. Die Betrachtung von Evaluationsnutzung in Vorstössen würde Aussagen darüber ermöglichen, welche Rolle systematisch generiertes Wissen bei der Wahrnehmung und Darstellung von Problemen und genereller dem Agenda-Setting spielt. Gleichzeitig damit verbunden sind auch Fragen zur Einflussnahme durch Lobbyarbeit (vgl. Varone et al. 2018). Zumal die vorliegende Untersuchung auf die Nutzung von Evaluationen fokussierte, stellt sich zudem die Frage nach der effektiven Bedeutung von Evaluationen und deren Nutzung - in der Forschung über Evaluation als Einfluss bezeichnet. Um verschiedene, mögliche Konsequenzen der Nutzung von Evaluationen im Parlament systematischer zu analysieren, wäre es lohnenswert, einen längeren 
Zeitraum zu betrachten. Dies gäbe auch Einblicke in die Frage, wie politische Parteien systematisch generiertem Wissen zu einer Frage gegenüberstehen und ob sich dies je nach (Macht-)Position und politischer Konjunktur verändert. Angesichts drängender, gesellschaftlicher Fragen dürfte zudem generell die parlamentarische Nutzung von systematisch generiertem Wissen in weiteren Politikfeldern wie der Migrations- oder der Klimapolitik interessieren. Gerade diese Politikfelder weisen darauf hin, dass die internationale Verflechtung sowie der technologische Fortschritt zusätzliche zu erforschende Kontextfaktoren oder -bedingungen der Nutzung von systematisch generiertem Wissen darstellen können. 


\section{Ausblick: Was ist die Rolle der Evaluation in den Parlamenten?}

Wie sind die vorliegenden Erkenntnisse nun einzuschätzen? Was bedeuten sie für die Rolle der Evaluation in den Parlamenten generell und in den Schweizer Parlamenten im Besonderen? Abschliessend sollen diese Fragen - bisweilen zugespitzt - aus einer skeptischeren oder einer optimistischeren Warte erläutert werden.

Skeptikerinnen und Skeptiker dürften die vorliegenden Ergebnisse als ernüchternd ansehen, da diese nicht auf eine systematische, routinemässige Verwendung von Evaluationen in den Parlamenten hinweisen. Die Evaluationskapazität der Parlamente scheint also begrenzt, die Parlamentsmitglieder orientieren sich nicht umfassend an systematisch generierten Informationen zu den Wirkungen staatlichen Handelns, wie dies die Modernisierungsbestrebungen des New Public Management oder der evidenzbasierten Politikgestaltung fordern. In einigen untersuchten Gesetzgebungsprozessen setzten sich die Parlamentsmitglieder zwar mit Evaluationen differenziert auseinander und diskutierten die Evaluationen in den Debatten, diese Fälle verfügen aber über eine besonders günstige Ausgangslage für ein solches Verhalten. So waren die Massnahmen der Fälle der Basis-/Grundstufe und des Steuersystems für energieeffiziente Fahrzeuge Gegenstand von umfassenden Evaluations- beziehungsweise Forschungsprojekten. Externe Fachpersonen vermittelten die Ergebnisse dieser Projekte häufig direkt anhand von Präsentationen. Zudem polarisierten beide Massnahmen und waren heiss diskutiert. Folglich war in diesen Fällen Nutzung wahrscheinlich, Skeptikerinnen und Skeptiker dürften sich gar fragen, ob die Verwendung von Evaluationen in diesen Fällen nicht unumgänglich oder selbstverständlich war. Schliesslich dürften Skeptikerinnen und Skeptiker die positiven Ergebnisse der Befragung generell hinterfragen: Sind Evaluationen oder systematisch generiertes Wissen nicht im Allgemeinen etwas Erstrebenswertes? Wieso sollte jemand deren Nutzung per se ablehnen? (vgl. Cairney 2016, 2).

Optimistinnen und Optimisten dürften dieser Sichtweise Argumente entgegenbringen, die sich auf funktionale Aspekte der parlamentarischen Entscheidungsprozesse beziehen und auf einer anderen Interpretation der Funktion von systematisch generiertem Wissen beruhen. Aufgrund der Spezialisierung in Parlamenten bedarf es so keiner Verwendung von Evaluationen durch alle Parlamentsmitglieder (vgl. Mooney 1991b, 1993, 2012; Songer 1988). Kommissionsmitglieder, dies zeigen die Fallstudien, setzten sich durchaus mit Evaluationen auseinander und verwiesen in den Plenumsdebatten darauf. Evaluationen sollten diesen Mitgliedern auch aus eigenem Interesse wichtig sein: Kommissionsgeschäfte bieten eine Möglichkeit, sich in der Fraktion, im Parlament und darüber hinaus zu profilieren (Mooney 1993, 195). Weiter kommen die Parlamente in schweizerischen Gesetzgebungsprozessen spät zum Zug. Wie die Fallstudien zur Schulsozialarbeit nahelegen, können Evaluationen auch für eine Gesetzesvorlage von Bedeutung sein, ohne im Parlament Verwendung zu finden (vgl. Frey 2012). Welchen Platz Evaluationen einnehmen können, ist bei teilweise detailhaften Beratungen zu Formulierungen der Gesetzesartikel ohnehin fraglich. Als Selbstverständlichkeit dürften die Optimistin- 
nen und Optimisten zudem die Verwendung von Evaluationen auch in den Fällen der Basis/Grundstufe oder dem Steuersystem für energieeffiziente Fahrzeuge nicht betrachten, selbst wenn die Ausgangslage die Wahrscheinlichkeit der Verwendung erhöhte. Gerade bei solchen heiss diskutierten Fragen dürfte Evaluationen im Gegensatz zu Ideologien, Interessen oder anderen Gesichtspunkten eine untergeordnete Rolle zukommen (vgl. Weiss 1995). Dass Evaluationen dennoch in den Diskurs einfliessen, würden Optimistinnen und Optimisten daher positiv werten. Dies impliziert, dass sich Parlamentsmitglieder zwar auf ihre Legitimität als Volksvertreterinnen und Volksvertreter abstützen, aber auch andere Aspekte berücksichtigen (vgl. Shulock 1999). Indem Evaluationen Teil des politischen Diskurses werden, würden Optimistinnen und Optimisten argumentieren, können sie auch die Qualität der parlamentarischen Entscheide erhöhen. Diese hängt nicht nur davon ab, ob die letztlich beschlossene Politik auf Evidenz aus Evaluationen basiert, sondern auch davon, wie das Parlament zu diesem Entscheid gekommen ist.

Als gemeinsamer Nenner dieser beiden Warten kann also festgestellt werden, dass sich die Mitglieder der schweizerischen Milizparlamente teilweise mit Evaluationen auseinandersetzen und Evaluationen Eingang in die Debatte finden. Von einer Durchdringung der parlamentarischen Prozesse mit Evaluationen kann aber keine Rede sein - die Befürchtung einer Technokratisierung ist unbegründet. Gleichzeitig sind Evaluationen dennoch fest etabliert in der Schweiz und auch von Relevanz im politischen Prozess. Wie verschiedene Studien zeigen, stützt sich die Verwaltung gerade auf Bundesebene auf Evaluationen, besonders bei der Umsetzung einer Politik, aber auch bei deren Revision oder Ausarbeitung (Balthasar 2007; Balthasar und Müller 2014; Frey 2012; Frey und Ledermann 2017; Ledermann 2014). Das Parlament nimmt jedoch eine wichtige Rolle im gesamten politischen Prozess ein, zumal dort demokratisch gewählte Volksvertreterinnen und Volksvertreter entscheiden.

Angesichts der Ressourcen, die für Evaluationen aufgewendet werden, und besonders angesichts des Anspruchs an wirkungsvolles Handeln im Parlament stellt sich dennoch die Frage, ob es Potenzial zur Steigerung der Nutzung von Evaluationen gäbe. Gemäss den vorliegenden Erkenntnissen verfügen die Evaluierenden selbst über gewisse Möglichkeiten, die Nutzung im Parlament zu fördern. Neben der generell wünschenswerten adressatengerechten Präsentation der wesentlichsten Ergebnisse weisen die Befunde darauf hin, dass der direkte Kontakt mit externen Fachpersonen ein besonders erfolgsversprechender Weg ist, durch den Evaluationen in den parlamentarischen Prozess gelangen. Demzufolge würde es eines besseren Zugangs der Evaluierenden zum Parlament oder zu einzelnen Mitgliedern bedürfen. Dies scheint gerade in der kleinräumigen Schweiz mit ihren Milizparlamenten möglich, wo die Parlamentsmitglieder in der Regel neben ihrem Amt auch beruflichen Tätigkeiten nachgehen. Zumal die Exekutive wesentliche Vorbereitungsarbeiten für die Milizparlamente übernimmt, scheint ein direkter Kontakt auch zur Verwaltung lohnenswert, wie andere Studien ebenso aufzeigen (vgl. Amara et al. 2004; Frey 2012).

Daneben deuten die vorliegenden Ergebnisse auf eine mögliche Steigerung der Nutzung in den Milizparlamenten durch die Professionalisierung des Amts und der Institution selbst. Die quantitative Analyse zeigt, dass Parlamentsmitglieder umso häufiger 
ihr Verständnis eines Themas mit Hilfe von Evaluationen verbessern, je mehr Zeit sie in ihr Amt investieren. Auf die Relevanz der Professionalisierung der Institution weisen die in Eberli und Bundi (2017) präsentierten Ergebnisse der Befragung hin: Demgemäss erfahren die Mitglieder der professionalisierteren Bundesversammlung häufiger durch die Parlamentsdienste von Evaluationen als die Mitglieder der Kantonsparlamente. Ein förderlicher Effekt der Professionalisierung scheint aber nur punktuell möglich, zumal die befragten Mitglieder der Bundesversammlung Evaluationen nicht generell häufiger nutzen als ihre Pendants auf Kantonsebene. Weiter zeigen Studien zum professionalisierten US-Kongress, dass auch deren Mitglieder systematisch generiertes Wissen unterschiedlich oft verwenden und dies auch von der Situation abhängt (vgl. Shulock 1999; Whiteman 1985b, 1995). Daher scheint der Beitrag der Professionalisierung zur Nutzung begrenzt, folgenreich dürfte er aber eher in den Kantonsparlamenten sein, deren Mitglieder wenig Zeit und kaum Unterstützung bei der Erledigung ihrer Aufgabe haben (Bundi et al. 2017).

Die Nutzung von Evaluationen in den schweizerischen Parlamenten, selbst wenn sie gefördert wird, betrifft schliesslich einen Teilaspekt der übergeordneten Frage der Bedeutung von systematisch generiertem Wissen in der Politik. Einerseits sind Evaluationen lediglich eine Form systematisch generierten Wissens. Evaluationen liefern Informationen zur Zielerreichung, Wirksamkeit oder Problemlösefähigkeit einer Politik und können damit einen Beitrag an die Politikgestaltung leisten. Andere Formen systematisch generierten Wissens sind ebenfalls relevant für die Politikgestaltung: Informationen etwa zur Art, Definition oder Grösse eines Problems können Grundlagen zur Frage liefern, wie dringend und auf welche Weise ein Problem angegangen werden sollte (vgl. Cairney 2016). Für eine Abwägung solcher Handlungsoptionen zur Politikgestaltung weiter relevant sind zudem Informationen oder Wissen zu den beteiligten politischen Akteurinnen und Akteure, zu politischen Prozessen und Institutionen sowie zu praktischen Fragen der Umsetzung (vgl. Head 2008a; Nutley et al. 2003). Anderseits sind an der Politikgestaltung nicht nur Parlamentsmitglieder, sondern auch diverse andere, staatliche, nicht-staatliche, subnationale, nationale oder internationale Akteurinnen und Akteure beteiligt. Entsprechend können Evaluationen nicht nur im Parlament, sondern durch diverse Akteurinnen und Akteure, auf unterschiedlichen Ebenen und zu verschiedenen Zeitpunkten des politischen Entscheidungsprozesses einfliessen. Folglich sind Evaluationen und parlamentarische Entscheidungsprozesse Teil eines Ganzen. Bestehen aber Evaluationen im von Interessen und Ideologien geprägten parlamentarischen Entscheidungsprozess, spricht dies auch für eine Bedeutung von Wissen in der Politik. 


\section{Literatur}

Achtnich, Peter. 2009. Vollzug der energetischen Massnahmen 2008. Zürich.

Achtnich, Peter, und Stefan Schwyn. 2006. Vollzug der energetischen Massnahmen 2005. Zürich.

Albaek, Erik. 1995. "Between Knowledge and Power: Utilization of Social Science in Public Policy Making." Policy Sciences 28 (1): 79-100.

Alkin, Marvin C., und Jean A. King. 2016. "The Historical Development of Evaluation Use.” American Journal of Evaluation 37 (4): 568-579.

Alkin, Marvin C., und Jean A. King. 2017. "Definitions of Evaluation Use and Misuse, Evaluation Influence, and Factors Affecting Use." American Journal of Evaluation 38 (3): 434-450.

Alkin, Marvin C., und Sandy M. Taut. 2003. "Unbundling evaluation use.” Studies in Educational Evaluation 29 (1): 1-12.

Amara, Nabil, Mathieu Ouimet, und Réjean Landry. 2004. "New Evidence on Instrumental, Conceptual, and Symbolic Utilization of University Research in Government Agencies." Science Communication 26 (1): 75-106.

Amt für Jugend und Berufsberatung. 2008. Jugendhilfe im Kanton Zürich 1918-2008: Zwischen Professionalität und politischem Kräftemessen. Zürich.

Amt für Jugend und Berufsberatung. 2011. Empfehlungen zur Einführung von SchulsozialarbeitGrundlagen und Umsetzungshilfen. Zürich.

Apollonio, Dorie E, und Lisa A Bero. 2017. "Interpretation and use of evidence in state policymaking: a qualitative analysis." BMJ Open 7 (2): Online open: https://doi.org/10.1136/ bmjopen-2016-012738.

Apollonio, Dorie E., und Lisa A. Bero. 2009. "Evidence and argument in policymaking: development of workplace smoking legislation." BMC Public Health 9 (1): Online open: https://doi.org/10.1186/1471-2458-9-189.

Arnold, Urs V. 2008. Das Parlament im Modell des New Public Managements: Parlamentarische Funktionen und Prozesse im Wandel. Books on Demand.

asa. 2007. Rabattmodell für die kantonale Motorfahrzengsteuer zur Förderung energie- und umwelteffizienter Personenwagen. Empfehlung der Vereinigung der Strassenverkehrsämter der Schweiz (asa).

Askim, Jostein. 2007. "How Do Politicians Use Performance Information? An Analysis of the Norwegian Local Government Experience." International Review of Administrative Sciences 73 (3): 453-472.

Askim, Jostein. 2008. "Determinants of Performance Information Utilization in Political Decision Making." In Performance Information in the Public Sector, hrsg. von van Dooren, Wouter, und Steven van de Walle. Basingstoke, New York: Palgrave Macmillan, 125-139.

Askim, Jostein. 2009. “The Demand Side of Performance Measurement: Explaining Councillors' Utilization of Performance Information in Policymaking." International Public Management Journal 12 (1): 24-47.

avenir social, und SSAV. 2010. Qualitätsrichtlinien für die Schulsozialarbeit. Bern.

Backer, T. E. 1991. "Knowledge Utilization: The Third Wave." Science Communication 12 (3): $225-240$. 
BADAC. 2016. BADAC - Die Schweizer Kantone und Städte online vergleichen. http://www.badac.ch/de/index.php [Stand 02.06.2014].

Baier, Florian. 2008. «Schulsozialarbeit.» In Schulische und schulnahe Dienste: Angebote, Praxis und fachliche Perspektiven, hrsg. von Baier, Florian, und Stefan Schnurr. Bern: Haupt, 87-120.

Bailer, Stefanie. 2011. "People's Voice or Information Pool? The Role of, and Reasons for, Parliamentary Questions in the Swiss Parliament." The Journal of Legislative Studies 17 (3): 302-314.

Bailer, Stefanie. 2014. "Interviews and Surveys in Legislative Research." In The Oxford Handbook of Legislative Studies, hrsg. von Martin, Shane, Thomas Saalfeld, und Kaare Strøm. New York: Oxford University Press, 167-193.

Bailer, Stefanie, Peter Meissner, Tamaki Ohmura, und Peter Selb. 2013. Seiteneinsteiger im Deutschen Bundestag. Wiesbaden: Springer VS.

Balthasar, Andreas. 2005. «Was ist Evaluation und für wen evaluieren wir?» LeGes - Gesetzgebung \& Evaluation 16 (1): 65-80.

Balthasar, Andreas. 2007. Institutionelle Verankerung und Verwendung von Evaluationen: Praxis und Verwendung von Evaluationen in der schweizerischen Bundesverwaltung. Zürich, Chur: Rüegger.

Balthasar, Andreas. 2009a. "Evaluationen in der Schweiz: Verbreitung und Verwendung.» In Evaluation. Ein systematisches Handbuch, hrsg. von Widmer, Thomas, Wolfgang Beywl, und Carlo Fabian. Wiesbaden: VS Verlag für Sozialwissenschaften.

Balthasar, Andreas. 2009b. "Institutional Design and Utilization of Evaluation: A Contribution to a Theory of Evaluation Influence Based on Swiss Experience." Evaluation Review 33 (3): 226-256.

Balthasar, Andreas. 2010. "Are there bases for evidence-based health policy in Switzerland? Factors influencing the extent of evaluation activity in health policy in the Swiss cantons." Evidence \& Policy: A Journal of Research, Debate and Practice 6 (3): 333-349.

Balthasar, Andreas. 2015. «L'utilisation de l'évaluation par l'administration fédérale.» In Regards croisés sur l'évaluation en Suisse, hrsg. von Horber-Papazian, Katia. Lausanne: Presses polytechniques et universitaires romandes, 115-132.

Balthasar, Andreas, und Franziska Müller. 2014. «Die Verbreitung evidenzbasierter und gleichstellungssensitiver Informationen in den Entscheidungsprozessen kantonaler Steuer- und Sozialtransferpolitik: eine quantitative Analyse.» Swiss Political Science Review 20 (1): 70-95.

Balthasar, Andreas, und Stefan Rieder. 2009. «Wo ist evidenzbasierte Politik möglich? Die Verbreitung von Evaluationen auf kantonaler Ebene.» In Demokratie als Leidenschaft. Festschrift für Prof. Wolf Linder zum 65. Geburtstag, hrsg. von Vatter, Adrian, Frédéric Varone, und Fritz Sager. Bern: Haupt, 403-429.

Balthasar, Andreas, Fritz Sager, und Thomas Widmer. 2017. "Einleitung.» In Evaluation im politischen System der Schweiz, hrsg. von Sager, Fritz, Thomas Widmer, und Andreas Balthasar. Zürich: NZZ Libro, 9-20.

Balthasar, Andreas, und Chantal Strotz. 2017. «Verbreitung und Verankerung von Evaluation in der Bundesverwaltung.» In Evaluation im politischen System der Schweiz, hrsg. von Sager, Fritz, Thomas Widmer, und Andreas Balthasar. Zürich: NZZ Libro, 89-117.

Bättig, Christoph, und Philippe Schwab. 2015. «La place de l'évaluation dans le cadre du contrôle parlementaire.» In Regards croisés sur l'évaluation en Suisse, hrsg. von Horber-Papazian, Katia. Lausanne: Presses Polytechniques et Universitaires Romandes, 1-23. 
Beach, Derek, und Rasmus Brun Pedersen. 2013. Process-Tracing Methods: Foundations and Guidelines. Ann Arbor: The University of Michigan Press.

Beck, Ulrich, und Wolfgang Bonß. 1985. "Was will und wozu dient 'Verwendungsforschung??» In 22. Deutscher Soziologentag 1984: Sektions- und Ad-hoc-Gruppen, hrsg. von Franz, HansWerner. Wiesbaden: VS Verlag für Sozialwissenschaften, 610-613.

Bell, Charles Gordon, und Charles M. Price. 1975. First Term: Study in Legislative Organization. Beverly Hills, London: SAGE Publications.

Best, Heinrich, und Lars Vogel. 2014. “The Sociology of Legislators and Legislatures.” In The Oxford Handbook of Legislative Studies, hrsg. von Martin, Shane, Thomas Saalfeld, und Kaare Strøm. New York: Oxford University Press, 57-81.

Beyme, Klaus von. 1984. Parteien in westlichen Demokratien. 2. Aufl. München: Piper.

Beywl, Wolfgang, und Thomas Widmer. 2009. «Evaluation in Expansion: Ausgangslage für den intersektoralen Dreiländer-Vergleich.» In Evaluation: Ein systematisches Handbuch, hrsg. von Widmer, Thomas, Wolfgang Beywl, und Carlo Fabian. Wiesbaden: VS Verlag für Sozialwissenschaften, 13-23.

Bieber, Tonia. 2014. "Cooperation or Conflict? Education Politics in Switzerland after the PISA Study and the Bologna Process." In Internationalization of Education Policy: A New Constellation of Statehood in Education?, hrsg. von Martens, Kerstin, Philipp Knodel, und Michael Windzio. London: Palgrave Macmillan, 179-201.

Biesta, Gert. 2010. "Valuing What We Measure or Measuring What We Value? On the Need to Engage with the Question of Purpose in Educational Evaluation, Assessment, and Measurement." In Evaluation, Bildung und Gesellschaft. Steuerungsinstrumente zwischen Anspruch und Wirklichkeit, hrsg. von Böttcher, Wolfgang, Jan Nikolas Dicke, und Nina Hogrebe. Münster: Waxmann Verlag, 35-46.

Bildungsdirektion des Kantons Zürich. 2003. Räume der Grundstufe. Zürich.

Bildungsdirektion des Kantons Zürich. 2012. Fachstelle für Schulbeurteilung: Jahresbericht 2010/ 2011.

Bimber, Bruce. 1991. "Information as a Factor in Congressional Politics." Legislative Studies Quarterly 16 (4): 585-605.

Bimber, Bruce. 1996. The Politics of Expertise in Congress: The Rise and Fall of the Office of Technology Assessment. New York: State University of New York Press.

Blatter, Joachim, Clara Bombach, und Roman Wiprächtiger. 2015. "Enhancing gender equity through evidence-based policymaking? Theorizing and tracing the use of systematic knowledge in family and tax policy reforms." European Policy Analysis 1 (1): 3-34.

Blum, Roger. 1978. «Rolle, Schwierigkeiten und Reform der kantonalen Parlamente.» In Annuaire suisse de science politique - Schweizerisches Jahrbuch für politische Wissenschaft. Bern: Haupt, $11-32$.

Bochsler, Daniel, Christophe Koller, Pascal Sciarini, Sylvie Traimond, und Ivar Trippolini. 2004. Die Schweizer Kantone unter der Lupe: Behörden, Personal, Finanzen. Bern: Haupt.

Bogenschneider, Karen, und Tom Corbett. 2010. Evidence-Based Policymaking: Insights From Policy-Minded Researchers and Research-Minded Policymakers. New York: Routledge. 
Bogenschneider, Karen, Olivia M. Little, und Kristen Johnson. 2013. "Policymakers' Use of Social Science Research: Looking Within and Across Policy Actors." Journal of Marriage and Family 75 (2): 263-275.

Borrás, Susana, und Steven Højlund. 2015. "Evaluation and policy learning: The learners' perspective." European Journal of Political Research 54 (1): 99-120.

Boswell, Christina. 2008. "The Political Functions of Expert Knowledge: Knowledge and Legitimation in European Union Immigration Policy." Journal of European Public Policy 15 (4): 471-488.

Boswell, Christina. 2009. The Political Uses of Expert Knowledge: Immigration Policy and Social Research. Cambridge, New York: Cambridge University Press.

Böttcher, Wolfgang, Jan Nikolas Dicke, und Nina Hogrebe, Hrsg. 2010. Evaluation, Bildung und Gesellschaft. Steuerungsinstrumente zwischen Anspruch und Wirklichkeit. Münster: Waxmann Verlag.

Boyer, John F., und Laura I. Langbein. 1991. "Factors Influencing the Use of Health Evaluation Research in Congress." Evaluation Review 15 (5): 507-532.

Brownson, Ross C., Elizabeth A. Dodson, Jon F. Kerner, und Sarah Moreland-Russell. 2016. "Framing research for state policymakers who place a priority on cancer." Cancer Causes \& Control 27 (8): 1035-1041.

Brun, Mathias E. 2003. Adressatengerechte Berichterstattung bei Leistungsaufträgen. Bern: Haupt.

Brun, Mathias E., und John Philipp Siegel. 2006. "What does appropriate performance reporting for political decision makers require? Empirical evidence from Switzerland." International Journal of Productivity and Performance Management 55 (6): 480-497.

Bundesamt für Statistik. 2014. Bildungsabschlüsse. Ausgabe 2014. Neuchâtel.

Bundesamt für Statistik. 2016. Statistik Schweiz-Bundesamt für Statistik. http://www.bfs.admin.ch [Stand 21.07.2016].

Bundesamt für Strassen ASTRA. 2016. Strassenfinanzierung. Zuständigkeiten. https:/www.astra.admin.ch/astra/de/home/themen/strassenfinanzierung/ zustaendigkeiten.hmtl [Stand 07.05.2016].

Bundesrat, Eidgenössisches Departement für Umwelt, Verkehr, Energie und Kommunikation UVEK. 2011. «Bundesrat verabschiedet neue Energieetikette für Personenwagen.»

Bundi, Pirmin. 2016. "What do we know about the demand for evaluation? Insights from the parliamentary arena." American Journal of Evaluation 37 (4): 522-541.

Bundi, Pirmin. 2018a. "Parliamentarians' strategies for policy evaluations." Evaluation and Program Planning 69 (3): 130-138.

Bundi, Pirmin. 2018b. "Varieties of accountability: How attributes of policy fields shape parliamentary oversight." Governance 31 (1): 163-183.

Bundi, Pirmin, Daniela Eberli, und Sarah Bütikofer. 2017. "Between Occupation and Politics. Legislative Professionalization in the Swiss Cantons.” Swiss Political Science Review 23 (1): 1-20.

Bundi, Pirmin, Daniela Eberli, und Sarah Bütikofer. 2018. «Zwischen Beruf und Politik: Die Professionalisierung in den Parlamenten.» In Das Parlament in der Schweiz. Macht und Ohnmacht der Volksvertretung, hrsg. von Vatter, Adrian. Zürich: NZZ Libro, 315-343.

Bundi, Pirmin, Daniela Eberli, Kathrin Frey, und Thomas Widmer. 2014. Parlamente und Evaluationen: Methodenbericht. Zürich. 
Bundi, Pirmin, Frédéric Varone, Roy Gava, und Thomas Widmer. 2016. "Self-Selection and Misreporting in Legislative Surveys." Political Science Research and Methods: Online: https://doi.org/10.1017/psrm.2016.35.

Bussmann, Werner. 2008. "The Emergence of Evaluation in Switzerland." Evaluation 14 (4): 499-506.

Bussmann, Werner. 2010. "Evaluation of Legislation: Skating on Thin Ice.” Evaluation 16 (3): 279-293.

Bütikofer, Sarah. 2013. «Mythos Milizparlament - vom Amt zum Beruf.» Parlament, Parlement, Parlamento 16 (3): 3-11.

Bütikofer, Sarah. 2014. Das Schweizer Parlament: eine Institution auf dem Pfad der Moderne. BadenBaden: Nomos.

Cairney, Paul. 2016. The Politics of Evidence-Based Policy Making. London: Palgrave Macmillan.

Cairney, Paul. 2018. “Three habits of successful policy entrepreneurs." Policy \& Politics 46 (2): 199-215.

Cairney, Paul, und Mikine Yamazaki. 2018. "A Comparison of Tobacco Policy in the UK and Japan: If the Scientific Evidence is Identical, Why is There a Major Difference in Policy?" Journal of Comparative Policy Analysis: Research and Practice 20 (3): 253-268.

Caplan, Nathan. 1979. “The Two-Communities Theory and Knowledge Utilization.” The American Behavioral Scientist 22 (3): 459-470.

Caplan, Nathan, Andrea Morrison, und Russell J. Stambaugh. 1975. The use of social science knowledge in policy decisions at the national level: A report to respondents. Ann Arbor: Institute for Social Research, University of Michigan.

Christie, Christina A., und Marvin C. Alkin. 1999. "Further reflections on evaluation misutilization." Studies in Educational Evaluation 25 (1): 1-10.

Cohen, Jacob. 1988. Statistical power analysis for the behavioral sciences. 2. Aufl. New York: Psychology Press.

Collier, David. 1993. "The Comparative Method." In Political Science: The State of Discipline II, hrsg. von Finifter, Ada W. Washington D.C.: American Political Science Association, 105-119.

Collier, David. 2011. “Understanding Process Tracing." PS: Political Science \& Politics 44 (4): 823-830.

Collier, David, und James Mahoney. 1996. "Insights and Pitfalls: Selection Bias in Qualitative Research.” World Politics 49 (1): 56-91.

COPLAN ibe. 1997. Evaluation der verbrauchsabhängigen Heizkostenabrechnung (VHKA): Vollzug und Wirkungszusammenhänge.

Cousins, J. Bradley. 2004. "Commentary: Minimizing Evaluation Misuse as Principled Practice." American Journal of Evaluation 25 (3): 391-397.

Cousins, J. Bradley, und Kenneth A. Leithwood. 1986. "Current Empirical Research on Evaluation Utilization." Review of Educational Research 56 (3): 331-364.

Cousins, J. Bradley, und Lyn M. Shulha. 2006. "A Comparative Analysis of Evaluation Utilzation and its Cognate Fields of Inquiry: Current Issues and Trends." In The SAGE Handbook of Evaluation, hrsg. von Shaw, Ian F., Jennifer C. Greene, und Melvin M. Mark. London: Sage Publications, 266-291. 
Damgaard, Erik. 1995. "How Parties Control Committee Members." In Parliaments and Majority Rule in Western Europe, hrsg. von Döring, Herbert. Frankfurt: Campus, 308-325.

Davies, Huw T.O, Sandra M. Nutley, und Peter C. Smith. 2000a. "Introducing evidence-based policy and practice in public services." In What Works? Evidence-Based Policy and Practice in Public Services, hrsg. von Davies, Huw T.O, Sandra M. Nutley, und Peter C. Smith. Bristol: The Policy Press, 1-11.

Davies, Huw T.O, Sandra M. Nutley, und Peter C. Smith, Hrsg. 2000b. What Works? Evidence-Based Policy and Practice in Public Services. Bristol: The Policy Press.

de Haan, Peter, Roman Bolliger, und Robert Ladner. 2010. Von heute nach morgen: Künftige Rollenbilder der Garagisten: im Auftrag des AGVS, Autogewerbeverband der Schweiz. Zollikon.

de Haan, Peter, Christoph Duthaler, und Anja Peters. 2006. Käufer des Hybridfahrzengs Toyota Prius im Vergleich mit Käufern von Toyota Corolla und Toyota Avensis: Forschungsbericht zum Projekt Hybridfahrzenge. Berichte zum Schweizer Autokaufverhalten Nr. 6. Zürich.

de Haan, Peter, Michel Müller, und Anja Peters. 2007a. Anreizsysteme beim Neuwagenkauf: Wirkungsarten, Wirksamkeit und Wirkungseffizienz. Berichte zum Schweizer Autokaufverhalten Nr. 14. Zürich.

de Haan, Peter, Michel Müller, Anja Peters, und Andrea Hauser. 2007b. Lenkungsabgaben zur Senkung des CO2-Auststosses beim Neuwagenkauf: Hintergrund, Mechanismen, Prognosen. Bern.

de Haan, Peter, und Anja Peters. 2005. Charakteristika und Beweggründe von Käufern des Toyota Prius 2: Forschungsbericht zum Projekt Hybridfahrzenge. Berichte zum Schweizer Autokaufverhalten Nr. 1. Zürich.

Delley, Jean-Daniel. 2005. «La commission externe d'évaluation des politiques publiques du canton de Genève.» LeGes - Gesetzgebung \& Evaluation 16 (1): 111-119.

Demaj, Labinot, Lukas Summermatter, und Kuno Schedler. 2012. Sage mir, was Du liest: Ergebnisbericht zum Informationsverhalten der Parlamentarierinnen und Parlamentarier der Kantone Aargau und Zürich. St. Gallen.

Deschouwer, Kris, Sam Depauw, und André Audrey. 2014. "Representing the People in Parliaments." In Representing the People: A Survey Among Members of Statewide and Substate Parliaments. Oxford: Oxford University Press, 1-18.

Drilling, Matthias. 2009. Schulsozialarbeit: Antworten auf veränderte Lebenswelten. 4. Aufl. Bern: Haupt.

Drilling, Matthias, und Carlo Fabian. 2010. «Schulsozialarbeit in der Schweiz und in Liechtenstein.» In Forschung zur Schulsozialarbeit: Stand und Perspektiven, hrsg. von Speck, Karsten, und Thomas Olk. Weinheim: Juventa-Verlag, 155-167.

Duncan, Otis Dudley, und Beverly Duncan. 1955. "A methodological analysis of segregation indexes." American Sociological Review 20 (2): 210-217.

Dunlop, Claire A., und Claudio Radaelli. 2018. "The lessons of policy learning: types, triggers, hindrances and pathologies." Policy \& Politics 46 (2): 255-272.

Dunn, William N. 1983. “Measuring Knowledge Use.” Science Communication 5 (1): 120-133.

Eberli, Daniela. 2015. "Tracing the Use of Evaluations in Legislative Processes in Swiss Cantonal Parliaments.” Konferenzpapier, ICPP Conference, Milan. 
Eberli, Daniela. 2018. "Tracing the use of evaluations in legislative processes in Swiss cantonal parliaments." Evaluation and Program Planning 69 (3): 139-147.

Eberli, Daniela, und Pirmin Bundi. 2017. «Parlament und Evaluation: Guts Meets Brain.» In Evaluation im politischen System der Schweiz. Entwicklung, Bedeutung und Wechselwirkung, hrsg. von Sager, Fritz, Thomas Widmer, und Andreas Balthasar. Zürich: NZZ Libro, 243-278.

Eberli, Daniela, Pirmin Bundi, Kathrin Frey, und Thomas Widmer. 2014. Befragung Parlamente und Evaluationen: Ergebnisbericht. Zürich.

Eberli, Daniela, Sarah Bütikofer, und Pirmin Bundi. 2018. «La professionalizzazione dei parlamenti cantonali.» In Milizia e professionismo nella politica svizzera, hrsg. von Pilotti, Andrea, und Oscar Mazzoleni. Locarno: Dadò, 75-102.

EDK-Ost. 2005. Zusammenfassung der Ersterhebung aus den Kantonen AG, SG, ZH, TG, GL, NW: Lernstand der Schülerinnen und Schüler. Befragungen beteiligter Personen. St. Gallen.

EDK-Ost. 2010. Projektschlussbericht 2010: Erziehung und Bildung in Kindergarten und Unterstufe im Rahmen der EDK-Ost und Partnerkantone (Projekt EDK-Ost 4bis8). Bern: Schulverlag plus.

Eidgenössisches Departement für Umwelt, Verkehr, Energie und Kommunikation UVEK. 2006. «Revision der energieEtikette für Personenwagen.»

Eidgenössisches Departement für Umwelt, Verkehr, Energie und Kommunikation UVEK. 2002. «Bundesrat beschliesst energieEtikette für Personenwagen.»

Eidgenössisches Departement für Umwelt, Verkehr, Energie und Kommunikation UVEK, und Bundesamt für Energie. 2010. «Bundesrat will Energieetikette für Personenwagen beibehalten und verbessern.»

Eisinga, Rob, Manfred te Grotenhuis, und Ben Pelzer. 2013. "The reliability of a two-item scale: Pearson, Cronbach, or Spearman-Brown?" International Journal of Public Health 58 (4): 637-642.

Ernst Basler Partner. 2010. Gesetz über die Besteuerung der Strassenfahrzeuge: Gutachten zu den "Ecotax»-und Volksvorschlag-Vorlagen: im Auftrag des Strassenverkehrs-und Schifffahrtsamt SVSA. Zollikon.

Erziehungsdirektion des Kantons Bern. 2009. Bildungsstrategie. Bern.

Erziehungsdirektion des Kantons Bern, und Amt für Kindergarten, Volksschule und Beratung. 2008. Schulsozialarbeit. Leitfaden zur Einführung und Umsetzung. Bern.

Esterling, Kevin M. 2004. The Political Economy of Expertise: Information and Efficiency in American National Politics. Ann Arbor: University of Michigan Press.

Esterling, Kevin M. 2007. "Buying Expertise: Campaign Contributions and Attention to Policy Analysis in Congressional Committees." American Political Science Review 101 (1): 93-109.

Esterling, Kevin M. 2011. “'Deliberative disagreement' in U.S. health policy committee hearings.” Legislative Studies Quarterly 36 (2): 169-198.

ETH. 2008. ECOTAX. Gutachten. Zürich.

EVP Kanton Bern. 2009. Vernehmlassungsantwort zur Revision des kantonalen Energiegesetzes (KEnG). Stellungnahme, 11.03.2009.

EVP Kanton Bern. 2011. Vernehmlassung zur Revision des Volksschulgesetzes VSG (REVOS 2012). Stellungnahme, 24.01.2011. 
Fabian, Carlo, Matthias Drilling, Caroline Müller, Bettina Galliker Schrott, und Sawako EggerSuetsgu. 2008. "Wirksamkeit von Schulsozialarbeit auf der Ebene der Schülerinnen und Schüler.» Soziale Innovation 3: 68-74.

Fabian, Carlo, Matthias Drilling, Caroline Müller, Bettina Galliker Schrott, und Sawako EggerSuetsgu. 2010. "Zur Wirksamkeit von Schulsozialarbeit auf der Ebene der Schülerinnen und Schüler.» In Forschung zur Schulsozialarbeit: Stand und Perspektiven, hrsg. von Speck, Karsten, und Thomas Olk. Weinheim: Juventa-Verlag, 197-208.

Fabian, Carlo, Caroline Müller, Bettina Galliker Schrott, und Matthias Drilling. 2007. Schulsozialarbeit und Früherfassung in der Stadt Thun: Schlussbericht der Evaluation. Basel.

Fankhauser, Silvia, Christian Merkelbach, und Marlyse Merazzi. 2011. Ecole enfantine $-1^{r e}$ et $2^{e}$ années primaires: projet-pilote de cycle élémentaire. Rapport final. Bern.

FDP Kanton Bern. 2009 a. Teilrevision Motorfahrzeugsteuergesetz zielt in die richtige Richtung aber! Pressemitteilung, 30.04.2009.

FDP Kanton Bern. 2009 b. Vernehmlassung Totalrevision kantonales Energiegesetz. Stellungnahme, 16.03.2009.

FDP Kanton Bern. 2011. Änderung Volksschulgesetz - Vernehmlassung. Stellungnahme, 01.02.2011.

FDP Kanton Zürich. 2009. Revision des Verkehrsabgabengesetzes (E-VAG). Stellungnahme.

FDP Kanton Zürich. 2010 a. Grundstufe: mit Wahlfreiheit und sauberer Evaluation schneller am Ziel. Pressemitteilung, 17.03.2010.

FDP Kanton Zürich. 2010 b. Umweltschutz statt Vorschriften. Pressemitteilung, 22.04.2010.

Feh Widmer, Antoinette. 2015. Parlamentarische Mitgliederfluktuation auf subnationaler Ebene in der Schweiz. Baden-Baden: Nomos.

Felber, Peter, und Erziehungsdirektion des Kantons Bern. 2009. Bericht "Jugend und Gewalt», an den Regierungsrat: Bericht des Projektteams an die Steuerungsgruppe vom 20. November 2009. Bern.

Fenno, Richard F. 1973. Congressmen in committees. 4. Aufl. Boston: Little, Brown.

Fischer, Frank, und John Forester. 1993. The Argumentative Turn in Policy Analysis and Planning. Durham, London: Duke University Press.

Florio, David H., Michael M. Behrmann, und Diane L. Goltz. 1979. "What Do Policy Makers Think of Educational Research \& Evaluation? "Or Do They"? Educational Evaluation and Policy Analysis 1 (6): 61-87.

Fornerod, Serge. 2001. «A quoi et à qui servent les évaluations? Une recherche sur la place des évaluations dans les processus de décision et d'apprentissage dans l'administration fédérale.» Mémoire de master (MPA), IDHEAP, Lausanne.

Freiburghaus, Dieter, und Willi Zimmermann. 1985. Wie wird Forschung politisch relevant? Erfahrungen in und mit den schweizerischen nationalen Forschungsprogrammen. Bern: P. Haupt.

Frey, Kathrin. 2010. "Revising Road Safety Policy: The Role of Systematic Evidence in Switzerland." Governance 23 (4): 667-690.

Frey, Kathrin. 2012. Evidenzbasierte Politikformulierung in der Schweiz: Gesetzesrevisionen im Vergleich. Baden-Baden: Nomos.

Frey, Kathrin, Vanessa Di Giorgi, und Thomas Widmer. 2016. «Die Evaluationstätigkeit in der Schweiz: Die SynEval『Datenbank.» LeGes - Gesetzgebung \& Evaluation 27 (3): 505-522. 
Frey, Kathrin, und Simone Ledermann. 2017. «Nutzung von Evaluationen in Regierung und Verwaltung» In Evaluation im politischen System der Schweiz, hrsg. von Sager, Fritz, Thomas Widmer, und Andreas Balthasar. Zürich: NZZ Libro, 211-242.

Frey, Kathrin, und Thomas Widmer. 2011. "Revising Swiss Policies: The Influence of Efficiency Analyses." American Journal of Evaluation 32 (4): 494-517.

Frey, Kathrin, und Thomas Widmer. 2013. «Vom Eigenen oder Fremden lernen bei der evidenzbasierten Politikgestaltung?» In Forschung über Evaluation: Bedingungen, Prozesse und Wirkungen, hrsg. von Hense, Jan, Stefan Rädiker, Wolfgang Böttcher, und Thomas Widmer. Münster: Waxmann, 165-185.

Fullerton, Andrew S. 2009. "A Conceptual Framework for Ordered Logistic Regression Models." Sociological Methods \& Research 38 (2): 306-347.

Furubo, Jan-Eric, Ray C. Rist, und Rolf Sandahl, Hrsg. 2002. International atlas of evaluation. New Brunswick: Transaction Publishers.

Gander, Heiri. 2017. "Committees, parliamentary questions and inquiry committees. A comparative view on oversight tools in Swiss cantons." Konferenzpapier, Conference of the ECPR Standing Group on Parliaments, Basel.

Gava, Roy, Frédéric Varone, André Mach, Steven Eichenberger, Julien Christe, und Corinne Chao-Blanco. 2017. “Interests groups in Parliament: Exploring MPs' interest affiliations (2000-2011).” Swiss Political Science Review 23 (1): 77-94.

George, Alexander L., und Andrew Bennett. 2005. Case Studies And Theory Development In The Social Sciences. Cambridge: MIT Press.

Gerring, John. 2007. Case study research: Principles and practices. New York: Cambridge University Press.

Gilligan, Thomas W., und Keith Krehbiel. 1987. "Collective Decisionmaking and Standing Committees: An Informational Rationale for Restrictive Amendment Procedures.” Journal of Law, Economics, and Organization 3 (2): 287-335.

Ginsburg, Alan, und Nancy Rhett. 2003. "Building a Better Body of Evidence: New Opportunities to Strengthen Evaluation Utilization.” American Journal of Evaluation 24 (4): 489-498.

Godet, Flavia, und Peter de Haan. 2007. Individuelle Entscheidungsstrategien beim Autokauf: Umgang mit widersprüchlichen Antworten zu Paarvergleichen. Forschungsbericht im Rahmen des Projekts "Entscheidungsfaktoren beim Kauf treibstoff-effizienter Neuwagen». Berichte zum Schweizer Autokaufverhalten Nr. 16. Zürich.

Gormley, William T. 1986. "Regulatory Issue Networks in a Federal System.” Polity 18 (4): 595-620.

Grossenbacher, Silvia. 2008. Das Projekt «EDK-Ost 4bis8» im nationalen und internationalen Kontext: Eine erste Bilanz. Aarau.

Grosser Rat des Kantons Bern. 2000a. Tagblatt des Grossen Rates des Kantons Bern. Aprilsession, 4. bis 5. April 2000.

Grosser Rat des Kantons Bern. 2000b. Tagblatt des Grossen Rates des Kantons Bern. Junisession, 6. Juni 2000.

Grosser Rat des Kantons Bern. 2004. Tagblatt des Grossen Rates des Kantons Bern. Aprilsession 2004, 21. April 2004. 
Grosser Rat des Kantons Bern. 2006. Tagblatt des Grossen Rates des Kantons Bern. Novembersession 2006, 23. November 2006.

Grosser Rat des Kantons Bern. 2007. Tagblatt des Grossen Rates des Kantons Bern. Septembersession 2007, 13. September 2007.

Grosser Rat des Kantons Bern. 2009a. Tagblatt des Grossen Rates des Kantons Bern. Novembersession 2009, 16. bis 18. November 2009.

Grosser Rat des Kantons Bern. 2009b. Tagblatt des Grossen Rates des Kantons Bern. Novembersession 2009, 18.-19. November 2009.

Grosser Rat des Kantons Bern. 2009c. Tagblatt des Grossen Rates des Kantons Bern. Novembersession 2009, 26. November 2009.

Grosser Rat des Kantons Bern. 2009d. Tagblatt des Grossen Rates des Kantons Bern. Septembersession 2009, 31. August 2009.

Grosser Rat des Kantons Bern. 2010a. Tagblatt des Grossen Rates des Kantons Bern. Novembersession 2010, 24. November 2010.

Grosser Rat des Kantons Bern. 2010b. Tagblatt des Grossen Rates des Kantons Bern. Novembersession 2010, 25. November 2010.

Grosser Rat des Kantons Bern. 2011a. Kosten und Nutzen von Bildungsreformen hinterfragen: Schulsozialarbeit. Motion. M 004/2011.

Grosser Rat des Kantons Bern. 2011b. Tagblatt des Grossen Rates des Kantons Bern. Märzsession 2011, 30. März 2011.

Grosser Rat des Kantons Bern. 2011c. Tagblatt des Grossen Rates des Kantons Bern. Novembersession 2011, 22.-23. November 2011.

Grosser Rat des Kantons Bern. 2011d. Tagblatt des Grossen Rates des Kantons Bern. Septembersession 2011, 5. September 2011.

Grosser Rat des Kantons Bern. 2012. Tagblatt des Grossen Rates des Kantons Bern. Märzsession 2012, 21. März 2012.

Grüne Kanton Bern. 2008. Stellungnahme zur Änderung des Gesetzes über die Besteuerung der Strassenfahrzeuge (BSFG). Stellungnahme, 02.04.2008.

Grüne Kanton Bern. 2009 a. Grüne loben Eggers Vorlage zum Energiegesetz. Pressemitteilung, 02.07.2009.

Grüne Kanton Bern. 2009 b. Vernehmlassung Energiegesetz des Kantons Bern (KEnG). Stellungnahme, 05.03.2009.

Grüne Kanton Bern. 2011. Vernehmlassung zur Teilrevision des Volksschulgesetzes. Stellungnahme, 20.01.2011.

Grüne Kanton Zürich. 2009. Vernehmlassung zur Revision von Strassengesetz und Verkehrsabgabengesetz: Grüne pochen auf Verursacherprinzip. Stellungnahme, 13.07.2009.

Grüne Kanton Zürich. 2010 a. Die Grünen verlangen Nachbesserungen. Pressemitteilung, 22.04.2010.

Grüne Kanton Zürich. 2010 b. Zur Revision Energiegesetz: Es besteht Nachbesserungsbedarf. Pressemitteilung, 18.03.2010.

Grüne Kanton Zürich. 2011 a. Der 30 jährige Kampf der Grünen gegen die Atomkraft ist am Ziel! Pressemitteilung, 05.07.2011. 
Grüne Kanton Zürich. 2011b. Grüne für Einführung der Grundstufe im ganzen Kanton Zürich. Pressemitteilung, 06.10.2011.

Grüne Partei der Schweiz. 2014. Geschichte der Grünen in der Schweiz. Bern.

Guston, David H., Megan Jones, und Lewis M. Branscomb. 1997. "The Demand for and Supply of Technical Information and Analysis in State Legislatures." Policy Studies Journal 25 (3): 451-469.

Haering, Barbara. 2012. "15 Jahre NPM in Kantonen.» Parlament, Parlement, Parlamento 15 (2): $11-13$.

Hall, Richard L., und Alan V. Deardorff. 2006. “Lobbying as Legislative Subsidy.” American Political Science Review 100 (1): 69-84.

Hammer, Stephan, und Markus Maibach. 1999. Finanzielle Anreize zur Förderung energieeffizienter Personenwagen: Bericht zu Handen der Energiedirektorenkonferenz. Zürich.

Hammer, Stephan, Markus Maibach, und Peter Marti. 2001. Instrumente zur Absenkung des spezifischen Treibstoffuerbrauchs von Personenwagen. Forschungsprogramm Energiewirtschaftliche Grundlagen. Bern.

Hardy, Vincent. 2016. "Evaluation and Utilization of Policy Information in the Canadian Parliament: The Influence of External Policy Actors." Canadian Political Science Review 9 (3): 99-130.

Hartmann Grass, Barbara. 2007. Schulsozialarbeit im Kanton Zürich aktueller Stand und Entwicklungsperspektiven. Referat. Dübendorf.

Head, Brian W. 2008a. "Three Lenses of Evidence-Based Policy." Australian Journal of Public Administration 67 (1): 1-11.

Head, Brian W. 2008b. "Wicked problems in public policy." Public Policy 3 (2): 101-118.

Head, Brian W. 2010. "Reconsidering Evidence-Based Policy: Key Issues and Challenges.” Policy and Society 29 (2): 77-94.

Head, Brian W. 2015. “Toward More “Evidence-Informed” Policy Making?” Public Administration Review 76 (3): 472-484.

Hedeker, Donald, und Robin J. Mermelstein. 1998. "A Multilevel Thresholds of Change Model for Analysis of Stages of Change Data.” Multivariate Behavioral Research 33 (4): 427-455.

Heierli, Claudia. 2000. «Die Stärke der Kommissionen in den 26 kantonalen Parlamenten: Arbeitsoder Redeparlamente?» Lizenziatsarbeit, Institut für Politikwissenschaft, Universität Bern.

Heintz, H. Theodore, und Hank C. Jenkins-Smith. 1988. "Advocacy coalitions and the practice of policy analysis.” Policy Sciences 21 (2): 263-277.

Henry, Gary T. 2000. “Why Not Use?” New Directions for Evaluation 2000 (88): 85-98.

Henry, Gary T., und Melvin M. Mark. 2003. "Beyond Use: Understanding Evaluation's Influence on Attitudes and Actions." American Journal of Evaluation 24 (3): 293-314.

Hense, Jan, Stefan Rädiker, Wolfgang Böttcher, und Thomas Widmer, Hrsg. 2013a. Forschung über Evaluation: Bedingungen, Prozesse und Wirkungen. Münster: Waxmann.

Hense, Jan, Stefan Rädiker, Thomas Widmer, und Wolfgang Böttcher. 2013b. «Notwendigkeit und Potenzial einer Forschung über Evaluation.» In Forschung über Evaluation: Bedingungen, 
Prozesse und Wirkungen, hrsg. von Hense, Jan, Stefan Rädiker, Thomas Widmer, und Wolfgang Böttcher. Münster: Waxmann, 7-17.

Hense, Jan, und Thomas Widmer. 2013. «Ein Überblick zum internationalen Stand der Forschung über Evaluationen.» In Forschung über Evaluation: Bedingungen, Prozesse und Wirkungen, hrsg. von Hense, Jan, Stefan Rädiker, Wolfgang Böttcher, und Thomas Widmer. Münster: Waxmann, 251-278.

Herbert, James Leslie. 2014. "Researching evaluation influence: a review of the literature." Evaluation Review 38 (5): 388-419.

Himmelsbach, Raffael. 2014. "Playing Second Fiddle: Expert Advice and Decision-Making in Switzerland." Dissertation, Institut d'études politiques et internationales, Université de Lausanne, Lausanne.

Hird, John A. 2005a. "Policy analysis for what? The effectiveness of nonpartisan policy research organizations." Policy Studies Journal 33 (1): 83-105.

Hird, John A. 2005b. Power, Knowledge, and Politics: Policy Analysis in the States. Washington, D.C: Georgetown University Press.

Hird, John A. 2009. "The Study and Use of Policy Research in State Legislatures.” International Regional Science Review 32 (4): 523-535.

Hirschi, Christian. 2009. «Ländervergleich Energie und Umwelt.» In Evaluation: Ein systematisches Handbuch, hrsg. von Widmer, Thomas, Wolfgang Beywl, und Carlo Fabian. Wiesbaden: VS Verlag für Sozialwissenschaften, 236-241.

Hofstetter, Carolyn Huie, und Marvin C Alkin. 2003. "Evaluation use revisited.” In International handbook of educational evaluation, hrsg. von Kellaghan, Thomas, und Daniel L. Stufflebeam. Dordrecht: Kluwer Academic Publishers, 197-222.

Högenauer, Anna-Lena, und Christine Neuhold. 2015. "National Parliaments after Lisbon: Administrations on the Rise?” West European Politics 38 (2): 335-354.

Holzer, Thomas, Christian Bolliger, und Wolf Linder. 2003. Metaevaluation ERKOS. Bern.

Horber-Papazian, Katia. 2006. «La place de l'évaluation des politiques publiques en Suisse.» In Contributions à l'action publique, hrsg. von Chappelet, Jean-Loup. Bern: Haupt, 131-144.

Horber-Papazian, Katia. 2011. "The Power of Illusion: Evaluative Information and Political Steering in Valais." In Evaluation: Seeking Truth Or Power?, hrsg. von Eliadis, Pearl, Jan-Eric Furubo, und Steve Jacob. New Brunswick: Transaction Publishers, 131-148.

Horber-Papazian, Katia, und Michael Bützer. 2011. "Dissemination of Evaluation Reports in Newspapers: The case of CEPP evaluations in Geneva, Switzerland." In Open to the Public: Evaluation in the Public Arena, hrsg. von Boyle, Richard, Jonathan D. Breul, und Peter DahlerLarsen. New Brunswick: Transaction Publishers, 43-66.

Hosmer, Jr David W., Stanley Lemeshow, und Rodney X. Sturdivant. 2013. Applied Logistic Regression. Hoboken: John Wiley \& Sons.

Howlett, Michael. 2009. "Governance Modes, Policy Regimes and Operational Plans: A MultiLevel Nested Model of Policy Instrument Choice and Policy Design.” Policy Sciences 42 (1): 73-89.

Ingold, Karin, und Muriel Gschwend. 2014. "Science in Policy-Making: Neutral Experts or Strategic Policy-Makers?” West European Politics 37 (5): 993-1018. 
Innvær, Simon, Gunn Vist, Mari Trommald, und Andrew Oxman. 2002. "Health policy-makers' perceptions of their use of evidence: a systematic review." Journal of health services research \& policy 7 (4): 239-244.

IPSO. 1986. Individuelle Heizkostenabrechnung nach Verbrauch: Zwischenbericht 1. Eine quantitativ-qualitative Evaluations-Studie zu den Heizkostenverteilsystemen der Siedlungen Limmat und Manegg. Zürich.

IPSO. 1989. Individuelle Heizkostenabrechnung nach Verbrauch: Synthesebericht, Studie im Auftrag der Stadt Zürich. Zürich.

Iseli, Daniel, und Roger Pfiffner. 2011a. Zusammenarbeit und Koordination: Offene Kinder- und Jugendarbeit und Schulsozialarbeit. Bern.

Iseli, Daniel, und Roger Pfiffner. 2011b. Zusammenarbeit und Koordination: Offene Kinder- und Jugendarbeit und Schulsozialarbeit. Kurzfassung. Bern.

Iten, Rolf, Stephan Hammer, Stefan Kessler, und Roman Frick. 2000. Evaluation der Berner Energiepolitik der 90er Jahre: Auswirkungen auf Energie und Beschäftigung. Bern.

Jackson-Elmoore, Cynthia. 2014. "Diversity, Information Sources and Sub-National Legislative Processes." Konferenzpapier, Annual Meeting of the Australian Political Studies Association, Sydney.

Jacob, Steve, Sandra Speer, und Jan-Eric Furubo. 2015. "The institutionalization of evaluation matters: Updating the International Atlas of Evaluation 10 years later.” Evaluation 21 (1): 6-31.

James, Thomas E., und Paul D. Jorgensen. 2009. "Policy Knowledge, Policy Formulation, and Change: Revisiting a Foundational Question.” Policy Studies Journal 37 (1): 141-162.

Janett, Daniel. 2001. «Parlamentarische Evaluationen und ihr Einfluss auf den politischen Entscheidungsprozess.» Konferenzpapier, SAGW-Kolloquium, Brienz.

Janett, Daniel. 2003a. «Erfolgskontrolle staatlichen Handelns durch das Parlament.» In Verwaltung im 21. Jahrhundert: Herausforderungen, Probleme, Lösungswege: 20. Kolloquium (2001) der Schweizerischen Akademie der Geistes- und Sozialwissenschaften, hrsg. von Schweizer, Rainer, Claude Jeanrenaud, Stephan Kux, und BEat Sitter-Liver. Fribourg: Paulusverlag, 97-109.

Janett, Daniel. 2003b. «Evaluation wird als Kontrollinstrument des Bundesparlaments immer wichtiger: Eine Replik auf Markus Spinatsch Beitrag im International Atlas of Evaluation.» LeGes - Gesetzgebung \& Evaluation 14 (1): 165-167.

Janett, Daniel. 2004. «Die Evaluationsfunktion des Bundesparlaments - eine Zwischenbilanz.» LeGes - Gesetzgebung \& Evaluation 15 (2): 137-150.

Janett, Daniel. 2009. «Parlamentarische Verwaltungskontrolle durch Evaluation - Ein Erfahrungsbericht aus der Schweiz.» In Parlamentarische Kontrolle und Ausgliederung, hrsg. von Bussjäger, Peter. Wien: Braunmüller, 117-134.

Jenkins-Smith, Hank C. 1988. "Analytical debates and policy learning: analysis and change in the federal bureaucracy." Policy Sciences 21 (2): 169-211.

Jenkins-Smith, Hank C., und Paul A. Sabatier. 1993. "The Dynamics of Policy-Oriented Learning." In Policy Change and Learning: An Advocacy Coalition Approach, hrsg. von Sabatier, Paul A., und Hank C. Jenkins-Smith. Boulder: Westview Press, 41-56.

Johnson, Carole, und Colin Talbot. 2007. "The UK Parliament and Performance: Challenging or Challenged?” International Review of Administrative Sciences 73 (1): 113-131. 
Johnson, Kelli, Lija O. Greenseid, Stacie A. Toal, Jean A. King, Frances Lawrenz, und Boris Volkov. 2009. "Research on Evaluation Use: A Review of the Empirical Literature From 1986 to 2005." American Journal of Evaluation 30 (3): 377-410.

Johnson, R. Burke. 1998. “Toward a theoretical model of evaluation utilization.” Evaluation and Program Planning 21 (1): 93-110.

Kantonaler Gewerbeverband Zürich. 2011. «KGV: Verkehrsabgabengesetz muss zurückgezogen werden.»

Kantonsrat Zürich. 1999. Protokoll des Zürcher Kantonsrates. 7. Sitzung, Montag 5. Juli 1999.

Kantonsrat Zürich. 2001a. Protokoll des Zürcher Kantonsrates. 89. Sitzung, Montag, 5. März 2001.

Kantonsrat Zürich. 2001b. Protokoll des Zürcher Kantonsrates. 103. Sitzung, Montag, 28. Mai 2001.

Kantonsrat Zürich. 2001c. Protokoll des Zürcher Kantonsrates. 107. Sitzung, Montag, 2. Juli 2001.

Kantonsrat Zürich. 2002. Protokoll des Zürcher Kantonsrates. 149. Sitzung, Dienstag 28. Mai.

Kantonsrat Zürich. 2004. Protokoll des Zürcher Kantonsrates. 78. Sitzung, Montag, 6. Dezember 2004.

Kantonsrat Zürich. 2006. Protokoll des Zürcher Kantonsrates. 149. Sitzung, Montag, 27. März 2006.

Kantonsrat Zürich. 2007. Protokoll des Zürcher Kantonsrates. 207. Sitzung, Montag, 2. April 2007.

Kantonsrat Zürich. 2008. Protokoll des Zürcher Kantonsrates. 80. Sitzung, Montag, 24. November 2008.

Kantonsrat Zürich. 2010a. Protokoll des Zürcher Kantonsrates. 175. Sitzung, Montag, 5. Juli 2010.

Kantonsrat Zürich. 2010b. Protokoll des Zürcher Kantonsrates. 195. Sitzung, Montag, 6. Dezember 2010.

Kantonsrat Zürich. 2011a. Protokoll des Zürcher Kantonsrates. 6. Sitzung, Montag, 6. Juni 2011.

Kantonsrat Zürich. 2011b. Protokoll des Zürcher Kantonsrates. 12. Sitzung, Montag, 11. Juli 2011.

Kantonsrat Zürich. 2011c. Protokoll des Zürcher Kantonsrates. 17. Sitzung, Montag, 12. September 2011.

Kantonsrat Zürich. 2011d. Protokoll des Zürcher Kantonsrates. 205. Sitzung, Montag, 7. Februar 2011.

Kantonsrat Zürich. 2011e. Protokoll des Zürcher Kantonsrates. 210. Sitzung, Montag, 14. März 2011.

Kantonsrat Zürich. 2011f. Protokoll des Zürcher Kantonsrates. 211. Sitzung, Montag, 21. März 2011.

Kantonsrat Zürich. 2011 g. Protokoll des Zürcher Kantonsrates. 218. Sitzung, Montag, 18. April 2011.

Kantonsrat Zürich. 2012a. Protokoll des Zürcher Kantonsrates. 50. Sitzung, Montag, 16. April 2012.

Kantonsrat Zürich. 2012b. Protokoll des Zürcher Kantonsrates. 61. Sitzung, Montag, 2. Juli 2012.

Keller, Mario. 2007. Der Energieverbrauch des Verkehrs 1990-2035: Ergebnisse der Szenarien I bis IV und der zugehörigen Sensitivitäten "BIP hoch», "Preise hoch» und "Klima wärmer». Bern.

Keller, Mario, Peter de Haan, René Zbinden, Jürg Grütter, und Walter Ott. 2002. CO2-Reduktion durch Beeinflussung der Treibstoffpreise. Bern.

Kerr, Henry H. 1981. Parlement et société en Suisse. Saint-Saphorin: Georgi.

Kessler, Stefan, und Rolf Iten. 2003. Wirkungen der kantonalen Energievorschriften im Gebäudebereich im Jahr 2002. Bern: Bundesamt für Energie. 
Kessler, Stefan, und Florian Kasser. 2008. Wirkung kantonaler Energiegesetze. Analyse der Auswirkungen gemäss Art. 20 EnG, Aktualisierung für das Jahr 2007. Bern: Bundesamt für Energie.

Kingdon, John W. 1995. Agendas, alternatives, and public policies. 2. Aufl. New York: Harper Collins College Publishers.

Kirkhart, Karen E. 2000. "Reconceptualizing Evaluation Use: An Integrated Theory of Influence." New Directions for Evaluation 2000 (88): 5-23.

Klöti, Ulrich. 1997. «Inhaltliche und methodische Anforderungen an wissenschafliche Politikevaluationen.» In Einführung in die Politikevaluation, hrsg. von Bussmann, Werner, Ulrich Klöti, und Peter Knoepfel. Basel, Frankfurt am Main: Helbling und Lichtenhahn, 39-57.

Klöti, Ulrich, und Gerald Schneider. 1989. Die Informationsbeschaffung des Gesetzgebers: Eine vergleichende Evaluationsstudie zur Bedeutung von Wirkungsanalysen im Schweizer Legiferierungsprozess. Grüsch: Verlag Rüegger.

Knoepfel, Peter, und Werner Bussmann. 1997. «Die öffentliche Politik als Evaluationsobjekt.» In Einführung in die Politikevaluation, hrsg. von Bussmann, Werner, Ulrich Klöti, und Peter Knoepfel. Basel, Frankfurt am Main: Helbling und Lichtenhahn, 58-77.

Knott, Jack, und Aaron Wildavsky. 1980. "If Dissemination Is the Solution, What Is the Problem?" Science Communication 1 (4): 537-578.

Kohler, Ulrich, und Frauke Kreuter. 2012. Datenanalyse mit Stata: Allgemeine Konzepte der Datenanalyse und ihre praktische Anwendung. München: Oldenbourg Wissenschaftsverlag.

Konferenz der Kantonalen Justiz- und Polizeidirektorinnen und -direktoren KKJPD. 2007. «Rabatte für sparsame Fahrzeuge.»

Konferenz Kantonaler Energiedirektoren (EnDK). 2000. Mustervorschriften der Kantone im Energiebereich (MuKEn): Ausgabe 2000: Von der EnDK anlässlich der Generalversammlung vom 24.08.2000 verabschiedet. Bern.

Konferenz Kantonaler Energiedirektoren (EnDK). 2008. Mustervorschriften der Kantone im Energiebereich (MuKEn): Ausgabe 2008: Von der EnDK anlässlich der Plenarsammlung vom 4. April verabschiedet. Bern.

Konferenz Kantonaler Energiedirektoren (EnDK). 2014. Mustervorschriften der Kantone im Energiebereich (MuKEn): Ausgabe 2014: Von der EnDK anlässlich der Plenarsammlung vom 9. Januar 2015 verabschiedet. Bern.

Konferenz Kantonaler Energiedirektoren (EnDK), und EnergieSchweiz. 2010. Stand der Energiepolitik in den Kantonen 2010. Bern.

Krehbiel, Keith. 1991. Information and legislative organization. Ann Arbor: University of Michigan Press.

Krüger, Paul, Alain M. Schoenenberger, Michael Derrer, und Claudio Bologna. 2001. Entschädigung und Infrastruktur der Parlamentsarbeit. Analytisches Profil über den Wert der parlamentarischen Arbeit. Studie im Auftrag der Staatspolitischen Kommission des Nationalrates. Genf: Eco'diagnostic.

Kübler, Daniel. 1999. "Ideas as Catalytic Elements for Policy Change: Advocacy Coalitions and Drug Policy in Switzerland.” In Public Policy and Political Ideas, hrsg. von Braun, Dietmar, und Andreas Busch. Cheltenham: Edward Elgar, 116-135.

Kübler, Daniel. 2001. "Understanding policy change with the advocacy coalition framework: an application to Swiss drug policy." Journal of European Public Policy 8 (4): 623-641. 
Kuckartz, Udo. 2010. Einführung in die computergestützte Analyse qualitativer Daten. 3. Aufl. Wiesbaden: VS Verlag für Sozialwissenschaften.

Kühnel, Steffen M., und Dagmar Krebs. 2010. «Multinomiale und ordinale Regression.» In Handbuch der sozialwissenschaftlichen Datenanalyse, hrsg. von Wolf, Christof, und Henning Best. Wiesbaden: VS Verlag für Sozialwissenschaften, 855-886.

Laakso, Markku, und Rein Taagepera. 1979. “'Effective’ Number of Parties: A Measure with Application to West Europe." Comparative Political Studies 12 (1): 3-27.

Lacy, Michael G. 2006. "An Explained Variation Measure for Ordinal Response Models With Comparisons to Other Ordinal R 2 Measures.” Sociological Methods \& Research 34 (4): 469-520.

Ladner, Andreas, und Raphaël Leuenberger. 2012. «Ergebnisse aus den BADAC-Erhebungen: Leichter Trend zu ständigen Parlamentskommissionen, Vielfalt der Kommissionensysteme existiert jedoch weiter.» Parlament, Parlement, Parlamento 15 (1): 4-5.

Lawrence, Natalia S., Jemma C. Chambers, Sinead M. Morrison, Sven Bestmann, Gerard O'Grady, Christopher D. Chambers, und Andrew P. Kythreotis. 2017. "The Evidence Information Service as a new platform for supporting evidence-based policy: a consultation of UK parliamentarians." Evidence \& Policy: A Journal of Research, Debate and Practice 13 (2): 275-316.

Ledermann, Simone. 2012. "Exploring the Necessary Conditions for Evaluation Use in Program Change." American Journal of Evaluation 33 (2): 159-178.

Ledermann, Simone. 2014. «Evidenz und Expertise im vorparlamentarischen Gesetzgebungsprozess: Die Rolle von Verwaltung und externen Experten.» Swiss Political Science Review 20 (3): 453-485.

Lester, James P. 1993. “The Utilization of Policy Analysis by State Agency Officials.” Science Communication 14 (3): 267-290.

Leviton, Laura C. 2003. "Evaluation Use: Advances, Challenges and Applications." American Journal of Evaluation 24 (4): 525-535.

Leviton, Laura C., und Edward F.X Hughes. 1981. "Research On the Utilization of Evaluations: A Review and Synthesis.” Evaluation Review 5 (4): 525-548.

Lienhard, Andreas, Daniel Schwarz, Fritz Sager, Reto Steiner, und Andreas Müller. 2011. Reform des Parlamentsrechts im Kanton Bern. Studie im Auftrag der Kommission Parlamentsrechtsrevision des Kantons Bern. Bern.

Lincoln, Yvonna S., und Egon G. Guba. 1980. "The Distinction Between Merit and Worth in Evaluation." Educational Evaluation and Policy Analysis 2 (4): 61-71.

Linder, Wolf, Conrad U. Brunner, Sandro Looser, Mila Trombitas, und Daniel Wittwer. 1990. Verbrauchsabhängige Heizkostenabrechnung, Förderung erneuerbarer Energien: Evaluation von Prozess und Wirkungen in drei Kantonen. Bern: Forschungszentrum für schweizerische Politik.

Lindquist, Evert A. 1990. “The Third Community, Policy Inquiry, and Social Scientists.” In Social Scientists, Policy, and the State, hrsg. von Brooks, Stephen, und Alain-G. Gagnon. New York: Praeger, 21-51.

Lomas, Jonathan, und Adalsteinn D. Brown. 2009. "Research and Advice Giving: A Functional View of Evidence-Informed Policy Advice in a Canadian Ministry of Health." Milbank Quarterly 87 (4): 903-926.

Long, J. Scott. 1997. Regression Models for Categorical and Limited Dependent Variables. Thousand Oaks: Sage Publications. 
Long, J. Scott, und Jeremy Freese. 2014. Regression models for categorical dependent variables using Stata. 3. Aufl. College Station: Stata Press.

Lundin, Martin, und PerOla Öberg. 2014. "Expert knowledge use and deliberation in local policy making." Policy Sciences 47 (1): 25-49.

Lüthi, Ruth. 1997. Die Legislativkommissionen der Schweizerischen Bundesversammlung: Institutionelle Veränderungen und das Verhalten von Parlamentsmitgliedern. Bern: P. Haupt.

Lüthi, Ruth. 2005. «Stärkere Parlamente dank ständigen Kommissionen? Referat für die Tagung der Schweizerischen Gesellschaft für Parlamentsfragen, 17.9.2005.» Parlament, Parlement, Parlamento 8 (3): 4-8.

Lüthi, Ruth. 2014. "Parlament.» In Handbuch der Schweizer Politik, hrsg. von Knoepfel, Peter, Yannis Papadopoulos, Pascal Sciarini, Adrian Vatter, und Silja Häusermann. Zürich: NZZ libro, 169-192.

Mabry, Linda. 2010. “The Responsibility of Evaluation.” In Evaluation, Bildung und Gesellschaft. Steuerungsinstrumente zwischen Anspruch und Wirklichkeit, hrsg. von Böttcher, Wolfgang, Jan Nikolas Dicke, und Nina Hogrebe. Münster: Waxmann Verlag, 17-33.

Mader, Luzius. 2005. «Artikel 170 der Bundesverfassung: Was wurde erreicht, was ist noch zu tun?» LeGes - Gesetzgebung \& Evaluation 16 (1): 29-37.

Mader, Luzius. 2009. «Die institutionelle Einbettung der Evaluationsfunktion in der Schweiz.» In Evaluation: Ein systematisches Handbuch, hrsg. von Widmer, Thomas, Wolfgang Beywl, und Carlo Fabian. Wiesbaden: VS Verlag für Sozialwissenschaften, 52-63.

Maestas, Cherie, Grant W. Neeley, und Lilliard E. Richardson. 2003. "The State of Surveying Legislators: Dilemmas and Suggestions." State Politics \& Policy Quarterly 3 (1): 90-108.

Mahoney, James, und Gary Goertz. 2004. "The Possibility Principle: Choosing Negative Cases in Comparative Research.” American Political Science Review 98 (04): 653-669.

Majone, Giandomenico. 1989. Evidence, Argument, and Persuasion in the Policy Process. New Haven: Yale University Press.

Malen, Betty, Michael J. Murphy, und Sue Geary. 1988. "The Role of Evaluation Information in Legislative Decision Making: A Case Study of a Loose Cannon on Deck." Theory Into Practice 27 (2): 111-125.

Manewitsch, Vladimir. 2013. «Statistische Methoden zur Analyse von Daten mit strukturell fehlenden Werten. Mit Anwendungen aus der Marktforschung.» Dissertation, FriedrichAlexander-Universität Erlangen, Nürnberg.

Mark, Melvin M. 2011. "Toward better research on-and thinking about-evaluation influence, especially in multisite evaluations." New Directions for Evaluation 2011 (129): 107-119.

Mark, Melvin M., und Gary T. Henry. 2004. "The Mechanisms and Outcomes of Evaluation Influence." Evaluation 10 (1): 35-57.

Marmot, Michael G. 2004. "Evidence based policy or policy based evidence?” BMJ 328 (7445): 906-907.

Marnoch, Gordon. 2008. The Performance Metrics Boom and Parliamentary Scrutiny and Evaluation: The Public Services Programme. Quality, Performance \& Delivery. ESRC Discussion Paper Series: No. 0810. Swindon.

Martin, Shane. 2014. “Committees.” In The Oxford Handbook of Legislative Studies, hrsg. von Martin, Shane, Thomas Saalfeld, und Kaare Strøm. New York: Oxford University Press, 352-368. 
Mattson, Ingvar, und Kaare Strøm. 1995. “Parliamentary Committees.” In Parliaments and Majority Rule in Western Europe, hrsg. von Döring, Herbert. Frankfurt: Campus, 249-306.

Mayring, Philipp. 2010. Qualitative Inhaltsanalyse: Grundlagen und Techniken. 11. aktual. und überarb. Aufl. Weinheim: Beltz.

McDavid, James C., und Irene Huse. 2012. "Legislator Uses of Public Performance Reports: Findings From a Five-Year Study." American Journal of Evaluation 33 (1): 7-25.

McKinsey \& Company. 2009. Swiss Greenhouse Gas Abatement Cost Curve. Zürich.

Michaels, Sarah, und Mike Gruszczynski. 2016. "Deliberating on Missouri River Water Diversions in Congressional Committee Hearings." Journal of Contemporary Water Research \& Education 158 (1): 132-147.

Milic, Thomas, Michael Hermann, Mario Nowak, und Lorenz Bosshardt. 2016. ParlamentarierInnen-Umfrage zur Nationalen Strategie gegen Krebs. Kurzbericht zur Umfrage. Zürich.

Miquel, Gerard Padro I., und James M. Snyder. 2006. "Legislative Effectiveness and Legislative Careers.” Legislative Studies Quarterly 31 (3): 347-381.

Montpetit, Éric. 2011. "Scientific Credibility, Disagreement, and Error Costs in 17 Biotechnology Policy Subsystems.” Policy Studies Journal 39 (3): 513-533.

Mooney, Christopher Z. 1991a. "Information Sources in State Legislative Decision Making." Legislative Studies Quarterly 16 (3): 445-455.

Mooney, Christopher Z. 1991b. "Peddling Information in the State Legislature: Closeness Counts." The Western Political Quarterly 44 (2): 433-444.

Mooney, Christopher Z. 1992. "Putting It on Paper: The Content of Written Information Used in State Lawmaking." American Politics Research 20 (3): 345-365.

Mooney, Christopher Z. 1993. "Strategic information search in state legislative decision making." Social Science Quarterly 74 (1): 185-198.

Mooney, Christopher Z. 2012. "Legislative Thinking: Responsibility, Motivation, and Cognitive Complexity in U.S. Statehouses." Konferenzpapier, APSA Annual Meeting, New Orleans.

Morshed, Alexandra B., Elizabeth A. Dodson, Rachel G. Tabak, und Ross C. Brownson. 2017. "Comparison of Research Framing Preferences and Information Use of State Legislators and Advocates Involved in Cancer Control, United States, 2012-2013.” Preventing Chronic Disease 14: Online: http://dx.doi.org/10.5888/pcd14.160292.

Moser, Urs, Domenico Angelone, Judith Hollenweger, und Alex Buff. 2011. Nach sechs Jahren Primarschule. Deutsch, Mathematik und motivational-emotionales Befinden am Ende der 6. Klasse. Zürich.

Moser, Urs, Nicole Bayer, und Simone Berweger. 2008. Summative Evaluation Grundstufe und Basisstufe: Zwischenbericht zuhanden der EDK-OST. Zürich.

Moser, Urs, Nicole Bayer, und EDK-Ost. 2010. Schlussbericht der summativen Evaluation: Lernfortschritte vom Eintritt in die Eingangsstufe bis zum Ende der 3. Klasse der Primarstufe. Bern: Schulverlag plus.

Moser, Urs, und Judith Hollenweger. 2008. Lernstandserhebung am Ende der 3. Klasse. Schlussbericht zuhanden der Bildungsdirektion. Zürich.

Moser, Urs, Margrit Stamm, und Judith Hollenweger. 2004. Lernstandserhebung 1. Klassen Kanton Zürich. Schlussbericht zuhanden der Bildungsdirektion. Zürich.

Müller, Stephan. 2004. Schulsozialarbeit im Kanton Zürich: Schlussbericht. Zürich. 
Müller, Stephan. 2005. Schulsozialarbeitsforschung im Kanton Zürich: Aktuelle Probleme und Entwicklungsperspektiven: Überarbeitete und ergänzte Fassung des Artikels in SozialAktuell 13/2005. Zürich.

Müller, Stephan, und Ruth Gurny. 2004. «Schulsozialarbeit im Kanton Zürich: Analyse eines Innovationsprozesses.» vpod bildungspolitik (137): 30-32.

Müller, Stephan, und Ivo Willimann. 2008. Die Schulsozialarbeit aus der Sicht der Schulen: Ergebnisse der Befragungen von Schulleitungen, Lehrpersonen, KindergärtnerInnen und HortleiterInnen: Zusammenfassung. Zürich.

Neuenschwander, Martin. 1995. «Kantonale Volksabstimmung vom 25. Juni. Argumente gegen das Energiegesetz.» Neue Zürcher Zeitung, 17.05.1995.

Neuenschwander, Peter, Daniel Iseli, und Renate Stohler. 2007. Bestandesaufnahme der Schulsozialarbeit im Kanton Bern. Bern.

Newman, Joshua, und Brian Head. 2015. "Beyond the two communities: a reply to Mead's 'why government often ignores research'” Policy Sciences 48 (3): 383-393.

Nideröst, Bruno. 2002. «Erfolgsbedingungen für Evaluationen.» LeGes - Gesetzgebung \& Evaluation 13 (1): 39-55.

Nordwestschweizerische Erziehungsdirektorenkonferenz. 2003. Schulsozialarbeit in den NW EDK-Kantonen. Prospektivbericht der Arbeitsgruppe SSA NW EDK. Aarau.

Nordwestschweizerische Erziehungsdirektorenkonferenz. 2004. Jahresbericht NW EDK 2003. Aarau.

Nuspliger, Kurt. 2005. «Der Stellenwert der Evaluation am Beispiel des Kantons Bern.» LeGes Gesetzgebung \& Evaluation 16 (1): 51-63.

Nutley, Sandra M., Sarah Morton, Tobias Jung, und Annette Boaz. 2010. "Evidence and policy in six European countries: diverse approaches and common challenges." Evidence \& Policy: A Journal of Research, Debate and Practice 6 (2): 131-144.

Nutley, Sandra M., Isabel Walter, und H.T.O Davies. 2007. Using evidence: How research can inform public services. Bristol, UK: The Policy Press.

Nutley, Sandra M., Isabel Walter, und Huw T.O Davies. 2003. "From Knowing to Doing: A Framework for Understanding the Evidence-into-Practice Agenda." Evaluation 9 (2): 125-148.

Nutley, Sandra M., und Jeff Webb. 2000. "Evidence and the policy process." In What Works? Evidence-Based Policy and Practice in Public Services, hrsg. von Davies, Huw T.O, Sandra M. Nutley, und Peter C. Smith. Bristol; UK: The Policy Press, 13-41.

Oh, Cheol H. 1997. "Issues for the new thinking of knowledge utilization: Introductory remarks." Knowledge and Policy 10 (3): 3-10.

Ökozentrum Langenbruck. 1995. Evaluation der verbrauchsabhängigen Heizkostenabrechnung: Vollzugsgrad und Wirkung auf den Verbrauch. Bern.

Ökozentrum Langenbruck. 1996. Evaluation der verbrauchsabhängigen Heizkostenabrechnung: Vollzugsgrad und Wirkung auf den Verbrauch, Aufdatierung des Berichts vom Juni 1995. Bern.

Oliver, Kathryn, Simon Innvar, Theo Lorenc, Jenny Woodman, und James Thomas. 2014. "A systematic review of barriers to and facilitators of the use of evidence by policymakers." BMC Health Services Research 14 (1): 1-12.

Orton, Lois, Ffion Lloyd-Williams, David Taylor-Robinson, Martin O'Flaherty, und Simon Capewell. 2011. "The Use of Research Evidence in Public Health Decision Making Pro- 
cesses: Systematic Review.” PLoS ONE 6 (7): Online open: https://doi.org/10.1371/journal. pone.0021704.

Parlamentarische Verwaltungskontrolle. 2005. Die drei «KMU-Tests» des Bundes: bekannt? genutzt? wirkungsvoll? Bericht zuhanden der Geschäftsprüfungskommission des Nationalrates. Bern.

Parsons, Wayne. 2002. "From Muddling Through to Muddling Up - Evidence Based Policy Making and the Modernisation of British Government." Public Policy and Administration 17 (3): 43-60.

Patashnik, Eric M., und Justin Peck. 2016. “Can Congress Do Policy Analysis?” In Governing in a Polarized Age: Elections, Parties, and Political Representation in America, hrsg. von Gerber, Alan S., und Eric Schickler. Cambridge: Cambridge University Press, 243-266.

Patton, Michael Quinn. 1985. “Six Honest Serving Men for Evaluation.” Konferenzpapier, 69th Annual Meeting of the American Educational Research Association, Chicago.

Patton, Michael Quinn. 1997. Utilization-focused evaluation: The new century text. 3. Aufl. Thousand Oaks: Sage Publications.

Pawson, Ray. 2006. Evidence-Based Policy: A Realist Perspective. London: SAGE Publications.

Pedrini, Seraina. 2014. "Deliberative Capacity in the Political and Civic Sphere." Swiss Political Science Review 20 (2): 263-286.

Pegan, Andreja. 2017. "The role of personal parliamentary assistants in the European Parliament." West European Politics 40 (2): 295-315.

Peters, Anja, Peter de Haan, und Michel Müller. 2006. Wave 2 der Grossbefragung «Mobilität und Autokauf»: Hintergrundbericht im Rahmen des Projekts "Entscheidungsfaktoren beim Kauf treibstoff-effizienter Neuwagen». Berichte zum Schweizer Autokaufverhalten Nr. 9. Zürich.

Peters, Anja, Heinz Gutscher, und Roland W. Scholz. 2011. "Psychological determinants of fuel consumption of purchased new cars." Transportation Research Part F: Traffic Psychology and Behaviour 14 (3): 229-239.

Pfister, Walter E., und Roland Ruffieux. 1975. "Parlament, Regierung und Verwaltung.» In Annuaire suisse de science politique, Schweizerisches Jahrbuch für Politische Wissenschaft. Bern: Haupt, 85-94.

Pilotti, Andrea. 2015. "The Historical Changes and Continuities of Swiss Parliamentary Recruitment.” Swiss Political Science Review 21 (2): 246-253.

Pilotti, Andrea. 2017. Entre démocratisation et professionnalisation: le Parlement suisse et ses membres de 1910 à 2016. Zürich, Genf: Seismo.

Pilotti, Andrea, André Mach, und Oscar Mazzoleni. 2010. «Les parlementaires suisses entre démocratisation et professionnalisation, 1910-2000.» Swiss Political Science Review 16 (2): 211-245.

Pollitt, Christopher. 2006. "Performance Information for Democracy: The Missing Link?” Evaluation 12 (1): 38-55.

Pollitt, Christopher, und Geert Bouckaert. 2004. Public management reform: a comparative analysis. Oxford: Oxford University Press.

Projektkommission 4bis8 der EDK-Ost und Partnerkantone. 2007. Leitideen zum Rahmenkonzept Grundstufe und Basisstufe. Arbeitspapier der Projektkommission 4 bis8 der EDK-Ost und Partnerkantone. St. Gallen. 
Quesel, Carsten. 2012. «Konkordanz, föderale Verflechtung und direktdemokratische Mobilisierung. Eine Fallstudie zur Bildungspolitik in der Schweiz.» In Konkordanzdemokratie: Ein Demokratietyp der Vergangenheit?, hrsg. von Köppl, Stefan, und Uwe Kranenpohl. Baden-Baden: Nomos, 293-316.

Rabe-Hesketh, Sophia, und Anders Skrondal. 2012. Multilevel and longitudinal modeling using Stata: Volume II: Categorical Responses, Counts, and Survival. 3. Aufl. College Station: Stata Press.

Radaelli, Claudio M. 1995. "The role of knowledge in the policy process." Journal of European Public Policy 2 (2): 159-183.

Raudla, Ringa. 2012. "The Use of Performance Information in Budgetary Decision-Making by Legislators: Is Estonia any different?” Public Administration 90 (4): 1000-1015.

Regierungsrat des Kantons Bern. 2008a. Kantonales Energiegesetz (KEnG). Antrag des Regierungsrates für die Vernehmlassung.

Regierungsrat des Kantons Bern. 2008b. Kantonales Energiegesetz (KEnG). Vortrag des Regierungsrates an den Grossen Rat zum kantonalen Energiegesetz.

Regierungsrat des Kantons Bern. 2008c. Vortrag des Regierungsrates an den Grossen Rat betreffend Gesetz über die Besteuerung der Strassenfahrzeuge (BSFG).

Regierungsrat des Kantons Bern. 2009a. Gesetz über die Besteuerung der Strassenfahrzeuge (BSFG). Antrag des Regierungsrates.

Regierungsrat des Kantons Bern. 2009b. Gesetz über die Besteuerung der Strassenfahrzeuge (BSFG). Gemeinsamer Antrag des Regierungsrates und der Kommission.

Regierungsrat des Kantons Bern. 2009c. Gesetz über die Besteuerung der Strassenfahrzeuge (BSFG). Gemeinsamer Antrag des Regierungsrates und der Kommission für die zweite Lesung.

Regierungsrat des Kantons Bern. 2009d. Kantonales Energiegesetz (KEnG). Gemeinsamer Antrag des Regierungsrates und der Kommission vom 21. Oktober 2009.

Regierungsrat des Kantons Bern. 2010a. Grossratsbeschluss betreffend den Volksvorschlag "Steuerliche Entlastung der Strassenfahrzeuge im Kanton Bern». Gemeinsamer Antrag des Regierungsrates und der Kommission.

Regierungsrat des Kantons Bern. 2010b. Volksschulgesetz (Änderung). Vortrag des Regierungsrates an den Grossen Rat zum Volksschulgesetz (VSG).

Regierungsrat des Kantons Bern. 2011a. Volksschulgesetz (VSG) (Änderung). Antrag des Regierungsrates.

Regierungsrat des Kantons Bern. 2011b. Volksschulgesetz (VSG) (Änderung). Gemeinsamer Antrag des Regierungsrates und der Kommission.

Regierungsrat des Kantons Zürich. 2003. Auszug aus dem Protokoll des Regierungsrates des Kantons Zürich. Sitzung vom 10. September 2003. 1337. Volksschule (Schulversuch, Grundstufe).

Regierungsrat des Kantons Zürich. 2006. Energieplanungsbericht. Bericht des Regierungsrates über die Energieplanung des Kantons Zürich. Zürich.

Regierungsrat des Kantons Zürich. 2007a. 4392-4398. Gesetzesänderungen zur Umsetzung der Neugestaltung des Finanzausgleichs und der Aufgabenteilung zwischen Bund und Kantonen (NFA) im Kanton Zürich. Anträge des Regierungsrates vom 11. April 2007, 18. April 2007 und 2. Mai 2007.

Regierungsrat des Kantons Zürich. 2007b. Auszug aus dem Protokoll des Regierungsrates des Kantons Zürich. Sitzung vom 14. November 2007. 1691. Schulversuch Grundstufe (Verlängerung). 
Regierungsrat des Kantons Zürich. 2008a. Auszug aus dem Protokoll des Regierungsrates des Kantons Zürich. Sitzung vom 9. Dezember 2008. 1961. Gesetz über die ambulante Kinder- und Jugendhilfe, Neuerlass (Vernehmlassung).

Regierungsrat des Kantons Zürich. 2008b. Bericht und Antrag des Regierungsrates an den Kantonsrat zur Einzelinitiative KR-Nr. 278/2006 betreffend Einführung der individuellen Heizkostenabrechnung vom 17. September 2008.

Regierungsrat des Kantons Zürich. 2009a. Auszug aus dem Protokoll des Regierungsrates des Kantons Zürich. 549. Finanzierung Strasseninfrastruktur und Verkehrsabgaben; Revision (Vernehmlassung).

Regierungsrat des Kantons Zürich. 2009b. Kinder-und Jugendhilfegesetz (KJHG). Antrag des Regierungsrates vom 16. Dezember 2009.

Regierungsrat des Kantons Zürich. 2009c. prima-Initiative Kantonale Volksinitiative für die Weiterentwicklung der Kindergartenstufe (vom 8. September 2009). ABl 20091884.

Regierungsrat des Kantons Zürich. 2010a. Auszug aus dem Protokoll des Regierungsrates des Kantons Zürich. Sitzung vom 7. Juli 2010. 1039. Schulversuch Grundstufe (Verlängerung).

Regierungsrat des Kantons Zürich. 2010b. Beschluss des Kantonsrates über die Volksinitiative "prima-Initiative (Kantonale Volksinitiative für die Weiterentwicklung der Kindergartenstufe). Antrag des Regierungsrates vom 7. Juli 2010.

Regierungsrat des Kantons Zürich. 2010c. Energiegesetz. Antrag des Regierungsrates vom 3. März 2010.

Regierungsrat des Kantons Zürich. 2010d. Verkehrsabgabengesetz. Antrag des Regierungsrates vom 14. April 2010.

Regierungsrat des Kantons Zürich. 2011a. Auszug aus dem Protokoll des Regierungsrates des Kantons Zürich: Sitzung vom 4. Mai 2011. 580. Änderung Verkehrsabgabengesetz (Regulierungsfolgeabschätzung im Sinne des Entlastungsgesetzes).

Regierungsrat des Kantons Zürich. 2011b. Beschluss des Kantonsrates über die Volksinitiative "prima-Initiative (Kantonale Volksinitiative für die Weiterentwicklung der Kindergartenstufe). Antrag des Regierungsrates vom 28. September 2011.

Regierungsrat des Kantons Zürich. 2013. «Ende des Volksrechts konstruktives Referendum» am 1. Mai 2013.»

Regierungsrat des Kantons Zürich. 2014. Geschäftsbericht und Rechnung 2013. Zürich.

Rich, Robert F. 1997. "Measuring knowledge utilization: Processes and outcomes.” Knowledge and Policy 10 (3): 11-24.

Rieder, Stefan, Christoph Schwenkel, und Beat Züsli. 2008. Konzept, Vollzug und Wirkung der verbrauchsabhängigen Heiz- und Warmwasserkostenabrechnung (VHKA).

Rieder, Stefan, und Thomas Widmer. 2007. Kantone im Wandel: Reformaktivitäten der Schweizer Kantone zwischen 1900 und 1999: Ursachen, Ausgestaltung und Konsequenzen. Bern: Haupt Verlag.

Rietig, Katharina. 2018. "The Links Among Contested Knowledge, Beliefs, and Learning in European Climate Governance: From Consensus to Conflict in Reforming Biofuels Policy.” Policy Studies Journal 46 (1): 137-159.

Riklin, Alois, Heidi Dohar, und Schweizerische Studienkommission der Eidgenössischen Räte «Zukunft des Parlaments». 1977. Parlamentsreform. Bern. 
Riklin, Alois, und Silvano Möckli. 1991. "Milizparlament?» In Das Parlament, "oberste Gewalt des Bundes»?, hrsg. von Bovey Lechner, Madeleine, Martin Graf, und Annemarie Huber-Hotz. Bern: Paul Haupt, 145-163.

Riklin, Alois, und Alois Ochsner. 1984. «Parlament.» In Handbuch Politisches System der Schweiz. Strukturen und Prozesse, hrsg. von Klöti, Ulrich. Bern, Stuttgart: Paul Haupt, 77-115.

Rissi, Christof, und Fritz Sager. 2013. "Types of Knowledge Utilization of Regulatory Impact Assessments: Evidence From Swiss Policymaking." Regulation \& Governance 7 (3): 348-364.

Ritter, Alison, Caitlin Elizabeth Hughes, Kari Lancaster, und Robert Hoppe. 2018. "Using the Advocacy Coalition Framework and Multiple Streams policy theories to examine the role of evidence, research and other types of knowledge in drug policy." Addiction.

Ritz, Adrian, und Fritz Sager. 2010. "Outcome-Based Public Management and the Balance of Powers in the Context of Direct Democracy.” Public Administration 88 (1): 120-135.

Roderick, J. A. Little, und Nathaniel Schenker. 1995. "Missing Data." In Handbook of Statistical Modeling for the Social and Behavioral Sciences, hrsg. von Arminger, Gerhard, Clifford C. Clogg, und Michael E. Sobel. New York: Plenum Press, 39-75.

Saalfeld, Thomas, und Kaare W. Strøm. 2014. "Political Parties and Legislators." In The Oxford Handbook of Legislative Studies, hrsg. von Martin, Shane, Thomas Saalfeld, und Kaare Strøm. New York: Oxford University Press, 371-398.

Sabatier, Paul A. 1988. "An advocacy coalition framework of policy change and the role of policy-oriented learning therein." Policy Sciences 21 (2-3): 129-168.

Sabatier, Paul A. 1993. "Policy Change over a Decade or More." In Policy Change and Learning: An Advocacy Coalition Approach, hrsg. von Sabatier, Paul A., und Hank C. Jenkins-Smith. Boulder: Westview Press, 13-39.

Sabatier, Paul A., und Hank C. Jenkins-Smith. 1993a. "The Advocacy Coalition Framework: Assessment, Revisions, and Implications for Scholars and Practitioners." In Policy Change and Learning: An Advocacy Coalition Approach, hrsg. von Sabatier, Paul A., und Hank C. Jenkins-Smith. Boulder: Westview Press, 211-235.

Sabatier, Paul A., und Hank C. Jenkins-Smith, Hrsg. 1993b. Policy Change and Learning: An Advocacy Coalition Approach. Boulder: Westview Press.

Sabatier, Paul A., und Christopher M. Weible. 2007. "The Advocacy Coalition Framework: Innovations and Clarifications." In Theories of the Policy Process, hrsg. von Sabatier, Paul A. Boulder: Westwing Press, 189-220.

Sabatier, Paul A., und David Whiteman. 1985. "Legislative Decision Making and Substantive Policy Information: Models of Information Flow." Legislative Studies Quarterly 10 (3): 395-421.

Sager, Fritz, Karin Ingold, und Andreas Balthasar. 2017a. Policy-Analyse in der Schweiz: Besonderheiten, Theorien, Beispiele. Zürich: NZZ Libro.

Sager, Fritz, Johanna Künzler, und Philipp Lutz. 2017b. «Das politische System der Schweiz und seine Kontaktpunkte zur Evaluation.» In Evaluation im politischen System der Schweiz, hrsg. von Sager, Fritz, Thomas Widmer, und Andreas Balthasar. Zürich: NZZ Libro, 23-48.

Sager, Fritz, und Christof Rissi. 2011. "The limited scope of policy appraisal in the context of referendum democracy - the case of regulatory impact assessment in Switzerland." Evaluation 17 (2): 151-163. 
Sager, Fritz, und Christian Rüefli. 2005. «Die Evaluation öffentlicher Politiken mit föderalistischen Vollzugsarrangements. Eine konzeptionelle Erweiterung des Stufenmodells und eine praktische Anwendung.» Swiss Political Science Review 11 (2): 101-129.

Sanderson, Ian. 2001. "Performance Management, Evaluation and Learning in 'Modern' Local Government." Public Administration 79 (2): 297-313.

Sanderson, Ian. 2002. "Evaluation, Policy Learning and Evidence-Based Policy Making." Public Administration 80 (1): 1-22.

Schafer, Joseph L, und John W Graham. 2002. "Missing Data: Our View of the State of the Art." Psychological methods 7 (2): 147-177.

Scharpf, Fritz W. 1975. Demokratietheorie zwischen Utopie und Anpassung. Kronberg: Scriptor.

Scharpf, Fritz W. 2000. Regieren in Europa. Effektiv und demokratisch? Frankfurt am Main: Campus.

Scharpf, Fritz W. 2006. Problem Solving Effectiveness and Democratic Accountability in the EU. Reihe Politikwissenschaft 107. Wien.

Schedler, Kuno, und Lukas Schmucki. 2009. "The Political Rationale of Administrative Reforms: Parliamentary Support of Output Control in Switzerland." Swiss Political Science Review 15 (1): $1-30$.

Schlager, Edella. 1995. "Policy making and collective action: Defining coalitions within the advocacy coalition framework." Policy Sciences 28 (3): 243-270.

Schlaufer, Caroline. 2018. "The contribution of evaluations to the discourse quality of newspaper content." Evaluation and Program Planning 69 (3): 157-165.

Schlaufer, Caroline, und Iris Stucki. 2017. «Die Nutzung wissenschaftlicher Evidenz in Abstimmungserläuterungen.» LeGes - Gesetzgebung \& Evaluation 28 (1): 69-80.

Schmidt, Nicolas. 2008. New-Public-Management im Parlament: Auswirkungen auf die Steuerungsfähigkeit in den Kantonen. Zürich, Chur: Rüegger.

Schrefler, Lorna. 2010. "The Usage of Scientific Knowledge by Independent Regulatory Agencies." Governance 23 (2): 309-330.

Schreier, Margrit. 2014. "Ways of Doing Qualitative Content Analysis: Disentangling Terms and Terminologies." 201415 (1): Art. 18.

Schwab, Philippe. 2005. «L'évaluation au service du Parlement?» LeGes - Gesetzgebung \& Evaluation 16 (1): 39-49.

Schweizerische Konferenz der kantonalen Erziehungsdirektoren (EDK). 1997. Bildung und Erziehung der vier-bis achtjährigen Kinder in der Schweiz. Eine Prospektive. Bern.

Schweizerische Konferenz der kantonalen Erziehungsdirektoren (EDK). 1999. Die Ausbildung von Lehrpersonen für die Basisstufe. Prospektivstudie. Stellungnahme. www.edudoc.ch [Stand 07.08.2016].

Schweizerische Konferenz der kantonalen Erziehungsdirektoren (EDK). 2000. Erste Empfehlungen zur Bildung und Erziehung der vier-bis achtjährigen Kinder in der Schweiz.

Schweizerische Konferenz der kantonalen Erziehungsdirektoren (EDK). 2016. HarmoS. http://www.edk.ch/dyn/11659.php [Stand 10.08.2016].

Schweizerische Koordinationsstelle für Bildungsforschung. 2010. Bildungsbericht 2010. Schweiz. Aarau. 
Sciarini, Pascal. 2014. "Processus législatif.» In Handbuch der Schweizer Politik: Manuel de la politique suisse, hrsg. von Knoepfel, Peter, Yannis Papadopoulos, Pascal Sciarini, Adrian Vatter, und Silja Häusermann. Zürich: Verlag Neue Zürcher Zeitung, 525-561.

Sciarini, Pascal, Frédéric Varone, Giovanni Ferro-Luzzi, Fabio Cappelletti, Vahan Garibian, und Ismail Muller. 2017. Étude sur le revenu et les charges des parlementaires fédéraux. Genf.

Scriven, Michael. 1991. Evaluation Thesaurus. Newbury Park: Sage Publications.

Seawright, Jason, und John Gerring. 2008. "Case Selection Techniques in Case Study Research: A Menu of Qualitative and Quantitative Options.” Political Research Quarterly 61 (2): 294-308.

Shaw, Malcolm T. 1979. "Conclusions.” In Committees in Legislatures: A Comparative Analysis, hrsg. von Lees, John David, und Malcolm T. Shaw. Durham: Duke University Press, 361-434.

Shulha, Lyn M., und J. Bradley Cousins. 1997. "Evaluation Use: Theory, Research, and Practice Since 1986." American Journal of Evaluation 18 (3): 195-208.

Shulock, Nancy. 1998. "Legislatures: Rational Systems or Rational Myths?” Journal of Public Administration Research and Theory 8 (3): 299-324.

Shulock, Nancy. 1999. "The paradox of policy analysis: If it is not used, why do we produce so much of it?" Journal of Policy Analysis and Management 18 (2): 226-244.

Smith, Steven S., und Christopher J. Deering. 1990. Committees in Congress. 2. Aufl. Washington: CQ Press.

Solesbury, William. 2001. Evidence Based Policy: Whence it Came and Where it's Going. ESRC UK Centre for Evidence Based Policy and Practice: Working Paper 1. London.

Sommer, Andrea. 2011. «Schlechte Noten für die Basisstufe.» Berner Zeitung, 13.11.2011.

Sommer, Heini. 1989. Volkswirtschaftliche Auswirkungen energiepolitischer Massnahmen des Kantons Bern: Studie 18 im Rahmen des Zweiten Energieberichts. Bern.

Songer, Donald R. 1988. "The Influence of Empirical Research: Committee vs. Floor Decision Making." Legislative Studies Quarterly 13 (3): 375-392.

SP Kanton Bern. 2008. Stellungnahme zur Änderung des Gesetzes über die Besteuerung der Strassenfahrzeuge (BSFG). Stellungnahme, 14.04.2008.

SP Kanton Bern. 2009. Stellungnahme zum Kantonalen Energiegesetz (KEnG). Stellungnahme, 12.03.2009.

SP Kanton Bern. 2011. Vernehmlassung zum Entwurf des teilrevidierten Volksschulgesetzes (VSG). Stellungnahme, 24.01.2011.

SP Kanton Zürich. 2009 a. Gesetz über die ambulante Kinder- und Jugendhilfe (KJHG). Vernehmlassungsantwort. Stellungnahme.

SP Kanton Zürich. 2009 b. Revision des Verkehrsabgabengesetzes (E-VAG). Stellungnahme.

Speck, Karsten, und Thomas Olk. 2010a. «Stand und Perspektiven der Wirkungs- und Nutzerforschung zur Schulsozialarbeit im deutschsprachigen Raum.» In Forschung zur Schulsozialarbeit: Stand und Perspektiven, hrsg. von Speck, Karsten, und Thomas Olk. Weinheim: Juventa-Verlag, 309-325.

Speck, Karsten, und Thomas Olk, Hrsg. 2010b. Forschung zur Schulsozialarbeit: Stand und Perspektiven. Weinheim: Juventa-Verlag. 
Speer, Sandra, Valerie Pattyn, und Bart de Peuter. 2015. "The growing role of evaluation in parliaments: holding governments accountable?” International Review of Administrative Sciences 81 (1): 37-57.

Spinatsch, Markus. 2002. "Evaluation in Switzerland: Moving toward a Decentralized System.” In International atlas of evaluation, hrsg. von Furubo, Jan-Eric, Ray C. Rist, und Rolf Sandahl. New Brunswick: Transaction Publishers, 375-391.

Staatskanzlei des Kantons Bern. 1993. Gesetze, Dekrete und Verordnungen des Kantons Bern.

Staatskanzlei des Kantons Bern. 2011. 13. Februar 2011. Kantonale Volksabstimmung. Botschaft des Grossen Rates des Kantons Bern.

Staatskanzlei des Kantons Bern. 2012. 23. September 2012. Kantonale Volksabstimmung. Botschaft des Grossen Rates des Kantons Bern.

Staatskanzlei des Kantons Bern. 2014. Tätigkeitsbericht des Ratssekretariats für das Jahr 2013. Bern.

Stamm, Margrit. 2002. Evaluation "Pilotversuch Grundstufe»: Zwischenbericht zuhanden der Bildungsdirektion des Kantons Zürich. Aarau.

Stamm, Margrit. 2003. Evaluation «Pilotversuch Grundstufe»: Schlussbericht zuhanden der Bildungsdirektion des Kantons Zürich. Aarau.

Stamm, Margrit. 2009. Frühkindliche Bildung in der Schweiz. Eine Grundlagenstudie im Auftrag der UNESCO-Kommission Schweiz. Fribourg: Universität Fribourg, Departement für Erziehungswissenschaften.

Ständerat, und Kommission für Umwelt, Raumplanung und Energie. 2012. 05.309s Kt.Iv. BE. Differenzierung der Motorfahrzengsteuer auf Bundesebene. Bericht der Kommission für Umwelt, Raumplanung und Energie vom 3. September 2012.

StatistischesAmt des KantonsZürich. 2016.Abstimmungsarchiv. http://www.wahlen-abstimmungen. zh.ch/internet/justiz_inneres/wahlen-abstimmungen/de/abstimmungen/abstimmungsarchiv. html?tag=30.11.2008 [Stand 10.08.2016].

Steffani, Winfried. 1979. Parlamentarische und präsidentielle Demokratie: Strukturelle Aspekte westlicher Demokratien. Opladen: Westdeutscher Verlag.

Stockbauer, Uta. 2000. «Was macht Evaluationen nützlich? Grundlagen und empirische Untersuchungen zum Thema Verwertung und Verwertbarkeit von Evaluationen.» Dissertation, Universität Salzburg, Salzburg.

Stöckli, Georg, und Rita Stebler. 2009. Unterricht und Entwicklung in der Grundstufe: Begleitstudie zum Grundstufenversuch im Kanton Zürich. Zürich.

Strebel, Michael. 2014. Exekutivföderalismus in der Schweiz? Einbezug der Parlamente bei interkantonalen Vereinbarungen. Baden-Baden: Nomos.

Stucki, Iris. 2016. "The use of evidence in public debates in the media: the case of Swiss direct-democratic campaigns in the health policy sector." Evidence \& Policy: A Journal of Research, Debate and Practice 12 (4): 487-504.

Stucki, Iris. 2018. "Evidence-based arguments in direct democracy: The case of smoking bans in Switzerland." Evaluation and Program Planning 69 (3): 148-156.

Summermatter, Lukas, und Labinot Demaj. 2012. «Die Nutzung integrierter Aufgaben- und Finanzpläne (IAFP): Wie Schweizer Kantonsparlamentarier den IAFP im Budgetprozess verwenden.» Verwaltung \& Management 18 (4): 171-179. 
SVP Kanton Bern. 2008. Vernehmlassung zur Änderung des Gesetzes über die Besteuerung der Strassenfahrzeuge: Weitere Anstrengungen bei den Fahrzeugsteuern dringend nötig. Stellungnahme, 15.04.2008.

SVP Kanton Bern. 2009 a. Besteuerung der Motorfahrzeuge: Keine Verzögerungstaktik bitte! Pressemitteilung, 30.04.2009.

SVP Kanton Bern. 2009 b. Energiegesetz: Die Regierung ignoriert einmal mehr berechtigte Kritik. Pressemitteilung, 02.07.2009.

SVP Kanton Bern. 2009 c. Vernehmlassung zur Totalrevision des kantonalen Energiegesetzes (KEnG). Stellungnahme, 13.03.2009.

SVP Kanton Bern. 2010. Zu den Basisstufen-Versuchen: Höhere Kosten ohne Zusatznutzen. Pressemitteilung, 28.06.2010.

SVP Kanton Bern. 2011. Vernehmlassungsantwort der SVP Kanton Bern zur Änderung des Volksschulgesetzes. Stellungnahme, 27.01.2011.

SVP Kanton Zürich. 2009. Gegen neue Abgaben und Gebühren im Strassenverkehr. Stellungnahme, 14.07.2009.

SVP Kanton Zürich. 2010. Gegen die Verschlechterung bestehender Gesetze. Pressemitteilung, 22.04.2010.

SVP Kanton Zürich. 2011. SVP lehnt neue, gewerbefeindliche Umweltabgabe ab. Pressemitteilung, 17.03.2011.

Tabuga, Aubrey D. 2017. "Knowledge utilization in policymaking: evidence from congressional debates in the Philippines." Journal of Asian Public Policy 10 (3): 302-317.

Trachsler, Ernst. 2009. Beratung im Schulversuch Grundstufe: Analyse betreffend Beratungstätigkeit, Qualitätsentwicklung und Know-how-Sicherung im Hinblick auf die Verlängerungsphase. Zürich.

Trees, Patrick, Christina Bundi Caldelari, und Céline Baumgartner. 2013. «Kanton Bern - Neues Parlamentsrecht.» Parlament, Parlement, Parlamento 16 (2): 45-57.

Trippolini, Ivar. 2007. «Strukturen, Rechte und Ressourcen der Kommissionen in den kantonalen Parlamenten: Situationsbeschrieb und Diskussionsbeitrag.» Parlament, Parlement, Parlamento 10 (1): 26-31.

Tschannen, Pierre, und Daniela Wyss. 2008. Delegationsfragen im Bereich der schadstoffbezogenen Motorfahrzeugsteuer. Bern.

Valovirta, Ville. 2002. "Evaluation Utilization as Argumentation.” Evaluation 8 (1): 60-80.

van Dooren, Wouter. 2004. "Supply and Demand of Policy Indicators." Public Management Review 6 (4): 511-530.

Van Dooren, Wouter. 2008. "Nothing New Under the Sun? Change and Continuity in the Twentieth-Century Performance Movements." In Performance Information in the Public Sector: How It Is Used, hrsg. von Dooren, Wouter van, und Steven Van de Walle. Basingstoke, New York: Palgrave Macmillan, 11-23.

Varone, Frédéric, Pirmin Bundi, und Roy Gava. 2018. "Policy evaluation in parliament: Interest groups as catalysts." International Review of Administrative Sciences. Online: https:// doi.org/10.1177/0020852317750461.

Varone, Frédéric, und Steve Jacob. 2004. «Institutionnalisation de l'évaluation et nouvelle gestion publique: Un état des lieux comparatif.» Revue internationale de politique comparée 11 (2): 271-292. 
Vatter, Adrian. 2002. Kantonale Demokratien im Vergleich: Entstehungsgründe, Interaktionen und Wirkungen politischer Institutionen in den Schweizer Kantonen. Opladen: Leske + Budrich.

Vatter, Adrian. 2014. Das politische System der Schweiz. Baden-Baden: Nomos.

Veall, Michael R, und Klaus F Zimmermann. 1992. "Pseudo囚R 2's in the ordinal probit model." Journal of mathematical sociology 16 (4): 333-342.

Vedung, Evert. 1997. Public policy and program evaluation. New Bruswick: Transaction Publishers.

Vedung, Evert. 2010. "Four Waves of Evaluation Diffusion.” Evaluation 16 (3): 263-277.

Vögeli-Mantovani, Urs, und Silvia Grossenbacher. 2005. Die Schulsozialarbeit kommt an! Aarau: Schweizerische Koordinationsstelle für Bildungsforschung.

Vogt, Franziska, Bea Zumwald, Christa Urech, Nadja Abt, Sonja Bischoff, Grazia Buccheri, und Mirjam Lehner. 2008. Formative Evaluation Grund-und Basisstufe: Zwischenbericht. St. Gallen.

Vogt, Franziska, Bea Zumwald, Christa Urech, Nadja Abt, und EDK-Ost. 2010. Schlussbericht der formativen Evaluation. Grund-/Basisstufe: Umsetzung, Unterrichtsenwicklung und Akzeptanz bei Eltern und Lehrpersonen. Bern: Schulverlag plus.

Volkswirtschaftsdirektion des Kantons Zürich. 2009. Revision Strassenfinanzierung (Strassengesetz und Verkehrsabgaben). Erläuterungen für die Vernehmlassung.

von Matt, Hans-Kaspar. 2002. Projekt Integrierte Schulsozialarbeit im Schulkreis Brunnmatt: Schlussbericht. Bern.

Wagner-Willi, Monika, und Patrik Widmer-Wolf. 2009. Kinder mit besonderem Förderbedarf in der Grundstufe: Schlussbericht zur Fallstudie INTEGRU zuhanden der Bildungsdirektion des Kantons Zürich. Zürich.

Webber, David J. 1986. "Explaining policymakers' use of policy information: The relative importance of the two-community theory versus decision-maker orientation." Science Communication 7 (3): 249-290.

Webber, David J. 1987. "Legislators' Use of Policy Information." American Behavioral Scientist 30 (6): 612-631.

Webber, David J. 1991. "The Distribution and Use of Policy Knowledge in the Policy Process." Knowledge and Policy 4 (4): 6-35.

Weible, Christopher M. 2008. "Expert-Based Information and Policy Subsystems: A Review and Synthesis.” Policy Studies Journal 36 (4): 615-635.

Weible, Christopher M., Andrew Pattison, und Paul A. Sabatier. 2010. "Harnessing expert-based information for learning and the sustainable management of complex socio-ecological systems." Environmental Science \& Policy 13 (6): 522-534.

Weible, Christopher M., Paul A. Sabatier, und Kelly McQueen. 2009. "Themes and Variations: Taking Stock of the Advocacy Coalition Framework.” Policy Studies Journal 37 (1): 121-140.

Weingast, Barry R., und William J. Marshall. 1988. "The Industrial Organization of Congress; or, Why Legislatures, Like Firms, Are Not Organized as Markets." Journal of Political Economy 96 (1): 132-163.

Weiss, Carol H. 1970. “The Politicization of Evaluation Research.” Journal of Social Issues 26 (4): 57-68.

Weiss, Carol H. 1977. "Research for Policy's Sake: The Enlightenment Function of Social Research.” Policy Analysis 3 (4): 531-545. 
Weiss, Carol H. 1979. “The Many Meanings of Research Utilization.” Public Administration Review 39 (5): 426-431.

Weiss, Carol H. 1980. "Knowledge Creep and Decision Accretion.” Science Communication 1 (3): 381-404.

Weiss, Carol H. 1981. "Measuring the Use of Evaluation." In Utilizing evaluation: concepts and measurement techniques, hrsg. von Ciarlo, James A. Beverly Hills: Sage Publications, 17-33.

Weiss, Carol H. 1987. "Congressional Committee Staffs (Do, Do Not) Use Analysis.” In Social Science Research and Government: Comparative Essays on Britain and the United States, hrsg. von Bulmer, Martin. Cambridge: Cambridge University Press, 94-112.

Weiss, Carol H. 1989. "Congressional Committees as Users of Analysis." Journal of Policy Analysis and Management 8 (3): 411-431.

Weiss, Carol H. 1990. "Evaluation for Decisions: Is Anybody There? Does Anybody Care?" In Debates on evaluation, hrsg. von Alkin, Marvin C., Michael Quinn Patton, und Carol H. Weiss. Newbury Park: Sage Publications, 171-184.

Weiss, Carol H. 1995. "The Four 'I's' of School Reform: How Interests, Ideology, Information and Institution Affect Teachers and Principals." Harvard Educational Review 65 (4): 571-593.

Weiss, Carol H. 1998. "Have We Learned Anything New About the Use of Evaluation?” American Journal of Evaluation 19 (1): 21-33.

Weiss, Carol H. 1999. "The Interface between Evaluation and Public Policy." Evaluation 5 (4): 468-486.

Weiss, Carol H., und Michael J. Bucuvalas. 1980. "Truth Tests and Utility Tests: DecisionMakers' Frames of Reference for Social Science Research." American Sociological Review 45 (2): 302-313.

White, John Kenneth. 2006. "What is a Political Party?” In Handbook of Party Politics, hrsg. von Katz, Richard S., und William Crotty. London: SAGE Publications Ltd, 6-15.

Whiteman, David. 1985a. "The Fate of Policy Analysis in Congressional Decision Making: Three Types of Use in Committees." Political Research Quarterly 38 (2): 294-311.

Whiteman, David. 1985b. "Reaffirming the Importance of Strategic Use: A Two-Dimensional Perspective on Policy Analysis in Congress." Science Communication 6 (3): 203-224.

Whiteman, David. 1995. Communication in Congress: members, staff, and the search for information. Lawrence: University Press of Kansas.

Whiteman, David. 1997. "Congress and Policy Analysis." Technological Forecasting and Social Change 54 (2-3): 177-189.

Widmer, Thomas. 1996. Fallstudien zur Meta-Evaluation Teil VI: Heizkostenabrechnung und erneuerbare Energien. digitalisierte Aufl. Zürich.

Widmer, Thomas. 2002. «Staatsreformen und Evaluation: Konzeptionelle Grundlagen und Praxis bei den Schweizer Kantonen.» Zeitschrift für Evaluation 1 (1): 101-114.

Widmer, Thomas. 2007. «Rahmenbedingungen und Praxis der Evaluation im schweizerischen Bundesstaat.» ÖHW: Das öffentliche Haushaltswesen in Österreich 48 (1-2): 69-93.

Widmer, Thomas. 2008. «Evaluationsansätze und ihre Effekte: Erfahrungen aus verschiedenen Politikfeldern.» In Wissenschaft unter Beobachtung: Effekte und Defekte von Evaluationen, hrsg. von Matthies, Hildegard, und Dagmar Simon. Wiesbaden: VS Verlag für Sozialwissenschaften, 267-287. 
Widmer, Thomas. 2009. "The contribution of evidence-based policy to the output-oriented legitimacy of the state." Evidence \& Policy: A Journal of Research, Debate and Practice 5 (4): 351-372.

Widmer, Thomas. 2017. «Geschichte der Evaluation im schweizerischen politischen System.» In Evaluation im politischen System der Schweiz, hrsg. von Sager, Fritz, Thomas Widmer, und Andreas Balthasar. Zürich: NZZ Libro, 51-66.

Widmer, Thomas, und Thomas De Rocchi. 2012. Kompaktwissen Evaluation: Grundlagen, Ansätze und Anwendungen. Zürich, Chur: Rüegger.

Widmer, Thomas, Daniela Eberli, Günter Ackermann, Lars Balzer, Pirmin Bundi, Christian Hirschi, Tanya Kasper, Peter Neuenschwander, Björn Neuhaus, Walter Rohrbach, Caroline Schlaufer, und Felix Strebel. 2016. «Forschung über Evaluation in der Schweiz: Stand und Aussichten.» LeGes - Gesetzgebung \& Evaluation 27 (3): 459-483.

Widmer, Thomas, Charles Landert, und Nicole Bachmann. 2000. Evaluations-Standards der schweizerischen Evaluationsgesellschaft (Seval-Standards). Genf; Bern: SEVAL.

Widmer, Thomas, und Peter Neuenschwander. 2004. "Embedding Evaluation in the Swiss Federal Administration: Purpose, Institutional Design and Utilization." Evaluation 10 (4): 388-409.

Widmer, Thomas, und Erwin Rüegg. 2005. «Konsequenzen von Staatsreformen für die demokratische Steuerungsfähigkeit: Vergleichende Analyse zu vier Schweizer Kantonen.» Politische Vierteljahresschrift 46 (1): 86-109.

Widmer, Thomas, Erwin Rüegg, und Peter Neuenschwander. 2001. Stand und Aussichten der Evaluation beim Bund (EvalBund). Schlussbericht zuhanden der Schweizerischen Bundeskanzlei. Zürich.

Wiesli, Reto, und Wolf Linder. 2000. Repräsentation, Artikulation und Durchsetzung kantonaler Interessen im Ständerat und Nationalrat: Studie im Auftrag der Parlamentsdienste der Schweizerischen Bundesversammlung. Bern.

Williams, Richard. 2006. "Generalized ordered logit/partial proportional odds models for ordinal dependent variables." The Stata Journal 6 (1): 58-82.

Williams, Richard. 2016. "Understanding and interpreting generalized ordered logit models." The Journal of Mathematical Sociology 40 (1): 7-20.

Winkelmann, Anja, und Peter Neuenschwander. 2010. Evaluation Projekt Schulsozialarbeit Kirchberg. Bern.

Winzen, Thomas. 2011. "Technical or Political? An Exploration of the Work of Officials in the Committees of the European Parliament." The Journal of Legislative Studies 17 (1): 27-44.

Wirths, Damien. 2017. "Procedural Institutionalization of the Evaluation Through Legal Basis: A New Typology of Evaluation Clauses in Switzerland.” Statute Law Review 38 (1): 23-39.

Wirths, Damien, und Katia Horber-Papazian. 2016. "Les clauses d'évaluation dans le droit des cantons suisses: leur diffusion, leur contenu et la justification à l'origine de leur adoption.» LeGes - Gesetzgebung \& Evaluation 27 (3): 485-502.

WK Kellogg Foundation. 2004. Logic model development guide. Michigan: WK Kellogg Foundation.

Wollmann, Hellmut. 2003. "Evaluation in public-sector reform: Towards a 'third wave' of evaluation?" In Evaluation in Public-sector Reform: Concepts and Practice in International Perspective, hrsg. von Wollmann, Hellmut. Cheltenham: Edward Elgar Publishing Limited, 1-11. 
Wurz, Jeannie. 2014. Swiss politicians: just don't call me a professional. https://www.swissinfo.ch/ eng/the-militia-system-_swiss-politicians--just-don-t-call-me-a-professional/41065618 [Stand 15.01.2016].

Yarbrough, Donald B. 2011. The Program Evaluation Standards: A Guide for Evaluators and Evaluation Users. 3. Aufl. Thousand Oaks; Calif: SAGE.

Yin, Robert K. 2014. Case study research: Design and methods. 5. Aufl. Thousand Oaks: Sage.

Yläoutinen, Sami, und Mark Hallerberg. 2009. "The Role of Parliamentary Committees in the Budgetary Process in the Central and Eastern European Countries." In Parlamente, Agendasetzung und Vetospieler: Festschrift für Herbert Döring, hrsg. von Ganghof, Steffen, Christoph Hönnige, und Christian Stecker. Wiesbaden: VS Verlag für Sozialwissenschaften, 147-173.

Z'graggen, Heidi. 2009. Die Professionalisierung von Parlamenten im historischen und internationalen Vergleich. Bern: Haupt.

Z'graggen, Heidi, und Wolf Linder. 2004. Professionalisierung der Parlamente im internationalen Vergleich: Studie im Auftrag der Parlamentsdienste der Schweizerischen Bundesversammlung. Institut für Politikwissenschaft, Universität Bern. Bern.

Zimmermann, Willi. 1990. «Un inventaire des évaluations au niveau fédéral.» In Évaluation des politiques publiques en Suisse. Pourquoi? Pour qui? Comment?, hrsg. von Horber-Papazian, Katia. Lausanne: Presses polytechniques et universitaires romandes, 79-88.

Zwaan, Pieter, Stijn van Voorst, und Ellen Mastenbroek. 2016. "Ex post legislative evaluation in the European Union: questioning the usage of evaluations as instruments for accountability." International Review of Administrative Sciences 82 (4): 674-693.

Zwier, Robert. 1979. "The Search for Information: Specialists and Nonspecialists in the U.S. House of Representatives." Legislative Studies Quarterly 4 (1): 31-42. 
Evaluationen beurteilen die Wirksamkeit einer Politik anhand wissenschaftlicher Methoden und liefern damit Grundlagen für wirkungsorientierte, fundierte Entscheide im Parlament. Obwohl fest etabliert in der Schweiz, ist die Rolle der Evaluation in den Parlamenten kaum erforscht. Gestützt auf eine schweizweite Befragung von Parlamentsmitgliedern und Studien kantonaler Gesetzgebungsprozesse befasst sich Daniela Eberli mit der Evaluationsnutzung und ihren Einflussfaktoren und trägt damit zur Frage bei, welche Bedeutung Wissen in den heutigen, modernen Parlamenten zukommt.

Daniela Eberli arbeitet als wissenschaftliche Mitarbeiterin am Institut für Politikwissenschaft der Universität Zürich. Neben ihren Forschungstätigkeiten zur evidenzbasierten Politikgestaltung und zu den Schweizer Parlamenten befasst sie sich seit mehreren Jahren im Rahmen von Auftragsprojekten mit der Evaluation von Konzepten oder Massnahmen, besonders in der Gesundheits- und Bildungspolitik.

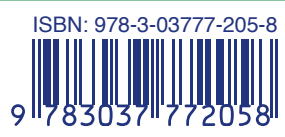

